OPEN ACCESS

\section{The ALICE experiment at the CERN LHC}

To cite this article: The ALICE Collaboration et al 2008 JINST 3 S08002

View the article online for updates and enhancements.

\section{Related content}

Performance of ALICE detector and electronics under first beam conditions G Aglieri Rinella and ALICE collaboration

- ALICE results from the first $\mathrm{Pb}--\mathrm{Pb}$ run at the CERN LHC

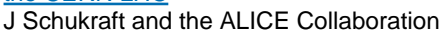

Performance of the ALICE VZERO system The ALICE collaboration

\section{Recent citations}

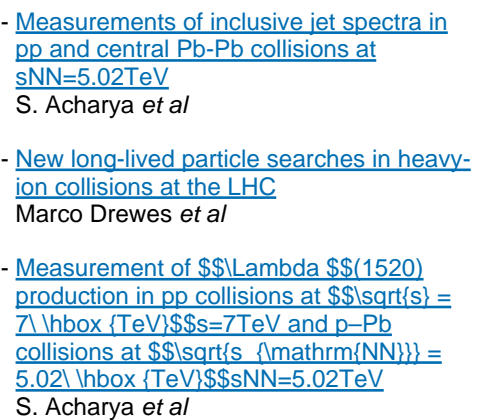




\section{The ALICE experiment at the CERN LHC}

\section{ALICE Collaboration}

ABSTRACT: ALICE (A Large Ion Collider Experiment) is a general-purpose, heavy-ion detector at the CERN LHC which focuses on QCD, the strong-interaction sector of the Standard Model. It is designed to address the physics of strongly interacting matter and the quark-gluon plasma at extreme values of energy density and temperature in nucleus-nucleus collisions. Besides running with $\mathrm{Pb}$ ions, the physics programme includes collisions with lighter ions, lower energy running and dedicated proton-nucleus runs. ALICE will also take data with proton beams at the top LHC energy to collect reference data for the heavy-ion programme and to address several QCD topics for which ALICE is complementary to the other LHC detectors. The ALICE detector has been built by a collaboration including currently over 1000 physicists and engineers from 105 Institutes in 30 countries. Its overall dimensions are $16 \times 16 \times 26 \mathrm{~m}^{3}$ with a total weight of approximately $10000 \mathrm{t}$. The experiment consists of 18 different detector systems each with its own specific technology choice and design constraints, driven both by the physics requirements and the experimental conditions expected at LHC. The most stringent design constraint is to cope with the extreme particle multiplicity anticipated in central $\mathrm{Pb}-\mathrm{Pb}$ collisions. The different subsystems were optimized to provide high-momentum resolution as well as excellent Particle Identification (PID) over a broad range in momentum, up to the highest multiplicities predicted for LHC. This will allow for comprehensive studies of hadrons, electrons, muons, and photons produced in the collision of heavy nuclei. Most detector systems are scheduled to be installed and ready for data taking by mid-2008 when the LHC is scheduled to start operation, with the exception of parts of the Photon Spectrometer (PHOS), Transition Radiation Detector (TRD) and Electro Magnetic Calorimeter (EMCal). These detectors will be completed for the high-luminosity ion run expected in 2010.

This paper describes in detail the detector components as installed for the first data taking in the summer of 2008 .

KEYWORDS: Instrumentation for heavy-ion accelerators; Instrumentation for particle accelerators and storage rings - high energy; Cherenkov and transition radiation; Gaseous detectors; Liquid detectors; Photon detectors for UV, visible and IR photons; Scintillators, scintillation and light emission processes; Solid state detectors; Calorimeters; Cherenkov detectors; $\mathrm{dE} / \mathrm{dx}$ detectors; Gamma detectors; Large detector systems for particle and astroparticle physics; Particle 
identification methods; Particle tracking detectors; Photon detectors for UV, visible and IR photons; Spectrometers; Time projection chambers; Timing detectors; Transition radiation detectors; Analysis and statistical methods; Computing; Data processing methods; Data reduction methods; Pattern recognition, cluster finding, calibration and fitting methods; Simulation methods and programs; Software architectures; Detector alignment and calibration methods; Detector cooling and thermo-stabilization; Detector design and construction technologies and materials; Detector grounding; Manufacturing; Overall mechanics design; Special cables; Voltage distributions. 


\section{The ALICE Collaboration}

K. Aamodt, ${ }^{1}$ A. Abrahantes Quintana, ${ }^{2}$ R. Achenbach, ${ }^{3}$ S. Acounis, ${ }^{4}$ D. Adamová, ${ }^{5}$ C. Adler, ${ }^{6}$ M. Aggarwal, ${ }^{7}$ F. Agnese,${ }^{8}$ G. Aglieri Rinella, ${ }^{9}$ Z. Ahammed, ${ }^{10}$ A. Ahmad, ${ }^{11}$ N. Ahmad, ${ }^{11}$ S. Ahmad, ${ }^{11}$ A. Akindinov, ${ }^{12}$ P. Akishin, ${ }^{13}$ D. Aleksandrov, ${ }^{14}$ B. Alessandro, ${ }^{15}$ R. Alfaro, ${ }^{16}$ G. Alfarone, ${ }^{15}$ A. Alici,,${ }^{17}$ J. Alme, ${ }^{18}$ T. Alt, ${ }^{3}$ S. Altinpinar,,${ }^{19}$ W. Amend,,${ }^{20}$ C. Andrei, ${ }^{21}$ Y. Andres, ${ }^{9}$ A. Andronic, ${ }^{19}$ G. Anelli, ${ }^{9}$ M. Anfreville, ${ }^{22}$ V. Angelov, ${ }^{3}$ A. Anzo, ${ }^{16}$ C. Anson, ${ }^{23}$ T. Anticić, ${ }^{24}$ V. Antonenko, ${ }^{14}$ D. Antonczyk,${ }^{19}$ F. Antinori, ${ }^{25}$ S. Antinori, ${ }^{17}$ P. Antonioli, ${ }^{26}$ L. Aphecetche, ${ }^{4}$ H. Appelshäuser, ${ }^{20}$ V. Aprodu, ${ }^{21}$ M. Arba, ${ }^{27}$ S. Arcelli, ${ }^{17}$ A. Argentieri, ${ }^{28}$ N. Armesto, ${ }^{29}$ R. Arnaldi, ${ }^{15}$ A. Arefiev, ${ }^{13}$ I. Arsene, ${ }^{1}$ A. Asryan, ${ }^{30}$ A. Augustinus, ${ }^{9}$ T.C. Awes, ${ }^{31}$ J. Äysto,${ }^{32}$ M. Danish Azmi, ${ }^{11}$ S. Bablock, ${ }^{18}$ A. Badalà, ${ }^{33}$ S.K. Badyal, ${ }^{34}$ J. Baechler, ${ }^{9}$ S. Bagnasco,${ }^{15}$ R. Bailhache, ${ }^{19}$ R. Bala,${ }^{34}$ A. Baldisseri, ${ }^{22}$ A. Baldit, ${ }^{35}$ J. Bán, ${ }^{36}$ R. Barbera, ${ }^{37}$ P.-L. Barberis, ${ }^{9}$ J.M. Barbet, ${ }^{4}$ G. Barnäfoldi, ${ }^{38}$ V. Barret,${ }^{35}$ J. Bartke, ${ }^{39}$ D. Bartos, ${ }^{21}$ M. Basile, ${ }^{17}$ V. Basmanov, ${ }^{40}$ N. Bastid, ${ }^{35}$ G. Batigne, ${ }^{4}$ B. Batyunya, ${ }^{13}$ J. Baudot, ${ }^{8}$ C. Baumann, ${ }^{41}$ I. Bearden, ${ }^{42}$ B. Becker,${ }^{27}$ J. Belikov, ${ }^{9}$

R. Bellwied, ${ }^{43}$ E. Belmont-Moreno, ${ }^{16}$ A Belogianni, ${ }^{44}$ S. Belyaev, ${ }^{14}$ A. Benato, ${ }^{25}$ J.L. Beney, ${ }^{4}$ L. Benhabib, ${ }^{4}$ F. Benotto, ${ }^{15}$ S. Beolé, ${ }^{45}$ I. Berceanu, ${ }^{21}$ A. Bercuci, ${ }^{19}$ E. Berdermann, ${ }^{19}$ Y. Berdnikov, ${ }^{46}$ C. Bernard, ${ }^{47}$ R. Berny, ${ }^{4}$ J.D. Berst, ${ }^{8}$ H. Bertelsen, ${ }^{42}$ L. Betev,${ }^{9}$ A. Bhasin,${ }^{48}$ P. Baskar, ${ }^{10}$ A. Bhati, ${ }^{7}$ N. Bianchi, ${ }^{49}$ J. Bielčik,,${ }^{50}$ J. Bielčiková, ${ }^{50}$ L. Bimbot, ${ }^{51}$ G. Blanchard,${ }^{35}$ F. Blanco, ${ }^{37}$ F. Blanco, ${ }^{52}$ D. Blau, ${ }^{14}$ C. Blume, ${ }^{20}$ S. Blyth, ${ }^{53}$ M. Boccioli, ${ }^{9}$ A. Bogdanov, ${ }^{54}$ H. Bøggild, ${ }^{42}$ M. Bogolyubsky,,${ }^{55}$ L. Boldizsár, ${ }^{38}$ M. Bombara, ${ }^{48}$ C. Bombonati, ${ }^{25}$ M. Bondila, ${ }^{32}$ D. Bonnet, ${ }^{8}$ V. Bonvicini, ${ }^{56}$ H. Borel, ${ }^{22}$ F. Borotto, ${ }^{15}$ V. Borshchov, ${ }^{57}$ Y. Bortoli, ${ }^{4}$ O. Borysov, ${ }^{56}$ S. Bose,${ }^{58}$ L. Bosisio, ${ }^{56}$ M. Botje,${ }^{59}$ S. Böttger, ${ }^{3}$ G. Bourdaud, ${ }^{4}$ O. Bourrion, ${ }^{47}$ S. Bouvier, ${ }^{4}$ A. Braem, ${ }^{9}$ M. Braun, ${ }^{30}$

P. Braun-Munzinger, ${ }^{19,107}$ L. Bravina, ${ }^{1}$ M. Bregant, ${ }^{56}$ G. Bruckner, ${ }^{9}$ R. Brun, ${ }^{9}$ E. Bruna,,${ }^{45}$ O. Brunasso, ${ }^{15}$ G.E. Bruno, ${ }^{60}$ D. Bucher, ${ }^{41}$ V. Budilov, ${ }^{13}$ D. Budnikov, ${ }^{40}$ H. Buesching,,${ }^{20}$ P. Buncic, ${ }^{9}$ M. Burns, ${ }^{9}$ S. Burachas,${ }^{14}$ O. Busch, ${ }^{6}$ J. Bushop,${ }^{59}$ X. Cai, ${ }^{61}$ H. Caines,${ }^{50}$ F. Calaon, ${ }^{25}$ M. Caldogno, ${ }^{25}$ I. Cali, ${ }^{9}$ P. Camerini,${ }^{56}$ R. Campagnolo, ${ }^{9}$ M. Campbell, ${ }^{9}$ X. Cao, ${ }^{62}$ G.P. Capitani, ${ }^{49}$ G. Cara Romeo,${ }^{26}$ M. Cardenas-Montes, ${ }^{52}$ H. Carduner, ${ }^{4}$ F. Carena, ${ }^{9}$ W. Carena, ${ }^{9}$ P. Cariola, ${ }^{63}$ F. Carminati, ${ }^{9}$ J. Casado, ${ }^{52}$ A. Casanova Diaz,${ }^{49}$ M. Caselle, ${ }^{63}$ J. Castillo Castellanos, ${ }^{22}$ J. Castor, ${ }^{35}$ V. Catanescu, ${ }^{21}$ E. Cattaruzza, ${ }^{56}$ D. Cavazza, ${ }^{26}$ P. Cerello, ${ }^{15}$ S. Ceresa,,${ }^{9}$ V. Černý, ${ }^{64}$ V. Chambert, ${ }^{51}$ S. Chapeland, ${ }^{9}$ A. Charpy, ${ }^{51}$ D. Charrier, ${ }^{4}$ M. Chartoire, ${ }^{65}$ J.L. Charvet, ${ }^{22}$ S. Chattopadhyay, ${ }^{58}$ S. Chattopadhyay, ${ }^{10}$ V. Chepurnov, ${ }^{13}$ S. Chernenko, ${ }^{13}$ M. Cherney ${ }^{23}$ C. Cheshkov, ${ }^{9}$ B. Cheynis, ${ }^{65}$ P. Chochula, ${ }^{9}$ E. Chiavassa, ${ }^{45}$ V. Chibante Barroso,,${ }^{9}$ J. Choi, ${ }^{66}$ 
P. Christakoglou, ${ }^{44}$ P. Christiansen,${ }^{67}$ C. Christensen, ${ }^{42}$ O.A. Chykalov ${ }^{57}$ C. Cicalo, ${ }^{27}$

L. Cifarelli-Strolin, ${ }^{17}$ M. Ciobanu, ${ }^{19}$ F. Cindolo,${ }^{26}$ C. Cirstoiu, ${ }^{9}$ O. Clausse, ${ }^{8}$ J. Cleymans, ${ }^{68}$

O. Cobanoglu, ${ }^{45}$ J.-P. Coffin, ${ }^{8}$ S. Coli, ${ }^{15}$ A. Colla, ${ }^{9}$ C. Colledani, ${ }^{8}$ C. Combaret,${ }^{65}$

M. Combet, ${ }^{22}$ M. Comets, ${ }^{51}$ G. Conesa Balbastre, ${ }^{49}$ Z. Conesa del Valle, ${ }^{4}$ G. Contin, ${ }^{56}$

J. Contreras, ${ }^{69}$ T. Cormier, ${ }^{43}$ F. Corsi, ${ }^{28}$ P. Cortese, ${ }^{70}$ F. Costa, ${ }^{26}$ E. Crescio, ${ }^{15}$ P. Crochet, ${ }^{35}$

E. Cuautle, ${ }^{71}$ J. Cussonneau, ${ }^{4}$ M. Dahlinger, ${ }^{19}$ A. Dainese, ${ }^{72}$ H.H. Dalsgaard, ${ }^{42}$ L. Daniel, ${ }^{48}$

I. Das, ${ }^{58}$ T. Das, ${ }^{10}$ A. Dash, ${ }^{73}$ R. Da Silva, ${ }^{9}$ M. Davenport, ${ }^{9}$ H. Daues, ${ }^{19}$ A. De Caro,${ }^{74}$

G. de Cataldo, ${ }^{63}$ J. De Cuveland, ${ }^{3}$ A. De Falco,${ }^{75}$ M. de Gaspari, ${ }^{6}$ P. de Girolamo, ${ }^{22}$

J. de Groot, ${ }^{9}$ D. De Gruttola, ${ }^{74}$ A. De Haas,${ }^{76}$ N. De Marco,${ }^{15}$ S. De Pasquale, ${ }^{74}$

P. De Remigis, ${ }^{15}$ D. de Vaux,${ }^{68}$ G. Decock, ${ }^{22}$ H. Delagrange, ${ }^{4}$ M. Del Franco, ${ }^{49}$

G. Dellacasa, ${ }^{70}$ C. Dell'Olio, ${ }^{60}$ D. Dell'Olio, ${ }^{60}$ A. Deloff, ${ }^{77}$ V. Demanov, ${ }^{40}$ E. Dénes, ${ }^{38}$

G. D'Erasmo, ${ }^{60}$ D. Derkach, ${ }^{30}$ A. Devaux,${ }^{35}$ D. Di Bari, ${ }^{60}$ A. Di Bartolomeo, ${ }^{74}$ C. Di Giglio,,${ }^{60}$

S. Di Liberto, ${ }^{78}$ A. Di Mauro, ${ }^{9}$ P. Di Nezza, ${ }^{49}$ M. Dialinas,${ }^{4}$ L. Diaz, ${ }^{71}$ R. Díaz Valdes,${ }^{32}$

T. Dietel, ${ }^{41}$ R. Dima, ${ }^{79}$ H. Ding, ${ }^{61}$ C. Dinca, ${ }^{21}$ R. Divià, ${ }^{9}$ V. Dobretsov, ${ }^{14}$ A. Dobrin, ${ }^{67}$

B. Doenigus, ${ }^{19}$ T. Dobrowolski, ${ }^{77}$ I. Domínguez, ${ }^{71}$ M. Dorn, ${ }^{3}$ S. Drouet, ${ }^{51}$ A.E. Dubey, ${ }^{10}$

L. Ducroux,${ }^{65}$ F. Dumitrache, ${ }^{15}$ E. Dumonteil, ${ }^{22}$ P. Dupieux,${ }^{35}$ V. Duta, ${ }^{21}$

A. Dutta Majumdar, ${ }^{58}$ M. Dutta Majumdar, ${ }^{10}$ Th. Dyhre, ${ }^{42}$ L. Efimov, ${ }^{13}$ A. Efremov, ${ }^{13}$

D. Elia, ${ }^{63}$ D. Emschermann, ${ }^{6}$ C. Engster, ${ }^{9}$ A. Enokizono,,${ }^{80}$ B. Espagnon,,${ }^{51}$ M. Estienne, ${ }^{8}$

A. Evangelista, ${ }^{26}$ D. Evans, ${ }^{48}$ S. Evrard, ${ }^{9}$ C.W. Fabjan, ${ }^{9}$ D. Fabris, ${ }^{25}$ J. Faivre, ${ }^{25}$

D. Falchieri, ${ }^{17}$ A. Fantoni, ${ }^{49}$ R. Farano, ${ }^{15}$ R. Fearick, ${ }^{68}$ O. Fedorov,${ }^{13}$ V. Fekete,${ }^{64}$ D. Felea, ${ }^{81}$

G. Feofilov, ${ }^{30}$ A. Férnandez Téllez, ${ }^{82}$ A. Ferretti, ${ }^{45}$ F. Fichera, ${ }^{33}$ S. Filchagin, ${ }^{40}$ E. Filoni, ${ }^{15}$

C. Finck, ${ }^{4}$ R. Fini, ${ }^{63}$ E.M. Fiore, ${ }^{60}$ D. Flierl,${ }^{9}$ M. Floris, ${ }^{75}$ Z. Fodor,${ }^{38}$ Y. Foka, ${ }^{19}$ S. Fokin,,${ }^{14}$

P. Force, ${ }^{35}$ F. Formenti, ${ }^{9}$ E. Fragiacomo,${ }^{83}$ M. Fragkiadakis, ${ }^{44}$ D. Fraissard,${ }^{9}$ A. Franco, ${ }^{63}$

M. Franco, ${ }^{63}$ U. Frankenfeld, ${ }^{19}$ U. Fratino, ${ }^{28}$ S. Fresneau, ${ }^{4}$ A. Frolov, ${ }^{109}$ U. Fuchs, ${ }^{9}$

J. Fujita, ${ }^{23}$ C. Furget, ${ }^{47}$ M. Furini, ${ }^{26}$ M. Fusco Girard, ${ }^{74}$ J.-J. Gaardhøje, ${ }^{42}$ A. Gabrielli, ${ }^{17}$

S. Gadrat, ${ }^{4}$ M. Gagliardi, ${ }^{45}$ A. Gago, ${ }^{69}$ L. Gaido, ${ }^{15}$ A. Gallas Torreira, ${ }^{63}$ M. Gallio, ${ }^{45}$

E. Gandolfi, ${ }^{17}$ P. Ganoti, ${ }^{44}$ M. Ganti, ${ }^{10}$ J. Garabatos, ${ }^{19}$ A. Garcia Lopez,,${ }^{9}$ L. Garizzo, ${ }^{25}$

L. Gaudichet, ${ }^{15}$ R. Gemme, ${ }^{70}$ M. Germain, ${ }^{4}$ A. Gheata, ${ }^{9}$ M. Gheata, ${ }^{9}$ B. Ghidini, ${ }^{60}$

P. Ghosh, ${ }^{10}$ G. Giolu, ${ }^{21}$ G. Giraudo, ${ }^{15}$ P. Giubellino, ${ }^{15}$ R. Glasow, ${ }^{41}$ P. Glässel, ${ }^{6}$

E.G. Ferreiro, ${ }^{29}$ C. Gonzalez Gutierrez, ${ }^{9}$ L.H. Gonzales-Trueba, ${ }^{16}$ S. Gorbunov, ${ }^{3}$

Y. Gorbunov, ${ }^{23}$ H. Gos, ${ }^{84}$ J. Gosset, ${ }^{22}$ S. Gotovac, ${ }^{85}$ H. Gottschlag, ${ }^{41}$ D. Gottschalk, ${ }^{3}$

V. Grabski, ${ }^{16}$ T. Grassi, ${ }^{9}$ H. Gray, ${ }^{53}$ O. Grebenyuk, ${ }^{76}$ K. Grebieszkow, ${ }^{84}$ C. Gregory, ${ }^{9}$

C. Grigoras, ${ }^{9}$ N. Grion, ${ }^{83}$ V. Grigoriev, ${ }^{54}$ A. Grigoryan, ${ }^{86}$ C. Grigoryan, ${ }^{9}$ S. Grigoryan,,${ }^{35}$

Y. Grishuk, ${ }^{12}$ P. Gros, ${ }^{67}$ J. Grosse-Oetringhaus, ${ }^{9}$ J.-Y. Grossiord, ${ }^{65}$ R. Grosso, ${ }^{9}$

B. Grynyov, ${ }^{87}$ C. Guarnaccia, ${ }^{74}$ F. Guber, ${ }^{88}$ F. Guerin, ${ }^{35}$ R. Guernane, ${ }^{35}$ M. Guerzoni, ${ }^{26}$

A. Guichard, ${ }^{35}$ M. Guida, ${ }^{74}$ G. Guilloux ${ }^{4}$ H. Gulkanyan, ${ }^{86}$ K. Gulbrandsen, ${ }^{42}$ T. Gunji, ${ }^{89}$

A. Gupta, ${ }^{34}$ V. Gupta, ${ }^{34}$ H.-A. Gustafsson, ${ }^{67}$ H. Gutbrod, ${ }^{19}$ C. Hadjidakis, ${ }^{49}$ M. Haiduc, ${ }^{81}$

G. Hamar, ${ }^{38}$ H. Hamagaki, ${ }^{89}$ J. Hamblen, ${ }^{90}$ J.C. Hansen, ${ }^{42}$ P. Hardy, ${ }^{22}$ D. Hatzifotiadou, ${ }^{26}$

J.W. Harris, ${ }^{50}$ M. Hartig, ${ }^{20}$ A. Harutyunyan, ${ }^{86}$ A. Hayrapetyan, ${ }^{86}$ D. Hasch, ${ }^{49}$ D. Hasegan, ${ }^{81}$

J. Hehner, ${ }^{19}$ N. Heine, ${ }^{41}$ M. Heinz,${ }^{50}$ H. Helstrup, ${ }^{91}$ A. Herghelegiu, ${ }^{21}$ S. Herlant, ${ }^{22}$

G. Herrera Corral, ${ }^{69}$ N. Herrmann, ${ }^{6}$ K. Hetland, ${ }^{91}$ P. Hille, ${ }^{1}$ H. Hinke,${ }^{20}$ B. Hippolyte, ${ }^{8}$

M. Hoch, ${ }^{9}$ H. Hoebbel, ${ }^{3}$ H. Hoedlmoser, ${ }^{9}$ T. Horaguchi, ${ }^{92}$ M. Horner, ${ }^{53}$ P. Hristov, ${ }^{9}$

I. Hřivnáčová, ${ }^{51}$ S. Hu, ${ }^{93}$ C. Hu Guo, ${ }^{8}$ T. Humanic, ${ }^{94}$ A. Hurtado,${ }^{52}$ D.S. Hwang, ${ }^{95}$ 
J.C. Ianigro, ${ }^{65}$ M. Idzik, ${ }^{15, a}$ S. Igolkin, ${ }^{9}$ R. Ilkaev, ${ }^{40}$ I. Ilkiv, ${ }^{77}$ M. Imhoff, ${ }^{8}$ P.G. Innocenti, ${ }^{9}$ E. Ionescu, ${ }^{21}$ M. Ippolitov, ${ }^{14}$ M. Irfan, ${ }^{11}$ C. Insa,,${ }^{35}$ M. Inuzuka, ${ }^{89}$ C. Ivan, ${ }^{76}$ A. Ivanov, ${ }^{30}$ M. Ivanov, ${ }^{19}$ V. Ivanov,${ }^{46}$ P. Jacobs, ${ }^{53}$ A. Jacholkowski, ${ }^{9}$ L. Jančurová, ${ }^{13}$ R. Janik, ${ }^{64}$ M. Jasper, ${ }^{59}$ C. Jena, ${ }^{73}$ L. Jirden, ${ }^{9}$ D.P. Johnson, ${ }^{96}$ G.T. Jones, ${ }^{48}$ C. Jorgensen, ${ }^{9}$ F. Jouve,,${ }^{35}$ P. Jovanović, ${ }^{48}$ A. Junique, ${ }^{9}$ A. Jusko,,${ }^{48}$ H. Jung, ${ }^{97}$ W. Jung, ${ }^{97}$ K. Kadija, ${ }^{23}$ A. Kamal,,${ }^{11}$ R. Kamermans, ${ }^{76}$ S. Kapusta, ${ }^{9}$ A. Kaidalov, ${ }^{12}$ V. Kakoyan, ${ }^{86}$ S. Kalcher, ${ }^{3}$ E. Kang, ${ }^{97}$ J. Kapitan, ${ }^{4}$ V. Kaplin, ${ }^{54}$ K. Karadzhev, ${ }^{14}$ O. Karavichev, ${ }^{88}$ T. Karavicheva, ${ }^{88}$ E. Karpechev,${ }^{88}$ K. Karpio, ${ }^{77}$ A. Kazantsev, ${ }^{14}$ U. Kebschull, ${ }^{3}$ R. Keidel,${ }^{90}$ M. Mohsin Khan, ${ }^{11}$ A. Khanzadeev, ${ }^{46}$ Y. Kharlov, ${ }^{55}$ D. Kikola, ${ }^{84}$ B. Kileng, ${ }^{91}$ D. Kim,${ }^{32}$ D.S. Kim, ${ }^{97}$ D.W. Kim, ${ }^{97}$ H.N. Kim, ${ }^{97}$ J.S. Kim, ${ }^{97}$ S. Kim, ${ }^{95}$ J.B. Kinson, ${ }^{48}$ S.K. Kiprich,${ }^{57}$ I. Kisel, ${ }^{3}$ S. Kiselev, ${ }^{12}$ A. Kisiel, ${ }^{84}$ T. Kiss, ${ }^{38}$ V. Kiworra, ${ }^{3}$ J. Klay, ${ }^{80}$ C. Klein Bösing, ${ }^{9}$ M. Kliemant, ${ }^{20}$ A. Klimov, ${ }^{14}$ A. Klovning, ${ }^{18}$ A. Kluge, ${ }^{9}$ R. Kluit, ${ }^{59}$ S. Kniege, ${ }^{20}$ R. Kolevatov, ${ }^{30}$ T. Kollegger, ${ }^{20}$ A. Kolojvari, ${ }^{30}$ V. Kondratiev, ${ }^{30}$ E. Kornas, ${ }^{39}$ E. Koshurnikov, ${ }^{13}$ I. Kotov, ${ }^{94}$ R. Kour, ${ }^{48}$ M. Kowalski, ${ }^{39}$ S. Kox, ${ }^{47}$ K. Kozlov, ${ }^{14}$ I. Králik, ${ }^{36}$ F. Kramer, ${ }^{20}$ I. Kraus, ${ }^{59}$ A. Kravčáková, ${ }^{36}$ T. Krawutschke, ${ }^{99}$ M. Krivda, ${ }^{48}$ E. Kryshen, ${ }^{46}$ Y. Kucheriaev, ${ }^{14}$ A. Kugler, ${ }^{5}$ C. Kuhn, ${ }^{8}$ P. Kuijer, ${ }^{59}$ L. Kumar, ${ }^{7}$ N. Kumar, ${ }^{7}$ P. Kumpumaeki, ${ }^{9}$ A. Kurepin, ${ }^{88}$ A.N. Kurepin ${ }^{88}$ S. Kushpil, ${ }^{5}$ V. Kushpil, ${ }^{5}$ M. Kutovsky, ${ }^{13}$ H. Kvaerno, ${ }^{1}$ M. Kweon, ${ }^{6}$ J.-C. Labbé, ${ }^{9}$ F. Lackner, ${ }^{9}$ P. Ladron de Guevara, ${ }^{52}$ V. Lafage, ${ }^{51}$ P. La Rocca, ${ }^{37}$ M. Lamont, ${ }^{50}$ C. Lara, ${ }^{3}$ D.T. Larsen, ${ }^{18}$ G. Laurenti, ${ }^{26}$ C. Lazzeroni, ${ }^{48}$ Y. Le Bornec, ${ }^{51}$ N. Le Bris, ${ }^{4}$ C. Le Gailliard, ${ }^{51}$ V. Lebedev, ${ }^{14}$ J. Lecoq, ${ }^{35}$ K.S. Lee,${ }^{97}$ S.C. Lee,${ }^{97}$ F. Lefévre, ${ }^{4}$ I. Legrand, ${ }^{21}$ T. Lehmann, ${ }^{6}$ L. Leistam, ${ }^{9}$ P. Lenoir, ${ }^{9}$ V. Lenti, ${ }^{63}$ H. Leon, ${ }^{16}$

I. Leon Monzon, ${ }^{100}$ P. Lévai, ${ }^{38}$ Q. Li, ${ }^{62}$ X. Li,${ }^{93}$ F. Librizzi, ${ }^{33}$ R. Lietava, ${ }^{48}$ N. Lindegaard, ${ }^{42}$ V. Lindenstruth, ${ }^{3}$ C. Lippmann, ${ }^{9}$ M. Lisa,,${ }^{94}$ O.M. Listratenko, ${ }^{57}$ F. Littel, ${ }^{8}$ Y. Liu, ${ }^{62}$ J. Lo, ${ }^{9}$ V. Lobanov, ${ }^{13}$ V. Loginov, ${ }^{54}$ M. López Noriega,,${ }^{9}$ R. López-Ramírez, ${ }^{82}$ E. López Torres, ${ }^{2}$ P.M. Lorenzo, ${ }^{9}$ G. Løvhøiden, ${ }^{1}$ S. Lu, ${ }^{93}$ W. Ludolphs, ${ }^{6}$ M. Lunardon, ${ }^{79}$ L. Luquin, ${ }^{4}$ S. Lusso, ${ }^{15}$ J-R. Lutz,${ }^{8}$ M. Luvisetto, ${ }^{26}$ V. Lyapin, ${ }^{32}$ A. Maevskaya, ${ }^{88}$ C. Magureanu, ${ }^{21}$ A. Mahajan, ${ }^{34}$ S. Majahan, ${ }^{34}$ T. Mahmoud, ${ }^{6}$ A. Mairani, ${ }^{101}$ D. Mahapatra, ${ }^{73}$ A. Makarov, ${ }^{13}$ I. Makhlyueva, ${ }^{9}$ M. Malek, ${ }^{51}$ T. Malkiewicz, ${ }^{32}$ D. Mal'Kevich, ${ }^{12}$ P. Malzacher, ${ }^{19}$ A. Mamonov, ${ }^{40}$ C. Manea, ${ }^{25}$ L.K. Mangotra, ${ }^{34}$ D. Maniero, ${ }^{25}$ V. Manko, ${ }^{14}$ F. Manso, ${ }^{35}$ V. Manzari, ${ }^{63}$ Y. Mao, ${ }^{61}$ A. Marcel, ${ }^{22}$ S. Marchini, ${ }^{25}$ J. Mareš, ${ }^{102}$ G.V. Margagliotti, ${ }^{56}$ A. Margotti, ${ }^{26}$ A. Marin, ${ }^{19}$ J.-C. Marin, ${ }^{9}$ D. Marras, ${ }^{27}$ P. Martinengo, ${ }^{9}$ M.I. Martínez,,${ }^{82}$ A. Martinez-Davalos, ${ }^{16}$ G. Martínez Garcia, ${ }^{4}$ S. Martini, ${ }^{25}$ A. Marzari Chiesa, ${ }^{45}$ C. Marzocca, ${ }^{28}$ S. Masciocchi, ${ }^{19}$ M. Masera, ${ }^{45}$ M. Masetti, ${ }^{17}$ N.I. Maslov, ${ }^{103}$ A. Masoni, ${ }^{27}$ F. Massera, ${ }^{26}$ M. Mast, ${ }^{9}$ A. Mastroserio,${ }^{60}$ Z.L. Matthews, ${ }^{48}$ B. Mayer, ${ }^{101}$ G. Mazza, ${ }^{15}$ M.D. Mazzaro,${ }^{25}$ A. Mazzoni, ${ }^{78}$ F. Meddi, ${ }^{104}$ E. Meleshko, ${ }^{14}$ A. Menchaca-Rocha, ${ }^{16}$ S. Meneghini, ${ }^{26}$ M. Meoni, ${ }^{9}$ J. Mercado Perez,${ }^{6}$ P. Mereu, ${ }^{15}$ O. Meunier,${ }^{22}$ Y. Miake, ${ }^{105}$ A. Michalon, ${ }^{8}$ R. Michinelli, ${ }^{26}$ N. Miftakhov, ${ }^{46}$ M. Mignone, ${ }^{15}$ K. Mikhailov, ${ }^{12}$ J. Milosevic, ${ }^{1}$ Y. Minaev ${ }^{13}$ F. Minafra, ${ }^{60}$ A. Mischke, ${ }^{76}$ D. Miśkowiec, ${ }^{19}$ V. Mitsyn,${ }^{13}$ C. Mitu,${ }^{81}$ B. Mohanty, ${ }^{10}$ D. Moisa, ${ }^{21}$ L. Molnar, ${ }^{38}$ M. Mondal, ${ }^{10}$ N. Mondal, ${ }^{10}$ L. Montaño Zetina, ${ }^{69}$ M. Monteno, ${ }^{15}$ M. Morando, ${ }^{79}$ M. Morel,${ }^{9}$ S. Moretto, ${ }^{79}$ Th. Morhardt,,${ }^{19}$ A. Morsch, ${ }^{9}$ T. Moukhanova, ${ }^{14}$ M. Mucchi, ${ }^{15}$ V. Muccifora, ${ }^{49}$ E. Mudnic, ${ }^{85}$ H. Müller, ${ }^{9}$ W. Müller, ${ }^{19}$ J. Munoz ${ }^{82}$ D. Mura,${ }^{27}$ L. Musa, ${ }^{9}$ J.F. Muraz,${ }^{47}$ A. Musso, ${ }^{15}$ R. Nania, ${ }^{26}$ B. Nandi, ${ }^{106}$ E. Nappi, ${ }^{63}$ F. Navach, ${ }^{60}$ S. Navin, ${ }^{48}$ T. Nayak, ${ }^{10}$ S. Nazarenko, ${ }^{40}$ G. Nazarov,${ }^{40}$ L. Nellen, ${ }^{71}$ 
F. Nendaz,${ }^{65}$ A. Nianine, ${ }^{14}$ M. Nicassio,${ }^{63}$ B.S. Nielsen, ${ }^{42}$ S. Nikolaev, ${ }^{14}$ V. Nikolic, ${ }^{24}$ S. Nikulin, ${ }^{14}$ V. Nikulin, ${ }^{46}$ B. Nilsen, ${ }^{94}$ M. Nitti, ${ }^{63}$ F. Noferini, ${ }^{26}$ P. Nomokonov, ${ }^{13}$ G. Nooren, ${ }^{76}$ F. Noto, ${ }^{37}$ D. Nouais,,${ }^{15}$ A. Nyiri, ${ }^{1}$ J. Nystrand, ${ }^{18}$ G. Odyniec, ${ }^{53}$ H. Oeschler, ${ }^{107}$ M. Oinonen, ${ }^{32}$ M. Oldenburg, ${ }^{9}$ I. Oleks, ${ }^{13}$ E.K. Olsen,,${ }^{42}$ V. Onuchin, ${ }^{55}$ C. Oppedisano, ${ }^{15}$ F. Orsini, ${ }^{22}$ A. Ortiz-Velázquez, ${ }^{71}$ C. Oskamp, ${ }^{76}$ A. Oskarsson, ${ }^{67}$ F. Osmic,,${ }^{9}$ L. Österman, ${ }^{67}$ I. Otterlund, ${ }^{67}$ G. Ovrebekk, ${ }^{18}$ K. Oyama, ${ }^{6}$ M. Pachr, ${ }^{108}$ P. Pagano, ${ }^{74}$ G. Paić, ${ }^{71}$ C. Pajares, ${ }^{29}$ S. Pal,${ }^{58}$ S. Pal, ${ }^{10}$ G. Pálla, ${ }^{38}$ A. Palmeri, ${ }^{33}$ G. Pancaldi, ${ }^{26}$ R. Panse, ${ }^{3}$ A. Pantaleo, ${ }^{63}$ G.S. Pappalardo, ${ }^{33}$ B. Pastirčák, ${ }^{36}$ C. Pastore, ${ }^{60}$ O. Patarakin,,${ }^{14}$ V. Paticchio,,${ }^{63}$ G. Patimo,${ }^{74}$ A. Pavlinov, ${ }^{43}$ T. Pawlak, ${ }^{84}$ T. Peitzmann, ${ }^{76}$ Y. Pénichot, ${ }^{22}$ A. Pepato, ${ }^{25}$ H. Pereira, ${ }^{22}$ D. Peresunko, ${ }^{14}$ C. Perez, ${ }^{69}$ J. Perez Griffo, ${ }^{52}$ D. Perini, ${ }^{9}$ D. Perrino, ${ }^{60}$ W. Peryt, ${ }^{84}$ A. Pesci,,${ }^{26}$ V. Peskov, ${ }^{9}$ Y. Pestov, ${ }^{109}$ A.J. Peters,${ }^{9}$ V. Petráček, ${ }^{108}$ A. Petridis, ${ }^{44}$ M. Petris, ${ }^{21}$ V. Petrov, ${ }^{43}$ V. Petrov, ${ }^{55}$ M. Petrovici, ${ }^{21}$ J. Peyré,${ }^{51}$ S. Piano,${ }^{83}$ A. Piccotti,${ }^{15}$ P. Pichot,${ }^{4}$ C. Piemonte, ${ }^{83}$ M. Pikna, ${ }^{64}$ R. Pilastrini, ${ }^{26}$ P. Pillot, ${ }^{4}$ O. Pinazza,${ }^{26}$ B. Pini, ${ }^{15}$ L. Pinsky,${ }^{94}$ V. Pinto Morais,${ }^{9}$ V. Pismennaya, ${ }^{13}$ F. Piuz, ${ }^{9}$ R. Platt,${ }^{48}$ M. Ploskon, ${ }^{20}$ S. Plumeri, ${ }^{8}$ J. Pluta, ${ }^{84}$ T. Pocheptsov, ${ }^{13}$ P. Podesta, ${ }^{71}$ F. Poggio, ${ }^{45}$ M. Poghosyan, ${ }^{86}$ T. Poghosyan, ${ }^{86}$ K. Polák, ${ }^{102}$ B. Polichtchouk, ${ }^{55}$ P. Polozov, ${ }^{12}$ V. Polyakov, ${ }^{46}$ B. Pommeresch, ${ }^{18}$ F. Pompei, ${ }^{43}$ A. Pop,${ }^{21}$ S. Popescu, ${ }^{3}$ F. Posa, ${ }^{60}$ V. Pospíśil, ${ }^{108}$ B. Potukuchi, ${ }^{34}$ J. Pouthas, ${ }^{51}$ S. Prasad, ${ }^{10}$ R. Preghenella,,${ }^{17}$ F. Prino, ${ }^{15}$ L. Prodan, ${ }^{21}$ G. Prono, ${ }^{22}$ M.A. Protsenko, ${ }^{57}$ C.A. Pruneau, ${ }^{43}$ A. Przybyla, ${ }^{19}$ I. Pshenichnov, ${ }^{88}$ G. Puddu, ${ }^{75}$ P. Pujahari, ${ }^{106}$ A. Pulvirenti, ${ }^{37}$ A. Punin, ${ }^{40}$ V. Punin,,${ }^{40}$ J. Putschke, ${ }^{50}$ J. Quartieri, ${ }^{74}$ E. Quercigh, ${ }^{9}$ I. Rachevskaya, ${ }^{83}$ A. Rachevski, ${ }^{83}$ A. Rademakers, ${ }^{9}$ S. Radomski, ${ }^{6}$ A. Radu, ${ }^{21}$ J. Rak, ${ }^{32}$ L. Ramello, ${ }^{70}$ R. Raniwala, ${ }^{110}$ S. Raniwala, ${ }^{110}$ O.B. Rasmussen, ${ }^{42}$ J. Rasson, ${ }^{53}$ V. Razin, ${ }^{88}$ K. Read,,${ }^{90}$ J. Real, ${ }^{47}$ K. Redlich, ${ }^{77}$ C. Reichling, ${ }^{3}$ C. Renard, ${ }^{4}$ G. Renault, ${ }^{42}$ R. Renfordt, ${ }^{20}$ A.R. Reolon, ${ }^{49}$ A. Reshetin, ${ }^{88}$ J.-P. Revol,,${ }^{9}$ K. Reygers, ${ }^{41}$ H. Ricaud, ${ }^{8}$ L. Riccati, ${ }^{15}$ R.A. Ricci, ${ }^{72}$ M. Richter, ${ }^{18}$ P. Riedler, ${ }^{9}$ L.M. Rigalleau, ${ }^{4}$ F. Riggi,${ }^{37}$ W. Riegler, ${ }^{9}$ E. Rindel,${ }^{51}$ J. Riso, ${ }^{43}$ A. Rivetti, ${ }^{15}$ M. Rizzi, ${ }^{26}$ V. Rizzi, ${ }^{63}$ M. Rodriguez Cahuantzi, ${ }^{82}$ K. Røed, ${ }^{91}$ D. Röhrich,,${ }^{18}$ S. Román-López, ${ }^{82}$ M. Romanato, ${ }^{25}$ R. Romita, ${ }^{60}$ F. Ronchetti, ${ }^{49}$ P. Rosinsky, ${ }^{9}$ P. Rosnet,, 35 S. Rossegger, ${ }^{9}$ A. Rossi,${ }^{56}$ V. Rostchin, ${ }^{46}$ F. Rotondo,${ }^{15}$ F. Roukoutakis, ${ }^{44}$ S. Rousseau, ${ }^{51}$ C. Roy, ${ }^{4}$ D. Roy, ${ }^{4}$ P. Roy,${ }^{58}$ L. Royer, ${ }^{35}$ G. Rubin,,${ }^{38}$ A. Rubio, ${ }^{2}$ R. Rui, ${ }^{56}$ I. Rusanov, ${ }^{6}$ G. Russo, ${ }^{74}$ V. Ruuskanen, ${ }^{32}$ E. Ryabinkin, ${ }^{14}$ A. Rybicki, ${ }^{39}$ S. Sadovsky, ${ }^{55}$ K. Šafařík, ${ }^{9}$ R. Sahoo, ${ }^{73}$ J. Saini, ${ }^{10}$ P. Saiz, ${ }^{9}$ S. Salur, ${ }^{50}$ S. Sambyal, ${ }^{34}$ V. Samsonov, ${ }^{46}$ L. Š́ndor, ${ }^{36}$ A. Sandoval, ${ }^{16}$ H. Sann, ${ }^{19 *}$ J.-C. Santiard,${ }^{9}$ R. Santo, ${ }^{41}$ R. Santoro,${ }^{60}$ G. Sargsyan, ${ }^{86}$ P. Saturnini, ${ }^{35}$ E. Scapparone, ${ }^{26}$ F. Scarlassara, ${ }^{79}$ B. Schackert, ${ }^{98}$ C. Schiaua, ${ }^{21}$ R. Schicker, ${ }^{6}$ T. Schioler, ${ }^{9}$ J.D. Schippers, ${ }^{59}$ C. Schmidt, ${ }^{19}$ H. Schmidt, ${ }^{19}$ R. Schneider, ${ }^{3}$ K. Schossmaier, ${ }^{9}$ J. Schukraft, ${ }^{9}$ Y. Schutz, ${ }^{4}$ K. Schwarz, ${ }^{19}$ K. Schweda, ${ }^{6}$ E. Schyns, ${ }^{9}$ G. Scioli, ${ }^{17}$ E. Scomparin, ${ }^{15}$ H. Snow ${ }^{48}$ S. Sedykh, ${ }^{19}$ G. Segato, ${ }^{79}$ S. Sellitto, ${ }^{74}$ F. Semeria, ${ }^{26}$ S. Senyukov, ${ }^{45}$ H. Seppänen, ${ }^{32}$ S. Serci, ${ }^{75}$ L. Serkin, ${ }^{16}$ S. Serra, ${ }^{26}$ T. Sesselmann, ${ }^{3}$ A. Sevcenco, ${ }^{81}$ I. Sgura, ${ }^{28}$ G. Shabratova, ${ }^{13}$ R. Shahoyan, ${ }^{9}$ E. Sharkov, ${ }^{12}$ S. Sharma, ${ }^{34}$ K. Shigaki, ${ }^{92}$ K. Shileev, ${ }^{63}$, P. Shukla, ${ }^{6}$ A. Shurygin, ${ }^{13}$ M. Shurygina, ${ }^{13}$ Y. Sibiriak, ${ }^{14}$ E. Siddi, ${ }^{27}$ T. Siemiarczuk, ${ }^{77}$ M.H. Sigward, ${ }^{8}$ A. Silenzi, ${ }^{17}$ D. Silvermyr, ${ }^{31}$ R. Silvestri, ${ }^{74}$ E. Simili, ${ }^{76}$ V. Simion, ${ }^{21}$ R. Simon, ${ }^{19}$ L. Simonetti, ${ }^{15}$, R. Singaraju, ${ }^{10}$ V. Singhal, ${ }^{10}$ B. Sinha, ${ }^{10}$ T. Sinha, ${ }^{58}$ M. Siska,${ }^{64}$ B. Sitár, ${ }^{64}$ M. Sitta, ${ }^{70}$ B. Skaali, ${ }^{1}$ P. Skowronski,${ }^{84}$ M. Slodkowski, ${ }^{84}$ N. Smirnov, ${ }^{50}$ L. Smykov, ${ }^{13}$ R. Snellings,,${ }^{59}$ W. Snoeys, ${ }^{9}$ C. Soegaard,,${ }^{42}$ J. Soerensen,,${ }^{42 *}$ 
O. Sokolov, ${ }^{16}$ A. Soldatov, ${ }^{14}$ A. Soloviev,${ }^{55}$ H. Soltveit,${ }^{6}$ R. Soltz, ${ }^{80}$ W. Sommer, ${ }^{20}$ C. Soos, ${ }^{9}$

F. Soramel, ${ }^{111}$ S. Sorensen, ${ }^{90}$ D. Soyk,${ }^{19}$ M. Spyropoulou-Stassinaki, ${ }^{44}$ J. Stachel, ${ }^{6}$

F. Staley, ${ }^{22}$ I. Stan, ${ }^{81}$ A. Stavinskiy, ${ }^{12}$ J. Steckert, ${ }^{9}$ G. Stefanini, ${ }^{9}$ G. Stefanek, ${ }^{77}$ T. Steinbeck, ${ }^{3}$

H. Stelzer, ${ }^{19}$ E. Stenlund,${ }^{67}$ D. Stocco,${ }^{45}$ M. Stockmeier, ${ }^{19}$ G. Stoicea, ${ }^{21}$ P. Stolpovsky,,${ }^{55}$

P. Strmeň, ${ }^{64}$ J.S. Stutzmann, ${ }^{4}$ G. Su, ${ }^{62}$ T. Sugitate,${ }^{92}$ M. Sumbera, ${ }^{5}$ C. Suire,${ }^{51}$ T. Susa,${ }^{24}$

K. Sushil Kumar, ${ }^{112}$ D. Swoboda, ${ }^{9}$ J. Symons,${ }^{53}$ I. Szarka, ${ }^{64}$ A. Szostak, ${ }^{68}$ M. Szuba, ${ }^{84}$

P. Szymanski, ${ }^{9}$ M. Tadel, ${ }^{9}$ C. Tagridis, ${ }^{44}$ L. Tan, ${ }^{62}$ D. Tapia Takaki, ${ }^{48}$ H. Taureg, ${ }^{9}$

A. Tauro, ${ }^{28}$ M. Tavlet, ${ }^{9}$ G. Tejeda Munoz, ${ }^{82}$ J. Thäder, ${ }^{3}$ R. Tieulent, ${ }^{65}$ P. Timmer, ${ }^{59}$

T. Tolyhy,${ }^{38}$ N. Topilskaya, ${ }^{88}$ C. Torcato de Matos, ${ }^{9}$ H. Torii, ${ }^{92}$ L. Toscano, ${ }^{15}$ F. Tosello, ${ }^{15}$

A. Tournaire, ${ }^{4}$ T. Traczyk, ${ }^{84}$ G. Tröger, ${ }^{3}$ W. Tromeur, ${ }^{65}$ D. Truesdale,,${ }^{94}$ W. Trzaska, ${ }^{32}$

G. Tsiledakis, ${ }^{19}$ E. Tsilis, ${ }^{44}$ A. Tsvetkov, ${ }^{14}$ M. Turcato, ${ }^{25}$ R. Turrisi, ${ }^{25}$ M. Tuveri, ${ }^{27}$ T. Tveter, ${ }^{1}$

H. Tydesjo, ${ }^{9}$ L. Tykarski, ${ }^{77}$ K. Tywoniuk, ${ }^{1}$ E. Ugolini, ${ }^{26}$ K. Ullaland, ${ }^{18}$ J. Urbán, ${ }^{36}$

G.M. Urciuoli, ${ }^{78}$ G.L. Usai, ${ }^{75}$ M. Usseglio, ${ }^{22}$ A. Vacchi, ${ }^{83}$ M. Vala, ${ }^{13}$ F. Valiev, ${ }^{30}$

P. Vande Vyvre, ${ }^{9}$ A. Van Den Brink, ${ }^{76}$ N. Van Eijndhoven, ${ }^{76}$ N. Van Der Kolk, ${ }^{59}$

M. van Leeuwen, ${ }^{53}$ L. Vannucci, ${ }^{72}$ S. Vanzetto,,${ }^{65}$ J.-P. Vanuxem, ${ }^{9}$ M.A. Vargas, ${ }^{82}$

R. Varma, ${ }^{106}$ A. Vascotto, ${ }^{9}$ A. Vasiliev, ${ }^{14}$ M. Vassiliou, ${ }^{44}$ P. Vasta, ${ }^{63}$ V. Vechernin, ${ }^{30}$

M. Venaruzzo, ${ }^{56}$ E. Vercellin,,${ }^{45}$ S. Vergara, ${ }^{82}$ W. Verhoeven, ${ }^{41}$ F. Veronese ${ }^{25}$ I. Vetlitskiy, ${ }^{12}$

R. Vernet, ${ }^{37}$ V. Victorov, ${ }^{55}$ L. Vidak, ${ }^{85}$ G. Viesti, ${ }^{79}$ O. Vikhlyantsev, ${ }^{40}$ Z. Vilakazi, ${ }^{68}$

O. Villalobos Baillie, ${ }^{48}$ A. Vinogradov, ${ }^{14}$ L. Vinogradov, ${ }^{30}$ Y. Vinogradov,${ }^{40}$ T. Virgili, ${ }^{74}$

Y. Viyogi, ${ }^{73}$ A. Vodopianov, ${ }^{13}$ G. Volpe, ${ }^{63}$ D. Vranic, ${ }^{19}$ J. Vrláková, ${ }^{36}$ B. Vulpescu, ${ }^{6}$

C. Wabnitz, ${ }^{8}$ V. Wagner, ${ }^{108}$ L. Wallet, ${ }^{9}$ R. Wan, ${ }^{61}$ Y. Wang, ${ }^{6}$ Y. Wang, ${ }^{61}$ R. Wheadon, ${ }^{15}$

R. Weis, ${ }^{3}$ Q. Wen, ${ }^{93}$ J. Wessels, ${ }^{41}$ J. Westergaard, ${ }^{42}$ J. Wiechula, ${ }^{19}$ A. Wiesenaecker, ${ }^{20}$

J. Wikne, ${ }^{1}$ A. Wilk, ${ }^{41}$ G. Wilk, ${ }^{77}$ C. Williams, ${ }^{26}$ N. Willis, ${ }^{51}$ B. Windelband, ${ }^{6}$ R. Witt, ${ }^{50}$

H. Woehri, ${ }^{27}$ K. Wyllie, ${ }^{9}$ C. Xu ${ }^{61}$ C. Yang,${ }^{61}$ H. Yang,${ }^{18}$ F. Yermia, ${ }^{15}$ Z. Yin, ${ }^{61}$ Z. Yin, ${ }^{9}$

B. Yun Ky, ${ }^{51}$ I. Yushmanov, ${ }^{14}$ B. Yuting, ${ }^{76}$ E. Zabrodin, ${ }^{1}$ S. Zagato, ${ }^{26}$ B. Zagreev, ${ }^{12}$

P. Zaharia, ${ }^{21}$ A. Zalite, ${ }^{46}$ G. Zampa, ${ }^{83}$ C. Zampolli, ${ }^{26}$ Y. Zanevskiy, ${ }^{13}$ A. Zarochentsev, ${ }^{30}$

O. Zaudtke, ${ }^{41}$ P. Závada, ${ }^{102}$ H. Zbroszczyk, ${ }^{84}$ A. Zepeda, ${ }^{69}$ V. Zeter, ${ }^{8}$ I. Zgura, ${ }^{81}$

M. Zhalov, ${ }^{46}$ D. Zhou, ${ }^{61}$ S. Zhou, ${ }^{93}$ G. Zhu, ${ }^{62}$ A. Zichichi, ${ }^{17}$ A. Zinchenko, ${ }^{13}$ G. Zinovjev, ${ }^{87}$

Y. Zoccarato, ${ }^{65}$ A. Zubarev, ${ }^{13}$ A. Zucchini, ${ }^{26}$ and M. Zuffa ${ }^{26}$

${ }^{1}$ Department of Physics, University of Oslo, Oslo, Norway

${ }^{2}$ Centro de Aplicaciones Tecnológicas y Desarrollo Nuclear (CEADEN), Madrid/Havana, Spain/Cuba

${ }^{3}$ Kirchhoff-Institut für Physik, Ruprecht-Karls-Universität Heidelberg, Heidelberg, Germany BMBF

${ }^{4}$ SUBATECH, Ecole des Mines de Nantes, Université de Nantes, CNRS/IN2P3, Nantes, France

${ }^{5}$ Academy of Sciences of the Czech Republic, Nuclear Physics Institute,

Řež/Prague, Czech Republic

${ }^{6}$ Physikalisches Institut, Ruprecht-Karls-Universität Heidelberg, Heidelberg, Germany BMBF

${ }^{7}$ Physics Department, Panjab University, Chandigarh, India

${ }^{8}$ IPHC, Université Louis Pasteur, CNRS/IN2P3, Strasbourg, France

${ }^{9}$ CERN, European Organization for Nuclear Reasearch, Geneva, Switzerland

${ }^{10}$ Variable Energy Cyclotron Centre, Kolkata, India

${ }^{11}$ Department of Physics Aligarh Muslim University, Aligarh, India

${ }^{12}$ Institute for Theoretical and Experimental Physics, Moscow, Russia 
${ }^{13}$ JINR, Joint Institute for Nuclear Research, Dubna,

${ }^{14}$ Russian Research Center Kurchatov Institute, Moscow, Russia

${ }^{15}$ Sezione INFN, Torino, Italy

${ }^{16}$ Instituto de Fisica, Universidad Nacional Autonoma de Mexico, Mexico City, Mexico

${ }^{17}$ Dipartimento di Fisica dell'Università and Sezione INFN, Bologna, Italy

${ }^{18}$ Department of Physics, University of Bergen, Bergen, Norway

${ }^{19}$ GSI, Gesellschaft für Schwerionenforschung GmbH, Darmstadt, Germany

${ }^{20}$ Institut für Kernphysik, Johann-Wolfgang-Goethe Universität Frankfurt, Frankfurt, Germany BMBF

${ }^{21}$ National Institute for Physics and Nuclear Engineering, Bucharest, Romania

${ }^{22}$ CEA, Centre d'Études Nucléaires, DAPNIA, Saclay, France

${ }^{23}$ Creighton University, Omaha Nebraska, Creighton, U.S.A.

${ }^{24}$ Rudjer Bošković Institute, Zagreb, Croatia

${ }^{25}$ Sezione INFN, Padova, Italy

${ }^{26}$ Sezione INFN, Bologna, Italy

${ }^{27}$ Sezione INFN, Cagliari, Italy

${ }^{28}$ Politecnico and Sezione INFN, Bari, Italy

${ }^{29}$ IGFAE, Universidad de Santiago de Compostela, Santiago de Compostela, Spain

${ }^{30}$ V. Fock Institute for Physics, St. Petersburg State University, St. Petersburg, Russia

${ }^{31}$ Oak Ridge National Laboratory, Oak Ridge, U.S.A.

${ }^{32}$ University of Jyväskylä, Helsinki/Jyväskylä, Finland

${ }^{33}$ Sezione INFN, Catania, Italy

${ }^{34}$ Physics Department, University of Jammu, Jammu, India

${ }^{35}$ LPC, Université Blaise Pascal, CNRS/IN2P3, Clermont-Ferrand, France

${ }^{36}$ Institute of Experimental Physics, Slovak Academy of Siences and Faculty of Science,

P.J. Šafárik University, Košice, Slovak Republic

${ }^{37}$ Dipartimento di Fisica dell'Università and Sezione INFN, Catania, Italy

${ }^{38}$ KFKI Research Institute for Particle and Nuclear Physics, Hungarian Academy of Sciences, Budapest, Hungary

${ }^{39}$ Henryk Niewodniczánski Institute of Nuclear Physics, High Energy Physics Department, Cracow, Poland

${ }^{40}$ Russian Federal Nuclear Center (VNIIEF), Sarov, Russia

${ }^{41}$ Institut für Kernphysik, Westfälische Wilhelms-Universität Münster, Münster, Germany BMBF

${ }^{42}$ University of Copenhagen, Niels Bohr Institute, Copenhagen, Denmark

${ }^{43}$ Wayne State University, Detroit, U.S.A.

${ }^{44}$ University of Athens, Physics Department, Athens, Greece

${ }^{45}$ Dipartimento di Fisica Sperimentale dell'Università and Sezione INFN, Torino, Italy

${ }^{46}$ Petersburg Nuclear Physics Institute, Gatchina, Russia

${ }^{47}$ LPSC, Université Joseph Fourier Grenoble 1, CNRS/IN2P3, Institut Polytechnique de Grenoble, Grenoble, France

${ }^{48}$ School of Physics and Space Research, University of Birmingham, Birmingham, U.K.

${ }^{49}$ Laboratori Nazionali di Frascati, INFN, Frascati, Italy

${ }^{50}$ Yale University, New Haven, U.S.A.

${ }^{51}$ IPNO, Université Paris-Sud, CNRS/IN2P3, Orsay, France 
${ }^{52}$ Centro de Investigaciones Energeticas Medioambientales y Tecnologicas (CIEMAT), Madrid/Havana, Spain/Cuba

${ }^{53}$ Lawrence Berkeley National Laboratory, Berkeley, U.S.A.

${ }^{54}$ Moscow Engineering Physics Institute, Moscow, Russia

${ }^{55}$ Institute for High Energy Physics, Protvino, Russia

${ }^{56}$ Dipartimento di Fisica dell'Università and Sezione INFN, Trieste, Italy

${ }^{57}$ Scientific Research Technological Institute of Instrument Engineering, Kharkov, Ukraine

${ }^{58}$ Saha Institute of Nuclear Physics, Kolkata, India

${ }^{59}$ National Institute for Nuclear and High Energy Physics (NIKHEF), Amsterdam, Netherlands

${ }^{60}$ Dipartimento Interateneo di Fisica "M. Merlin" and Sezione INFN, Bari, Italy

${ }^{61}$ Hua-Zhong Normal University, Wuhan, China

${ }^{62}$ Hua-Zhong University of Science and Technology, Wuhan, China

${ }^{63}$ Sezione INFN, Bari, Italy

${ }^{64}$ Comenius University, Faculty of Mathematics, Physics and Informatics, Bratislava, Slovak Republic

${ }^{65}$ IPNL, Université de Lyon, CNRS/IN2P3, Lyon, France

${ }^{66}$ Sejong University, Department of Physics, Kangnung/Pohang, Republic of Korea

${ }^{67}$ Division of Experimental High Energy Physics, University of Lund, Lund, Sweden

${ }^{68}$ University of Cape Town, Physics Department, Cape Town, South Africa

${ }^{69}$ Centro de Invextigacion y de Estudios Avanzados (CINVESTAV) Mexico, Mexico City and Merida, Mexico

${ }^{70}$ Dipartimento di Scienze e Tecnologie Avanzate dell'Università del Piemonte Orientale and Gruppo Collegato INFN, Alessandria, Italy

${ }^{71}$ Instituto de Ciencias Nucleares, Universidad Natcional Autonoma de Mexico, Mexico City, Mexico

${ }^{72}$ Laboratori Nazionali di Legnaro, INFN, Legnaro, Italy

${ }^{73}$ Institute of Physics, Bhubaneswar, India

${ }^{74}$ Dipartimento di Fisica "E.R. Caianiello" dell'Università and Sezione INFN, Salerno, Italy

${ }^{75}$ Dipartimento di Fisica dell'Università and Sezione INFN, Cagliari, Italy

${ }^{76}$ Subatomic Physics Department, Utrecht University, Utrecht, Netherlands

${ }^{77}$ Soltan Institute for Nuclear Studies, Warsaw, Poland

${ }^{78}$ Sezione INFN, Roma, Italy

${ }^{79}$ Dipartimento di Fisica dell'Università and Sezione INFN, Padova, Italy

${ }^{80}$ Lawrence Livermore National Laboratory, Livermore, U.S.A.

${ }^{81}$ Institute of Space Sciences ISS, Bucharest, Romania

${ }^{82}$ Benemerita Universidad Autonoma de Puebla, Puebla, Mexico

${ }^{83}$ Sezione INFN, Trieste, Italy

${ }^{84}$ Warsaw University of Technology, Warsaw, Poland

${ }^{85}$ Technical University of Split FESB, Split, Croatia

${ }^{86}$ Yerevan Physics Institute, Yerevan, Armenia

${ }^{87}$ Bogolyubov Institute for Theoretical Physics, Kiev, Ukraine

${ }^{88}$ Institute for Nuclear Research, Academy of Science, Moscow, Russia

${ }^{89}$ University of Tokyo, Tokyo, Japan 
${ }^{90}$ University of Tennessee, Knoxville, U.S.A.

${ }^{91}$ Bergen University College, Faculty of Engineering, Bergen, Norway

${ }^{92}$ Hiroshima University, Hiroshima, Japan

${ }^{93}$ China Institute of Atomic Energy, Beijing, China

${ }^{94}$ NSF, Department of Physics, Ohio State University, Columbus OH, U.S.A.

${ }^{95}$ Sejong University, Department of Physics, Seoul, Republic of Korea

${ }^{96}$ Ohio Supercomputer Centre, Columbus, U.S.A.

${ }^{97}$ Kangnung National University, Kangnung/Pohang, Republic of Korea

${ }^{98}$ Fachhochschule Worms Zentrum für Technologietransfer und Telekommunikation ZTT,

Worms, Germany BMBF

${ }^{99}$ Fachhochschule Köln, Köln, Germany BMBF

${ }^{100}$ Universidad Autonoma de Sinaloa, Culiacan, Mexico

${ }^{101}$ University of Houston, Houston, U.S.A.

${ }^{102}$ Academy of Sciences of the Czech Republic (ASCR), Institute of Physics, Prague, Czech Republic

${ }^{103}$ National Scientific Centre, Kharkov Institute of Physics and Technology, Kharkov, Ukraine

${ }^{104}$ Dipartimento di Fisica dell'Università 'Sapienza' and Sezione INFN, Roma, Italy

${ }^{105}$ University of Tsukuba, Tsukuba, Japan

${ }^{106}$ Indian Institute of Technology, Mumbai, India

${ }^{107}$ Institut für Kernphysik, Technische Universität Darmstadt, Darmstadt, Germany BMBF

${ }^{108}$ Czech Technical University of Prague CTU, Prague, Czech Republic

${ }^{109}$ Budker Institute for Nuclear Physics, Novosibirsk, Russia

${ }^{110}$ Physics Department, University of Rajasthan, Jaipur, India

${ }^{111}$ Dipartimento di Fisica and INFN Sezione di Trieste, Udine, Italy

${ }^{112}$ Bhabha Atomic Research Centre, Mumbai, India

${ }^{a}$ Now at Faculty of Physics and Nuclear Techniques, AGH University of Science and Technology, Cracow, Poland

${ }^{*}$ Deceased

Corresponding author: Hans-Ake Gustafsson (Hans-Ake.Gustafsson@hep.lu.se) 


\section{Contents}

The ALICE Collaboration iii

1 Introduction 1

1.1 ALICE experiment 1

1.2 Design considerations 2

1.2.1 Physics observables 2

1.2.2 Performance specification 2

1.3 Detector layout 6

1.3.1 Tracking detectors 6

1.3.2 Particle identification 6

$\begin{array}{lll}\text { 1.3.3 Electromagnetic calorimeters } & 7\end{array}$

$\begin{array}{lll}\text { 1.3.4 Muon spectrometer } & 7\end{array}$

$\begin{array}{lll}\text { 1.3.5 } & \text { Forward and trigger detectors } & 8\end{array}$

$\begin{array}{lll}\text { 1.3.6 Trigger and data acquisition } & 8\end{array}$

\section{Experimental area}

2.1 Underground area and surface facilities 9

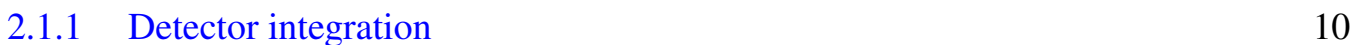

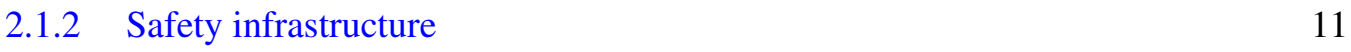

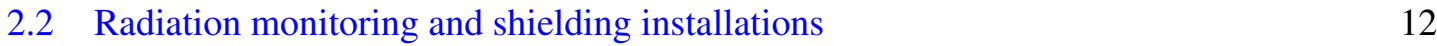

2.3 Magnets 13

$\begin{array}{lll}2.3 .1 & \text { Solenoid } & 13\end{array}$

$\begin{array}{ll}\text { 2.3.2 Dipole } & 13\end{array}$

2.4 Beam pipe 14

$\begin{array}{lll}2.5 & \text { Survey and alignment } & 17\end{array}$

3 Central detectors $\quad \mathbf{1 8}$

3.1 Inner Tracking System (ITS) 18

3.1.1 Silicon Pixel Detector (SPD) 22

3.1.2 Silicon Drift Detector (SDD) 37

3.1.3 Silicon Strip Detectors (SSD) 46

3.2 Time-Projection Chamber (TPC) 54

$\begin{array}{lll}\text { 3.2.1 Design considerations } & 54\end{array}$

3.2.2 Detector layout $\quad 55$

3.2.3 Front-end electronics and readout $\quad 63$

3.3 Transition Radiation Detector (TRD) 66

3.3.1 Design considerations $\quad 66$

$\begin{array}{lll}3.3 .2 & \text { Detector layout } & 68\end{array}$

$\begin{array}{lll}\text { 3.3.3 TRD performance } & 70\end{array}$

$\begin{array}{lll}\text { 3.3.4 Readout electronics } & 72\end{array}$ 
$\begin{array}{lll}3.4 & \text { Time-Of-Flight (TOF) detector } & 74\end{array}$

$\begin{array}{lll}\text { 3.4.1 Design considerations } & 74\end{array}$

$\begin{array}{lll}3.4 .2 & \text { Detector layout } & 75\end{array}$

$\begin{array}{lll}\text { 3.4.3 Front-end electronics and readout } & 81\end{array}$

3.5 High-Momentum Particle Identification Detector (HMPID) 83

$\begin{array}{lll}\text { 3.5.1 Design considerations } & 83\end{array}$

$\begin{array}{lll}3.5 .2 & \text { Detector layout } & 83\end{array}$

$\begin{array}{lll}\text { 3.5.3 Front-end electronics and readout } & 87\end{array}$

3.6 PHOton Spectrometer (PHOS) 87

$\begin{array}{lll}\text { 3.6.1 Design considerations } & 87\end{array}$

$\begin{array}{lll}\text { 3.6.2 Detector layout } & 88\end{array}$

3.6.3 Front-end electronics and readout $\quad 91$

3.7 ElectroMagnetic CALorimeter (EMCal) 92

$\begin{array}{ll}\text { 3.7.1 Design considerations } & 92\end{array}$

3.7.2 Mechanical issues 93

3.7.3 Strip modules and super modules $\quad 96$

$\begin{array}{ll}\text { 3.7.4 Module physical parameters } & 97\end{array}$

$\begin{array}{llr}\text { 3.7.5 } & \text { Module optical system } & 98\end{array}$

$\begin{array}{lll}3.7 .6 & \text { Readout and trigger } & 100\end{array}$

$\begin{array}{lll}\text { 3.7.7 EMCal calibration } & 101\end{array}$

$3.8 \quad$ ALICE COsmic Ray DEtector (ACORDE) 102

$\begin{array}{lll}3.8 .1 & \text { Design considerations } & 102\end{array}$

$\begin{array}{lll}3.8 .2 & \text { Detector layout } & 102\end{array}$

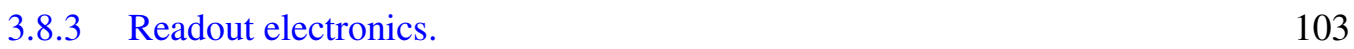

4 Muon spectrometer $\quad \mathbf{1 0 5}$

$\begin{array}{ll}\text { 4.1 Design considerations } & 105\end{array}$

$\begin{array}{lll}4.2 & \text { Detector layout } & 106\end{array}$

$\begin{array}{lll}4.3 \text { Absorbers } & 107\end{array}$

$\begin{array}{lll}4.4 & \text { Tracking system } & 109\end{array}$

$\begin{array}{lll}4.5 & \text { Trigger system } & 112\end{array}$

5 Forward detectors $\quad \mathbf{1 1 5}$

$\begin{array}{lll}5.1 & \text { Zero Degree Calorimeter (ZDC) } & 115\end{array}$

$\begin{array}{lll}\text { 5.1.1 Introduction } & 115\end{array}$

$\begin{array}{lll}5.1 .2 & \text { Detector layout } & 115\end{array}$

$\begin{array}{ll}\text { 5.1.3 Signal transmission and readout } & 119\end{array}$

$\begin{array}{ll}\text { 5.1.4 Monitoring and calibration } & 119\end{array}$

$\begin{array}{lll}5.2 & \text { Photon Multiplicity Detector (PMD) } & 120\end{array}$

$\begin{array}{ll}\text { 5.2.1 Design considerations } & 120\end{array}$

$\begin{array}{lll}5.2 .2 & \text { Detector layout } & 121\end{array}$

$\begin{array}{ll}\text { 5.2.3 Front-End Electronics and readout } & 124\end{array}$

$\begin{array}{lll}5.3 & \text { Forward Multiplicity Detector (FMD) } & 125\end{array}$ 
$\begin{array}{lll}\text { 5.3.1 Design considerations } & 125\end{array}$

$\begin{array}{lll}\text { 5.3.2 Detector layout } & 126\end{array}$

$\begin{array}{lll}\text { 5.3.3 Front-end electronics and readout } & 129\end{array}$

$\begin{array}{ll}\text { 5.3.4 Detector response } & 131\end{array}$

$\begin{array}{lll}5.4 & \text { V0 detector } & 132\end{array}$

$\begin{array}{lll}\text { 5.4.1 Design considerations } & 132\end{array}$

$\begin{array}{lll}5.4 .2 & \text { Detector layout } & 133\end{array}$

$\begin{array}{lll}\text { 5.4.3 Front-end electronics } & 134\end{array}$

$\begin{array}{lll}5.5 & \text { T0 detector } & 136\end{array}$

$\begin{array}{lll}\text { 5.5.1 Design considerations } & 136\end{array}$

$\begin{array}{lll}\text { 5.5.2 Detector layout } & 136\end{array}$

5.5.3 Fast electronics and readout 136

6 Trigger, online and offline computing 139

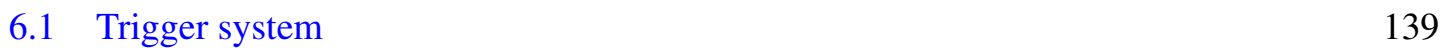

$\begin{array}{ll}\text { 6.1.1 Design considerations } & 139\end{array}$

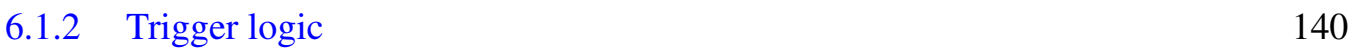

$\begin{array}{lll}\text { 6.1.3 Trigger inputs and classes } & 144\end{array}$

$\begin{array}{lll}6.1 .4 & \text { Trigger data } & 144\end{array}$

6.1.5 Event rates and rare events $\quad 145$

6.2 Data AcQuisition (DAQ) System 146

$\begin{array}{lll}\text { 6.2.1 Design considerations } & 146\end{array}$

$\begin{array}{ll}\text { 6.2.2 System architecture } & 147\end{array}$

$\begin{array}{ll}\text { 6.2.3 System flexibility and scalability } & 150\end{array}$

$\begin{array}{lll}\text { 6.2.4 Event rates and rare events } & 151\end{array}$

6.2.5 Data challenges 151

6.3 High-Level Trigger (HLT) 153

6.3.1 Introduction 153

6.3.2 Architecture 154

6.3.3 Cluster management 156

$\begin{array}{lll}\text { 6.3.4 Software architecture } & 157\end{array}$

6.3.5 HLT interfaces to other online systems and offline 159

6.3.6 Online data processing 160

$\begin{array}{ll}6.4 & \text { Offline computing } \\ & 162\end{array}$

$\begin{array}{lll}\text { 6.4.1 Introduction } & 162\end{array}$

$\begin{array}{ll}\text { 6.4.2 Computing model } & 163\end{array}$

$\begin{array}{lll}\text { 6.4.3 Distributed computing } & 167\end{array}$

$\begin{array}{lll}6.4 .4 & \text { AliRoot framework } & 170\end{array}$

$\begin{array}{ll}\text { 6.4.5 The quasi-online environment } & 180\end{array}$

7 Control System $\quad 183$

$\begin{array}{lll}7.1 & \text { Detector Control System (DCS) } & 183\end{array}$

$\begin{array}{lll}7.1 .1 & \text { Introduction } & 183\end{array}$ 
7.1.2 Design strategy and system architecture

7.1.3 System implementation and operation

$\begin{array}{lll}7.2 & \text { Experiment Control System (ECS) } & 192\end{array}$

7.2.1 Requirements 192

$\begin{array}{lll}\text { 7.2.2 } & \text { System architecture } & 193\end{array}$

7.2.3 Interfaces to the online systems 195

$\begin{array}{lll}7.3 & \text { Online Detector Calibration } & 197\end{array}$

8 Performance 199

$\begin{array}{lll}8.1 & \text { Track and vertex reconstruction } & 200\end{array}$

8.1.1 Primary vertex determination 200

$\begin{array}{lll}8.1 .2 & \text { Track reconstruction } & 201\end{array}$

8.1.3 Secondary-vertex finding 205

$\begin{array}{lll}8.2 & \text { Particle identification } & 207\end{array}$

8.2.1 Charged-hadron identification 207

8.2.2 Electron identification $\quad 212$

$\begin{array}{lll}8.3 & \text { Neutral-particle detection } & 212\end{array}$

$\begin{array}{ll}8.4 \text { Muon detection } & 215\end{array}$

$\begin{array}{lr}\text { ALICE acronym list } & 219\end{array}$

$\begin{array}{ll}\text { Bibliography } & 226\end{array}$ 


\section{Chapter 1}

\section{Introduction}

\subsection{ALICE experiment}

ALICE (A Large Ion Collider Experiment) is a general-purpose, heavy-ion detector at the CERN LHC which focuses on QCD, the strong interaction sector of the Standard Model. It is designed to address the physics of strongly interacting matter and the quark-gluon plasma at extreme values of energy density and temperature in nucleus-nucleus collisions. It will allow a comprehensive study of hadrons, electrons, muons, and photons produced in the collision of heavy nuclei $(\mathrm{Pb}-$ $\mathrm{Pb}$ ), up to the highest multiplicities anticipated at the LHC. The physics programme also includes collisions with lighter ions and at lower energy, in order to vary energy density and interaction volume, as well as dedicated proton-nucleus runs. Data taking during proton-proton runs at the top LHC energy will provide reference data for the heavy-ion programme and address a number of specific strong-interaction topics for which ALICE is complementary to the other LHC detectors.

The first conceptual ideas for a general-purpose, heavy-ion detector at the LHC were formulated in a workshop, sponsored by ECFA, at the end of 1990 [1]. The ALICE concept evolved via the Expression of Interest [2] and a Letter of Intent [3] towards the Technical Proposal [4] and its Addenda [5-7]. The experiment was approved in 1997 and the designs of the different detector systems are described in detail in a number of Technical Design Reports [8-19]. The expected detector performance and the physics reach, based on detailed simulations, are summarized in the Physics Performance Report [20, 21]. ${ }^{1}$

The ALICE detector is built by a collaboration including currently over 1000 physicists and engineers from 105 Institutes in 30 countries. Its overall dimensions are $16 \times 16 \times 26 \mathrm{~m}^{3}$ with a total weight of approximately $10000 \mathrm{t}$. ALICE consists of a central barrel part, which measures hadrons, electrons, and photons, and a forward muon spectrometer. The central part covers polar angles from $45^{\circ}$ to $135^{\circ}$ and is embedded in a large solenoid magnet reused from the L3 experiment at LEP. From the inside out, the barrel contains an Inner Tracking System (ITS) of six planes of high-resolution silicon pixel (SPD), drift (SDD), and strip (SSD) detectors, a cylindrical Time-Projection Chamber (TPC), three particle identification arrays of Time-of-Flight (TOF), Ring Imaging Cherenkov (HMPID) and Transition Radiation (TRD) detectors, and two electromagnetic

\footnotetext{
${ }^{1}$ The ALICE detector was described previously in the PPR and some figures and tables are reproduced in this article with permission from the publisher.
} 
calorimeters (PHOS and EMCal). All detectors except HMPID, PHOS, and EMCal cover the full azimuth. The forward muon arm $\left(2^{\circ}-9^{\circ}\right)$ consists of a complex arrangement of absorbers, a large dipole magnet, and fourteen planes of tracking and triggering chambers. Several smaller detectors (ZDC, PMD, FMD, T0, V0) for global event characterization and triggering are located at small angles. An array of scintillators (ACORDE) on top of the L3 magnet is used to trigger on cosmic rays. Table 1.1 and figures 1.1 , and 1.2 summarize the acceptance and location of the various detector systems.

Most detector systems will be installed and ready for data taking by mid 2008 when the LHC is scheduled to start operation, with the exception of parts of PHOS ( 1 out of 5 modules installed), TRD (4 out of 18), PMD, and EMCal (construction started in 2008). These detectors will be completed for the high-luminosity ion runs expected in 2010 and after.

\subsection{Design considerations}

\subsubsection{Physics observables}

As the single dedicated heavy-ion experiment at the LHC, ALICE is a general-purpose detector addressing a broad range of observables which were typically covered at previous accelerators (AGS, SPS, RHIC) by a suite of more specialized experiments. A comprehensive overview of the physics requirements as well as the expected performance of ALICE is given in the Physics Performance Reports [20, 21] and briefly summarized below according to the aspect of the collision on which they provide some information.

Global event features such as multiplicity and transverse or zero-degree energy flow define the geometry, i.e. impact parameter, shape and orientation of the collision volume, and number of interacting nucleons. Nuclear modifications to the parton distribution function can be extracted by comparing global event features and, more directly, specific hard processes (e.g. direct photons, heavy flavours in pp, pA and A-A collisions.

Heavy flavour production, and jet fragmentation will probe parton kinematics and energy loss in the plasma phase. Elliptic flow is sensitive to plasma properties like shear viscosity and equation of state. Promt photons can reveal the thermal radiation from the early phase. Quarkonia production probes deconfinement and parton recombination whereas resonance parameters (masses, branching ratios) is sensitive to chiral symmetry restoration. Particle ratios and $p_{\mathrm{t}}$ distributions are governed by thermodynamical properties and hydrodynamical evolution at and around the phase transition. Particle interferometry measures the space time evolution of the collision, and nonstatistical fluctuations would be a sign of critical phenomena in the vicinity of a first-order phase transition.

\subsubsection{Performance specification}

The choice and design of ALICE is driven by the physics requirements as well as by the experimental conditions expected in nucleus-nucleus collisions at the LHC. The most stringent design constraint is the extreme particle multiplicity, which could be up to three orders of magnitude larger than in typical pp interactions at the same energy and a factor two to five still above the highest 
Table 1.1: Summary of the ALICE detector subsystems. The acceptance in $\eta$ is calculated from the nominal interaction point and is $360^{\circ}$ in azimuth, unless noted otherwise. The position is the approximate distance from the interaction point to the face of the detector and corresponds to the radius for barrel detectors (inner and outer radius for the TPC and TRD) or the position along the beam ( $z$ coordinate) for the others. The dimension corresponds to the total area covered by active detector elements. 'Channels' is the total number of independent electronic readout channels. In case a detector is subdivided, the numbers refer to the individual components (e.g. pixel layers 1 and 2, muon tracking stations $1-5$ ).

\begin{tabular}{|c|c|c|c|c|}
\hline Detector & Acceptance $(\eta, \phi)$ & Position (m) & Dimension $\left(\mathrm{m}^{2}\right)$ & Channels \\
\hline ITS layer 1,2 (SPD) & $\pm 2, \pm 1.4$ & $0.039,0.076$ & 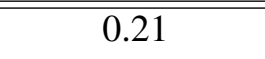 & $9.8 \mathrm{M}$ \\
\hline ITS layer 3,4 (SDD) & $\pm 0.9, \pm 0.9$ & $0.150,0.239$ & 1.31 & 133000 \\
\hline ITS layer 5,6 (SSD) & $\pm 0.97, \pm 0.97$ & $0.380,0.430$ & 5.0 & $2.6 \mathrm{M}$ \\
\hline TPC & $\begin{array}{l} \pm 0.9 \text { at } \mathrm{r}=2.8 \mathrm{~m} \\
\pm 1.5 \text { at } \mathrm{r}=1.4 \mathrm{~m}\end{array}$ & $0.848,2.466$ & $\begin{array}{c}\text { readout } 32.5 \mathrm{~m}^{2} \\
\text { Vol. } 90 \mathrm{~m}^{3}\end{array}$ & $\overline{5557568}$ \\
\hline TRD & \pm 0.84 & $2.90,3.68$ & 716 & $1.2 \mathrm{M}$ \\
\hline TOF & \pm 0.9 & 3.78 & 141 & 157248 \\
\hline HMPID & $\pm 0.6,1.2^{\circ}<\phi<58.8^{\circ}$ & 5.0 & 11 & 161280 \\
\hline PHOS & $\pm 0.12,220^{\circ}<\phi<320^{\circ}$ & 4.6 & 8.6 & 17920 \\
\hline EMCal & $\pm 0.7,80^{\circ}<\phi<187^{\circ}$ & 4.36 & 44 & 12672 \\
\hline ACORDE & $\pm 1.3,-60^{\circ}<\phi<60^{\circ}$ & 8.5 & 43 & 120 \\
\hline \multicolumn{5}{|l|}{ Muon Spectrometer } \\
\hline Tracking station 1 & \multirow[t]{5}{*}{$-2.5<\eta<-4.0$} & -5.36 & 4.7 & \multirow[t]{5}{*}{$1.08 \mathrm{M}$} \\
\hline Tracking station 2 & & -6.86 & 7.9 & \\
\hline Tracking station 3 & & -9.83 & 14.4 & \\
\hline Tracking station 4 & & -12.92 & 26.5 & \\
\hline Tracking station 5 & & -14.22 & 41.8 & \\
\hline Trigger station 1 & \multirow[t]{2}{*}{$-2.5<\eta<-4.0$} & -16.12 & 64.6 & \multirow[t]{2}{*}{21000} \\
\hline Trigger station 2 & & -17.12 & 73.1 & \\
\hline ZDC:ZN & $|\eta|<8.8$ & \pm 116 & $2 \times 0.0049$ & 10 \\
\hline ZDC:ZP & $\begin{array}{c}6.5<|\eta|<7.5 \\
-9.7^{\circ}<\phi<9.7^{\circ}\end{array}$ & \pm 116 & $2 \times 0.027$ & 10 \\
\hline ZDC:ZEM & $\begin{array}{c}4.8<\eta<5.7, \\
-16^{\circ}<\phi<16^{\circ} \text { and } \\
164^{\circ}<\phi<196^{\circ}\end{array}$ & 7.25 & $2 \times 0.0049$ & 2 \\
\hline PMD & $2.3<\eta<3.7$ & 3.64 & 2.59 & 2221184 \\
\hline FMD disc 1 & $3.62<\eta<5.03$ & inner: 3.2 & & \\
\hline FMD disc 2 & $1.7<\eta<3.68$ & $\begin{array}{l}\text { inner: } 0.834 \\
\text { outer: } 0.752\end{array}$ & 0.266 & 51200 \\
\hline FMD disc 3 & $-3.4<\eta<-1.7$ & $\begin{array}{c}\text { inner:- }-0.628 \\
\text { outer:-0752 }\end{array}$ & & \\
\hline$\overline{\mathrm{V} \text { V0A }}$ & $2.8<\eta<5.1$ & 3.4 & 0.548 & 32 \\
\hline V0C & $-1.7<\eta<-3.7$ & -0.897 & 0.315 & 32 \\
\hline T0A & $4.61<\eta<4.92$ & 3.75 & 0.0038 & 12 \\
\hline T0C & $-3.28<\eta<-2.97$ & -0.727 & 0.0038 & 12 \\
\hline
\end{tabular}




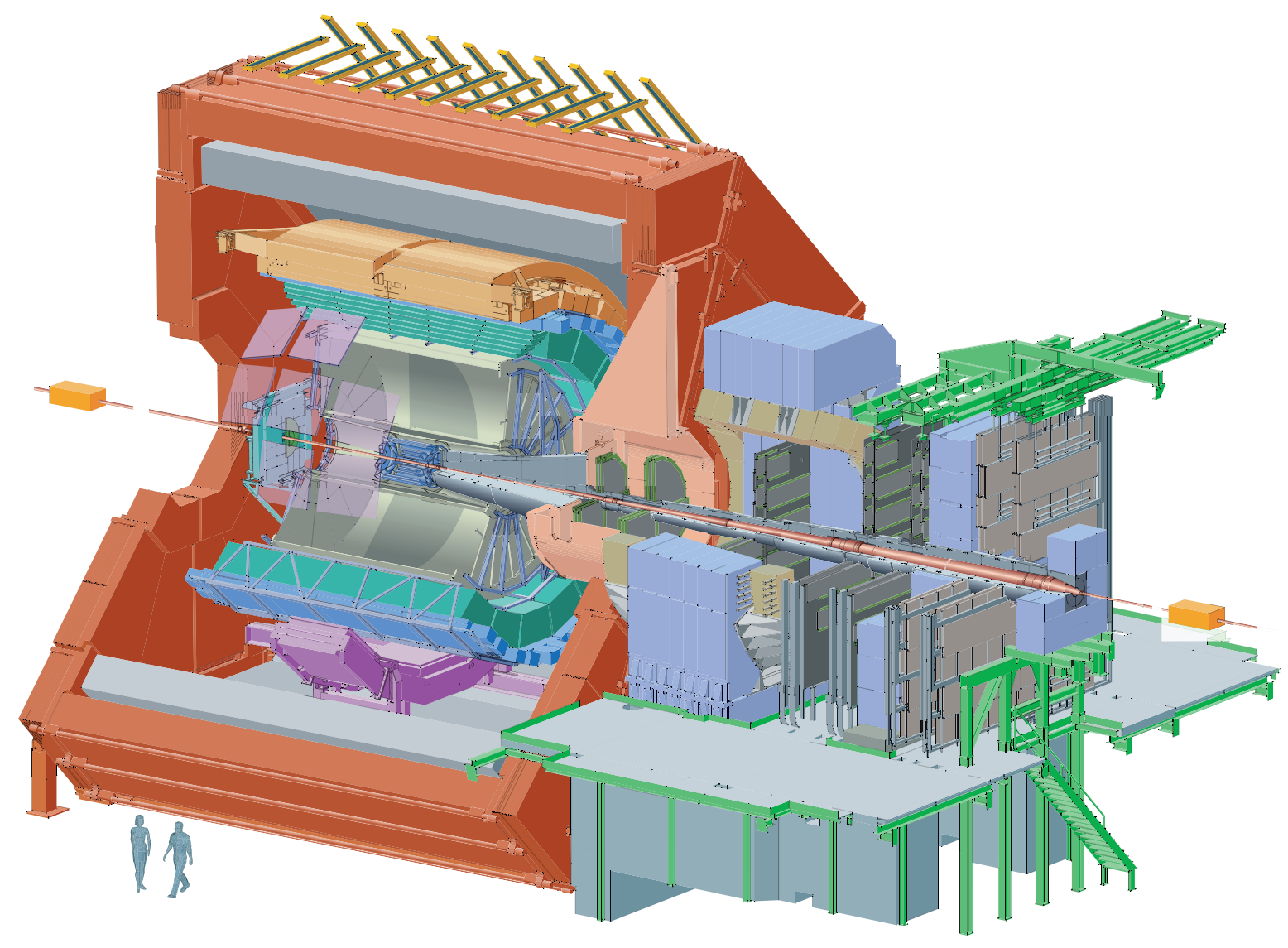

Figure 1.1: ALICE schematic layout.

multiplicities measured at RHIC. Originally, estimates for the charged particle multiplicity density at mid-rapidity in central $\mathrm{Pb}-\mathrm{Pb}$ collisions spanned the range from $\mathrm{d} N / \mathrm{d} \eta=2000$ up to almost $\mathrm{d} N / \mathrm{d} \eta=8000$. More recent extrapolations from RHIC measurements point to significantly lower values of $\mathrm{d} N / \mathrm{d} \eta=1500-4000$. The design of ALICE was optimized for a value of about $\mathrm{d} N / \mathrm{d} \eta=$ 4000 , but tested with simulations up to twice that amount. The tracking was made particularly safe and robust by using mostly three-dimensional hit information with many points (up to 150) in a moderate field of $0.5 \mathrm{~T}$. A large dynamic range is required for momentum measurement, spanning more than three orders of magnitude from tens of $\mathrm{MeV} / \mathrm{c}$ (collective effects at large length scales, good acceptance for resonance decays) to well over $100 \mathrm{GeV} / c$ (jet physics). This is achieved with a combination of very low material thickness to reduce multiple scattering at low $p_{\mathrm{t}}\left(13 \% \mathrm{X}_{0}\right.$ up to the end of the TPC) and a large tracking lever arm of up to $3.5 \mathrm{~m}$ to guarantee a good resolution at high $p_{\mathrm{t}}$. Particle Identification (PID) over much of this momentum range is essential, as many observables are either mass or flavour dependent. ALICE employs essentially all known PID techniques: specific ionization energy loss $\mathrm{d} E / \mathrm{d} x$, time-of-flight, transition and Cherenkov radiation, electromagnetic calorimetry, muon filters, and topological decay reconstruction.

ALICE will concentrate on physics at or close to midrapidity, i.e. the region of lowest baryon density and maximum energy density. The acceptance has to be sufficient to cover particle decays even at low momentum, jet fragmentation, and to study some variables on an event-by-event basis, 

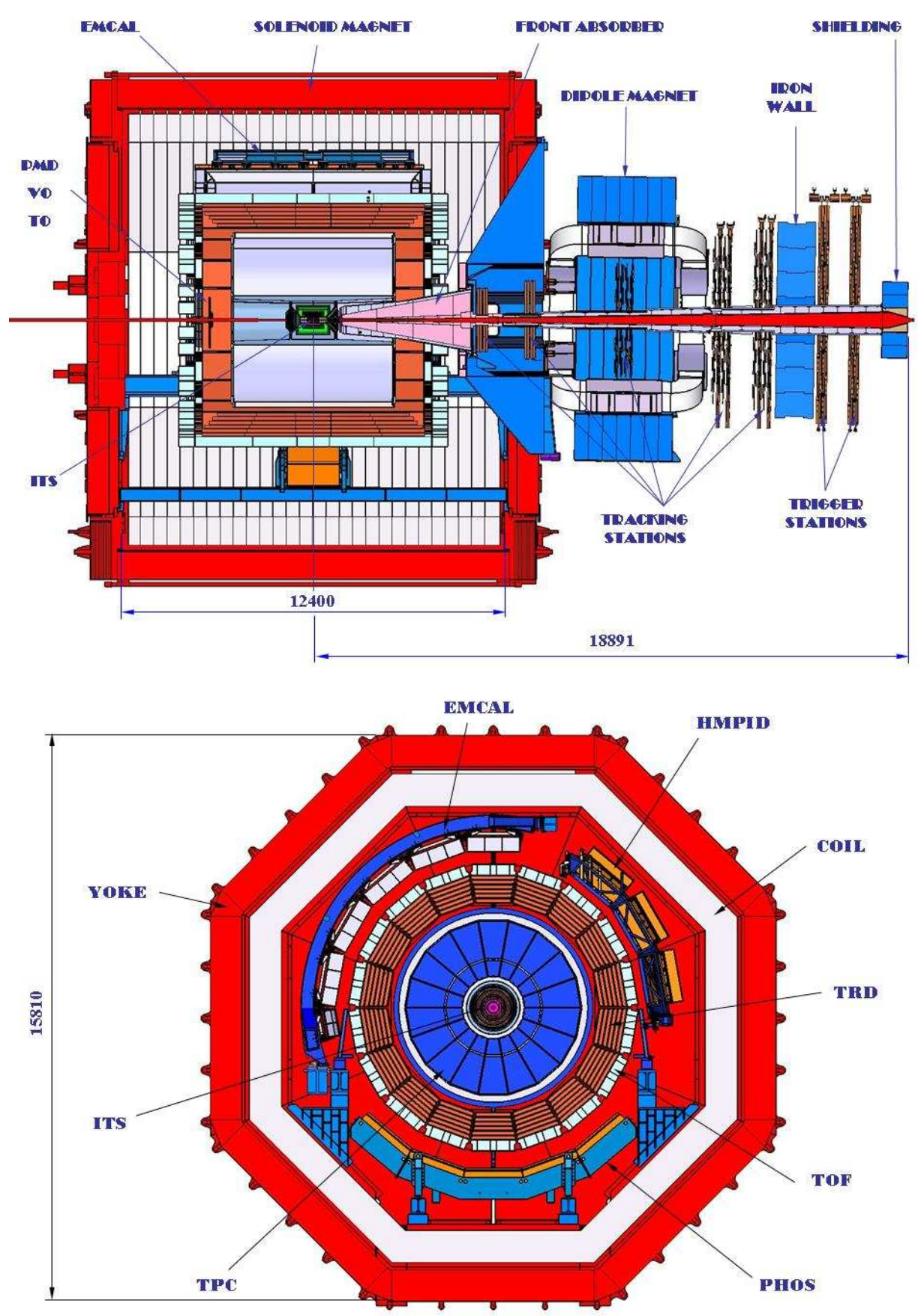

Figure 1.2: ALICE 2D cut views along the $y z$ direction (upper part) and along the $x y$ direction (lower part). The ALICE coordinate system is defined as follows: $x$-axis is perpendicular to the mean beam direction, aligned with the local horizontal and pointing to the accelerator centre; $y$ axis is perpendicular to the $x$-axis and to the mean beam direction, pointing upward; $z$-axis is parallel to the mean beam direction. The positive $z$-axis is pointing in the direction opposite to the muon spectrometer; see [22]. 
requiring typically several thousand reconstructed particles per event. Therefore the instrumented part is concentrated over 2 units in rapidity around mid-rapidity for the barrel detectors and covers 1.5 units in rapidity at small angles for the muon measurement. The interaction rate with nuclear beams at $\mathrm{LHC}$ is low $(10 \mathrm{kHz}$ for $\mathrm{Pb}-\mathrm{Pb})$ and radiation doses are moderate $(<3000 \mathrm{~Gy})$, allowing the use of slow but high-granularity detectors like TPC and SDD. Rare signals are enriched with selective triggers operating at several levels wherever possible (jets, high $p_{\mathrm{t}}$ electrons, muons, photons). With the expected integrated $\mathrm{Pb}-\mathrm{Pb}$ luminosity of $0.5 \mathrm{nb}^{-1}$ per year, the kinematic reach for example for jets within the acceptance of the EMCAL will exceed $200 \mathrm{GeV}$ and the muon arm will collect some $10000 \Upsilon$ decays. A number of rare signals like heavy flavour decays are however very difficult to select at the trigger level in heavy ion reactions; these require then a large sample of minimum bias or central collisions and a high bandwidth Data Acquisition system $(1.3 \mathrm{~GB} / \mathrm{s}$ to permanent storage) to collect of the order of a few $10^{7}$ events in the few weeks of LHC operation dedicated to ions each year.

\subsection{Detector layout}

\subsubsection{Tracking detectors}

Tracking in the central barrel is divided into the Inner Tracking System (ITS), a six-layer, silicon vertex detector, and the Time-Projection Chamber (TPC). The Transition Radiation Detector (TRD) will also be used for tracking in the central region improving the $p_{\mathrm{t}}$ resolution at high momentum.

The basic functions of the inner tracker are: i) secondary vertex reconstruction of heavy flavour and strange particle decays, ii) particle identification and tracking of low-momentum particles, and iii) improvement of the impact parameter and momentum resolution. Because of the high particle density, the innermost four layers need to be truly two-dimensional devices, i.e. silicon pixel and silicon drift detectors. The outer layers are equipped with double-sided silicon microstrip detectors. The four outer layers have analog readout for independent particle identification via $\mathrm{d} E / \mathrm{d} x$ in the non-relativistic region, which provides the ITS with stand-alone capability as a low- $p_{\mathrm{t}}$ particle spectrometer.

The need for efficient and robust tracking has led to the choice of a TPC as the main tracking detector. In spite of its drawbacks concerning speed and data volume, only such a conservative and redundant tracking device can guarantee reliable performance at order 10000 charged particles within the acceptance. The inner radius of the TPC is determined by the maximum acceptable hit density, the outer radius of $2.5 \mathrm{~m}$ by the length required for achieving $\mathrm{d} E / \mathrm{d} x$ resolution of better than 5-7\%. With this resolution the TPC can serve, in addition to tracking, as a detector for particle identification in the region of the relativistic rise, up to momenta of order $50 \mathrm{GeV} / c$. The designs of the readout chambers and electronics, as well as the choice of the operating gas, are optimized for good double-track resolution and minimal space charge induced distortions.

\subsubsection{Particle identification}

Particle identification over a large part of the phase space and for many different particles is an important design feature of ALICE with several detector systems dedicated to PID: the TOF array 
is optimized for large acceptance and average momenta; it covers the central barrel over an area of $140 \mathrm{~m}^{2}$ with 160000 individual cells at a radius of close to $4 \mathrm{~m}$. The requirement for an affordable system with a large number of channels, needed to keep the occupancy at or below $10 \%$, as well as state-of-the-art time resolution of better than $100 \mathrm{ps}$, was solved with the development of a novel type of gas detector, the Multigap Resistive Plate Chamber. The HMPID detector is a single-arm, $10 \mathrm{~m}^{2}$ array of proximity focusing ring imaging Cherenkov counters with liquid radiator and solid CsI photocathode evaporated on the segmented cathode of multiwire proportional chambers. It extends the hadron identification capabilities toward higher momenta in about $10 \%$ of the barrel acceptance. The Transition Radiation Detector will identify electrons above $1 \mathrm{GeV} / \mathrm{c}$ to study production rates of quarkonia and heavy quarks near mid-rapidity. It consists of six layers of $\mathrm{Xe} / \mathrm{CO}_{2}$-filled time expansion wire chambers following a composite foam and fibre radiator and includes a distributed tracklet processor in the front-end electronics for triggering purposes. A hadron rejection of order 100 in central collisions is required to bring the background from misidentified hadrons below the level of real electrons.

\subsubsection{Electromagnetic calorimeters}

Photons, spanning the range from thermal emission to hard QCD processes, as well as neutral mesons are measured in the small single-arm, high-resolution and high-granularity PHOS electromagnetic calorimeter. It is located far from the vertex $(4.6 \mathrm{~m})$ and made of dense scintillating crystals $\left(\mathrm{PbWO}_{4}\right)$ in order to cope with the large particle density. $\mathrm{PbWO}_{4}$ has both a small Moliere radius and sufficient light output to measure the lowest energies of interest with good resolution. A set of multiwire chambers in front of PHOS acts as a charged particle veto (CPV). The interaction and energy loss of high-energy partons in dense matter will play an essential role in the study of nuclear collisions at the LHC. In order to enhance the capabilities for measuring jet properties, a second electromagnetic calorimeter (EMCal) will be installed in ALICE starting in 2008. The EM$\mathrm{Cal}$ is a $\mathrm{Pb}$-scintillator sampling calorimeter with longitudinal wavelength-shifting fibres, read out via avalanche photo diodes. Much larger than PHOS, but with lower granularity and energy resolution, it is optimized to measure jet production rates and fragmentation functions in conjunction with the charged particle tracking in the other barrel detectors.

\subsubsection{Muon spectrometer}

The forward muon arm is primarily designed to measure the production of heavy-quark resonances $\left(\mathrm{J} / \Psi, \psi^{\prime}, \Upsilon, \Upsilon^{\prime}, \Upsilon^{\prime \prime}\right)$ with a mass resolution sufficient to separate all states. It is located at small angles $\left(2^{\circ}-9^{\circ},-4<\eta<-2.4\right)$ to provide good acceptance down to zero transverse momentum and a manageable background from hadron decays. It consists of a composite absorber $\left(\approx 10 \lambda_{\text {int }}\right)$, made with layers of both high- and low- $Z$ materials starting $90 \mathrm{~cm}$ from the vertex, a large dipole magnet with a $3 \mathrm{Tm}$ field integral placed outside the L3 magnet, and ten planes of very thin, highgranularity, cathode strip tracking stations. A second muon filter $\left(\approx 7 \lambda_{\text {int }}\right.$ of iron) at the end of the spectrometer and four planes of Resistive Plate Chambers are used for muon identification and triggering. The spectrometer is shielded by a dense conical absorber tube, of about $60 \mathrm{~cm}$ outer diameter, which protects the chambers from secondary particles created in the beam pipe. 


\subsubsection{Forward and trigger detectors}

A number of small and specialized detector systems are used for triggering or to measure global event characteristics. The event time is measured with very good precision $(<25 \mathrm{ps})$ by the T0 detector; two sets of 12 Cherenkov counters (fine mesh photomultipliers with fused silicon radiator) mounted around the beam pipe. Two arrays of segmented scintillator counters, called V0 detector, are used as minimum bias trigger and for rejection of beam-gas background. An array of 60 large scintillators (ACORDE) on top of the L3 magnet will trigger on cosmic rays for calibration and alignment purposes, as well as for cosmic ray physics. The Forward Multiplicity Detector (FMD) provides multiplicity information over a large fraction of the solid angle $(-3.4<\eta<-1.7$ and $1.7<\eta<5$ ). Charged particles are counted in rings of silicon strip detectors located at three different positions along the beam pipe. The Photon Multiplicity Detector (PMD) measures the multiplicity and spatial distribution of photons event-by-event in the region $2.3<\eta<3.7$. It consists of two planes of gas proportional counters with cellular honeycomb structure, preceded by two lead converter plates of 3 radiation lengths each. Two sets of two compact calorimeters each will be used to measure and trigger on the impact parameter of the collision (Zero Degree Calorimeter ZDC). They are made of tungsten (neutron calorimeters, $\mathrm{ZN}$ ) and brass (proton calorimeters, ZP) with embedded quartz fibres, and located on both sides in the machine tunnel, about $116 \mathrm{~m}$ from the interaction region. In addition, two small electromagnetic calorimeters (ZEM) are installed on one side, $7 \mathrm{~m}$ from the vertex, to improve the centrality selection.

\subsubsection{Trigger and data acquisition}

The hardware trigger in ALICE combines the input from detectors with fast trigger capability (T0, V0, ZDC, SPD, TOF, TRD, PHOS, EMCal, Muons, ACORDE). It operates at several levels to satisfy the individual timing requirements of the different detectors, as the ALICE electronics is in general not pipelined: a pretrigger activates the TRD electronics shortly after each interaction $(<$ $900 \mathrm{~ns}$ ) while two further levels ( $\mathrm{L} 0$ at $1.2 \mu \mathrm{s}$ and $\mathrm{L} 1$ at $6.5 \mu \mathrm{s}$ ) reduce the event rate depending on the trigger inputs. A final trigger (L2 at about $100 \mu \mathrm{s}$ ) is issued after the end of the drift time in the TPC, the slowest detector in ALICE.

The trigger includes a flexible protection against pile-up and an event priority scheme which optimises both the acceptance of rare triggers and the overall throughput of accepted events. The software-based High-Level Trigger (HLT) is a farm of up to 1000 multiprocessor PCs which will subject essentially complete events to a detailed on-line analysis. Its main task is to select or reject events and to reduce the event size by either selecting only a fraction of the data for readout (region of interest) or by compressing the complete event information. The relatively short LHC heavyion runs determine the main feature of the data acquisition system, i.e., the very large bandwidth of $1.25 \mathrm{~GB} / \mathrm{s}$ to permanent storage which is required to collect a sufficient number of events. It combines a custom optical data link, used throughout the experiment, with commodity equipment (PCs and network switches) in a highly parallel and scalable architecture. 


\section{Chapter 2}

\section{Experimental area}

\subsection{Underground area and surface facilities}

The ALICE detector and area facilities are located at Point 2 of the LHC accelerator tunnel in the commune of St. Genis-Pouilly, France. It is comprised of several surface buildings and a shaft which gives access to the underground installations, see figure 2.1. A large assembly hall, equipped with an overhead crane of $63 \mathrm{t}$ capacity houses the ALICE main workshop and a $150 \mathrm{~m}^{2}$ permanent clean room. The primary gas supply and services like electricity, cooling and ventilation are distributed from the dedicated service buildings at the surface.

The SX2 assembly hall, is built over the PX24 access shaft to the underground cavern, see figure 2.1. This large hall is equipped with an overhead crane with $65 \mathrm{t}$ capacity which is used to transfer equipment between the surface and the UX25 cavern via the shaft PX24. The building contains also the power supply for the L3 solenoid and a $80 \mathrm{~kW}$ Uninteruptible Power Supply (UPS) for the experiment.

The beam line is situated $44 \mathrm{~m}$ underground as shown in figure 2.1. The floor of the UX25 cavern is $52 \mathrm{~m}$ underground and there is $28 \mathrm{~m}$ of overburden above the detector. The detector operations are controlled and supervised from the ALICE control room in the SX2 surface building. A large part of the electronics for the ALICE detector is housed in four floors of counting rooms, which are suspended inside the PX24 shaft. The counting rooms are separated from the underground cavern by a concrete shielding and therefore accessible during machine operation. Some of the gas systems for the detector are installed on top of this shielding.

Personnel access to the experimental cavern is through the PX24 shaft. Equipment for the experiment reaches the cavern through the PX24 shaft or alternatively through a machine access shaft. The cavern is equipped with an overhead crane using two hooks of $20 \mathrm{t}$ capacity each. Two levels of gangways cover the full length of the UX25 cavern on both sides. Racks for trigger, electronics, gas systems and services are located on the cavern floor and along the cavern walls on the upper gangways.

Three distinct areas, the A side, centre, and $\mathrm{C}$ side, can be identified in the cavern. On the $\mathrm{A}$ side, the low-beta triplet is installed on a concrete platform. The space underneath this platform is used for racks and cooling systems installations. This part of the cavern also contains a magnet for compensation of the muon dipole field. This magnet is installed on a removable platform in order 


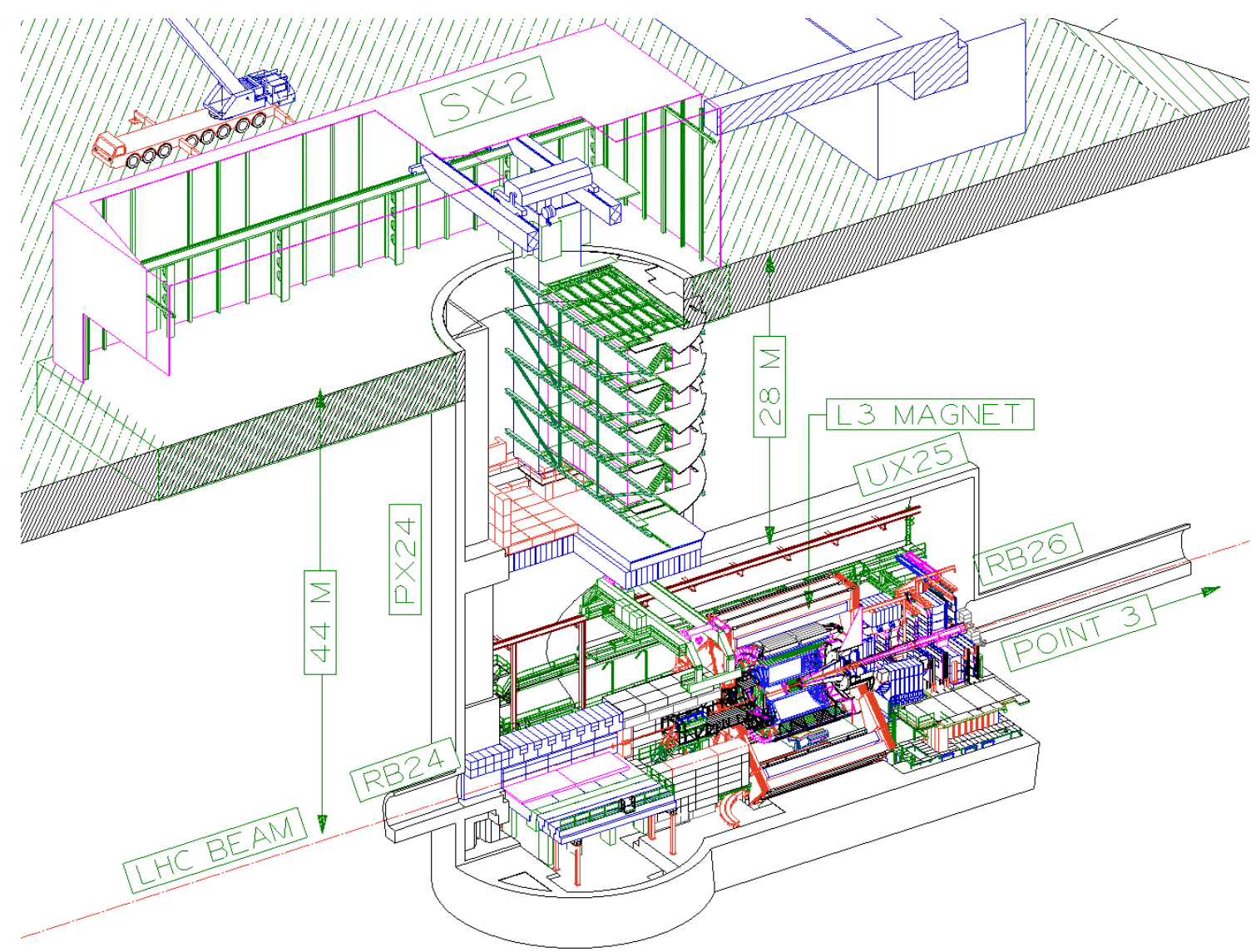

Figure 2.1: The general implementation of the ALICE experiment at Point 2 of the LHC tunnel.

to allow access for installation and maintenance of the detectors. The central part of the cavern is occupied by the L3 solenoid magnet which houses the ALICE central detectors. The dipole magnet and the forward muon system are located on the $\mathrm{C}$ side of the cavern. Below the dipole magnet, a rack area for the time critical trigger detectors is installed.

\subsubsection{Detector integration}

The general layout of the ALICE experiment is shown in figure 2.2. The detector is composed of 18 sub-detectors and their associated systems for power supply, cooling, gas, detector control, detector safety, trigger and data acquisition.

The central part of ALICE is enclosed in the L3 solenoid, which has an internal length of $12.1 \mathrm{~m}$ and a radius of $5.75 \mathrm{~m}$.

The ITS, TPC, TRD and TOF detectors are supported inside the L3 solenoid magnet by the so-called space-frame. The space-frame is a cylindrical, $7 \mathrm{~m}$ long and $8.5 \mathrm{~m}$ diameter stainless steel construction. This structure rests on two $12 \mathrm{~m}$ long rails, which span the length of the solenoid magnet. The rails are attached to the octagonal frame which supports the magnet 'doors' on each side of the magnet. There is a second pair of rails for the PHOS detector and a third pair for the EMCal detector. The HMPID is mounted on a cradle, which is attached to the space-frame. The 


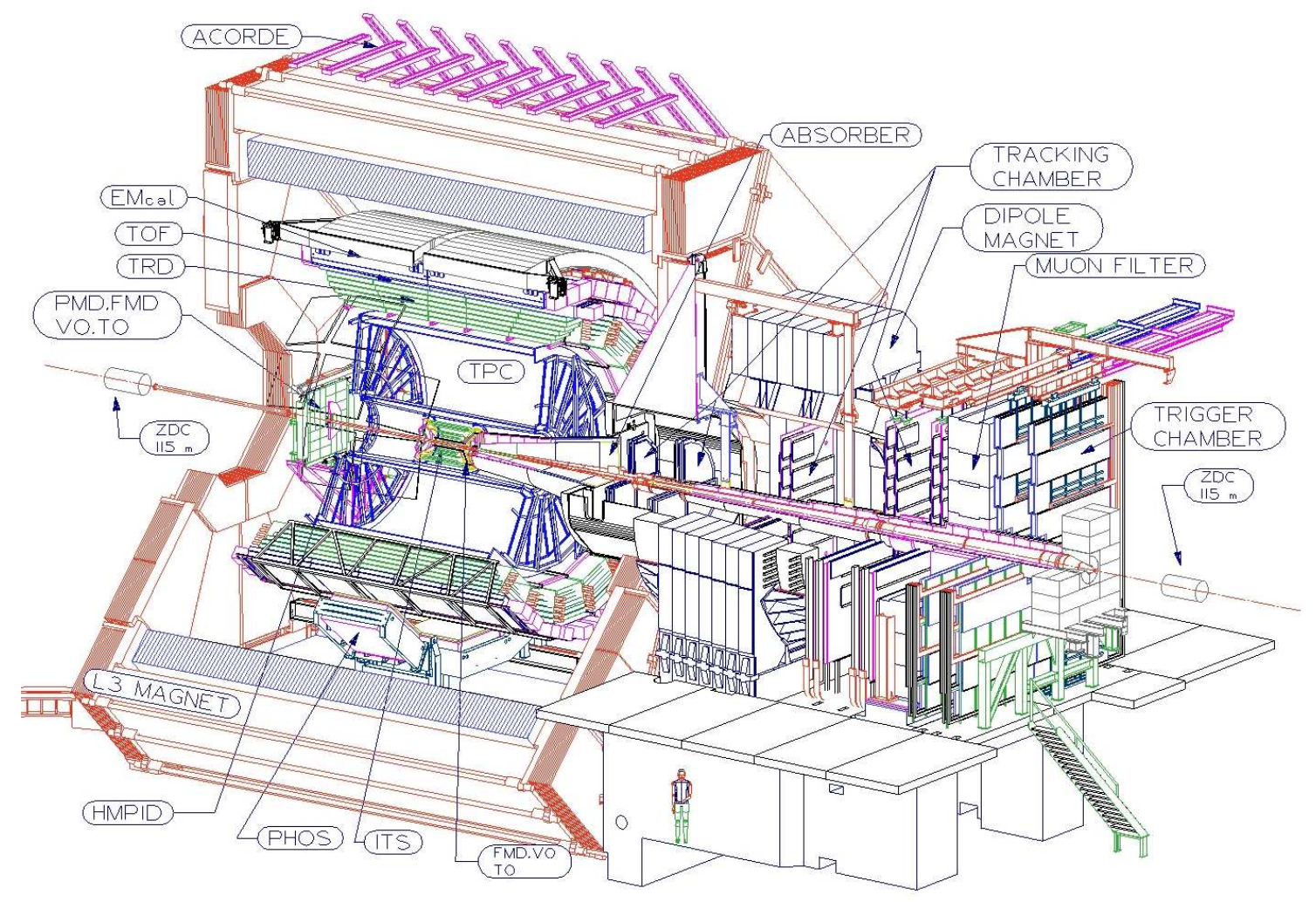

Figure 2.2: General view of the ALICE detector.

rails can connect to removable extensions on the A side in order to allow the displacement of the structures for installation or maintenance.

In addition there are large structures installed on both sides of the space-frame which serve as supports for services. The services on the $\mathrm{C}$ side of the experiment are leaving the solenoid through gaps in the permanently closed magnet door. The services on the A side of the experiment must be removable and are therefore fixed to a structure called the mini-frame which can be removed for maintenance of the ALICE detectors.

All support structures inside the solenoid field volume are made of non-magnetic materials. The layout of the support structures follows the 18 fold segmentation of the TPC. The structural beams are placed in the insensitive regions between the readout chambers of the TPC detector.

A concrete beam shield around the beamline dominates the space between the L3 solenoid and the A section of the LHC tunnel. The muon arm spectrometer is installed on the C side of the solenoid. It is comprised of a normal conducting dipole magnet and five stations of tracking chambers. Particles emitted in the direction of the muon arm are absorbed in the muon arm absorber (figure 2.2), which reaches into the L3 solenoid, and a $300 \mathrm{t}$ iron filter wall.

\subsubsection{Safety infrastructure}

Fire detection in large volumes such as surface halls and the underground cavern is ensured by roofmounted permanently installed smoke detectors. Fire detection in parts of the detector is ensured 
by a sniffer system. This system can be modified according to the layout of the detector elements. Both systems can generate level 2 and 3 alarms. Alarm level 3 is sent directly to the fire brigade for intervention. Alarms level 2 from the sniffer can be handled by the Detector Control System (DCS) which would cut electrical power and flammable gas in the detector. The racks on the C side are equipped with individual smoke detectors and a $\mathrm{CO}_{2}$ fire-extinguishing system.

Air is also sampled, by the sniffer system, for oxygen deficiency hazard in some restricted areas such as the L3 magnet and the rack area on the $\mathrm{C}$ side. In case of lack of oxygen during an access in the given area, the evacuation alarm will be triggered.

The closed volumes of the solenoid magnet are connected to a nitrogen-flushing system. In the event of a fire, nitrogen is channeled by the ventilation systems directly to these areas.

The surface gas building and the gas racks in UX25 are monitored by permanently-installed flammable-gas detectors. Flammable gas detection in the detector environment is ensured by the sniffer system.

The ALICE experiment fully complies with the CERN rules and regulations in matters of safety [23]

\subsection{Radiation monitoring and shielding installations}

Radiation monitors for the dose surveillance of material and equipment are installed in the solenoid magnet, on the A side of the experiment. Additional radiation monitors are installed in the cavern to monitor the induced activity and warn people entering during shut-downs. Monitors are also installed in the PX24 and SX2 to monitor the stray radiation during LHC operation. Fixed monitors survey the air and water release.

The radiation environment in the experimental cavern was simulated for the planned running scenario of the ALICE experiment. Running with pp, low- and high-mass ion-ion collisions over a ten year period was assumed. Beam-beam interactions, beam-gas interactions and miss-injected beams were considered as potential radiation sources. Table 2.1 shows doses and fluences for different central detectors [24]. The dose at the location of the inner layer of the ITS pixel detector is estimated to $2.7 \mathrm{kGy}$ with a $1 \mathrm{MeV}$ neutron equivalent fluency of $3.5 \times 10^{12} \mathrm{~cm}^{-2}$ for the ten years operation scenario. The doses at the different rack locations inside the cavern are at most of the order of $5 \mathrm{~Gy}$ or substantially lower. The neutron fluencies are typically $10^{3} \mathrm{~cm}^{-2}$ with a maximum at $10^{8} \mathrm{~cm}^{-2}$ for one location.

The $2.4 \mathrm{~m}$ thick PX24 shielding plug is not sufficient to shield the produced radiation during nominal operation of LHC. A permanent shielding from $1.6 \mathrm{~m}$ thick concrete blocks is therefore put into place around the beam line as an extension of the machine tunnel over the length of the LHC elements.

The $10 \mathrm{~m}$ long gap between this permanently installed shielding and the front of the L3 solenoid is covered by $1.6 \mathrm{~m}$ thick concrete shielding blocks which are removable over a length of $7.5 \mathrm{~m}$ in order to open the solenoid doors and to gain access to the central detectors for installation and removal of modules.

The radiation from the LHC machine towards the experiment is shielded by combined steel $(0.8 \mathrm{~m})$ and concrete $(1.0 \mathrm{~m})$ walls in the LHC tunnel to either side of the UX25 cavern. These plugs fill the tunnel cross-section around the machine magnets and the Cryogenic-Ring Line (QRL). 
Table 2.1: Doses and neutron fluences in central detectors [24].

\begin{tabular}{|l|c|c|c|}
\hline System & Radius $(\mathrm{cm})$ & Dose $(\mathrm{Gy})$ & $\mathrm{h}-\Phi\left(\mathrm{cm}^{-2}\right) 1 \mathrm{MeV}$ n-equ \\
\hline \hline SPD1 & 3.9 & $2.7 \times 10^{3}$ & $3.5 \times 10^{12}$ \\
SPD2 & 7.6 & $6.8 \times 10^{2}$ & $1.3 \times 10^{12}$ \\
SDD1 & 14 & $2.5 \times 10^{2}$ & $5.5 \times 10^{11}$ \\
SDD2 & 24 & $1.2 \times 10^{2}$ & $3.2 \times 10^{11}$ \\
SSD1 & 40 & $5.0 \times 10^{1}$ & $2.3 \times 10^{11}$ \\
SSD2 & 45 & $3.0 \times 10^{1}$ & $2.0 \times 10^{11}$ \\
TPC(in) & 78 & $1.6 \times 10^{1}$ & $1.5 \times 10^{11}$ \\
TPC(out) & 278 & $2.2 \times 10^{0}$ & $4.5 \times 10^{10}$ \\
TRD & 320 & $1.8 \times 10^{0}$ & $2.6 \times 10^{10}$ \\
TOF & 350 & $1.2 \times 10^{0}$ & $2.0 \times 10^{10}$ \\
PHOS & 460 & $5.0 \times 10^{-1}$ & $1.7 \times 10^{10}$ \\
HMPID & 460 & $5.0 \times 10^{-1}$ & $1.7 \times 10^{10}$ \\
\hline
\end{tabular}

\subsection{Magnets}

The experiment includes a solenoid magnet previously used in the L3 experiment of LEP which houses the central detectors and a dipole magnet situated next to the solenoid which is part of the forward muon spectrometer.

A dipole magnet with resistive coils and a horizontal field perpendicular to the beam axis is an integral part of the muon spectrometer arm. The required field integral in the forward direction is $3 \mathrm{Tm}$. The tracking stations are located up to $14 \mathrm{~m}$ from the IP.

\subsubsection{Solenoid}

The L3 solenoid magnet is a room temperature solenoid with an octagonal aluminium coil cooled by demineralised water via external cooling circuits. The octagonal steel flux return yoke is closed by pole cap 'doors'. The magnet was put into operation in 1988 for the L3 LEP Experiment [25].

The elevation of the LHC beam axis by $300 \mathrm{~mm}$ with respect to LEP results in an eccentric position of the L3 solenoid, which was centered on the LEP interation point. As an improvement measure additional steel elements (the so-called 'plug') were inserted to reduce the diameter of the hole in the L3 doors and compensate the off axis effect. The field variations in the volume of the detectors, up to $2.5 \mathrm{~m}$ in radius and $\pm 2.5 \mathrm{~m}$ along the axis from IP, are below $2 \%$ of the nominal field value. This is a reduction by a factor of two as compared to the original L3 field quality.

The main characteristics of the L3 solenoid are given in table 2.2

\subsubsection{Dipole}

The dipole magnet [26] is placed $7 \mathrm{~m}$ from the interaction vertex, at some $10 \mathrm{~cm}$ distance from the L3 solenoid. 
Table 2.2: L3 solenoid magnet characteristics.

\begin{tabular}{|l|c|c|}
\hline Parameter & Value & Unit \\
\hline \hline Nom. flux density & 0.50 & $\mathrm{~T}$ \\
Ampere turns & 5.04 & $\mathrm{MA}$ \\
Operating current & 30.00 & $\mathrm{kA}$ \\
Stored energy & 150 & $\mathrm{MJ}$ \\
Power & 4.20 & $\mathrm{MW}$ \\
Number of turns & 168 & \\
Inner radius of coil & 5930 & $\mathrm{~mm}$ \\
Avg. radius of coil & 6375 & $\mathrm{~mm}$ \\
Total weight & 7800 & $\mathrm{t}$ \\
Overall dimensions $(\mathrm{D} \times \mathrm{L})$ & $15.8 \times 14.1$ & $\mathrm{~m}$ \\
\hline
\end{tabular}

The size (free gap between poles $3.5 \mathrm{~m}$, height of the yoke $9 \mathrm{~m}$, total weight about $900 \mathrm{t}$ ) is defined by the requirements on the angular acceptance of the spectrometer. The magnetic flux density $\left(\mathrm{B}_{\text {nom }}=0.67 \mathrm{~T}, 3 \mathrm{Tm}\right.$ field integral between IP and muon filter) is defined by the requirements on the mass resolution.

The magnet yoke is constructed from 28 low-carbon steel modules made for cost reasons from existing steel stacks which consist of $3 \mathrm{~cm}$ thick steel sheets welded to each other. The vertical poles are oriented at an angle of $9^{\circ}$ with respect to the vertical symmetry plane leaving a free gap between the poles of $2.972-3.956 \mathrm{~m}$.

The two Saddle type coils have semi-cylindrical coil ends. They are constructed from hollow aluminium conductor with square cross-section of $25.5 \mathrm{~cm}^{2}$ and an internal hole for cooling with demineralised water at a rate of some $130 \mathrm{~m}^{3} / \mathrm{hr}$. Each coil is assembled from 3 sub-coils with 4 layers of 14 turns each. They delimit the overall length of the magnet to $5 \mathrm{~m}$. The distance of the centre of the dipole yoke from the IP is $9.87 \mathrm{~m}$. The main characteristics of the ALICE dipole are shown in table 2.3

The magnet was installed in its final position on a $3 \mathrm{~m}$ high reinforced concrete platform. The close distance between the solenoid magnet and the dipole leads to a strong magnetic force (estimated at $120 \mathrm{t}$ ) between the two magnets. Measurements, at full power, do not indicate any displacement of the magnet structures. The stray field in the vicinity of the magnet attenuates rather rapidly to less than 50 Gauss at the level of the gangways.

\subsection{Beam pipe}

The beam vacuum system represents the main interface between the experiment and the LHC machine. It must therefore fulfil a dual set of requirements.

The ALICE experimental requirements include maximum transparency to particles, limited beam-gas backgrounds and conformity with the environmental and installation constraints.

The accelerator requirements include safe operation of the machine, adequate beam aperture and dynamic vacuum conditions compatible with ultimate LHC performance. 
Table 2.3: Dipole magnet characteristics.

\begin{tabular}{|l|c|c|}
\hline Parameter & Meas. Value & Unit \\
\hline Ampere turns & $/$ & $\mathrm{MA}$ \\
Operating current & 6.00 & $\mathrm{kA}$ \\
Coil voltage & 597 & $\mathrm{~V}$ \\
Power & 3.58 & $\mathrm{MW}$ \\
Inductance & 1.00 & $\mathrm{H}$ \\
Stored energy & 18 & $\mathrm{MJ}$ \\
Diff. pressure & 12 & $\mathrm{Bar}$ \\
Flow rate & 130 & $\mathrm{~m}^{3} / \mathrm{h}$ \\
Diff. temperature & 24 & $\mathrm{C}$ \\
Mag. field $\mathrm{B}_{\mathrm{x}}($ center of coils $)$ & 0.666 & $\mathrm{~T}$ \\
Field integral $\mathrm{B}_{\mathrm{x}}(-13.8 \mathrm{~m}<z<-6 \mathrm{~m})$ & 3.04 & $\mathrm{Tm}$ \\
\hline
\end{tabular}

The vacuum system is a fully in-situ baked UHV system, pumped by a combination of lumped sputter-ion and distributed Non-Evaporable Getter (NEG) pumps. The NEG system consists of a thin sputtered coating along the whole internal surface of the vacuum chambers. The average vacuum pressure has been estimated to be $10^{-8} \mathrm{~Pa}$ for $\mathrm{pp}$ run and $10^{-9} \mathrm{~Pa}$ for $\mathrm{Pb}$-ion run with nominal parameters.

The ALICE beam pipes, ranging from $19 \mathrm{~m}$ of either side of the IP consist of 3 sections (figure 2.3). The Central section (CS) beryllium beam pipe is a $4 \mathrm{~m}$ long beryllium section with internal diameter $58 \mathrm{~mm}$ and $0.8 \mathrm{~mm}$ thick wall. This part is protected by a polyimide wrapping about $80 \mu \mathrm{m}$ thick and extends along the beam from $-3585 \mathrm{~mm}<z<365 \mathrm{~mm}$. The $\mathrm{C}$ side section in the absorber consists of 3 chambers of conical stainless steel tubes which are up to $450 \mathrm{~mm}$ in diameter. The A side section uses standard LHC machine components and consists of 4 copper tubes. The main parameters of the beampipe are summarized in table 2.4

To simplify the assembly procedure, an all-metal valve with RF-bridge is located between the $\mathrm{CS}$ and A sections. Each vacuum chamber has its fixed point towards the A-side, and towards the $\mathrm{C}$-side a bellows or compensator module allowing for thermal expansions and relative beam pipe movements.

The main pump used to eliminate gases in the system is the NEG pump. After activation by heating under vacuum to above $200^{\circ} \mathrm{C}$, the NEG film gives a high distributed pumping speed for most gases. Gases not pumped by the NEG system are removed by sputter-ion pumps.

The vacuum chambers are designed to resist stress induced by vacuum and the weight of the mechanical components. However, especially the Beryllium is not able to support stresses induced by offsets during detector opening and closing procedures. The chambers will therefore be vented to neon gas at atmospheric pressure, purified to the ppb level by a special developed gas purifying system. Neon is not pumped by the NEG system, so the beam vacuum system can be made operational at the end of a short intervention by simply re-pumping the neon gas.

All chambers are permanently equipped with bake-out heaters for periodic re-activation of the NEG coating, with the exception of the central section. 

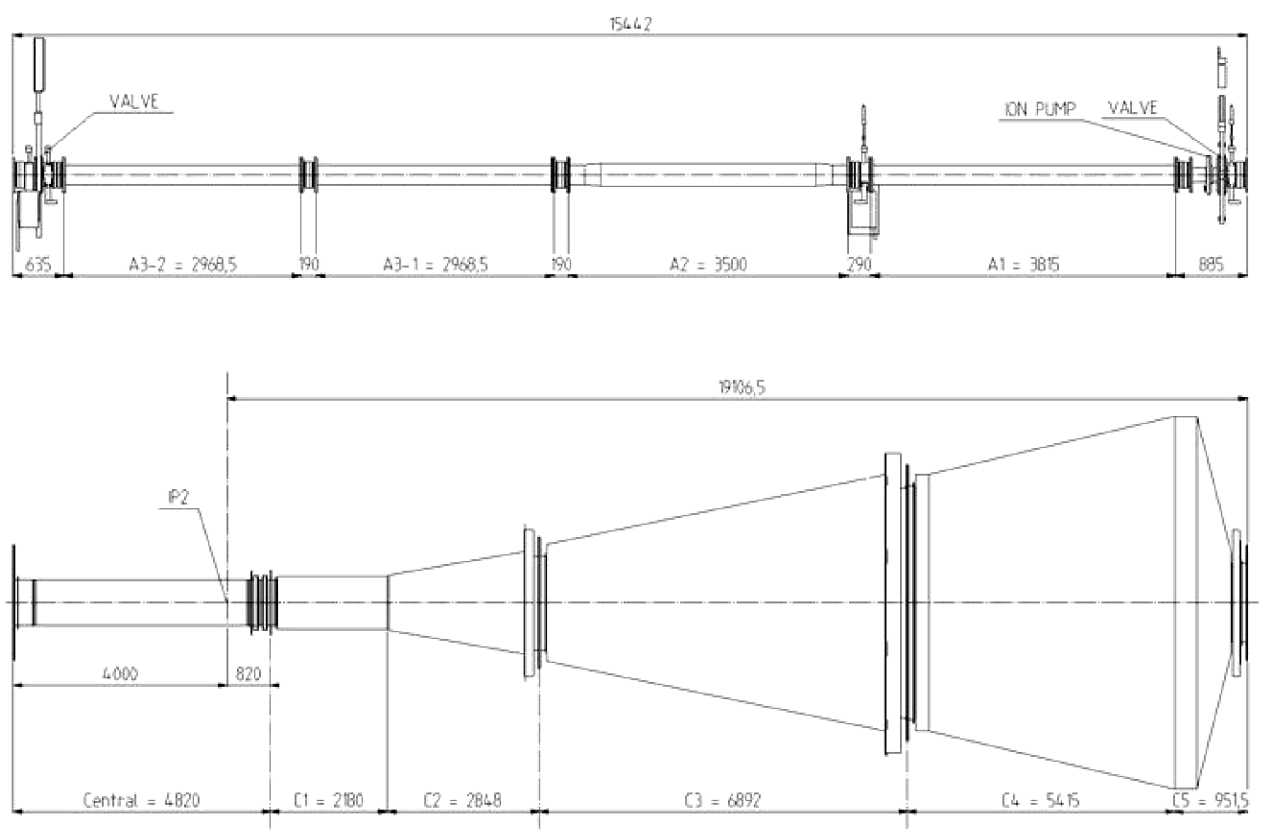

Figure 2.3: Layout of the ALICE vacuum chamber.

Table 2.4: Dimensions and materials of the ALICE vacuum chamber.

\begin{tabular}{|l|c|c|c|c|c|}
\hline Name & $\begin{array}{c}\text { Length } \\
\mathrm{mm}\end{array}$ & $\begin{array}{c}\text { min. ID } \\
\mathrm{mm}\end{array}$ & $\begin{array}{c}\text { max. ID } \\
\mathrm{mm}\end{array}$ & $\begin{array}{c}\text { Angle } \\
\mathrm{deg}\end{array}$ & Material \\
\hline \hline $\mathrm{A} / 1$ & 3815 & 80 & 80 & 0 & Copper \\
$\mathrm{A} / 2$ & 3500 & 60 & 96 & 0 & Copper oval chamber \\
$\mathrm{A} / 3-1$ & 2968.5 & 80 & 80 & 0 & Copper \\
$\mathrm{A} / 3-2$ & 2968.5 & 80 & 80 & 0 & Copper \\
\hline Central & 4800 & 58 & 58 & 0 & Beryllium, Stainless steel \\
\hline $\mathrm{C} / 1$ & 2180 & 58 & 58 & 0 & Stainless steel \\
$\mathrm{C} / 2$ & 2848 & 58 & 120 & 0.7 & Stainless steel \\
$\mathrm{C} / 3$ & 6892 & 120 & 300 & 0.8 & Stainless steel \\
$\mathrm{C} / 4$ & 5415 & 300 & 450 & 0.9 & Stainless steel \\
$\mathrm{C} / 5$ & 951.5 & 100 & 450 & 15 & Stainless steel \\
\hline
\end{tabular}




\subsection{Survey and alignment}

The survey and alignment requirements for the ALICE detector elements can be separated into two categories. First, all sub-detectors and the beam pipe must be placed at the nominal position within a specified tolerance, typically of the order of $\mathrm{mm}$, which is verified by standard survey techniques during installation and monitored with dedicated equipment during operation. The second category consists of requirements concerning the relative alignment of detector elements and the stability of their position during operation, typically of the order of $<50 \mu \mathrm{m}$ for the muon system, which is monitored by optical alignment devices, and $<10 \mu \mathrm{m}$ for the ITS, which is ensured by a rigid carbon-fibre structure.

The alignment strategy of the central detectors in the L3 magnet is determined by the mechanical interconnections of the subdetectors. The ITS detector, consisting of the Silicon Pixel Detector (SPD) at inner radius, the Silicon Drift Detector (SDD) at intermediate radius and Silicon Strip Detector (SSD) at outer radius, has a nominal clearance of $5 \mathrm{~mm}$ to the central beampipe. The SPD, SDD and SSD detector elements are positioned on a rigid carbon-fibre structure with an accuracy of better than $100 \mu \mathrm{m}$. The relative position of these elements is not monitored by external devices and all alignment questions are studied with particle tracks. The beam pipe is fixed to this ITS barrel structure on both sides with 4 steel wires at a tension of $50 \mathrm{~N}$. This guaranties a stable relative position of the beam pipe and the ITS detector.

The ITS barrel itself is held by two fixation points on the inner TPC cylinder, which together with a third point on the bottom of the barrel fix the relative position of the ITS and the TPC. The weight of the ITS causes a sag of about $0.7 \mathrm{~mm}$ of the inner TPC drum. The relative position of the ITS and the TPC is monitored by an optical alignment system with a precision of $<20, \mu \mathrm{m}$. The TPC is supported by two rails which are fixed inside the space-frame, while the space-frame itself is standing on rails which are fixed on each end of the L3 magnet.

The positioning of the ITS-TPC inside the L3 solenoid is determined by the requirement that the central beam pipe must be placed within $\pm 5 \mathrm{~mm}$ of the nominal beam axis and that the angle between the ITS-TPC axis and the magnetic field axis is less than $1 \mathrm{mrad}$. The installation of the TOF and the TRD modules on the space-frame causes a deformation of the space-frame of up to $5 \mathrm{~mm}$ and a sag of the rails supporting the space-frame of up to $10 \mathrm{~mm}$. In order to monitor the beam-pipe position and space-frame deformation during detector installation and operation, an optical monitoring system is installed. The used BCAM [27] system has a relative angular resolution of $5 \mu \mathrm{rad}$. In case the $5 \mathrm{~mm}$ tolerance is violated, the entire space-frame is moved to place the beam pipe back in the nominal position.

In the muon spectrometer the particle momentum is measured by 5 tracking stations which are not mechanically conneted. The relative position of these stations is monitored to $<50 \mu \mathrm{m}$ with a dedicated system of BCAMs. 


\section{Chapter 3}

\section{Central detectors}

\subsection{Inner Tracking System (ITS)}

The main tasks of the Inner Tracking System (ITS) are to localize the primary vertex with a resolution better than $100 \mu \mathrm{m}$, to reconstruct the secondary vertices from the decays of hyperons and $\mathrm{D}$ and $\mathrm{B}$ mesons, to track and identify particles with momentum below $200 \mathrm{MeV} / c$, to improve the momentum and angle resolution for particles reconstructed by the Time-Projection Chamber (TPC) and to reconstruct particles traversing dead regions of the TPC. The ITS therefore contributes to practically all physics topics addressed by the ALICE experiment, as discussed in detail in [20].

The ITS surrounds the beam pipe, for which it provides the mechanical support so that no relative movement will take place during operation. The beam pipe is a $800 \mu \mathrm{m}$-thick beryllium cylinder of $6 \mathrm{~cm}$ outer diameter, coaxial with the ITS detector layers. As shown schematically in figure 3.1 the ITS consists of six cylindrical layers of silicon detectors, located at radii between 4 and $43 \mathrm{~cm}$. It covers the rapidity range of $|\eta|<0.9$ for all vertices located within the length of the interaction diamond ( $\pm 1 \sigma$, i.e. $\pm 5.3 \mathrm{~cm}$ along the beam direction). The number, position and segmentation of the layers were optimized for efficient track finding and high impact-parameter resolution. In particular, the outer radius is determined by the necessity to match tracks with those from the TPC, and the inner radius is the minimum allowed by the radius of the beam pipe. The first layer has a more extended pseudo-rapidity coverage $(|\eta|<1.98)$ to provide, together with the Forward Multiplicity Detectors (FMD), continuous coverage for the measurement of chargedparticles multiplicity. The detectors and front-end electronics are held by lightweight carbon-fibre structures. The geometrical dimensions and the technology used in the various layers of the ITS are summarized in table 3.1 .

Because of the high particle density expected in heavy-ion collisions at LHC, (as many as 50 particles per $\mathrm{cm}^{2}$ have been predicted for the inner layer), and in order to achieve the required impact parameter resolution, Silicon Pixel Detectors (SPD) have been chosen for the innermost two layers, and Silicon Drift Detectors (SDD) for the following two layers. The two outer layers, where the track density is expected to be below one particle per $\mathrm{cm}^{2}$, are equipped with double-sided Silicon micro-Strip Detectors (SSD). The four outer layers have analogue readout and therefore can be used for particle identification via $\mathrm{d} E / \mathrm{d} x$ measurement in the non-relativistic $\left(1 / \beta^{2}\right)$ region. The analogue readout has a dynamic range large enough to provide the $\mathrm{d} E / \mathrm{d} x$ measurement for low- 


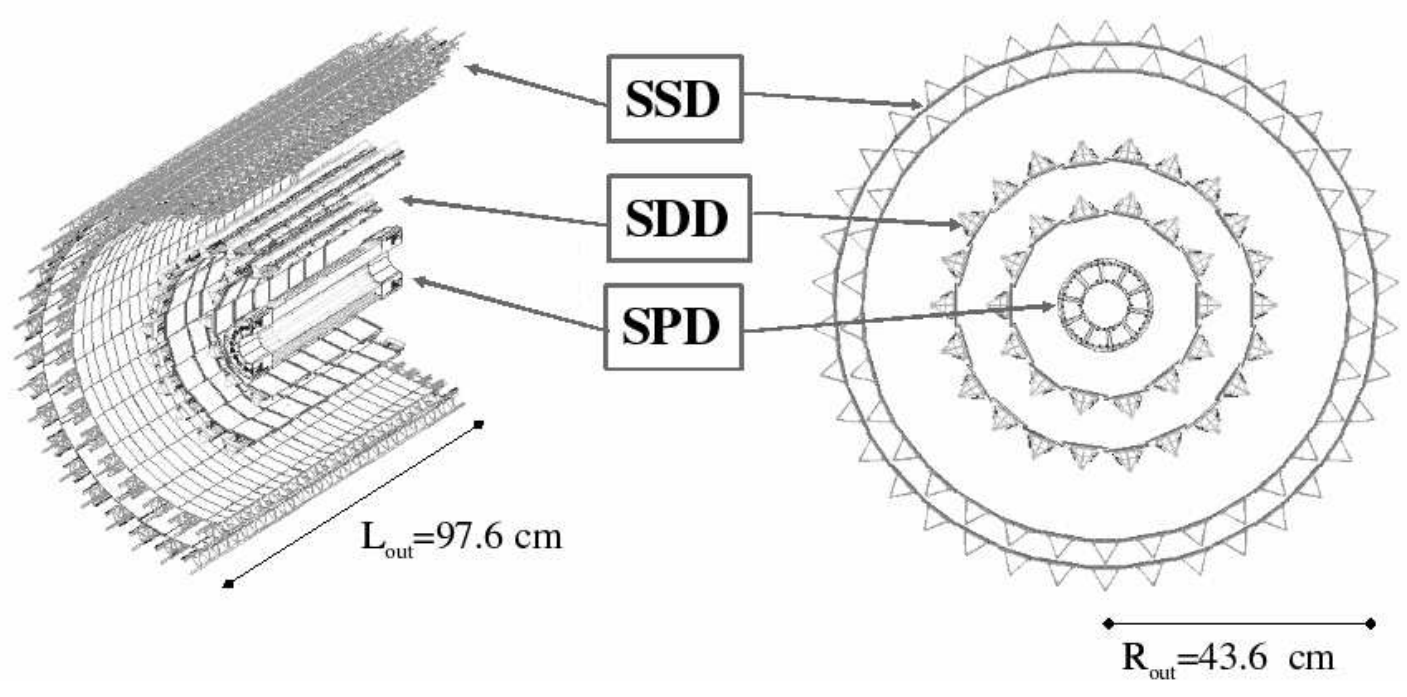

Figure 3.1: Layout of the ITS.

Table 3.1: Dimensions of the ITS detectors (active areas).

\begin{tabular}{|c|c|c|c|c|r|}
\hline Layer & Type & $r(\mathrm{~cm})$ & $\pm z(\mathrm{~cm})$ & Area $\left(\mathrm{m}^{2}\right)$ & Channels \\
\hline \hline 1 & pixel & 3.9 & 14.1 & 0.07 & 3276800 \\
2 & pixel & 7.6 & 14.1 & 0.14 & 6553600 \\
3 & drift & 15.0 & 22.2 & 0.42 & 43008 \\
4 & drift & 23.9 & 29.7 & 0.89 & 90112 \\
5 & strip & 38.0 & 43.1 & 2.20 & 1148928 \\
6 & strip & 43.0 & 48.9 & 2.80 & 1459200 \\
\hline \multicolumn{6}{|c|}{ Total area } \\
\hline \multicolumn{6}{|c|}{}
\end{tabular}

momentum, highly ionising particles, down to the lowest momentum at which tracks can still be reconstructed. This feature gives the ITS stand-alone capability as a low- $p_{\mathrm{t}}$ particle spectrometer. The main parameters for each of the three detector types are summarized in table 3.2. The overall cooling system of the ITS outer layers has been designed [28-30] to satisfy the stringent requirements of the TPC in terms of temperature stability and uniformity.

The momentum and impact parameter resolution for low-momentum particles are dominated by multiple scattering effects in the material of the detector; therefore the amount of material in the active volume has been kept to a minimum. The silicon detectors used to measure ionisation densities (drift and strips) must have a minimum thickness of approximately $300 \mu \mathrm{m}$ to provide acceptable signal-to-noise ratio. In addition, detectors must overlap to cover the solid angle entirely. Therefore, the detectors effective thickness amounts to $0.4 \%$ of $X_{0}$. The additional material in the active volume, i.e. electronics, cabling, support structure, and cooling system, has been kept at a comparable effective thickness (table 3.3). The values in the table are for the detector as built, and they are remarkably close to the goals set at the time of the design [11]. Figure 3.2 shows the integral of the material traversed by a particle crossing perpendicularly the ITS as a function 
Table 3.2: Parameters of the various detector types. A module represents a single sensor element.

\begin{tabular}{|lr|c|c|c|}
\hline \multicolumn{1}{|c|}{ Parameter } & & Silicon Pixel & Silicon Drift & Silicon Strip \\
\hline \hline Spatial precision $\mathrm{r} \varphi$ & $(\mu \mathrm{m})$ & 12 & 35 & 20 \\
Spatial precision $z$ & $(\mu \mathrm{m})$ & 100 & 25 & 830 \\
Two track resolution $\mathrm{r} \varphi$ & $(\mu \mathrm{m})$ & 100 & 200 & 300 \\
Two track resolution $z$ & $(\mu \mathrm{m})$ & 850 & 600 & 2400 \\
Cell size & $\left(\mu \mathrm{m}^{2}\right)$ & $50 \times 425$ & $202 \times 294$ & $95 \times 40000$ \\
Active area per module & $\left(\mathrm{mm}^{2}\right)$ & $12.8 \times 69.6$ & $72.5 \times 75.3$ & $73 \times 40$ \\
Readout channels per module & & 40960 & $2 \times 256$ & $2 \times 768$ \\
Total number of modules & & 240 & 260 & 1698 \\
Total number of readout channels & $(\mathrm{k})$ & 9835 & 133 & 2608 \\
Total number of cells & $(\mathrm{M})$ & 9.84 & 23 & 2.6 \\
Max. occupancy for central Pb-Pb (inner layer) & $(\%)$ & 2.1 & 2.5 & 4 \\
Max. occupancy for central Pb-Pb (outer layer) & $(\%)$ & 0.6 & 1.0 & 3.3 \\
Power dissipation in barrel & $(\mathrm{W})$ & 1350 & 1060 & 850 \\
Power dissipation end-cap & $(\mathrm{W})$ & 30 & 1750 & 1150 \\
\hline
\end{tabular}

Table 3.3: ITS material budget traversed by straight tracks perpendicularly to the detector surface. Units are percentages of radiation length.

\begin{tabular}{|l|c|c|c|c|c|c|}
\hline \multirow{2}{*}{ Detector } & \multicolumn{2}{c|}{ Pixel } & \multicolumn{2}{c|}{ Drift } & \multicolumn{2}{c|}{ Strip } \\
& Inner & Outer & Inner & Outer & Inner & Outer \\
\hline \hline Layer & 1.14 & 1.14 & 1.13 & 1.26 & 0.83 & 0.86 \\
Thermal shield/Support & & 0.52 & 0.25 & & 0.53 & \\
\hline Total & \multicolumn{4}{|c|}{$7.18(7.26$ including Air) } \\
\hline
\end{tabular}

of radius. In figure 3.3 the total material encountered by a perpendicular track crossing the ITS is shown versus the azimuthal angle. The inevitable variations due to the structure of the ladders and their overlaps remain within an acceptable range.

As discussed in [20], the relative momentum resolution achievable with the ITS is better than $2 \%$ for pions with transverse momentum between $100 \mathrm{MeV} / c$ and $3 \mathrm{GeV} / c$.

The granularity of the detectors was optimized to cope with a track density of 8000 tracks per unit of rapidity at mid-rapidity, the upper limit of theoretical predictions. Under these conditions, the ITS would detect simultaneously more than 15000 tracks. Keeping the system occupancy low, at the level of a few per cent, requires several million effective cells in each layer of the ITS. As shown in table 3.2, the occupancy is roughly constant in the different ITS layers. The ITS detectors have a spatial resolution of the order of a few tens of $\mu \mathrm{m}$, with the best precision ( $12 \mu \mathrm{m})$ for the detectors closest to the primary vertex. They thus provide a resolution on the impact-parameter measurement adequate for heavy-flavoured particle detection (better than $60 \mu \mathrm{m}$ in the $r \varphi$ plane for $p_{\mathrm{t}}>1 \mathrm{GeV} / c$ ). In addition, for momenta larger than $3 \mathrm{GeV} / c$ the spatial precision of the ITS is an essential element of the momentum resolution. 


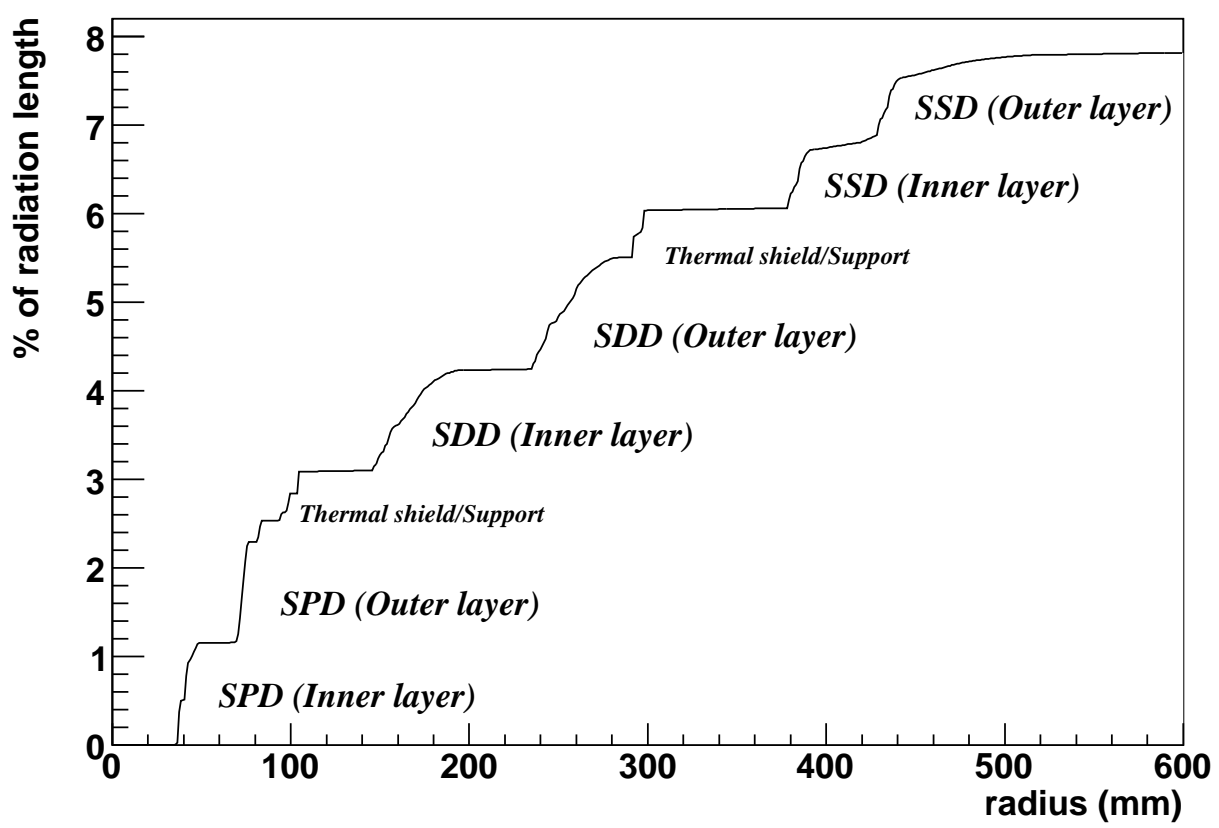

Figure 3.2: Integral of material thickness traversed by a perpendicular track originating at the primary vertex versus radius.

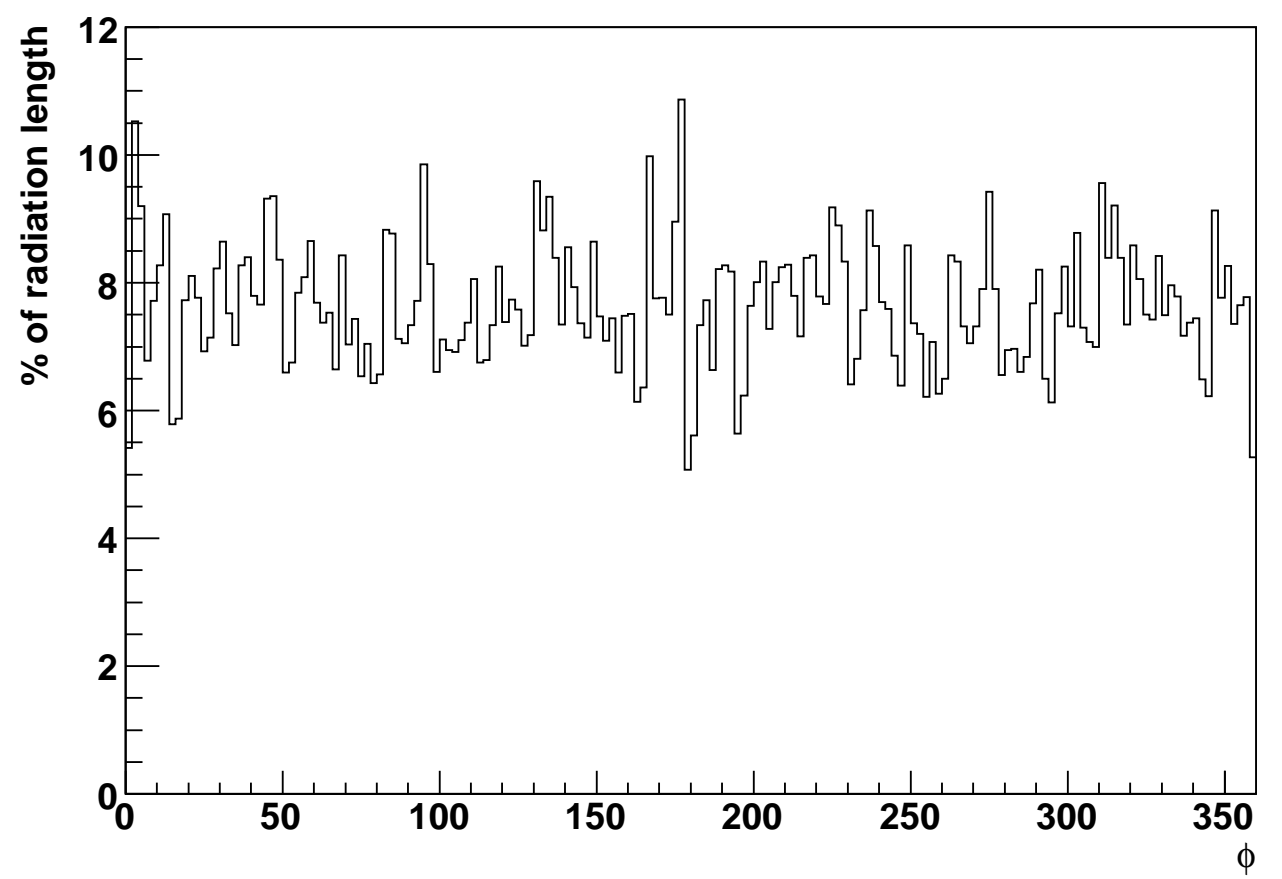

Figure 3.3: Material thinkness traversed by a perpendicular track originating at the primary vertex versus azimuthal angle. 
For the ITS, the total dose expected during the lifetime of the experiment (see section 2.2) varies from a few krad (tens of Gy) for the outer parts of the ITS to about $270 \mathrm{krad}$ ( $2.7 \mathrm{kGy}$ ) for the inner parts [20]. Although these levels are not very severe, compared, for instance, with the levels in ATLAS and CMS, all components used in the ITS design were tested for their radiation hardness to levels exceeding significantly the expected doses, and full radiation tolerant technologies have been used throughout the system for critical components, such as the front end electronics.

\subsubsection{Silicon Pixel Detector (SPD)}

\subsubsection{SPD system overview}

The Silicon Pixel Detector (SPD) constitutes the two innermost layers of the ITS. It is a fundamental element for the determination of the position of the primary vertex as well as for the measurement of the impact parameter of secondary tracks originating from the weak decays of strange, charm, and beauty particles [20]. The SPD will operate in a region where the track density could be as high as 50 tracks $/ \mathrm{cm}^{2}$, and in relatively high radiation levels: in the case of the inner layer, the integrated levels (10 years, standard running scenario) of total dose and fluence are estimated to be $\approx 2.7 \mathrm{kGy}$ and $\approx 3.5 \times 10^{12} \mathrm{n} / \mathrm{cm}^{2}$ ( $1 \mathrm{MeV}$ neutron equivalent), respectively [31]. The SPD design implements several specific solutions to minimize the material budget. The average material traversed by a straight track perpendicular to the detector surface is $\approx 1 \% X_{0}$ per layer.

The SPD is based on hybrid silicon pixels, consisting of a two-dimensional matrix (sensor ladder) of reverse-biased silicon detector diodes bump-bonded to readout chips. Each diode is connected through a conductive solder bump to a contact on the readout chip corresponding to the input of an electronics readout cell. The readout is binary: in each cell, a threshold is applied to the pre-amplified and shaped signal and the digital output level changes when the signal is above a set threshold. This technique had already been successfully applied in the WA97 and NA57 experiments at CERN [32]. The basic detector module is the half-stave. Each half-stave consists of two ladders, one Multi-Chip Module (MCM) and one high density aluminium/polyimide multi-layer interconnect. The ladder consists of a silicon sensor matrix bump bonded to 5 frontend chips. The sensor matrix includes $256 \times 160$ cells measuring $50 \mu \mathrm{m}(r \varphi)$ by $425 \mu \mathrm{m}(z)$. Longer sensor cells are used in the boundary region to ensure coverage between readout chips. The sensor matrix has an active area of $12.8 \mathrm{~mm}(r \varphi) \times 70.7 \mathrm{~mm}(z)$. The front-end chip reads out a sub-matrix of $256(r \varphi) \times 32(z)$ detector cells. The thickness of the sensor is $200 \mu \mathrm{m}$, the smallest that can be achieved with an affordable yield in standard processes. The thickness of the readout chip is $150 \mu \mathrm{m}$; the readout wafers are thinned after bump deposition, before bump bonding. The two ladders are attached and wire bonded to the high density aluminium/polyimide interconnect (pixel bus). A $200 \mu \mathrm{m}$ clearance between the short edges allows for dicing tolerances and ease of assembly. The pixel bus carries data/control bus lines and power/ground planes. The multi-chip-module, wire bonded to the pixel bus and located at the end of the half-stave, controls the front-end electronics and is connected to the off-detector readout system via optical fibre links.

Two half-staves are attached head-to-head along the $z$ direction to a carbon-fibre support sector, with the MCMs at the two ends, to form a stave. Each sector supports six staves: two on the 


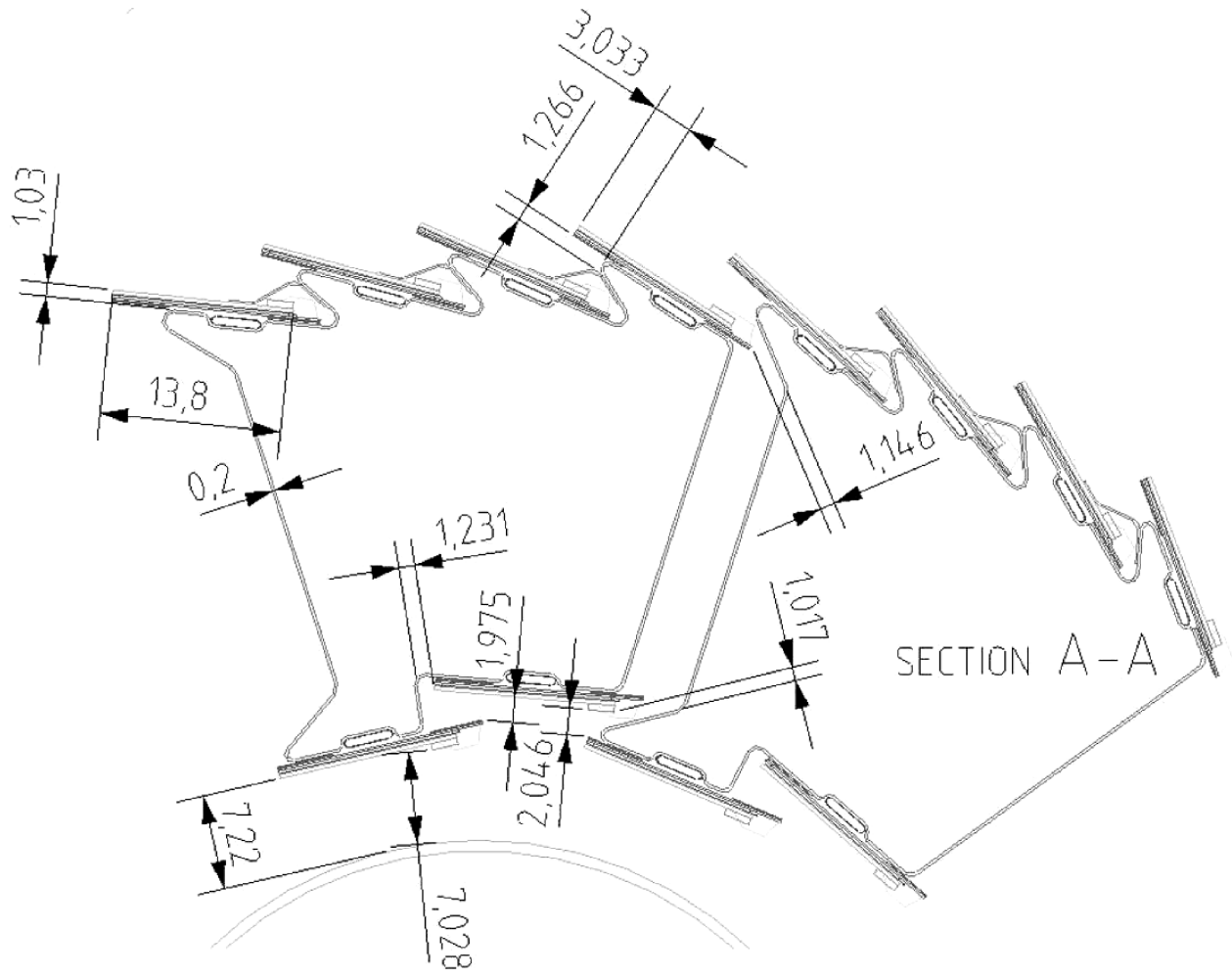

Figure 3.4: Carbon-fibre support of the Si-pixel staves.

inner layer and four on the outer layer. Ten sectors are then mounted together around the beam pipe to close the full barrel. In total, the SPD (60 staves) includes 240 ladders with 1200 chips for a total of $9.8 \times 10^{6}$ cells. The inner (outer) SPD layer is located at an average distance of $3.9 \mathrm{~cm}(7.6 \mathrm{~cm})$ from the beam axis; the clearances between the detector modules are very small (figure 3.4). The power dissipated in the front-end electronics is $\approx 1.35 \mathrm{~kW}$. The cooling system is of the evaporative type and is based on $\mathrm{C}_{4} \mathrm{~F}_{10}$. The sectors are equipped with cooling capillaries embedded in the sector support and running underneath the staves (one per stave). The heat transfer from the frontend chips is assured with high thermal conductivity grease. The SPD barrel is surrounded by an Al-coated carbon-fibre external shield to prevent radiation of heat towards the SDD layers.

Figure 3.5 shows one half-barrel assembled and ready to be integrated in the pixel mechanics. Figure 3.6 shows the SPD installed around the beryllium beam pipe in the experiment.

\subsubsection{Pixel chip and sensor, ladder, bus and front-end electronics}

The ALICE pixel readout chip is a mixed signal ASIC developed in an IBM $0.25 \mu \mathrm{m}$ CMOS process (6 metal layers) with radiation-tolerant layout design [33]. Each chip contains 8192 readout cells of $50 \mu \mathrm{m} \times 425 \mu \mathrm{m}$ arranged in 32 columns and 256 rows. The size of the chip is $13.5 \mathrm{~mm} \times$ $15.8 \mathrm{~mm}$ including internal DACs, JTAG controller, chip controls and wire bonding pads. The chip clock frequency is $10 \mathrm{MHz}$. A detailed description of the chip architecture can be found in [34, 35]. Each pixel cell contains a preamplifier-shaper with leakage current compensation, followed by a 


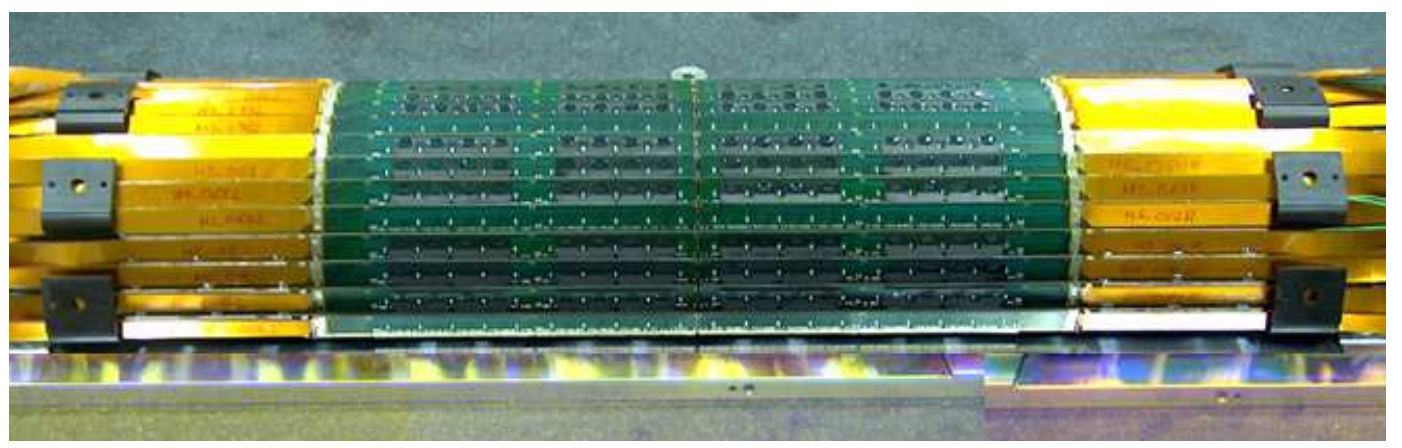

Figure 3.5: Half barrel assembled on reference table.

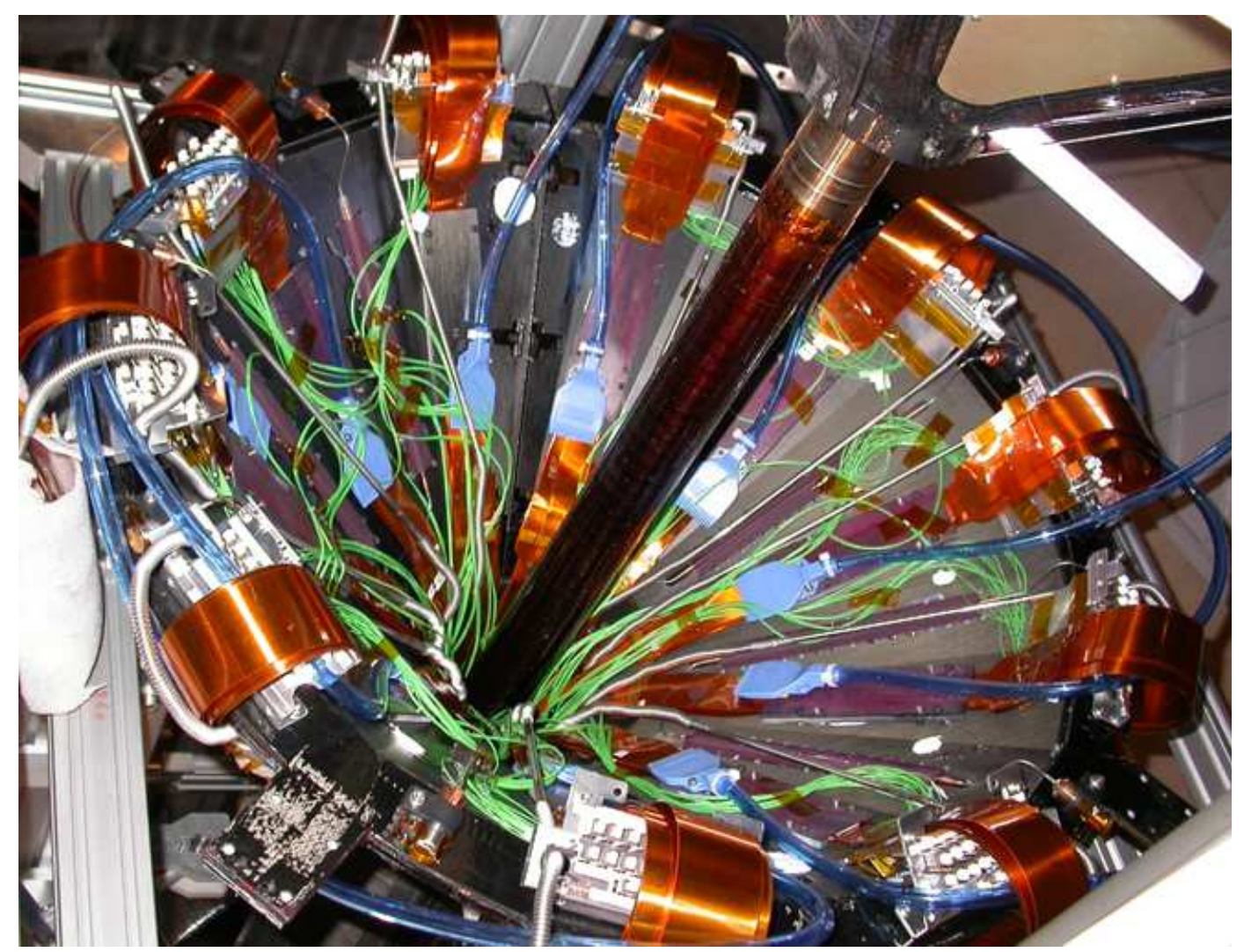

Figure 3.6: The SPD installed around the beryllium beam pipe.

discriminator. A signal above threshold results in a logical 1 which is propagated through a delay line during the L1 trigger latency $(\approx 6 \mu \mathrm{s})$. A four-hit-deep multi-event buffer in each cell allows derandomization of the event arrival times. Upon arrival of the L1 trigger, the logical level present at the end of the delay line is stored in the first available buffer location. The power consumption per chip is $\approx 990 \mathrm{~mW}$. Electrical tests indicated a mean minimum threshold of $\approx 1000$ electrons and a spread of $\approx 200$ electrons r.m.s. [36]. 
Table 3.4: Main specifications of the ALICE SPD front-end chip.

\begin{tabular}{|l|c|}
\hline Cell size & $50 \mu \mathrm{m}(r \varphi) \times 425 \mu \mathrm{m}(z)$ \\
Number of cells & $256(r \varphi) \times 32(z)$ \\
Minimum threshold & $1000 e$ \\
Threshold uniformity & $200 e$ \\
L1 latency & up to $51 \mu \mathrm{s}$ \\
Operating clock frequency & $10 \mathrm{MHz}$ \\
Radiation tolerance & in excess of $10 \mathrm{Mrad}$ \\
\hline
\end{tabular}

The pixel chip includes many operation parameters that are remotely adjustable. The on-chip global registers include 42 8-bit DACs that adjust current and voltage bias references, L1 trigger delay, global threshold voltage, and leakage current compensation. In each pixel cell a 3-bit register allows individual tuning of the threshold; there is also provision to enable the test pulse input and to mask the cell. All configuration parameters are controlled through the JTAG bus via the digital PILOT chip.

Each pixel chip generates a pulse (Fast-OR) whenever a pixel cell (or a group of them) detects a particle signal above threshold. The Fast-OR is used to self-trigger the front-end in test mode and to implement a prompt physics trigger as described in section 3.1.1.9.

The pixel chip has proven to be insensitive to a total ionization dose (TID) exceeding $10 \mathrm{Mrad}$. The measured cross-section for Single-Event Effects (SEU) is $\approx 3 \times 10^{-16} \mathrm{~cm}^{2}$, corresponding to an upset rate of $0.1 \mathrm{bit} / \mathrm{hour}$ in the full barrel for $\mathrm{Pb}-\mathrm{Pb}$ collisions at nominal luminosity. The main specifications of the ALICE SPD front-end chip are summarized in table 3.4.

A detailed protocol was developed for testing and classifying the readout chips into three classes. Only class I chips are used for flip-chip bonding. These chips show full electrical functionality, normal current consumption and less than $1 \%$ faulty pixels. Class II chips are fully functional, but have more than $1 \%$ pixel defects. Class III chips show a serious defect, such as an excess in current consumption or a JTAG malfunction. Each wafer ( $200 \mathrm{~mm}$ diameter) contains 86 ALICE pixel chips. A total of 58 wafers were tested. An average yield of 45 class I chips per wafer was found. The pixel chip wafers are thinned down from $725 \mu \mathrm{m}$ native thickness to $150 \mu \mathrm{m}$ thickness to reduce the overall material budget in the SPD. Wafer thinning through backside grinding is done by the bump bonding supplier (VTT Center for Microelectronics, Espoo, Finland) after deposition of the solder bumps, which are $\approx 20 \mu \mathrm{m}$ in diameter.

The pixel sensors have an active size of $12.8 \mathrm{~mm} \times 70.7 \mathrm{~mm}$. They are produced on $200 \mu \mathrm{m}$ thick, high resistivity n-type silicon wafers supplied by CANBERRA (CANBERRA Semiconductor, B-2250 Olen, Belgium) and ITCirst (ITCirst, 38100 Povo di Trento, Italy). Five readout chips are flip chip bonded to one sensor ( $200 \mu \mathrm{m}$ thick) using Pb-Sn solder bump bonds. The performance of each ladder is evaluated using the on-chip test pulse generator as well as a radioactive source. The Fast-OR functionality is verified. According to the test results the ladders are classified into three classes [37]. Only class I ladders are used for half-stave mounting. They have less than $1 \%$ defects per chip and a total leakage current of less than $2 \mu \mathrm{A}$ at nominal operating voltage of $50 \mathrm{~V}$. A rework procedure was developed by VTT in order to replace chips that show an unaccept- 


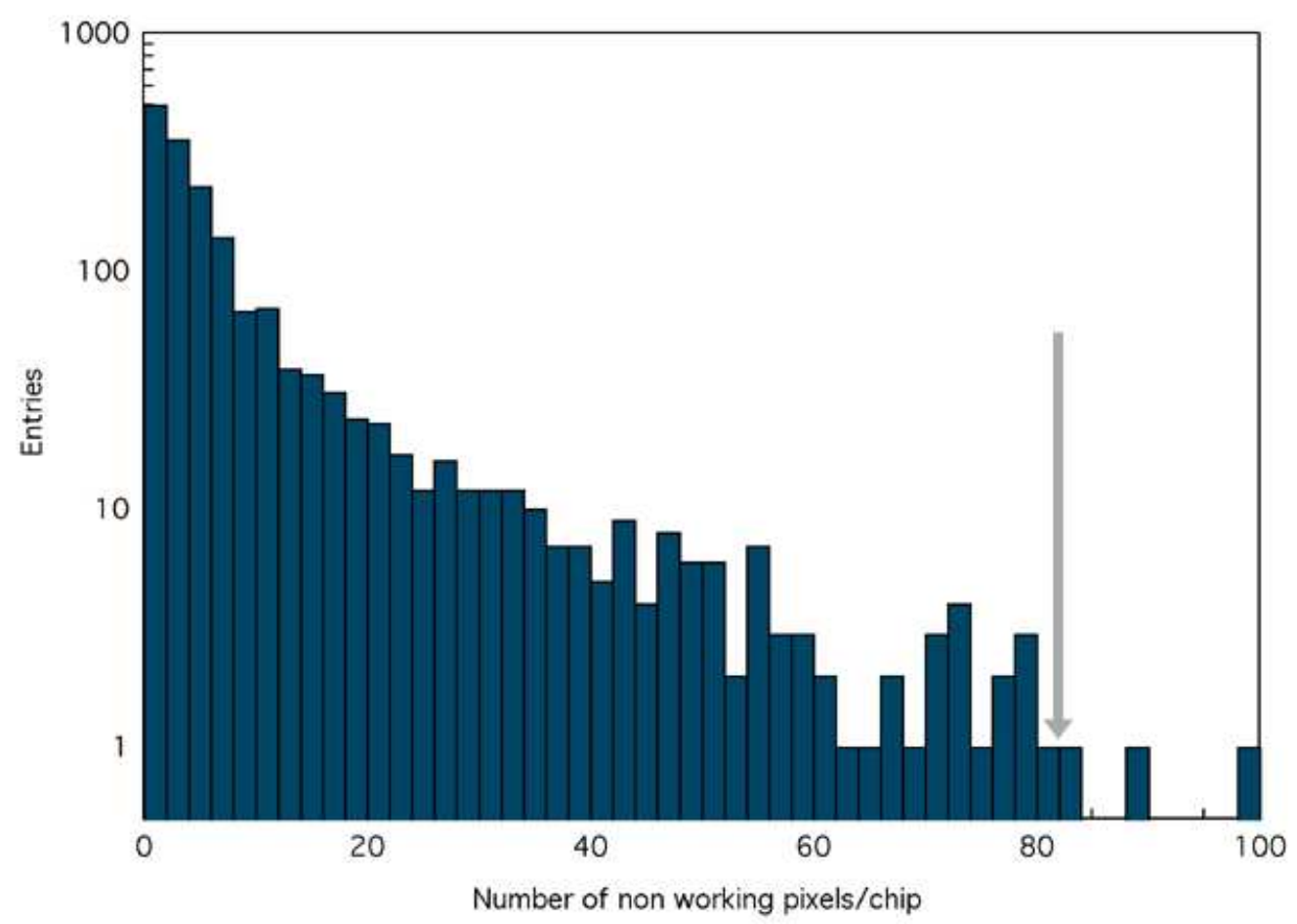

Figure 3.7: Number of pixel defects per chip in class 1 ladders. The arrow corresponds to the 1\% cut acceptance.

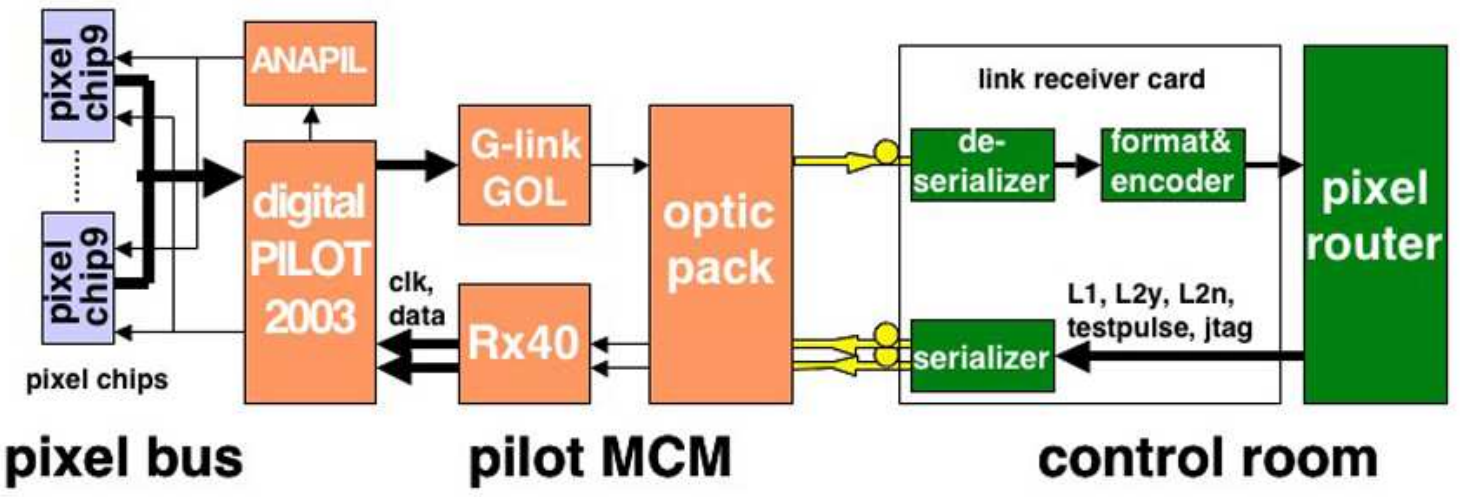

Figure 3.8: Block diagram of the electronic system.

able number of defects after bump bonding. A total of 445 ladders were tested, with a class I yield of $77 \%$ (including reworked units). Figure 3.7 shows the number of defects per chip of all class I ladders.

A schematic diagram of the SPD electronics system is shown in figure 3.8. The pixel bus (figure 3.9) is a 5-metal layer sequential build-up (SBU) substrate (aluminium/polyimide).

The use of aluminium in place of copper is dictated by the low-mass requirements; it is not an industrial standard and has required a custom development (CERN TS-DEM Workshop) [38]. Two $50 \mu \mathrm{m}$ thick layers are used as LV power/ground planes. The other three layers carry data/control lines; two of these layers are made by vacuum deposition and are $\approx 7 \mu \mathrm{m}$ thick (see figure 3.9). 


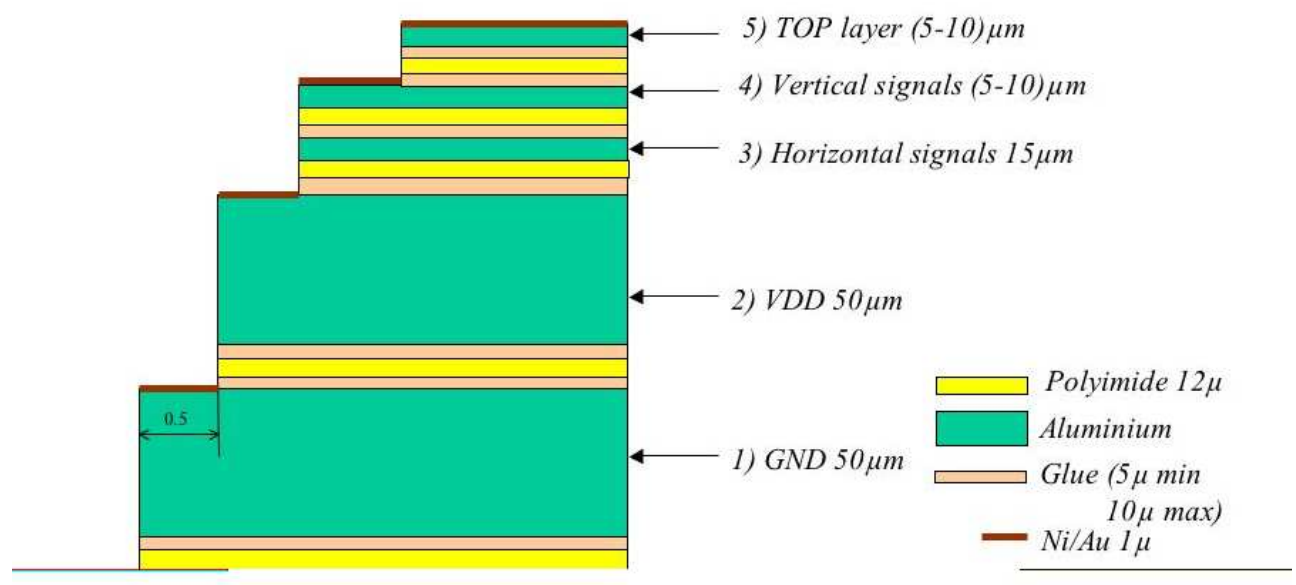

Figure 3.9: Pixel bus layers structure.

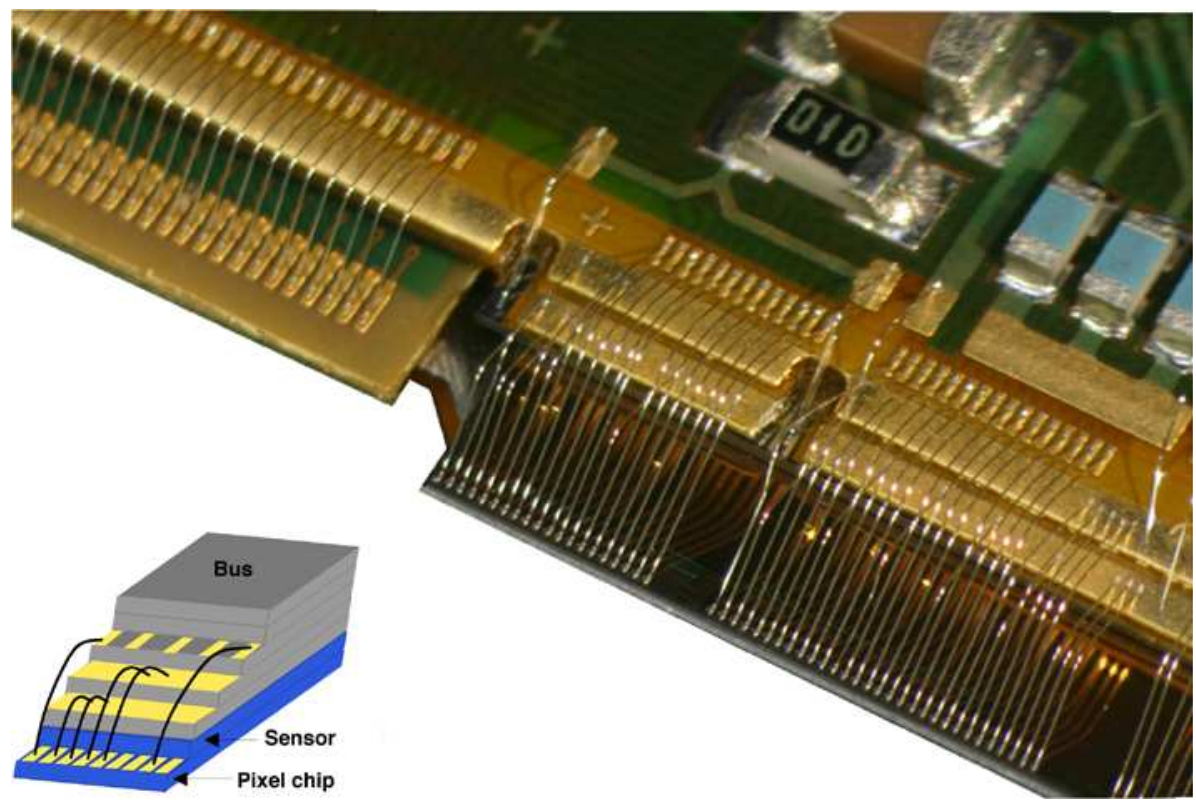

Figure 3.10: Wire bonding of ladders to pixel bus.

The edges of adjacent layers are step-wise receded to make room for wire bonding pads and to allow connections to the power and ground planes without using vias. The overall thickness of the pixel bus is $\approx 280 \mu \mathrm{m}$. A picture of the wire bonding of ladders to the bus is shown in figure 3.10. The MCM (figure 3.11) performs the readout of a half-stave. It includes the digital PILOT, the analogue PILOT and the gigabit optical link driver GOL, all developed in the same IBM $0.25 \mu \mathrm{m}$ CMOS process used for the pixel chip, see [39-41].

The digital PILOT receives serial trigger, configuration data and clock from the off-detector electronics via two PIN diodes in the optical package and the receiver chip RX40 (see figure 3.8 on the preceding page). The digital PILOT initiates the pixel chip read-out, performs data multiplexing 


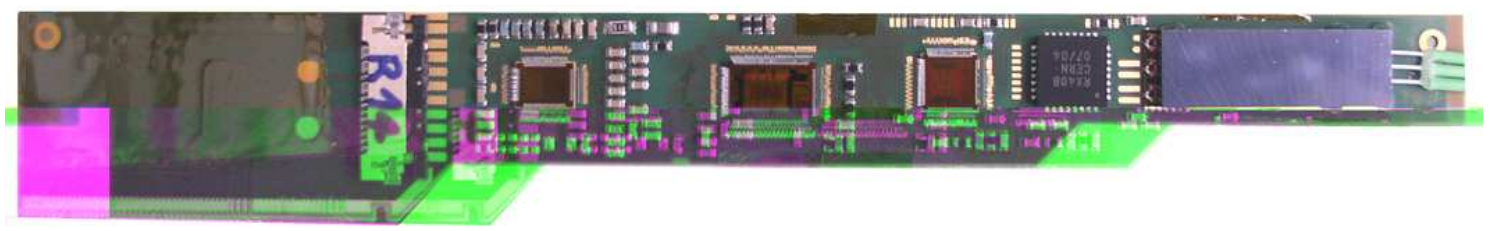

Figure 3.11: Multi-Chip Module (MCM). Left to right: wire bonds connecting the MCM ASICs via the pixel bus to the read-out chips, MCM ASICs, optical package with three optical fibres.

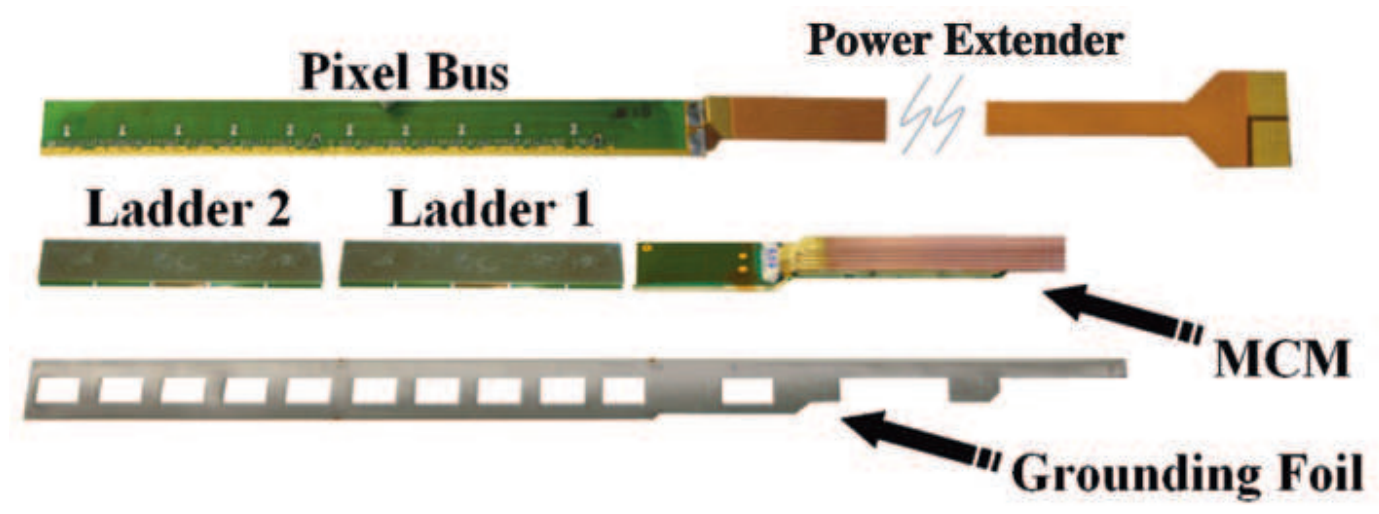

Figure 3.12: Half-stave assembly.

and sends the data to the G-link compatible $800 \mathrm{Mbit} / \mathrm{s}$ serializer GOL, which drives the laser in the optical package. The analogue PILOT contains DACs that generate precision reference levels for the pixel chips and an ADC that reads a Pt1000 temperature sensor chain on the half-stave.

The MCM is based on a 5-metal-layer sequential build-up substrate (polyimide-copper). The floorprint is $110 \mathrm{~mm} \times 12 \mathrm{~mm}$. The thickness is less than $1.5 \mathrm{~mm}$; this was achieved by mounting bare die ASICs and by the development of a custom optical package that is $1.2 \mathrm{~mm}$ thick (STMicroelectronics, Milano, Italy). As the MCM processes and transmits digital data at $800 \mathrm{Mbit} / \mathrm{s}$ and also provides reference voltages to the pixel read-out chips with a precision of $10 \mathrm{mV}$, the layout has required special care. The MCM data/signal lines are wired bonded to the pixel bus. Power is supplied to the pixel bus and the MCM via two independent flexible copper/polyimide laminates (power extenders).

\subsubsection{Half-stave assembly}

The half-stave assembly requires the attachment and wire bonding of the two ladders and the multichip module onto the high density aluminium-polyimide multi-layer interconnect (pixel bus) (figure 3.12). A grounding foil, consisting of an aluminium-polyimide laminate ( $25 \mu \mathrm{m}$ and $50 \mu \mathrm{m}$ thick, respectively), is included to provide electrical isolation with respect to the carbon fibre. The grounding foil has openings for the thermal coupling of the chip substrates to the cooling ducts via thermal grease pads. The cooling ducts run in grooves embedded in the carbon-fibre support.

The micrometric alignment of the half-stave components is performed with a Mitutoyo coordinate measuring machine (Crysta Apex 9166) equipped with custom developed tools and jigs 
for vacuum-suction handling of the components. The glue is the two-component epoxy adhesive Eccobond 45. It is electrically insulating and has adequate thermal conductivity. It can be cured at room temperature; the flexibility can be controlled by the amount of Catalyst 15 used. In our case a mixture of glue and catalyst in the $1: 1$ ratio was used.

The half-stave assembly is a three step procedure based on micrometric components alignment and gluing. The tight dimensional tolerances of the SPD require that the thickness and the planarity of the half-stave be kept under control very carefully during every production step. A few $\mu \mathrm{m}$ precision in the alignment of components and planarity better than $100 \mu \mathrm{m}$ were obtained.

In the first step the two ladders and the MCM are aligned with respect to one another and are glued to the grounding foil. In the second step the pixel bus is aligned and glued on top of them. The third and final step is the ultrasonic wire-bonding connection of the pixel bus to the front-end chips and the MCM. There are more than 1,100 bonds on each half-stave. The quality of the component alignment and gluing is crucial for the reliability and robustness of the wire-bonds. Details on the half-stave assembly procedure can be found in [42-44].

After assembly, each half-stave is extensively tested at nominal working temperature (Peltier coolers). The protocol includes functionality and performance tests such as minimum threshold, threshold uniformity, noisy pixels, dead pixels and Fast-OR. A thorough test is essential since the replacement of a malfunctioning half-stave on a sector is difficult and risky, as it could result in damage to a neighbouring half-stave. The half-staves are then shipped to the facility where the mounting on sector is performed. They are tested again on reception to check for any damage due to transport and handling.

\subsubsection{Sector assembly}

In view of the stringent requirements on the relative positioning of the components, material budget and mechanical handling, specific tools and techniques were developed for mounting the halfstaves on sectors. A detailed description of the SPD barrel sector assembly procedure can be found in [45]. The main steps of the procedure can be summarized as follows:

- the half-staves are tested on reception at the clean room facility where the system for mounting them onto the $200 \mu \mathrm{m}$ thick Carbon-Fibre Support Sectors (CFSS, see next section) is installed;

- the planarity of the surface on which the half-staves are to be attached is measured for compliance with specifications $(100 \mu \mathrm{m})$. A thin layer $(\approx 150 \mu \mathrm{m})$ of thermal grease is dispensed in rectangular pads, each pad corresponds to the back side of a pixel chip. The choice of the thermal grease (AOS 52029, AOS Thermal Compounds, NJ, USA) was made based on its mechanical, thermal and radiation hardness characteristics;

- two half-staves are then aligned to form a stave and positioned on the CFSS using a vacuum holding tool. The average uncertainty in the relative positioning of the two half-staves was found to be better than $20 \mu \mathrm{m}$ with respect to the nominal position; 


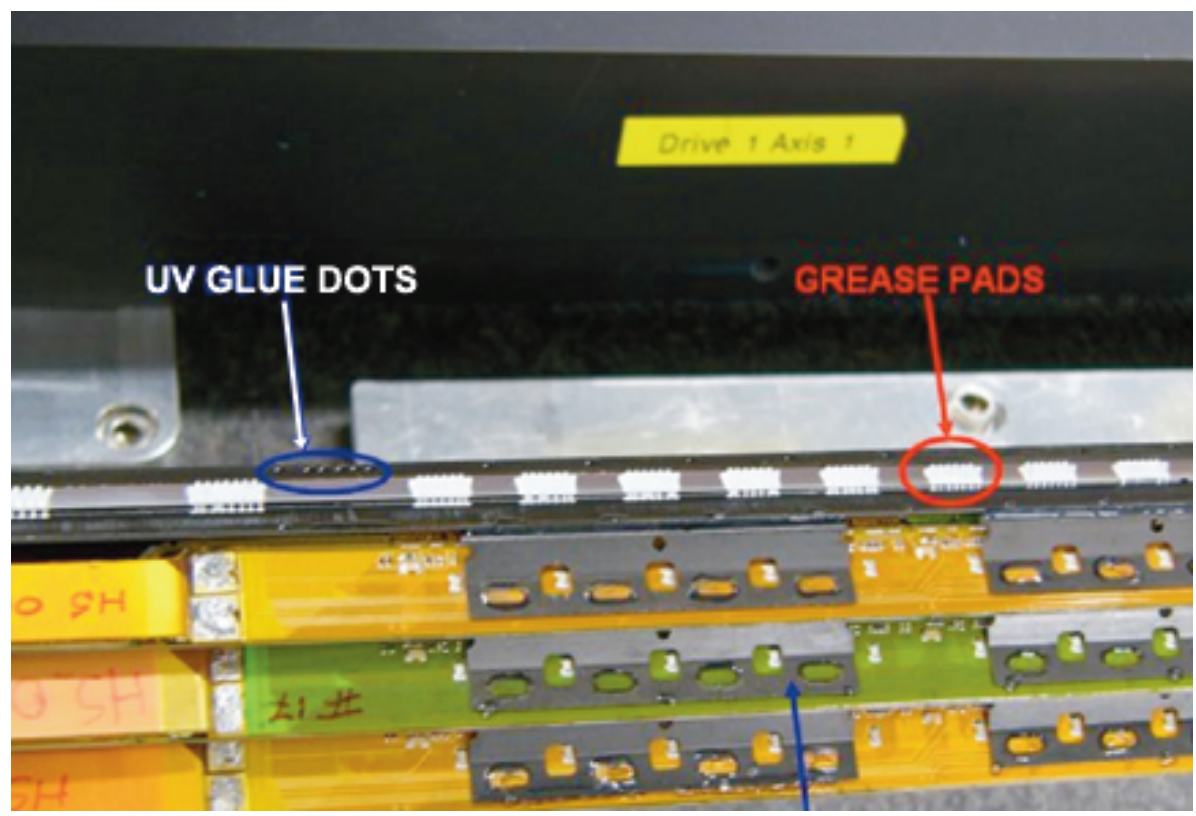

Figure 3.13: In the upper plane of the CFSS, thermal grease pads on the cooling duct and UV dots are visible. Such material is dispensed before the assembly of the half-staves. In the lower CFSS planes, three already assembled half-staves are shown. The half-staves are attached to the CFSS with carbon fibre clips and UV glue dots.

- the aligned stave is finally glued to the CFSS using a UV curable glue (Norland NEA 123, Norland Products Inc., NJ, USA). The upper part of the stave is fixed using carbon fibre clips, to protect the wire-bonding and to ensure good mechanical stability, as shown in figure 3.13.

The assembly of the whole SPD barrel was completed with a yield of $100 \%$ (all accepted halfstaves were assembled on the CFSS without any loss or damage). For two half-staves, additional thermal grease had to be injected during the test phase in order to improve the thermal contact.

\subsubsection{Carbon-Fibre Support Sector (CFSS)}

The CFSS is the mechanical support structure of the barrel with an embedded cooling system. The design provides a direct thermal contact between the cooling duct and the silicon chip backplane. This allows to maximise the thermal coupling between the stave and the cooling system and to avoid problems of anisotropic heat conductivity typical of carbon fibre structures. The shape of the groove for the cooling duct together with the shape of the CFSS also provide a very high local stiffness. The global and local deformations of the CFSS are expected to be of the order of $1 \mu \mathrm{m}$ or less in the service conditions. The CFSS are made by winding two layers of unidirectional highmodulus, $100 \mu \mathrm{m}$-thick carbon fibre tapes, with fibres parallel and perpendicular to the beam axis, around a metallic mandrel. In order to obtain a good surface finishing quality metallic countermandrels are also used. The wall thickness of the CFSS is about $200 \mu \mathrm{m}$ in the sensitive region increasing to $600 \mu \mathrm{m}$ at the ends in order to allow the positioning of the mechanical references and of the components needed for the global assembly of the sectors. The global thickness of 
the support end-sections is locally increased to $1.8 \mathrm{~mm}$ in the connections area, in order to allow for an adequate pin length. All the coupling pins and pin seats are made in carbon fibre in order to minimise the use of high- $Z$ material near the sensitive volume. The CFSS have a parilene deposition (about $10 \mu \mathrm{m}$ thick) in order to increase the DC electrical insulation and to also avoid the accidental release of debris from the CFSS surface. The CFSS qualification is performed by means of a 3D survey machine, with software that allows direct comparison with the 3D CAD model of the components. The planarity, the angular distortion and the difference between the nominal and the measured dimensions are precisely determined. Since the half-stave structure is a combination of materials with a non-negligible mismatch of thermal expansion coefficients, the global $\Delta \mathrm{T}$ from the assembly reference temperature was minimized and the half-staves are firmly attached to the stiff carbon-fibre structure by means of UV glue tags and carbon-fibre clips. Possible deformations do not exceed $10 \mu \mathrm{m}$ (in any direction) with stresses well within acceptable limits. Further details can be found in [46].

\subsubsection{Cooling}

The major contribution to the on-detector power dissipation is due to front-end chips; they generate a heat load of $\approx 23 \mathrm{~W}$ (nominal) in each stave. The design of the cooling system was driven by several constraints such as low material budget, long-term stability against corrosion, chemical compatibility, minimal temperature gradients, cooling duct temperature above the dew point. Several possible solutions based on different coolants were considered [47]. The final choice of an evaporative system with $\mathrm{C}_{4} \mathrm{~F}_{10}$ as coolant was found to fulfil all the requirements. The $\mathrm{C}_{4} \mathrm{~F}_{10}$ follows a Joule-Thomson cycle (rapid expansion at constant enthalpy and subsequent evaporation). The liquid, overcooled and compressed by a pump, is brought to the coexistence phase inside the cooling duct by a pressure drop inside the capillaries $(0.5 \mathrm{~mm}$ internal diameter, $550 \mathrm{~mm}$ long). Heat abduction through phase transition takes place inside the cooling tube at $15-18^{\circ} \mathrm{C}(1.9-2.0 \mathrm{bar})$; a compressor raises then the pressure pushing the gas towards a condenser, where the liquid phase is re-established by heat transfer to cold water $\left(\approx 6^{\circ} \mathrm{C}\right)$. The evaporation temperature can be controlled by regulating the pressure in the return line, setting then the coexistence conditions of the mixed phase. Each stave is put in thermal contact with the cooling duct mounted in a groove on the CFSS by a thermal grease layer (see section 3.1.1.5).

The cooling duct is obtained using Phynox tubes with a wall thickness of $40 \mu \mathrm{m}$ and an initial diameter of $2.6 \mathrm{~mm}$, squeezed down to flat profile with an overall thickness of $600 \mu \mathrm{m}$ in the thin dimension. Each sector is equipped with cooling collectors at the two ends, one functioning as an inlet and the other as an outlet for the whole sector. Extensive corrosion tests were performed on tubes, together with tests to optimize the choice of surface treatment and of fitting materials.

In the final test, detectors are turned on at nominal power. An infrared (IR) camera is used to measure the temperature distribution on the external part of the pixel matrix (i.e. the bus), on the MCM and on the power extenders (copper-polyimide laminates). Residual gaps due to mechanical tolerances of the surfaces in contact can locally affect the quality of thermal coupling. The average operating temperature of the half-staves is in the range of $25^{\circ} \mathrm{C}$ to $30^{\circ} \mathrm{C}$. The temperature profile measured on a half-stave with the IR camera is shown in figure 3.14. The temperature does not exceed $35{ }^{\circ} \mathrm{C}$ and the local variations are limited to a few ${ }^{\circ} \mathrm{C}$. 

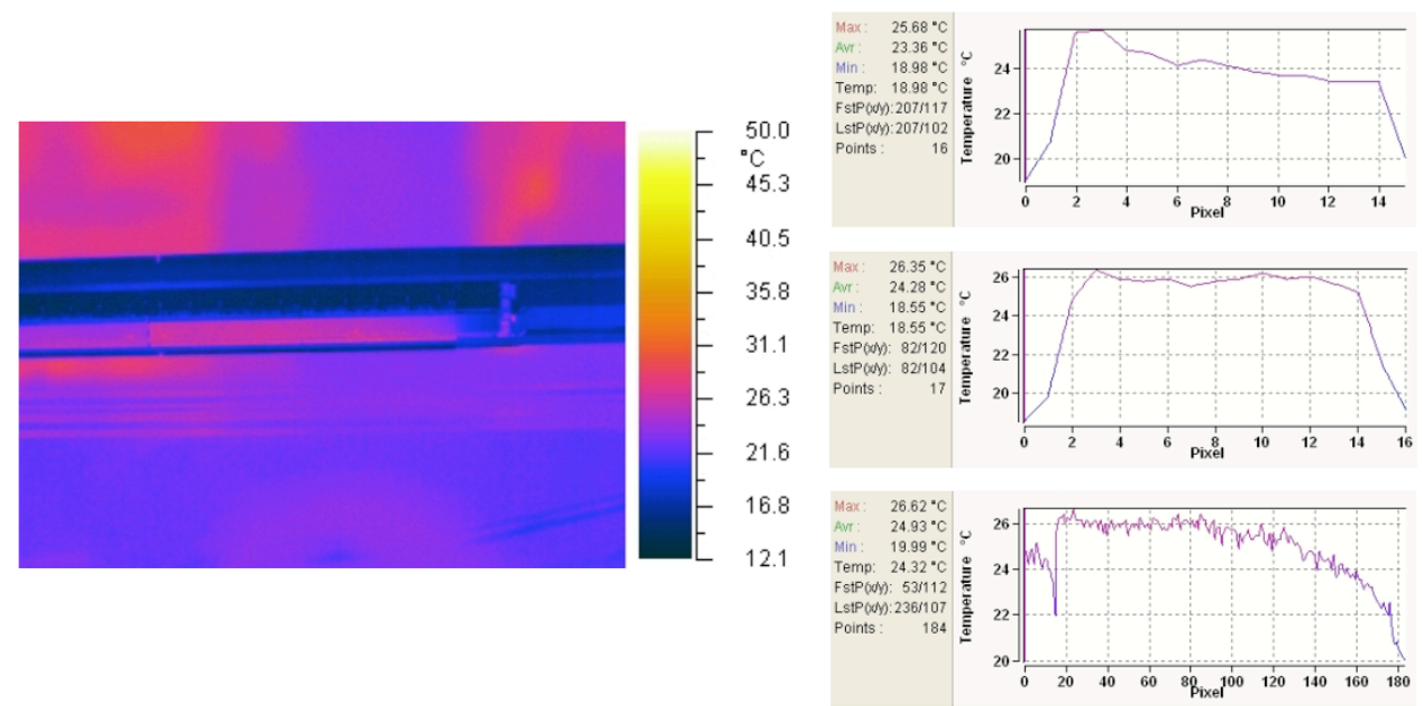

Figure 3.14: Left: infrared image of an SPD half-stave during operation. Right: temperature profiles - transversal across the ladders (top two plots) and longitudinal along the full half-stave (bottom plot).

In normal operation, if a sudden failure of the cooling were to occur, the temperature at the half-stave would increase at a rate of $1{ }^{\circ} \mathrm{C} / \mathrm{s}$. Continuous monitoring and a fast, reliable safety interlock on each half-stave are therefore mandatory. They are based on Pt1000 temperature transducers mounted on the pixel bus, next to the pixel chips. Two daisy chains of 5 transducers each (interleaved positions) provide redundant measurements of the average temperature. One chain is read out in the MCM, the other is hard-wired to the remote interlock system, based on a programmable logic controller (PLC) that is part of the detector control and safety system. Temperature values are logged. If the temperature reaches a preset threshold, the low-voltage power supply is promptly switched off by the safety interlock and an alarm is generated.

\subsubsection{Power supply and grounding}

The low-voltage power supply system is based on CAEN A3009, (CAEN, Viareggio, Italy) dc-dc converter modules, installed in racks in the experimental area at a distance of $\approx 40 \mathrm{~m}$ from the detector. Each half-sector (6 half-staves) is powered by one module, which has 12 independent floating outputs; 2 outputs are used for each half-stave, one for the pixel chips (1.85V/5.5A), the other one for the MCM (2.6V/0.5A). Remote sensing is used throughout. In each half-stave, the pixel chip/MCM supply return lines are shorted and define the half-stave ground. In the CAEN A3009, all return lines are connected via $10 \mathrm{k} \Omega$ resistors to a power supply reference ground that is connected to the ALICE ground (on the absorber and the space frame).

The detector bias voltage ( $50 \mathrm{~V}$ typically at start of detector operation) is provided by CAEN A1519 modules located in the control room at a distance of $\approx 120 \mathrm{~m}$ from the detector.

One module ( 12 channels) is used for each sector. The two sensor ladders in one half-stave share one high voltage module output but are connected by one coaxial cable each to the HV- 


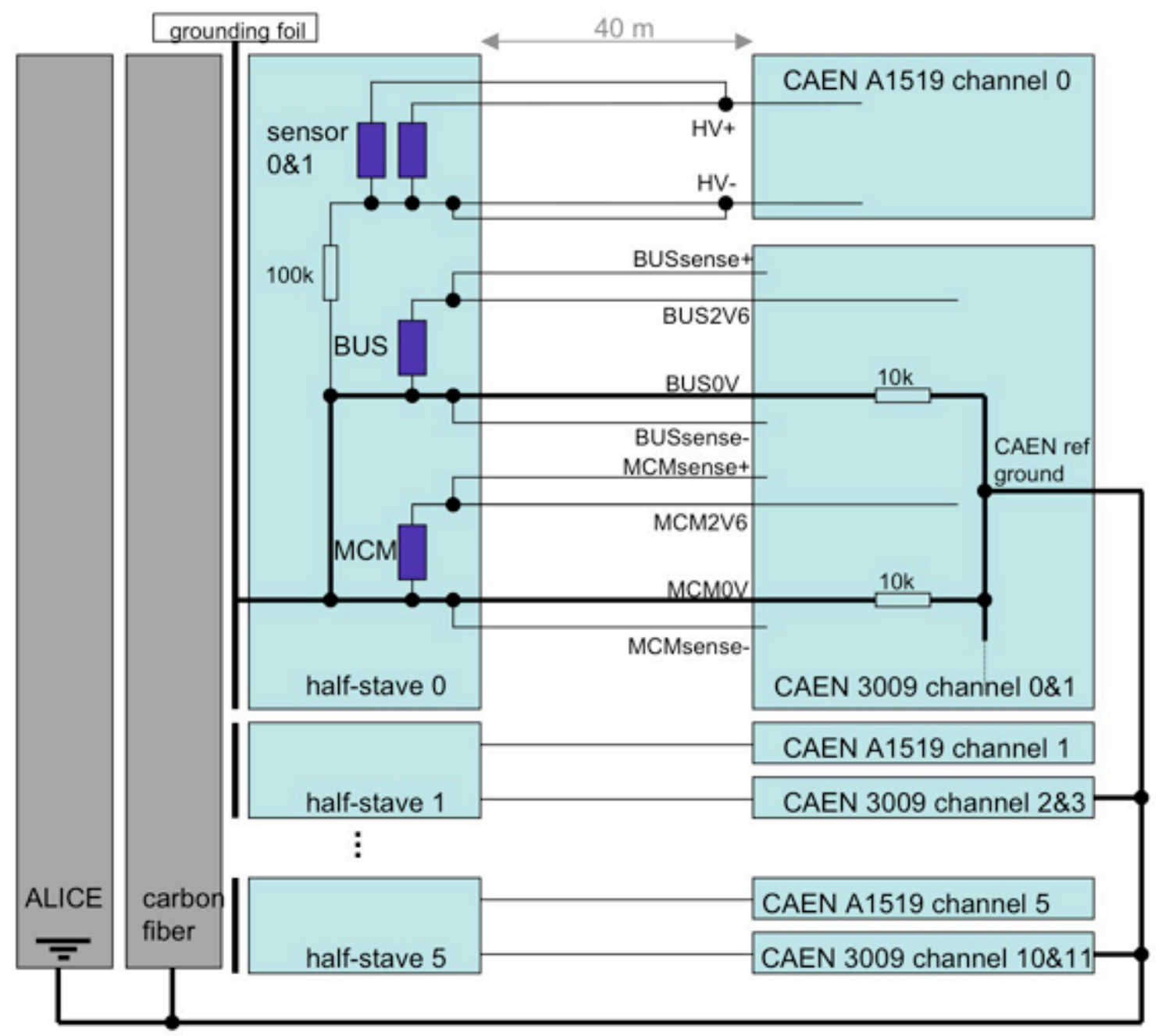

Figure 3.15: Power supply and grounding scheme.

module in the control room. This allows the individual connection of a sensor ladder to the bias voltage. The return line of the high voltage is connected to the half-stave ground via a $100 \mathrm{k} \Omega$ resistor. The half-stave ground is isolated from the carbon-fibre support by means of the grounding foil. The carbon-fibre support itself is connected to the ALICE ground.

Figure 3.15 illustrates the power supply and grounding scheme.

\subsubsection{Readout and Calibration}

After the reception of the L1 trigger signal, pixel hit data are extracted from the digital delay line and stored in one of the four multi-event buffers in the pixel chip. Upon arrival of the second level trigger (L2), the data contained in the multi-event buffer locations corresponding to the first (oldest) L1 trigger are loaded onto the output shift registers. Then, for each chip, the data from the 256 rows of cells are shifted out during 256 cycles of a $10 \mathrm{MHz}$ clock. At each cycle, a 32-bit word containing the hit pattern from one chip row is output on the 32-bit data bus where it is processed 
by the MCM and sent optically to the read-out electronics located in the control room. One pixel chip is read out in $25.6 \mu \mathrm{s}$. The 10 chips on each half-stave are read out sequentially. The 120 half-staves are read out in parallel. In ion beams operation, the dead time introduced by the readout of the SPD is estimated to be less than $10 \%$ in the worst case, corresponding to ALICE running with Ar-Ar beams at high luminosity, with an $\mathrm{L} 1$ rate of $2.5 \mathrm{kHz}$.

The SPD off-detector read-out electronics is located in the control room [41]. Twenty $9 \mathrm{U}$ VME- and FPGA-based electronics cards (routers) contain each 3 plug-in type daughter cards (link receiver cards). The link receiver cards are connected via optical fibres to two half-staves. The link receivers zero-suppress and re-format the hit data to an ALICE off-line analysis compatible format. The routers multiplex data from the six half-staves into one ALICE optical Detector Data Link (DDL) and attach trigger and status information. Each of the 20 router cards provides the interface to the trigger, DAQ and DCS/ECS for 6 half-staves. The trigger information is delivered by the Trigger, Timing and Control (TTC) system via optical fibres. Each router is interfaced to the DAQ via one DDL. The 20 DDLs are connected to Local Data Collectors (LDCs) housed in 4 PCs. The data access from the DCS/ECS to the routers is established via the router VME ports. The same port also allows monitoring and copying the data flow during data taking.

Detector calibration is performed using the on-chip test pulse generator [34, 35]. The test pulse amplitude is controlled by the analogue PILOT chip and is programmable. The pulse can be sent to individual pixel cells or to clusters of them. The SPD DCS is required for executing the calibration procedures [48].

\subsubsection{Fast-OR pixel trigger}

Each pixel chip provides a Fast-OR digital pulse when one or more of the pixels in the matrix are hit. The Fast-OR signals of the 10 chips on each of the 120 half-staves are read by the PILOT chip ([39-41]) and transmitted every $100 \mathrm{~ns}$ on the 120 optical links that are also used for the data readout. The Fast-OR allows the implementation of a unique prompt trigger capability. The pre-processed Fast-OR data can be used to contribute to the Level 0 trigger decision in the ALICE Central Trigger Processor (CTP). This feature is very useful in particular in the case of events with very low multiplicities in pp runs. Various trigger algorithms including the Fast-OR are being investigated [49]. These studies have shown that background rejection in pp interactions and event selection in heavy-ions runs is significantly improved by using the Fast-OR information in the L0-trigger decision.

The pixel trigger signal generated by the Fast-OR processor must reach the CTP within $\approx$ $800 \mathrm{~ns}$ of the interaction in order to meet the latency requirements of the L0-trigger electronics [50]. Short connections and minimal processing overhead are therefore essential. However, some constraints deriving from the pixel chip design have to be taken into account. The Fast-OR output is synchronized with the $10 \mathrm{MHz}$ pixel system clock, hence the signal is effectively integrated over $100 \mathrm{~ns}$ corresponding to 1 bunch-crossing in the case of operation with heavy ions, or 4 consecutive bunch-crossings in pp operation. All 120 half-staves are synchronized to the same integration period covering the same set of bunch-crossings in order to limit this ambiguity when all Fast-OR signals are simultaneously processed and to minimize the latency. In the ALICE trigger system, the bunch-crossing ambiguity can be resolved by considering the coincidence between the pixel trigger signal and the ALICE V0 detector signal. 


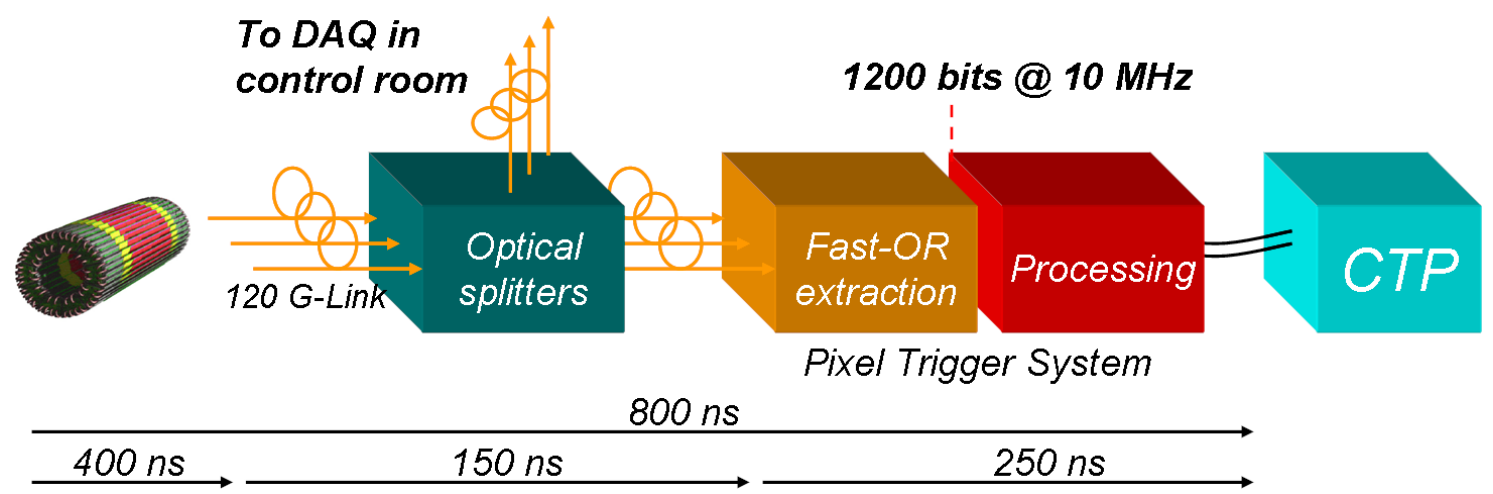

Figure 3.16: Pixel trigger system architecture.

The pixel trigger system architecture is shown in figure 3.16. A full description of the pixel trigger system is given in [51]. The development of the system was challenging due to the low latency and extreme compactness required. Two-way optical splitters equipping the optical fibres outgoing from the detector allow the simultaneous transmission of the readout and trigger data to the readout system and to the pixel trigger system respectively. The pixel trigger system consists of a set of ten receiver boards that deserialize the optical data streams and extract the 1200 FastOR bits received every $100 \mathrm{~ns}$ from the detector. Each receiver board includes a customized 12 channels optical parallel receiver module [52], 12 G-Link compatible deserializer ASICs and a FPGA to tap the Fast-OR signals from the twelve $800 \mathrm{Mb} / \mathrm{s}$ input channels. The receiver boards are all connected as mezzanines onto a motherboard and provide the 1200 Fast-OR bits as simultaneous inputs to the processing unit on the motherboard, a fast FPGA with a large number of pins and large logic space. Various algorithms, based on global multiplicity or on predefined topologies, can be implemented as Boolean logic functions of the Fast-OR bits. The algorithm to process the FastOR bits is implemented in programmable hardware to allow fast execution, upgrading and remote reconfiguration. The result of the pixel trigger algorithm implemented on the FPGA is directly fed to the ALICE CTP.

The implementation on a large FPGA of some of the proposed trigger algorithms was completed. The behavioural simulations showed that even the most complex function among the proposed ones could be evaluated in less than $15 \mathrm{~ns}$ in a Xilinx Virtex 4 device. The critical delays in the system are associated with the Fast-OR data deserialization, extraction and transfer between the peripheral FPGAs and the processing unit. The overall latency budget of $800 \mathrm{~ns}$ can be subdivided among each of the processes along the data flow. The on-detector electronics takes $400 \mathrm{~ns}$ from the time of the collision to transmit the Fast-OR bits. The shortest path for the optical fibres from the detector to the location of the pixel trigger system is $\approx 37 \mathrm{~m}$. This implies a signal propagation delay of $\approx 185 \mathrm{~ns}$. Therefore only $\approx 215 \mathrm{~ns}$ are left for the deserialization, extraction and processing phases. The system is fully developed, and its compliance with these constraints was verified. 


\subsubsection{Tests of the sector, half-barrel and Fast-OR with cosmics}

A dedicated test and integration facility was set up at CERN within the Departmental Silicon Facility (DSF) clean room area (class 100000 ). The facility includes the complete readout chain and trigger/DAQ system, the final LV and HV power supplies and cables, the cooling plant and the detector control system and safety interlock to allow testing one complete half-barrel. The facility was used to develop and test major parts of the system. Each sector is tested individually before its integration into a half-barrel. The complete test sequence is repeated for each of the two half-barrels.

A thermal survey of each sector was performed using a thermal imaging camera together with the measurement of the Pt1000 transducer chains mounted on each half-stave. The average temperature on the half-stave in normal operating conditions was $\approx 28^{\circ} \mathrm{C}$.

On each sector, all the half-staves were tested following a well defined procedure for a full functionality check. Power supply and leakage currents were measured. The on-chip test pulse was used to determine the minimum threshold, the homogeneity of the matrix response and the number of noisy pixels. No degradation of the minimum threshold compared to the half-stave tests was found. The total number of noisy pixels in all 10 sectors is $\approx 50$. Sector tests were also carried out using a ${ }^{90} \mathrm{Sr}$ radioactive source to identify non-working pixels. The bump bonding yield was in agreement with the corresponding measurements on half-staves.

On a subset of half-staves mounted on a sector the Fast-OR trigger setting was adjusted to carry out dedicated runs with cosmic rays. For this purpose the sector was oriented for maximum vertical acceptance. A similar test was later carried out on part of one half-barrel. In a 6 hour continuous run, in which the trigger was based on the coincidence of the Fast-OR of the inner and outer layer, approximately 7000 events were collected. The observed distribution of clusters in the two layers and the clusters correlation along the sector axis were compatible with the expectations, considering that the limited test time available did not allow the optimization of uniformity response and Fast-OR DACs settings required for maximum efficiency.

\subsubsection{Results from beam tests}

SPD prototype assemblies were tested in proton- and pion- secondary-beams at the SPS [53, 54] in the years 2002 and 2003. The experimental setup consisted of a movable assembly under test placed between two fixed doublets of pixel planes of the same type. The doublets were used as the reference and tracking system. Measurements were done at different threshold settings and orientations of the test device with respect to the beam line. The configuration of the tracking doublets, together with a detailed cluster analysis of the hits, allowed a study of the intrinsic spatial precision of the planes under test and their detection efficiency $[55,56]$.

At normal track incidence and standard threshold (200 DAC value, corresponding to about 3000 electrons [57]), mainly single and double pixel clusters occurred. After subtracting the uncertainty on the telescope prediction, the global detector precision (mixing all cluster patterns) along the short pixel side $\left(y\right.$-axis) was $\sigma_{\text {pixel }}(y)=(11.1 \pm 0.2) \mu \mathrm{m}$. In a wide plateau around this threshold setting the detector efficiency was above $99 \%$.

Data runs were taken in 2002 with the detector under test tilted by $0,5,10,15,20,25$ and 30 degrees and with thresholds set at 185, 200 and 210 DAC units (increasing DAC values correspond 

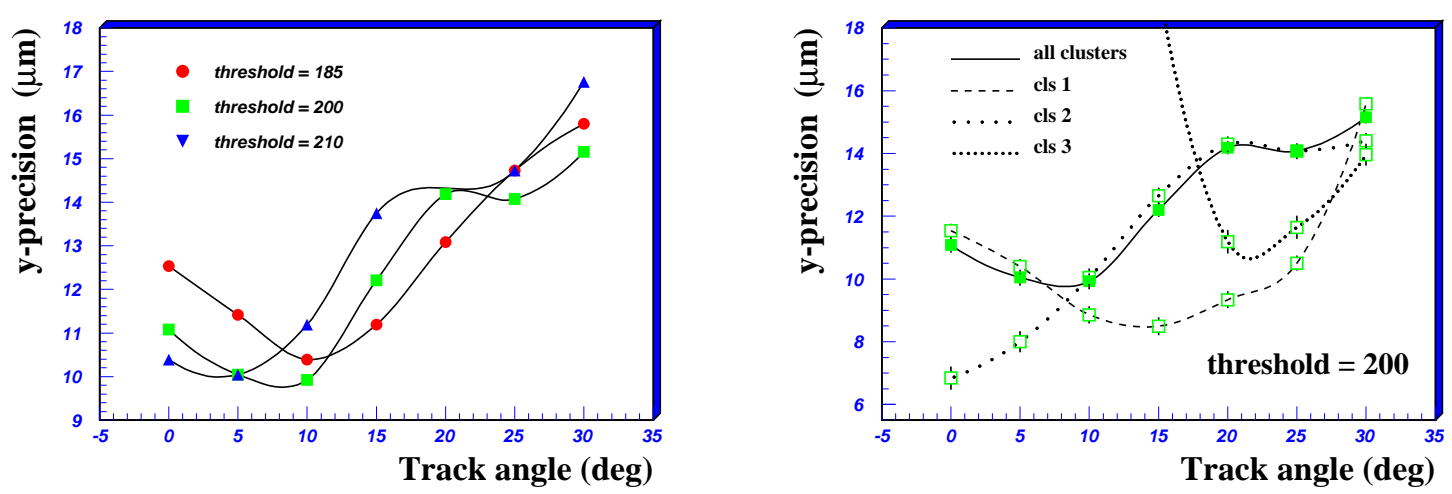

Figure 3.17: Test-beam results on the intrinsic precision in the $y$ coordinate as a function of the track incidence angle on the detector: at different threshold settings (left) and at 200 DAC units threshold for the main cluster patterns separately (right).

to decreasing effective threshold). Results of the study on the spatial precision as a function of the track incidence angles are shown in figure 3.17. The plot on the left side shows that the highest spatial precision is obtained at an incidence angle in the range $5-10^{\circ}$; for each threshold this occurs at the angle for which the fractions of single and double pixel clusters are roughly equal. The precision also degrades for all the threshold settings with the increasing track angle, as expected when higher cluster topologies are involved. The plot on the right side shows the intrinsic precision, together with the contributions due to the main pixel cluster patterns, in the case of 200 DAC threshold setting. These results are particularly useful in the tuning of the tracking errors to be associated to the SPD clusters, in simulations as well as in the analysis of experimental data.

\subsubsection{Silicon Drift Detector (SDD)}

The Silicon Drift Detectors (SDD) [11] equip the two intermediate layers of the ITS, where the charged particle density is expected to reach up to $7 \mathrm{~cm}^{-2}$. They have very good multitrack capability and provide two out of the four $\mathrm{d} E / \mathrm{d} x$ samples needed for the ITS particle identification.

\subsubsection{Sensor layout}

The ALICE SDDs were produced from very homogeneous high-resistivity $(3 \mathrm{k} \Omega \mathrm{cm}) 300 \mu \mathrm{m}$ thick Neutron Transmutation Doped (NTD) silicon [58]. As shown in figure 3.18, they have a sensitive area of $70.17(r \varphi) \times 75.26(z) \mathrm{mm}^{2}$ and a total area of $72.50 \times 87.59 \mathrm{~mm}^{2}$. The sensitive area is split into two drift regions by the central cathode strip to which a $\mathrm{HV}$ bias of $-2.4 \mathrm{kV}$ is applied. In each drift region, and on both detector surfaces, $291 \mathrm{p}^{+}$cathode strips, with $120 \mu \mathrm{m}$ pitch, fully deplete the detector volume and generate a drift field parallel to the wafer surface. To keep the biasing of the collection region independent on the drift voltage, a second bias supply of $-40 \mathrm{~V}$ is added. The overall detector performance, when averaged over its entire area, does not depend significantly on the applied bias voltage in a fairly large range from $-1.65 \mathrm{kV}$ to $-2.4 \mathrm{kV}$, so the precise value of bias voltage to be applied during long term operation is to be adapted to the specific running conditions. The degrading of the high voltage to the zero potential of the detector boundary 

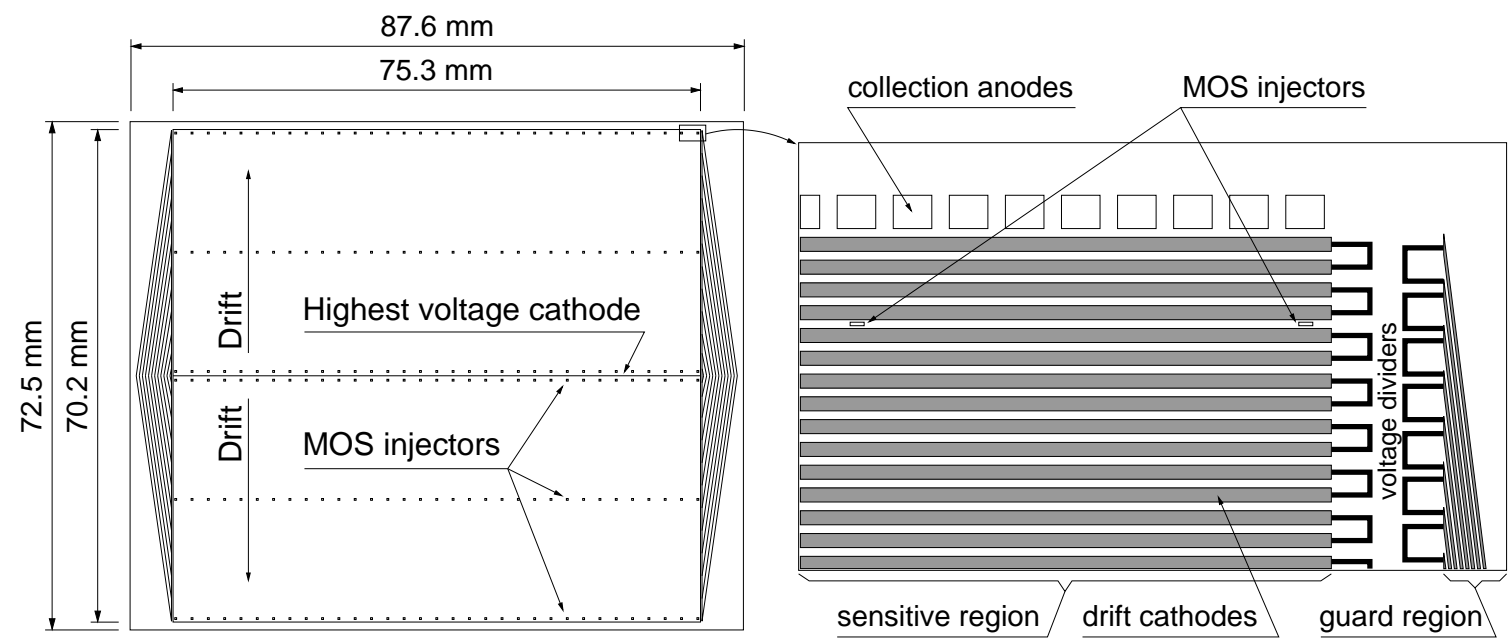

Figure 3.18: Layout of the ALICE SDD. The sensitive area is split into two drift regions by the central, highest voltage, cathode. Each drift region has one row of 256 collection anodes and three rows of 33 point-like MOS charge injectors for monitoring the drift velocity. Drift and guard regions have independent built-in voltage dividers.

is implemented by two insensitive guard regions biased by 145 cathode strips with $32 \mu \mathrm{m}$ pitch. To improve the detector reliability, all the drift and guard regions have their own built-in voltage dividers. Their total power dissipation is $1 \mathrm{~W}$ per detector and is removed by an appropriate air circulation system. Each drift region has 256 collection anodes with $294 \mu \mathrm{m}$ pitch and three rows of 33 MOS charge injectors $\left(20 \times 100 \mu \mathrm{m}^{2}\right.$ each) to monitor the drift velocity which depends on temperature: $v_{\text {drift }} \propto T^{-2.4}$, [59], which gives a $0.8 \% / \mathrm{K}$ variation at room temperature. They will be triggered at regular intervals during the gaps in the LHC orbit.

At the bias voltage of $-2.4(-1.65) \mathrm{kV}$ the drift velocity is $8.1(5.6) \mu \mathrm{m} / \mathrm{ns}$. Since the frontend electronics samples the signal of each anode at a frequency of $40.08 \mathrm{MHz}$, the size of the sensitive element (cell) is $294 \times 202(130) \mu \mathrm{m}^{2}$, corresponding to $89.1(138.4) \times 10^{3}$ cells per detector, which are readout by 512 electronic channels ( 256 at each end).

The space precision along the drift direction $(r \varphi)$, as obtained during beam tests of full-size prototypes, is better than $38 \mu \mathrm{m}$ over the whole detector surface. The precision along the anode axis $(z)$ is better than $30 \mu \mathrm{m}$ over $94 \%$ of the detector surface and reaches $60 \mu \mathrm{m}$ close to the anodes, where a fraction of clusters affects only one anode. The average values are $35 \mu \mathrm{m}$ and $25 \mu \mathrm{m}$ respectively [60]. The detection efficiency is larger than $99.5 \%$ for amplitude thresholds as high as 10 times the electronic noise.

The double-track resolution was calculated using the detailed simulation program, included in AliRoot, see section 6.4 after its preliminary tuning based on the single-track results from beam tests [61]. Due to charge diffusion during the drift process, the double-track resolution is a function of the drift time for a given separation efficiency. The relative distance at which two clusters are disentangled with a $70 \%$ efficiency grows almost linearly from $600 \mu \mathrm{m}$ near the anodes to $800 \mu \mathrm{m}$ at the maximum drift distance [62]. The average double-track resolution measured in the beam test for tracks perpendicular to the SDD plane is $570 \mu \mathrm{m}$ for $70 \%$ resolution efficiency [63]. The main parameters of the ALICE SDD are summarised in table 3.5. 
Table 3.5: The main characteristics of the ALICE silicon drift detectors.

\begin{tabular}{|l|c|}
\hline Sensitive area & $70.17 \times 75.26 \mathrm{~mm}^{2}$ \\
Total area & $72.50 \times 87.59 \mathrm{~mm}^{2}$ \\
Collection anodes (readout channels) & $2 \times 256$ \\
Anode pitch & $294 \mu \mathrm{m}$ \\
Operating voltage & -1.65 to $-2.4 \mathrm{kV}$ \\
Nominal bias of the collection region & $-40 \mathrm{~V}$ \\
Drift velocity & 5.6 to $8.1 \mu \mathrm{m} / \mathrm{ns}$ \\
Maximum drift time & 4.3 to $6.3 \mu \mathrm{s}$ \\
Cell size at drift velocity $8.1 \mu \mathrm{m} / \mathrm{ns}$ & $294 \times 202 \mu \mathrm{m}^{2}$ \\
Cells per detector at drift velocity $8.1 \mu \mathrm{m} / \mathrm{ns}$ & $2 \times 256 \times 174$ \\
Total number of cells $(260$ SDDs) & $23.10^{6}$ \\
Average resolution along the drift $(r \varphi)$ & $35 \mu \mathrm{m}$ \\
Average resolution along the anode $(z)$ & $25 \mu \mathrm{m}$ \\
Detection efficiency & $99.5 \%$ \\
Average double-track resolution at $70 \%$ efficiency at max. field & $700 \mu \mathrm{m}$ \\
\hline
\end{tabular}

\subsubsection{Front-end electronics and readout}

The SDD front-end electronics is based on three types of ASICs. The first two, PASCAL and AMBRA, are assembled on the front-end hybrid, shown in figure 3.19 on its assembly jig. PASCAL contains three functional blocks: preamplifier, analogue storage and Analogue-to-Digital Converter (ADC) [64-67]. AMBRA, which receives data from PASCAL, is a digital four-event buffer which performs data derandomisation, baseline equalization on an anode-by-anode basis and 10-bit to 8bit non-linear data compression. AMBRA also sends the data to the third ASIC, CARLOS, which is a zero-suppressor and data-compressor mounted on one of the end-ladder boards [68, 69]. The three ASICs have been designed using radiation-tolerant layout technique (enclosed gate geometry) based on a commercial CMOS deep-submicron process $(0.25 \mu \mathrm{m})$. The PASCAL chip designed with this technology has proven to be insensitive to total ionisation dose up to $300 \mathrm{kGy}$. The average power dissipation of each PASCAL-AMBRA front-end channel is about $6 \mathrm{~mW}$.

The signal generated by an SDD anode feeds the PASCAL amplifier-shaper which has a peaking time of $40 \mathrm{~ns}$ and a dynamic range of $32 \mathrm{fC}$ (the charge released by an 8-MIP particle hitting near the anode). The amplifier is DC coupled to the sensor and is able to cope with a leakage current of 500nA per anode. The amplifier output is sampled at $40.08 \mathrm{MHz}$ by a ring analogue memory with 256 cells per anode. This is the mode of operation in the idle state of the front-end. When an L0 trigger is received, the SDD BUSY is immediately set and, after a programmable delay which accounts for the L0 latency $(1.2 \mu \mathrm{s})$ and the maximum detector drift time $(\sim 5 \mu \mathrm{s})$, the analogue memories are frozen. The BUSY being still set, their contents are then digitised by a set of 10-bit linear successive-approximation ADCs which write the data into one of the free AMBRA buffers. On a run-by-run basis, PASCAL can be programmed to use half of this nominal frequency for the sampling, thus reducing the amount of data and, therefore, the subsystem readout dead-time. Analysis of beam test data and simulation have shown that the cost in terms of both spatial resolution and double-track resolution is negligible. The advantages of an A/D 

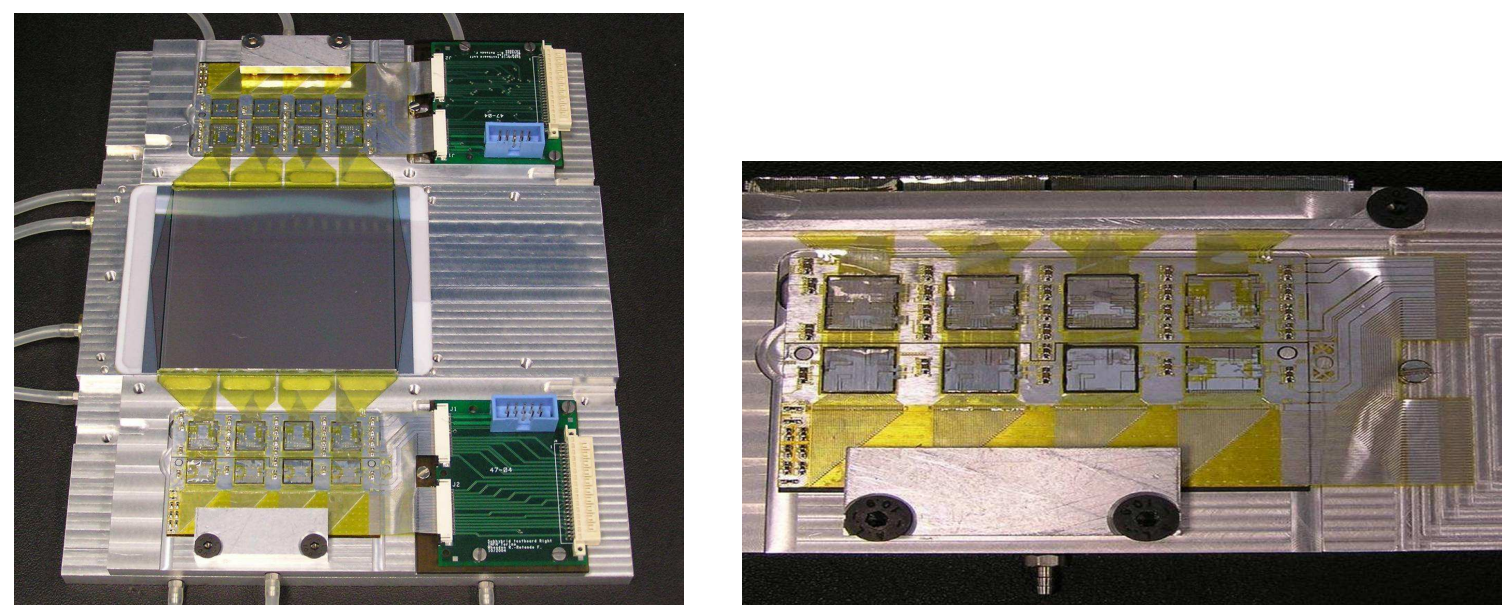

Figure 3.19: Left: a Silicon Drift Detector on the assembly jig with the two front-end hybrids connected, via their test extensions, to test interface circuits. Right: a single hybrid on its assembly jig: the four chips on the top row are the PASCALs, the ones on the bottom are the AMBRAs.
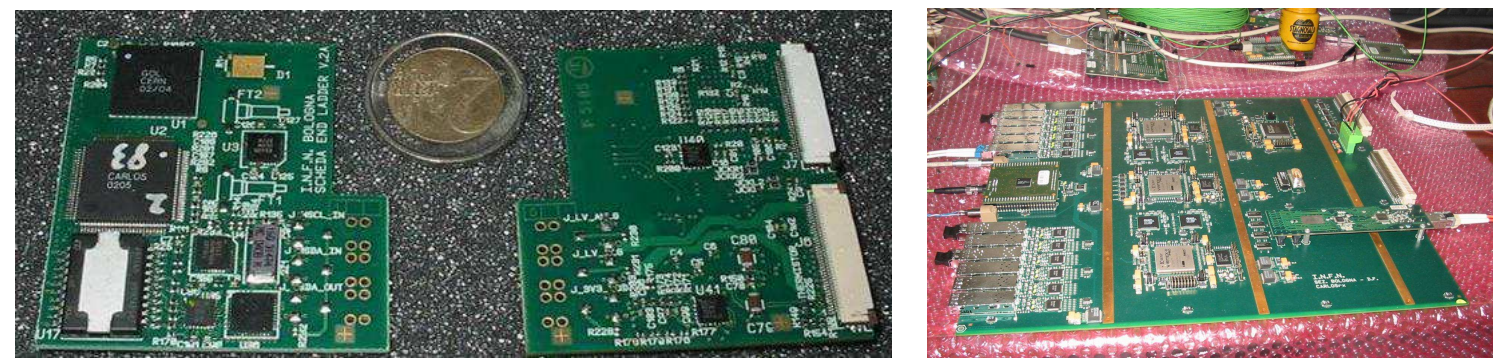

Figure 3.20: Left: photograph of a CARLOS end-ladder board, with the GOL and CARLOS chips visible on the left. Right: photograph of a CARLOS-rx board.

conversion on board of the front-end chip are the noise immunity during signal transmission, and the possibility of inserting a multiple-event buffer to derandomise the data and, therefore, to slow down the transfer rate to the DAQ system. This greatly reduces the material budget of the cabling. The digitisation lasts for about $230 \mu \mathrm{s}(120 \mu \mathrm{s}$ in half-frequency mode) and can be aborted by the absence of the L1 trigger or by the arrival of an L2-reject signal; in both cases, the frontend electronics resets the SDD BUSY and returns to the idle state within $100 \mathrm{~ns}$. On the successful completion of the analogue-to-digital conversion the SDD BUSY is reset if at least one buffer is still available in the AMBRAs. As soon as the conversion is completed, all the AMBRAs transmit the data in parallel to the CARLOS chips on the end-ladders, an operation which takes $1.23 \mathrm{~ms}(0.61 \mathrm{~ms}$ in half-frequency mode) if 192 samples per anode are read out. By means of a 2-dimensional 2thresholds algorithm and with no additional dead time, the CARLOS chips reduce the SDD event size from the raw $24.4 \mathrm{MB}$ by more than one order of magnitude. They also format data and feed the GOL ASICs [70] which in turn drive the optical links. The board on which the CARLOS and GOL chips are mounted is shown in figure 3.20 (the chips are visible on the left). In the counting room, 24 VME boards, CARLOS-rx [71], concentrate the data coming from the 260 SDDs into 24 DDL (Detector Data Link) [72] channels and embody the trigger information in the data flow. 
Table 3.6: Main parameters of the SDD front-end electronics.

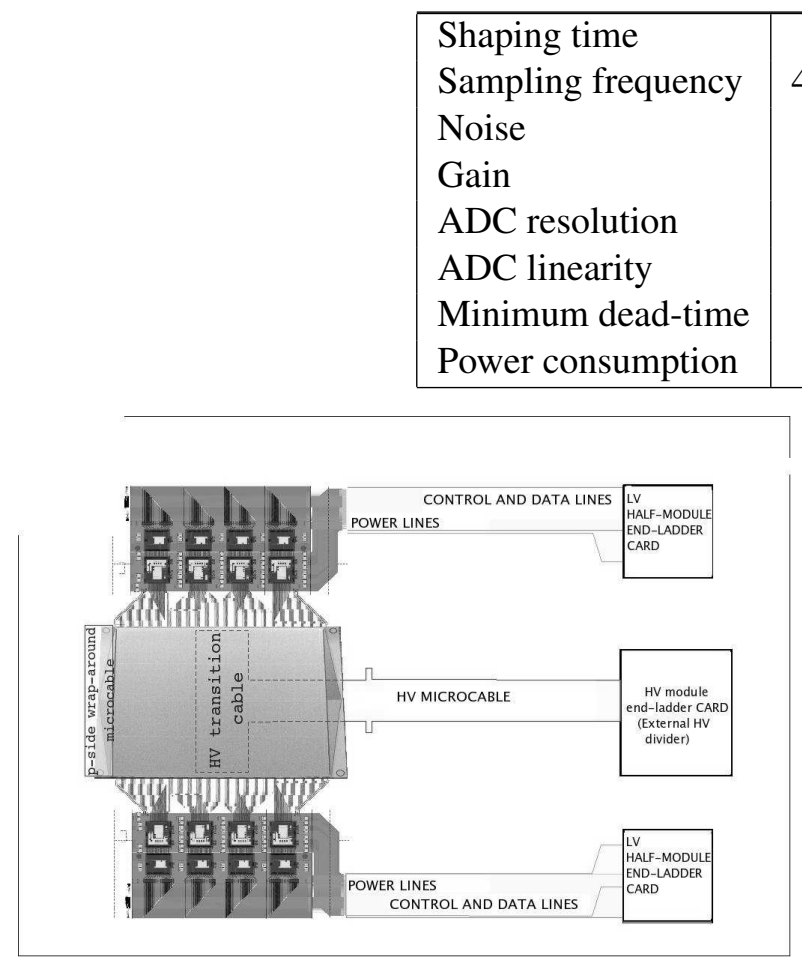

\begin{tabular}{|c|}
$40 \mathrm{~ns}$ \\
$40 \mathrm{MHz}$ or $20 \mathrm{MHz}$ \\
350 electrons rms \\
$50 \mathrm{mV} / \mathrm{fC}$ \\
$10 \mathrm{bit}$ \\
better than $1 \%$ \\
$129 \mu \mathrm{s}$ \\
$6 \mathrm{~mW} / \mathrm{channel}$
\end{tabular}

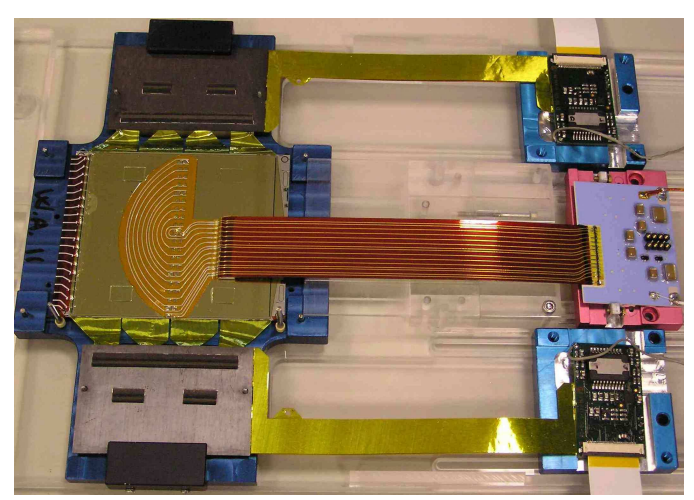

Figure 3.21: Scheme (left) and photograph (right) of a SDD module.

CARLOS-rx, shown in figure 3.20, also uploads in parallel the configuration parameters received over the DDL to the ladder electronics it controls, and monitors the error-flag words embedded in the data flow by the CARLOS chips in order to signal potential Single-Event Upsets (SEU) on the ladder electronics.

To allow the full testability of the readout electronics at the board and system levels, the three ASICs embed a JTAG standard interface. In this way it was possible to test each chip after the various assembly stages. The same interface is used to download control information into the chips before the data taking. A programmable test pulse generator on PASCAL allows for a fast, yet detailed, test of the whole chain for calibration purposes.

The average noise of the front-end electronics is $2 \mathrm{ADC}$ counts rms, which corresponds to an Equivalent Noise Charge (ENC) of 350 electrons. This value measured at the system level is close to that obtained with a single chip (320 electrons) [73].

Table 3.6 summarizes the main parameters of the SDD front-end electronics.

\subsubsection{SDD modules}

A SDD module (see figure 3.21) consists of one silicon drift detector and two front-end hybrids, each connected to the corresponding end-ladder LV board. A micro-cable, specially designed to carry high voltage (up to over $2.4 \mathrm{kV}$ ), connects the detector to the HV end-ladder board. All the assembly and test steps were performed in-house [74]. A photograph of the final assembly is shown in figure 3.21 . 
The detector bias voltage is provided by specially designed flexible printed circuit called "micro-cables". The connections to the central bias cathode and to the injector lines is provided by a micro-cable called 'transition cable', glued on the detector p-side. The bias lines are wire bonded to the corresponding bonding pads. The high voltage is then brought to the $\mathrm{n}$-side using the so called 'wrap-around' cable. The transition cable is Tape Automatic Bonded (TAB) to the long HV cable connected to the corresponding HV end-ladder board. Each sensor was carefully tested and after being accepted for module production. It was equipped with transition and wrap-around cables and sent to module assembly. The transition cable and the wrap-around cable are clearly visible in figure 3.21, on the centre of the sensor and on its left-hand side respectively.

After wafer-testing, with a yield of $78 \%$ for AMBRA and $89 \%$ for PASCAL [73], thinning to $150 \mu \mathrm{m}$ and dicing, the front end chips were $\mathrm{TAB}$ bonded to aluminium multilayer printed circuits called 'chip-cables', one chip-cable per PASCAL-AMBRA pair. This circuit provides connections of data lines between AMBRA and PASCAL as well as the connections to the sub-hybrid circuit. After testing, the bondings on the chip-cables were protected by a thin layer of glue.

After this encapsulation process, chip-cables were glued four by four on a sub-hybrid circuit, in turn glued onto a rigid carbon-fibre heat-dissipater called heat-bridge, which clips to the cooling tubes running along both sides of a ladder to ensure good thermal contact. Each hybrid was then tested before and after a series of thermal cycles from 20 to $65^{\circ} \mathrm{C}$ and the bonds encapsulated with protective glue.

Testing at the different assembly stages was facilitated by the presence of extensions to the micro-cables interfacing to the test equipment, which were later removed before further assembly. Such test extensions are visible in figure 3.19 on page 40, which shows a sensor being connected to its two front-end hybrids.

Finally, the module was completed by the assembly of the long cables which connect it to the LV and HV boards, both sitting at the end of the ladders. These micro-cables have typical polyimide and aluminium thicknesses varying from 12 to $20 \mu \mathrm{m}$, leading to total thicknesses of the multilayer assembly of $220 \mu \mathrm{m}$ for the digital cable and $150 \mu \mathrm{m}$ for the analogue one. The high voltage cable is $340 \mu \mathrm{m}$ thick.

The LV boards ensure the signal interfaces and Low Voltage distribution, carry the radiationhard low voltage regulators, the LVDS signal-receivers, the interface with the DCS and the ancillary circuitry to drive the MOS charge injectors, while the HV boards carry a support High-Voltage divider. The development of the long micro-cable was particularly delicate since it was designed to minimize material while keeping excellent High-Voltage insulation, signal quality and power dissipation.

The complexity of the assembly and test procedures (over 20 process steps were necessary) led to an average time of 3 days to have the complete module ready for final test with laser.

This test was developed to get a complete characterization of each individual module prior to assembly in the ladders. By mounting the fully assembled module on a moving stage and generating charge at known positions with a laser, each sensor was mapped with over 100000 points, providing a detailed description of its response. In particular, this method allows for the generation of correction tables compensating for doping fluctuations, which affect a subset of the detectors [75, 76], defects in the integrated voltage divider and noisy or dead channels. 

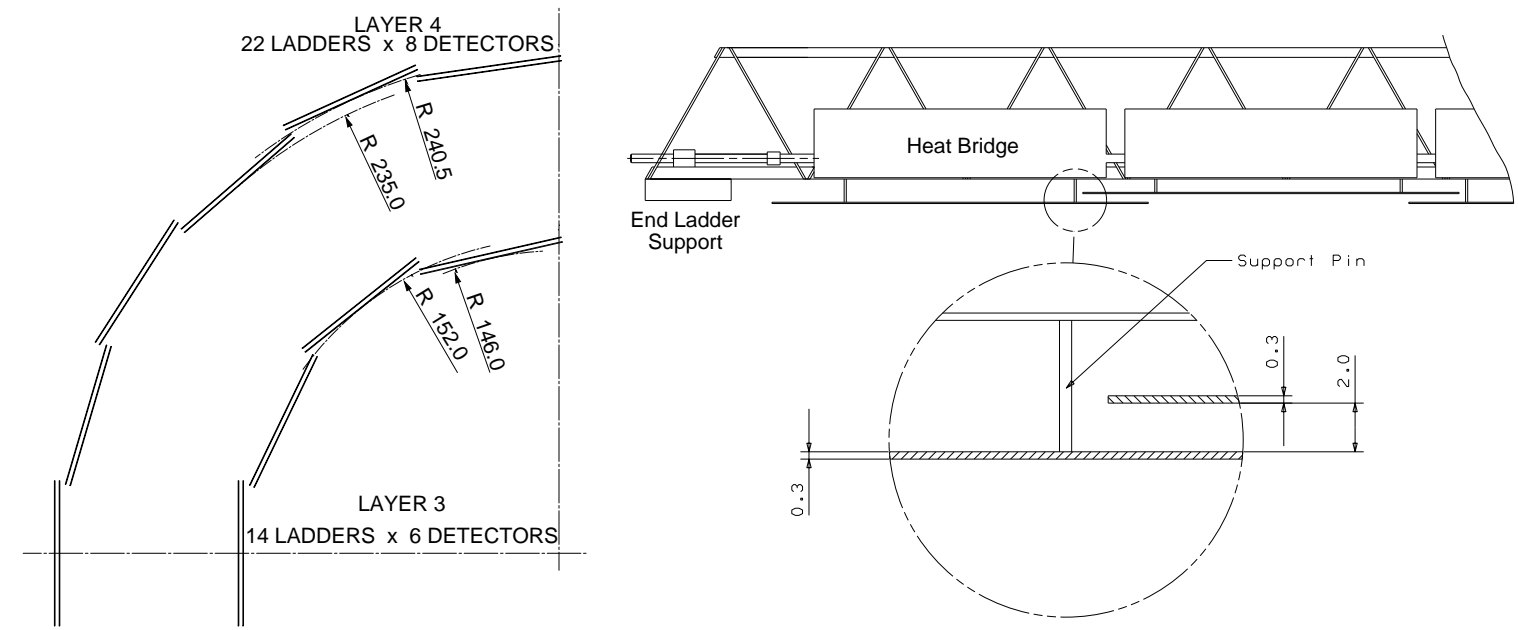

Figure 3.22: The SDDs are mounted at different radii in both $\mathrm{r} z$ and $\mathrm{r} \phi$ planes to obtain the full coverage in the acceptance region. Units are millimetres.

Table 3.7: Main parameters of the ALICE SDD layers and ladders.

\begin{tabular}{|l|c|c|}
\hline & layer 3 & layer 4 \\
\hline \hline Detectors per ladder & 6 & 8 \\
Ladders per layer & 14 & 22 \\
Detectors per layer & 84 & 176 \\
Ladder sensitive half-length (cm) & 22.16 & 29.64 \\
Average layer radius (cm) & 15.03 & 23.91 \\
Ladder space-frame weight (g) & 11 & 15 \\
Weight of ladder components (g) & 87 & 121 \\
\hline
\end{tabular}

\subsubsection{SDD ladders}

The SDDs modules are mounted on linear structures called ladders. There are 14 ladders with six modules each on layer 3 , and 22 ladders with eight modules each on layer 4. Modules and ladders are assembled (figure 3.22) to have an overlap of the sensitive areas larger than $580 \mu \mathrm{m}$ in both $r \varphi$ and $z$ directions. This ensures full angular coverage for vertices located within the interaction diamond, $\pm \sigma=10.6 \mathrm{~cm}$, and for $p_{\mathrm{t}}>35 \mathrm{MeV} / c$.

The main geometrical parameters of the SDD layers and ladders are summarised in table 3.7. The ladder space frame is a lightweight truss made of Carbon-Fibre Reinforced Plastic (CFRP) and has a protective coating against humidity absorption. The sagging of the space frame under the load of the ladder components applied to the mid point of the truss was evaluated with a Finite Element Analysis model and verified by measurement. When the load acts perpendicularly to the detector plane the sagging is $21 \mu \mathrm{m}$; when it acts parallel to the detector plane the sagging is $7 \mu \mathrm{m}$. All ladders were measured individually, and only those with sags smaller than $40 \mu \mathrm{m}$ were accepted for mounting. 

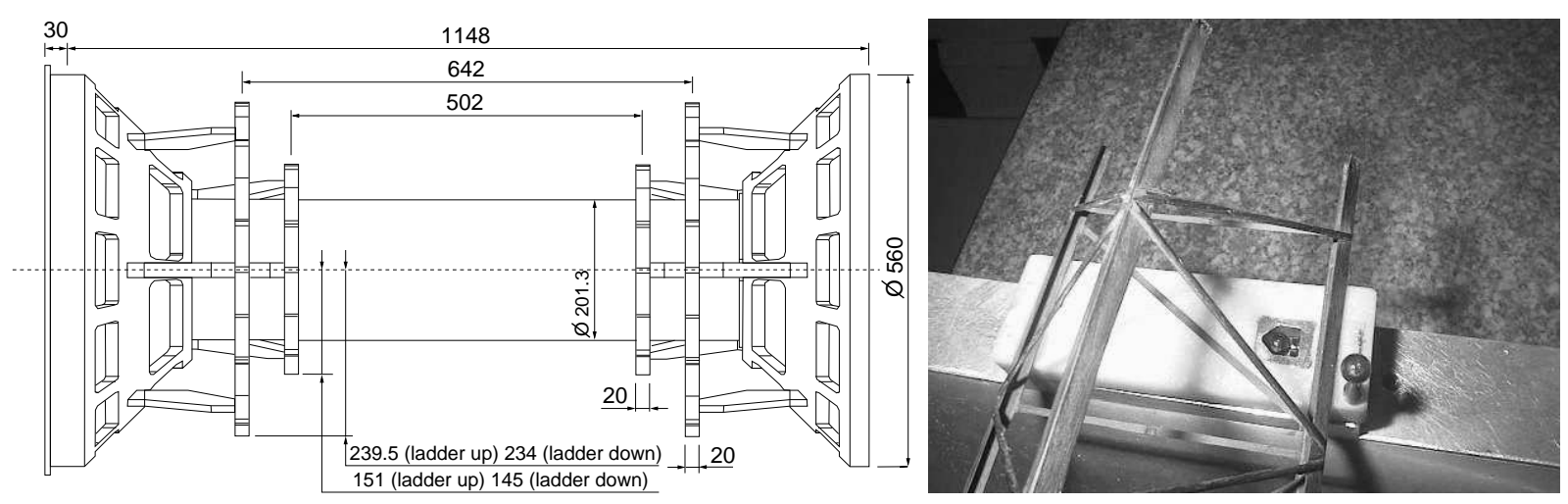

Figure 3.23: Left: support structure of the SDD ladders; units are millimetres. Right: ladder foot with its $\mathrm{V}$-shaped nut and the reference ruby sphere which is pressed against the nut by a spring. The ruby sphere glued onto the ladder foot is used to verify the ladder position once the assembly of a layer is completed.

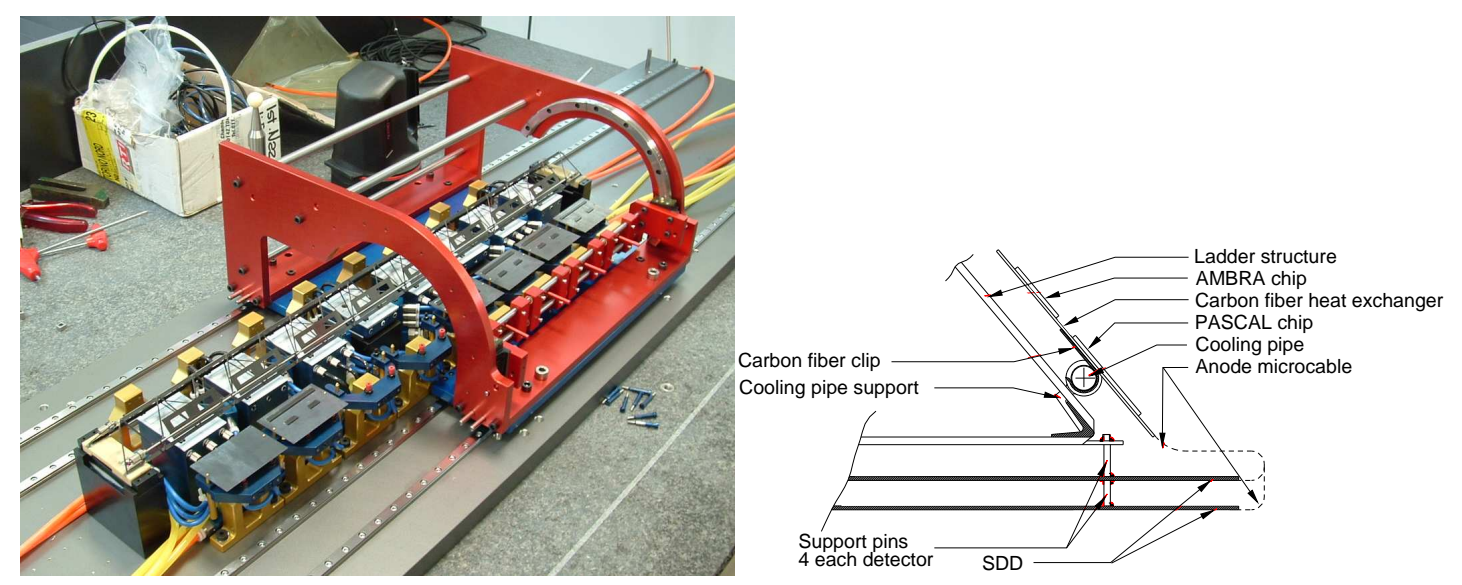
support rings (figure 3.23). The cones provide the links to the outer Silicon Strip Detector barrel and have windows for the passage of the SDD services. The support rings are mechanically fixed to the cones and bear reference ruby spheres for the ladder positioning. The accuracy of the re-positioning of the ladders after a dismount-mount sequence was measured to be better that $10 \mu \mathrm{m}$ full width.

The detectors are attached to the support structure using ryton pins and have their anode rows parallel to the ladder axis $(z)$. The front-end electronics, assembled on two hybrid circuits, one per anode row, is glued with a thermo-conductive compound on rigid carbon fibre heat-exchangers, which in turn are clipped to the cooling pipes running along the ladder structure (figure 3.24). The pipes are made of MP35N alloy and have $80 \mu \mathrm{m}$ wall thickness and $2 \mathrm{~mm}$ inner diameter. The coolant is demineralised water and the system is designed for underpressure operation. The connections between the detectors, the front-end hybrids and the end-ladder boards, and the connection for the detector biasing are all provided with flexible aluminium-polyimide micro-cables that are 

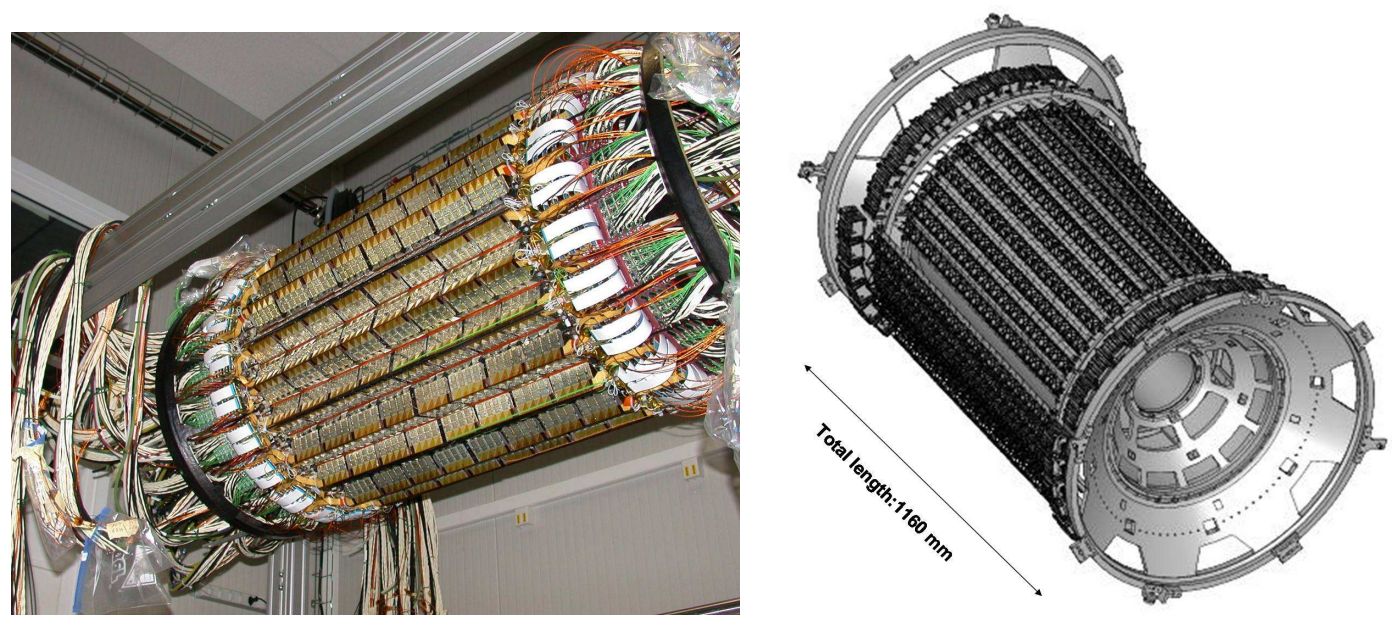

Figure 3.25: Left: the SDD detector completely assembled, ready to be integrated with the Silicon Strips. Right: the CAD desgin of the SDD layers, showing the support cones and the ladders of the two layers.

TAB bonded. Before the hybrids were clipped to the cooling pipes, the positions of the detectors were measured with respect to the reference ruby spheres glued to the ladder feet. The whole procedure of assembly of detector modules to the ladder frame was performed using a precision measurement machine and a set of specially-designed tools, which allowed the assembly of one ladder in approximately one day. Figure 3.24 shows a ladder during assembly and the assembly tool. Each ladder, once assembled and measured, was completely tested. The few modules with problems which could not be fixed on the ladder were removed and replaced. Although the MOS injectors have proven to be an excellent monitoring instrument for the drift velocity, the cooling system of the SDD layers has been designed with extreme care to provide a temperature stability of $0.1 \mathrm{~K}$, undergoing not only detailed simulations and prototype tests, but also the construction of a full scale model of the entire system, which allowed the verification and optimization of the proposed solutions $[28,30]$. The final system is a combination of two independent underpressure water circuits, one coupled to the front-end electronics via the pipes running along the ladders and the other coupled to the readout electronics, LV and HV boards via pipes embedded in the end-ladder structure. The water cooling is complemented by a moderate air flow preventing the creation of hot spots while providing additional cooling to the sensors [28]. To ensure proper operation and the persistence of the underpressure conditions, each of the 13 SDD cooling circuits is controlled by four pressure regulators and monitored by 4 flow-meters and 6 pressure sensors. Depending on its position, a cooling circuit cools two or four ladders (and the related end-ladders). This kind of grouping has been imposed by the limited available space for the piping.

In figure 3.25 the completely assembled SDD detectors are shown, prior to insertion in the SSD detectors, together with a CAD drawing of the SDD layers, showing ladders from both layers and the support cones. 
Table 3.8: SSD system parameters (see text).

\begin{tabular}{|l|c|l|}
\hline component layer & layer 5 & layer 6 \\
\hline \hline Radius & 378 and $384 \mathrm{~mm}$ & 428 and $434 \mathrm{~mm}$ \\
Sensitive area & $2.2 \mathrm{~m}^{2}$ & $2.8 \mathrm{~m}^{2}$ \\
Number of ladders & 34 & 38 \\
Modules/ladder & 22 & 25 \\
Total modules & 748 & 950 \\
\hline Total weight & \multicolumn{2}{|c|}{$1.24 \mathrm{kN}$} \\
\hline
\end{tabular}

\subsubsection{Silicon Strip Detectors (SSD)}

\subsubsection{Overview}

The outer layers of the ITS are crucial for the matching of tracks from the TPC to the ITS. They provide a two dimensional measurement of the track position. In addition they provide $\mathrm{d} E / \mathrm{dx}$ information to assist particle identification for low-momentum particles. The system is optimized for low mass in order to minimise multiple scattering.

Both outer layers use double sided Silicon Strip Detectors (SSD) [77]. The detection modules [78] consist of one sensor each, connected to two hybrids with six HAL25 [79] chips each. All interconnections between the sensor and the electronics in the detection module are made using aluminium on polyimide cables (micro-cables) [80]. Minimization of the material budget of the mechanical support for the detection modules is achieved by using linear Carbon Fibre Composite (CFC) material [81] for all support structures in the active volume. The cooling system is also optimized $[29,30]$ in line with the zero heat balance required for all ALICE detectors. The application of $300 \mu \mathrm{m}$ thick CFC with high thermal conductivity as miniature motherboards [30] (stiffeners) significantly reduces the material needed for the support and cooling of the front-end chips [30]. The modules are assembled on ladders [81] of the same design as those supporting the SDD. These ladders are one module wide and up to 25 modules long along the beam direction. The 72 ladders, carrying a total of 1698 modules, are mounted on CFC support cones in two concentric cylinders. For each layer the ladders are mounted in two slightly different radii such that full azimuthal coverage is obtained. The modules are cooled by water running through two thin ( $40 \mu \mathrm{m}$ wall thickness) phynox tubes along each ladder [30, 82].

The electronic signals from the modules are AC-coupled and buffered in custom made electronics, the EndCap Modules (ECM) [83] located at each end of each ladder. The analogue to digital conversion of the signals performed in the Front-End ReadOut Modules (FEROM) [84] located outside the ALICE magnet, see figure 3.26.

The technology of each component was chosen corresponding to the radiation environment and magnetic field in which they need to operate. The segmentation of the power, cooling and readout system avoids failure of significant parts of the system due to a single point failure. Down to the level of the front-end chips malfunctioning components can be isolated to maintain system integrity. The main geometrical parameters of this system are listed in table 3.8. A detailed description of the SSD system design can be found in [11]. 


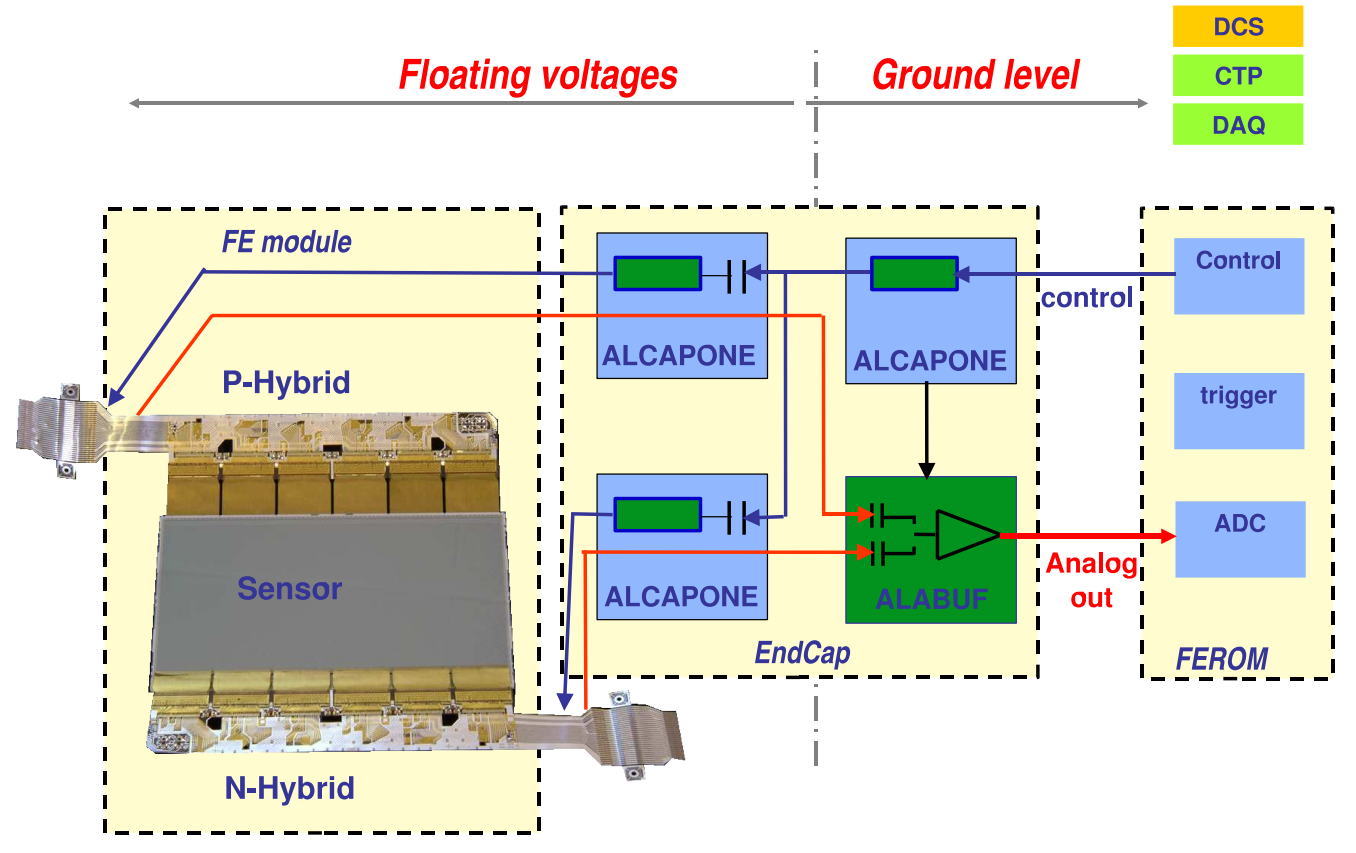

Figure 3.26: Schematic overview of the SSD readout system. The detection module is located in the active volume. The endcap is located at the end of the ladder, just outside the active volume and the FEROM is located outside the ALICE magnet (see text).

Table 3.9: SSD sensor parameters. The sensors were produced by three different suppliers (Canberra, ITC and Sintef) according to the same specification.

\begin{tabular}{|l|c|l|c|}
\hline Sensor area & $75 \times 42 \mathrm{~mm}^{2}$ & Active area & $73 \times 40 \mathrm{~mm}^{2}$ \\
Sensor thickness & $300 \mu \mathrm{m}$ & Strip pitch & $95 \mu \mathrm{m}$ \\
Strips per side & 768 & Stereo angle & $35 \mathrm{mrad}$ \\
Strip tilt p-side & $+7.5 \mathrm{mrad}$ & Strip tilt n-side & $-27.5 \mathrm{mrad}$ \\
Operating voltage & $20-90 \mathrm{~V}$ & Bias+guard current & $\leq 7 \mu \mathrm{A}$ \\
\hline
\end{tabular}

\subsubsection{Detection module}

The sensors are $300 \mu \mathrm{m}$ thick and they have 768 strips on each side with a pitch of $95 \mu \mathrm{m}$. The stereo angle is $35 \mathrm{mrad}$ which is a compromise between stereo view and reduction of ambiguities resulting from high particle densities. The stereo angle is obtained by defining strips with an angle of $7.5 \mathrm{mrad}$ with respect to the sensor short edge on the p-side and with an angle of $27.5 \mathrm{mrad}$ on the $\mathrm{n}$-side. Sensors are mounted with the strips nearly parallel to the magnetic field in order to optimize the resolution in the bending direction, see table 3.9. The sensor p-side (n-side) of layer 5 (layer 6) faces the interaction region. This results in four almost equally spaced strip orientations in the two layers, which significantly reduces the number of ambiguities seen by the tracking software.

The hybrids are obtained by connecting six HAL25 front-end chips [79] to an aluminium on polyimide hybrid circuit (flex) which is supported by the stiffener. The chips are glued directly 
Table 3.10: HAL25 front-end chip parameters.

\begin{tabular}{|l|c|}
\hline Non-linearity & $<5 \%$ \\
Readout & sequential analogue samples \\
Readout speed & $10 \mathrm{MHz}$ at $20 \mathrm{pF} / 200 \Omega$ \\
Supply & $+2.5 \mathrm{~V}$ single supply \\
Dissipation & $340 \mu \mathrm{W} /$ channel \\
Internal parameter control & JTAG \\
Size & $3.8 \mathrm{x} 11 \mathrm{~mm}^{2}$ \\
Thickness & $150 \mu \mathrm{m}$ \\
Technology & $0.25 \mu \mathrm{m}$ IBM CMOS, 3 metal layers \\
Design & mixed analogue and digital radtol ASIC \\
Analogue channels & 128 \\
Input connection pitch & $80 \mu \mathrm{m}$ \\
Output connection pitch & $125 \mu \mathrm{m}$ \\
ENC with sensor connected & $<400 e^{-}$ \\
Shaping time & $1.2-2.4 \mu \mathrm{s}$ adjustable \\
Output & $14 \mathrm{MIP}$ \\
Range & $250 \mu \mathrm{A}$ bipolar differential current \\
\hline
\end{tabular}

on the stiffener which provides a thermal conductivity about 1.3 times better than copper while keeping the multiple scattering to a minimum (about $0.03 \% X_{0}$ ). Each stiffener is connected to the cooling tubes by small aluminium clamps. The flex consists of two layers of $30 \mu \mathrm{m}$ thick aluminium traces on a $20 \mu \mathrm{m}$ thick polyimide foil. These layers are glued together and the electrical connections between them are made using single point tape automated bonding (spTAB) [85]. The flex hosts power lines as well as digital and analogue lines for driving and reading the chips. The decoupling capacitors and transmission line termination resistors are soldered onto the flex.

The HAL25 is a mixed analogue and digital ASIC realized in IBM $0.25 \mu \mathrm{m}$ CMOS technology, see table 3.10 .

It is designed to be radiation tolerant up to several Mrad. It includes 128 analogue channels. Each channel has a preamplifier to convert the positive or negative charge input from the sensor into an analogue voltage step whose magnitude is proportional to the charge. Its input range is about 300000 electrons, corresponding to about 14 MIPS in this set-up. The preamplifier is followed by a shaping circuit whose shaping time is remotely adjustable between $1.4 \mu \mathrm{s}$ and $2.4 \mu \mathrm{s}$. The analogue peak value is stored in a sample and hold circuit controlled by an external HOLD signal generated in the FEROM, see section 3.1.3.4. The analogue samples are shifted out serially through an integrated analogue multiplexer at a maximum speed of $10 \mathrm{MHz}$ under control of a clock and token generated in the FEROM. All internal parameters of the HAL25 can be set through a JTAG bus. For testing purposes the HAL25 contains an internal pulser and boundary scan registers.

All connections between the chips and the flex and between the chips and the sensor are made by chip-cables connected via spTAB. Chip-cables consist of $14 \mu \mathrm{m}$ thick aluminium traces supported by a $10 \mu \mathrm{m}$ thick polyimide foil [80]. They allow for an easy testing of the chip before 


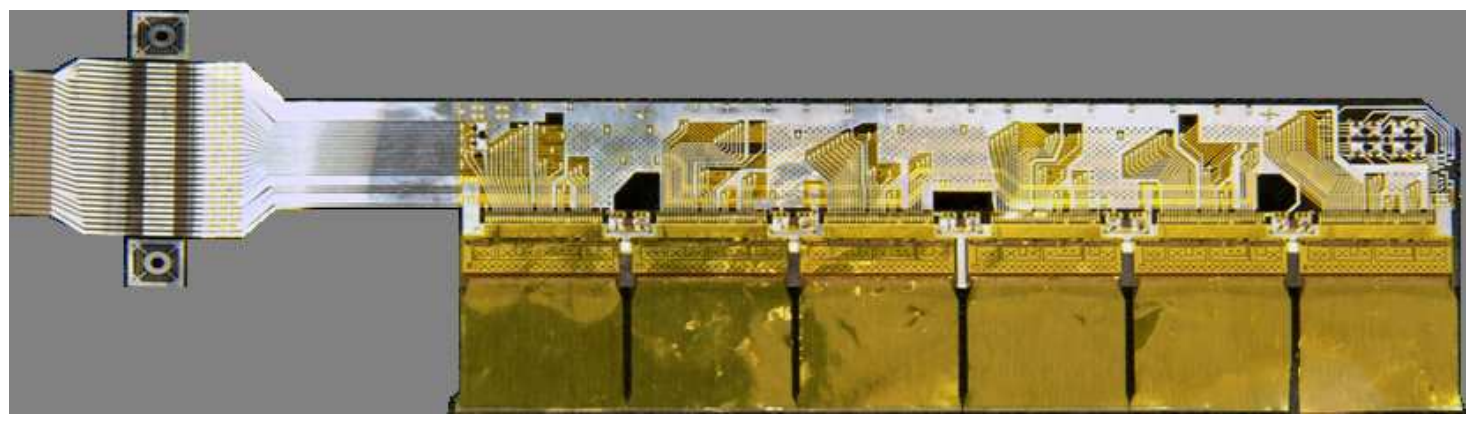

Figure 3.27: Photograph of a hybrid. Each hybrid was tested individually, using the connection area at the left side of the flex. This part was cut off before connecting to the sensor. The lower part of the photograph shows the six chip-cables which will connect the HAL25 chips to the sensor. The chips themselves are covered by the chip-cables and not visible in this picture. The aluminium clamps connecting to the cooling tubes are on the reverse side of the hybrid.

further use. The chip-cables also function as a pitch adapter between the sensor pads ( $95 \mu \mathrm{m}$ pitch) and the chip input pads ( $80 \mu \mathrm{m}$ pitch). In addition the use of a flexible connection between sensor and electronics makes it possible to fold the hybrids on top of the sensor, creating a compact object. The detection modules are mounted on $1 \mathrm{~m}$ long carbon fibre supports [81] such that the active areas of the sensors overlap $0.9 \mathrm{~mm}$ in the $z$-direction. Each module is connected to the support by three pins made of G10. A photograph of a hybrid is shown in figure 3.27.

\subsubsection{Endcaps}

The detection modules are connected to the Front-End ReadOut Module (FEROM) by means of the endcap modules [83]. These modules are placed at both ends of each ladder.

Each endcap module controls the detector modules on a half ladder, the number of modules depending on which layer and which side. Due to the limited available space $\left(7 \times 7 \times 5 \mathrm{~cm}^{3}\right)$ the electronics is miniaturized and active cooling is necessary. The electronics is able to survive Single Event Effects (SEE) and about $500 \mathrm{~Gy}$ of total ionizing dose. It is built around two ASIC's in the same IBM $0.25 \mu \mathrm{m}$ CMOS technology as the HAL25. The control functions for the front-end and the endcap, like power regulation and latch-up protection (over current), signal coupling, readout control and error detection are integrated in the ALCAPONE chip. The ALABUF includes drivers for the analogue signals and switches between the analogue signals from the $\mathrm{p}$-side and $\mathrm{n}$-side of the detection modules.

The electronics that reads out each side of the sensor, operate at a different potential, defined by the sensor bias. This sensor bias voltage is provided symmetrically with respect to ground potential. Therefore, all signals are connected via AC coupling to each detector side, see also figure 3.26 on page 47. Each hybrid has its own ALCAPONE with a power regulator that has a programmable voltage, but a fixed current limit. The HAL25 front-end chips need to be configured via the JTAG serial bus. This bus is distributed by the ALCAPONE chips. The endcap itself is also controllable via the same JTAG connection.

At the start of the readout, a token is sent by the FEROM to all the modules, first to the 
p-hybrids and then to the n-hybrids. Analogue switches in the ALABUF multiplex the analogue signals from the $\mathrm{p}$-side and $\mathrm{n}$-side of the detection module to create a single differential signal per module which is transmitted to the ADC's in the FEROM. The endcap is connected to the outside via a custom made cable which provides the LVDS control signals for the readout, the JTAG communication, the detector bias, and the low power supply voltages from the floating power supplies. The analogue outputs are also transmitted via this cable bundle. All signals on this cable are differential to minimize interference and are at safe ground potential, because the AC coupling is done completely inside the endcap.

\subsubsection{Readout}

The main task of the Front-End ReadOut Module (FEROM) [84] is to digitize the 2.6 million analogue samples from the front-end modules, keeping up with the trigger rate in ALICE. This is achieved by digitizing the signals from each of the 1698 detection modules in parallel. The detection modules themselves can store only one analogue sample per input. They serialize the 1536 analogue samples before sending them to the FEROM.

The FEROM system, see figure 3.28, consists of 8 crates each containing 216 analogue-todigital converters (AD modules) and two interface cards (LINK modules) connected to the data acquisition system, the detector control system and the central trigger processor. These crates are located in the cavern just outside the ALICE magnet. They connect to the endcaps via approximately $40 \mathrm{~m}$ long twisted pair cables.

Upon an L0 trigger the FEROM activates the HOLD signal with an adjustable delay, matching the shaping time of the amplifiers in the HAL25 on the detection module, see section 3.1.3.2.

If the event is accepted by the L1 trigger decision the FEROMs generate the control signals needed for the readout of the detection modules. The analogue samples of the two hybrids of each module are shifted out. With the chosen readout rate of $10 \mathrm{MHz}$ the total conversion time for one event is less than $160 \mu \mathrm{s}$. The design of the front-end electronics does not allow overlapping L0 triggers before an L2 trigger decision is made. In addition the BUSY of the readout system remains set for $40 \mu \mathrm{s}$ after completion of a readout cycle in order to allow the ladder electronics to quiet down. Therefore the readout rate is limited to about $5 \mathrm{kHz}$. In case the zero suppression in the FEROM is switched off or the occupancy increases above $6 \%$ the readout rate is limited by the bandwidth of the optical link to the data acquisition system at $25 \mathrm{MB} / \mathrm{s}$ per FEROM crate. The expected occupancy for the most central events in $\mathrm{Pb}-\mathrm{Pb}$ collisions is below $3 \%$.

In case the event is rejected by a L2 trigger decision a fast-clear signal is sent to the endcaps and to the front-end chips and the obtained digital and analogue information is discarded. In order not to overload the data acquisition system the digitized data are corrected for pedestals and zerosuppressed for each sample individually. The remaining data are stored in an event buffer and sent to the data acquisition system asynchronously. The FPGA based design allows easy upgrading of the algorithm, when needed. Currently an additional algorithm to suppress baseline distortion and its implementation is being studied so that it can be installed should the experimental environment prove different from the laboratory environment.

The detector control system communicates with the FEROM using the JTAG protocol. The JTAG chain can be used to check interconnections inside the FEROM and the connections between 


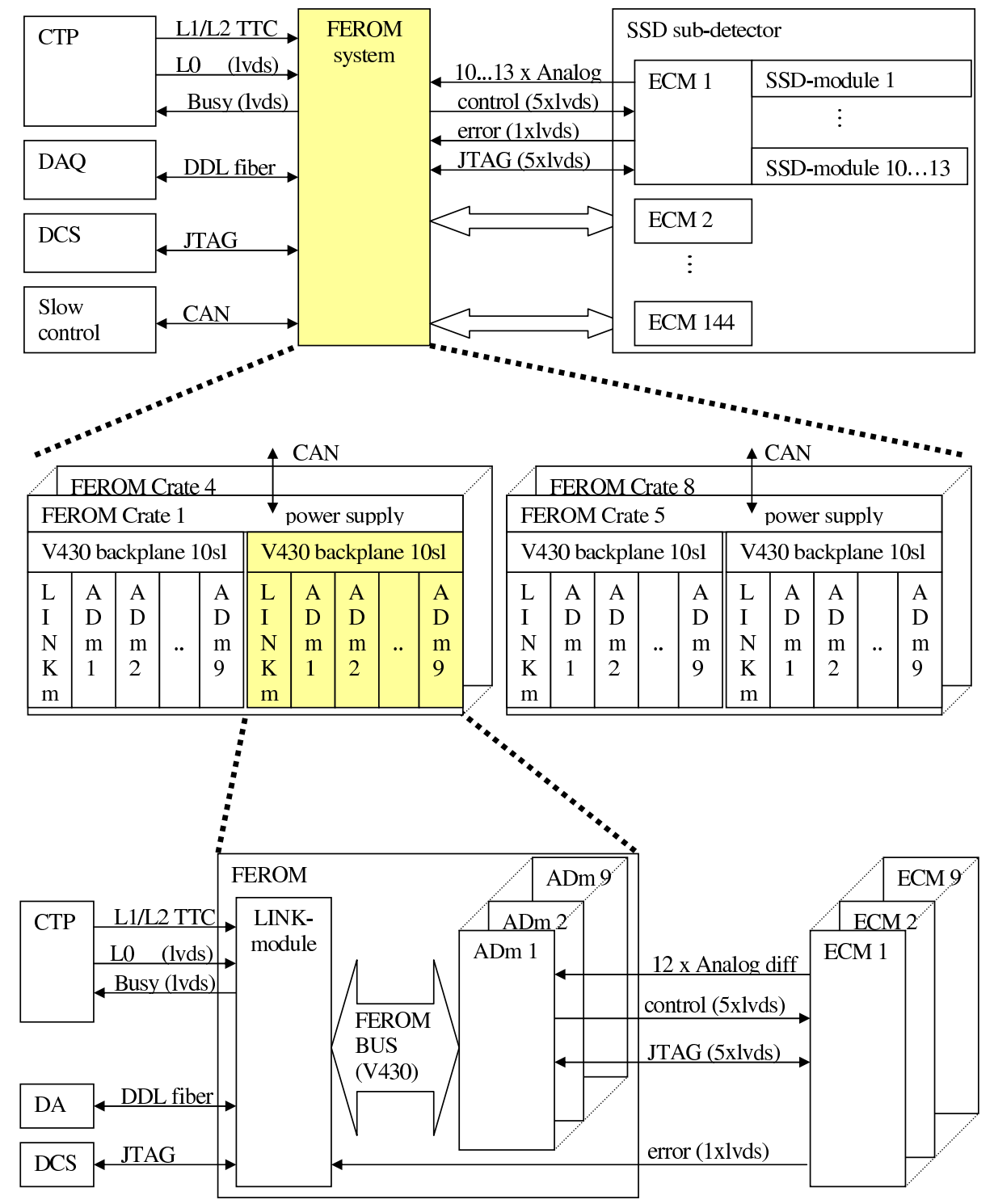

Figure 3.28: The embedding of the FEROM between the ALICE data acquisition and control system (left) and the SSD detector system (right) is shown in the top part of this figure. The central part shows the configuration of 8 FEROM crates, each equipped with AD modules and LINK modules. The bottom section shows the internal communication between the 9 AD modules and the single LINK module in each crate.

the FEROM and the endcap. Each endcap can include the corresponding HAL25 chips in the JTAG chain when needed.

As the FEROMs are installed in the cavern and therefore inaccessible during beam-time, much emphasis was put on high reliability. Each card in a FEROM crate has a multidrop addressable JTAG switch. In this way the scope of a single point failure is reduced to the branch behind the switch. Each card serves up to 12 front-end modules, corresponding to about $0.7 \%$ of the SSD system. Special precautions were taken to reduce SEU problems due to the $0.1 \mathrm{~Gy}$ irradiation 
dose expected. In case the neutron dose outside the ALICE magnet proves to be much higher than expected, an upgrade based on the much more radiation tolerant ACTEL flash FPGA instead of the currently used Xilinx SRAM FPGA is already prepared. A dedicated addressing protocol with TTL comparators, a parity check over the measured data and poly switch fuses on each module limit the consequence of single event effects. Furthermore, each backplane in a FEROM crate is split in two and each half is connected with a separate data link to the DAQ system.

\subsubsection{Power and cooling}

All the sensors mounted on the same half-ladder are biased in parallel by means of two power supply channels belonging respectively to a positive and a negative polarity power supply. These are CAEN power supplies(A3501P and A3501N) which are connected in series to provide the sensors with a bias voltage which is symmetric with respect to a reference ground connected to the central point. The central point is connected to ground in each endcap. The signal ground is connected to the safety ground at the input of the ADC modules in the FEROM. The ladder structure is connected to the endcap signal ground but otherwise floating with respect to the rest of the mechanical structure.

Each hybrid floats upon the potential level of the sensor side to which the hybrid is connected. The voltage needed for operating the hybrids $(2.5 \mathrm{~V})$ is supplied by regulators in the endcap which are powered at $4 \mathrm{~V}$ by low-voltage cards (CAEN A3602). Two channels of the low-voltage cards are used to power respectively the p-side and n-side hybrids of a half-ladder, providing a typical current of $2 \mathrm{~A}$. The third low-voltage channel is used to power the endcap electronics, which is referenced to ground. The powering of both hybrid and endcaps electronics makes use of sense wires to correct for the voltage drop in the connecting cables.

For homogeneity purposes all three low-voltage channels are identical, even though the power needed by the endcap electronics is lower. The choice of using floating power supplies helps in reducing possible common mode phenomena and allows a unique reference ground thereby preventing the occurrence of ground loops.

Both the bias and the low-voltage cards are part of the CAEN EASY system, which is designed to operate in high radiation environment, being able to tolerate moderate radiation doses and to operate inside intense magnetic fields. This choice was necessary since the power supplies will be sitting just outside the L3 magnet in an area where the magnetic field will be as high as 300 Gauss and where the integrated dose of order $0.1 \mathrm{~Gy}$. The power supply modules will be located in the experimental area while the monitoring and control will be made using an interface card (CAEN-A1676A) sitting in a mainframe (CAEN-SY1527) located in the counting room.

The segmentation of the power supplies allows us to switch on each half-ladder individually. In addition each hybrid on each detection module can be switched on individually by the endcaps. Therefore the malfunction of one module will not influence the operation of the others. A short in the power cabling outside the ITS will also affect at most one half-ladder.

The average power dissipated inside the barrel is $2.2 \mathrm{~kW}$ of which one third in the detection modules. The actual power depends mainly on the readout rate. Outside the barrel up to $1 \mathrm{~kW}$ is dissipated in the cables for which no special cooling is provided. 
Table 3.11: SSD performance.

\begin{tabular}{|l|c|l|c|}
\hline Spatial precision $\mathrm{r} \phi$ & $20 \mu \mathrm{m}$ & Spatial precision $\mathrm{z}$ & $820 \mu \mathrm{m}$ \\
Material budget L5 & $0.84 \%$ & Material budget L6 & $0.84 \%$ \\
Support cone & $0.53 \%$ & readout duration & $180 \mu \mathrm{s}$ \\
Dead channels & $<3 \%$ & S/N for 1 MIP & $>30$ \\
\hline
\end{tabular}

Each ladder has two cooling pipes operated in counter flow with the endcap in series with the return on its side. The ladders are grouped into 8 segments corresponding to the segmentation of the FEROMs. The power supplies are interlocked with temperature sensors on each endcap. The water system is designed as a leak proof system; it operates below atmospheric pressure. A moderate airflow is provided to even out moisture and temperature gradients inside the SSD volume. Tests and simulations $[29,30]$ have shown that the SSD can operate in a thermal neutral way with the water temperature about $5 \mathrm{~K}$ below ambient temperature.

\subsubsection{Performance}

The spatial resolution of the SSD system is determined by the $95 \mu \mathrm{m}$ pitch of the sensor readout strips and the charge-sharing between those strips (see table 3.11). Without making use of the analogue information the r.m.s spatial resolution is $27 \mu \mathrm{m}$. Beam tests have shown that a spatial resolution of better than $20 \mu \mathrm{m}$ in the $\mathrm{r} \phi$ direction can be obtained by analysing the charge distribution within each cluster. In the direction along the beam the spatial resolution is $820 \mu \mathrm{m}$.

Almost one half of the material budget of the SSD layers consists of the sensors. The sensors amount to $0.36 \%$ of radiation length; the remainder is distributed between mechanics $(0.20 \%)$, cabling $(0.16 \%)$, and electronics components $(0.11 \%)$ for a total thickness in each layer of only $0.84 \% X_{0}$. The hybrids and cabling on the ladders were reduced to the minimum amount of material required for an efficient heat transfer between the electronics and the cooling tubes and for an acceptable electrical resistance in the cabling. The cylinder connecting the two cones which support the ladders adds $0.53 \% X_{0}$. Thus, the average amount of material seen by particles traversing the two layer SSD at $\theta=90^{\circ}$ is $2.2 \% X_{0}$.

The positions of the detection modules referred to the ladder ends were measured for every ladder before mounting. The sag of a ladder due to gravity was modelled and checked by measurements [86] on selected ladders to be less than $50 \mu \mathrm{m}$. The position of the ladder mounting points was measured relative to the ITS reference points before mounting the ladders. Using all these data, the module positions on the completed SSD are expected to be known within $60 \mu \mathrm{m}$. However, the procedure of precisely checking the module's positions of the completed SSD is based on real tracks.

The efficiency of the SSD system is mainly determined by the number of defective channels and the signal to noise ratio (SNR) obtained in the final system. Beam tests on single modules and laboratory tests with the finally produced ladders have shown that a SNR of better than 30 is achieved in all modules in the final system. In figure 3.29 a typical response of a module to cosmics is shown. For details of the cluster reconstruction method see [87]. The number of non-working channels, i.e. high noise or no digital communication is about $3 \%$. 

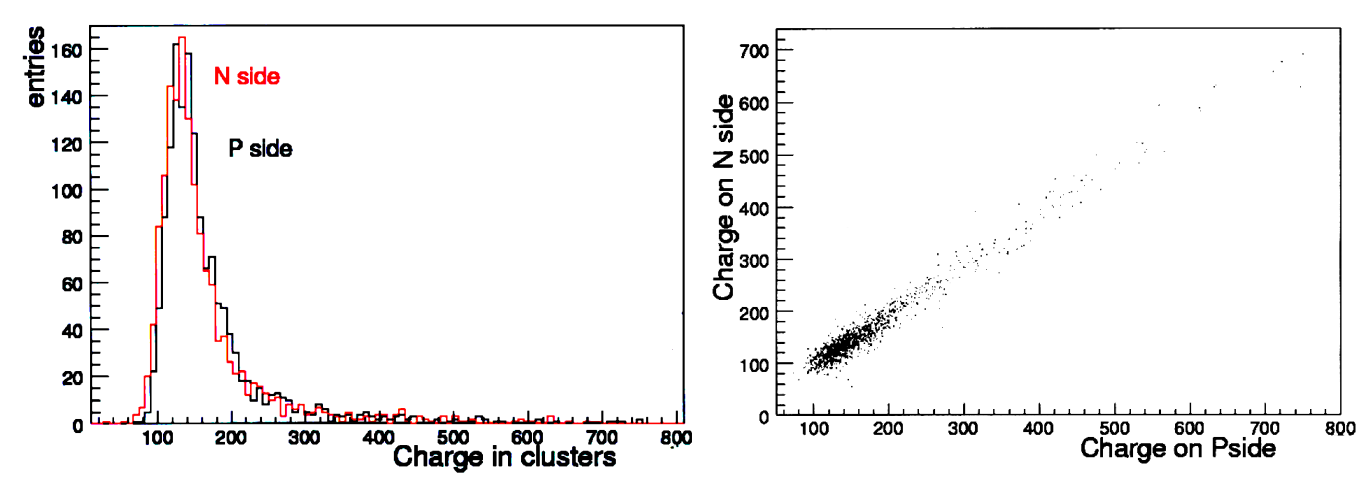

Figure 3.29: Typical response of a module to cosmics. The left panel shows a histogram of the total signal measured at the ADC for the reconstructed clusters. The right panel shows the correlation between the signals measured at the $\mathrm{P}$ and $\mathrm{N}$ sides of the sensor.

\subsection{Time-Projection Chamber (TPC)}

\subsubsection{Design considerations}

The Time-Projection Chamber (TPC) [14] is the main tracking detector of the central barrel and is optimised to provide, together with the other central barrel detectors, charged-particle momentum measurements with good two-track separation, particle identification, and vertex determination, see [14] and chapter 8 of this document. In addition, data from the central barrel detectors are used to generate a fast online High-Level Trigger (HLT) for the selection of low cross section signals, see section 6.3 .

The phase space covered by the TPC in pseudo-rapidity is $|\eta|<0.9$ for tracks with full radial track length (matches in ITS, TRD, and TOF detectors); for reduced track length (at reduced momentum resolution), an acceptance up to about $|\eta|=1.5$ is accessible. The TPC covers the full azimuth (with the exception of the dead zones). A large $p_{\mathrm{t}}$ range is covered from low $p_{\mathrm{t}}$ of about $0.1 \mathrm{Gev} / \mathrm{c}$ up to $100 \mathrm{GeV} / c$ with good momentum resolution, see chapter 8 .

At the $\mathrm{Pb}-\mathrm{Pb}$ design luminosity of the LHC, an interaction rate of $8 \mathrm{kHz}$ is expected, of which $10 \%$ are to be considered as central collisions. In the TPC design phase, before RHIC data allowed a better extrapolation to LHC energy, an extreme charge particle multiplicity density of $\mathrm{d} N_{\mathrm{ch}} / \mathrm{d} \eta=$ 8000 was assumed, which would result in 20000 charged primary and secondary tracks in the TPC acceptance, an unprecedented track density for a TPC. These extreme multiplicities set new demands on the design which were addressed by extensive R\&D activities. Careful optimisation of the TPC design finally resulted in maximum occupancies (defined as the ratio of the number of readout pads and time bins above threshold to all pads and time bins) of about $40 \%$ at the innermost radius and $15 \%$ at the outermost radius for the quoted extreme multiplicity.

It is expected that in $\mathrm{Pb}-\mathrm{Pb}$ runs the TPC can be operated at central collision rates of up to $200 \mathrm{~Hz}$. While there is not much operational experience with large TPCs at these rates, the current load on the readout chambers is not excessive. Simulations have shown that, at this rate, the space charge due to the ion feed-back during gate-open time starts to be comparable to the space charge due to the ionisation in the TPC drift volume itself, resulting in tracking distortions of order a few 
$\mathrm{mm}$. The true space-charge limit, however, might eventually depend on the background conditions due to the beam quality. Offline corrections for the space charge are expected to recover part of the resolution loss and should thus extend this limit.

For proton-proton runs, the memory time of the TPC is the limiting factor for the luminosity due to the $\sim 90 \mu$ s drift time. At a pp luminosity of about $5 \times 10^{30} \mathrm{~cm}^{-2} \mathrm{~s}^{-1}$, with a corresponding interaction rate of about $350 \mathrm{kHz}$, 'past' and 'future' tracks from an average of $60 \mathrm{pp}$ interactions are detected together with the triggered event; the detected multiplicity corresponds to about 30 minimum-bias pp events. The total occupancy, however, is lower by more than an order of magnitude than in $\mathrm{Pb}-\mathrm{Pb}$ collisions, since the average pp multiplicity is about a factor $10^{3}$ lower than the $\mathrm{Pb}-\mathrm{Pb}$ multiplicity for central collisions [4]. Tracks from pile-up events can be eliminated because they point to the wrong vertex.

The space charge, due to both ionisation in the drift volume and to the ion feed-back during gate-open time, is about one order of magnitude lower than for $\mathrm{Pb}-\mathrm{Pb}$, and thus not seen as a problem. Trigger rates of up to $1 \mathrm{kHz}$ seem to be realistic.

Before installation of the TPC in the ALICE cavern in January 2007, beam tests were performed with an inner readout chamber in a special setup [88] and the completed detector was commissioned sector-by-sector with laser and cosmic muon tracks triggered by ACORDE (see chapter 3.8), so that essentially all the design features underwent a serious test. As a result, the main specifications as laid down in the TPC TDR [14] were verified by actual measurements. In the following, we briefly review design features and components of the TPC.

\subsubsection{Detector layout}

The TPC design is 'conventional' in overall structure but innovative in many aspects. A summary of its main parameters is presented in table 3.12. The TPC is cylindrical in shape; the active volume has an inner radius of about $85 \mathrm{~cm}$, an outer radius of about $250 \mathrm{~cm}$, and an overall length along the beam direction of $500 \mathrm{~cm}$.

The detector is made of a large cylindrical field cage, filled with $90 \mathrm{~m}^{3}$ of $\mathrm{Ne} / \mathrm{CO}_{2} / \mathrm{N}_{2}$ (90/10/5), in which the primary electrons are transported over a distance of up to $2.5 \mathrm{~m}$ on either side of the central electrode to the end plates. Multi-wire proportional chambers with cathode pad readout are mounted into 18 trapezoidal sectors at each end plate.

The TPC layout is shown in several figures: figure 3.30 shows a 3D cut-open view of the field cage. Details of the support rods and mylar strips for the 3 bottom sectors of one half of the TPC are shown in figure 3.31. figure 3.32 is a cut along the beam ( $y z)$, figure 3.33 is a cut perpendicular to the beam $(x y)$ at one of the two, identical end plates.

Field cage. The field cage is based on a design with a central high-voltage electrode and two opposite axial potential dividers which create a highly uniform electrostatic field in the common gas volume, see figures 3.30 to 3.33. The central electrode is a stretched aluminized Mylar foil of $22 \mu \mathrm{m}$ thickness to satisfy the requirement of minimal material near $90^{\circ}$ relative to the beam direction. The electrical potential in the drift region is defined by aluminised Mylar strips wound around 18 inner and outer support rods which also contain two resistive potential dividers (inner and outer), see figure 3.31. The rods are aligned with the dead zones in-between the readout chambers. 
Table 3.12: Synopsis of TPC parameters.

\begin{tabular}{|c|c|}
\hline Pseudo-rapidity coverage & $\begin{array}{l}-0.9<\eta<0.9 \text { for full radial track length } \\
-1.5<\eta<1.5 \text { for } 1 / 3 \text { radial track length }\end{array}$ \\
\hline Azimuthal coverage & $360^{\circ}$ \\
\hline Radial position (active volume) & $848<r<2466 \mathrm{~mm}$ \\
\hline Radial size of vessel (outer dimensions) & $606.5<r<2780 \mathrm{~mm}$ \\
\hline Radial size of vessel (drift gas volume) & $788<r<2580 \mathrm{~mm}$ \\
\hline Length (active volume) & $2 \times 2500 \mathrm{~mm}$ \\
\hline Segmentation in $\varphi$ & 18 sectors \\
\hline Segmentation in $r$ & 2 chambers per sector \\
\hline Total number of readout chambers & $2 \times 2 \times 18=72$ \\
\hline $\begin{array}{l}\text { Inner readout chamber geometry } \\
\text { pad size } \\
\text { pad rows } \\
\text { total pads }\end{array}$ & $\begin{array}{c}\text { trapezoidal, } 848<r<1321 \mathrm{~mm} \text { active area } \\
4 \times 7.5 \mathrm{~mm} \quad(\varphi \times r) \\
63 \\
5504 \\
\end{array}$ \\
\hline $\begin{array}{l}\text { Outer readout chamber geometry } \\
\text { pad size } \\
\text { pad rows } \\
\text { total pads }\end{array}$ & $\begin{array}{l}\text { trapezoidal, } 1346<r<2466 \mathrm{~mm} \text { active area } \\
6 \times 10 \text { and } 6 \times 15 \mathrm{~mm} \quad(\varphi \times r) \\
64+\quad 32=96 \quad \text { (small and large pads) } \\
5952+4032=9984 \quad \text { (small and large pads) }\end{array}$ \\
\hline $\begin{array}{l}\text { Detector gas } \\
\text { Gas volume } \\
\text { Drift length } \\
\text { Drift field } \\
\text { Drift velocity } \\
\text { Maximum drift time } \\
\text { Total HV } \\
\text { Diffusion } \\
\end{array}$ & $\begin{array}{c}\mathrm{Ne} / \mathrm{CO}_{2} / \mathrm{N}_{2} \quad 90 / 10 / 5 \\
90 \mathrm{~m}^{3} \\
2 \times 2500 \mathrm{~mm} \\
400 \mathrm{~V} / \mathrm{cm} \\
2.7 \mathrm{~cm} / \mu \mathrm{s} \\
92 \mu \mathrm{s} \\
100 \mathrm{kV} \\
D_{\mathrm{L}}=D_{\mathrm{T}}=220 \mu \mathrm{m} / \sqrt{\mathrm{cm}}\end{array}$ \\
\hline Material budget & $X / X_{0}=3.5 \%$ near $\eta=0$ \\
\hline $\begin{array}{l}\text { Front-End Cards (FEC) } \\
\text { Readout Control Unit (RCU) scheme } \\
\text { Total RCUs } \\
\text { Total pads — readout channels }\end{array}$ & $\begin{array}{c}121 \text { per sector } \times 36=4356 \\
6 \text { per sector, } 18 \text { to } 25 \text { FEC per RCU } \\
216 \\
557568\end{array}$ \\
\hline $\begin{array}{l}\text { Pad occupancy (for } \mathrm{d} N / \mathrm{d} y=8000) \\
\text { Pad occupancy (for } \mathrm{pp} \text { ) }\end{array}$ & $\begin{array}{cc}40 \text { to } 15 \% & \text { inner / outer radius } \\
5 \text { to } 2 \times 10^{-4} & \text { inner / outer radius }\end{array}$ \\
\hline $\begin{array}{l}\text { Event size } \quad(\text { for } \mathrm{d} N / \mathrm{d} y=8000) \\
\text { Event size } \quad(\text { for } \mathrm{pp}) \\
\text { Total bandwidth } \\
\text { Trigger rate limits }\end{array}$ & $\begin{array}{c}\sim 90 \mathrm{MB} \quad(\text { conditions see text }) \\
\sim 1-4 \mathrm{MB} \text { (depending on pile-up and noise threshold) } \\
\sim 30 \mathrm{~GB} / \mathrm{s} \\
300 \mathrm{~Hz} \mathrm{Pb-Pb} \text { central events } \\
1000 \mathrm{~Hz} \text { proton-proton events }\end{array}$ \\
\hline $\begin{array}{l}\text { ADC } \\
\text { sampling frequency } \\
\text { time samples } \\
\text { Conversion gain }\end{array}$ & $\begin{array}{c}10 \mathrm{bit} \\
5-10 \mathrm{MHz} \\
500-1000 \\
6 \text { ADC counts / fC }\end{array}$ \\
\hline $\begin{array}{r}\text { Position resolution }(\sigma) \text { in } r \varphi \\
\text { in } z\end{array}$ & $\begin{array}{c}1100 \text { to } 800 \mu \mathrm{m} \text { inner / outer radii } \\
1250 \text { to } 1100 \mu \mathrm{m}\end{array}$ \\
\hline $\begin{array}{r}\mathrm{d} E / \mathrm{d} x \text { resolution, isolated tracks } \\
\mathrm{d} N / \mathrm{d} y=8000\end{array}$ & $\begin{array}{l}5.0 \% \\
6.8 \%\end{array}$ \\
\hline
\end{tabular}




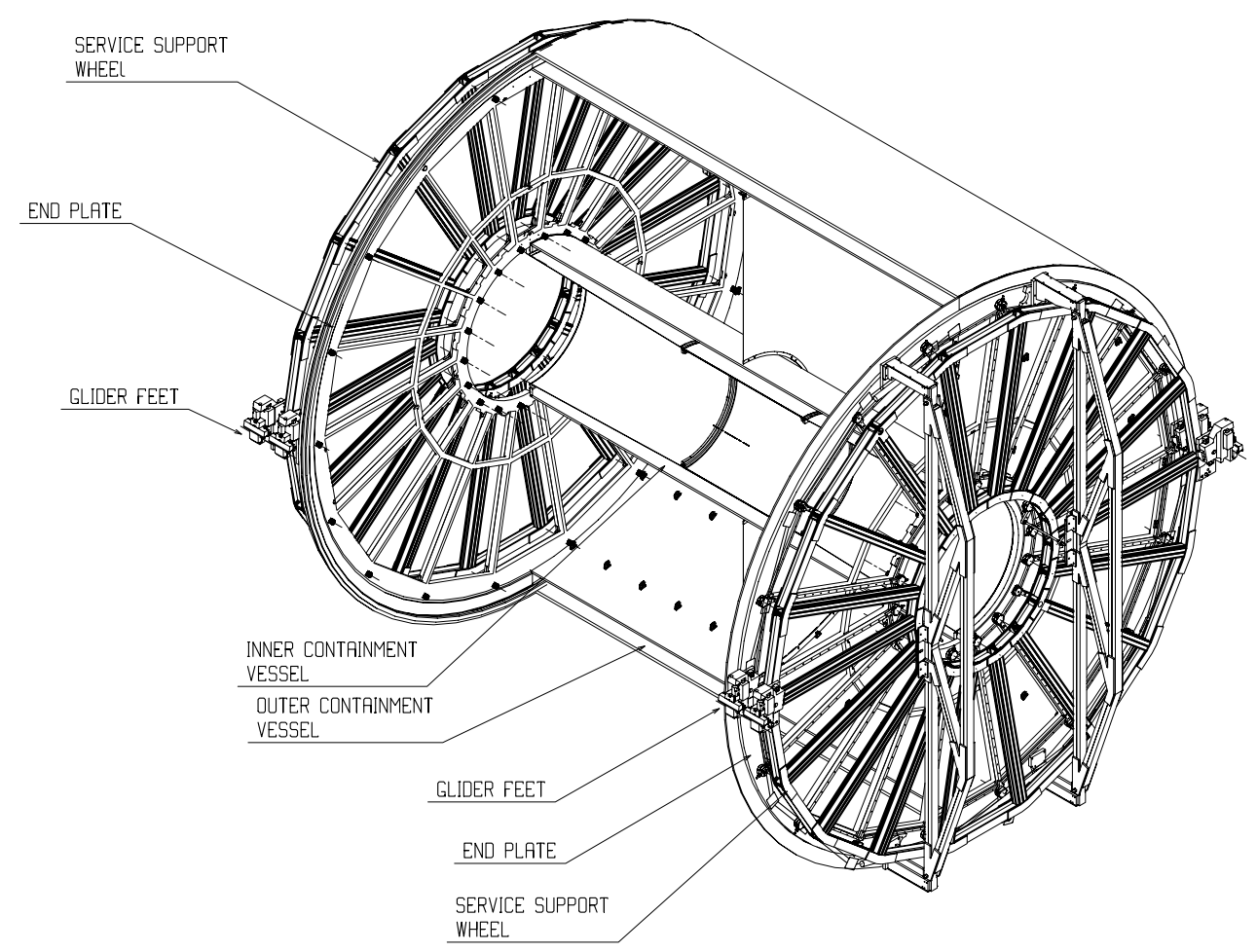

Figure 3.30: 3D view of the TPC field cage and service support wheels, without readout chambers. The containment vessels are cut open. The support rods and mylar strips are not shown, see figure 3.31 for these details.

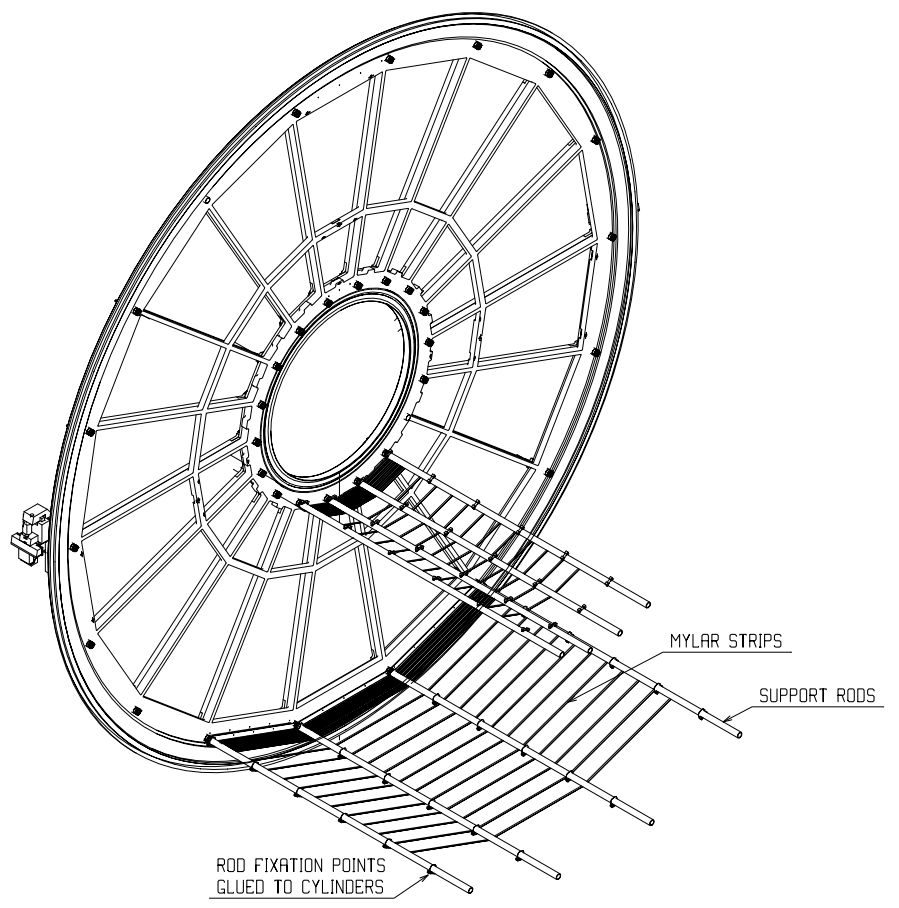

Figure 3.31: Detail of the field cage structure for three sectors at the bottom, showing the support rods and the mylar strips (near the end plate, the first few strips are all shown, further on for clarity only a few. There are 166 strips in total on each side). One inner and one outer rod contain a resistive divider. 


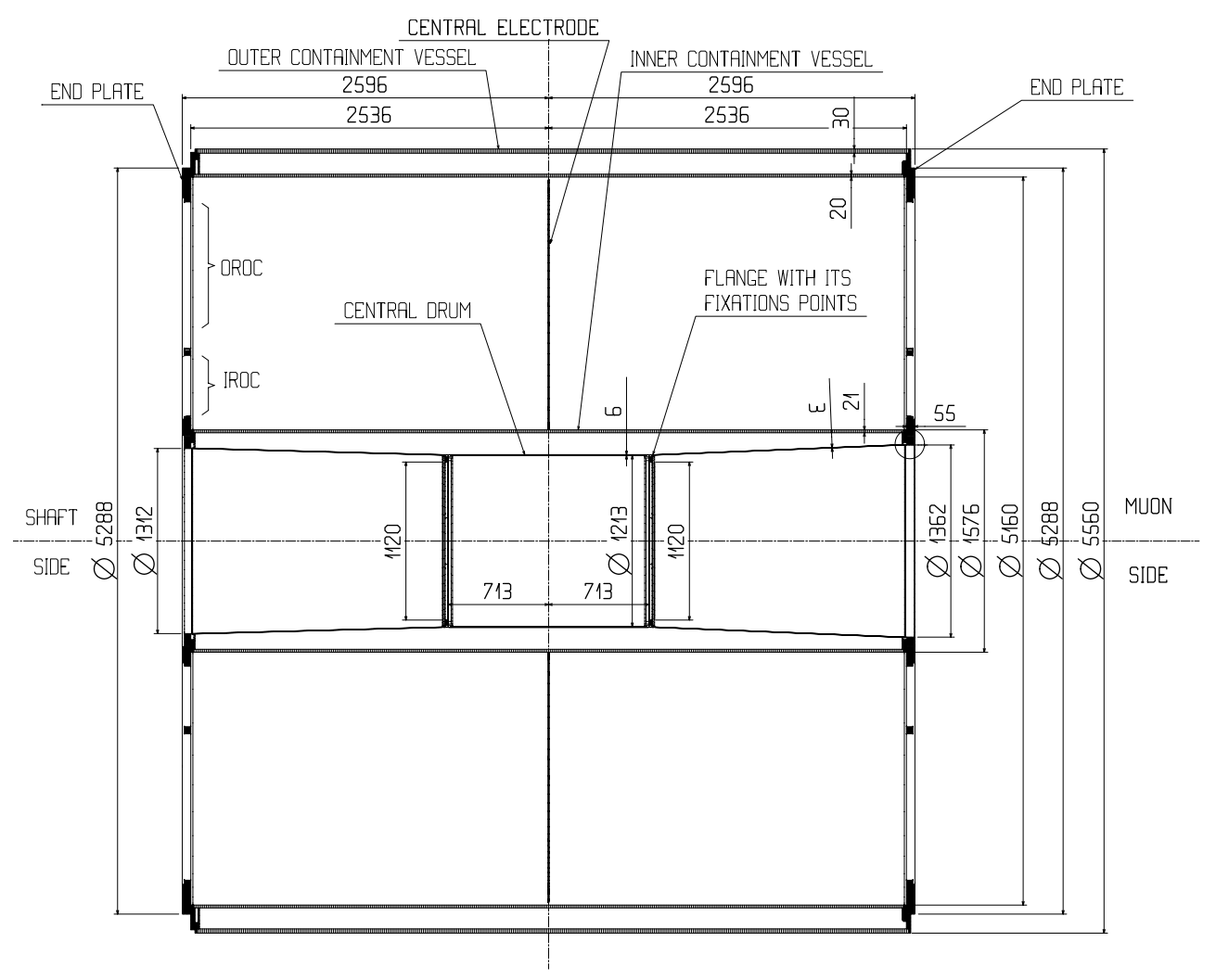

Figure 3.32: Cross section along the beam axis of the TPC field cage with some dimensions.

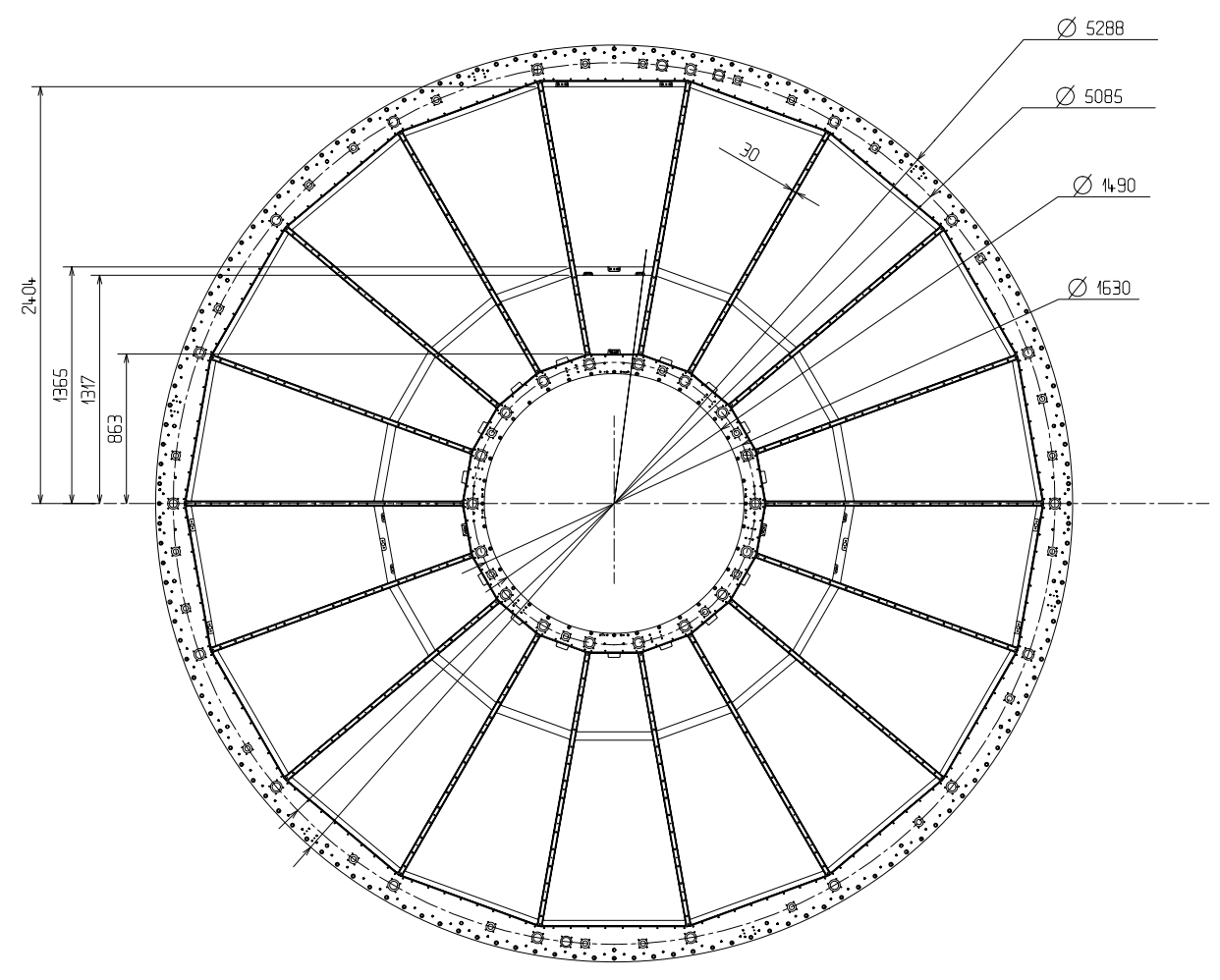

Figure 3.33: Layout of the TPC end plate. 
Because of the $\mathrm{Ne} / \mathrm{CO}_{2} / \mathrm{N}_{2}$ gas mixture used in the TPC, the field cage is operated at high voltage gradients, of about $400 \mathrm{~V} / \mathrm{cm}$, with a high voltage of $100 \mathrm{kV}$ at the central electrode, which results in a maximum drift time of about $90 \mu \mathrm{s}$.

An insulating gas envelope of $\mathrm{CO}_{2}$ in containment vessels surrounds the field cage. The field cage and containment volumes are each constructed from two concentric cylinders, sealed by the end plate on either side. To provide high structural integrity against gravitational and thermal loads while keeping the material budget low, composite materials were used. Hence the mechanical stability and precision is guaranteed to be about $250 \mu \mathrm{m}$. The coplanarity of the central electrode and of the entrance grids of the readout chambers have been carefully surveyed and adjusted to this precision by individual shimming of the readout chamber mounts.

Drift gas system. The drift gas $\mathrm{Ne} / \mathrm{CO}_{2} / \mathrm{N}_{2}(90 / 10 / 5)$ is optimised for drift speed, low diffusion, low radiation length and hence low multiple scattering, small space-charge effect, and ageing and stability properties. Mixtures containing $\mathrm{CH}_{4}$ and $\mathrm{CF}_{4}$ were rejected due to their ageing properties. The $\mathrm{N}_{2}$ admixture improves the quenching and allows higher maximum gas gains [89]. The drawback of $\mathrm{Ne} / \mathrm{CO}_{2}$ is that this mixture is a 'cold' gas, with a steep dependence of drift velocity on temperature [90]. For this reason, the TPC is aiming for a thermal stability with $\Delta T \leq 0.1 \mathrm{~K}$ in the drift volume, see below.

The gas system circulates and purifies the gas mixture, with very low fresh gas injection. The pressure follows the ambient pressure. The $\mathrm{CO}_{2}$ and $\mathrm{N}_{2}$ fractions are kept stable to $0.1 \%$, necessary to ensure stable drift velocity and gas gain of the readout chambers. The $\mathrm{O}_{2}$ impurity as achieved with a reduced flow during commissioning is extrapolated to be about $1 \mathrm{ppm}$ or lower in the final installation in the cavern, limiting the signal reduction due to attachment for the maximum drift length of $2.5 \mathrm{~m}$ to $<5 \%$.

Readout chambers. The readout chambers instrument the two end plates of the TPC cylinder with an overall active area of $32.5 \mathrm{~m}^{2}$ [91-93]. The chambers are multi-wire proportional chambers with cathode pad readout. Because of the radial dependence of the track density, the readout is segmented radially into two readout chambers with slightly different wire geometry adapted to the varying pad sizes mentioned below. The radial range of the active area is from $84.8 \mathrm{~cm}$ to $132 \mathrm{~cm}$ (and from $134.6 \mathrm{~cm}$ to $246.6 \mathrm{~cm}$ ) for the inner (and outer) chamber, respectively. Note that the active area of the readout chambers are larger than the openings of the end plate in figure 3.33, as seen, e.g., in figure 3.36. This design optimizes active area; it required, however, the mounting of the readout chamber from the inside. This was accomplished with a mounting tool that allowed insertion of a chamber through the end plates into the field cage, rotating and positioning of the chamber once inside, and finally engaging the chamber in its proper orientation with the mount points on the end plate from inside. The inactive areas between neighbouring inner chambers are aligned with those between neighbouring outer chambers. Such an arrangement optimises the momentum precision for detected high-momentum tracks but has the drawback of creating cracks in the acceptance - in about $10 \%$ of the azimuthal angle the detector is non-sensitive. The readout chambers are made of standard wire planes, i.e. they consist of a grid of anode wires above the pad plane, a cathode wire plane, and a gating grid, see figures 3.34 and 3.35 showing cuts along and perpendicular to the wires for the inner readout chamber. They combine, however, a very 


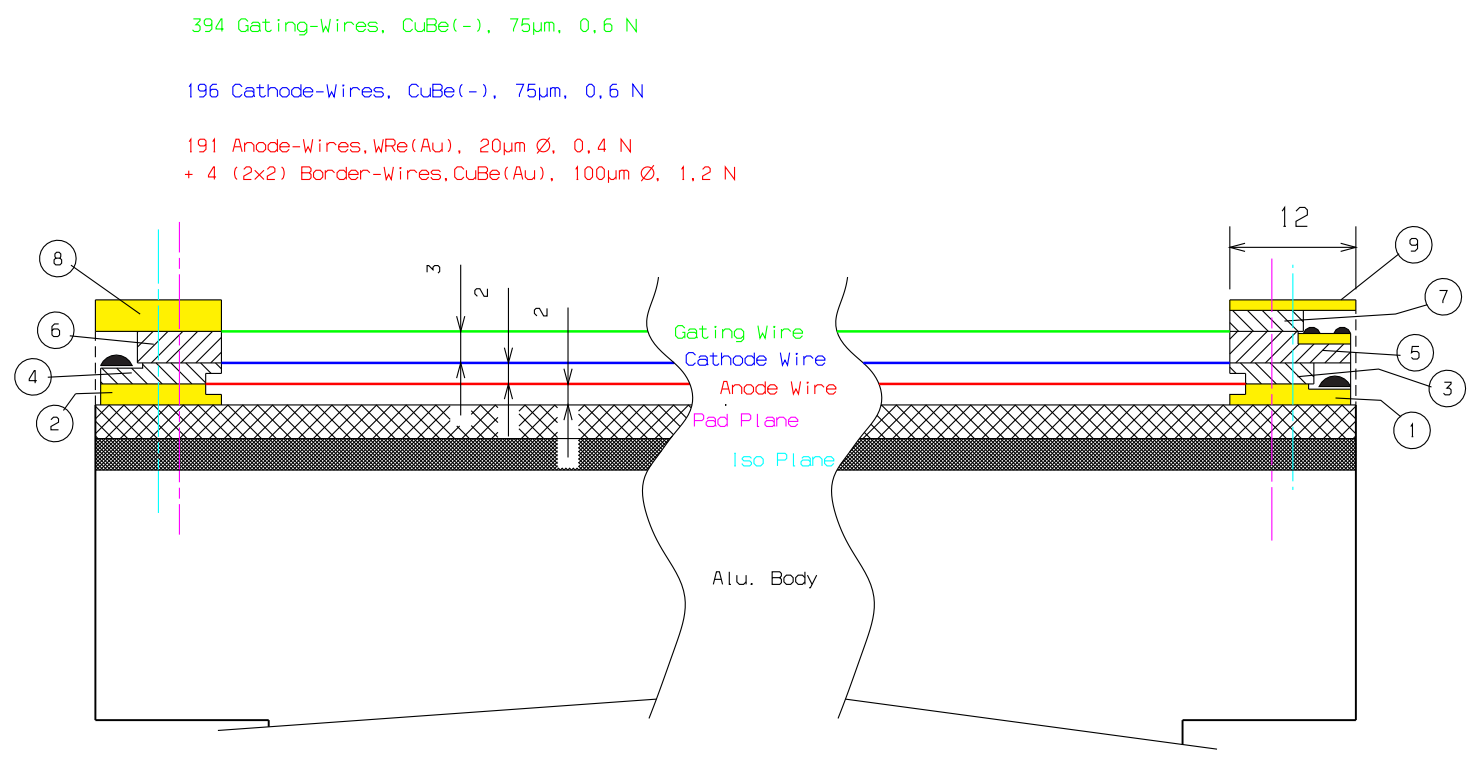

Figure 3.34: Cross section of inner readout chamber along wires, with wire diameters and wire tensions.

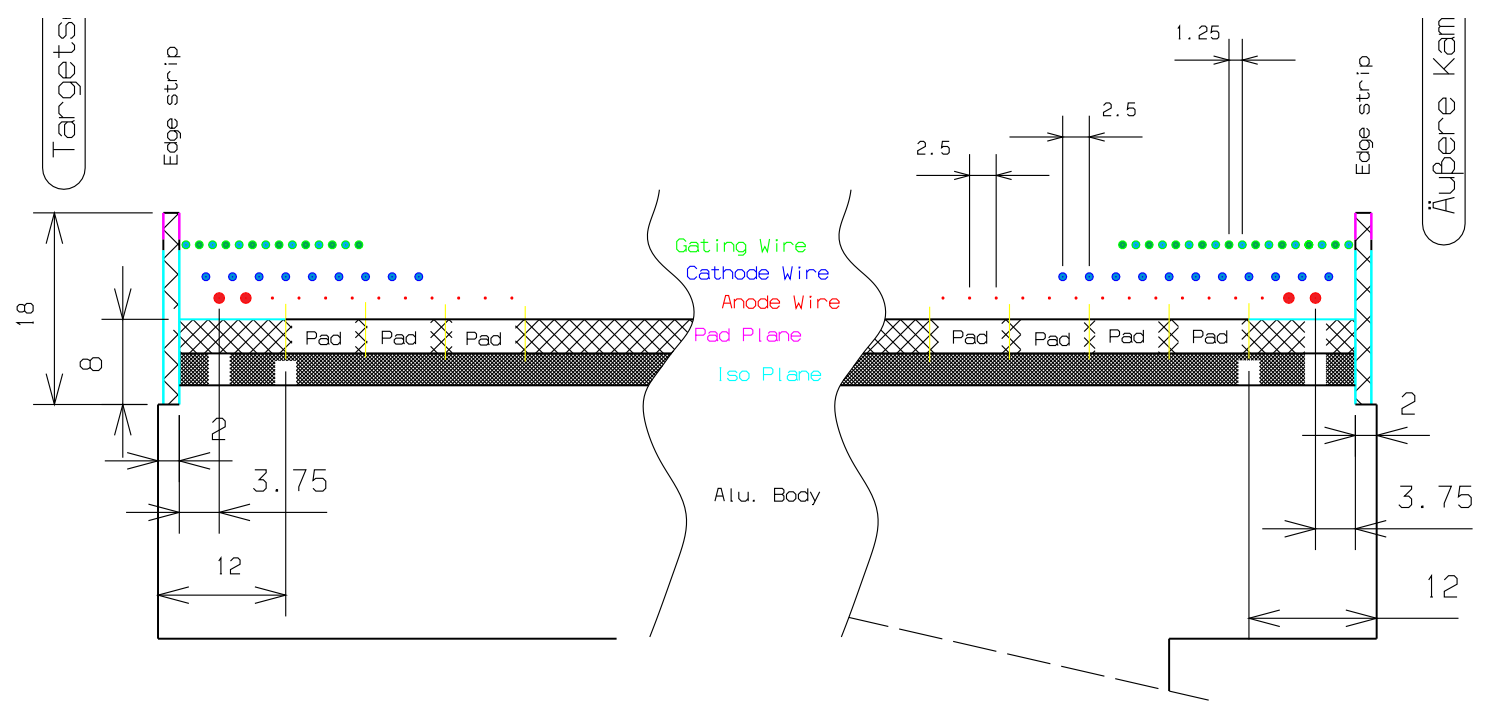

Figure 3.35: Cross section of inner readout chamber perpendicular to wires.

tight electrode configuration (anode-to-cathode distance 2 and $3 \mathrm{~mm}$ for inner and outer readout chamber, respectively) with unprecedented operating gain (up to 20000 ).

To keep the occupancy as low as possible and to ensure the necessary $\mathrm{d} E / \mathrm{d} x$, position and two-track resolution, there are about 560000 readout pads of three different sizes: $4 \times 7.5 \mathrm{~mm}^{2}$ in the inner chambers, $6 \times 10 \mathrm{~mm}^{2}$ and $6 \times 15 \mathrm{~mm}^{2}$ in the outer chambers.

The mechanical mount of the readout chambers is a 3-point kinematical mount. The seal to the end plate is a flexible foil pressed against O-rings both on the chambers and the end plate, see figure 3.36. Coplanarity was checked by survey and adjusted (using individually machined spacers for the 3-point mounts) with an accuracy of $\leq 250 \mu \mathrm{m}$. 


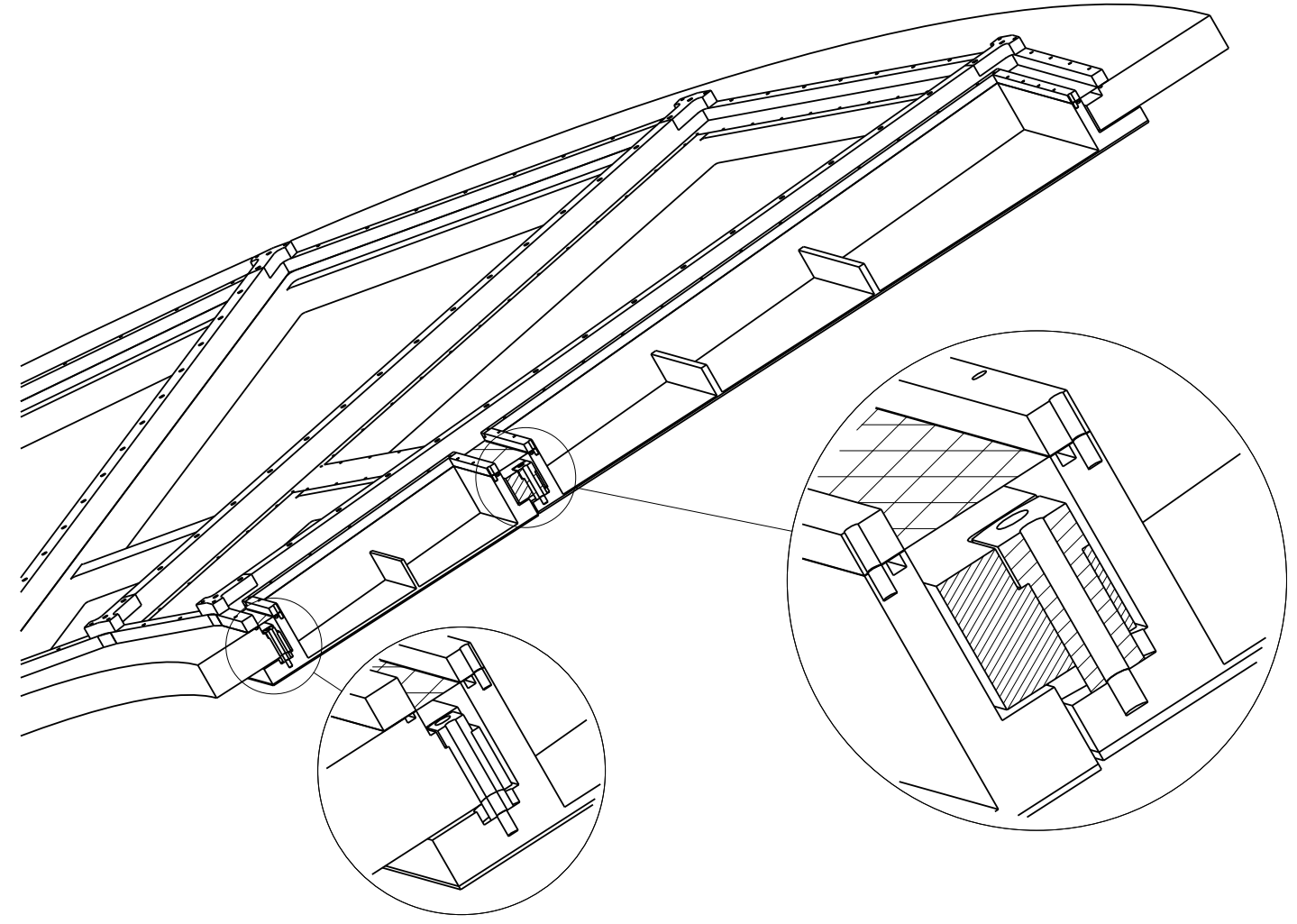

Figure 3.36: Cut of end plate with details of readout chamber mount points and seal with a foil (cross-hatched). The pad plane side of the chambers faces downwards in the drawing, it is larger than the end plate opening. Mounting is thus from the inside (see text).

Gating. The readout chambers are normally closed by a gating grid for electrons coming from the drift volume and for ions from the amplification region. The gate is opened only by the L1 trigger ( $6.5 \mu \mathrm{s}$ after the collision) for the duration of one drift-time interval, i.e. of about $90 \mu \mathrm{s}$ (see section 6.1). This helps to prevent space charge due to positive ions from drifting back from the multiplication region for non-triggered interactions and background. It has been verified that the closed gate reduces the drift of electrons by a factor better than $10^{5}$.

Laser calibration. The laser system [94] provides straight tracks distributed over all regions of the drift space. On each end plate, a laser beam of about $2 \mathrm{~cm}$ diameter is distributed to 6 of the 18 outer support rods of the field cage. At $2 \times 4 z$-positions, these beams are deflected by micro-mirror bundles, producing 7 fine laser rays of about $1 \mathrm{~mm}$ diameter traversing the drift volume perpendicular to the beam axis at strategic angles. This system is used for precise position inter-calibration for the readout chambers and will allow online monitoring of temperature and space-charge distortions, both of the order of a few $\mathrm{mm}$. The laser can be triggered with a rate of about $10 \mathrm{~Hz}$.

Cooling and temperature control. The requirement of $0.1 \mathrm{~K}$ thermal uniformity in the TPC drift volume, in the presence of thermal gradients inside the ALICE solenoid of about $5 \mathrm{~K}$ according to simulations, requires an elaborate system of heat screens and cooling circuits: (i) a heat screen 


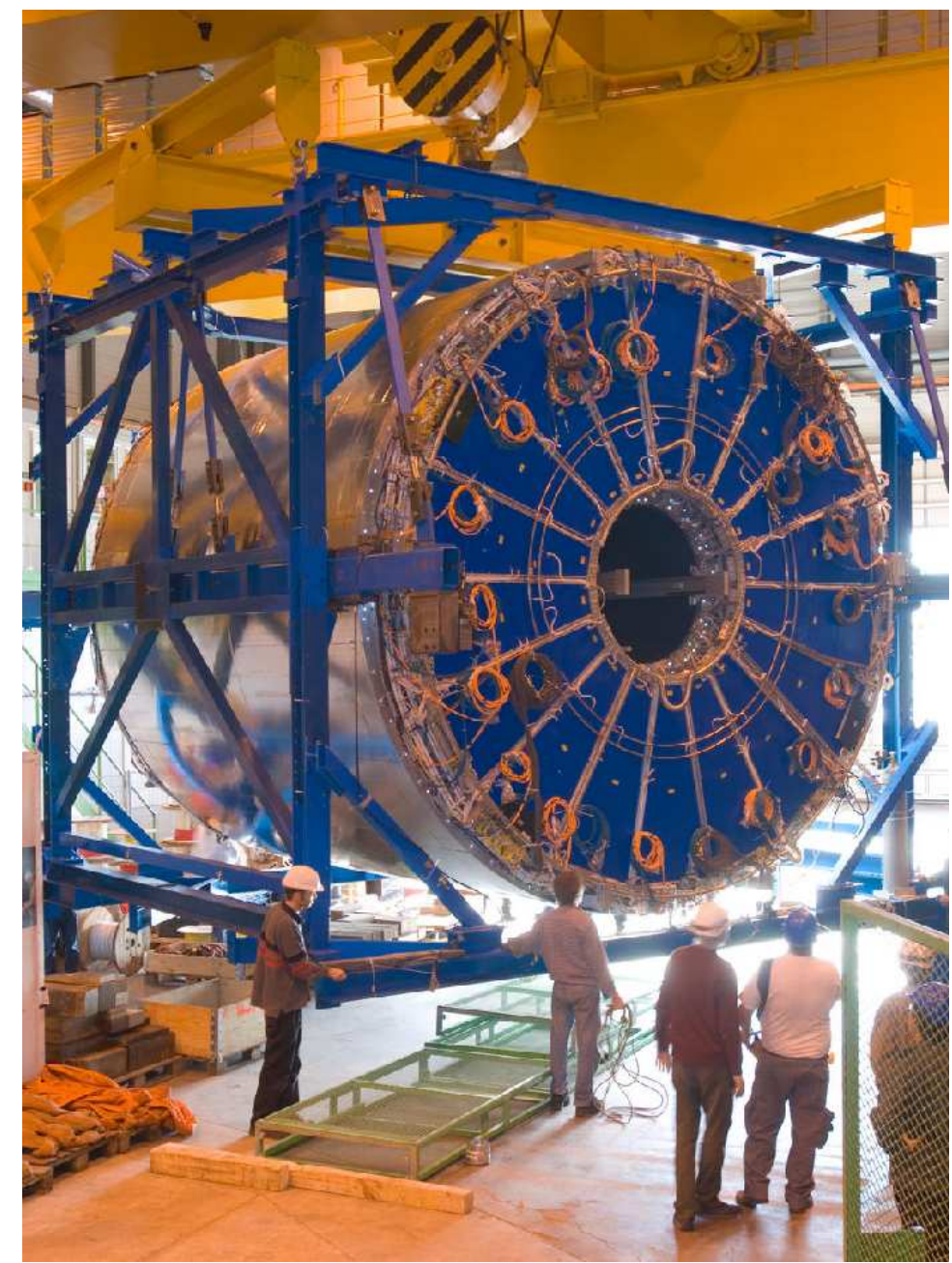

Figure 3.37: The TPC as assembled above ground on its way to the ALICE cavern.

at the outer radius toward the TRD, (ii) heat screens at the inner radius shielding from the ITS services, (iii) heat screens in the readout chamber bodies shielding from the FEE heat dissipation, (iv) cooling of the front-end electronics itself, (v) cooling of the resistive potential dividers, (vi) a heat screen at the FEE towards the outside. All these circuits are leakless i.e. operated at pressure below ambient. In the case of the resistive potential divider of the $100 \mathrm{kV}$ drift voltage very high resistivity water is used. For material budget reasons, no extra heat shield is used in the central region around the ITS barrel; the ITS controls its surface temperature there itself.

Integration into ALICE. The TPC field cage, which weighs $8 \mathrm{t}$ including the end plates and readout chambers, occupies the central opening of the spaceframe. The photograph in figure 3.37 shows the TPC assembled above ground on its transport to the ALICE cavern. The TPC is mounted inside the spaceframe on a rail system, with 4 feet gliding on Teflon. In the installation phase of the ITS and beam line, and possibly in future service periods, the TPC is partially retracted from the spaceframe by about $5 \mathrm{~m}$ to a 'parking position', allowing access to the area around the interaction point (see figure 3.38). 


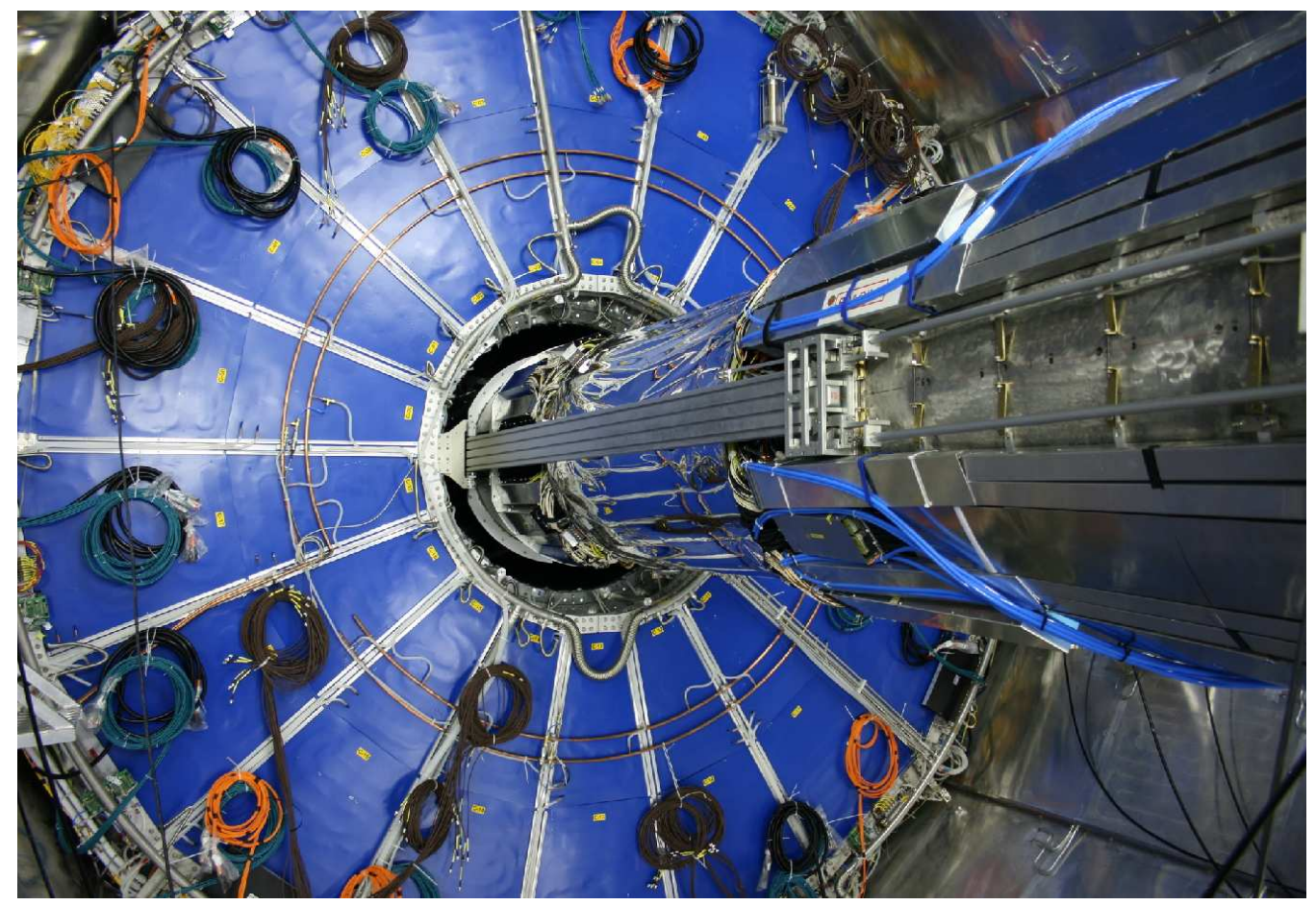

Figure 3.38: The TPC in the spaceframe, seen from the muon absorber side, at the "parking position' which allows access to the ITS. The ITS is supported by temporary rails that are finally removed. Note the thermal screen towards the TRD.

The front-end electronics and the services are not directly mounted on the TPC end plates but in two separate structures called service support wheels which cover the end plates and are independently supported by the TPC rails. This avoids loading the readout chambers with the weight of the electronics and services ( $1 \mathrm{t}$ for the readout cards alone on each side) and reduces thermal coupling to the drift volume. The ITS occupies the inner free radius of the TPC field cage, see chapter 3.1. The weight of the ITS is supported at two points by the innermost shell of the field cage.

\subsubsection{Front-end electronics and readout}

The front-end electronics has to read out the charge detected by about 560000 pads located on the readout chambers at the TPC end caps. These chambers deliver on their pads a current signal with a fast rise time (less than $1 \mathrm{~ns}$ ), and a long tail due to the motion of the positive ions. The amplitude, which is different for the different pad sizes, has a typical value of $7 \mu \mathrm{A}$. The signal is delivered on the detector impedance, to a very good approximation a pure capacitance of the order of a few $\mathrm{pF}$.

A single readout channel is comprised of three basic functional units, see block diagram of figure 3.39: i) a charge sensitive amplifier/shaper (PASA); ii) a 10-bit $25 \mathrm{MHz}$ low-power ADC; and iii) a digital circuit that contains a shortening filter for the tail cancellation, baseline subtraction 


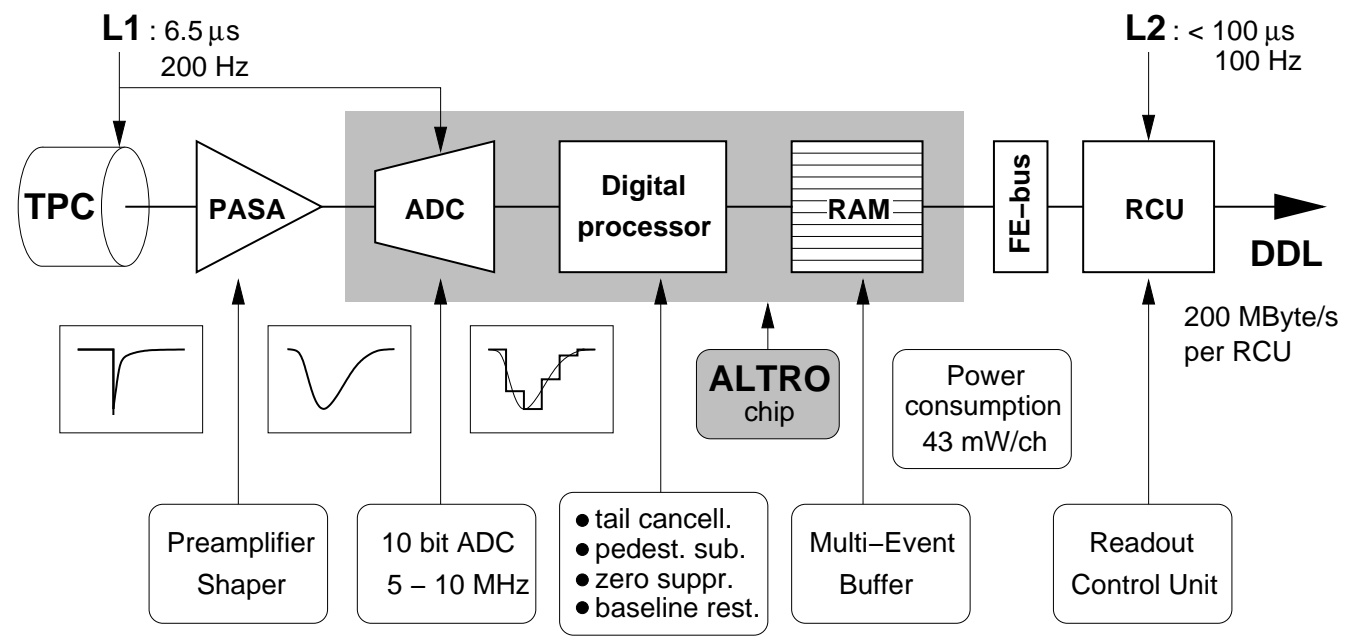

Figure 3.39: Block diagram of the TPC front-end electronics.

and zero-suppression circuits, and a multiple-event buffer. A front-end card (FEC) contains 128 channels. For the subsequent readout via a front-end bus and RCU see below.

The charge collected on the TPC pads is amplified and integrated by a low-input impedance amplifier. It is based on a Charge Sensitive Amplifier (CSA) followed by a semi-Gaussian pulse shaper of the $4^{\text {th }}$ order. These analogue functions are realized by a custom integrated circuit (PASA), implemented in $0.35 \mu \mathrm{m}$ CMOS technology, which contains 16 channels with a power consumption of $12 \mathrm{~mW}$ per channel. The circuit has a conversion gain of $12 \mathrm{mV} / \mathrm{fC}$ and a dual output dynamic range of $2 \times 1 \mathrm{~V}$ with a linearity of $0.2 \%$. It produces a pulse with a rise time of $150 \mathrm{~ns}$ and a shaping time (FWHM) of 190 ns. The single channel has a noise value (r.m.s.) of $\sim 550 e$ at the typical input capacity of $12 \mathrm{pF}$ and a channel-to-channel cross-talk below $-60 \mathrm{~dB}$. Immediately after the PASA, a 10-bit pipelined ADC (one per channel) samples the signal at a rate of $5-10 \mathrm{MHz}$. The digitized signal is then processed by a set of circuits that perform the baseline subtraction, tail cancellation, zero-suppression, formatting and buffering. The ADC and the digital circuits are contained in a single chip named ALTRO [95, 96] (ALice Tpc ReadOut). The ALTRO chip integrates 16 channels, each of them consisting of a 10-bit, $25 \mathrm{MHz}$ ADC, a pipelined Digital Processor and a multi-event buffer memory. When an L1 trigger is received, 500 or $1 \mathrm{k}$ time samples are temporarily stored in the multi-event buffer. Upon L2 trigger arrival the event is frozen, otherwise it is overwritten by the next event. The Digital Processor, running at the sampling frequency, implements several algorithms that are used to condition and shape the signal. After digitization, the Baseline Correction Unit I is able to perform channel-to-channel gain equalization and to correct for possible non-linearity and baseline drift of the input signal. It is also able to adjust DC levels and to remove systematic spurious signals by subtracting a pattern stored in a dedicated memory. The next processing block is an 18-bit, fixed-point arithmetic, $3^{\text {rd }}$ order Tail Cancellation Filter [97]. The latter is able to suppress the signal tail, within $1 \mu \mathrm{s}$ after the pulse peak, with an accuracy of 1 LSB. Since the coefficients of this filter are fully programmable, the circuit is able to cancel a wide range of signal-tail shapes. Moreover, these coefficients can be set independently for each channel and are re-configurable. This feature allows a constant quality of the output signal 
regardless of ageing effects on the detector and channel-to-channel fluctuations. The subsequent processing block, Baseline Correction Unit II, applies a baseline correction scheme based on a moving average filter. This scheme removes non-systematic perturbations of the baseline that are superimposed on the signal. At the output of this block, the signal baseline is constant with an accuracy of 1 LSB. Such accuracy allows an efficient zero-suppression procedure, which discards all data below a programmable threshold, except for a specified number of pre- and post-samples around each pulse. This produces a limited number of non-zero data packets, thus reducing the overall data volume. Each data packet is formatted with its time stamp and size information in such a way that reconstruction is possible afterwards. The output of the Data Processor is sent to a Data Memory of $5 \mathrm{kB}$, able to store up to 8 full events. The data is read out from the chip at $40 \mathrm{MHz}$ through a 40-bit wide bus, yielding a total bandwidth of $200 \mathrm{MB} / \mathrm{s}$. Moreover, the readout speed and the ADC sampling frequency are independent. Therefore, the readout frequency does not depend on the bandwidth of the input signal being acquired. The ALTRO chip is implemented in the ST $0.25 \mu \mathrm{m}$ HCMOS-7 process.

The readout chain up to the ALTRO chip is contained in the Front-End Cards [93] (FEC), which are plugged in support frames mounted to the service support wheel. Each FEC contains 128 channels ( 8 PASA and 8 ALTRO chips), and is connected to the cathode plane by means of 6 flexible cables. One row of FECs (up to 25) is controlled by a Readout Control Unit (RCU) [98], which interfaces the FECs to the DAQ, the trigger, and the Detector Control System (DCS) [99]. The RCU broadcasts the trigger information to the individual FEC modules and controls the readout procedure. Both functions are implemented via a custom bus, based on low-voltage signalling technology (GTL), which provides a data bandwidth of $200 \mathrm{MB} / \mathrm{s}$. The interfacing of the RCU modules to the Trigger and to the DAQ follows the standard data acquisition architecture of the experiment. In summary, for each of the 36 TPC sectors, the front-end electronics consists of 121 FECs in 6 rows, 6 RCUs, and 6 Detector Data Links (DDL).

The event sizes and rates quoted in table 3.12 are for the 1000 time-sample option. After zero-suppression and data encoding, the event size from the TPC for a central $\mathrm{Pb}-\mathrm{Pb}$ collision will be about $90 \mathrm{MB}$ at a charged multiplicity of $\mathrm{d} N_{\mathrm{ch}} / \mathrm{d} \eta=8000$, or $30 \mathrm{MB}$ at $\mathrm{d} N_{\mathrm{ch}} / \mathrm{d} \eta=2500$. For the 500-time-sample option, the event sizes reduce to $60 \%$ (less than a factor 2 due to the constant coding overhead). For pp-events, the data volume is very sensitive to the zero-suppression threshold. For a threshold at $3 \sigma, 1.4 \mathrm{MB}$ of the data volume arise from the noise alone, the pp track data itself are less than $100 \mathrm{kB}$ (both including coding). The data rate capabilities of the TPC readout, with a total bandwidth of $27 \mathrm{~GB} / \mathrm{s}$ measured in commissioning, allow transfer of 300 central $\mathrm{Pb}-\mathrm{Pb}$ events/s for $\mathrm{d} N_{\mathrm{ch}} / \mathrm{d} \eta=8000$. This exceeds the recording capabilities of the DAQ (see chapter 6.2) by far. The recorded data volume can be reduced using the 'region-of-interest' option of the trigger, reading out only a few sectors of the TPC.

In order to increase the physics potential of ALICE especially for jet and electron physics, rare signals like dielectron pair candidates have to be enriched in the trigger mix. Therefore an 'intelligent' readout is available via a HLT processor farm, which will operate on the raw data shipped through optical links. The HLT allows lossless data compression, selective readout of electron candidates identified by the TRD (the 'region-of-interest' option of the trigger), as well as online track finding and tracking of the whole TPC. Details of the intelligent readout including strategies for its implementation are discussed in section 6.3. 


\subsection{Transition Radiation Detector (TRD)}

\subsubsection{Design considerations}

The main purpose of the ALICE Transition Radiation Detector (TRD) [16] is to provide electron identification in the central barrel for momenta above $1 \mathrm{GeV} / c$. Below this momentum electrons can be identified via specific energy loss measurement in the TPC. Above $1 \mathrm{GeV} / c$ transition radiation (TR) from electrons passing a radiator can be exploited in concert with the specific energy loss in a suitable gas mixture to obtain the necessary pion rejection capability. In conjunction with data from the ITS and the TPC it is possible to study the production of light and heavy vector-meson resonances and the dilepton continuum both in $\mathrm{pp}$ as well as in $\mathrm{Pb}-\mathrm{Pb}$ collisions. Exploiting the excellent impact parameter resolution of the ITS it is furthermore possible to reconstruct open charm and open beauty in semi-leptonic decays.

The TRD was designed to derive a fast trigger for charged particles with high momentum. It is part of the Level 1 trigger and can significantly enhance the recorded $\Upsilon$-yields, high- $p_{\mathrm{t}} J / \psi$, the high-mass part of the dilepton continuum as well as jets.

The design parameters of the TRD are the following:

Pion rejection capability This is governed by the signal-to-background ratio in the measurement of $J / \psi$ production and its $p_{\mathrm{t}}$ dependence. This led to the design goal for the pion rejection capability of a factor 100 for momenta above $1 \mathrm{GeV}$ in central $\mathrm{Pb}-\mathrm{Pb}$ collisions/c [6]. The measurement of the lighter vector-mesons and the determination of the continuum between the $J / \psi$ and the $\Upsilon$ will only be feasible when this level of rejection can be reached.

Position and momentum resolution It is crucial to be able to match to the TPC in order to exploit the combined momentum resolution leading to an overall mass resolution of about 100 $\mathrm{MeV} / \mathrm{c}^{2}$ at the $\Upsilon$-mass (for $\mathrm{B}=0.4 \mathrm{~T}$ ). The required pointing accuracy for electrons needs to be on the level of a fraction of a TPC pad. The anticipated momentum resolution of the TRD of $3.5(4.7) \%$ at $5 \mathrm{GeV} / c$ (depending on multiplicity) see table 3.13 will crucially determine the sharpness of the trigger threshold in $p_{\mathrm{t}}$ as well as the capability to reject fake tracks.

Radiation length It has to be minimized in order to reduce Bremsstrahlung leading to incorrect momentum determination or loss of electrons and to reduce photon conversions resulting in increased occupancy as well as incorrect matching.

Detector granularity In the bending direction this is governed by the desired momentum resolution and in longitudinal direction by the need to correctly identify and track electrons through all layers of the detector even at the largest anticipated multiplicities. For the desired quality of the reconstructed dilepton pair signal (mostly dictated by the tracking efficiency) this leads to pads with an average area of about $6 \mathrm{~cm}^{2}$, a tracking efficiency of $90 \%$ can thus be achieved for single tracks.

Including secondary particles this leads to a maximum occupancy of $34 \%$ at the highest simulated multiplicity density of $\mathrm{dN}_{\mathrm{ch}} / \mathrm{d} \eta=8000$. 
Table 3.13: Summary of TRD parameters.

\begin{tabular}{|c|c|}
\hline $\begin{array}{l}\text { Pseudo-rapidity coverage } \\
\text { Azimuthal coverage } \\
\text { Radial position }\end{array}$ & $\begin{array}{l}-0.84<\eta<0.84 \\
360^{\circ} \\
2.90<r<3.68 \mathrm{~m}\end{array}$ \\
\hline $\begin{array}{l}\text { Total number of detector modules } \\
\text { Largest/smallest module } \\
\text { Active detector area } \\
\text { Radiator } \\
\text { Radial detector thickness }\end{array}$ & $\begin{array}{l}540 \text { arranged in } 18 \text { super modules } \\
113 \times 145 \mathrm{~cm}^{2}\left(91 \times 122 \mathrm{~cm}^{2}\right. \\
683 \mathrm{~m}^{2} \\
\text { fibre/foam sandwich, } 4.8 \mathrm{~cm} \text { per layer } \\
X / X_{0}=23.4 \%\end{array}$ \\
\hline $\begin{array}{l}\text { Module segmentation } \\
\text { Typical pad size } \\
\text { Total number of pads }\end{array}$ & $\begin{array}{ll}144 \text { in } \varphi & 12-16 \text { in } z \\
0.7 \times 8.8 \mathrm{~cm}^{2} & \\
1.18 \times 10^{6} & \end{array}$ \\
\hline $\begin{array}{l}\text { Detector gas } \\
\text { Gas volume } \\
\text { Depth of drift region } \\
\text { Depth of amplification region } \\
\text { Drift field } \\
\text { Drift velocity } \\
\text { Longitudinal/transverse diffusion } \\
\text { Lorentz angle }\end{array}$ & $\begin{array}{l}\mathrm{Xe} / \mathrm{CO}_{2}(85 \% / 15 \%) \\
27.2 \mathrm{~m}^{3} \\
3 \mathrm{~cm} \\
0.7 \mathrm{~cm} \\
0.7 \mathrm{kV} / \mathrm{cm} \\
1.5 \mathrm{~cm} / \mu \mathrm{s} \\
\mathrm{D}_{L}=250 \mu \mathrm{m} / \sqrt{\mathrm{cm}} \quad \mathrm{D}_{T}=180 \mu \mathrm{m} / \sqrt{\mathrm{cm}} \\
8^{\circ} \text { at magnetic field } 0.4 \mathrm{~T}\end{array}$ \\
\hline $\begin{array}{l}\text { Number of readout channels } \\
\text { Time samples in } r \text { (drift) } \\
\text { ADC } \\
\text { Number of multi-chip modules } \\
\text { Number of readout boards }\end{array}$ & $\begin{array}{l}1.18 \times 10^{6} \\
20 \\
10 \mathrm{bit}, 10 \mathrm{MHz} \\
70848 \\
4104\end{array}$ \\
\hline $\begin{array}{l}\text { Pad occupancy for } \mathrm{d} N_{c h} / \mathrm{d} \eta=8000 \\
\begin{array}{lr}\text { Pad occupancy in pp } \\
\text { Space-point resolution at } 1 \mathrm{GeV} / c \quad \text { in } r \varphi \\
\text { in } z\end{array} \\
\begin{array}{l}\text { Momentum resolution } \\
\text { Pion suppression at } 90 \% \text { electron efficiency }\end{array}\end{array}$ & $\begin{array}{l}34 \% \\
2 \times 10^{-4} \\
400(600) \mu \mathrm{m} \text { for } \mathrm{d} N_{c h} / \mathrm{d} \eta=2000\left(\mathrm{~d} N_{c h} / \mathrm{d} \eta=8000\right) \\
2 \mathrm{~mm}(\text { offline }) \\
\delta p / p=2.5 \% \oplus 0.5 \%(0.8 \%) p /(\mathrm{GeV} / c) \\
\text { for } \mathrm{d} N_{c h} / \mathrm{d} \eta=2000\left(\mathrm{~d} N_{c h} / \mathrm{d} \eta=8000\right) \\
\text { better than } 100 \text { for } \mathrm{p}=1-6 \mathrm{GeV} / c\end{array}$ \\
\hline $\begin{array}{l}\text { Event size for } \mathrm{d} N_{c h} / \mathrm{d} \eta=8000 \\
\text { Event size for } \mathrm{pp} \\
\text { Trigger rate limit }\end{array}$ & $\begin{array}{l}11 \mathrm{MB} \\
6 \mathrm{kB} \\
100 \mathrm{kHz}\end{array}$ \\
\hline
\end{tabular}



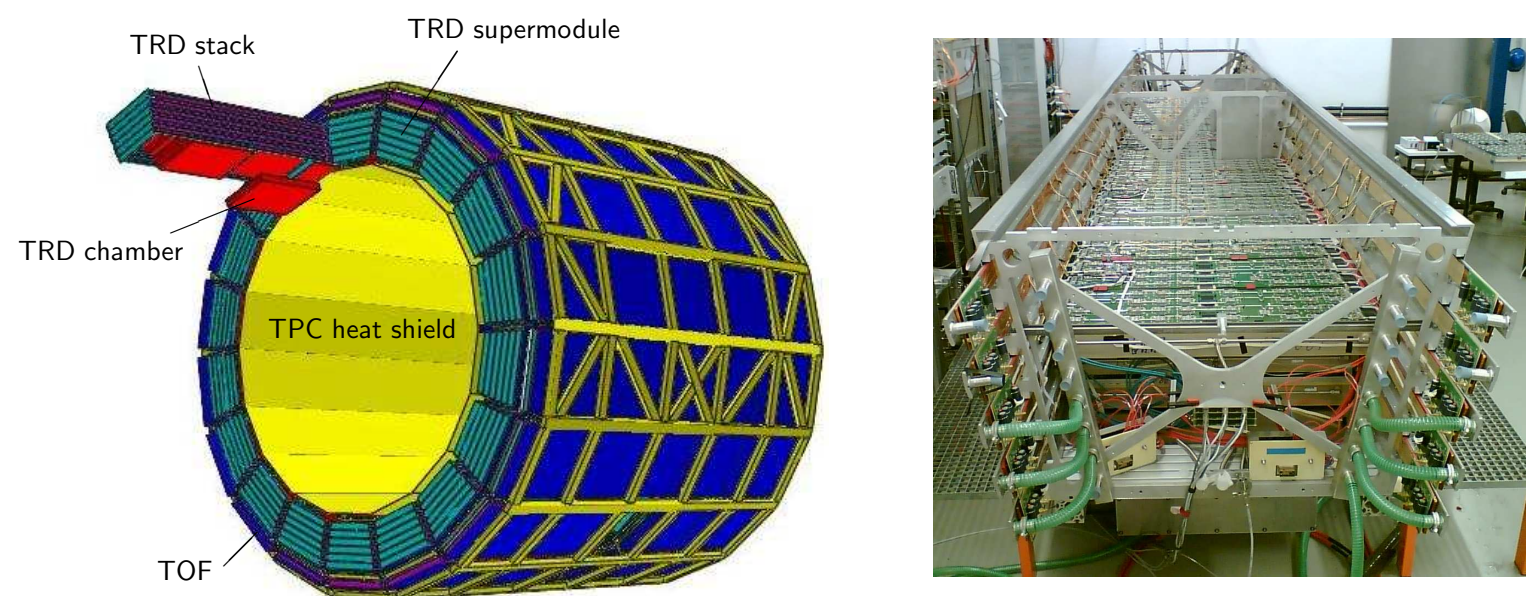

Figure 3.40: Left panel: schematic drawing of the TRD layout in the ALICE space frame. Shown are 18 super modules each containing 30 readout chambers (red) arranged in five stacks of six layers. One chamber has been displaced for clarity. On the outside the TRD is surrounded by the Time-Of-Flight (TOF) system (dark blue). On the inside the heat shield (yellow) towards the TPC is shown. Right panel: super module during assembly with the first three layers installed.

\subsubsection{Detector layout}

The final design of the TRD is depicted in figure 3.40. The TRD consists of 540 individual readout detector modules. They are arranged into 18 super modules (right panel of figure 3.40) each containing 30 modules arranged in five stacks along $\mathrm{z}$ and six layers in radius. In longitudinal $(z)$ direction the active length is $7 \mathrm{~m}$, the overall length of the entire super module is $7.8 \mathrm{~m}$, its total weight is $1650 \mathrm{~kg}$.

Each detector element consists of a carbon fibre laminated Rohacell/polypropylene fibre sandwich radiator of $48 \mathrm{~mm}$ thickness, a drift section of $30 \mathrm{~mm}$ thickness, and a multi-wire proportional chamber section $(7 \mathrm{~mm})$ with pad readout. The pad planes are supported by a honeycomb carbonfibre sandwich back panel $(22 \mathrm{~mm})$. While very light, the panel and the radiator provide enough mechanical rigidity of the chamber to cope with overpressure up to 1 mbar to ensure a deformation of less than $1 \mathrm{~mm}$. The entire readout electronics is directly mounted on the back panel of the detector. Including the water cooling system the total thickness of a single detector layer is $125 \mathrm{~mm}$. In the bending plane $(r \varphi)$ each pad row consists of 144 pads. The central chambers consist of 12 , all others of 16 pad rows. This leads to an overall channel count of $1.18 \times 10^{6}$. The total active area subtended by the pads is $716 \mathrm{~m}^{2}$. The operating conditions of the detector are summarized in table 3.13 .

Cross-sectional views of one TRD chamber together with average signals are shown in figure 3.41. Ionizing radiation produces electrons in the counting gas $\left(\mathrm{Xe} / \mathrm{CO}_{2}(85: 15)\right)$. Particles exceeding the threshold for transition radiation production $(\gamma \approx 1000)$ will in addition produce about $1.45 \mathrm{X}$-ray photons in the energy range of 1 to $30 \mathrm{keV}$. X-rays in this energy regime are efficiently converted by the high- $\mathrm{Z}$ counting gas with the largest conversion probability at the very beginning of the drift region. All electrons from ionization energy loss and X-ray conversions will drift towards the anode wires. After gas amplification in the vicinity of the anode wires the signal is induced on the readout pads. Signals of a typical track are shown in the inset of the central 

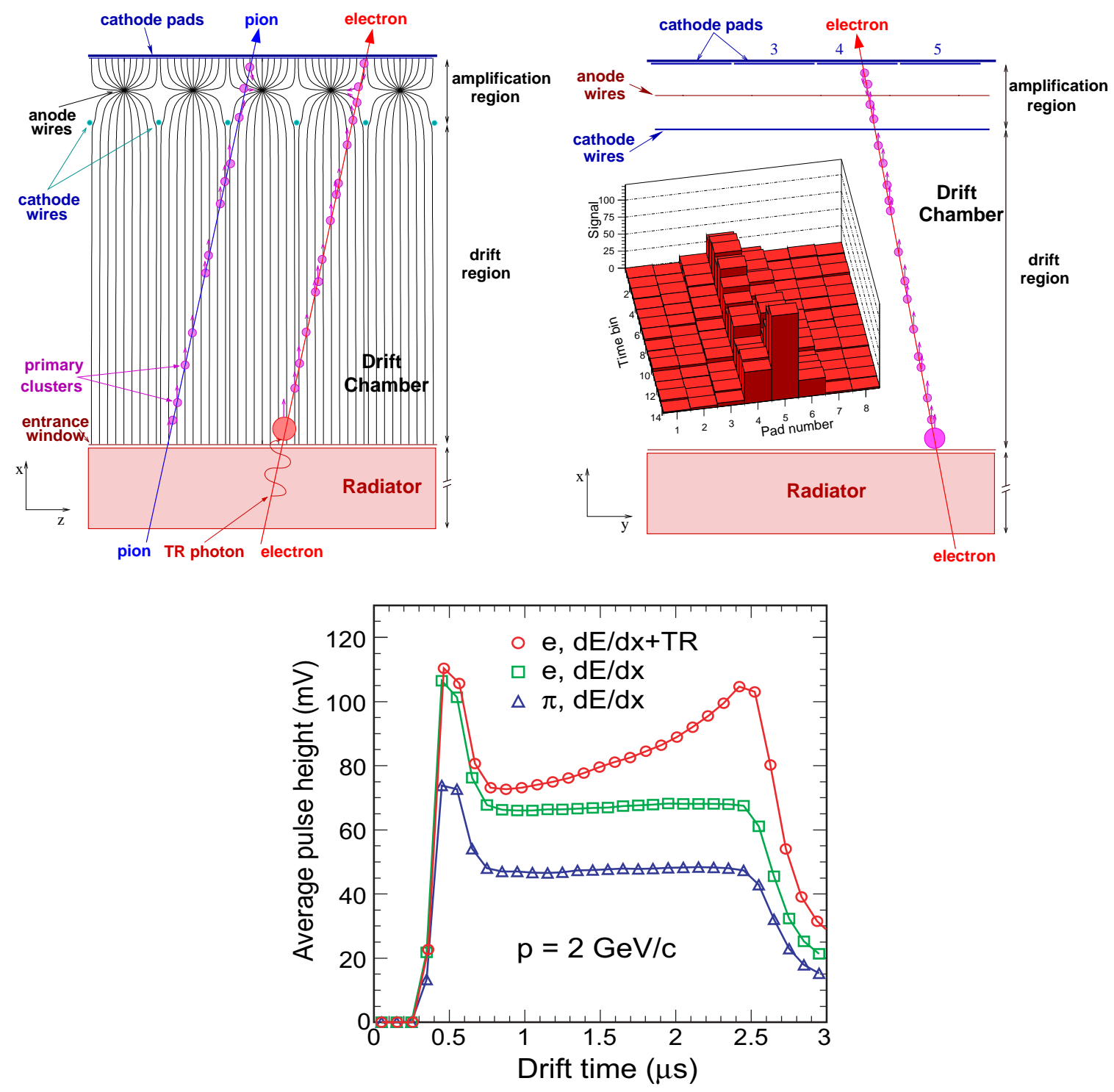

Figure 3.41: Schematic cross-sectional view of a detector module in $r z$-direction (top left panel) and $r \varphi$-direction (top right panel). The inset shows the charge deposit from an inclined track which is used for momentum reconstruction. The bottom panel shows the average pulse height as a function of drift time for pions (triangles), electrons without a radiator (squares) and electrons with a radiator (circles) for $2 \mathrm{GeV} / c$ particles.

panel of figure 3.41. The inclination of the track in the bending direction is a direct measure of its transverse momentum. For particles with a momentum of $2 \mathrm{GeV} / c$ the average amplitude of the cathode pad signal versus drift time is shown in the right panel. Two effects lead to an efficient discrimination between electrons and pions: i) the increased specific energy loss of electrons compared to pions at this momentum (difference between triangles and squares) and ii) the absorption of transition radiation generated by electrons (circles) predominantly at the beginning of the drift section corresponding to large drift times. 


\subsubsection{TRD performance}

As outlined above the performance of the TRD is characterized by its particle identification capability and its tracking performance.

\subsubsection{Electron identification}

The figure of merit for the TRD in terms of electron identification is its power to reject pions at a given electron efficiency (we choose 90\%). This is usually expressed in terms of the pion efficiency (we aim at 1\%). A parameterization of the measured amplitude spectra as a function of drift time and momentum both for electrons and pions provides the necessary likelihood distributions [100] that allow to evaluate the electron identification performance.

Specific energy loss. Measurements of the specific energy loss of electrons and pions of $2 \mathrm{GeV} / \mathrm{c}$ momentum in the $\mathrm{Xe} / \mathrm{CO}_{2}$ gas mixture are shown in figure 3.42 along with simulations. A correct understanding of the particle separation capability relies on a precise understanding of the details in the specific energy deposit of electrons and pions. Figure 3.42 demonstrates the level of agreement that has been achieved in the simulations. In that context it has been shown that a correct description of the escape probability of energetic $\delta$-electrons is needed to describe the tails of the energy distributions as well as their momentum dependence [101].

Transition radiation. The radiator is a composite structure using different inhomogeneous materials. The front and back face consist of $8 \mathrm{~mm}$ Rohacell foam covered with $0.1 \mathrm{~mm}$ carbon fibre laminate and $25 \mu \mathrm{m}$ aluminized mylar. It is filled with irregular polypropylene fibre mats (average fibre diameter $20 \mu \mathrm{m}$ ). The transition radiation production of this structure has been evaluated in prototype tests following [103]. The production of transition radiation sets in at an electron momentum of $0.5 \mathrm{GeV} / c$ and levels off at about $2 \mathrm{GeV} / c$ where on average 1.45 transition radiation photons are produced of which 1.25 are detected per incident electron [102]. The measured transition radiation energy spectra along with simulations are shown in figure 3.43.

Pion rejection. The resulting pion rejection capability was determined as a function of particle momentum. The results are shown in figure 3.44 for two different sets of data. One was obtained using six full-size TRD chambers ("big stack"), the other are extrapolations of results obtained with smaller prototype chambers ("small DCs"). Plotted is the percentage of pions erroneously identified as electrons versus momentum at an electron efficiency of $90 \%$. The analysis was based on the likelihood of total deposited charge (L-Q). Along with the measurements, results from simulations are shown applying two-dimensional likelihoods on total deposited charge and position (L-Q1,Q2) as well as analysis based on neural networks (NN) [100]. These methods provide a factor 2-5 increase of the pion rejection capability compared to the simple likelihood analysis on total deposited charge. 


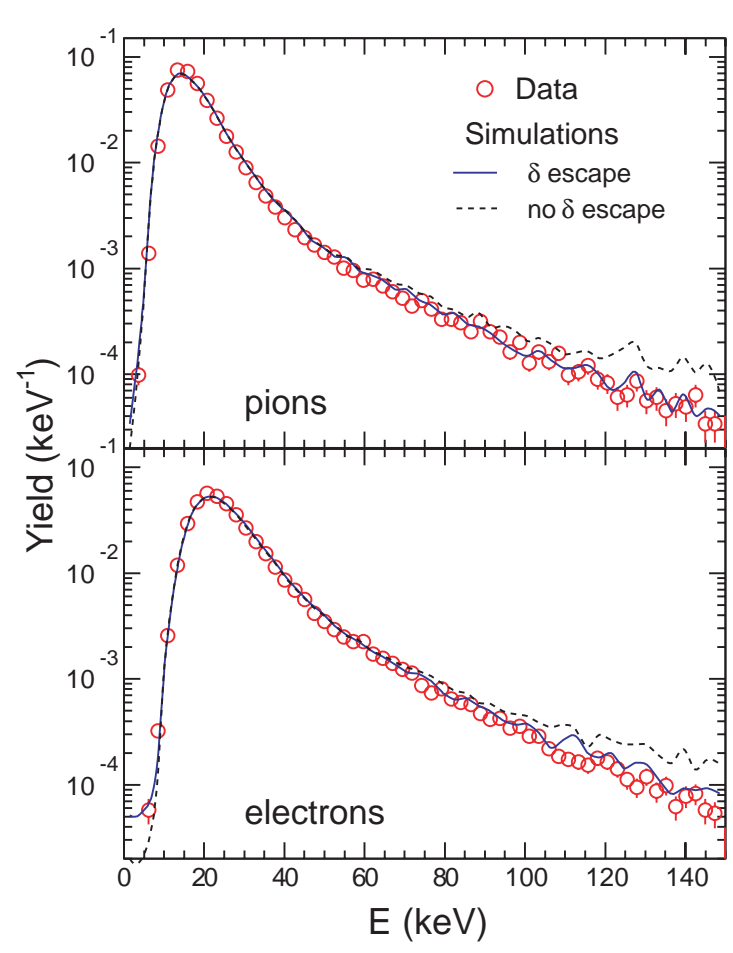

Figure 3.42: Measurement of the specific energy deposit of $2 \mathrm{GeV} / c$ pions (top) and electrons (bottom) in the nominal $\mathrm{Xe}, \mathrm{CO}_{2}$ gas mixture along with simulations [101].

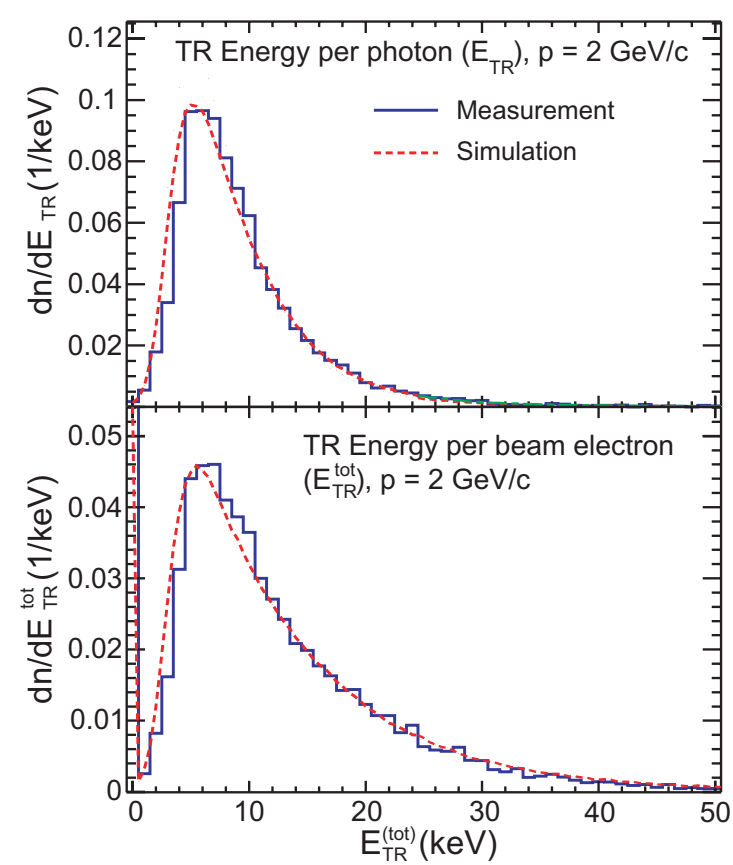

Figure 3.43: Measurement and simulation of the transition radiation spectrum from $2 \mathrm{GeV} / c$ electrons with the ALICE TRD radiator sandwich. The top panel shows the energy distribution of TR photons, the bottom panel the response per incident electron [102].

\subsubsection{Tracking}

The chief tracking device in ALICE is the TPC. The needed tracking capability of the TRD is derived from the requirement of a reasonably sharp trigger threshold for individual particles in the range of up to $p_{\mathrm{t}} \approx 10 \mathrm{GeV} / c\left(\delta p_{\mathrm{t}} / p_{\mathrm{t}} \approx 5 \%\right.$ at $\left.5 \mathrm{GeV} / c\right)$. For particles with large momentum the tracking through the six layers of the TRD improves the overall momentum resolution of the tracking through the central barrel, see chapter 8 .

Position resolution. The aforementioned tracking requirements in the nominal magnetic field of $0.5 \mathrm{~T}$, require a position resolution for each time bin of $\sigma_{y} \lesssim 400 \mu \mathrm{m}$ and a resulting angular resolution per layer of $\sigma_{\varphi} \lesssim 1^{\circ}$. The achievable respective resolutions were measured and are a function of the signal-to-noise ratio [104]. They are shown in figure 3.45. At a signal-to-noise ratio of about 40 the detector meets the requirements.

Momentum resolution. Using the above position and angular resolutions the stand-alone tracking resolution of the TRD can be derived for different momenta as a function of multiplicity density. For momenta below $2 \mathrm{GeV} / c$ the stand-alone momentum resolution of the TRD is around $\delta p_{\mathrm{t}} / p_{\mathrm{t}} \approx 2.5-3 \%$ with little multiplicity dependence. Through the inclusion of the TRD into the 


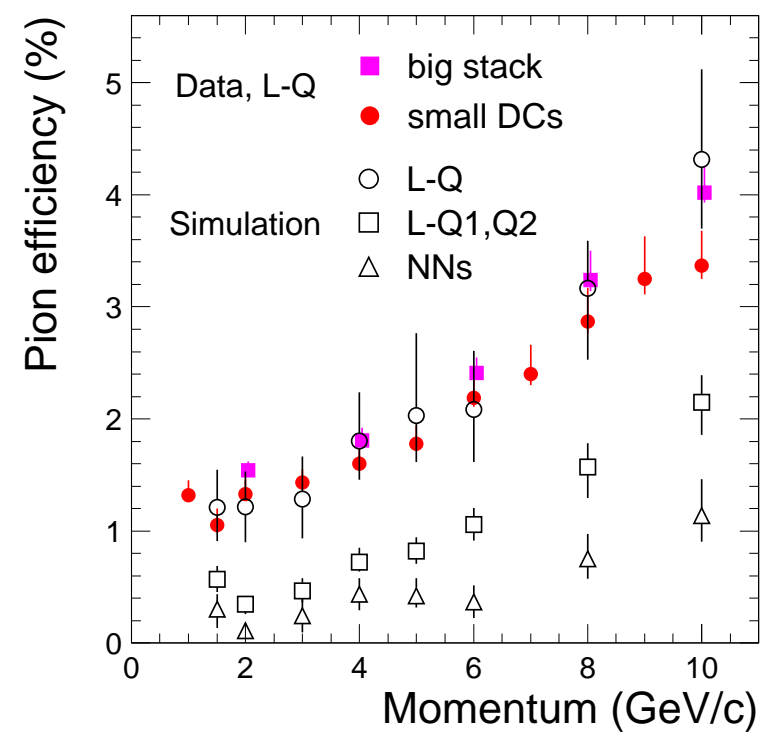

Figure 3.44: Measured pion efficiency as a function of beam momentum applying likelihood on total deposited charge (L-Q) (full symbols) measured with a stack of six chambers and smaller test chambers. Results are compared to simulations (open symbols) for $90 \%$ electron efficiency and six layers. These simulations were extended to two-dimensional likelihood on deposited charge and position (LQ1,Q2) and neural networks (NN) [100].

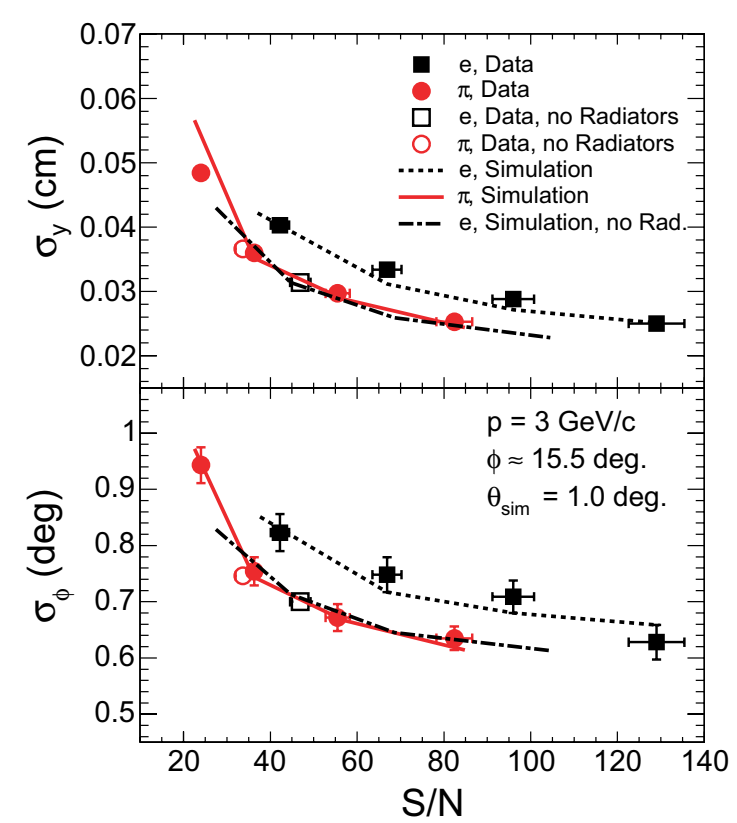

Figure 3.45: Measurements and simulations of the position (top) and angular resolution as a function of the signal-to-noise ratio. Open (full) symbols refer to electrons and pions measured with (without) radiator in front of the drift chamber along with the respective simulations [104].

tracking in the central barrel an overall momentum resolution of better than $5 \%$ can be obtained up to momenta of about $100 \mathrm{GeV}$, see chapter 8 .

\subsubsection{Readout electronics}

\subsubsection{General layout}

An overview of the TRD readout electronics is shown in figure 3.46. The electronics, including the optical serializers ORI (two per chamber), is directly mounted on the backside of the detector modules. The data are transmitted to the Global Tracking Unit through 60 optical fibres per super module. The GTU either passes the data directly to the DAQ via the Detector Data Link (DDL) or it further analyzes the data in order to derive a fast Level-1 trigger decision. In that case individual tracklets from different layers of a stack are combined to determine the multiplicity of high- $p_{\mathrm{t}}$ particles or to detect high-momentum $\mathrm{e}^{+} \mathrm{e}^{-}$-pairs. At Level-1 this trigger is transmitted to the Central Trigger Processor (CTP). 


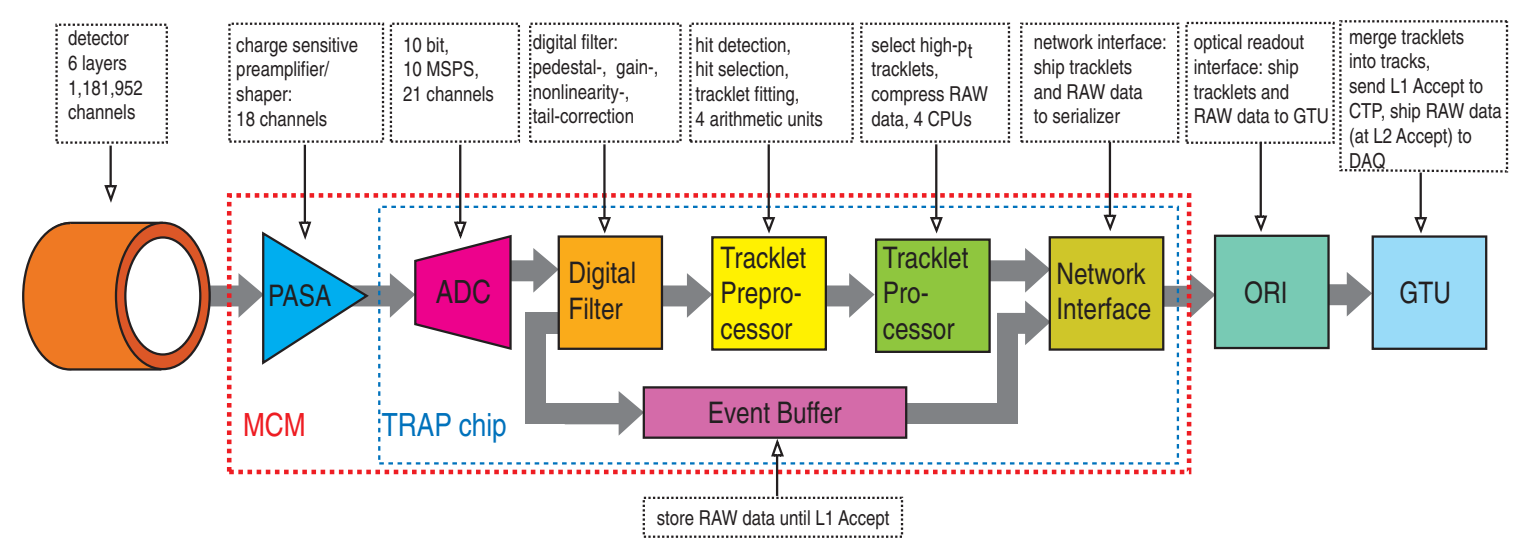

Figure 3.46: Schematic overview of the readout electronics of the TRD.

\subsubsection{Detector electronics}

Most of the on-detector readout electronics are realized as ASICs. Two such chips, an 18-channel full-custom preamplifier shaper (PASA), and a so-called Tracklet Processor (TRAP) were integrated into a multi-chip module (MCM). The PASA [105] is a folded cascode with differential output (120 ns shaping time, $850 e$ ENC for an input capacitance of $25 \mathrm{pF}, 12.4 \mathrm{mV} / \mathrm{fC}$ gain, and $12 \mathrm{~mW} /$ channel power consumption). It was realized using the AMS $0.35 \mu \mathrm{m}$ CMOS process. Each PASA has 18 input and 21 output channels. For reconstruction of tracklets the extra output channels are fed into the analogue inputs of the TRAPs on neighbouring MCMs to allow for continuous charge sharing across MCM boundaries.

The Tracklet Processor is a mixed signal design realized in the UMC $0.18 \mu \mathrm{m}$ process. It comprises 21 ADCs, digital filters [106], event buffers, and processing units [107] that allow to calculate the inclination of a track in the bending direction as well as the total charge deposited along the track (Local Tracking Unit - LTU). This feature allows to identify high- $p_{\mathrm{t}}$ particles at the trigger level. Evaluation of the deposited energy will furthermore allow to tag possible electron candidates at the trigger level.

\subsubsection{Global Tracking Unit (GTU)}

After preprocessing of the analogue signals as described above, the resulting track segments from the different detector layers have to be matched in three dimensions for transverse momentum reconstruction. Based on the data of all 1.2 million analogue channels, the reconstruction has to be performed within $6.1 \mu$ s to derive the Level-1 trigger decision. The entire trigger timing sequence involving the LTU and the GTU is depicted in figure 3.47.

The massively parallel hardware architecture of the GTU is capable of processing up to 20 000 track segments within $2 \mu \mathrm{s}$. The core of the GTU, along with a custom bus system, is the so-called Track Matching Unit (TMU). It is an FPGA-based system utilizing PCI and 12 fibreoptical SFP transceiver interfaces gathering the data from a stack of six chambers. It is realized as a CompactPCI plug-in card. The main FPGA is a Xilinx Virtex-4 FX chip which includes an integrated multi-gigabit serializer/ deserializer and PowerPC processor blocks. 


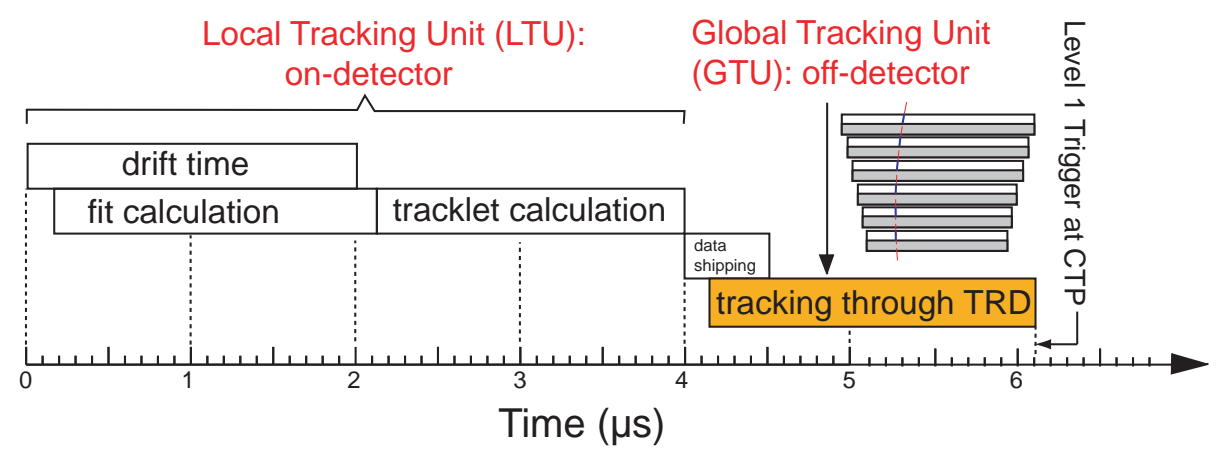

Figure 3.47: Trigger timing diagram for the generation of a high- $p_{\mathrm{t}}$ trigger.

\subsubsection{Detector Control System (DCS)}

The entire configuration, monitoring, and control of the detector electronics is realized on a single DCS board with an autonomous power supply. There is one DCS board per chamber. The boards comprise an Altera FPGA with ARM9 core, flash memory, Ethernet-, JTAG-, and $\mathrm{I}^{2} \mathrm{C}$-interfaces, 8 analogue and 8 digital inputs as well as 8 digital outputs.

The board reshapes and distributes the central clock signal received via an on-board Trigger Timing Controller (TTC) to all MCMs on the detector. All internal clocks of the MCMs are derived from this central clock. The configuration of different filter and gain parameters as well as zero suppression conditions used for the reconstruction of the tracklets within the Local Tracking Unit (LTU) are handled by the DCS board. It also monitors the temperatures and currents of all individual MCMs. Through its external control lines it is able to shutdown voltage regulators in case of over-temperature or over-current. Moreover, the DCS board can reroute the so-called Slow Control Serial Network in the configuration chain which allows us to bypass faulty MCMs without loss of data from entire readout boards.

The states of all services (low voltage, high voltage, gas, cooling) as well as all states generated or monitored by the DCS board are implemented as Finite-State Machines (FSM) which are operated and controlled via PVSS.

\subsection{Time-Of-Flight (TOF) detector}

\subsubsection{Design considerations}

The Time-Of-Flight (TOF) detector [15] is a large area array that covers the central pseudo-rapidity region $(|\eta| \lesssim 0.9)$ for Particle IDentification (PID) in the intermediate momentum range, below about $2.5 \mathrm{GeV} / c$ for pions and kaons, up to $4 \mathrm{GeV} / c$ for protons, with a $\pi / \mathrm{K}$ and $\mathrm{K} / \mathrm{p}$ separation better than $3 \sigma[21,108]$. The TOF, coupled with the ITS and TPC for track and vertex reconstruction and for $\mathrm{d} E / \mathrm{d} x$ measurements in the low-momentum range (up to about $1 \mathrm{GeV} / c$ ), will provide event-by-event identification of large samples of pions, kaons, and protons. In addition, at the inclusive level, identified kaons will allow invariant mass studies, in particular the detection of open heavy-flavoured states and vector-meson resonances such as the $\phi$ meson. Details on the physics 
observables that can be addressed and the expected performance are detailed in $[4,21,108,109]$ and in section 8 of this document.

A large-coverage TOF detector, operating efficiently, with an excellent intrinsic response and an overall occupancy not exceeding the 10-15\% level at the highest predicted charged-particle density of $\mathrm{d} N / \mathrm{d} \eta=8000$ was required; see [21]. This led to the current design with more than $10^{5}$ independent TOF channels. Since a large area had to be covered, a gaseous detector was chosen. In the framework of the LAA project at CERN an intensive R\&D programme has shown that the best solution for the TOF detector was the Multi-gap Resistive-Plate Chamber (MRPC) [110, 111].

The key aspect of these chambers is that the electric field is high and uniform over the full sensitive gaseous volume of the detector. Any ionisation produced by a traversing charged particle immediately starts a gas avalanche process which generates the observed signals on the pick-up electrodes. Unlike other types of gaseous detectors, there is no drift time associated with the movement of the electrons to a region of high electric field. Thus the time jitter of these devices is caused by the fluctuations in the growth of the avalanche.

The main advantages of the MRPC technology [112] with respect to other parallel-plate chamber designs are that: i) the chamber operates at atmospheric pressure; ii) the signal is the analogue sum of signals from many gaps, so there is no late tail and the charge spectrum is not of an exponential shape - it has a peak well separated from zero; iii) the resistive plates quench the streamers so there are no sparks, thus high-gain operation becomes possible; iv) the construction technique is in general rather simple and makes use of commercially available materials.

The final tests of several MRPC multicell strips from mass production confirmed that these devices indeed reach an intrinsic time resolution better than about $40 \mathrm{ps}$ and an efficiency close to $100 \%$.

\subsubsection{Detector layout}

The detector covers a cylindrical surface of polar acceptance $\left|\theta-90^{\circ}\right|<45^{\circ}$. It has a modular structure corresponding to 18 sectors in $\varphi$ and to 5 segments in $z$ direction.

The whole device is inscribed in a cylindrical shell with an internal radius of $370 \mathrm{~cm}$ and an external one of $399 \mathrm{~cm}$. The whole device thickness corresponds to $30 \%$ of a radiation length.

The basic unit of the TOF system is a 10-gap double-stack MRPC strip (figure 3.48) $122 \mathrm{~cm}$ long and $13 \mathrm{~cm}$ wide, with an active area of $120 \times 7.4 \mathrm{~cm}^{2}$ subdivided into two rows of 48 pads of $3.5 \times 2.5 \mathrm{~cm}^{2}$. An overall view of a strip is shown in figure 3.49 . The strips are placed inside gastight modules (which also act as Faraday cages) and are positioned transversely to the beam direction. Five modules of three different types are needed to cover the full cylinder along the $z$ direction.

They all have the same structure and width $(128 \mathrm{~cm})$ but differ in length. The actual dimensions and overall design are defined in such a way that the joining areas of the modules are aligned with the dead areas of the other detectors projected from the interaction point, thus creating a configuration of minimal disturbance for the external detectors. The length of the central module is $117 \mathrm{~cm}$, the intermediate ones $137 \mathrm{~cm}$, and the external ones $177 \mathrm{~cm}$. The overall TOF barrel length is $741 \mathrm{~cm}$ (active region).

The granularity of the TOF detector is dictated by the requirement of identifying, on an eventby-event basis, as many charged particles as possible, even at the maximum predicted chargedparticle multiplicity density of $\mathrm{d} N_{\mathrm{ch}} / \mathrm{d} \eta=8000$. Detailed simulation studies have shown that, with 

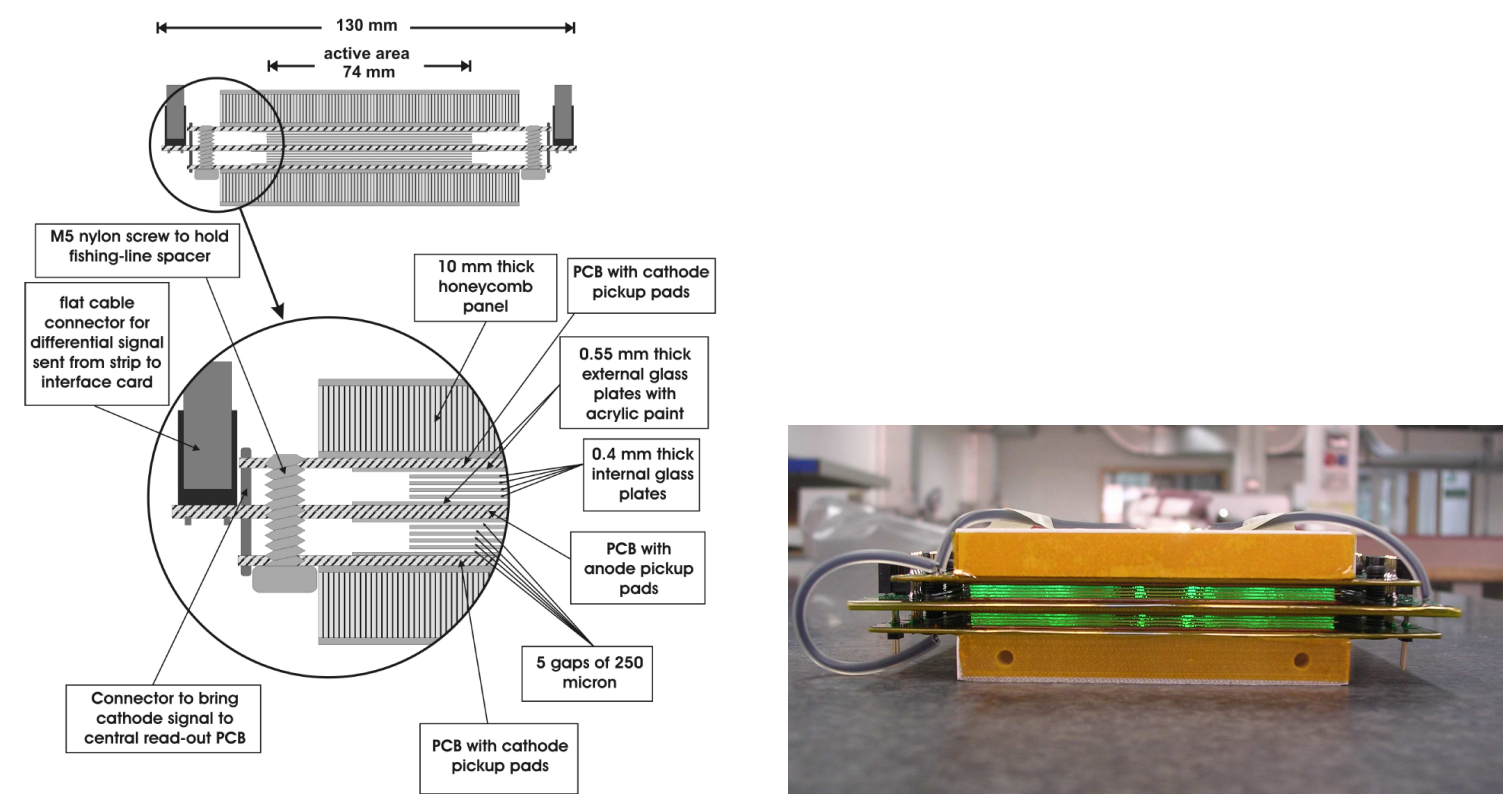

Figure 3.48: Schematic cross section (left) and photograph (right) of a 10-gap double-stack MRPC strip.

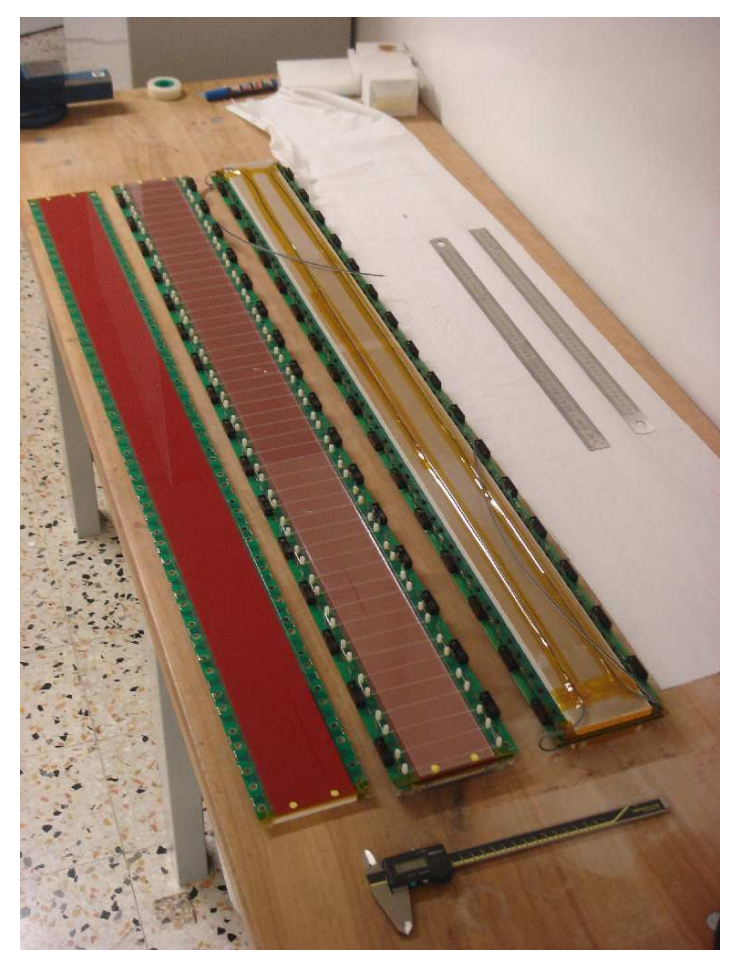

Figure 3.49: Photograph of an MRPC strip during (left and centre) and after (right) construction.

the chosen pad size of $3.5 \times 2.5 \mathrm{~cm}^{2}$ and the tilted-strip geometry, the occupancy of the detector is $\sim 14 \%$, at the highest charged-particle density (including secondary particles) with a magnetic field of $0.5 \mathrm{~T}$. Lower values of particle density result in linearly lower occupancy. 
In order to minimise the transversal path of the incident particles through the chamber strips, a special tilted positioning of the strips was devised. This reduces the number of very oblique transversal paths that can create a sharing effect of the signal among adjacent pads, thereby increasing the occupancy and the time jitter of the detected signals. The angle of the strips with respect to the axis of the cylinder is progressively increasing from $0^{\circ}$ in the central part $\left(\theta=90^{\circ}\right)$ of the detector to $45^{\circ}$ in the extreme part of the external module $\left(\theta=45^{\circ}\right)$. This arrangement makes the median zone of a strip perpendicular to a radius coming from the interaction point, thus minimising the angle of the incoming particles with respect to the normal direction. In order to avoid dead areas, adjacent strips were overlapped inside the modules so that the edge of the active area of one pad is aligned with the edge of the next one. This gives the possibility of creating a full active area with no geometrical dead zones. The modules were designed in such a way as to avoid any loss of the sensitive area, along the $z$-axis. The only dead area is due to the unavoidable presence of the supporting spaceframe structure.

Every module of the TOF detector consists of a group of MRPC strips (15 in the central, 19 in the intermediate and in the external modules) closed inside a box that defines and seals the gas volume and supports the external front-end electronics and services. An aluminium-honeycomb plate is the backbone of the module and gives the necessary mechanical stiffness to the system. The honeycomb plate is $1.5 \mathrm{~cm}$ thick including two aluminium skins, each $0.1 \mathrm{~cm}$ thick. A cover connected to the backbone by means of screws and standard sealing O-Ring closes the total gas volume; it has to withstand the overpressure required for gas circulation inside the chamber (less than 3 mbar). The cover is made of fibreglass $0.3 \mathrm{~cm}$ thick, reinforced with moulded ribs, protruded by $1.8 \mathrm{~cm}$; there are 6 ribs in the central module, 9 in the intermediate and 8 in the external ones. A $0.04 \mathrm{~cm}$ fibreglass web, covered by a $20 \mu \mathrm{m}$ aluminium layer, is glued to the inside surface of the cover in order to create an electromagnetic shielding. Inside the gas volume, fixed perpendicularly to the honeycomb plate, there are two macrolon plates, $0.5 \mathrm{~cm}$ thick, to which the MRPC strips are attached. The system turns out to be very simple and allows for a fast insertion of the strips at whatever angle is needed. Holes that accommodate feed-through for the signal cables coming from the readout pads, the HV connectors and the gas inlet and outlet, are machined into the honeycomb plate. The signal feed-through is made of a PCB having on one side connectors receiving the cables coming from the strips inside the gas volume and, on the other side, connectors that accommodate the front-end electronic cards, see figure 3.50. The PCB is glued to the honeycomb plate via special fibreglass gaskets which are inserted into the holes. The volume containing the electronic cards, input and output cables, gas pipes, water cooling pipes and radiators is closed by a $0.15 \mathrm{~cm}$ aluminium cover. This allows access to the electronics with no disturbance to the active part of a module.

The complete TOF system consists of 90 modules. Three central modules in front of PHOS have not been installed in order to reduce the amount of material for this high resolution electromagnetic calorimeter in three of its five modules. The five modules in a row are located inside a supermodule framework of longitudinal and transverse aluminium beams for each of the 18 sectors. Pairs of horizontal and vertical rollers are attached to the supermodule body permitting the insertion of the supermodule into the spaceframe sector where two rails are fixed. Each supermodule has an overall length of $\sim 930 \mathrm{~cm}$ and a weight of $\sim 1.4 \mathrm{t}$. A schematic layout of one supermodule inside the ALICE spaceframe and a photo of an assembled supermodule are shown in figure 3.51. An overview of the TOF detector parameters is shown in table 3.14 . 


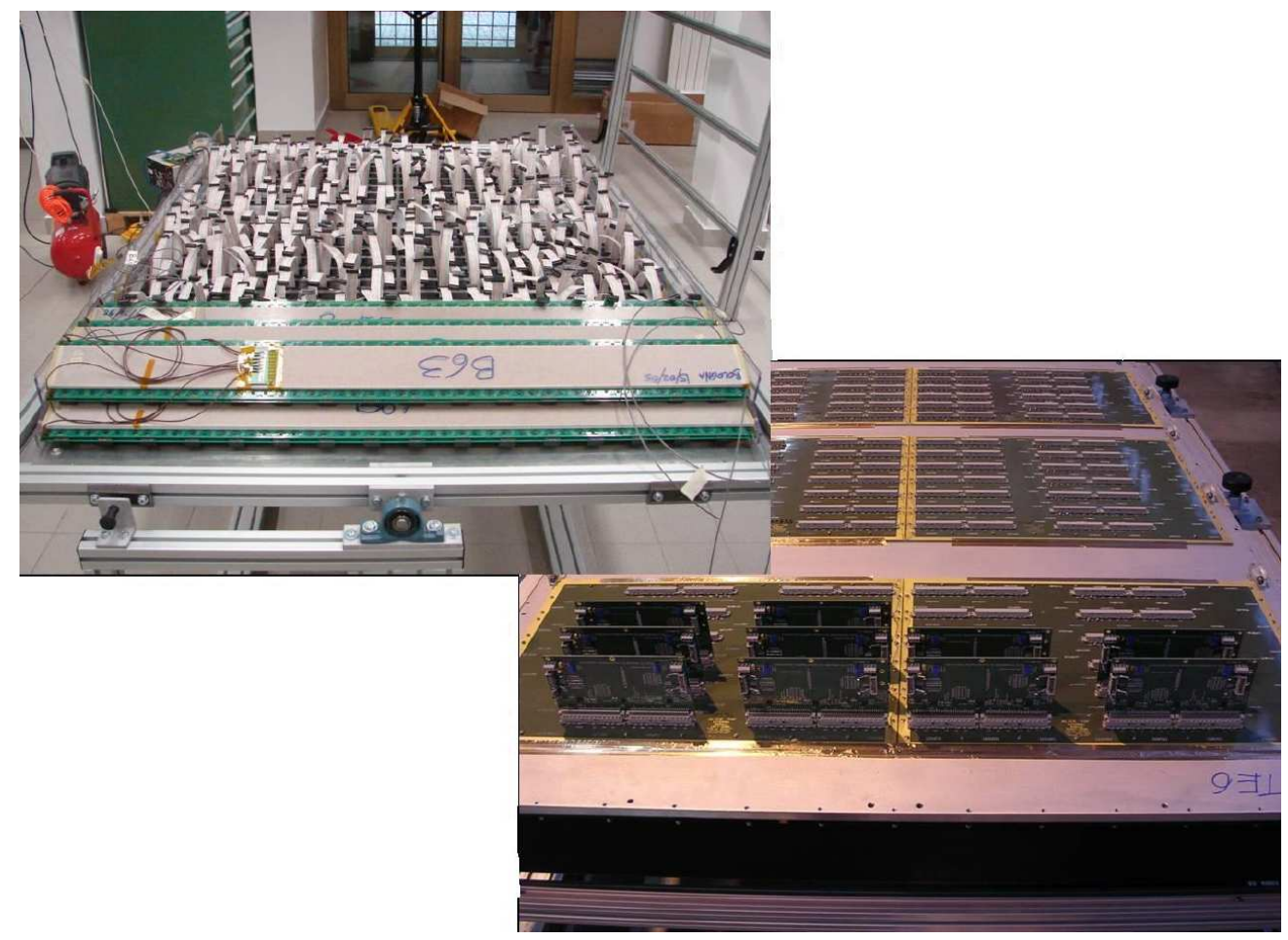

Figure 3.50: Photographs of a TOF module during assembly. The strips are installed inside the gas volume (upper) and the FEA cards are plugged onto the PCB connectors (lower).

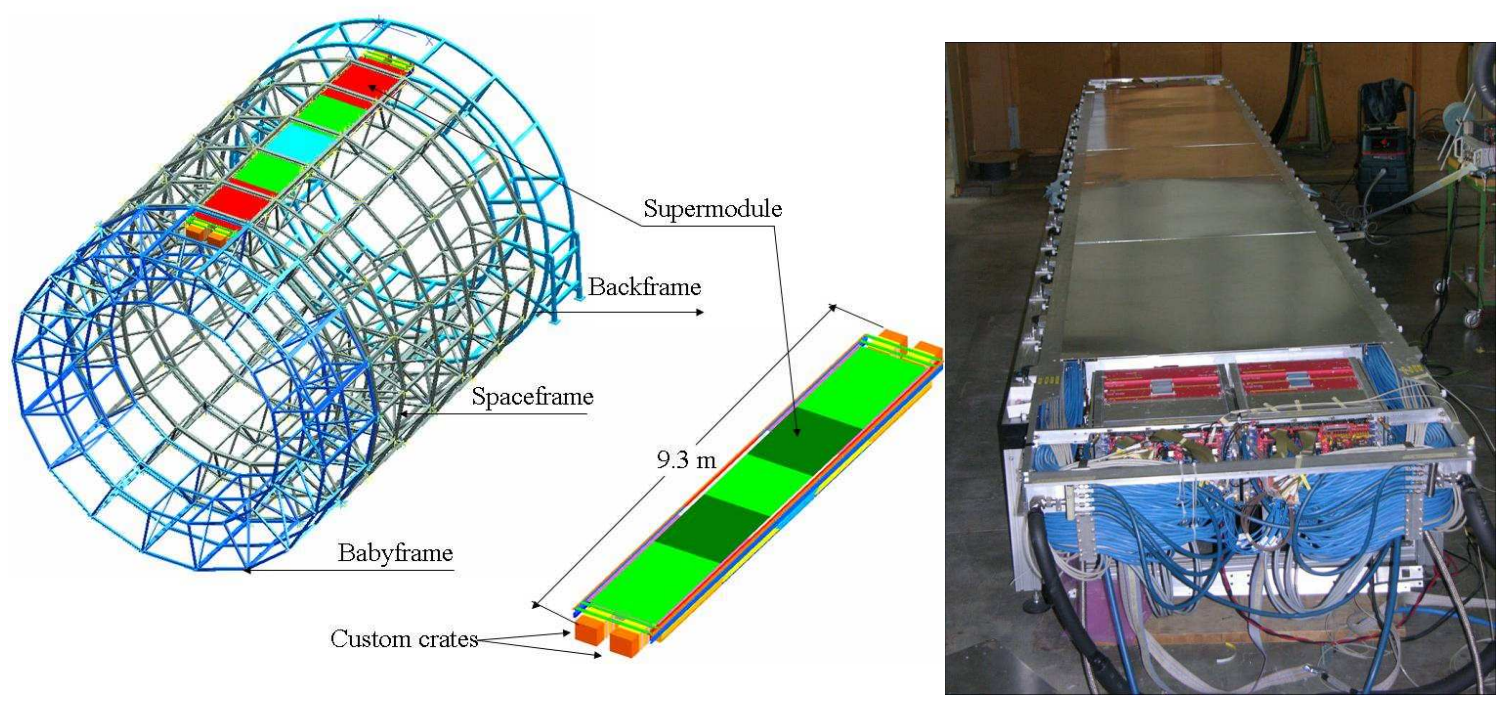

Figure 3.51: Schematic drawing of one TOF supermodule, consisting of 5 modules, in the ALICE spaceframe (left panel). A supermodule after assembly; in the foreground, the two custom crates with readout electronics (right panel). 
Table 3.14: Overview of TOF parameters.

\begin{tabular}{|c|c|}
\hline Pseudo-rapidity coverage & $-0.9<\eta<0.9$ \\
\hline Azimuthal coverage & $360^{\circ}$ \\
\hline Radial position & $370<r<399 \mathrm{~cm}$ \\
\hline Length (active region) & $741 \mathrm{~cm}$ \\
\hline Segmentation in $\varphi$ & 18-fold \\
\hline Segmentation in $z$ & 5 -fold \\
\hline Total number of modules & 90 \\
\hline Central module (A) & $117 \times 128 \mathrm{~cm}^{2}$ \\
\hline Intermediate module (B) & $137 \times 128 \mathrm{~cm}^{2}$ \\
\hline External module $(\mathrm{C})$ & $177 \times 128 \mathrm{~cm}^{2}$ \\
\hline Detector active area (total area) & $141 \mathrm{~m}^{2}\left(171 \mathrm{~m}^{2}\right)$ \\
\hline Detector radial thickness & $X / X_{0}=29.5 \%$ \\
\hline Number of MRPC strips per module & $15(\mathrm{~A}), 19(\mathrm{~B}), 19(\mathrm{C})$ \\
\hline Number of readout pads per MRPC strip & 96 \\
\hline Module segmentation in $\varphi$ & 48 pads \\
\hline Module segmentation in $z$ & $30(\mathrm{~A}), 38(\mathrm{~B}), 38(\mathrm{C})$ pads \\
\hline Readout pad geometry & $3.5 \times 2.5 \mathrm{~cm}^{2}$ \\
\hline Total number of MRPC strips & 1638 \\
\hline Total number of readout pads & 157248 \\
\hline Detector gas & $\mathrm{C}_{2} \mathrm{H}_{2} \mathrm{~F}_{4}(90 \%), \mathrm{i}-\mathrm{C}_{4} \mathrm{H}_{10}(5 \%), \mathrm{SF}_{6}(5 \%)$ \\
\hline Gas volume & $17.5 \mathrm{~m}^{3}$ \\
\hline Total flow rate & $1 \mathrm{~m}^{3} / \mathrm{h}$ \\
\hline Working overpressure & $<3$ mbar \\
\hline Fresh gas flow rate & $0.02 \mathrm{~m}^{3} / \mathrm{h}$ \\
\hline Number of readout channels & 157248 \\
\hline Number of front-end analogue chips (8-ch) & 19656 \\
\hline Number of front-end boards & 6552 \\
\hline Number of HPTDC chips (8-ch, 24.4 ps bin width) & 20520 \\
\hline Number of HPTDC readout boards (TRM) & 684 \\
\hline Number of readout boards (DRM) and crates & 72 \\
\hline Occupancy for $\mathrm{d} N_{\mathrm{ch}} / \mathrm{d} \eta=8000$ & $14 \%(B=0.5 \mathrm{~T})$ \\
\hline Occupancy for pp & $10^{-4}(B=0.5 \mathrm{~T})$ \\
\hline$\pi, \mathrm{K}$ identification range (with contamination $<20 \%$ ) & $0.5-3.0 \mathrm{GeV} / c$ \\
\hline p identification range (with contamination $<15 \%$ ) & $0.5-6.0 \mathrm{GeV} / c$ \\
\hline e identification range in $\mathrm{pp}$ (with contamination $<10 \%$ ) & $0.3-0.5 \mathrm{GeV} / c$ \\
\hline Event size for $\mathrm{d} N_{\mathrm{ch}} / \mathrm{d} \eta=8000$ & $110 \mathrm{kB}$ \\
\hline Event size for $\mathrm{pp}$ & $24 \mathrm{kB}$ \\
\hline
\end{tabular}



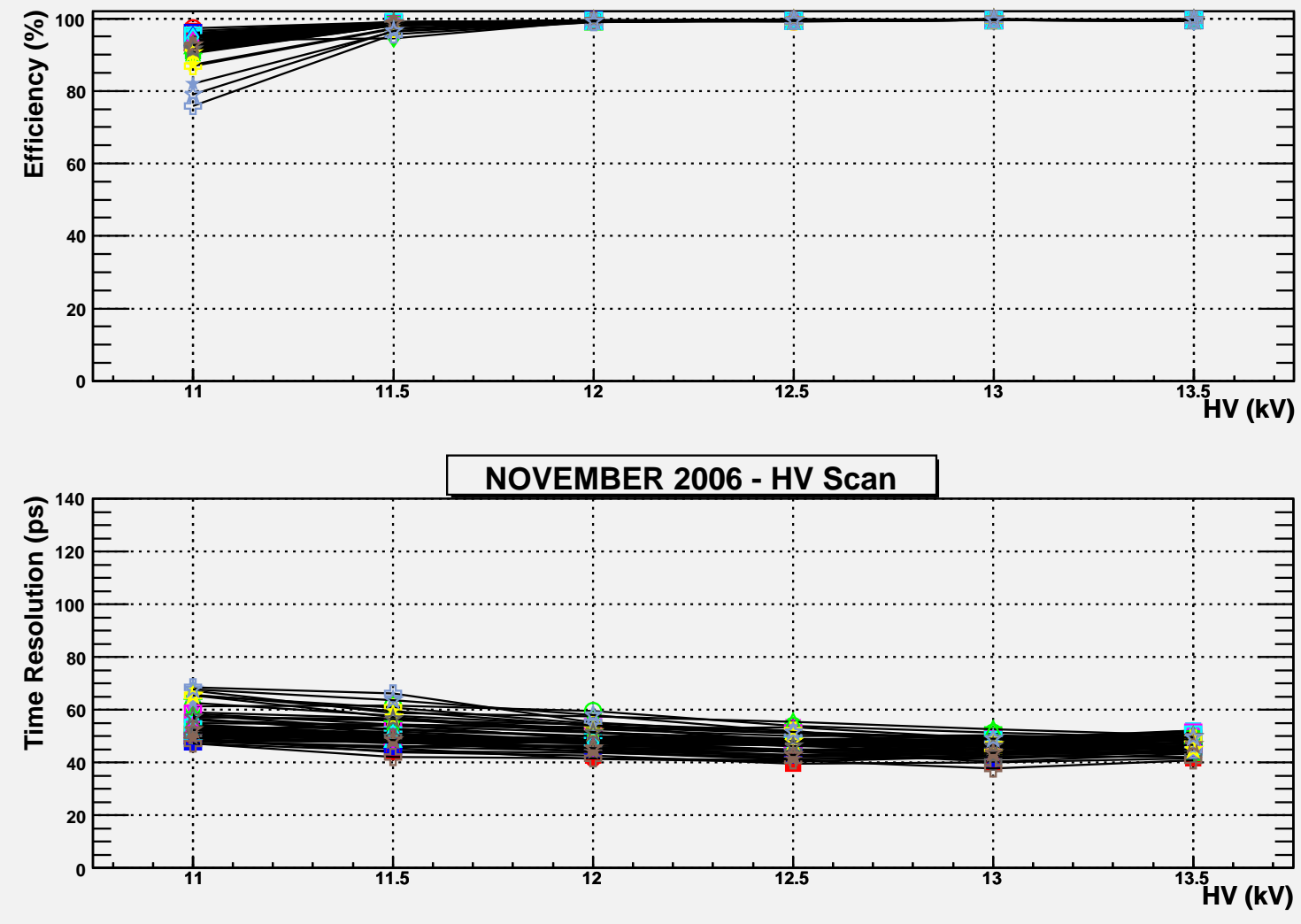

Figure 3.52: Efficiency and time resolution as a function of high voltage for a sample of massproduction MRPC strips. The operating voltage is $13 \mathrm{kV}$.

Results obtained at the CERN/PS T10 test-beam with ten MRPC strips, chosen randomly from two years of mass-production, and with the full electronic chain are shown in figure 3.52 and figure 3.53 for the efficiency and the time resolution. Similar results were obtained over a sample of several tens of pre-production strips. This demonstrates a detector of full efficiency and an intrinsic time resolution better than $40 \mathrm{ps}$.

The contribution of the full electronic chain (FEA, TRM, CPDM: see next section) to the overall time resolution of the detector has been measured to be about $38 \mathrm{ps}$.

Extensive tests [113] were performed at the CERN PS and Gamma Irradiation Facility (GIF) to study different gas mixtures, ageing effects and rate capability. The gas mixture was composed of $\mathrm{C}_{2} \mathrm{H}_{2} \mathrm{~F}_{4}(90 \%), \mathrm{i}_{-} \mathrm{C}_{4} \mathrm{H}_{10}(5 \%), \mathrm{SF}_{6}(5 \%)$. No ageing effect was observed after 3.5 times the dose foreseen for the 'standard running scenario' in the first 10 years of LHC operation, and a rate capability far in excess of the $50 \mathrm{~Hz} / \mathrm{cm}^{2}$ maximum expected rate at the ALICE experiment was demonstrated.

Details on the TOF detector mass-production procedures and results are available in [114]. 

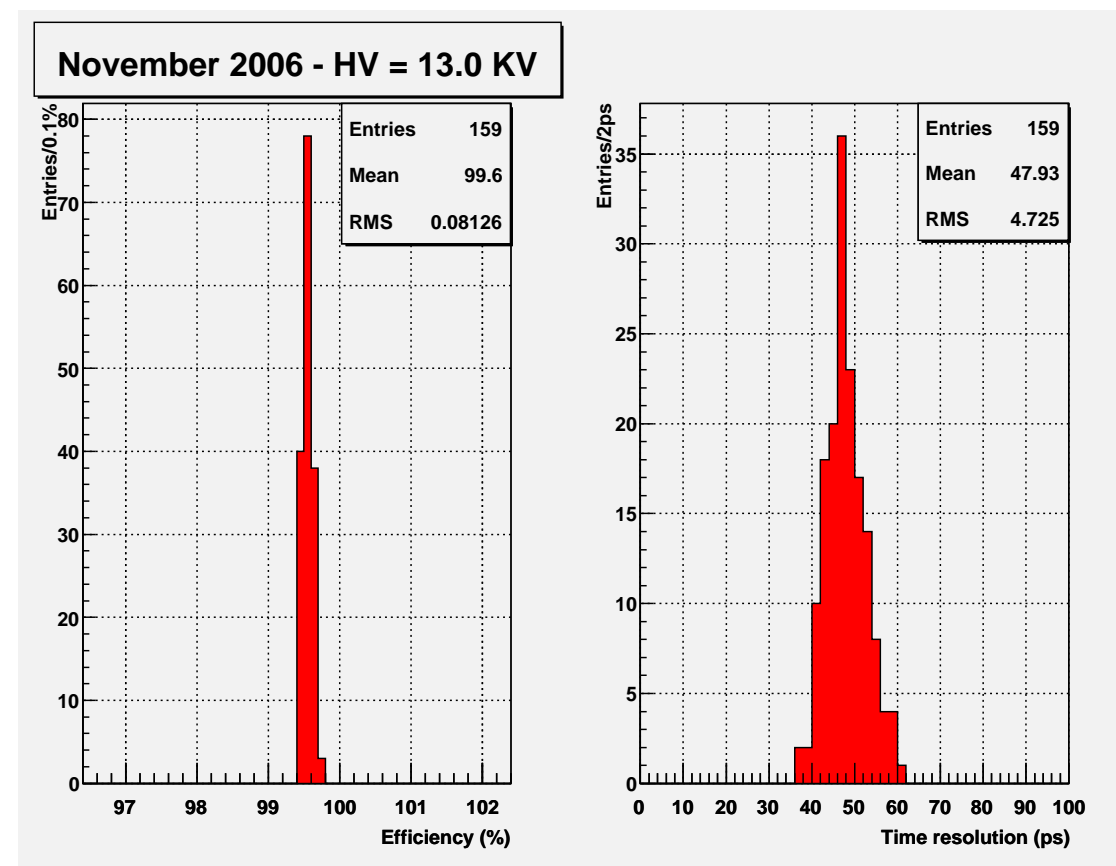

Figure 3.53: Efficiency and time resolution for a sample of mass-production MRPC strips measured in several positions (pads) along and across each strip, at fixed high voltage.

\subsubsection{Front-end electronics and readout}

An overview of the TOF electronics is shown in figure 3.54. The front-end electronics for the TOF were designed to comply with the basic characteristics of the MRPC detector, i.e. very fast differential signals from the anode and cathode readout pads and intrinsic time resolution better than 40 ps.

During the R\&D phase of the detector a solution based on commercial chips (a very fast nondifferential amplifier and a comparator) was adopted. In 2002 an ASIC chip ('NINO') in CMOS $0.25 \mu \mathrm{m}$ technology was developed and tested at PS in 2003 with excellent results [115], thus allowing a substantial reduction of the power dissipation (now at about $40 \mathrm{~mW} / \mathrm{channel}$ ). All the stages (input amplifier and comparator stages) of the 8-channel ASIC are fully differential, its input impedance and capacitance are matched to the transmission line and the peaking time is smaller than $1 \mathrm{~ns}$.

The basic Front-End Analogue card (FEA) contains 3 ASIC chips (24 channels) with a common threshold regulation; it is connected to the PCB interface cards on top of the honeycomb support plate of a module. The LVDS output signals, routed along the two sides of the supermodule, carry the information of the hit time (leading edge) and of the Time-Over-Threshold (TOT), related to the input charge, that is needed for the time-slewing correction.

The readout electronics, located in custom crates at both ends of a supermodule, consists of 9 (right side crate) or 10 (left side crate) TRM (TDC Readout Module) cards and one DRM (Data Readout Module) card in each crate. The TRM card houses the HPTDC [116] (High Performance TDC) 8-channel chips that are used in the very high resolution mode (24.4 ps bin width). Each 


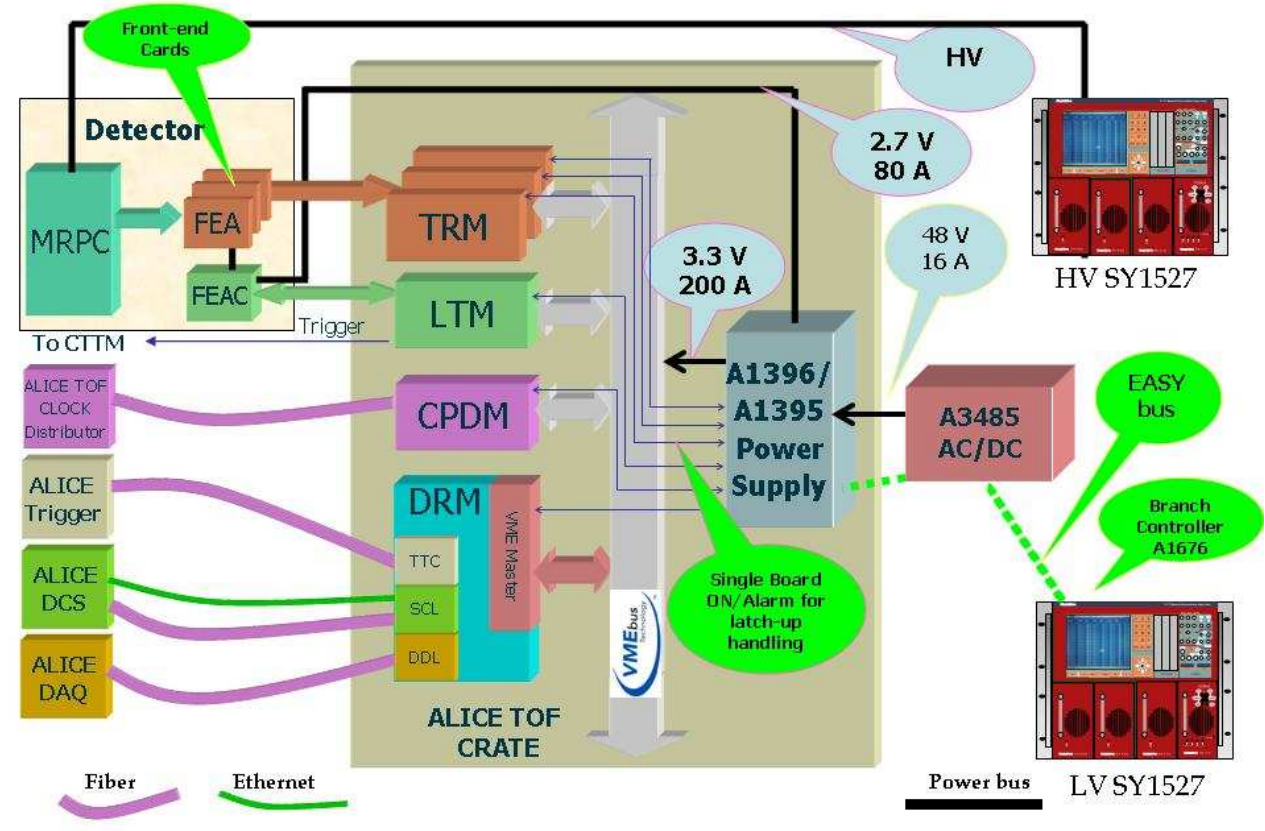

Figure 3.54: Schematic overview of the TOF electronics.

TRM card contains 30 HPTDC chips, i.e. 240 channels, corresponding to the readout pads of 2.5 MRPC strips. The DRM card is the TOF interface to the ALICE DAQ system, it reads and encodes the data from the TRM cards and sends them to the DAQ via the DDL optical link. The DRM card receives the trigger information (Level 1, Level 2-accept, Level 2-reject) from the CTP (Central Trigger Processor) via the TTCrx (Timing Trigger and Control receiver) chip and performs a slowcontrol function with a dedicated FPGA.

Two other electronic cards are located in the custom crates. The CPDM (Clock and Pulser Distribution Module) card distributes to the TRM, DRM and LTM (see below) cards a clock signal with a very low jitter (17 ps) and to the MRPC strips a pulser signal for calibration and monitoring purposes.

The LTM (Local Trigger Module) card, besides setting and monitoring the thresholds of the FEA ASICs, monitoring the FEA low voltage and the temperature inside the electronics volume of a module, collects the 48 OR signals from each pair of FEAs. The purpose of these signals is twofold: it sends a fast pretrigger to the Transition Radiation Detector (TRD) and provides a Level 0 (L0) trigger to select Ultra Peripheral Collisions (UPC) (see section 6.10.3 of [21]), Minimum Bias (MB) events in pp collisions, and Cosmic muons both for central detectors calibration and for cosmic-ray physics (see section 6.11 .5 of [21]).

A large electronics board, the CTTM (Cosmic and Topology Trigger Module), located under the muon spectrometer platform, receives the LTM signals, asserts the L0 and L1 triggers and sends them to the CTP. 


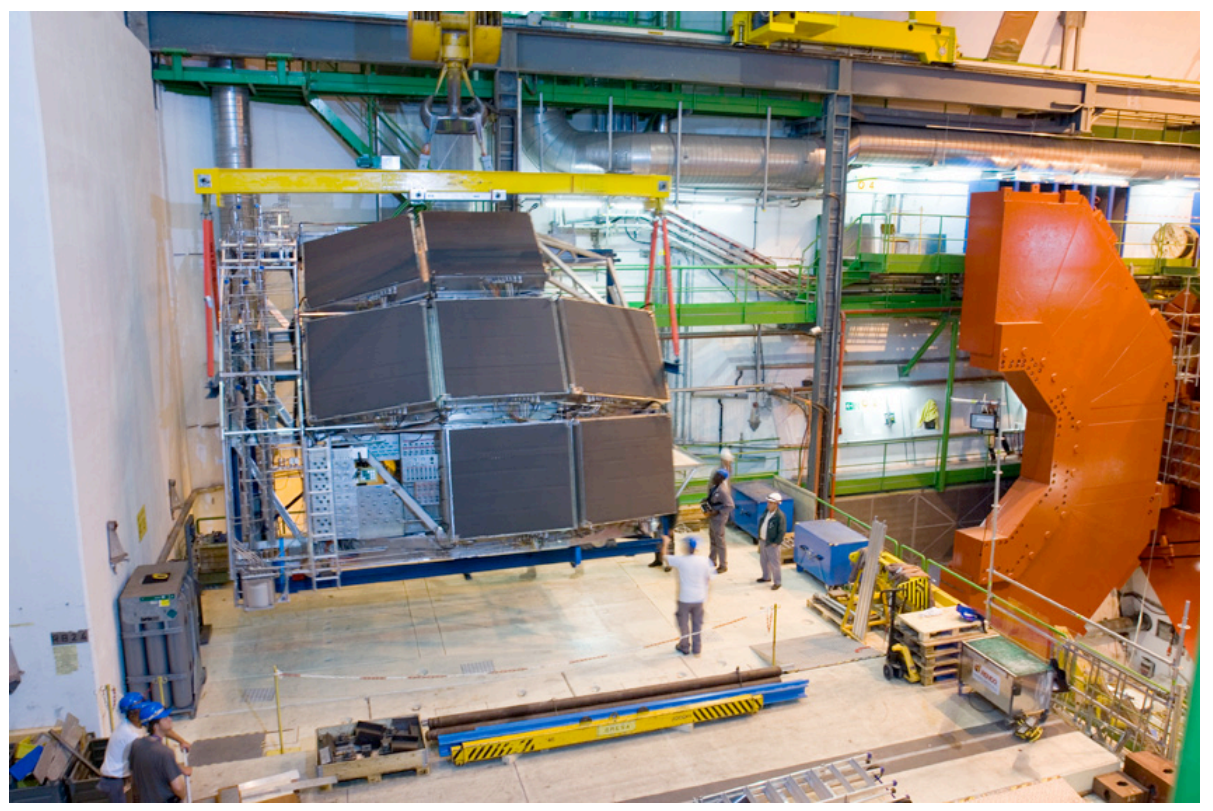

Figure 3.55: View of the seven modules of the HMPID mounted on the cradle.

\subsection{High-Momentum Particle Identification Detector (HMPID)}

\subsubsection{Design considerations}

The High-Momentum Particle Identificaton Detector (HMPID) [8], is dedicated to inclusive measurements of identified hadrons at $p_{\mathrm{t}}>1 \mathrm{GeV} / c$. The aim is to enhance the PID capability of ALICE by enabling identification of charged hadrons beyond the momentum interval attainable through energy-loss (in ITS and TPC) and time-of-flight measurements (in TOF). The detector was optimised to extend the useful range for $\pi / \mathrm{K}$ and $\mathrm{K} / \mathrm{p}$ discrimination, on a track-by-track basis, up to $3 \mathrm{GeV} / c$ and $5 \mathrm{GeV} / c$, respectively, see chapter 8 . The HMPID was designed as a single-arm array with an acceptance of $5 \%$ of the central barrel phase space. The geometry of the detector was optimized with respect to the particle yields in pp and heavy-ion collisions at LHC energies, and with respect to the large opening angle required for two-particle correlation measurements, see section 6.3 of [21]. In addition the identification of light nuclei and anti-nuclei (d, t, $\left.{ }^{3} \mathrm{He}, \alpha\right)$ at high transverse momenta in the central rapidity region can also be performed with the HMPID [117].

\subsubsection{Detector layout}

The HMPID is based on proximity-focusing Ring Imaging Cherenkov (RICH) counters and consists of seven modules of about $1.5 \times 1.5 \mathrm{~m}^{2}$ each, mounted in an independent support cradle (figure 3.55) [118]. The cradle is fixed to the spaceframe at the two o'clock position.

The radiator, which defines the momentum range covered by the HMPID, is a $15 \mathrm{~mm}$ thick layer of low chromaticity $\mathrm{C}_{6} \mathrm{~F}_{14}$ (perfluorohexane) liquid with an index of refraction of $n=1.2989$ at $\lambda=175 \mathrm{~nm}$ corresponding to $\beta_{\min }=0.77$ (i.e. a momentum threshold $p_{\text {th }}=1.21 \mathrm{~m}$, where $m$ is the particle mass). Cherenkov photons, emitted by a fast charged particle traversing the radiator, 


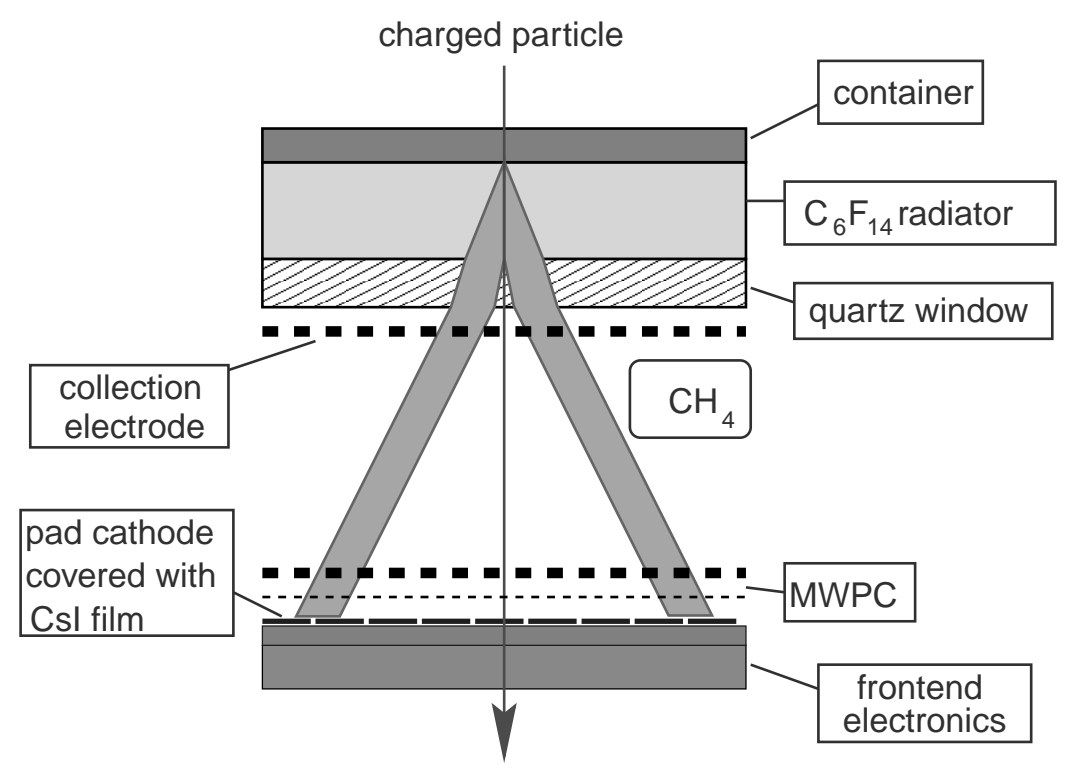

Figure 3.56: Working principle of a RICH detector employing CsI thin films deposited onto the cathode plane of a MWPC. The Cherenkov cone refracts out of the liquid radiator of $\mathrm{C}_{6} \mathrm{~F}_{14}$ and expands in the proximity volume of $\mathrm{CH}_{4}$ before reaching the MWPC photon detector. Electrons released by ionising particles in the proximity gap are prevented to enter the MWPC volume by a positive polarisation of the collection electrode close to the radiator.

are detected by a photon counter (figure 3.56) which exploits the novel technology of a thin layer of CsI deposited onto the pad cathode of a Multi-Wire Pad Chamber (MWPC). The HMPID, with a surface of about $11 \mathrm{~m}^{2}$, is the largest scale application of this technique [119].

A relevant feature of the adopted design for the HMPID photo-detector is the open geometry, which suppresses the electrode elements that in TMAE photo-detectors were specifically implemented to prevent spurious avalanches from feedback photons. Each HMPID module consists of a stack of five independent frames made out of a stabilised aluminium alloy (peralumin) which minimises out-gassing into the chamber active volume. The photo-detector, filled with pure methane and operated at ambient temperature and pressure, is closed on one side by an end-flange which supports six independent CsI photocathode panels, $64 \times 40 \mathrm{~cm}^{2}$ large, segmented into pads of $8 \times 8.4 \mathrm{~mm}^{2}$. On the opposite side, a honeycomb panel supports three $\mathrm{C}_{6} \mathrm{~F}_{14}$ radiator vessels placed at a distance of $80 \mathrm{~mm}$ from the anode wire plane. The gas tightness is ensured by soft O-rings placed in grooves in the chamber frames.

The assembly procedure of module elements fixes the wire-chamber gap ( $2 \mathrm{~mm}$ anode-cathode distance), with a precision of $50 \mu \mathrm{m}$, and the parallelism between radiator trays and photon detector within an accuracy better than $100 \mu \mathrm{m}$. The MWPC anode plane is made of gold-plated tungstenrhenium anode wires, $20 \mu \mathrm{m}$ in diameter, spaced by $4.2 \mathrm{~mm}$. They are soldered on a G-10 printed board with a precision of $0.1 \mathrm{~mm}$ and a tension of $50 \mathrm{~g}$. On both edges of the anode plane, the wires are made thicker to resist the boundary discontinuity of the electrostatic field. The stability of the anode wires against electrostatic forces is ensured by implementing a support line structure in Macor between the pad cathode and the anode plane. 
Cathode and collection wire planes are made out of $100 \mu \mathrm{m}$ diameter gold-plated $\mathrm{Cu} / \mathrm{Be}$ wires stretched at $200 \mathrm{~g}$ per wire by using crimping pins. Stiffness and flatness of pad cathodes is obtained by gluing together two multilayer printed circuit boards on an aluminium frame. The photo-converter is a $300 \mathrm{~nm}$ thick layer of CsI, evaporated by the Joule effect. During the photocathode production the quality cut was set such to allow for the detection of at least 16 resolved clusters per ring (for $\beta=1$ ). The performance of the selected 42 photocathodes allows, on average, for the detection of more than 20 resolved clusters. The production technique and quality evaluation of the 42 photo-cathodes equipping the seven HMPID modules are fully described in [120].

The liquid radiator containers consist of trays of $1330 \times 413 \mathrm{~mm}^{2}$ made out of glass-ceramic material (NEOCERAM), thermally compatible (thermal coefficient $0.5 \times 10^{-6}{ }^{\circ} \mathrm{C}^{-1}$ ) with the fused silica plates used as UV-transparent windows. The thickness and size of the tray's elements were carefully optimised by investigating the best compromise between the detector total radiation length and the perfluorohexane hydrostatic pressure: the quartz window is $5 \mathrm{~mm}$ thick, while the NEOCERAM base plate is $4 \mathrm{~mm}$ thick. To withstand the hydrostatic pressure, thirty cylindrical spacers are glued on the NEOCERAM bottom plate on one side and the quartz window on the other side. They consist of fused silica rods, $10 \mathrm{~mm}$ diameter, arranged in three rows of 10 equally spaced elements.

The radiator trays are supported by a stiff composite panel, consisting of a $50 \mathrm{~mm}$ thick layer of Rohacell sandwiched between two thin $0.5 \mathrm{~mm}$ layers of aluminium. Connections to the liquid radiator inlet and outlet pipe work are obtained by gluing flexible stainless steel bellows on the opposite edges of the NEOCERAM tray, the outlet (inlet) always being at the highest (lowest) location.

The radiator is $15 \mathrm{~mm}$ thick, a choice based on detailed MC simulations and test beam results [121]. Thus it is possible to operate the detector at a low gain (less than $10^{5}$ ) under stable conditions of operation while achieving good performance (see refs. [122, 123]). The high photon yield coupled with the improved quantum efficiency of the photo-cathodes will be used to tune the detector gain adapting it to the specific running conditions. A low gain can be used in ionion collisions, minimising the photon feedbacks and MIPs contribution to the occupancy, while in proton-proton runs, where the much lower track density eases the pattern recognition, higher gain can be used to improve the efficiency. A positive voltage of $2050 \mathrm{~V}$, applied to the anodes, while cathodes are grounded, provides a total 'nominal' gas gain of $4 \times 10^{4}$.

A liquid circulation system was implemented to purify $\mathrm{C}_{6} \mathrm{~F}_{14}$, fill and empty the twenty-one radiator trays at a constant flow, independently, remotely and safely. Due to the inaccessibility of the detector during the run and the fragility of the radiator trays, a system based on a gravity flow principle was chosen owing to its intrinsically safe nature. Since $\mathrm{C}_{6} \mathrm{~F}_{14}$ is not available in a highpurity grade form, filters are implemented in the circulation system in order to remove contaminants (mainly water and oxygen) and achieve the best transparency in the UV region where the detector operates. The full description of the liquid system can be found in [122]. The total HMPID gas volume is $1.4 \mathrm{~m}^{3}$ of pure $\mathrm{CH}_{4}$. It is split equally into the seven independent $\mathrm{RICH}$ modules by supplying each one of them individually with a gas flow rate such that five volume changes are obtained per day while maintaining an operating pressure of a few mbar above ambient pressure. When not operational, for instance during machine shutdown, the detector is flushed with argon. During the detector pre-commissioning prior to installation in the experimental cavern, a leak was detected in one (out of 21) of the radiator trays. Due to the little impact on the physics potential of 
Table 3.15: Synopsis of HMPID parameters.

\begin{tabular}{|l|c|}
\hline Pseudo-rapidity coverage & $-0.6<\eta<0.6$ \\
Azimuthal coverage & $1.2^{\circ}<\phi<58.8^{\circ}$ \\
Radial position & $5 \mathrm{~m}$ \\
Total number of modules & 7 \\
\hline Detector active area & $10.7 \mathrm{~m}^{2}$ \\
Detector thickness radially & $X / X_{0}=18 \%$ \\
\hline Radiator thickness & $15 \mathrm{~mm}$ \\
Radiator medium & liquid $\mathrm{C}_{6} \mathrm{~F}_{14}$ \\
Refractive index & 1.2989 at $175 \mathrm{~nm}$ \\
Independent radiator trays & 3 per module $(21$ total) \\
$\beta$ threshold & 0.77 \\
Detector gas & $\mathrm{CH}$ \\
Gas volume & $1.4 \mathrm{~m}^{3}$ \\
\hline MWPC anode-cathode gap & $2 \mathrm{~mm}$ \\
Operating voltage & $2050 \mathrm{~V}$ \\
Photon converter & Caesium Iodide $(\mathrm{CsI})$ \\
CsI thickness & $300 \mathrm{~nm}$ \\
Quantum Efficiency (QE) & $25 \%$ at $175 \mathrm{~nm}$ \\
Number of Photo-Cathodes (PC) & 42 \\
Number of pads/PC & $3840(161280 \mathrm{total})$ \\
Pad size & $8.0 \times 8.4=67.2 \mathrm{~mm}{ }^{2}$ \\
Front-end chip (GASSIPLEX-07-3) & 10080 \\
Peaking Time & $1.2 \mu \mathrm{s}$ \\
Readout chip (DILOGIC-3) & 3360 \\
Front-end cards & 3360 \\
Readout cards & 672 \\
ADC (12-bit) & 3360 \\
Power consumption/module & $12 \%$ \\
Number of DDL & $10 \mathrm{MHz}\left(\mathrm{maximum}^{2}\right.$ \\
Multiplexing frequency & $5 \mu \mathrm{s}$ at $10 \mathrm{MHz}$ \\
Multiplexing time & $<300 \mu \mathrm{s}$ \\
Readout time (for 12\% occupancy) & $<0.1 \mathrm{MB}$ \\
\hline Event size (for 12\% occupancy) & 161280 \\
Number of readout channels & $15 \mathrm{~kW})$ \\
\hline Occupancy (dN $\mathrm{ch} / \mathrm{d} \eta=8000)$ & \\
\hline
\end{tabular}

the detector (5\% loss in the acceptance) and the non-negligible risks, including delays, involved in the reparation, it was decided to install the detector inside the ALICE solenoid with 20 operational radiators while flushing with methane the leaking one.

The parameters of the detector are summarised in table 3.15. 


\subsubsection{Front-end electronics and readout}

The front-end electronics is based on two dedicated ASIC chips, GASSIPLEX [124] and DILOGIC [125], successfully developed in the framework of the HMPID project in the ALCATELMIETEC $0.7 \mu \mathrm{m}$ technology. The GASSIPLEX-07 chip is a 16-channel analogue multiplexed low-noise signal processor working in TRACK\&HOLD mode. It features a dedicated filter to compensate for the long ion drift tail, a semi-Gaussian shaper and internal protection against discharges. The noise on detector is found to be $1000 e$ r.m.s. During the commissioning before installation the number of dead or noisy pads was found to be less than 200 out of 161280 pads. In the HMPID application the GASSIPLEX analogue output is presented to the input of a commercial 12-bit ADC (AD9220ARS). The multiplexing level is 3 chips (48 channels) per ADC at a maximum frequency of $10 \mathrm{MHz}$. The digitization and zero-suppression time does not depend on the occupancy, being determined only by the multiplexing rate. The highest foreseen luminosity $\left(5 \times 10^{30} \mathrm{~cm}^{2} \mathrm{~s}^{-1}\right.$, interaction rate of about $\left.200 \mathrm{kHz}\right)$ in proton-proton interactions is acceptable for the HMPID since the front-end electronics has a baseline recovery better than $0.5 \%$ after $5 \mu s$. Moreover, the low multiplicity per event makes event overlap negligible.

The DILOGIC chip is a sparse data scan readout processor providing zero suppression and pedestal subtraction with individual threshold and pedestal values for up to 64 channels. Several chips are daisy-chained on the same 18-bit output bus. Asynchronous read/write operations are allowed. Data are read out via the standard ALICE Detector Data Link (DDL). The ALICE DDL Source Interface Unit (SIU) is housed, together with the TTCrq mezzanine board, in the Readout and Control Board (RCB) which interfaces the detector readout electronics with the DAQ and Trigger subsystems. Two RCBs boards are required to operate one HMPID module. The readout time after L2 arrival (for $12 \%$ occupancy) is of the order of $300 \mu \mathrm{s}$. Since momentum information is vital to exploit the HMPID detector, only events for which the TPC information is available are of interest and the HMPID can perfectly cope with the readout rates foreseen for the TPC.

\subsection{PHOton Spectrometer (PHOS)}

The PHOton Spectrometer (PHOS, $[9,20]$ ) is a high-resolution electromagnetic spectrometer covering a limited acceptance domain at central rapidity. The main physics objectives are the test of thermal and dynamical properties of the initial phase of the collision extracted from low $p_{\mathrm{t}}$ direct photon measurements and the study of jet quenching through the measurement of high- $p_{\mathrm{t}} \pi^{0}$ and $\gamma$-jet correlations.

\subsubsection{Design considerations}

The high particle multiplicity in nuclear collisions requires a dense, highly segmented calorimeter with small Molière radius at a large distance from the interaction point in order to keep the cell occupancy at a manageable level of about $10--20 \%$. A good energy and position resolution improves the signal to background ratio for meson identification, in particular at low $p_{\mathrm{t}}$ where the combinatorial background is very large. The identification of photons requires high discrimination power against charged hadrons, neutrons and anti-neutrons. Topological analysis of the shower 
development in the electromagnetic calorimeter, time of flight measurement and charged particle identification provide the discriminating criteria (see [21], section 5 and [126]). The required performance is met through a high granularity electromagnetic calorimeter, timing resolution of the individual detector cells of the order of a few ns and a charged particle detector in front of the calorimeter. Direct photons are discriminated against decay photons either through shower shape analysis at high $p_{\mathrm{t}}$ or through invariant mass analysis at low $p_{r m t}$.

The high-energy resolution and granularity is provided by using dense scintillator material (lead-tungstate, $\mathrm{PbWO}_{4}$ ) of $20 \mathrm{X}_{0}$ with high photo-electron yield. The energy resolution of PHOS is measured to be [127]:

$$
\frac{\sigma_{E}}{E[\mathrm{GeV}]}=\sqrt{\left(\frac{0.0130 \pm 0.0007}{E[\mathrm{GeV}]}\right)^{2}+\left(\frac{0.0130 \pm 0.0007}{\sqrt{E[\mathrm{GeV}]}}\right)^{2}+(1.12 \pm 0.3)^{2}}
$$

The high spatial resolution is achieved by choosing the dimension of the individual detection cells of the order of the Molière radius of the scintillator. The position resolution of PHOS is measured to be [9]:

$$
\sigma_{x, y}[\mathrm{~mm}]=\sqrt{\left(\frac{3.26}{\sqrt{\mathrm{E}[\mathrm{GeV}]}}\right)^{2}+0.44^{2}} .
$$

The resulting two-photon invariant mass resolution at the $\pi^{0}$ peak is $3.5 \%$.

The required timing resolution is achieved by use of fast scintillator and preamplifier. The timing resolution which can be reached with PHOS is about $2 \mathrm{~ns}$ at energies above $1.5 \mathrm{GeV}$.

The large dynamic range $(0.005-80 \mathrm{GeV})$ is achieved by selecting an appropriate detector length that minimizes shower leakage for the highest particle-energies without deteriorating the energy resolution for the lowest particle energies due to light attenuation.

\subsubsection{Detector layout}

PHOS is designed as a single-arm high-resolution high-granularity electromagnetic spectrometer consisting of a highly segmented electromagnetic calorimeter (PHOS) and a Charged-Particle Veto (CPV) detector. A synopsis of the parameters of PHOS is given in table 3.16. PHOS is subdivided into five independent PHOS+CPV units, called PHOS modules. It is positioned on the bottom of the ALICE setup at a distance of $460 \mathrm{~cm}$ from the interaction point. After its final installation it will cover approximately a quarter of a unit in pseudo-rapidity, $0.12 \leq \eta \leq 0.12$, and $100^{\circ}$ in azimuthal angle.

\subsubsection{Electromagnetic calorimeter (PHOS)}

Each PHOS module is segmented into 3584 detection cells arranged in 56 rows of 64 cells. The detection cell consists of a $22 \times 22 \times 180 \mathrm{~mm}^{3}$ lead-tungstate crystal, $\mathrm{PbWO}_{4}$ (PWO), coupled to a $5 \times 5 \mathrm{~mm}^{2}$ Avalanche Photo-Diode (APD) followed by a low-noise preamplifier [128]. The APD and the preamplifier are integrated in a common body glued onto the end face of the crystal (figure 3.57). The crystals are assembled in strip units of two rows of eight detection cells (figure 3.57). The 16 analog signals from one strip unit are fed into a T-shaped connector, connected to the shaper/digitizer/trigger electronics. 
Table 3.16: Summary of PHOS parameters.

\begin{tabular}{|l|c|}
\hline Coverage in pseudo-rapidity & $-0.12 \leq \eta \leq 0.12$ \\
Coverage in azimuthal angle & $220^{\circ}<\phi<320^{\circ}$ \\
Distance to interaction point & $4600 \mathrm{~mm}$ \\
Modularity & Five modules of $1.2 \times 1.4 \mathrm{~m}^{2}$ \\
\hline PHOS & \\
Material & Lead-tungstate crystals (PWO) Crystal \\
Crystal dimensions & $22 \times 22 \times 180 \mathrm{~mm}^{3}$ \\
Depth in radiation length & 20 \\
Number of crystals & 920,3584 crystals per module \\
Total area, crystal volume & $8.6 \mathrm{~m}^{2}, 1.5 \mathrm{~m}^{3}$ \\
Total crystal weight & $12.5 \mathrm{t}$ \\
Operating temperature & $-25^{\circ} \mathrm{C}$ \\
\hline CPV & $80 \% \mathrm{Ar} / 20 \% \mathrm{CO}_{2}$ \\
Gas & $0.05 X_{0}$ \\
Thickness & $8.6 \mathrm{~m}^{2}$ \\
Active area & $30 \mu \mathrm{m}$ \\
Wire diameter & 256 \\
Number of wires per module & $5.65 \mathrm{~mm}^{2}$ \\
Wire pitch & 7168 \\
Pad size, pad inter-distance & $22 \times 10.5 \mathrm{~mm}^{2}, 0.6 \mathrm{~mm}$ \\
Number of pads per module & \\
\hline
\end{tabular}

To increase the light yield of the PWO crystals (temperature coefficient $-2 \%$ per ${ }^{\circ} \mathrm{C}$ ), the PHOS modules are operated at a temperature of $-25^{\circ} \mathrm{C}$ stabilized with a precision of $0.3^{\circ} \mathrm{C}$. The crystals are located inside a cold enclosure, whereas the readout electronics are located outside this enclosure. The temperature is monitored by resistive temperature sensors of thickness $30-50 \mu \mathrm{m}$, inserted in the gap between crystals.

A calibration system using Light Emitting Diodes (LED) and stable current generators monitors every PHOS detection cell.

\subsubsection{Charged-Particle Veto detector}

The Charged-Particle Veto (CPV) detector is a Multi-Wire Proportional Chamber (MWPC) with cathode-pad readout $[129,130]$. Its charged-particle detection efficiency is better than $99 \%$. The spatial resolution of the reconstructed impact point is about $1.54 \mathrm{~mm}$ along the beam direction and $1.38 \mathrm{~mm}$ across the beam.

The CPV is placed on top of the PHOS modules at a distance of about $5 \mathrm{~mm}$. The material budget is less than $5 \%$ of $X_{0}$. The active volume of $14 \mathrm{~mm}$ thickness is filled with a gas mixture $80 \% \mathrm{Ar} / 20 \% \mathrm{CO}_{2}$ at a pressure slightly (1 mbar) above atmospheric pressure.

The cathode plane is segmented into 7168 pads of size $22 \times 10.5 \mathrm{~mm}^{2}$ with an inter-pad distance of $0.6 \mathrm{~mm}$. The largest dimension is aligned along the wires and the total active area of the CPV module is about $1.8 \mathrm{~m}^{2}$. 

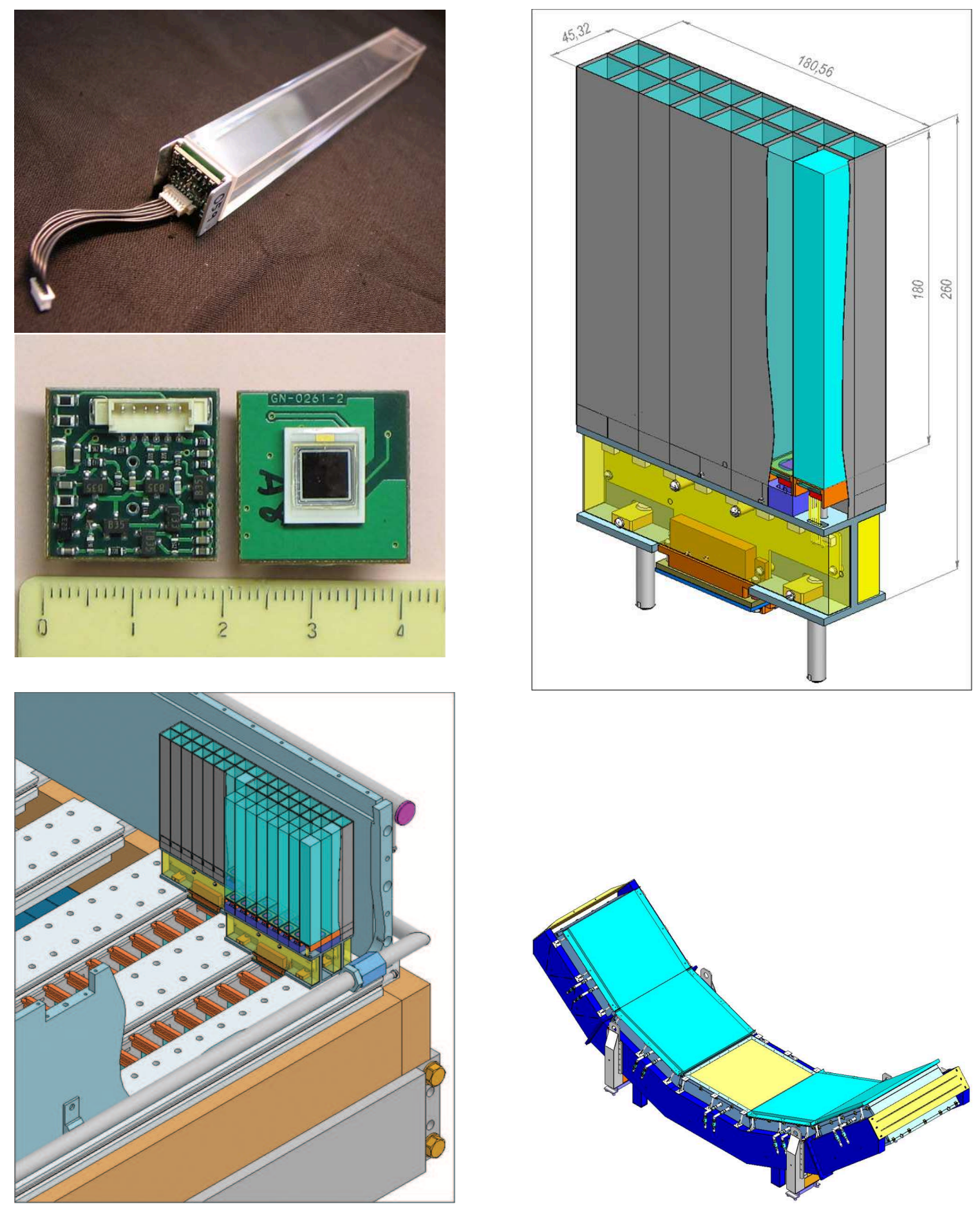

Figure 3.57: Left top: Crystal detector unit with glued photo-detector; photo-detector, APD mounted on the preamplifier substrate. Right top: Strip unit containing $8 \times 2$ crystal detector units. Left bottom: PHOS module with strip units installed onto cooling plates. Right bottom: 5 PHOS modules. 
Table 3.17: Summary of PHOS front-end electronic parameters.

\begin{tabular}{|l|c|}
\hline Least count energy single channel & $5-10 \mathrm{MeV}$ \\
Dynamic range & $0.005-80 \mathrm{GeV}$ \\
Energy channels & 'high' and 'low' gains \\
Timing resolution & around $2 \mathrm{~ns}$ at $2 \mathrm{GeV}$ \\
Trigger & $\mathrm{L} 0, \mathrm{~L} 1$ \\
max channel counting rate & $1 \mathrm{kHz}$ \\
in $\mathrm{Pb}-\mathrm{Pb}$ & $10 \mathrm{~Hz}$ \\
in pp & individual bias setting \\
APD gain control & \\
\hline
\end{tabular}

\subsubsection{Front-end electronics and readout}

A substantial effort was made for PHOS to investigate alternative shaper designs and shaping times. In the case of PHOS, a primary consideration is optimum performance for measurement of low energy photons, in the region around $1 \mathrm{GeV}$, of interest for observation of thermal photon radiation from the Quark Gluon Plasma. With the good intrinsic resolution of $\mathrm{PbWO}_{4}$, care must be taken to minimize the electronic contribution to the noise. This can be done by a judicious choice of the shaping time of the amplifier. For a detector capacitance of $\mathrm{C}_{D}=100 \mathrm{pF}$ as for the Hamamatsu S8664-55 APD, the results indicated an electronic noise minimum of about $300 e$ for a shaping time of about $2 \mu \mathrm{s}$. The competing consideration of a reasonably good timing measurement motivated a final choice of $1 \mu$ s shaping time for PHOS.

\subsubsection{PHOS front-end electronics}

The PHOS electronic chain includes energy digitization and programmable trigger logic generating L0 and L1 triggers to ALICE. Each input channel supplies two shaper channels, one with 'low' and one with 'high' amplification, digitized in separate ADCs. The gains of the APDs are tuned to equalize the channel response by means of a control system where the bias is set individually for each APD. The timing information is derived offline from the shape of the energy pulse. The main physics requirements for the front-end electronics are summarized in table 3.17. There are 4 electronic channels per PHOS crystal: high and low energy gain, fast trigger logic and APD bias control. The total number of electronic channels for the five PHOS modules is 89600 . The main parameters of the preamplifier are [127]: sensitivity $1 \mathrm{~V} / \mathrm{pC}$, max. input charge $8 \mathrm{pC}$, ENC around $400 \mathrm{e}$ for $C_{\mathrm{APD}}=100 \mathrm{pF}$, and power dissipation $62 \mathrm{~mW}$. The shaping amplifier has a time constant of $1.0 \mu \mathrm{s}$. The electronic noise in the output signal corresponds to around $10 \mathrm{MeV}$. The front-end electronics of one PHOS module is segmented (see figure 3.58) into 112 FEE boards each processing the signal from 32 crystals, eight trigger boards (TRU) each covering a region of $28 \times 16$ crystals and 4 readout controller units (RCUs) serving two branches of 14 FEE boards through a GTL bus. The trigger algorithm calculates in parallel all $4 \times 4$ partial energy sums of the digitized fast energy signals from the trigger region. This algorithm is implemented in a FPGA, programmable via the JTAG input. To control the gain of the energy channels, the APD bias control system regulates the bias voltage of each of the APDs with an precision of $0.2 \mathrm{~V}$. 


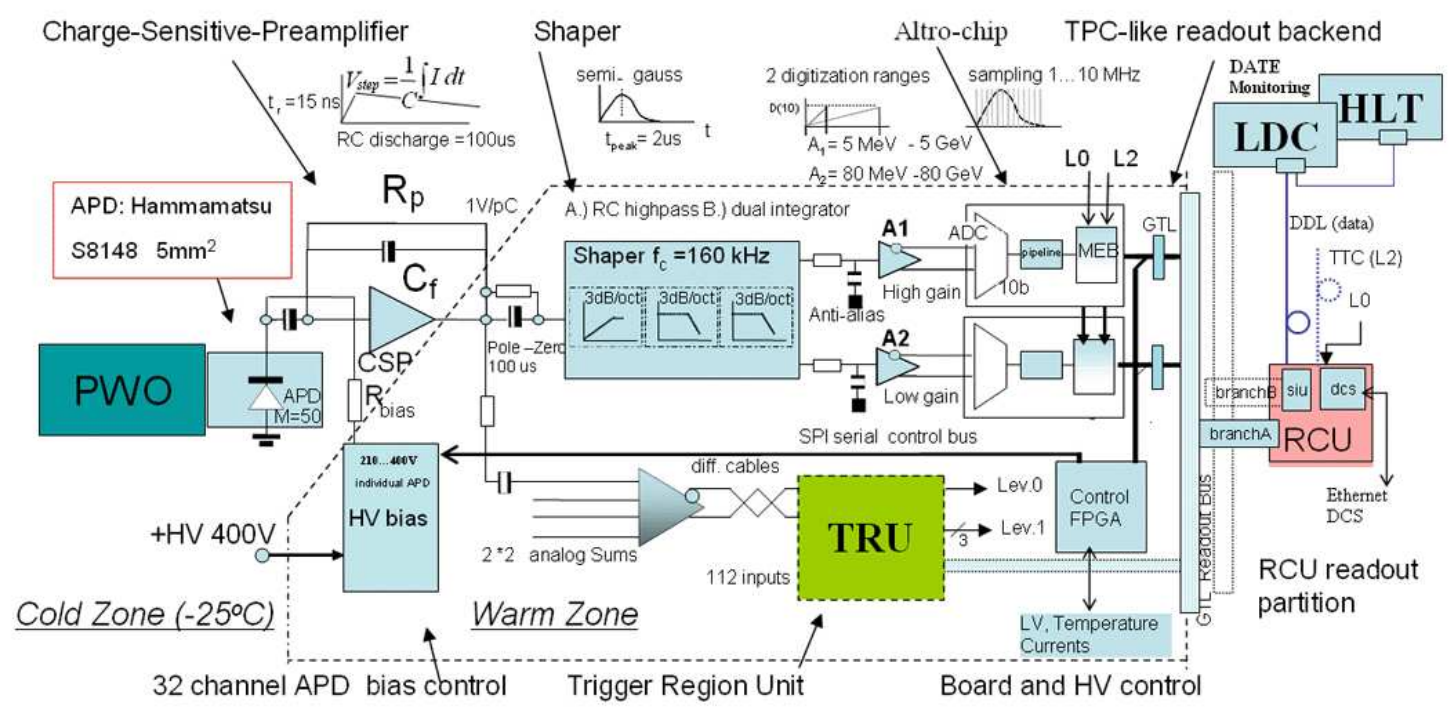

Figure 3.58: Layout of the PHOS electronics.

\subsubsection{CPV front-end electronics}

The CPV pad electronics is identical to the one used for the ALICE HMPID. Three kinds of electronics cards are used. The amplifier shapers for 48 pad channels are organized like the three GASSIPLEX cards, placed on top of the pad plane. 32 series of two Multi-Chip Module (MCM) cards and one Column-Memory and Read-Write (CMRW) protocol card are mounted on the Bus card, installed at the periphery of the pad plane, i.e. outside of the sensitive area of the CPV module.

\subsubsection{Readout}

The readout is based on the concept and modules developed for the TPC detector see section 3.2. A readout controller unit (RCU) transfers formatted event data over the ALICE Digital Data Link (DDL) to the ALICE DAQ system. The RCU operations are executed by means of firmware in the on-board FPGA. The firmware includes a Detector Control System (DCS) card with processor core for handling the Ethernet connection to the ALICE detector control system. The data and control interfaces between the RCU and the TRUs of the front-end electronics are implemented by means of a GTL bus.

\subsection{ElectroMagnetic CALorimeter (EMCal)}

\subsubsection{Design considerations}

The construction of a large ElectroMagnetic Calorimeter (EMCal) [7] began in 2008 with the aim to enable ALICE to explore in detail the physics of jet quenching (interaction of energetic partons with dense matter) over the large kinematic range accessible in heavy-ion collisions at the LHC [20].

The scope and basic design parameters of the calorimeter were chosen to match the physics performance requirements of the high- $p_{\mathrm{t}}$ physics goals [21]. The EMCal is a large $\mathrm{Pb}$-scintillator 
sampling calorimeter with cylindrical geometry, located adjacent to the ALICE magnet coil at a radius of $\sim 4.5$ metres from the beam line. It covers $|\eta| \leq 0.7$ and $\Delta \phi=107^{\circ}$, and is positioned approximately opposite in azimuth to the high-precision ALICE Photon-Spectrometer (PHOS) calorimeter. The size of the EMCal is constraint by the available free space and the maximum weight which can be supported by the L3 magnet.

The choice of a large-acceptance, moderate-resolution electromagnetic calorimeter provides a cost-effective pathway into jet physics in ALICE. The EMCal increases the electromagnetic calorimeter coverage of ALICE by nearly an order of magnitude. It provides a fast and efficient trigger (L0, L1) for hard jets, photons and electrons, allowing ALICE to exploit fully the luminosity of the LHC. The EMCal also measures the neutral energy component of jets, enabling full jet reconstruction in all collision systems, from $\mathrm{pp}$ to $\mathrm{Pb}-\mathrm{Pb}$. The combination of the EMCal, the excellent ALICE charged particle tracking capabilities, and the modest ALICE magnetic-field strength, is a preferred configuration for jet reconstruction in the high-background environment of heavy-ion collisions, allowing the optimization of background rejection while preserving the crucial jet-quenching signals at moderate transverse momentum. The EMCal in ALICE in conjunction with the TPC has good jet energy resolution in $\mathrm{Pb}-\mathrm{Pb}$ collisions, and excellent sensitivity to the full range of jet-quenching effects expected at the LHC.

The emphasis on high $p_{\mathrm{t}}$ means that the intrinsic energy resolution of the EMCal can be modest and the detector granularity can be coarser, with moderately high occupancy. These design criteria point toward the choice of a sampling-calorimeter design, similar to those that were built for STAR and PHENIX to study high- $p_{\mathrm{t}}$ phenomena in heavy-ion collisions at RHIC and then in the LHC-B experiment at the LHC.

\subsubsection{Mechanical issues}

The overall design of the EMCal is heavily influenced by its location within the ALICE L3 magnet. Figure 3.59 shows a schematic integration drawing of the end view of the ALICE central barrel. The EMCal is to be located inside the large room-temperature L3 magnet within a cylindrical integration volume $112 \mathrm{~cm}$ deep sandwiched between the ALICE central-detectors space frame (housing the TPC, TRD and TOF) and the magnet coils. Due to the installation of the PHOS carriage below the ALICE TPC and the HMPID above the ALICE TPC, the EMCal is limited to a region of about $107^{\circ}$ in azimuth above the TPC adjacent to the HMPID.

The EMCal support structure bridges the azimuthal gap between two dedicated rails mounted to the ends of the L3 magnet yoke. These rails support the full, $100 \mathrm{t}$ weight of the detector. The EMCal is installed into the support structure as twelve super module units, with the lower two super modules being of one-third size.

Small azimuthal gaps $(\sim 3 \mathrm{~cm})$ between super modules facilitate installation and alignment. These gaps are positioned in line with the TPC sector boundaries. Along these sector boundaries there is substantial additional structural material required for the support of the TPC and other detectors that would significantly degrade any electromagnetic measurements made in these gaps. Thus, the gaps create no additional loss of electromagnetic acceptance. Detailed simulations show that these gaps have no significant influence on the measured jet energy or jet resolution. 


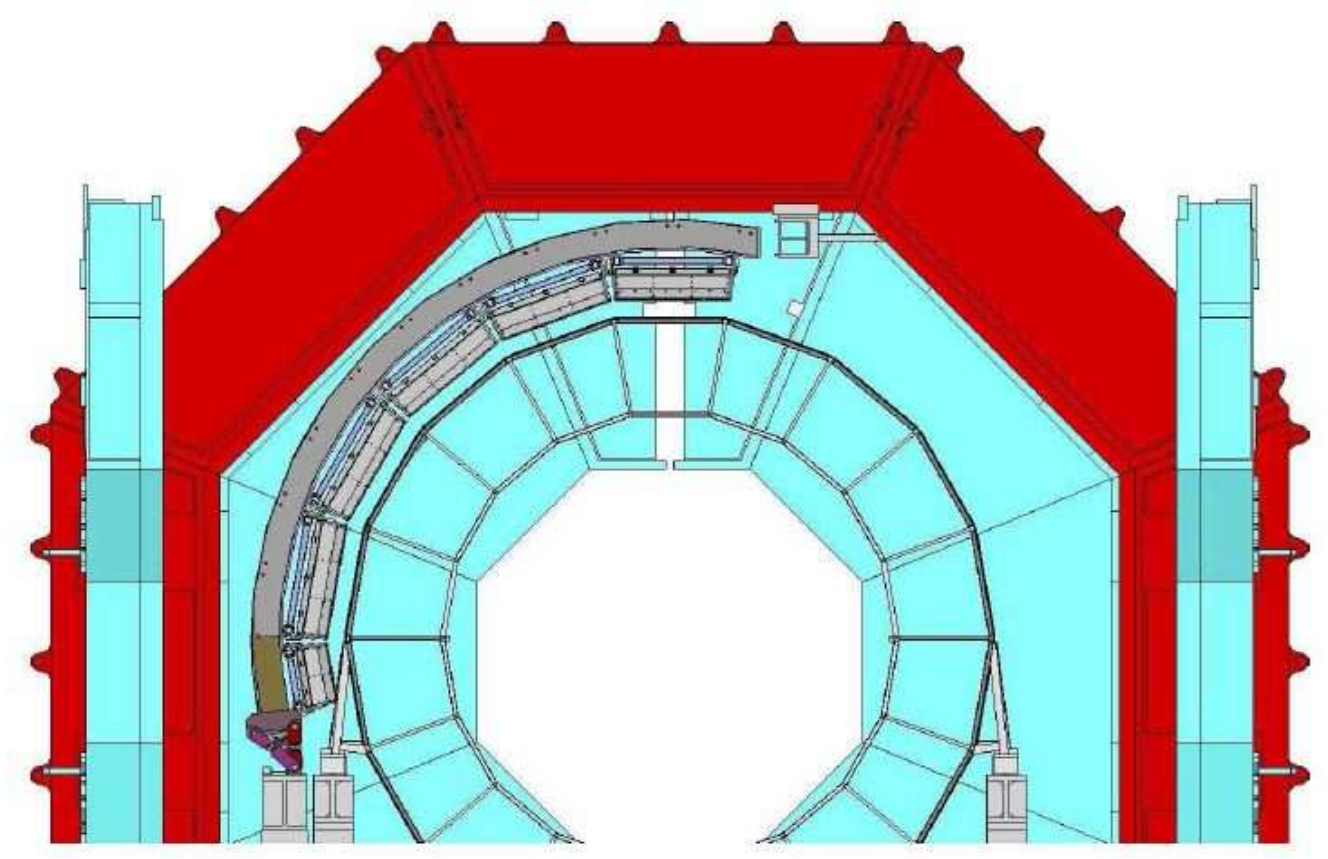

Figure 3.59: Schematic integration drawing of the end view of the ALICE central barrel.

The chosen technology is a layered $\mathrm{Pb}$-scintillator sampling calorimeter with a longitudinal pitch of $1.44 \mathrm{~mm} \mathrm{~Pb}$ and $1.76 \mathrm{~mm}$ scintillator with longitudinal wavelength-shifting fibre light collection (Shashlik). The detector is segmented into 12288 towers, each of which is approximately projective in $\eta$ and $\phi$ to the interaction vertex. The towers are grouped into super modules of two types: 'full size' which span $\Delta \eta=0.7$ and $\Delta \phi=20^{\circ}$, and 'one-third size' which span $\Delta \eta=0.7$ and $\Delta \phi=7^{\circ}$. There are 10 full-size and 2 one-third-size super modules in the full-detector acceptance The super module is the basic structural unit of the calorimeter. These are the units handled as the detector is moved below ground and rigged during installation into their final resting place in the L3 magnet.

Each full-size super module (see figure 3.60) is assembled from $12 \times 24=288$ modules arranged in 24 strip modules of 12 modules each (see below). Each module has a rectangular cross section in the $\phi$ direction and a trapezoidal cross section in the $\eta$ direction with a full taper of 1.5 degrees. The resultant assembly of stacked strip modules is approximately projective with an average angle of incidence at the front face of a module of less than 2 degrees in $\eta$ and less than 10 degrees in $\phi$.

The smallest building block of the calorimeter is the individual module. A cross section of a single module is shown in figure 3.61. Each individual module contains $2 \times 2=4$ towers built up from 76 alternating layers of $1.44 \mathrm{~mm} \mathrm{~Pb}$ (Pure $\mathrm{Pb}$-standard mill spec.) and 77 layers of $1.76 \mathrm{~mm}$ polystyrene base, injection molded scintillator (BASF143E $+1.5 \% \mathrm{pTP}+0.04 \% \mathrm{POPOP}$ ) with an intrinsic light output of 50\% Anthracene [131]. To best account for inactive materials in the space immediately before the calorimeter, the first layer of the detector is scintillator.

White, acid free, bond paper serves as a diffuse reflector on the scintillator surfaces and, in addition, provides the required degree of static friction between the calorimeter layers. The scintillator edges are treated with a $\mathrm{TiO}_{2}$ loaded reflector to improve the transverse optical uniformity 


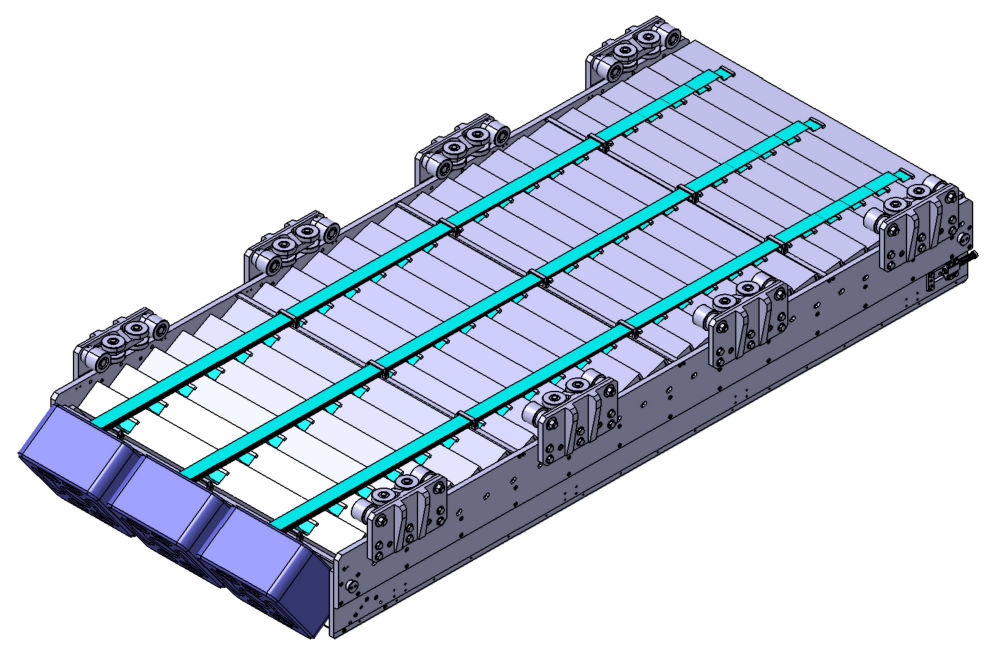

Figure 3.60: EMCAL super module.

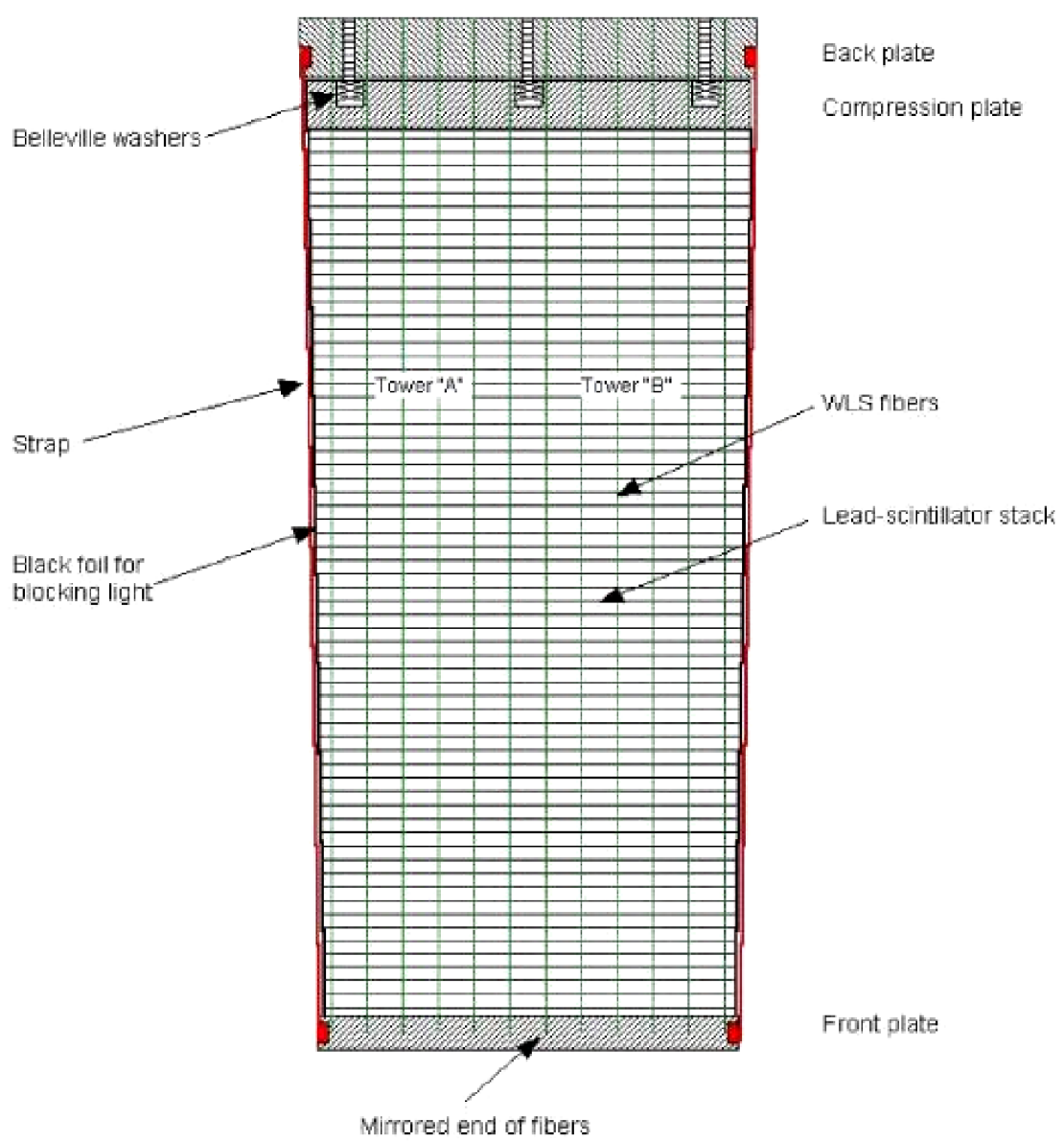

Figure 3.61: Cross sectional view of a single EMCal module. This view shows the 1.5 degree tapered cross section of the module. The principal components of the module are indicated. The size of module is $\sim 12 \mathrm{~cm} \times 12 \mathrm{~cm} \times 24.6 \mathrm{~cm}$. 
within a single tower. Each scintillator edge also receives an over-coating of black paint to provide tower to tower optical isolation better than $99 \%$. The total thickness of the reflective and optical isolating material on the scintillator edges is $\sim 50 \mu \mathrm{m}$. Before completing the final mechanical closure of the module, it is wrapped in a thin layer of black adhesive paper to improve the light tightness.

All modules in the calorimeter are mechanically and dimensionally identical. The front-face dimensions of the towers are $6 \times 6 \mathrm{~cm}^{2}$ resulting in individual tower acceptance of $\Delta \eta \times \Delta \phi \sim$ $0.014 \times 0.014$.

The $\mathrm{Pb}$-scintillator stack in a module is secured in place by the static friction between individual layers under the load of an internal pressure of $2 \mathrm{~kg} / \mathrm{cm}^{2}$. The module is closed by a skin of $150 \mu \mathrm{m}$ thick stainless steel on all four transverse surfaces. Flanges are welded to the ends of the stainless steel skin (straps) to permit secure attachment to the front and rear plates of the module. This thin stainless skin plus the optical treatment of the scintillator edges is the only inert material between the active tower volumes. The internal pressure in the module is stabilized against thermal effects, mechanical relaxation and long term flow of the $\mathrm{Pb}$ and/or polystyrene by a customized array of five non-linear spring sets (Belleville washers) per module. In this way, each module is a self supporting unit with a stable mechanical lifetime of more than 20 years with a large safety factor on the final compressing force. This compressed module design allows the detector to be completely supported from its rear surface.

\subsubsection{Strip modules and super modules}

An array of 12 modules in the $\phi$ direction and a strong back (which links them) form an EMCal 'strip module'. Like the individual module, the strip module is a self-supporting unit. The super module, discussed above, is made from 24 strip modules mounted in $\eta$ direction. The strong back, an aluminium beam, which integrates the modules into the strip module, functions as the mechanical support for modules and a stiff structural element of the super module structure. It provides protection for the optical fibres, structural mount for the light guide, APD and charge-sensitive preamplifier housing, and a light-tight enclosure of these elements.

The super module shown in figure 3.60 is the largest building block of the calorimeter. Starting with 288 individual EMCal modules which are rather compact and heavy, a stiff super module structure was designed that performs with very small deflections in any $\phi$ orientation yet does not require extensive, heavy external stiffening components that would reduce the available volume for the active detector.

The concept adopted for the ALICE EMCAL is a super module 'crate' which functions not as a box for the individual modules, but rather as an integrated structure in which the individual elements (strip modules) contribute to the overall stiffness. The super module crate thus functions as a large I-beam in which the aluminium flanges of the beam are the longitudinal sides of the crate and the 24 transverse rows of strip modules together form the 'core'. With $1444 \mathrm{~mm}$ spaced flanges, this configuration gives to the super module a good stiffness for any $\phi$ orientation. 


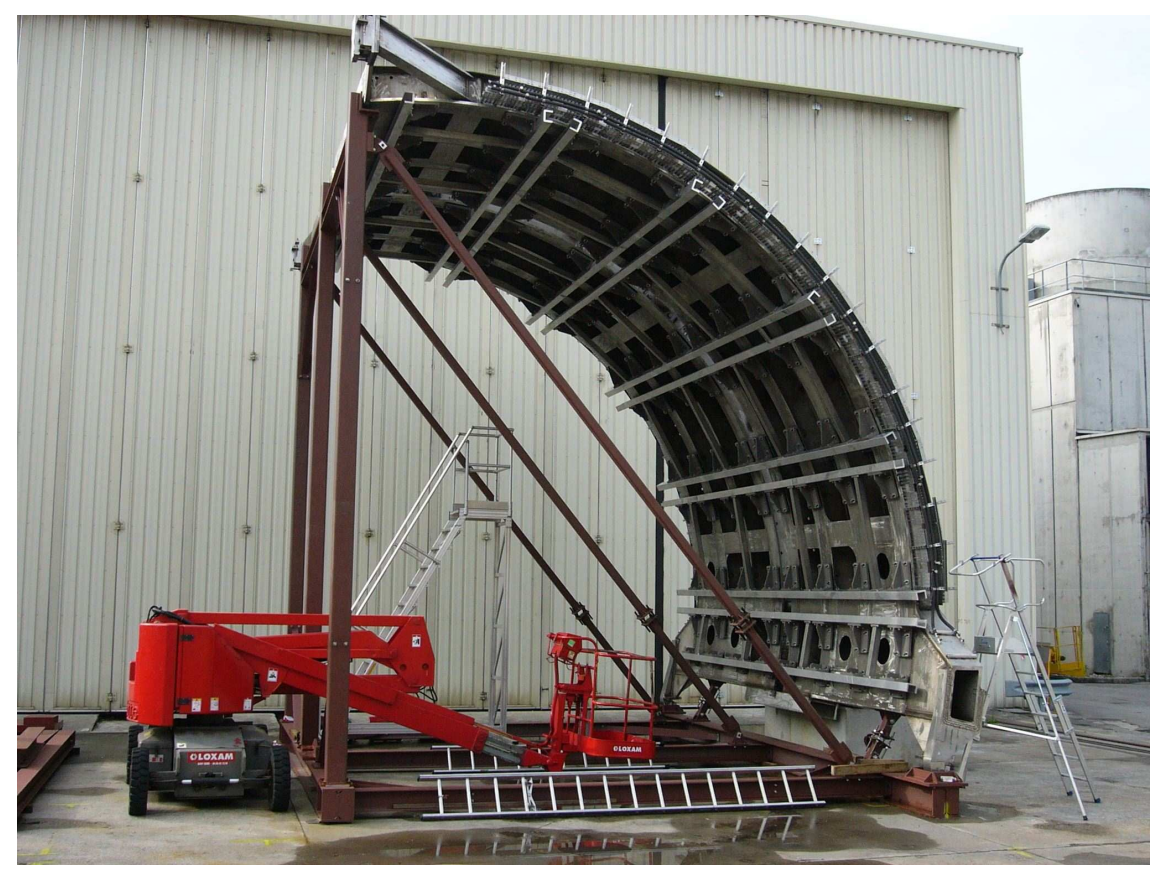

Figure 3.62: EMCal support structure and the associated rail supports for super modules is shown on its assembly fixture, above ground at the ALICE site (Point 2).

The interface between the super module crate and the EMCal support structure (figure 3.62) is achieved with a set of rails mounted on the inner surface of the EMCal support structure and rollers fitted to carriages mounted on super module crates (see figure 3.60). Each super module slides into its resting place inside the ALICE L3 magnet on two U-shaped aluminium rails. This system allows installation of super modules as needed during the annual LHC shutdown period until the full EMCal is in place.

\subsubsection{Module physical parameters}

The average density of the detector's active volume is $5.68 \mathrm{~g} / \mathrm{cm}^{3}$ which results from a $1: 1.22 \mathrm{~Pb}$ to scintillator ratio by volume. This results in a compact detector, consistent with the EMCal integration volume at the chosen detector thickness of 20.1 radiation lengths $\left(20.1 X_{0}\right)$. In simulations, this number of radiation lengths gives a maximum deviation from linearity (due mainly to shower leakage) of $1.5 \%$ for the most probable energy response in the range up to $100 \mathrm{GeV}$ photons.

The impact of detector energy resolution on the proposed physics programme was studied extensively. Given the main focus on jet physics and the significant non-detector contributions to the energy resolution in the heavy-ion environment, the calorimeter resolution is not very critical. Simulations and test-beam measurements indicate that the final detector resolution [7] will be better than $10 \% / \sqrt{E}$ which is completely adequate to accomplish the proposed scientific programme. The physical characteristics of the EMCal are summarized in table 3.18. 
Table 3.18: The EMCal physical parameters.

\begin{tabular}{|l|c|}
\hline Quantity & Value \\
\hline Tower size (at $\eta=0)$ & $6.0 \times 6.0 \times 24.6 \mathrm{~cm}^{3}$ active \\
Tower size & $\Delta \phi \times \Delta \eta=0.0143 \times 0.0143$ \\
Sampling ratio & $1.44 \mathrm{~mm} \mathrm{~Pb} / 1.76 \mathrm{~mm}$ scintillator \\
Number of layers & 77 \\
Effective radiation length $X_{0}$ & $12.3 \mathrm{~mm}$ \\
Effective moliere radius $R_{M}$ & $3.2 \mathrm{~cm}$ \\
Effective density & $5.68 \mathrm{~g} / \mathrm{cm}^{3}$ \\
Sampling fraction & 10.5 \\
Number of radiation length & 20.1 \\
Number of towers & 12,672 \\
Number of modules & 3,168 \\
Number of super modules & 10 full size, 2 half size \\
Weight of super modules & 7.7 metric tons (full size) \\
Total coverage & $\Delta \phi=107^{\circ},-0.7 \leq \eta \leq 0.7$ \\
\hline
\end{tabular}

\subsubsection{Module optical system}

Scintillation photons produced in each tower are captured by an array of 36 Kuraray Y-11 (200 M), double-clad, wavelength-shifting (WLS) fibres that run longitudinally through the $\mathrm{Pb} / \mathrm{scintillator}$ stack. Each fibre terminates in an aluminized mirror at the front face of the module and is integrated into a polished, circular group of 36 at the photo-sensor end at the back of the module. Because the tower transverse shape deviates slightly from square as a function of longitudinal depth, a fibre pattern was chosen which has exactly the same aspect ratio as the mechanical tower shape at a depth close to the electromagnetic shower maximum. This has the effect of making the fibre pattern uniform across tower boundaries when weighted by the shower energy deposition.

The mirroring on the distant end of the fibre creates an approximate compensation for the effects of the finite attenuation lengths within the fibre making the longitudinal response of the detector quite uniform. This is most significant for the shorter attenuation lengths associated with cladding light that is quite important in this rather short detector. The properties of the selected fibres are given in table 3.19.

The fibre bundle in a single tower terminates in a $6.8 \mathrm{~mm}$ diameter disk and connects to the APD photo sensor through a short light guide/diffuser with a square cross section of $7 \mathrm{~mm} \times$ $7 \mathrm{~mm}$ that tapers slowly down to $4.5 \mathrm{~mm} \times 4.5 \mathrm{~mm}$ as it mates (glued) to the $5 \mathrm{~mm} \times 5 \mathrm{~mm}$ active area of the APD.

Figure 3.63 shows 4 pre-fabricated fibre bundles inserted into the towers of a single prototype module. In this picture all of the module rear enclosing and structural elements are omitted so that the wavelength shifting fibres may be seen as they converge to the light guide (inside the black plastic tube) and finally to mate with the APD and charge sensitive preamplifier.

The selected photo sensor is the Hamamatsu S8664-55 Avalanche Photo Diode. This photodiode has a peak spectral response at a wavelength of $585 \mathrm{~nm}$ compared to an emission peak of 
Table 3.19: Characteristics of the EMCal module wavelength shifting fibres.

\begin{tabular}{|l|c|}
\hline Quantity & Value \\
\hline WLS fibre & Y-11 (200) M-DC \\
Manufacturer & Kuraray \\
WLS Fluor & K27 200 mg \\
Absorption peak & $430 \mathrm{~nm}$ \\
Emission peak & $476 \mathrm{~nm}$ \\
Decay time & $7 \mathrm{~ns}$ \\
Core material & PS \\
Refractive index of core & 1.59 \\
Inner cladding & PMMA \\
Refractive index of cladding & 1.49 \\
Outer cladding & FP \\
Refractive index of outer cladding & 1.42 \\
Long fibre attenuation length & $3.5 \mathrm{~m}$ \\
Fibre diameter & $1.0 \mathrm{~mm}$ \\
\hline
\end{tabular}

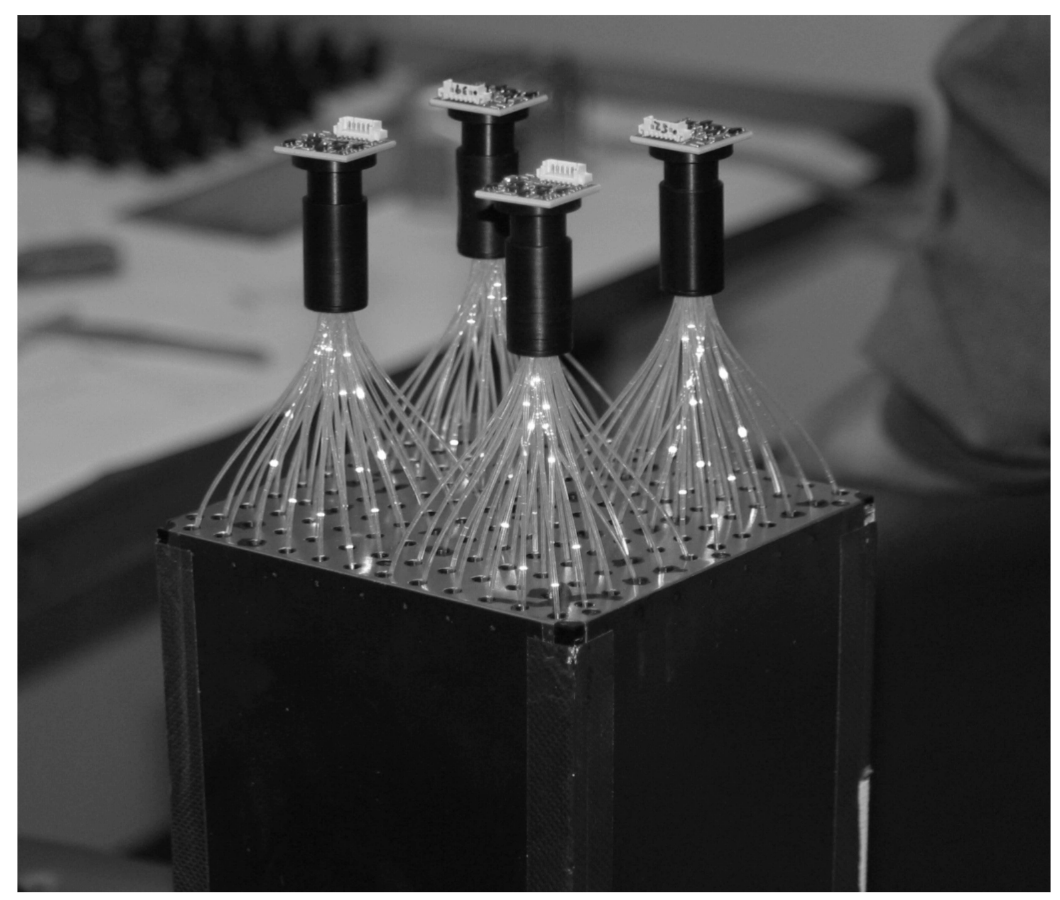

Figure 3.63: Prototype EMCal module. The back enclosing structure is removed so that the converging, wavelength shifting fibres are visible from each of the 4 towers. The charge sensitive preamps are shown integrated directly to the APD (not visible).

$476 \mathrm{~nm}$ for the Y-11 fibres. However, both the spectral response and the quantum efficiency of the APD are quite broad with the latter dropping from the maximum by only 5\% at the WLS fibre emission peak. At this wavelength, the manufacturer's specification gives a quantum efficiency of $80 \%$. 


\subsubsection{Readout and trigger}

Both the selected APD and the charge sensitive preamp are identical to those used for the PHOS calorimeter, described in section 3.6. The light yield, per unit of energy, deposit in the EMCal is similar to that of the PHOS $[9,127]$. Since the electronic noise performance requirements of the EMCal are less stringent than those of PHOS (due to the larger intrinsic energy resolution of the EMCal), the PHOS readout electronics were adopted for the EMCal readout, with only minor modification. The only significant difference with the PHOS readout is the difference in the FEE amplifier due to the chosen dynamic range, the EMCal light yield, and the amplifier shaping time.

\section{Dynamic range}

Based on the expected annual yield of photons and $\pi^{0}$ at high $p_{\mathrm{t}}$, the full scale energy range for an EMCAL tower is chosen to be $250 \mathrm{GeV}$ as compared to $80 \mathrm{GeV}$ for PHOS, well beyond the expected maximum photon or $\pi^{0}$ energy. Setting the EMCAL full-scale energy range to $250 \mathrm{GeV}$ sets the Least Significant Bit (LSB) on the lower of the two overlapping gain ranges to $250 \mathrm{MeV}$ (10-bits) with the corresponding maximum energy on the high-gain range at $16 \mathrm{GeV}$ and least-significant bit at $16 \mathrm{MeV}$.

\section{Light yield}

An important parameter of the EMCal readout is the light output to the APD per MeV of energy deposited in a tower. The test-beam measurements with the EMCal prototype modules gave a measured light yield of about 5 photo-electrons/MeVfrom the APD at gain $\mathrm{M}=1$, similar to the PHOS which produces 4.4 photo-electrons $/ \mathrm{MeV}$.

\subsubsection{Shaper time constant optimization}

Several considerations motivate a much shorter shaping time for the EMCal than the one for PHOS. First, simulations of central $\mathrm{Pb}-\mathrm{Pb}$ collisions within the AliRoot offline framework and using HIJING event generator (see chapter 6.4) indicate that the EMCal, with its hydrogen-rich scintillator, will be affected by a large slow-neutron contribution that has a tail extending for hundreds of ns after the collision. Second, the number of ALTRO chip digitizer samples recorded is dictated by the total shaped pulse width. With the approximately ten times coarser EMCal granularity, the occupancies will be correspondingly higher in EMCal than PHOS. With the goal to keep the total data volume per Readout Control Unit (RCU) similar to that of PHOS, the number of EMCal samples will be reduced to keep the product of occupancy $\times$ no. samples similar. This can be achieved with a shaping time of about $100 \mathrm{~ns}$. With $100 \mathrm{~ns}$ shaping time the voltage noise will dominate such that one expects a total electronics noise contribution of about $1500 e$ for the APD+preamplifier+shaper chain, corresponding to an electronics noise contribution of about $12 \mathrm{MeV}$ per EMCal tower.

The significance of this electronic noise is best illustrated relative to the estimated energy fluctuations due to late-neutron background. Simulations of the EMCal response for central $\mathrm{Pb}-\mathrm{Pb}$ collisions have shown that there is a large background energy deposit predominantly from late neutrons produced in secondary interactions in the surrounding materials of the ALICE experiment [132]. The time distribution of the background has a long tail with arrival times at the 
calorimeter extending for several hundreds of ns after the collision. (Note that this background is generated by the primary central $\mathrm{Pb}-\mathrm{Pb}$ collision, and hence it is a centrality dependent effect, expected to scale with the multiplicity of produced particles). The result indicates that as short a shaping time as feasible should be used in order to minimize this background contribution to the energy measurement. Without any additional timing cut, the average background energy deposit is $36 \mathrm{MeV}$ per tower, i.e. several times greater than the expected electronics noise contribution per tower with $100 \mathrm{~ns}$ shaping time.

\subsubsection{EMCal trigger}

The PHOS readout, which was adopted for the EMCal, includes a Trigger Region Unit card (TRU) [133] on each GTL readout bus that provides sliding $4 \times 4$ tower sums for generation of a Level-0 and three Level-1 triggers with programmable thresholds for simultaneous low-, mid-, and high-energy shower trigger outputs. All of the trigger outputs generated locally from each TRU are logically 'OR'ed and input to the ALICE Central Trigger Processor (CTP). The PHOS trigger was designed to trigger efficiently on photons and electrons and will serve that same purpose equally well for the EMCal. The PHOS TRU will be used for the EMCal with only minor modifications. First, because the EMCal input connections to the FEE cards do not have exactly the same geometrical mapping as for PHOS, the TRU FPGA code will be modified to take into account the different tower mapping of PHOS and the EMCal.

The PHOS TRU single shower trigger provides a good leading-photon, leading- $\pi^{0}$ trigger that also triggers efficiently on high-energy jets. However, because a single-shower trigger will have a bias toward jets including a leading $\pi^{0}$ it is of interest to have the capability to trigger on larger regions of EMCAL towers.

For this purpose a Summary Trigger Unit (STU) will take input from the EMCal TRU modules and perform energy sums over larger regions of the EMCal. The PHOS TRU design was modified for this purpose to provide raw trigger sum output that can input to the STU to form trigger sums over larger regions that cross TRU boundaries. Also, the STU will use the available raw data in order to improve the single shower-trigger by TRU boundaries for $4 \times 4$ tower sums. The STU will also receive particle multiplicity information from the V0 trigger detector that will allow to apply centrality dependent single shower and jet triggers. At the end of the trigger sequence (Level 2) the STU will provide, via sums DDL link, all raw trigger sums to the DAQ and HLT.

\subsubsection{EMCal calibration}

The initial pre-calibration of the EMCal will be performed at the time of super module assembly using the 'mip' peak obtained with cosmic-ray particles triggered by scintillator arrays above and below the super module. The individual APD voltages will be adjusted to match the cosmics mip peak in each tower to bring all towers to the same relative gain. Tests of the planned cosmics calibration procedure using prototype EMCal modules following beam-test measurements demonstrated that the cosmics mip peak can provide relative gain matching of each tower with an rms variation of less than $10 \%$. This level of initial gain matching is required to provide a uniform single-shower trigger response. Ultimately the absolute energy scale for each tower will be 


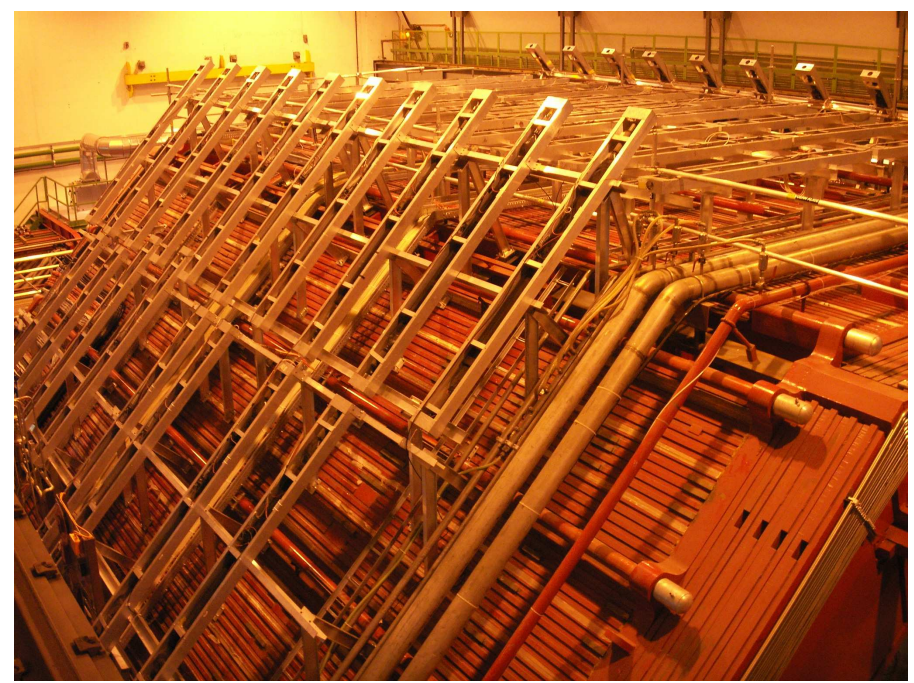

Figure 3.64: Photograph of the ACORDE scintillator module array on the upper faces of the magnet yoke in the ALICE pit. An ACORDE module unit includes two overlapping scintillator counters, each with $190 \times 20 \mathrm{~cm}^{2}$ effective area, and $10 \mathrm{~mm}$ thickness, plus two PMTs placed at the end of each scintillator, with optic guide and iron magnetic shielding. All components are attached to an aluminium structure, assembling a $40 \mathrm{~kg}$ robust box. There are twenty modules on each one of the three upper faces.

obtained using physics data from proton-proton collisions. This will be extracted using a combination of measurements of the mip peak, electron $\mathrm{E} / \mathrm{p}$ ratio, and $\pi^{0}$ mass for showers centered on each tower.

\subsection{ALICE COsmic Ray DEtector (ACORDE)}

\subsubsection{Design considerations}

ACORDE, the ALICE cosmic ray detector, is an array of plastic scintillator counters placed on the upper surface of the L3 magnet. It plays a two-fold role in ALICE: i) the first task is to provide a fast (Level-0) trigger signal, for the commissioning, calibration and alignment procedures of some of the ALICE tracking detectors; ii) it will also detect, in combination with the TPC, TRD and TOF, single atmospheric muons and multi-muon events (so-called muon bundles) thus allowing us to study high-energy cosmic rays in the energy region of the knee in the cosmic ray spectrum. A feasibility and performance study is detailed in [20, 21, 134, 135].

\subsubsection{Detector layout}

An ACORDE module consists of two scintillator counters, each with $190 \times 20 \mathrm{~cm}^{2}$ effective area, placed on top of each other and read out in coincidence (figure 3.64). With this setup a uniform efficiency higher than $90 \%$ along the whole length of a test module is achieved. Details of the construction and performance of this counters are explained in [134]. The ACORDE scintillator 
Table 3.20: Synopsis of ACORDE parameters.

\begin{tabular}{|c|c|}
\hline $\begin{array}{l}\text { Pseudo-rapidity coverage } \\
\text { Azimuthal coverage } \\
\text { Radial position }\end{array}$ & $\begin{array}{l}-1.3<\eta<1.3 \\
-60^{\circ}<\phi<60^{\circ} \\
r=8.5 \mathrm{~m}\end{array}$ \\
\hline Total number of detector modules & 60 \\
\hline Module size & $300 \times 26 \times 10 \mathrm{~cm}^{3}$ \\
\hline Active detector area & $190 \times 20 \mathrm{~cm}^{2}$ \\
\hline Radial detector thickness & $X / X_{0}=4.7 \%$ \\
\hline Module segmentation & 30 in $\varphi$ \\
\hline Number of readout channels & 120 \\
\hline Number of readout boards & 60 \\
\hline Trigger time delay electronics for & $220 \mathrm{~ns}$ \\
\hline multi-coincidence & $270 \mathrm{~ns}$ \\
\hline TRD wake-up & $170 \mathrm{~ns}$ \\
\hline Rate for cosmic single muon events & $4.5 \mathrm{~Hz} / \mathrm{m}^{2}$ \\
\hline Rate for cosmic 5-fold coincidence module events & $5 \times 10^{-3} \mathrm{~Hz}$ \\
\hline
\end{tabular}

module array, which includes 60 scintillator counter modules placed on top of the ALICE magnet, is shown in figure 3.64. ACORDE provides a fast Level-0 trigger signal to the Central Trigger Processor, when atmospheric muons impinge upon the ALICE detector. The signal is used for the calibration, alignment and performance of several ALICE tracking detectors, mainly the TPC, TOF, HMPID and ITS. The operational Cosmic Ray Trigger is delivering trigger signals independent of the LHC beam. Table 3.20 summarize the operation conditions of this detector.

The typical rate for single atmospheric muons reaching the ALICE detector is relatively low (4.5 Hz/m ${ }^{2}$, on top of the magnet), the rate for multi-muon events is expected to be much lower (less than $10^{-3} \mathrm{~Hz} / \mathrm{m}^{2}$ ). This is statistically sufficient for the study of these type of events, provided we can trigger and store tracking information from cosmic muons parallel to the ALICE normal data taking with colliding beams. Atmospheric muons need an energy of at least $17 \mathrm{GeV}$ to reach the ALICE hall, while the upper energy limit for reconstructed muons in the TPC will be near $2 \mathrm{TeV}$, at a magnetic field intensity of $0.5 \mathrm{~T}$. This allows us to measure and analyze the atmospheric muon momentum spectra in a wide range $(0.1-2 \mathrm{TeV})$ with a very high precision.

\subsubsection{Readout electronics.}

The ACORDE electronics was designed to fulfil the following requirements: i) produce the single muon trigger signal and provide a rough spatial position when atmospheric muons hit the ALICE magnet. This information will be used to calibrate the ALICE TPC detector and other tracking detectors; ii) provide a multi-coincidence trigger signal and store kinematics information from cosmic muons. Given the low rate of this trigger (less than $10^{-3} \mathrm{~Hz}$ ), it would greatly benefit from running in parallel with ALICE normal data taking; iii) provide the wake-up signal to the ALICE Transition Radiation Detector (TRD), and iv) supervise the performance of the ACORDE scintillator counter array. 


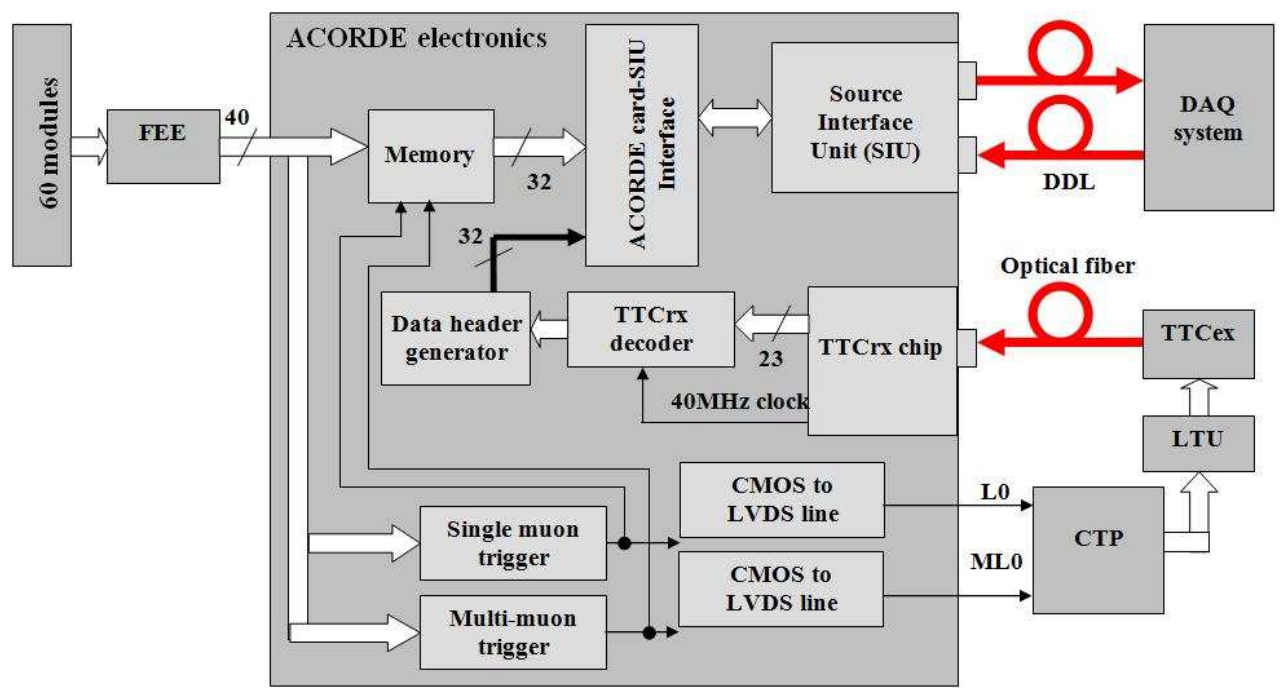

Figure 3.65: Block diagram of the ACORDE electronics system.

The ACORDE electronics (figure 3.65) is formed by 60 FEE Cards, one per ACORDE module; and the 'ACORDE OR' Card to generate the TRD wake up signal. This card receives the 60 coincidence LVDs signals coming from the FEE cards. The final component is the Main Card, which contains the electronics to receive the 120 LVDS signals coming from 120 scintillator. This card produces the single and the multi-coincidence trigger signals and provides the connectivity to the ALICE trigger and DAQ systems. 


\section{Chapter 4}

\section{Muon spectrometer}

Muon detection is performed in the pseudo-rapidity region $-4.0<\eta<-2.5$ by the muon spectrometer. With this detector, the complete spectrum of heavy-quark vector-mesons reasonances (i.e. $\mathrm{J} / \psi, \psi^{\prime}, \Upsilon, \Upsilon^{\prime}$ and $\Upsilon^{\prime \prime}$ ), as well as the $\phi$ meson, will be measured in the $\mu^{+} \mu^{-}$decay channel. The simultaneous measurement of all the quarkonia species with the same apparatus will allow a direct comparison of their production rate as a function of different parameters such as transverse momentum and collision centrality. In addition to vector mesons, the unlike-sign dimuon continuum up to masses around $10 \mathrm{GeV} / c^{2}$ will be measured. Since at LHC energies the continuum is expected to be dominated by muons from the semi-leptonic decay of open charm and open beauty, it will be possible to study the production of open (heavy) flavours with the muon spectrometer. Heavy-flavour production in the region $-2.5<\eta<-1$ will be accessible through measurement of $e-\mu$ coincidences, where the muon is detected by the muon spectrometer and the electron by the TRD.

The muon spectrometer will participate in the general ALICE data taking for $\mathrm{Pb}-\mathrm{Pb}$ collisions at luminosity $\mathscr{L}=10^{27} \mathrm{~cm}^{-2} \mathrm{~s}^{-1}$. The situation is different for intermediate-mass ion collisions (e.g. Ar-Ar). In this case, beside a general ALICE run at low luminosity, $\mathscr{L}=10^{27} \mathrm{~cm}^{-2} \mathrm{~s}^{-1}$, to match the TPC rate capability, a high-luminosity run at $\mathscr{L}=10^{29} \mathrm{~cm}^{-2} \mathrm{~s}^{-1}$ is also foreseen, to improve the $\Upsilon$ counting statistics. For the high-luminosity run, the muon spectrometer will take data together with a limited number of ALICE detectors (ZDC, SPD, PMD, T0, V0 and FMD) able to sustain the event rate. These detectors allow the determination of the collision centrality.

\subsection{Design considerations}

The main design criteria of the spectrometer were driven by the following considerations:

As the accuracy of dimuon measurements is statistics limited (at least for the $\Upsilon$ family), the spectrometer's geometrical acceptance was chosen as large as possible. In addition, a large acceptance down to zero $p_{\mathrm{t}}$ is required for measuring direct $\mathrm{J} / \psi$ production. At high $p_{\mathrm{t}}$ a large fraction of J/ $\psi$ 's is produced via b-decay [136]; based on Tevatron measurements [137] the contribution from b-decay to the total $\mathrm{J} / \Psi$ yield is $\approx 10 \%$ for $p_{\mathrm{t}}<3-4 \mathrm{GeV} / \mathrm{c}$ and then it increases linearly to $\approx 40 \%$ for $p_{\mathrm{t}}$ around $15-18 \mathrm{GeV} / c$. Since muon identification in the heavy-ion environment is only feasible for muon momenta above about $4 \mathrm{GeV} / \mathrm{c}$ because of the large amount of material 


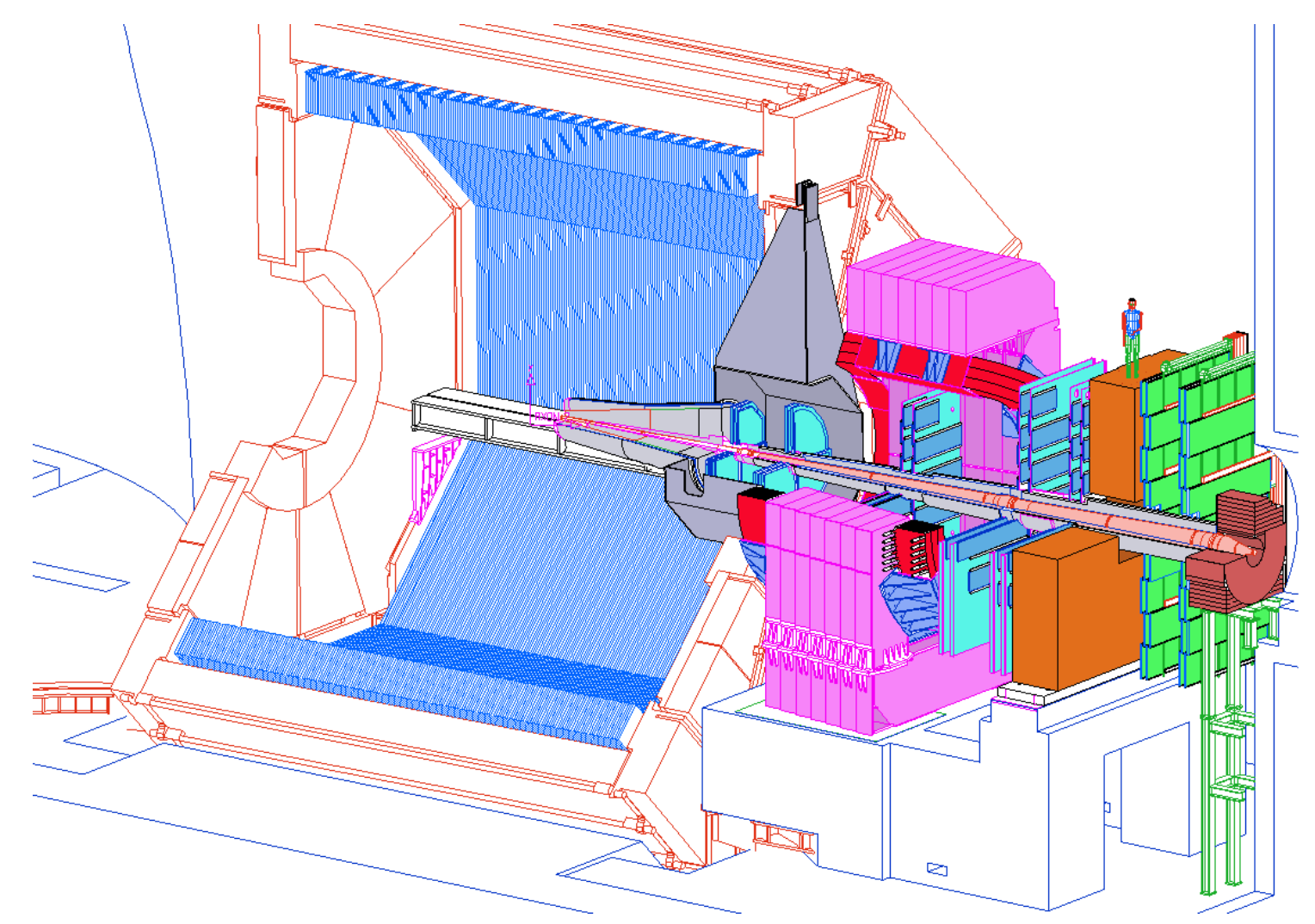

Figure 4.1: Layout of the muon spectrometer.

(absorber) required to reduce the flux of hadrons, measurement of low- $p_{\mathrm{t}}$ charmonia is possible only at small angles (i.e. at large rapidities) where muons are Lorentz boosted.

A resolution of $100 \mathrm{MeV} / c^{2}$ in the $10 \mathrm{GeV} / c^{2}$ dimuon invariant-mass region is needed to resolve the $\Upsilon, \Upsilon^{\prime}$ and $\Upsilon^{\prime \prime}$ resonances. This requirement determined the bending strength of the spectrometer magnet as well as the spatial resolution of the muon tracking system. In addition, multiple scattering is minimized by a careful optimization of the absorber and very thin detector planes.

The tracking and trigger detectors of the spectrometer have to cope with the high particle multiplicity expected in heavy-ion collision at LHC energies and have therefore a very high granularity read-out. The spectrometer is equipped with a selective dimuon trigger system to match the maximum trigger rate handled by the DAQ [138].

\subsection{Detector layout}

The muon spectrometer is designed to detect muons in the polar angular range $171^{\circ}-178^{\circ}$. This interval, a compromise between acceptance and detector cost, corresponds to the pseudo-rapidity range of $-4.0 \leq \eta \leq-2.5$.

The layout of the muon spectrometer is shown in figures 4.1 and 4.2. The spectrometer consists of the following components: a passive front absorber to absorb hadrons and photons from the interaction vertex; a high-granularity tracking system of 10 detection planes; a large dipole 


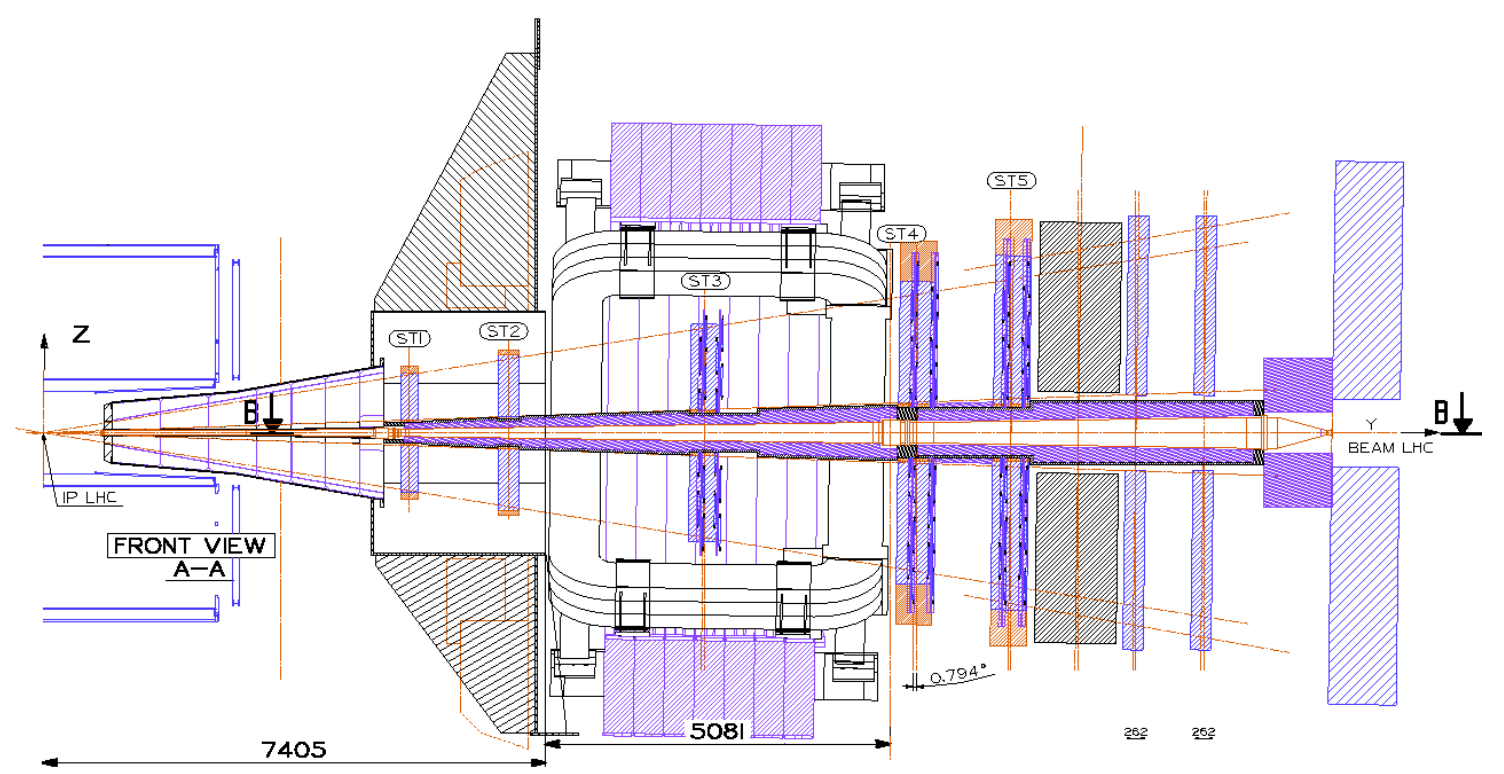

Figure 4.2: Muon spectrometer longitudinal section; according to the adopted numbering scheme station 1 (ST1) is the closest to the central barrel.

magnet; a passive muon-filter wall, followed by four planes of trigger chambers; an inner beam shield to protect the chambers from primary and secondary particles produced at large rapidities.

The main challenge for the muon spectrometer results from the high particle multiplicity per event rather than from the event rate, which is modest. Great care was taken both in the design of the absorbers (which have to provide strong absorption of the hadron flux coming from the interaction vertex) and of the detectors (which must be able to sustain the remaining high multiplicity). To optimize the spectrometer layout, simulations with FLUKA [139], C95 [140] and GEANT3 [141] were carried out. The particle yields predicted by the HIJING [142] event generator and multiplied by an extra (safety) factor of two were used as input. The main parameters of the muon spectrometer are summarised in table 4.1 and details on the dipole magnet are given in section 2.3.2 It is important to note that the muon spectrometer relies on the V0 detector as a fast interaction trigger to make the system more robust against background from beam-gas interactions in particular during the proton-proton run at nominal beam intensity [143]. A High-Level Trigger (HLT) for dimuons will reduce, by a factor four to five, the need in bandwidth and data storage.

\subsection{Absorbers}

The front absorber, whose length is $4.13 \mathrm{~m}\left(\sim 10 \lambda_{\text {int }}, \sim 60 X_{0}\right)$, is located inside the solenoid magnet. The fiducial volume of the absorber is made predominantly out of carbon and concrete to limit small-angle scattering and energy loss by traversing muons. At the same time, the front absorber is designed to protect other ALICE detectors from secondaries produced within the absorbing material itself [144]. The spectrometer is shielded throughout its length by a dense absorber tube surrounding the beam pipe. The tube (beam shield) is made of tungsten, lead and stainless steel. It has a conical geometry to reduce background particle interaction along the length of the spectrometer. While the front absorber and the beam shield are sufficient to protect the tracking chambers, 
Table 4.1: Summary of the main characteristics of the muon spectrometer.

\begin{tabular}{|c|c|}
\hline Muon detection & \\
\hline Polar, azimuthal angle coverage & $171^{\circ} \leq \theta \leq 178^{\circ}, 360^{\circ}$ \\
\hline Minimum muon momentum & $4 \mathrm{GeV} / \mathrm{c}$ \\
\hline Pseudo-rapidity coverage & $-4.0<\eta<-2.5$ \\
\hline Front absorber & \\
\hline Longitudinal position (from IP) & $-5030 \mathrm{~mm} \leq z \leq-900 \mathrm{~mm}$ \\
\hline Total thickness (materials) & $\left(\sim 10 \lambda_{\text {int }}, \sim 60 X_{0}\right)$ (carbon-concrete-steel) \\
\hline Dipole magnet & \\
\hline Nominal magnetic field, field integral & $0.67 \mathrm{~T}, 3 \mathrm{Tm}$ \\
\hline Free gap between poles & $2.972-3.956 \mathrm{~m}$ \\
\hline Overall magnet length & $4.97 \mathrm{~m}$ \\
\hline Longitudinal position (from IP) & $-z=9.94 \mathrm{~m}$ (centre of the dipole coils) \\
\hline Tracking chambers & \\
\hline No. of stations, no. of planes per station & 5,2 \\
\hline Longitudinal position of stations & $-z=5357,6860,9830,12920,14221 \mathrm{~mm}$ \\
\hline Anode-cathode gap (equal to wire pitch) & $2.1 \mathrm{~mm}$ for st. $1 ; 2.5 \mathrm{~mm}$ for st. $2-5$ \\
\hline Gas mixture & $80 \% \mathrm{Ar} / 20 \% \mathrm{CO}_{2}$ \\
\hline Pad size st. 1 (bending plane) & $4.2 \times 6.3,4.2 \times 12.6,4.2 \times 25.2 \mathrm{~mm}^{2}$ \\
\hline Pad size st. 2 (bending plane) & $5 \times 7.5,5 \times 15,5 \times 30 \mathrm{~mm}^{2}$ \\
\hline Pad size st. 3, 4 and 5 (bending plane) & $5 \times 25,5 \times 50,5 \times 100 \mathrm{~mm}^{2}$ \\
\hline Max. hit dens. st. $1-5$ (central $\mathrm{Pb}-\mathrm{Pb} \times 2$ ) & $5.0,2.1,0.7,0.5,0.6 \cdot 10^{-2}$ hits $/ \mathrm{cm}^{2}$ \\
\hline Spatial resolution (bending plane) & $\simeq 70 \mu \mathrm{m}$ \\
\hline Tracking electronics & \\
\hline Total no. of FEE channels & $1.08 \times 10^{6}$ \\
\hline Shaping amplifier peaking time & $1.2 \mu \mathrm{s}$ \\
\hline Trigger chambers & \\
\hline No. of stations, no. of planes per station & 2,2 \\
\hline Longitudinal position of stations & $-z=16120,17120 \mathrm{~mm}$ \\
\hline Total no. of RPCs, total active surface & $72, \sim 140 \mathrm{~m}^{2}$ \\
\hline Gas gap & single, $2 \mathrm{~mm}$ \\
\hline Electrode material and resistivity & Bakelite $^{\mathrm{TM}}, \rho=2-8 \times 10^{9} \Omega \mathrm{cm}$ \\
\hline Gas mixture & $\mathrm{Ar} / \mathrm{C}_{2} \mathrm{H}_{2} \mathrm{~F}_{4} / \mathrm{i}$-buthane $/ \mathrm{SF}_{6}(50.5 / 41.3 / 7.2 / 1)$ \\
\hline Pitch of readout strips (bending plane) & 10.6, 21.2, $42.5 \mathrm{~mm}$ (for trigger st. 1 ) \\
\hline Max. strip occupancy bend. (non bend.) plane & $3 \%(10 \%)$ in central $\mathrm{Pb}-\mathrm{Pb}$ \\
\hline Maximum hit rate on RPCs & $3(40) \mathrm{Hz} / \mathrm{cm}^{2}$ in $\mathrm{Pb}-\mathrm{Pb}(\mathrm{Ar}-\mathrm{Ar})$ \\
\hline Trigger electronics & \\
\hline Total no. of FEE channels & $2.1 \times 10^{4}$ \\
\hline No. of local trigger cards & $234+8$ \\
\hline
\end{tabular}


additional protection is needed for the trigger chambers. For this reason the muon filter, i.e. an iron wall $1.2 \mathrm{~m}$ thick $\left(\sim 7.2 \lambda_{\text {int }}\right)$, is placed after the last tracking chamber, in front of the first trigger chamber. The front absorber and muon filter stop muons with momentum less than $4 \mathrm{GeV} / c$.

\subsection{Tracking system}

Tracking chambers. The tracking chambers were designed to achieve a spatial resolution of about $100 \mu \mathrm{m}$, necessary for an invariant-mass resolution of the order of $100 \mathrm{MeV} / \mathrm{c}^{2}$ at the $\Upsilon$ mass $[145,146]$. In addition they can operate at the maximum hit density of about $5 \times 10^{-2} \mathrm{~cm}^{-2}$ expected in central $\mathrm{Pb}-\mathrm{Pb}$ collisions, where a few hundred particles are expected to hit the muon chambers. The tracking system covers a total area of about $100 \mathrm{~m}^{2}$. All these requirements were fulfilled by the use of cathode pad chambers. They are arranged in five stations: two are placed before, one inside and two after the dipole magnet. Each station is made of two chamber planes. Each chamber has two cathode planes, which are both read out to provide two-dimensional hit information. ${ }^{1}$ The first station is located right behind the absorber to measure the exit points of the muons as precisely as possible. To keep the occupancy at about $5 \%$, a fine-granularity segmentation of the readout pads is needed. For instance, pads as small as $4.2 \times 6.3 \mathrm{~mm}^{2}$ were used for the region of the first station close to the beam pipe, where the highest multiplicity is expected. Since the hit density decreases with the distance from the beam, larger pads are used at larger radii, keeping the total number of channels at about one million.

Multiple scattering of the muons in the chambers is minimized by using composite materials (e.g. carbon fibre). The chamber thickness corresponds to about $0.03 X_{0}$. Because of the different size of the stations, (ranging from few square metres for station 1 to more than $30 \mathrm{~m}^{2}$ for station 5) two different designs were adopted. The first two stations are based on a quadrant structure [149], with the readout electronics distributed on their surface (see figure 4.3). For the other stations, a slat architecture, was chosen (see figure 4.4). The maximum size of the slat is $40 \times 280 \mathrm{~cm}^{2}$ and the electronics is implemented on the side of the slats. The slats and the quadrants overlap to avoid dead zones on the detector.

Extensive tests on several prototypes and on pre-production tracking chambers have shown that these detectors achieve the required performances [150, 151]. In particular, space resolutions of the order of $70 \mu \mathrm{m}$ were measured.

Geometry monitoring system. The alignment of the spectrometer tracking chambers is crucial in order to achieve the required invariant-mass resolution of $100 \mathrm{MeV} / c^{2}$ at the $\Upsilon$ mass. Dedicated runs without magnetic field will be carried out at the beginning of each data taking period in order to align the ten tracking chambers with straight muon tracks, thus determining the initial geometry of the system. The displacements and deformations of the tracking chambers with respect to the initial geometry (due to different reasons, including switching on the magnetic field) will be measured and recorded during data taking by the Geometry Monitoring System (GMS). The requirement is to monitor the position of all the tracking chambers with a resolution better than $40 \mu \mathrm{m}$.

\footnotetext{
${ }^{1}$ Additional information about the clusterizer and tracking algorithms of the tracking system can be found in references $[147,148]$
} 


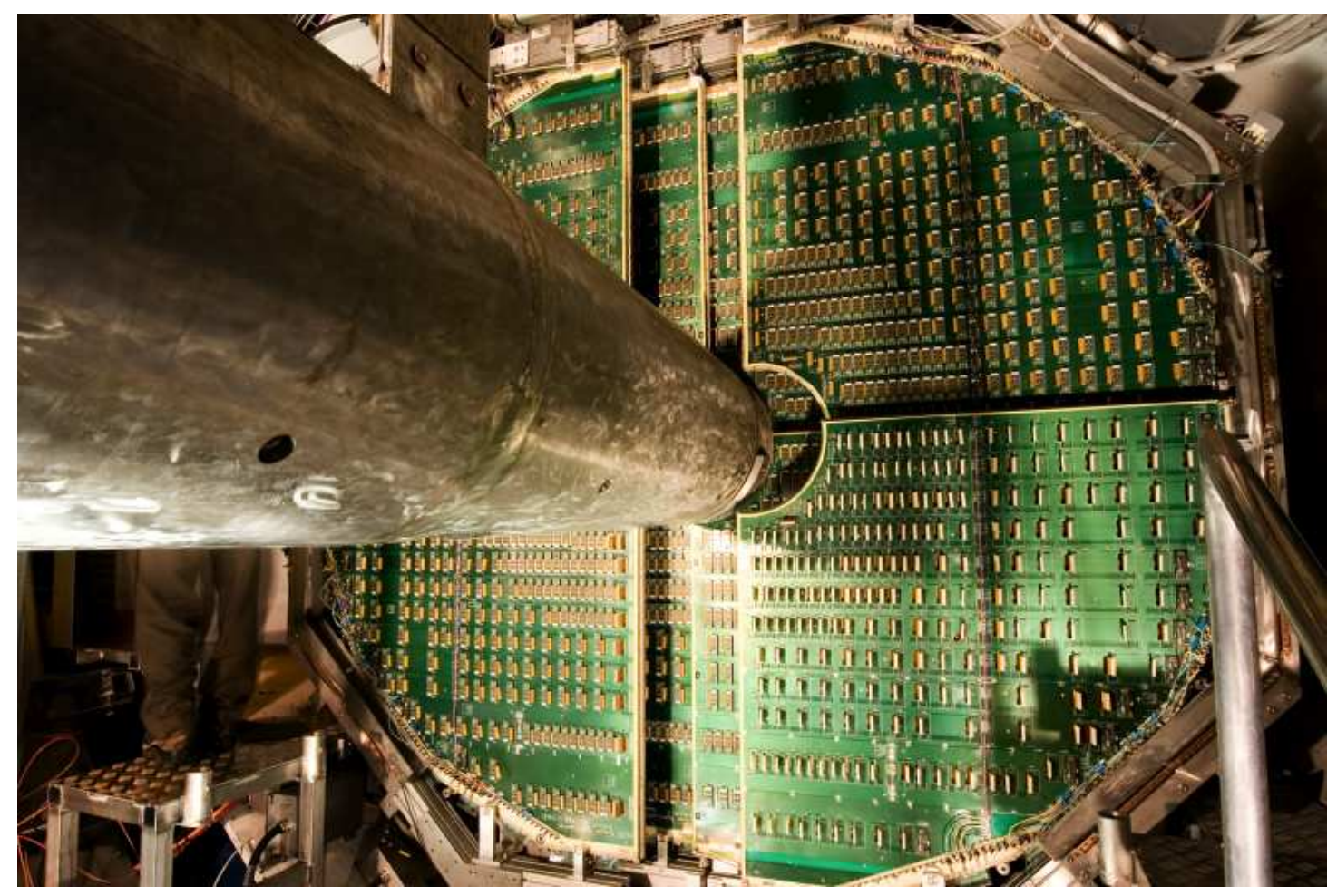

Figure 4.3: Layout of station 2 of tracking system. Readout electronics is distributed on the surface of a quadrant structure.

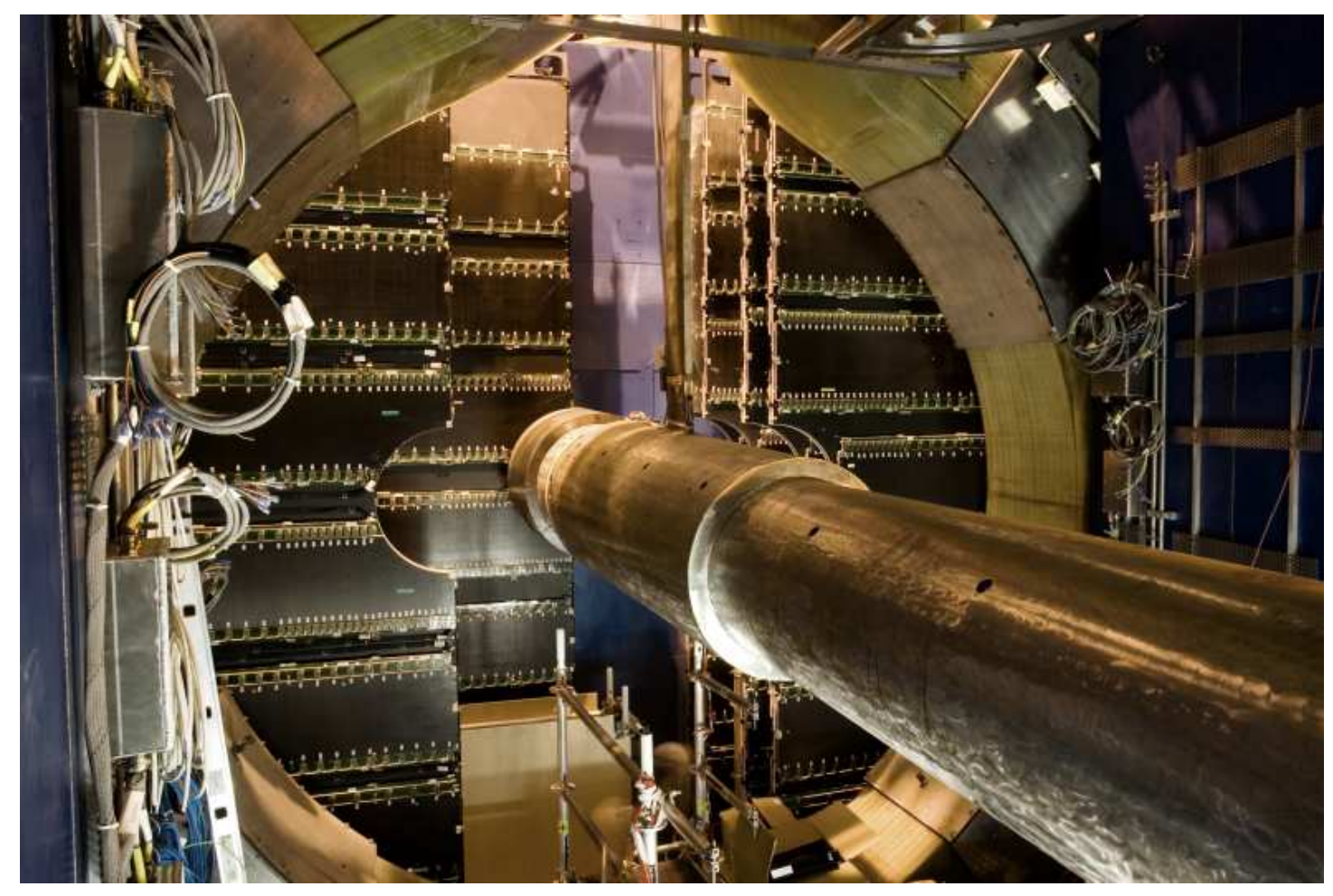

Figure 4.4: Layout of stations 4 and 5 of tracking system. 
The GMS is an array of 460 optical sensors which are installed on platforms placed at each corner of the tracking chambers. Two different types of optical devices were adopted: the BCAM and the Proximity [152]. A detailed description of these devices can be found in [153]. In both cases, the image of an object is projected on a CCD sensor through a lens. The analysis of the recorded image provides a measurement of the displacement. The most relevant difference between the two systems is represented by the object (i.e. the light source) used. For the BCAM, which is a long-range system, the object is a pair of point-like LEDs, while it is a coded mask for the Proximity, which is used for small distances.

The GMS consists of three parts. The first one is the Longitudinal Monitoring System (LMS). Its role is to monitor the relative positions between the chamber planes. It is composed of BCAM lines linking two neighbouring stations, and Proximity lines linking the two chambers of each station. The second part is the Transverse Monitoring System (TMS). It monitors the flatness of the chamber support planes and is made of BCAM optical lines. Finally, the last part of the system is represented by the External Monitoring System (EMS). It is composed of eight BCAM lines linking the last chamber to the cavern walls to monitor the displacement of the whole spectrometer.

A complete simulation of the full system, including environment effects, was performed to evaluate the overall GMS performance [154]. The performance of a part of the system was studied in a laboratory test, with a full-scale mock-up of three half-planes of chambers 6,7 and 8 . The test demonstrated that the GMS is able to measure transverse displacements from the initial position with an accuracy of $7 \mu \mathrm{m}$ [155]. In this test, the effects of thermal gradients in the space between the chambers were investigated as well. When gradients similar to the ones expected in ALICE are generated, the measured resolution on transverse displacement turns out to be $23 \mu \mathrm{m}$. Such a value is well within specifications, including some safety margin.

Tracking electronics. The design of the electronics of the tracking system was driven by two main requirements: to read about one million channels up to a rate of the order of $\mathrm{kHz}$ and to achieve a space resolution of the tracking chambers of at least $100 \mu \mathrm{m}$. The electronics chain is divided in three parts: the front-end boards, called MANU (MAnas NUmérique), the readout system, called CROCUS (Cluster Read Out Concentrator Unit System) and the interface with the general ALICE trigger, called TCI (Trigger Crocus Interface) [156].

Great care was taken in the design of the front-end electronics and boards to achieve a noise as low as $\sim 1000 e$, keeping at the same time the material budget as low as possible to minimize multiple scattering [12]. The front-end electronics is based, for all the tracking stations, on a 16channel chip called MANAS (Multiplexed ANALogic Signal processor) including the following functionalities: charge amplifier, filter, shaper, and track \& hold. The digitization of the signal is done on board. The channels of four of these chips are fed into a 12-bit ADCs, read out by the Muon Arm Readout Chip (MARC). This allows communication with the readout and performs zero suppression. This chain is mounted on front-end boards (MANUs). About 17000 MANU cards are necessary to treat the 1.08 millions channels of the tracking system. Since the gain of each channel has to be precisely known to achieve the required space resolution, the parameters of each channel are checked just after the assembly of the boards. They will be controlled in periodic calibration runs during the data taking and stored for use in offline track reconstruction. 
The PATCH (Protocol for ALICE Tracking Chambers) buses provide the connection between the MANUs and the CROCUS crate. Each chamber is read out by two CROCUS, leading to a total number of 20 CROCUS. During the acquisition phase, the main tasks of the CROCUS are to concentrate and to format the data from the chambers, to transfer them to the DAQ and to dispatch the trigger signals. These crates also allow the control of the FEE and of the calibration processes. All these tasks are carried out in the CROCUS through a DSP and FPGA farm. Each CROCUS crate houses five frontal (FRT) data readout boards, which perform the first data concentration stage. Each FRT drives up to 10 PATCH buses and collects the data sent by the MANUs. The data from the FRTs are transferred to the data concentrator (CRT) board where they are encapsulated and then sent to the DAQ. So, a CROCUS is able to read up to 50 PATCH buses with a chamber occupancy up to $5 \%$ and with rates of the order of $\mathrm{kHz}$.

The trigger signals, coming from the Central Trigger Processor (CTP), are distributed to the FEE through the CROCUS by the TCI. The main goals of the TCI are to decode the trigger signal, to generate the L1 reject in the FFT board (Frontal Fan-out Trigger) and to manage the busy signals of all the CROCUS crates. All these signals are sent to the twenty CROCUS via five FTD (Frontal Trigger Dispatching) cards.

All the components of the muon tracking electronics were intensively tested in laboratory, with beams and with cosmic rays [157-159]. In addition, radiation tests were successfully carried out. The FEE was tested with a proton beam [160] at a radiation level exceeding that for 10 years of ALICE running time by a factor of two. The CROCUS was tested using a neutron source. This test has shown that the number of SEU (Single Event Upset) in the worst case (first tracking station) will not exceed one SEU every two hours of data taking [161]. These performances fully satisfy the requirements of ALICE.

\subsection{Trigger system}

General considerations. In central $\mathrm{Pb}-\mathrm{Pb}$ collisions, about eight low- $p_{\mathrm{t}}$ muons from $\pi$ and $\mathrm{K}$ decays are expected to be detected per event in the spectrometer. To reduce to an acceptable level the probability of triggering on events where these low- $p_{\mathrm{t}}$ muons are not accompanied by the high$p_{\mathrm{t}}$ ones emitted in the decay of heavy quarkonia (or in the semi-leptonic decay of open charm and beauty), a $p_{\mathrm{t}}$ cut has to be applied at the trigger level on each individual muon [162]. Two programmable cuts (low- $p_{\mathrm{t}}$ and high- $p_{\mathrm{t}}$ cuts), are performed in parallel by the trigger electronics [163]. The values of the $p_{\mathrm{t}}$ thresholds can range from $\sim 0.5$ to $\sim 2 \mathrm{GeV} / c$. The following six trigger signals are delivered to the ALICE Central Trigger Processor (CTP), less than $800 \mathrm{~ns}$ after interaction, at $40 \mathrm{MHz}$ frequency: at least one single muon track above low- (high-) $p_{\mathrm{t}}$ cut; at least two unlike-sign muon tracks, each of them above low- (high-) $p_{\mathrm{t}}$ cut; at least two like-sign muon tracks, each of them above low- (high-) $p_{\mathrm{t}}$ cut.

The threshold for the low- $p_{\mathrm{t}}$ and high- $p_{\mathrm{t}}$ cuts represents a compromise between background rejection and signal detection efficiency in the mass regions of the $J / \psi$ and $\Upsilon$ resonances, respectively. 

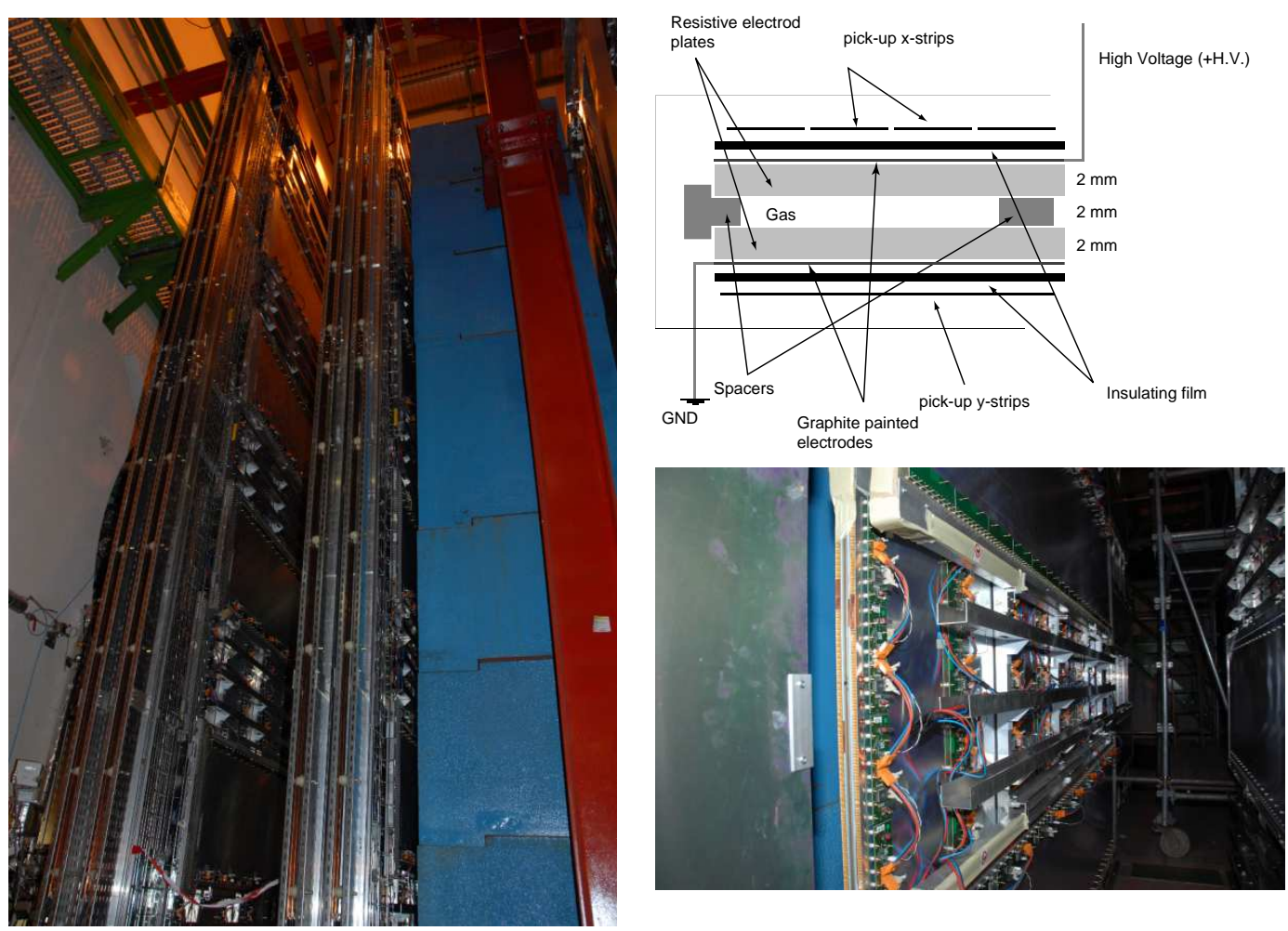

Figure 4.5: Left: view of the two trigger stations sitting behind the muon filter. Right-top: schematic view of an RPC cross section. Right-bottom: an individual RPC module equipped with the Front-End Electronics.

Trigger detector. To perform the $p_{\mathrm{t}}$ selection, a position-sensitive trigger detector with space resolution better than $1 \mathrm{~cm}$ is required. This resolution is achieved by Resistive Plate Chambers (RPCs) operated in streamer mode [164]. The trigger system consists of four RPC planes arranged in two stations, one metre apart from each other, placed behind the muon filter. Each plane consists of $18 \mathrm{RPC}$ modules and the typical size of a module is about $70 \times 300 \mathrm{~cm}^{2}$, corresponding to a total active area of about $140 \mathrm{~m}^{2}$ [165]. The RPC electrodes are made of low-resistivity Bakelite $\left(\rho \sim 3 \times 10^{9} \Omega \mathrm{cm}\right.$ ), to attain the needed rate capability (maximum expected value about $40 \mathrm{~Hz} / \mathrm{cm}^{2}$ for Ar-Ar high-luminosity runs). To improve the smoothness of the electrode surface, these are coated with linseed oil. The $(x, y)$ coordinates of the RPC hits are read out by segmented strips with pitch and length increasing with their distance from the beam axis.

Extensive tests were carried out to study the long-term behaviour of small-sized RPC prototypes operated in streamer mode. It was shown that RPCs are able to tolerate several ALICE years of data taking with heavy-ion beams [166]. It is worth noting that the detectors will take data for different colliding systems, resulting in a wide range of working conditions and requirements, in particular concerning position resolution and detector lifetime. The possibility of working in avalanche mode with the same front-end electronics was investigated in several beam and ageing tests [167] showing that this mode of operation is well suited for pp runs, where the requirements on the detector lifetime are more severe than in heavy-ion runs.

An overall view of the two trigger stations mounted at their final location is shown in figure 4.5. In the same figure an individual RPC module is shown as well. 
Trigger electronics. The RPCs are equipped with dual-threshold front-end discriminators [168] (ADULT), which are adapted to the timing properties of the detector and reach the necessary time resolution (1-2 ns) for the identification of the bunch crossing. From the discriminators, the signals are sent to the trigger electronics based on programmable circuits working in pipeline mode at $40 \mathrm{MHz}$. The trigger electronics is organized in 3 levels: local, regional and global. The local trigger algorithm of each local board searches for a single track pointing approximately back to the primary interaction vertex, using the information of the four RPC detector planes. Hits in at least 3 detector planes out of 4 are required to define a track, both in the bending and non-bending plane. The non-bending plane algorithm is very effective for background rejection. In the bending plane, the track deviation relative to a particle with infinite momentum is computed. Subsequent cuts on this deviation, performed by means of on-board look-up tables, allow delivering the low- and high$p_{\mathrm{t}}$ trigger signals on single muons. Finally, the regional and the global levels gather the signals of all local boards and deliver the single muon, like-sign and unlike-sign dimuon triggers of the whole detector. The initial information from the detectors as well as the information at various stages of the local, regional and global algorithms are stored for readout in the corresponding boards. 


\section{Chapter 5}

\section{Forward detectors}

\subsection{Zero Degree Calorimeter (ZDC)}

\subsubsection{Introduction}

The number of participant nucleons is the observable most directly related to the geometry of A-A collisions. It can be estimated by measuring the energy carried in the forward direction (at $0^{\circ}$ relative to the beam direction) by non-interacting (spectator) nucleons. In ALICE, spectator nucleons are detected by means of Zero-Degree Calorimeters (ZDC). If all the spectators are detected, the number of participants is given by:

$$
\begin{aligned}
E_{\mathrm{ZDC}}(\mathrm{TeV}) & =2.76 \times N_{\text {spectators }} \\
N_{\text {participants }} & =A-N_{\text {spectators }}
\end{aligned}
$$

where $2.76 \mathrm{TeV}$ is the energy per nucleon of the $\mathrm{Pb}$ beam at the LHC. However, such a simple estimate is no longer valid at a collider since not all the spectator nucleons can be detected (see section 5.1.2.2). The centrality information provided by the ZDC is also used for triggering at Level 1 (L1). Finally, the ZDC being also a position-sensitive detector, can give an estimate of the reaction plane in nuclear collisions.

\subsubsection{Detector layout}

In ALICE two sets of hadronic ZDCs are located at $116 \mathrm{~m}$ on either side of the Interaction Point (IP). In addition, two small electromagnetic calorimeters (ZEM) are placed at about $7 \mathrm{~m}$ from the IP, on both sides of the LHC beam pipe, opposite to the muon arm (see figure 5.1).

Spectator protons are spatially separated from neutrons by the magnetic elements of the LHC beam line. Therefore, each ZDC set is made by two distinct detectors: one for spectator neutrons $(\mathrm{ZN})$, placed between the beam pipes at $0^{\circ}$ relative to the $\mathrm{LHC}$ axis, and one for spectator protons (ZP), placed externally to the outgoing beam pipe on the side where positive particles are deflected. The ZN and ZP are installed on lifting platforms, in order to lower them out of the horizontal beam plane (where the radiation levels are highest) when not in use (see figure 5.2). 


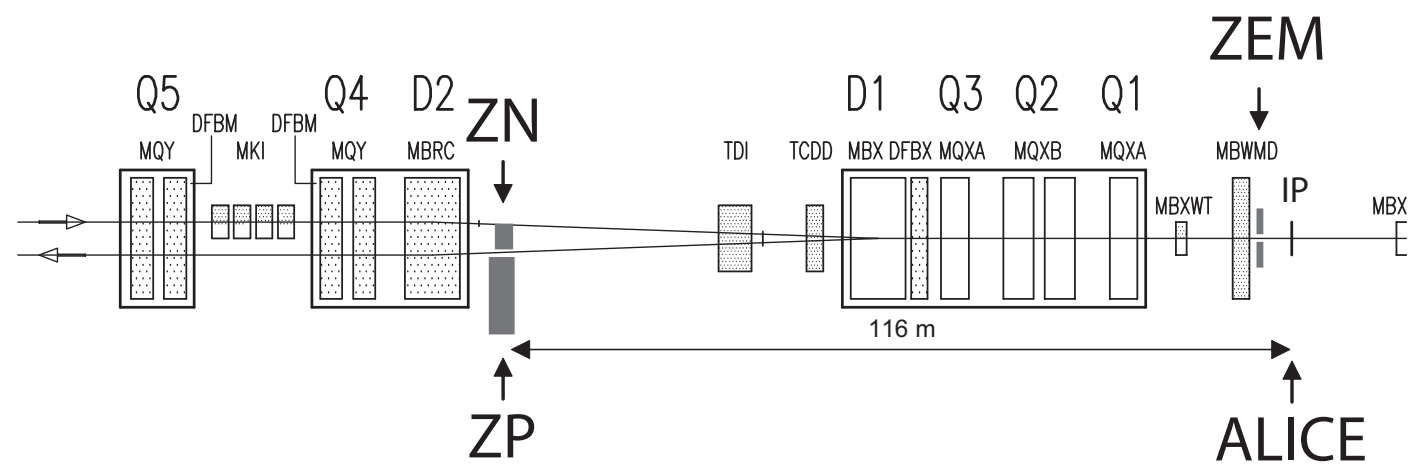

Figure 5.1: Schematic top view of the side of the ALICE beam line opposite to the muon arm. The locations of the neutron (ZN), proton (ZP) and forward electromagnetic (ZEM) calorimeters are shown. The position of the beam line dipoles (Dx) and quadrupoles (Qx) are also indicated.

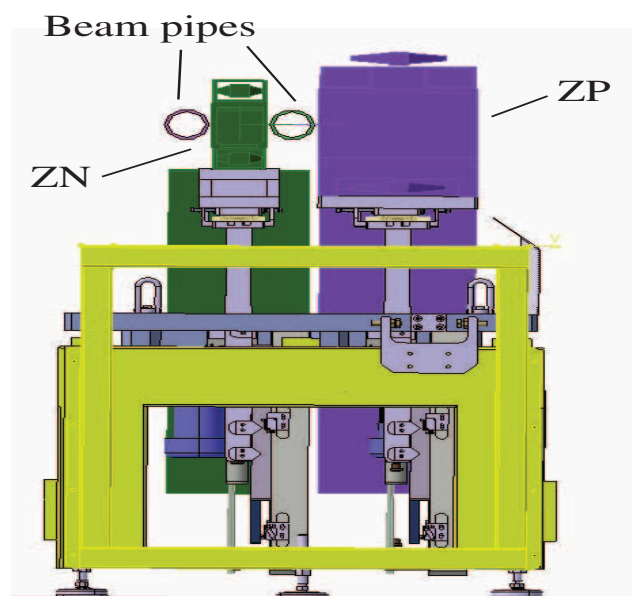

Figure 5.2: Front view of one ZDC set placed on the lifting platform in data-taking position.

\subsubsection{Hadron calorimeters}

The hadronic ZDCs are quartz fibres sampling calorimeters [169]; the shower generated by incident particles in a dense absorber (the so-called 'passive' material) produces Cherenkov radiation in quartz fibres ('active' material) interspersed in the absorber.

Due to the small amount of available space (particularly for the neutron calorimeter, whose transverse size can not be larger than $7 \mathrm{~cm}$ ), the detectors are very compact. For this reason a very dense $\mathrm{W}$-alloy was used as passive material for $\mathrm{ZN}$, to maximise the containment of the showers. For the proton calorimeter there are no such stringent space constraints; moreover, the spectator proton's spot has a wide spatial distribution $[10,170]$. Therefore, a larger detector made out of brass was constructed [171].

The passive material is a stack of metallic plates, grooved along the beam direction; quartz fibres are placed inside the grooves. The fibre spacing is smaller than the radiation length of the absorber, in order to avoid electron absorption in the passive material leading to non-uniformity 


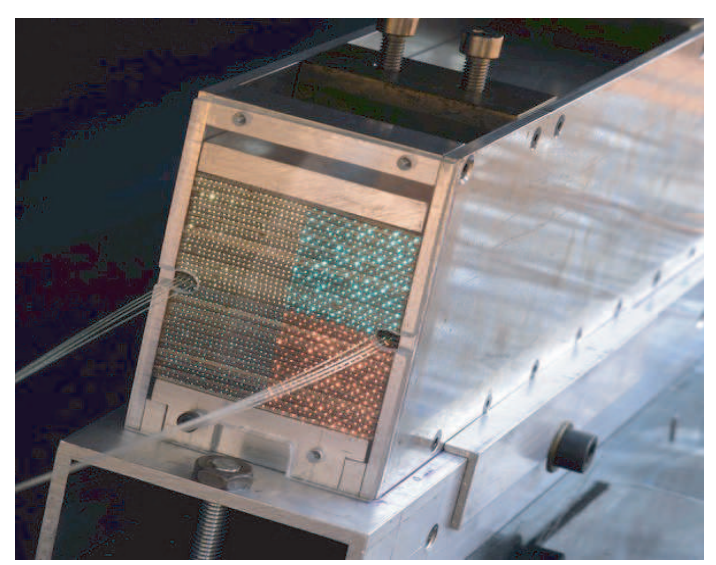

Figure 5.3: Front face of the $\mathrm{ZN}$ calorimeter; the quartz fibres connecting the monitoring laser system to PMTs are visible.

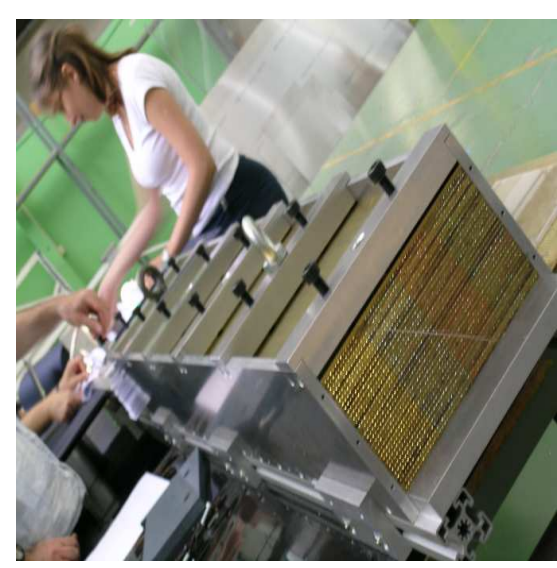

Figure 5.4: Front face of the ZP calorimeter.

Table 5.1: Dimensions and main characteristics of the detectors.

\begin{tabular}{|l|c|c|c|}
\hline & ZN & ZP & ZEM \\
\hline \hline Dimensions $\left(\mathrm{cm}^{3}\right)$ & $7.04 \times 7.04 \times 100$ & $12 \times 22.4 \times 150$ & $7 \times 7 \times 20.4$ \\
Absorber & tungsten alloy & brass & lead \\
$\rho_{\text {absorber }}\left(\mathrm{g} \mathrm{cm}^{-3}\right)$ & 17.6 & 8.5 & 11.3 \\
Fibre core diameter $(\mu \mathrm{m})$ & 365 & 550 & 550 \\
Fibre spacing $(\mathrm{mm})$ & 1.6 & 4 & not applicable \\
Filling ratio & $1 / 22$ & $1 / 65$ & $1 / 11$ \\
Length (in $X_{0}$ units) & 251 & 100 & 35.4 \\
Length (in $\lambda_{\mathrm{I}}$ units) & 8.7 & 8.2 & 1.1 \\
Number of PMTs & 5 & 5 & 1 \\
\hline
\end{tabular}

in the response. The optical read-out is divided into four independent towers. One out of every two fibres is sent to a single PMT, while the remaining fibres are sent to four PMTs, each one collecting the light from a single tower. The fibres emerge from the rear face of the calorimeter, are gathered together in bundles, and routed toward the PMTs, which are located $50 \mathrm{~cm}$ from the end of the calorimeters, out of the beam horizontal plane. The front faces of the $\mathrm{ZN}$ and $\mathrm{ZP}$ can be seen in figure 5.3 and 5.4, respectively. Table 5.1 summarizes the main characteristics of the detectors (further details can be found in [10]).

The ZDCs will operate in a harsh radiation environment, up to $10^{4} \mathrm{~Gy} /$ day at a luminosity of $10^{27} \mathrm{~cm}^{-2} \mathrm{~s}^{-1}$. For this reason, quartz fibres were chosen, due to their intrinsic radiation hardness $[172,173]$. In particular, we have used fibres with a pure silica core, silica fluorinated cladding and a hard polymer coat. Their numerical aperture is 0.22 .

Cherenkov calorimeters are known to provide a very fast signal, due to the intrinsic velocity of the emission process, and have very low sensitivity to induced radioactivity thanks to their threshold behaviour. 
Therefore, the limiting factors for their rate capability are the time response of the light detectors and the cable length. Beam tests with the $\mathrm{ZN}$ have shown that the FWHM of the detector signal, at the output of a Hamamatsu R329-02 photomultiplier tube (PMT), is of the order of $5 \mathrm{~ns}$. After $200 \mathrm{~m}$ of low-loss cable, we estimate a FWHM of less than $20 \mathrm{~ns}$. Given the expected collision frequency of $8 \mathrm{kHz}$ for $\mathrm{Pb}-\mathrm{Pb}$ hadronic collisions, or even the much higher rate of the electromagnetic dissociation process (predicted to be of the order of $0.1 \mathrm{MHz}$ ), we do not foresee any pile-up in the ZDCs.

The physics performance of the detector is related to the resolution on the number of spectator nucleons hitting the calorimeters, which in turn depends on the energy resolution of the ZDCs. Tests of the ZN were performed with a hadron beam at the SPS, at energies between 50 and $150 \mathrm{GeV}$. The calorimeter resolution, as a function of energy, is given by $(\sigma / E)^{2}=(2.57 \pm$ $0.03 / \sqrt{E(\mathrm{GeV})})^{2}+(0.103 \pm 0.006)^{2}[174]$. Extrapolating this result to LHC energy $(2.76 \mathrm{TeV})$ we get an expected resolution of $\sim 11.4 \%$. For $\mathrm{ZP}$, beam tests provided a resolution given by $(\sigma / E)^{2}=(2.37 \pm 0.02 / \sqrt{E(\mathrm{GeV})})^{2}+(0.125 \pm 0.002)^{2}$, leading to $\sigma \sim 13 \%$ at LHC energy [175]. All these results are in fair agreement with detailed simulations performed within the AliRoot offline framework, see chapter 6.4.

Thanks to its segmentation, the $\mathrm{ZN}$ measures the position of the centroid of the incoming spectator neutrons. The resolution on the impact point of a $100 \mathrm{GeV}$ hadron hitting the front face of the calorimeter is $\sim 3 \mathrm{~mm}$ [176]. A similar resolution is expected at LHC energy. When the number of spectator nucleons increases, a significant improvement is expected.

Finally, the expected resolution on the measurement of the $\mathrm{Pb}-\mathrm{Pb}$ collision impact parameter, based on the aforementioned simulation, is of the order of $1 \mathrm{fm}$ [177].

\subsubsection{Electromagnetic calorimeter}

In very peripheral A-A collisions, a significant number of spectator nucleons is bound into fragments having a charge-to-mass ratio similar to the one of $\mathrm{Pb}$. These fragments stay in the beam pipes and therefore cannot be detected by ZDCs. As a consequence, we can have a small amount of energy in the ZDCs for central events, where the number of spectators is small, but also for very peripheral events.

To distinguish the two classes of events, two electromagnetic calorimeters (ZEM) complement the hadronic ZDCs. They measure, event by event, the energy of particles emitted at forward rapidity (mainly photons generated from $\pi^{0}$ decays). Since this energy increases monotonically with the collision centrality, its value can help discriminating between central and peripheral collisions.

The detection technique is similar to the one used for the hadronic calorimeters: the absorber is made of 40 lead plates, each one $3 \mathrm{~mm}$ thick, with quartz fibres sandwiched in layers between the plates. The fibre cores have a diameter of $550 \mu \mathrm{m}$ and the active to passive volume ratio is about $1 / 11$. The ZEM fibres and plates are oriented at $45^{\circ}$ with respect to the LHC axis. This choice maximises the detector response, because Cherenkov light production has a pronounced peak around $45^{\circ}$ [172]. An air light guide positioned on top of the detector collects the light from the fibres up to a photomultiplier. 
The calorimeter dimensions are $7 \times 7 \times 20.4 \mathrm{~cm}^{3}$, and the total absorber length amounts to $35.4 X_{0}$. In this way showers generated by impinging particles are fully contained [178] in the calorimeter which covers the pseudo-rapidity range $4.8<\eta<5.7$.

The energy resolution of the ZEM was estimated with simulations using the HIJING [142] event generator. The results show that in central collisions $(b<2 \mathrm{fm})$ the total incident energy on the two electromagnetic calorimeters is about $7 \mathrm{TeV}$, while in peripheral interactions $(b \sim 10 \mathrm{fm})$ it is of the order of $1.5 \mathrm{TeV}$. A resolution:

$$
\frac{\sigma}{E}=\frac{0.69}{\sqrt{E(\mathrm{GeV})}}
$$

has been obtained with a detector prototype tested at SPS energies. Extrapolating this resolution to the LHC conditions, we expect an energy resolution $<1 \%$ for central collisions, increasing up to $1.8 \%$ for peripheral events. Central events can be selected by requiring a small amount of energy in the hadronic calorimeters and a significant energy deposition in the ZEMs.

\subsubsection{Signal transmission and readout}

The analogue signals from the calorimeter's PMTs are transmitted through $215 \mathrm{~m}$ long low-loss coaxial cables to the closest counting room. They are then fanned out, sending an output to the trigger logic and another one to the readout chain. For the trigger, signals from the ZDCs and the ZEMs are summed separately and then discriminated. An appropriate combination of these signals will provide three ALICE L1 triggers, defining different centrality intervals (central events, semi-central events, and minimum bias events). For the readout, each analogue signal from the photomultipliers will be sent to commercial ADC modules hosted in a VME crate. When a Level 0 (L0) trigger is received, the ZDC readout electronics will start to convert the signals and make them available for the ALICE DAQ if a L1 trigger is received.

\subsubsection{Monitoring and calibration}

Two monitoring procedures are implemented for the ZDCs. The first is based on the detection of cosmic rays crossing the absorber material, by means of scintillators placed above and below the calorimeters. These events induce a very small amount of Cherenkov light, that may give a single photoelectron signal in the PMTs. With appropriate statistics, it is possible to measure the single photoelectron peak, directly related to the PMT gain.

The second procedure is based on a pulsed laser source. A small bunch of fibres sends the laser light to a subsample of the fibres inside the calorimeter. The peak of the resulting amplitude distribution from the calorimeters' PMTs depends on the PMT gain and on the light transmission efficiency of the fibres. Another fibre brings the light directly to a reference PMT, monitoring the stability of the laser. With these two procedures it is possible to monitor both the PMT gain and the light transmission efficiency in the fibres, which is expected to deteriorate with the radiation dose. 


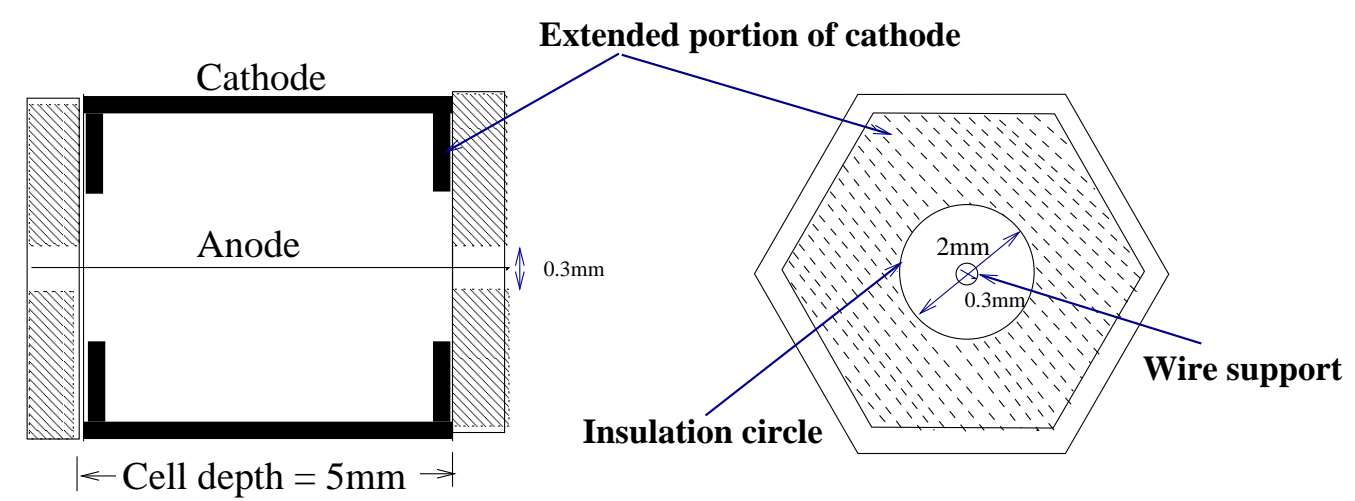

Figure 5.5: Schematic diagram of the cross section of a unit cell of the PMD.

\subsection{Photon Multiplicity Detector (PMD)}

\subsubsection{Design considerations}

The multiplicity and spatial $(\eta-\phi)$ distribution of photons in the forward pseudo-rapidity region of $2.3 \leq \eta \leq 3.7$ are measured by the Photon Multiplicity Detector (PMD) [13]. These measurements also provide estimations of transverse electromagnetic energy and the reaction plane on an eventby-event basis. The measurement of photon multiplicity gives important information in terms of limiting fragmentation, order of phase transition, the equation of state of matter and the formation of disoriented chiral condensates.

Because of the large particle density in the forward region, calorimetric techniques for photon measurements are not feasible. The PMD uses the preshower method where a three radiation length thick converter ( $1.5 \mathrm{~cm}$ thick lead with a $0.5 \mathrm{~cm}$ stainless steel backing) is sandwiched between two planes of highly granular gas proportional counters. The granularity and the converter thickness are optimized for high particle density so that the overlap of photon showers is minimal. The information from the detector plane placed in front of the converter is used as charged particle veto (CPV) and the preshower data from the second detector plane is used for photon identification.

The sensitive element of the detector consists of large arrays of gas proportional counters in a honeycomb cellular structure. The basic detector design was based on a detailed simulation which took into account the proper electrical field configurations within the chamber, the interaction of the particles in the gas and the generation of signals and reconstruction of physics observables. The basic cell is a honeycomb shaped cathode extended towards a $20 \mu \mathrm{m}$ thick gold-plated tungsten wire kept at a ground potential at the centre of each cell. The schematic diagram of the unit cell is shown in figure 5.5.

The granularity of the PMD was optimised given the requirements of low occupancy, and high efficiency and purity of photon detection, on an event-by-event basis, at the maximum predicted charge particle multiplicity density $\left(\mathrm{d} N_{\mathrm{ch}} / \mathrm{d} \eta=8000\right)$. The cell cross-section and depth are $0.22 \mathrm{~cm}^{2}$ and $0.5 \mathrm{~cm}$ respectively, optimised on the basis of detailed simulation and test beam results. The diameters of the insulation circle and the wire support are $2 \mathrm{~mm}$ and $0.3 \mathrm{~mm}$, respectively.

The response of the detector to charged particles was studied using a $5 \mathrm{GeV} / c$ pion beam. The left panel of figure 5.6 shows the efficiency of the detector as a function of the detector oper- 

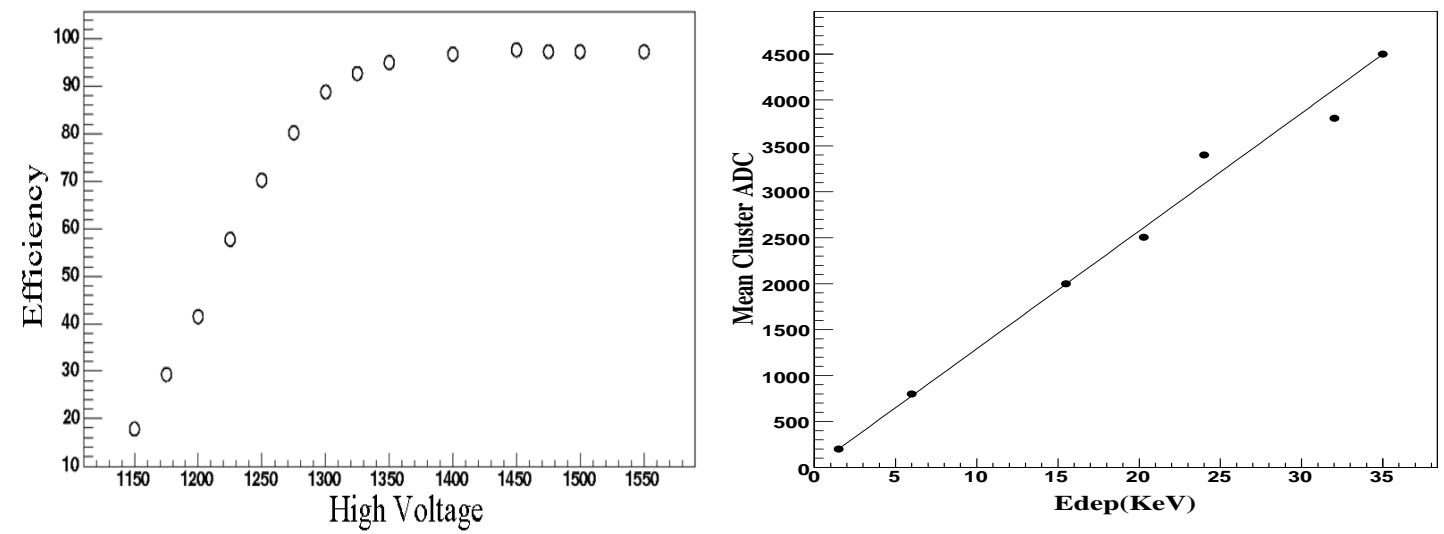

Figure 5.6: Left panel: efficiency of PMD module for charged particles as a function of operating voltage. Right panel: calibration relation between the energy deposition in terms of the measured mean cluster ADC counts versus the calculated values (in $\mathrm{keV}$ ) for the preshower plane.

ating voltages. The optimal operating voltage for the detector is -1400 Volts which forms part of the plateau region of the proportional zone. The efficiency is about $96 \%$ for charged pions at this voltage.

The calibration of the preshower PMD was obtained by performing an energy scan with electron beams in the range $1-5 \mathrm{GeV}$ and using $\mathrm{Pb}$ converters of different thicknesses. This is required to produce simulated data in ADC units in which the effects of the detector and readout electronics are properly folded in. The right panel of figure 5.6 shows the calibration relation for an operating voltage of 1400 Volts. The response of the detector is linear in the studied range of the energy deposition.

\subsubsection{Detector layout}

The PMD chambers are fabricated in the form of modules consisting of 4608 honeycomb cells. Each module is a gas tight enclosure and care is taken for high voltage isolation of each of the modules. Each plane of the PMD is made up of 24 modules as shown in figure 5.7. Two different types of modules (A-type and B-type) are employed, consisting of an array of $48 \times 96$ honeycomb cells configured in two different arrangements. Figure 5.8 shows the photographs of the two types of modules. Each module is served by separate high voltage and low voltage supplies.

The PMD is assembled in two equal halves. Each half has independent cooling, gas supply and electronics accessories. The PMD is supported from a stainless steel girder which forms part of the baby space frame in the forward region, provision being made for both $x$ - and $z$ - movements. The two halves can be moved on the girder to bring them together (as shown in figure 5.7) for data taking operation or separated for servicing. The girder itself can be moved on the baby space frame to insert the PMD in the solenoid magnet.

Table 5.2 gives an overview of PMD parameters. 


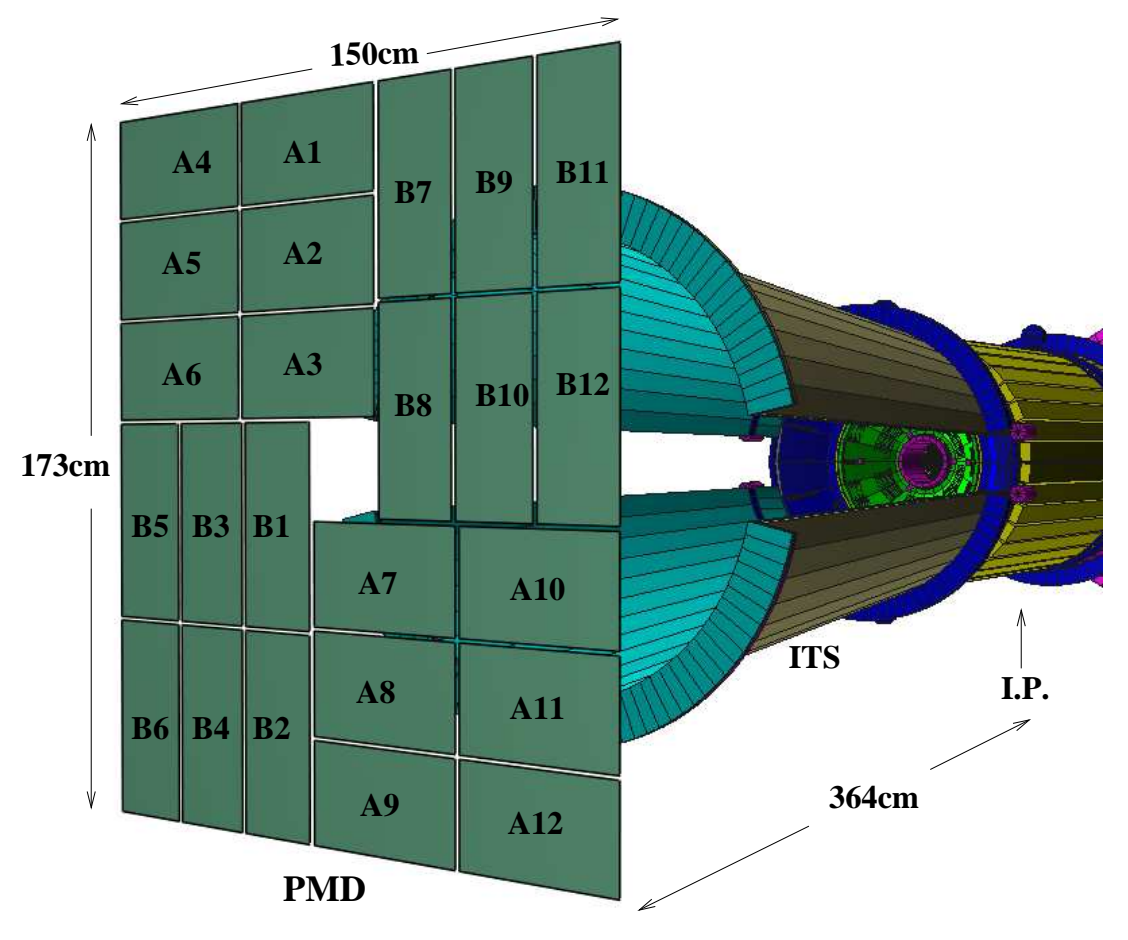

Figure 5.7: The PMD position and layout in ALICE shown with respect to the ITS. Each plane of the PMD is made up of 24 modules, half of which are of A-type and the other half of B-type.

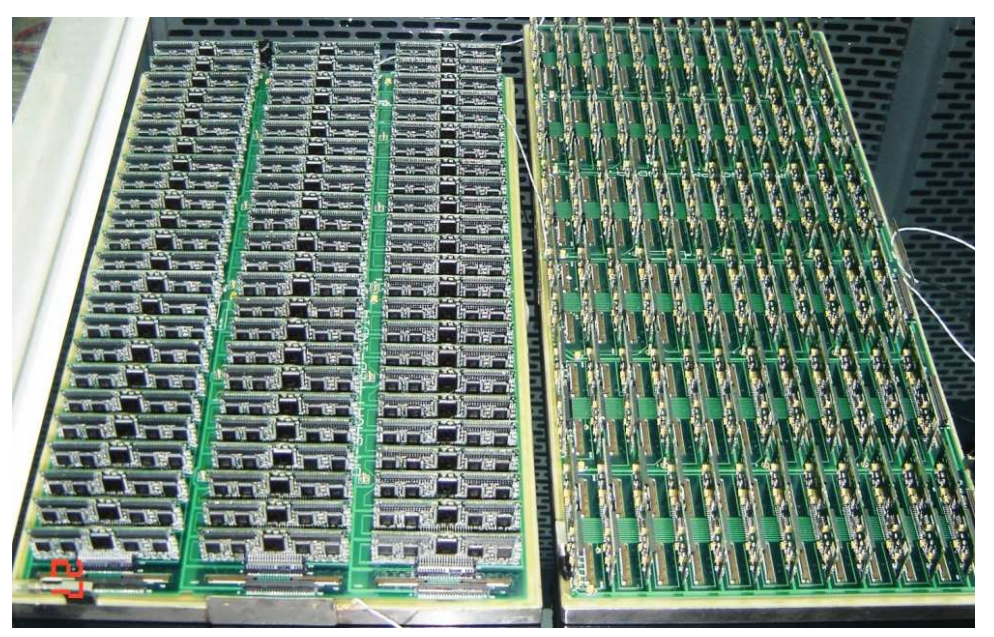

Figure 5.8: Photographs of two types of PMD modules showing the boundary frames and the connectors with the FEE boards mounted. 
Table 5.2: Summary of design and operating parameters of the PMD.

\begin{tabular}{|l|c|}
\hline Pseudo-rapidity coverage & $2.3 \leq \eta \leq 3.7$ \\
Azimuthal coverage & $360^{\circ}$ \\
Distance from vertex & $3.64 \mathrm{~m}$ \\
Detector active area & $2.59 \mathrm{~m}^{2}$ \\
Detector weight & $1200 \mathrm{~kg}$ \\
\hline Number of planes & two (Veto and Preshower) \\
Converter & $3 X_{0}(1.5 \mathrm{~cm} \mathrm{~Pb}+0.5 \mathrm{~cm} \mathrm{SS})$ \\
Hexagonal cell cross section & $0.22 \mathrm{~cm}^{2}$ \\
Hexagonal cell depth (gas thickness) & $0.5 \mathrm{~cm}^{\circ}$ \\
Detector gas & $\mathrm{Ar} / \mathrm{CO}_{2}(70 \% / 30 \%)$ \\
Operating voltage & $-1400 \mathrm{~V}$ \\
Charged-particle detection efficiency & $96 \%$ \\
\hline Number of cells in one module & 4608 \\
Number of modules per plane & 24 \\
Total number of cells & 221184 \\
Number of HV channels & 48 \\
\hline Number of FEE boards & 3456 \\
Number of CROCUS crates & 6 \\
Number of DDL channels & 6 \\
\hline Cell occupancy for d $N_{\mathrm{ch}} / \mathrm{d} \eta=8000$ for veto plane & $13 \%$ \\
Cell occupancy for d $N_{\mathrm{ch}} / \mathrm{d} \eta=8000$ for preshower plane & $28 \%$ \\
Average photon reconstruction efficiency & $54 \%$ \\
Average purity of photon sample & $65 \%$ \\
\hline Event size for d $N_{\mathrm{ch}} / \mathrm{d} \eta=8000$ & $0.12 \mathrm{MB}$ \\
Average time for one event readout & $100 \mu \mathrm{s}$ \\
\hline
\end{tabular}




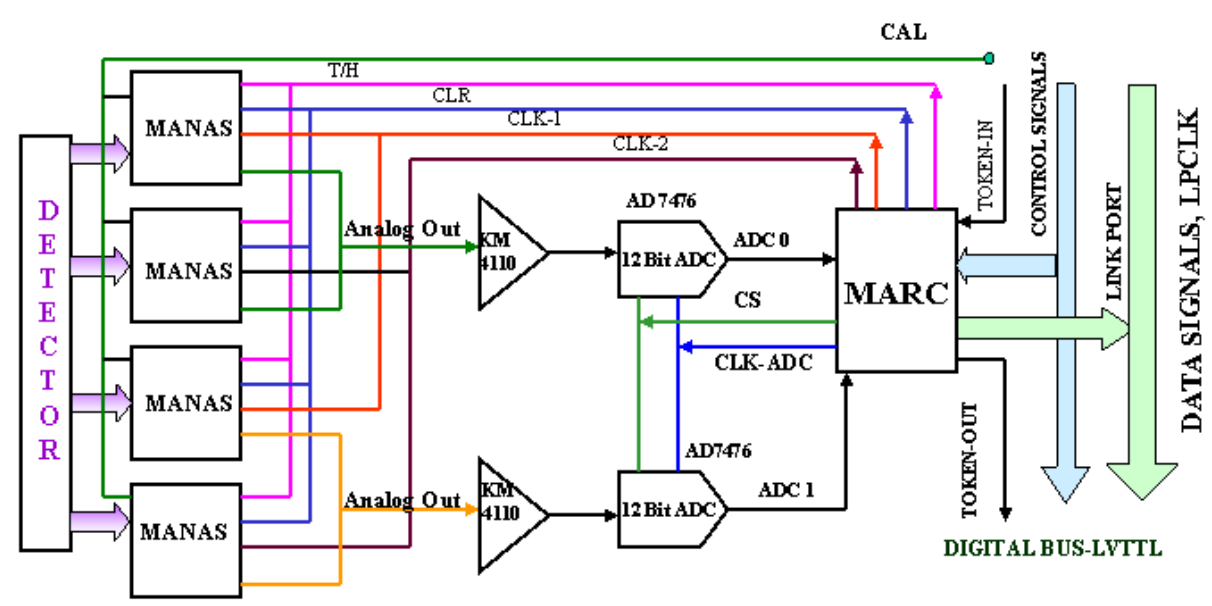

Figure 5.9: Front-End Electronics architecture of the PMD.
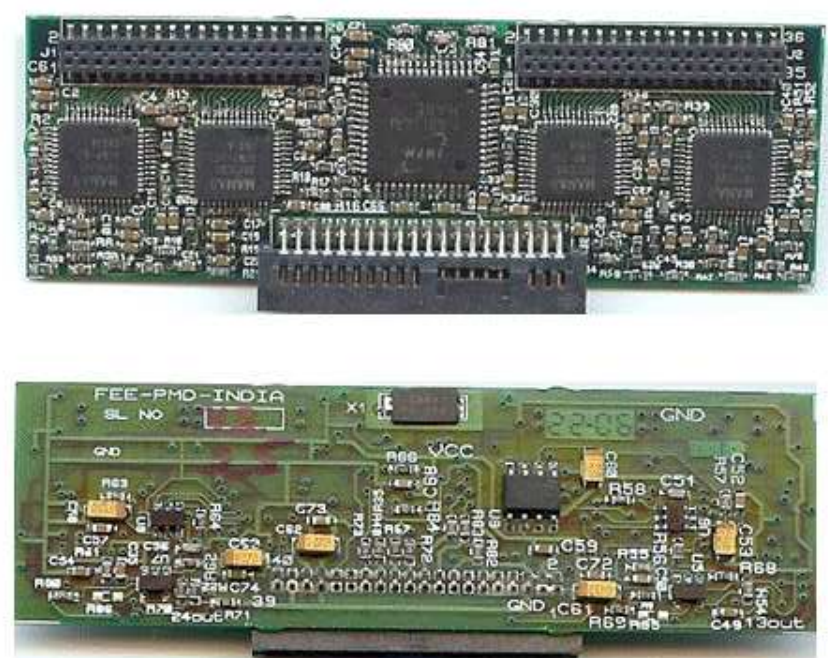

Figure 5.10: Photograph showing both sides of the Front-End Electronics board of the PMD. Four MANAS chips along with the MARC are clearly seen.

\subsubsection{Front-End Electronics and readout}

The schematics of the Front-End Electronics for the PMD is shown in figure 5.9, which is similar to the setup for the tracking chambers of ALICE muon spectrometer [12]. A photograph of the FEE board is shown in figure 5.10.

The signals from the anode wires for a group of 64 cells within a matrix of 4 rows and 16 columns are connected to two 32-pin FRC connectors by a flexible cable which connects to a FEE board at the other end. The signals are processed using the MANAS chip which handles 16 channels providing multiplexed analogue outputs. Each FEE board consists of four MANAS chips, two 12-bit ADCs, and a custom built ASIC called MARC chip which controls all 64 channels. The MARC chip controls 4 MANAS chips, two ADCs and performs zero suppression of the data. 
A set of FEE boards are read out using Digital Signal Processors (DSP). The MARC chip communicates with DSP through 4-bit bus. The DSPs are handled through a cluster readout system (CROCUS). The main objectives of the CROCUS are to gather and concentrate the information coded on the FEE and pass to the DAQ, drive the FEE boards via patch bus controllers, receive and distribute the trigger signals, and perform the calibration of the detector. Each CROCUS crate can handle 50 patch-buses. Each patch-bus handles one chain of FEE boards.

The readout chain is designed considering the occupancies in the chains and the restrictions on chain length as dictated by faithful signal transmission. The chain arrangement for CPV and preshower planes are done differently. Each readout chain in the preshower plane has 12 FEE boards. Each quadrant of the preshower plane has a total of 36 chains and is serviced by one CROCUS crate and one DDL channel. For the CPV plane the number of FEE boards in a chain is 24 for long type modules and a mix of 24 and 12 for short type modules. The total number of chains in each half of the CPV plane is 42 and serviced by one CROCUS and one DDL channel. The total number of DDL channels for PMD is six.

\subsection{Forward Multiplicity Detector (FMD)}

\subsubsection{Design considerations}

The main functionality of the Forward Multiplicity Detector (FMD) is to provide charged-particle multiplicity information in the pseudo-rapidity range $-3.4<\eta<-1.7$ and $1.7<\eta<5.0$. Figure 5.11 shows the combined pseudo-rapidity coverage of the FMD and ITS pixel detector seen from the nominal vertex position. The overlap between the FMD silicon rings and the ITS inner pixel layer provides redundancy and cross-checks of measurements between subdetectors and ensures that continuous coverage for a distribution of vertices along the $z$-axis. Additionally, high radial detector segmentation allows for the study of multiplicity fluctuations on an event-by-event basis while azimuthal segmentation allows for the determination of the reaction plane for each event and the analysis of flow within the FMD's pseudo-rapidity coverage.

The segmentation of the FMD was chosen such that, on average, one charged particle would occupy each strip for central events. Simulations of central $\mathrm{Pb}-\mathrm{Pb}$ collisions with $\mathrm{d} N_{\mathrm{ch}} / \mathrm{d} \eta \approx 8000$ in the mid-rapidity region were used to study the FMD design parameters [18]. Contributions from secondary interactions in the beampipe, ITS, T0 detector, and V0 detector as well as in services and support structures increase the number of particles impinging on the FMD. For these events, the average hit density over the whole FMD was one charged particle while no individual strip had an average hit density greater than three charged particles. Peripheral A-A collisions and pp collisions produce significantly lower hit densities. The FMD is designed to allow up to 20 minimum-ionizing particles (MIPs) in a single strip before saturating.

The segmentation chosen for the detector (strips) and the single layer geometry results from a compromise between the desired performance (multiplicity resolution) and the necessity to minimize the overall cost and complexity of the detector. The system readout time $(>1.2 \mu \mathrm{s})$ does not allow the FMD to serve as a multiplicity trigger and, therefore, provides only offline analysis information. 

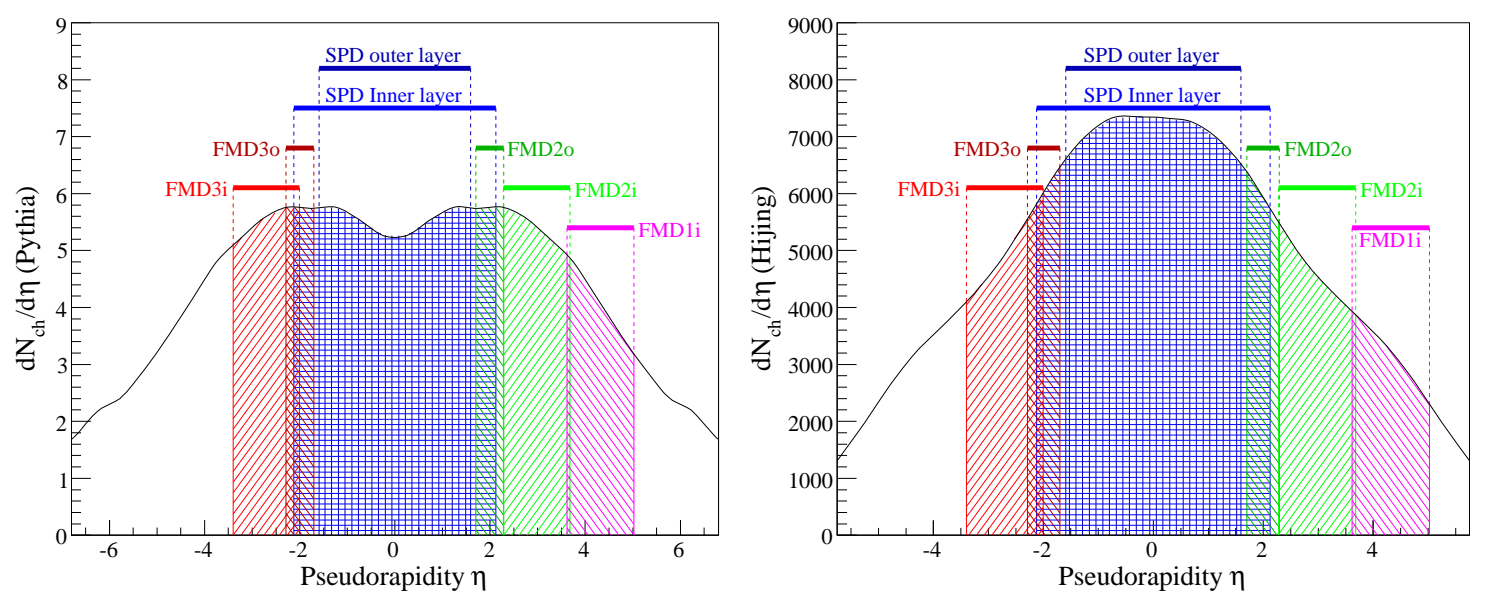

Figure 5.11: Pseudo-rapidity coverage of the FMD rings ( 1 inner, 2 inner and outer, and 3 inner and outer) along with the two ITS pixel layers seen from the nominal vertex position overlaid on simulated multiplicity distributions to illustrate which regions are measured by each detector. The left multiplicity distribution is produced by the PYTHIA event generator [179] with pp collisions at $\sqrt{s}$ $=14 \mathrm{TeV}$, while the right multiplicity distribution is produced by the HIJING event generator [142] with $\mathrm{Pb}-\mathrm{Pb}$ collisions at $\sqrt{s_{N N}}=5.5 \mathrm{TeV}$.

\subsubsection{Detector layout}

Figure 5.12 shows the location of each FMD ring in ALICE as well as the basic layout of the silicon sensors within an FMD ring. FMD2 and FMD3 each consist of both an inner and an outer ring of silicon sensors and are located on either side of the ITS detector. FMD2 and FMD3 are positioned to have approximately the same acceptance, however, the presence of the T0 detector necessitated a different placement for the FMD3 inner ring. Another ring, FMD1, was placed further from the interaction point opposite to the muon spectrometer to extend the charged particle multiplicity coverage. The upper limit on this additional coverage (determined to be at $\eta=5.0$ ) is constrained by the increasing number of secondary particle contributions at very forward rapidity.

Each detector ring consists of 10 (for an inner ring) or 20 (for an outer ring) silicon sensors. The inner radius of the rings is constrained by the beampipe radius while the outer radius is constrained by the inner radius of the TPC. The radial span (distance from inner radius to outer radius) is limited by the $15 \mathrm{~cm}$ diameter wafers from which the silicon sensors are made. Two silicon sensor types were fabricated. Inner sensors consist of two azimuthal sectors each with 512 silicon strips. The radii of the inner strips range from $4.2 \mathrm{~cm}$ to $17.2 \mathrm{~cm}$. Outer sensors also consist of two azimuthal sectors each with 256 silicon strips with radii ranging from $15.4 \mathrm{~cm}$ to $28.4 \mathrm{~cm}$. Each ring (inner and outer) contains 10240 silicon strips giving the full FMD a total of 51200 silicon strips to be read out. An assembled FMD ring system (FMD3) is shown in figure 5.13. An overview of the FMD design parameters is given in table 5.3. 


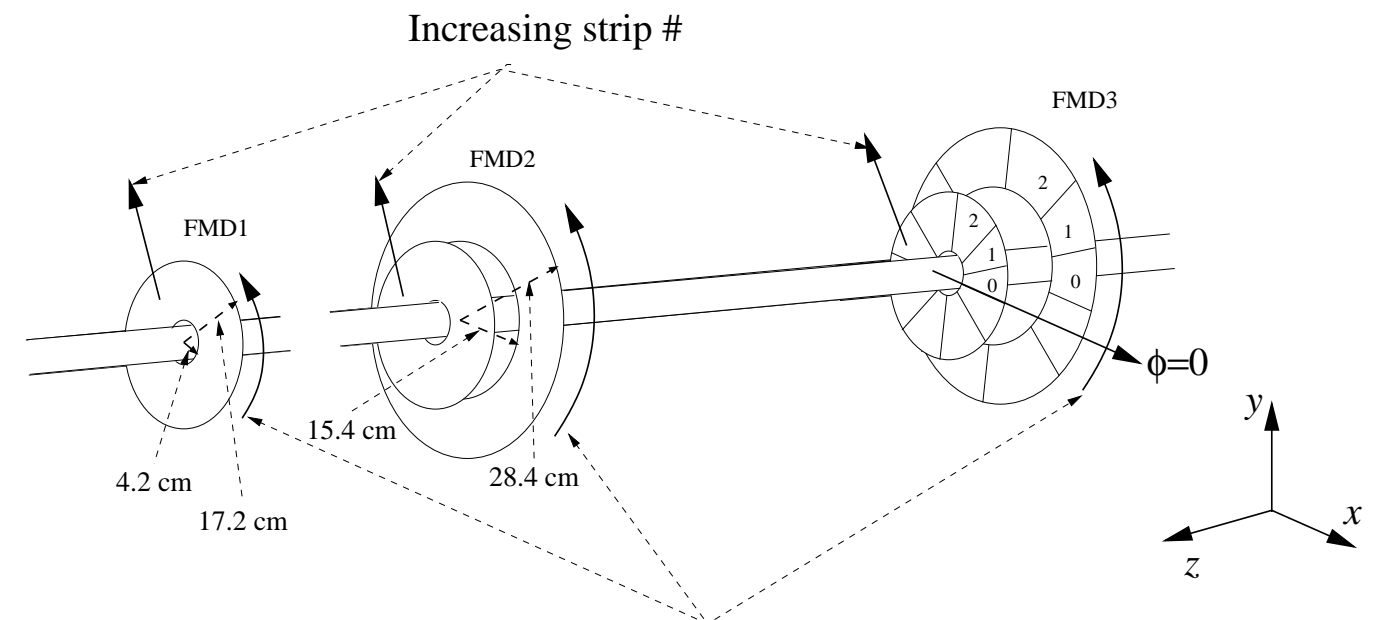

Increasing sector \#

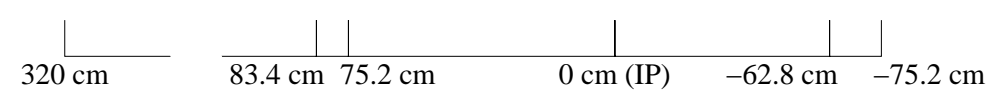

Figure 5.12: Layout of the FMD rings in the ALICE experiment. FMD3 and FMD2 are located on each side of the ITS detector while FMD1 is much further away from the interaction point (IP).

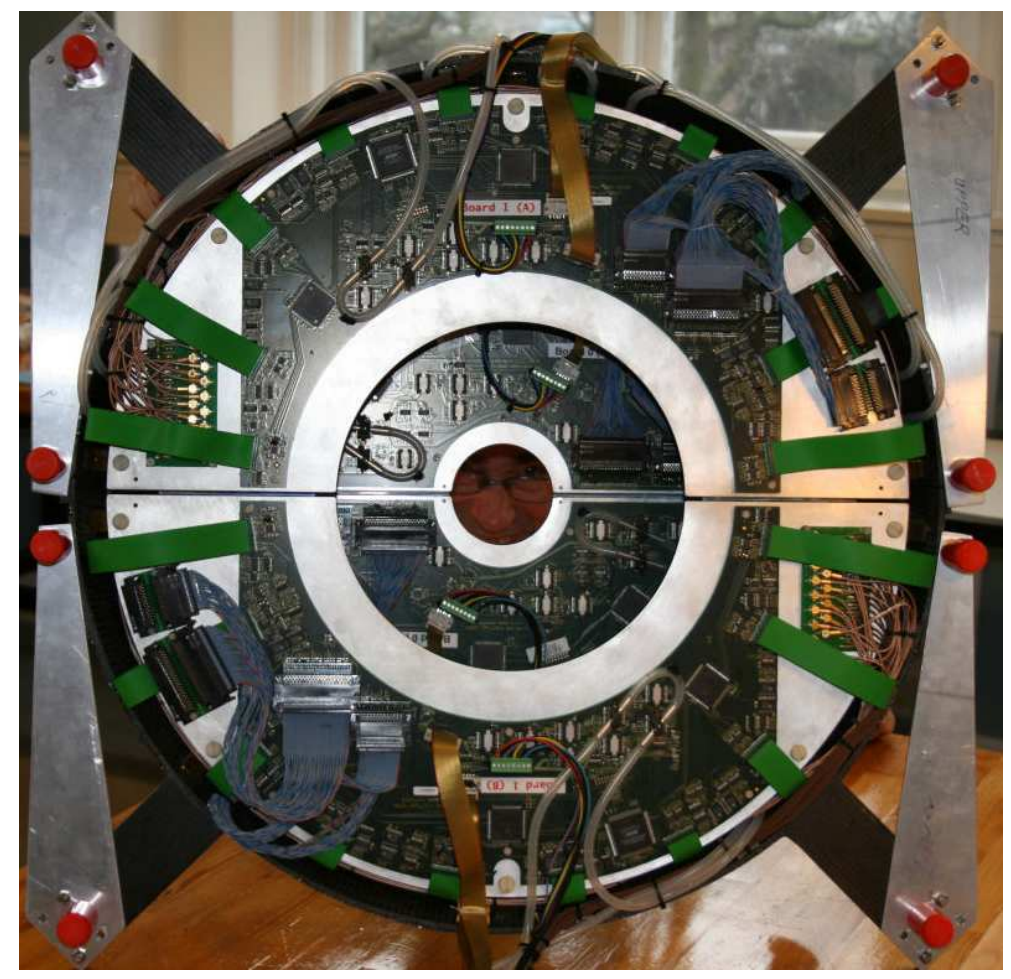

Figure 5.13: Assembled FMD3 detector. The side of the detector points away from the interaction point. Two outer digitizer boards can be seen while the two inner digitizer boards are partially covered. Each of the green cables connects a silicon module mounted on the other side of the support plate to its respective digitizer board. 
Table 5.3: Overview of FMD parameters.

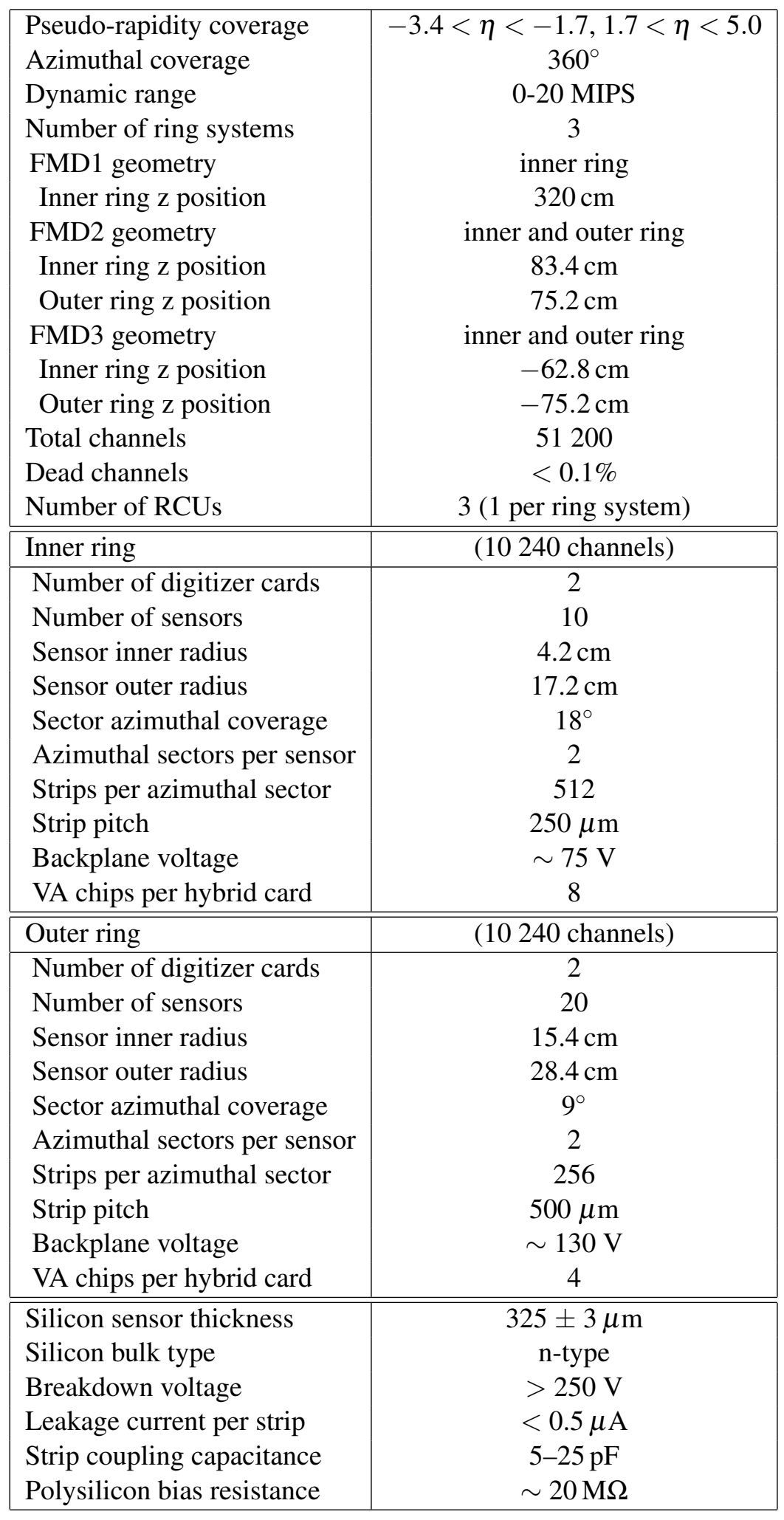




\subsubsection{Front-end electronics and readout}

The signals from each silicon strip in the FMD must be collected and transferred for processing. Figure 5.14 shows the components of an inner FMD module which detects the particles and performs the initial collection of this information. A hybrid PC card with dimensions slightly smaller than the silicon sensor is attached to the sensor by gluing thin ceramic spacers between them to form a silicon module. Bonding pads exist along the perimeter of both the silicon sensor and hybrid PC card to allow small bond wires to connect the signals from the silicon sensor to readout electronics. To achieve the best signal resolution, amplification of the signal must be done as close as possible to the detector element. A VA preamplifier chip [180] is placed directly on the hybrid PC card to amplify and shape the detected signal. This preamplifier chip has low noise (250-350 ENC for the FMD detector load) and a gain allowing for signals up to 20 MIPs to be read out before saturating. Since these chips are located adjacent to the silicon detectors, they will receive the same radiation dose as the silicon detectors. The version of the VA chip used for the FMD is made with $0.35 \mu \mathrm{m}$ AMS technology in order to resist to radiation levels well beyond $1 \mathrm{Mrad}$ [181]. Each VA chip has input lines for individual amplification and shaping of 128 signals. An inner silicon module therefore requires eight VA chips while an outer silicon module requires four. A 40-pin connector on the hybrid PC card provides enough connections for power to the VA chips, control of readout, and dedicated lines for each VA chip's differential readout of the amplified signals. A separate high-voltage connector is necessary for supplying the backplane voltage to reverse-bias the silicon sensor and deplete the silicon bulk. The hybrid PC card provides filtration circuitry to reduce noise on the high voltage line. A small wire connection from the hybrid PC card is looped around the edge of the sensor and attached with conducting glue to the back side of the silicon sensor. Typical high voltage values for the inner sensors are around $75 \mathrm{~V}$ while outer sensors require around $130 \mathrm{~V}$.

Further electronics is needed to digitize the signals and control readout. An electronics board (termed 'digitizer board') was built for this purpose. The digitizer board has the same shape as a silicon half-ring and is mounted on the backside of the low mass honeycomb support plate of the silicon modules. Short readout cables connect five inner modules to an inner digitizer card or 10 outer modules to an outer digitizer card. The digitizer card provides the low voltage to power the silicon modules as well as controlling readout. The main components of a digitizer card are the ALTRO chip [96] (three per digitizer card), each used for digitizing the signal from one or two silicon modules, and an FPGA chip, which controls the readout of the silicon modules as well as monitoring services for temperature, voltage and current.

The ALTRO chip is a fast ADC chip developed by the ALICE TPC group. It allows analogueto-digital conversion to be done on the detector, thereby avoiding long cables for analogue signals. The use of the ALTRO chip in the FMD allows further electronics in the readout chain to be common with the TPC. An FPGA was used as a low-cost means for controlling readout and monitoring. Additionally, the use of an FPGA allows changes to be made to the readout and monitoring algorithm without having access to the physical hardware. For complicated readout procedures, such as calibration, the FPGA can be programmed.

A schematic diagram of the readout chain is shown in figure 5.15. An L0 trigger arrives directly at the board controller on the digitiser card to signify that a collision has occurred. The board controller then waits the time necessary (which is approximately $2 \mu$ s after the bunch crossing) for 

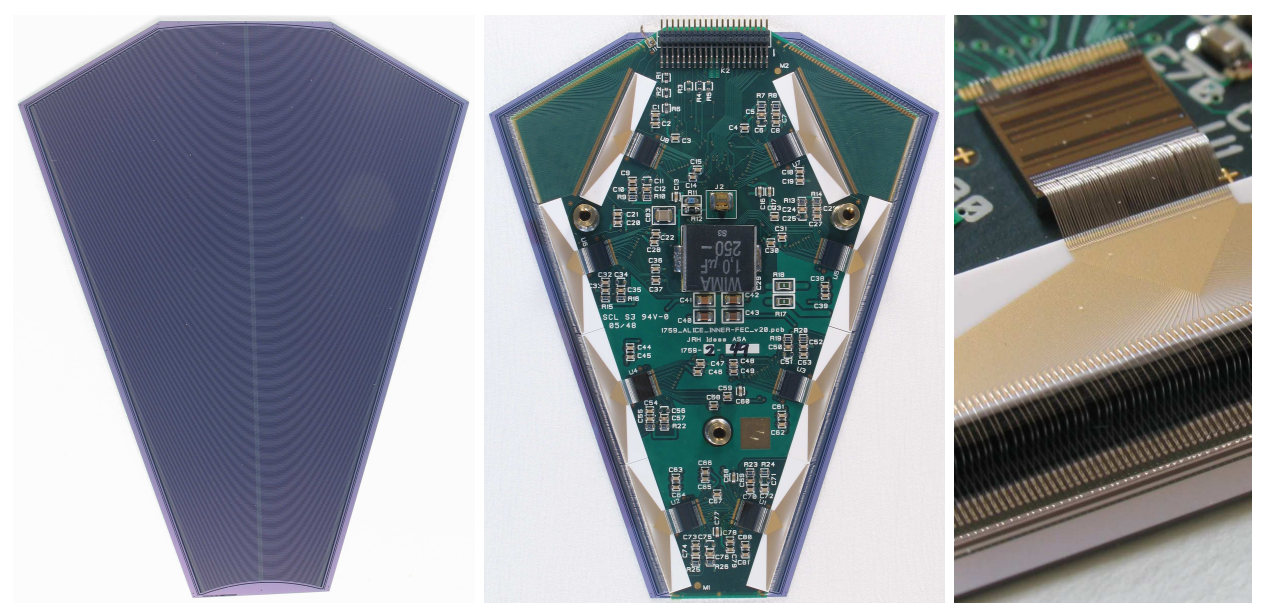

Figure 5.14: The components of an inner silicon module are shown. At left is a silicon sensor with two sectors each containing 512 silicon strips which detect charged particles via their energy loss. The middle picture shows an inner FMD silicon module where the hybrid readout card is mechanically attached to and separated from the hybrid PC card via ceramic spacers. The rightmost picture is an enlarged view of the edge of this FMD module. Bond wires are attached from each strip of the silicon sensor to an input on the edge of the hybrid PC card. A pitch adapter adjusts the separation of readout lines to the separation required by the readout chip. The readout chip collects the charge from the signals deposited by the charged particles and holds the analogue value for each channel until further readout occurs.

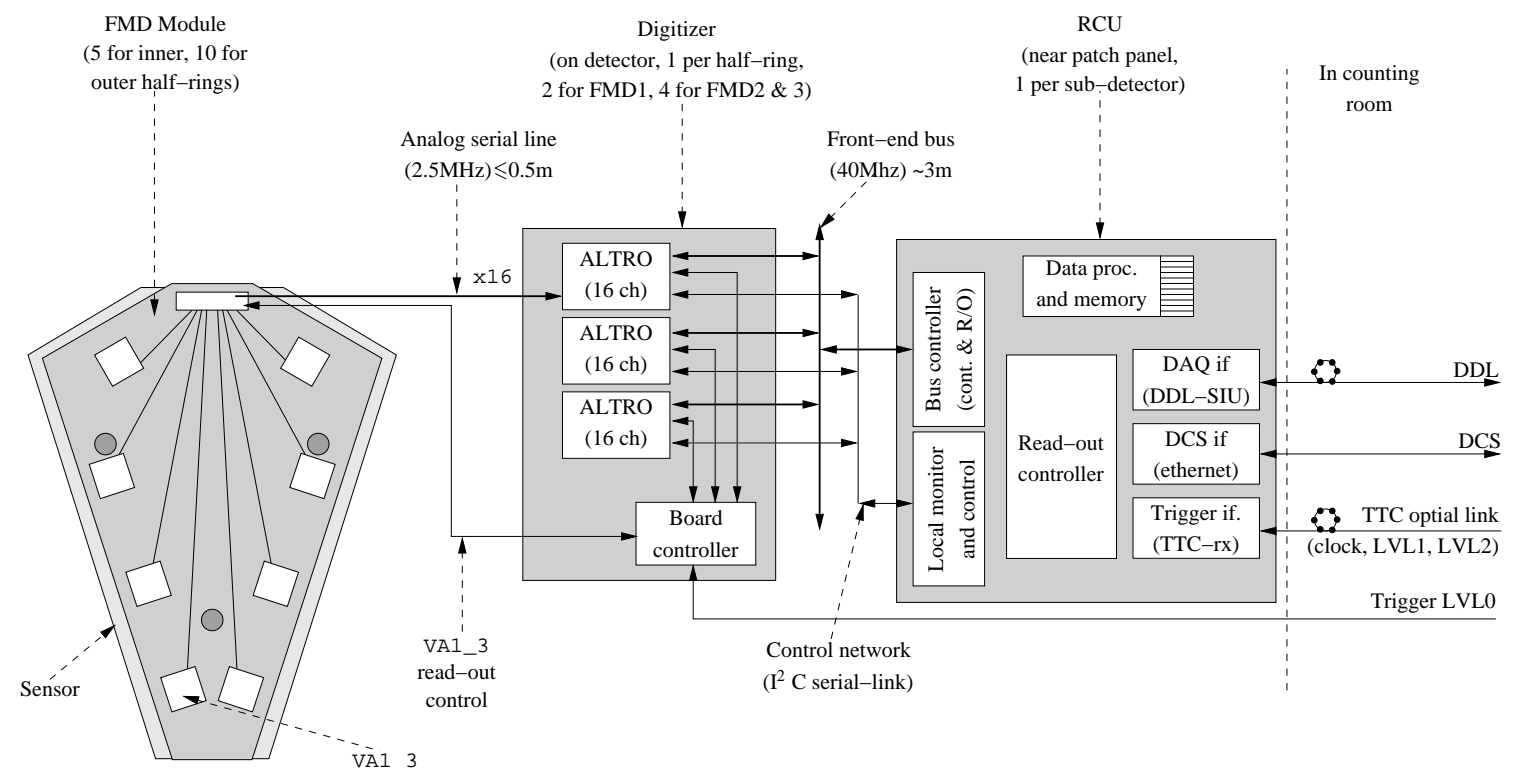

Figure 5.15: Diagram of readout chain for the FMD. The board controller on the digitizer card controls the readout sequence of the silicon modules. Analogue data is received at the ALTRO chips and digitized. The readout control unit (RCU) coordinates the transfer of the digitized data from the ALTRO chips to the DAQ system. 

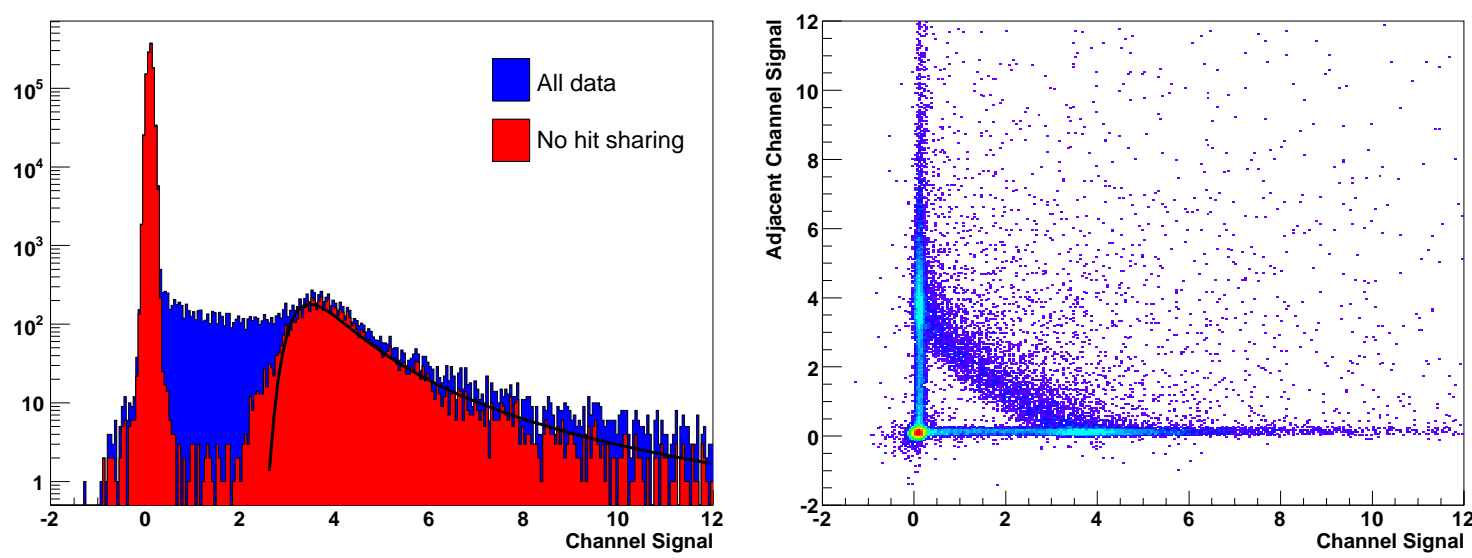

Figure 5.16: The distribution of signals from a single silicon strip is shown on the left. The blue distribution corresponds to all signals seen in that strip. A large set of signals having energies between the pedestal peak and the MIP peak is seen in this distribution. The correlation between the signal seen in this strip and an adjacent strip is shown on the right. A clear band is seen with signals whose sum of energy between the two strips is 1 MIP. These shared hits can be removed by requiring the adjacent channels to have only noise (by making a maximum energy cut) to reveal the actual detector response of Landau distributed signals (shown by the fit).

the VA chip to have the peak response after shaping of the collected signal's charge. At that time, the board controller directs all VA chips to hold the analogue levels of the signals it currently has. If the event passes the L1 criteria (approximately $6 \mu$ s after the bunch crossing), an L1 signal is sent to the board controller via the RCU [182]. The board controller then manages the serial readout of the 128 signals from each VA chip to the ALTRO where it is digitized and stored in one of four or eight buffers. Each VA chip is read out in parallel during this process. After the data has been transferred to the ALTRO buffers or if an L1 signal was never received, the hold of the analogue signals in the VA chip is released and another L0 signal can be accepted as long as there are empty ALTRO buffers. Thereafter, if the event passes the L2 criteria (on the order of $88 \mu$ s after the bunch crossing, controlled by the TPC readout time), a L2 signal is sent through the board controller to the ALTRO chips directing them to use the next buffer in the next event (if one exists). If no L2 signal is received, the ALTRO buffer is overwritten by the next event's readout. Transfer of data from the ALTRO buffers to the DAQ system is controlled by the RCU and done serially for all data in all ALTRO buffers for that event on a $40 \mathrm{MHz}$ bus that can transfer data nominally at $160 \mathrm{MB} / \mathrm{s}$ and maximally at $200 \mathrm{MB} / \mathrm{s}$.

\subsubsection{Detector response}

Figure 5.16 shows the calibrated response for a single FMD strip to charged particles accumulated over many events. 
The large peak at zero is generated from events where no particle has deposited energy in this detector strip and the width of that peak arises from the intrinsic noise of this detector strip along with the noise accumulated during readout. While the energy per unit length deposited in a silicon detector is described by a Landau distribution, the total energy deposited in a detector strip also depends on the angle of the particle relative to the detector. Additionally, some particles share their deposited energy among multiple detector strips. After these shared hits are removed from the distribution in figure 5.16, a peak is clearly seen separated from the noise with a long tail with increasing energy. A fit of this peak to a Landau distribution yields the most probable energy loss, a minimum-ionizing particle (1 MIP), as a result. Deviations of the fit from a Landau distribution are due to incomplete removal of all shared hits. The ratio of this value to the noise (termed the signalto-noise ratio) determines the ease of distinguishing between hit and not-hit strips. Measurements performed with the FMD [183] have shown outer sensors to have an average signal-to-noise ratio of 23:1 while inner sensors have an average ratio of 40:1, far in excess of the design criteria of 10:1.

\subsection{V0 detector}

\subsubsection{Design considerations}

The V0 detector [18] is a small angle detector consisting of two arrays of scintillator counters, called VOA and VOC, which are installed on either side of the ALICE interaction point.

This detector has several functions. It provides minimum-bias triggers for the central barrel detectors in pp and A-A collisions. These triggers are given by particles originating from initial collisions and from secondary interactions in the vacuum chamber elements. As the dependence between the number of registered particles on the $\mathrm{V} 0$ arrays and the number of primary emitted particles is monotone, the $\mathrm{V} 0$ serves as an indicator of the centrality of the collision via the multiplicity recorded in the event. Cuts on the number of fired counters and on the total charge can be applied to achieve rough centrality triggers. There are three such triggers, the multiplicity, semi-central and central triggers.

In practice and during normal operation, both arrays are required (AND mode) to provide triggers, namely: Minimum Bias trigger (MB), Multiplicity Trigger (MT), semi-Central Trigger (CT1) and Central Trigger (CT2). An OR mode can also be adopted. In pp collisions, the efficiency for the detection of at least one charged particle detected in both sides is about $75 \%$ when no secondary particle is taken into account. It raises up to $84 \%$ when the environment effects are introduced $[18,49]$.

Interactions of protons with the residual gas of the vacuum chamber generate tracks through the ALICE subdetectors. Minimum Bias p-Gas trigger (PG) can help to eliminate false events [184]. Moreover, a large background trigger rate is expected in the muon spectrometer trigger chambers. The absence of Minimum Bias Trigger from VOC alone (OR mode of MB trigger), is a good signal to reject a large part of these false muon triggers [185].

Finally, the V0 detector participates in the measurement of luminosity in pp collisions with a good precision of about 10\%. Detailed studies can be found in [186]. 
Table 5.4: V0A and V0C arrays. Pseudo-rapidity and angular acceptances (deg.) of the rings.

\begin{tabular}{|c|c|c|c|c|}
\hline \multirow{2}{*}{ Ring } & \multicolumn{2}{|c|}{ V0A } & \multicolumn{2}{c|}{ V0C } \\
& $\eta_{\max } / \eta_{\min }$ & $\theta_{\min } / \theta_{\max }$ & $\eta_{\min } / \eta_{\max }$ & $\theta_{\max } / \theta_{\min }$ \\
\hline 0 & $5.1 / 4.5$ & $0.7 / 1.3$ & $-3.7 /-3.2$ & $177.0 / 175.3$ \\
1 & $4.5 / 3.9$ & $1.3 / 2.3$ & $-3.2 /-2.7$ & $175.3 / 172.4$ \\
2 & $3.9 / 3.4$ & $2.3 / 3.8$ & $-2.7 /-2.2$ & $172.4 / 167.5$ \\
3 & $3.4 / 2.8$ & $3.8 / 6.9$ & $-2.2 /-1.7$ & $167.5 / 159.8$ \\
\hline
\end{tabular}
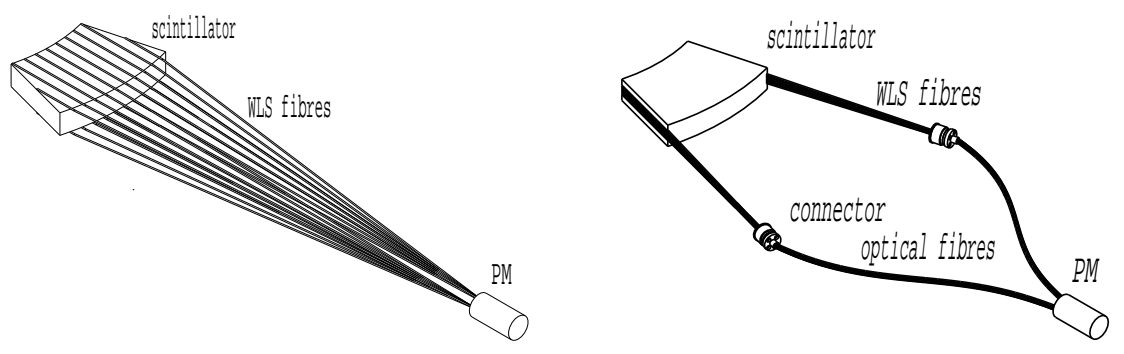

Figure 5.17: Schematic design of the V0A (left) and V0C (right) detection elements.

\subsubsection{Detector layout}

The V0A detector is located $340 \mathrm{~cm}$ from the vertex on the side opposite to the muon spectrometer whereas V0C is fixed to the front face of the hadronic absorber, $90 \mathrm{~cm}$ from the vertex. They cover the pseudo-rapidity ranges $2.8<\eta<5.1$ (V0A) and $-3.7<\eta<-1.7$ (V0C) and are segmented into 32 individual counters each distributed in four rings (table 5.4).

Extensive tests [187] were performed to choose the best design for the elementary counters and the easiest integration of the disks in the system. The material consists of $\mathrm{BC} 404^{1}$ scintillating material (2.5 and $2.0 \mathrm{~cm}$ in thickness for V0A and V0C respectively) with $1 \mathrm{~mm}$ in diameter BCF9929A Wave-Length Shifting (WLS) fibres. ${ }^{1}$ The fibres spaced by $1 \mathrm{~cm}$ are embedded in the two transverse faces of the segments following the 'megatile' technique [188] for the V0A array (figure 5.17). There are 32 elementary counters arranged in 4 rings and 8 sectors of $45^{\circ}$. The fibres are grouped by layers of 9 units and glued along the two radial edges of the segments for the V0C array (figure 5.17). There are 48 elementary counters of this type distributed following two inner rings of 8 counters and two outer rings of 16 counters. The latter ones are connected 2 by 2 to make an unique detection element. A picture of each array is shown in figure 5.18.

The radiation effects on V0, located close to IP and at small angles, were of concern and investigated [189]. Results of irradiations of elements show that it could be necessary to replace some of them due to the loss of signal. Therefore, spare inner V0C elements are foreseen if large irradiation effects require their replacement.

The light is guided to the photo-multiplier system H6153-70MOD from Hamamatsu. ${ }^{2}$ The

\footnotetext{
${ }^{1}$ Bicron, Saint-Gobain Industrial Ceramics, P.O. Box 3093, 3760 DB Soest, The Netherlands.

${ }^{2}$ Hamamatsu Photonics, 360 Foothill Rd Bridgewater, NJ 08807, U.S.A.
} 

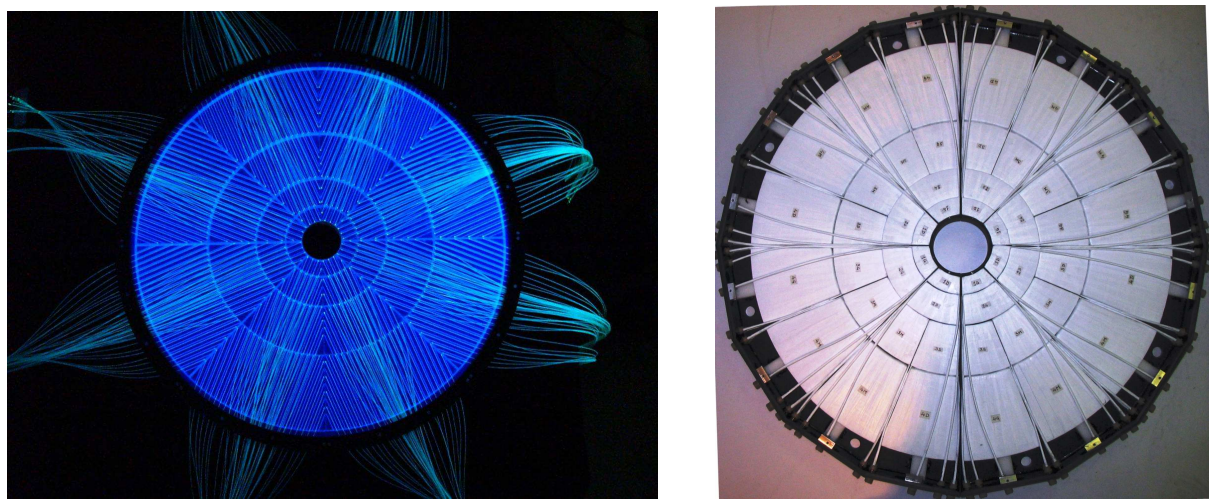

Figure 5.18: Front view of VOA (left) and VOC (right) arrays.
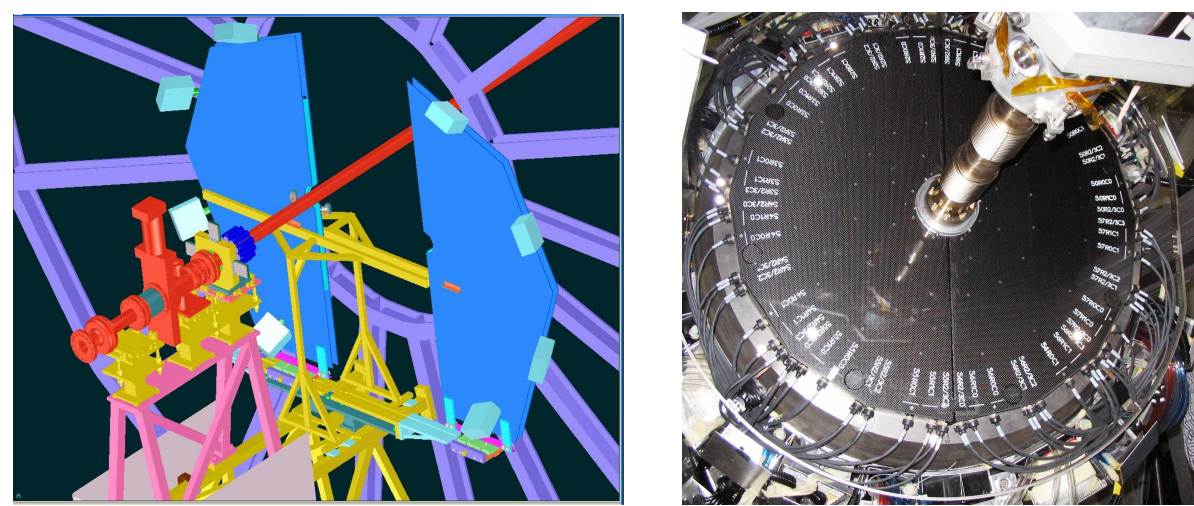

Figure 5.19: V0A 3D drawing (left) and V0C picture (right).

PMTs are fixed on the V0A disk holder in groups of 4 units and connected directly to the WLS fibres. They are installed on the absorber in groups of 8 units for the VOC and connected to counters through Mitsubishi ${ }^{3}$ optical fibres 3.22 m long. A picture of each device is shown in figure 5.19.

\subsubsection{Front-end electronics}

Two signals are delivered to the Front-End Electronics (FEE). The first one is unchanged, the second one amplified by a factor 10. Signal charge and arrival time relatively to the LHC bunch clock are measured for the 32 channels of both arrays. The time resolution of the individual counters is better than $1 \mathrm{~ns}$. Two types of triggers are provided from each array [18]. The first one is based on pre-adjusted time windows in coincidence with the time signals from the counters. Minimum Bias, Beam-Gas and Multiplicity Triggers are obtained by this method. The second type of triggers is based on the total charge collected by the arrays. The two Centrality Triggers are built starting from these quantities.

The readout and data acquisition architecture is designed to be compatible with the different running modes and with the trigger rates [18]. The triggers listed in section 5.4.1 and combinations of some of them, are available. Only five triggers which are chosen according to specificities of collisions and recorded data are sent to the Central Trigger Processor (CTP).

\footnotetext{
${ }^{3}$ Mitsubishi PMMA fibres distributed by Promic, 46 rue de la Pierre Plantée, 42650 Saint-Jean Bonnefonds, France.
} 


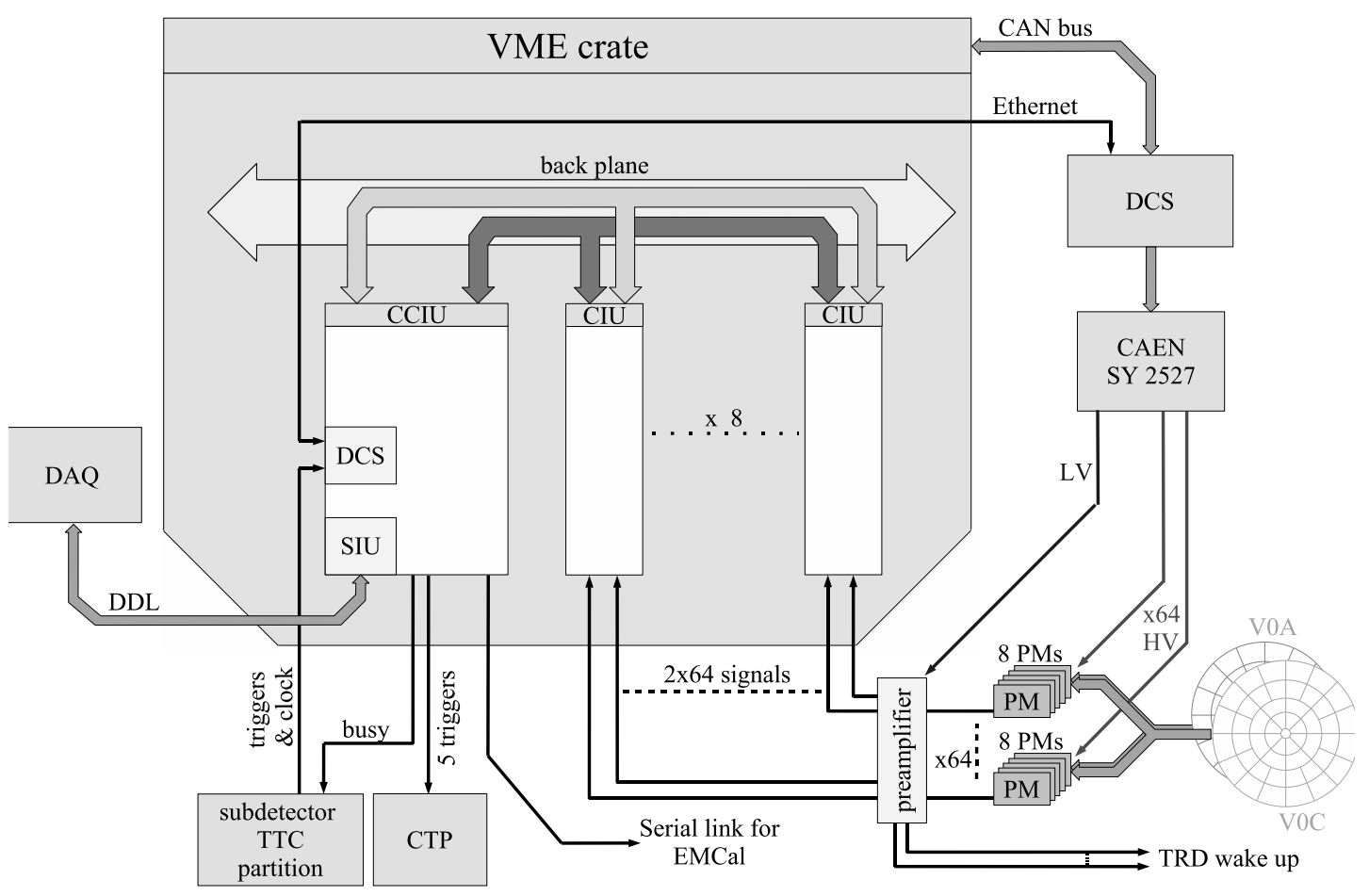

Figure 5.20: V0 Front-End Electronics architecture.

The trigger generation, readout, and data acquisition chain of the V0 consist of two types of $9 \mathrm{U}$ boards located in one $9 \mathrm{U}$ VME crate and linked by a full custom-made back plane (figure 5.20).

The Channel Interface Unit (CIU) board performs the PMT's anode signal integration (dual charge integrator), the digitization of the time, the pre-processing for the generation of the various triggers, and the data storage during a L0 and a L2 trigger. The two main components of this board are the threshold discriminator and the HPTDC [190], a Time-to-Digital Converter. Each CIU board processes anode signals from the eight photo-multipliers of a V0 ring. There are four CIU boards per array.

The Channel Concentrator Interface Unit (CCIU) board distributes the clock to elements of the FEE, generates the calibration triggers, the five final trigger and busy signals, collects and organizes the data from CIU boards, and provides several interfaces with the Experiment Control System. The three main blocks of the CCIU board are (i) the FPGA for the data handling (data collection on the back plane, data preparation for several interfaces), (ii) the SIU mezzanine, interface between DAQ and FEE, and (iii) the DCS mezzanine, interface between FEE and DCS (through an ethernet link) for the V0 slow control and between FEE and the TTC partition.

Irradiation effects were evaluated with low energy proton beam. The number of Single Event Upsets (SEU) induced in a FPGA chip was determined as a function of the flux of particles. Considering the position of the FEE in the cavern, no deterioration of electronics components is expected during 10 years of ALICE running [191]. 


\subsection{T0 detector}

\subsubsection{Design considerations}

The T0 detector $[18,192,193]$ was designed with the following objectives: First, to generate a start time (T0) for the TOF detector. This timing signal corresponds to the real time of the collision (plus a fixed time delay) and is independent of the position of the vertex. The required precision of this signal is about $50 \mathrm{ps}$ (r.m.s.). Then, to measure the vertex position (with a precision $\pm 1.5 \mathrm{~cm}$ ) for each interaction and to provide a L0 trigger when the position is within the preset values. This will discriminate against beam-gas interactions. The T0 can also generate an early 'wake-up' signal to the TRD, prior to L0. In addition, T0 provides redundancy to the V0 counters and can generate minimum bias (one or both arrays hit) and multiplicity triggers (semi-central and central).

Since the T0 detector generates the earliest (L0) trigger signals, they must be generated online without the possibility of any offline corrections. The offline corrections are applied for non-trigger signals, such as the time reference for the TOF detector. The dead time of the detector should be less than the bunch-crossing period in pp collisions ( $25 \mathrm{~ns})$.

\subsubsection{Detector layout}

The detector consists of two arrays of Cherenkov counters, 12 counters per array. Each Cherenkov counter is based on a Russian made fine-mesh photomultiplier tube PMT-187, $30 \mathrm{~mm}$ in diameter, $45 \mathrm{~mm}$ long. Each PMT is optically coupled to a quartz radiator $20 \mathrm{~mm}$ in diameter and $20 \mathrm{~mm}$ thick. One of the arrays, T0-C in figure 5.21 , is placed $72.7 \mathrm{~cm}$ from the nominal vertex. Such a small distance had to be chosen because of the space constraints imposed by the front cone of the muon absorber and other forward detectors. The pseudo-rapidity range of T0-C is $-3.28 \leq$ $\eta \leq-2.97$. On the opposite side of the Interaction Point (IP), the distance of the array (T0-A in figure 5.21) is about $375 \mathrm{~cm}$ - comfortably far from the congested central region. The T0-A is grouped together with the other forward detectors (FMD, V0, and PMD) and covers the pseudorapidity range of $4.61 \leq \eta \leq 4.92$. In the radial (transverse) direction both $\mathrm{T} 0$ arrays are placed as close to the beam pipe as possible to maximize triggering efficiency.

The triggering efficiency of the detector for minimum bias pp collisions, estimated by Monte Carlo simulations, is about $40 \%$ for all inelastic processes at $14 \mathrm{TeV}$. The individual efficiencies of T0-A and T0-C are 50\% and 59\%, respectively. The triggering efficiency in heavy-ion collisions is, due to the high multiplicities, practically $100 \%$. The basic parameters of the T0 detector are summarized in table 5.5.

\subsubsection{Fast electronics and readout}

The T0 electronics consists of the front-end electronics, located close to the arrays, inside the so-called shoeboxes, and the main $\mathrm{T} 0$ electronics, placed inside the T0 electronic racks, already outside of the L3 magnet, close to the racks assigned to ALICE trigger electronics. All amplitude, timing and trigger signals will be digitized and stored by the ALICE DAQ.

The T0-C and T0-A arrays generate timing signals, which feed directly to the TRD, to be used as a pre-trigger 'wake-up' signal. Due to the length of the connecting cables the coincidence 


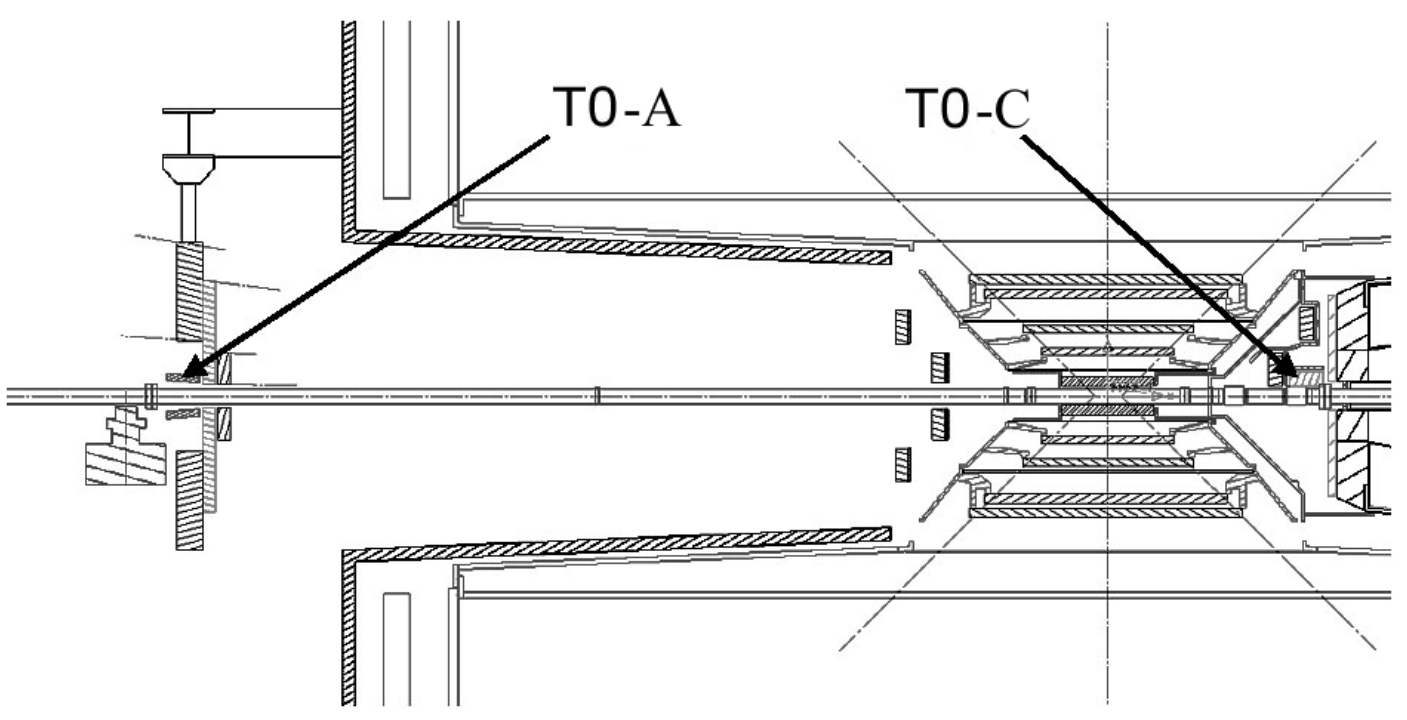

Figure 5.21: The layout of T0 detector arrays inside ALICE.

Table 5.5: Overview of the T0 detector parameters.

\begin{tabular}{|l|c|c|}
\hline Parameters & T0-A & T0-C \\
\hline \hline$z$-position $(\mathrm{cm})$ & +375 & -72.7 \\
$r$-position $(\mathrm{cm})$ & 6.5 & 6.5 \\
Number of Cherenkov counters & 12 & 12 \\
Pseudorapidity coverage & $+4.61 /+4.92$ & $-3.28 /-2.97$ \\
Detector active area $\left(\mathrm{cm}^{2}\right)$ & 37.7 & 37.7 \\
Efficiency with beam pipe $(\%)$ & 50 & 59 \\
\hline Efficiency with beam pipe of both arrays in co-incidence $(\%)$ & 40 \\
Time resolution of each PMT module $(\mathrm{ps})$ & 37 \\
Online vertex position resolution $(\mathrm{cm})$ & 1.5 \\
\hline
\end{tabular}

requirement for the 'wake-up' is implemented not in the T0 electronic racks, but directly at the TRD level.

The T0 timing signal is generated online by a mean timer. The position of the T0 signal on the time axis is equal to $(\mathrm{T} 0-\mathrm{C}+\mathrm{T} 0-\mathrm{A}) / 2+\mathrm{T}_{\text {delay }}$, where $\mathrm{T}_{\text {delay }}$ is the fixed delay of the analogue mean timer.

The position of the vertex is measured as $\mathrm{T} 0-\mathrm{A}-\mathrm{T} 0-\mathrm{C}$ and this value is sent to a digital discriminator with preset upper and lower limits thus providing the $\mathrm{T}_{\text {vertex }}$ trigger signal.

$\mathrm{The} \mathrm{T}_{\text {semi-central }}$ and $\mathrm{T} 0_{\text {central }}$ multiplicity trigger signals are generated by 2 discriminator levels applied to the linear sum of the amplitudes from all the detectors in the array.

In order to achieve the very high online time resolution of about $50 \mathrm{ps}$, the T0 detector is equipped with sophisticated fast timing electronics [194]. The most crucial elements of the T0 electronics are: the timing discriminators, the mean timer, and the module generating $\mathrm{T} 0_{\text {vertex }}$ signal for the trigger. All these components have dead time below $25 \mathrm{~ns}$, good stability and long-term 


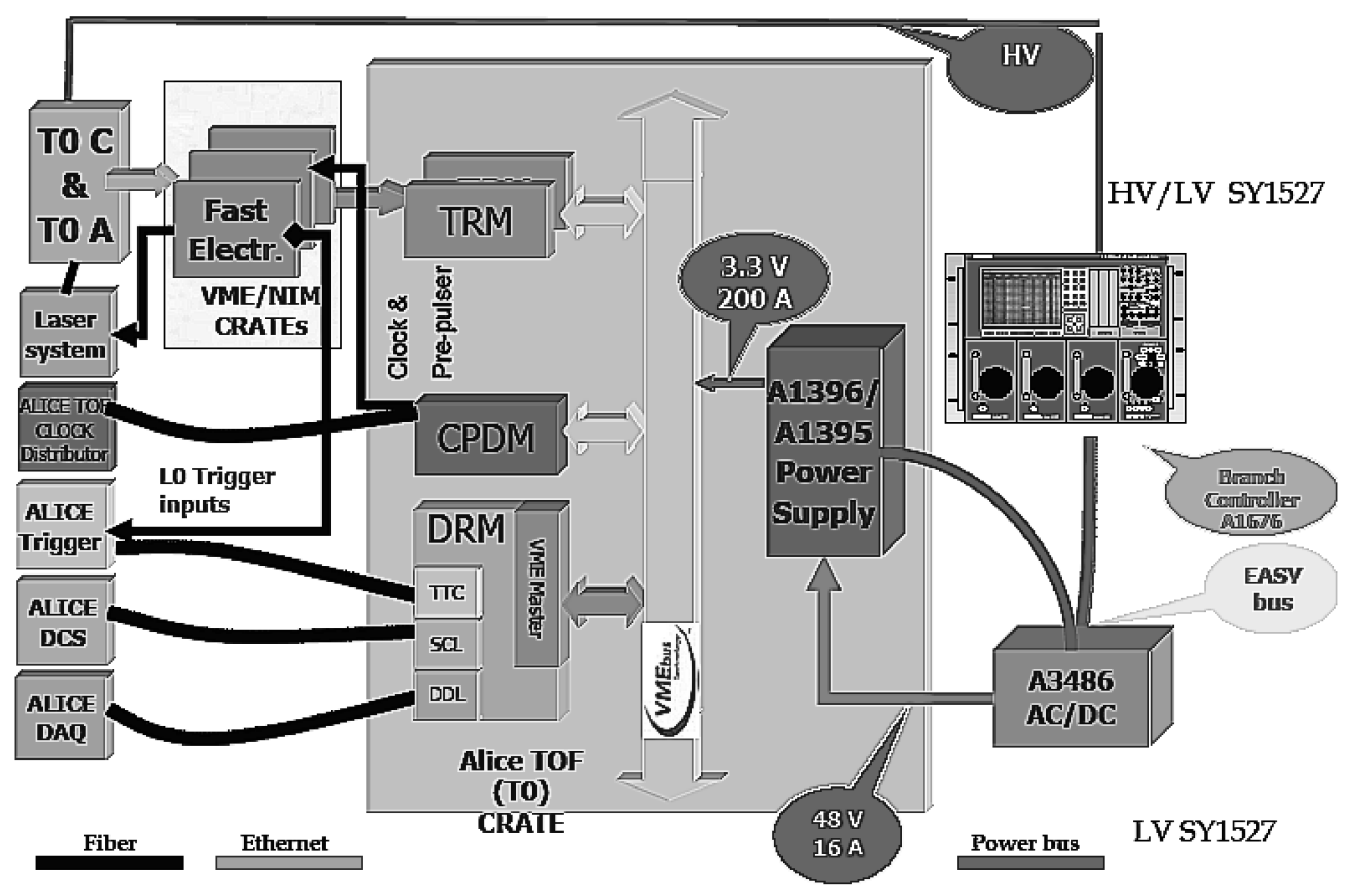

Figure 5.22: Block diagram of $\mathrm{T} 0$ hardware.

reliability. The modules were tested using a picosecond diode laser [195], and PS beams at CERN.

The only commercial unit in the full fast electronics chain is a Canberra Constant Fraction Discriminator model 454. It operates over the range of input amplitudes $50-3000 \mathrm{mV}$, corresponding to a dynamic range 1-60 MIP. The measured time walk, that has to be corrected offline, is 120 ps. The parameters of the mean timer and the $\mathrm{T}_{\text {vertex }}$ module were evaluated using two Cherenkov counters, emulating the two T0 arrays of PMTs, and $6 \mathrm{GeV} / c$ negative pion and kaon beams from CERN PS. The online position of the mean timer output signal in this set-up was stable on the time axis within \pm 10 ps. The time resolution of each PMT module was measured to be about $\sigma=37 \mathrm{ps}$. The vertex position resolution of the trigger module was also very good $(\sigma=1.5 \mathrm{~cm})$ in the full range of allowed positions.

The readout electronics, shown in the centre of figure 5.22, consists of CPDM (Clock and Pulse Distribution module), TRM (TDC Readout Module) and DRM (Data Readout Module) cards. The CPDM card is used to distribute 40MHz low-jitter LHC clock to TRM, DRM and to main T0 electronics. The TRM card houses the HPTDC, used by T0 in very high resolution mode (24.4 ps bin width). Each TRM card contains 30 HPTDC chips. All modules are quite similar to those used for the TOF detector. The time and amplitude for each of the 24 PMTs and the main signals produced by T0 electronics (interaction time, vertex, T0-C, T0-A, linear sum of amplitudes and 2 multiplicity signals) will be stored. The DRM card is the T0 interface to the ALICE DAQ system, it reads and encodes the data from the TRM cards and sends them to the ALICE DAQ via the DDL optical link. The DRM card receives the trigger information from the CTP via the TTCrx chip and performs a slow-control function with a dedicated CPU. 


\section{Chapter 6}

\section{Trigger, online and offline computing}

\subsection{Trigger system}

\subsubsection{Design considerations}

The ALICE Central Trigger Processor (CTP) [17, 196-198] is designed to select events having a variety of different features at rates which can be scaled down to suit physics requirements and the restrictions imposed by the bandwidth of the Data Acquisition (DAQ) system, see section 6.2, and the High-Level Trigger (HLT), see section 6.3. The challenge for the ALICE trigger is to make optimum use of the component detectors, which are busy for widely different periods following a valid trigger, and to perform trigger selections in a way which is optimised for several different running modes: ion ( $\mathrm{Pb}-\mathrm{Pb}$ and several lighter species), $\mathrm{pA}$, and $\mathrm{pp}$, varying by almost two orders of magnitude in counting rate.

The first response from the trigger system has to be fast to suit the detector requirements. The principal design requirement for the tracking detectors is to be able to cope with the large multiplicities in $\mathrm{Pb}-\mathrm{Pb}$ collisions (interaction rate $8 \mathrm{kHz}$ at $\mathscr{L}=10^{30} \mathrm{~cm}^{-2} \mathrm{~s}^{-1}$ ). In some cases this has led to the use of non-pipelined 'track and hold' electronics (e.g. for those detectors using the GASSIPLEX [124] front-end chip) and these can require a strobe at $1.2 \mu \mathrm{s}$. To achieve this, the 'fast' part of the trigger is split into two levels: a Level 0 (L0) signal, which reaches detectors at $1.2 \mu \mathrm{s}$, but which is too fast to receive all the trigger inputs, and a Level 1 (L1) signal arriving at $6.5 \mu \mathrm{s}$, which picks up all remaining fast inputs. Note that the CTP decisions are made in $100 \mathrm{~ns}$, with the rest of the L0 latency coming from the generation time for the trigger input signals and from the cable delays.

Another feature of the ALICE environment is that the high multiplicities of $\mathrm{Pb}-\mathrm{Pb}$ collisions make events containing more than one central collision unreconstructable. For this reason, 'pastfuture protection' (see below) is an important part of the ALICE trigger. A final level of the trigger (Level 2, L2) waits for the end of the past-future protection interval ( $88 \mu \mathrm{s})$ to verify that the event can be taken. This interval can also be used for running trigger algorithms.

The CTP consists of seven different types of $6 \mathrm{U}$ VME boards housed in a single VME crate. The signals are distributed to the detectors using the Local Trigger Unit (LTU). Transmission of trigger signals to each detector is mediated by one of these boards. In addition, the boards can be decoupled from the CTP and used to emulate CTP signals for testing purposes. Emulation of 
trigger sequences using the LTU is an important part of the testing and commissioning of detector electronics.

\subsubsection{Trigger logic}

The number of trigger inputs and classes required for ALICE (24 L0 inputs, 24 L1 inputs, 12 L2 inputs, 50 trigger classes - see below) means that the trigger logic requires some restrictions, since a simple enumeration of all outcomes (look-up table approach) is not feasible. An investigation of the trigger conditions actually required for ALICE shows that these can be accommodated by logic involving three states of the inputs (asserted, negated, not relevant), coupled by ANDs. Negated inputs are rarely used, so they are available for only six out of the fifty trigger classes, the rest allowing the two requirements asserted and not relevant only.

The definition of an interaction, the basis of the minimum bias trigger, needs an OR of different detector inputs, owing to lower trigger efficiencies in pp interactions because of lower multiplicities. In order to do this, four specific L0 inputs are selected for use in a look-up table for which any arbitrary logic can be applied. Two independent functions can be defined. These derived functions can be used as additional conditions with any other L0 input in defining the overall L0 conditions. Note that these same four inputs also define two further independent functions which are used as the 'interaction' signal in the past-future protection circuits.

We define a trigger class in terms of the logical condition demanded for the inputs, the set of detectors required for readout (a maximum of six combinations, called detector clusters, can be defined at any one time), the past-future protection requirements (which are in fact determined by the detector set) and in addition scaling factors, handling of Region-of-Interest (RoI) data and handling of rare triggers. These features are described in more detail below.

Table 6.1 gives a list of trigger inputs for L0 and table 6.2 those for L1. The suffices ' $m b$ ', 'sc' and 'ce' refer to the centrality conditions for minimum bias, semi-central and central events respectively. In table 6.1 the 'ZDC diss. single arm' trigger input is one which gives a rough indication of an electromagnetic dissociation signal, based on a single arm of the ZDC. It is confirmed by the 'ZDC diss' signal in table 6.2, which is much more selective. TOF provides two cosmic ray trigger inputs, one for a single vertical muon, and one for several muons simultaneously. Inputs relevant for a specific data taking period must therefore be selected using a patch panel. Table 6.3 gives the list of trigger classes for pp interactions at LHC startup.

The trigger inputs for V0, TRD and SPD are not given in detail. For V0 there are four basic signals, corresponding to hits on either side of the interaction region in the time windows for incoming beam-gas and outgoing colliding-beam interactions, the latter classified according to multiplicity. The V0 electronics offers all these signals and certain Boolean combinations of them, making sixteen signals in all. Any five of these can be sent to the CTP using programmable logic.

Note that the 'TRD pre-trigger' input fulfils a different function from the other trigger inputs. It is formed by copies of the T0, V0 and TOF inputs sent in parallel to the TRD, where they serve to 'wake-up' the TRD electronics, which otherwise is in a standby state. Only if the TRD pretrigger is sent, the TRD is ready to accept triggers, or to be read out. Therefore, the confirmatory TRD pre-trigger signal (interaction detected AND TRD in ready state) is a prerequisite for any class for which TRD triggers or readout are required. The other TRD L0 inputs in fact reflect 
Table 6.1: List of $\mathrm{L} 0$ trigger inputs for $\mathrm{Pb}-\mathrm{Pb}$ and $\mathrm{pp}$ collisions. The $*$ indicates when the input is implemented.

\begin{tabular}{|r|c|l|c|c|c|c|l|c|c|}
\hline No. & Det. & \multicolumn{1}{|c|}{ Description } & Pb-Pb & pp & No. & Det. & Description & Pb-Pb & pp \\
\hline \hline 1 & MTR & DM like high $p_{\mathrm{t}}$ & $*$ & $*$ & 21 & TOF & High Multiplicity & & $*$ \\
2 & & DM unlike high $p_{\mathrm{t}}$ & $*$ & $*$ & 22 & V0 & V01 & $*$ & $*$ \\
3 & & DM like low $p_{\mathrm{t}}$ & $*$ & $*$ & 23 & & V02 & $*$ & $*$ \\
4 & & DM unlike low $p_{\mathrm{t}}$ & $*$ & $*$ & 24 & & V03 & $*$ & $*$ \\
5 & & DM single & $*$ & $*$ & 25 & & V04 & $*$ & $*$ \\
6 & T0 & T0 vertex & & $*$ & 26 & & V05 & $*$ & $*$ \\
7 & & T0C & $*$ & 27 & TRD & Pretrigger & $*$ & $*$ \\
8 & & T0A & $*$ & 28 & & TRD2 & & \\
9 & & T0 sc & $*$ & & 29 & & TRD3 & & \\
10 & & T0 ce & $*$ & $*$ & 31 & & TRD4 & & \\
11 & PHO & PHOS signal & & & TRD5 & & \\
12 & ZDC & ZDC diss. single arm & $*$ & & 32 & SPD & SPD MB & & $*$ \\
13 & EMC & EMCAL single shower & $*$ & $*$ & 33 & & SPD2 & & \\
14 & ACO & ACORDE single muon & $*$ & $*$ & 34 & & SPD3 & & \\
15 & & multi-muon & $*$ & $*$ & 35 & & SPD4 & & \\
16 & TOF & cosmic vert. muon & $*$ & $*$ & 36 & & SPD5 & & \\
17 & & cosmic multi-muon & $*$ & $*$ & 37 & & SPD6 & & \\
18 & & MB & & $*$ & 38 & & SPD7 & & \\
19 & & ultraperipheral $(\rho)$ & & $*$ & 39 & & SPD8 & & \\
20 & & ultraperipheral $(\mathrm{J} / \Psi)$ & & $*$ & 40 & & SPD9 & & \\
& & & & & 41 & & SPD10 & & \\
\hline
\end{tabular}

different requirements on the pre-trigger signals. In particular, different combinations of TOF signals (indicating activity at central rapidity) in combination with no activity in V0 can be used to select double diffractive events in pp interactions, while hits in T0 and V0 can be used to signal 'normal' events. The TRD also offers trigger signals at L1, which are 'true' TRD inputs in that they derive from the signals in the TRD detector. The exact allocation of trigger logic will be based on charged-track $p_{\mathrm{t}}$ cuts and electron identification in combination with multiplicity requirements (e.g. like sign high- $p_{\mathrm{t}}$ dielectron pair, four or more high- $p_{\mathrm{t}}$ tracks, identified electron, etc.).

The pixel detector (SPD) also provides trigger signals at L0 level, though its latency does not allow its use in the TRD pre-trigger. Several inputs are reserved for SPD triggers, where, in addition to simple multiplicity requirements, other signals, such as triggers with tracks directed towards the HMPID. These can be set up in a programmable way to run in parallel with the main multiplicity-based trigger selections.

Table 6.3 gives an example of a set of possible trigger class conditions (called trigger descriptors). The signal names 'V0or', 'BEAMGASor' and 'BEAMGASc' correspond to possible settings of the five V0 signals; 'V0or' means a hit in the appropriate time window on either side 
Table 6.2: List of $\mathrm{L} 1$ trigger inputs for $\mathrm{Pb}-\mathrm{Pb}$ and $\mathrm{pp}$ collisions. The $*$ indicates when the input is implemented.

\begin{tabular}{|r|c|l|c|c|}
\hline No. & Det. & \multicolumn{1}{|c|}{ Description } & Pb-Pb & pp \\
\hline \hline 1 & PHO & PHOS low $\mathrm{E}_{\mathrm{t}}$ & $*$ & $*$ \\
2 & & ${\text { PHOS med } \mathrm{E}_{\mathrm{t}}}$ & $*$ & $*$ \\
3 & & PHOS high $\mathrm{E}_{\mathrm{t}}$ & $*$ & $*$ \\
4 & TOF & jet trigger & & $*$ \\
5 & TRD & TRD1 & $*$ & $*$ \\
6 & & TRD2 & $*$ & $*$ \\
7 & & TRD3 & $*$ & $*$ \\
8 & & TRD4 & $*$ & $*$ \\
9 & & TRD5 & $*$ & $*$ \\
10 & & TRD6 & $*$ & $*$ \\
11 & ZDC & ZDC mb & $*$ & $*$ \\
12 & & ZDC sc & $*$ & $*$ \\
13 & & ZDC ce & $*$ & $*$ \\
14 & & ZDC diss. & $*$ & \\
15 & EMC & EMCAL single shower low & $*$ & $*$ \\
16 & & EMCAL single shower med & $*$ & $*$ \\
17 & & EMCAL single shower high & $*$ & $*$ \\
18 & & EMCAL jet & $*$ & $*$ \\
\hline
\end{tabular}

of V0, and 'BEAMGASor', 'BEAMGASc' to signals in the time windows for beam gas on either side (suffix 'or') or coming from the C-side (suffix ' $c$ '). 
Table 6.3: List of trigger classes with trigger conditions. The symbols $\oplus$ and $\odot$ indicate OR and AND respectively.

\begin{tabular}{|c|l|l|}
\hline Number & Description & \multicolumn{1}{|c|}{ Condition } \\
\hline \hline 1 & MB1 & V0or $\oplus$ PIXELor $\odot \overline{\text { BEAMGASor }}$ \\
2 & MB2 & V0or $\odot$ PIXELor $\odot$ BEAMGASor \\
3 & MB3 & V0and $\odot$ PIXELor $\odot \overline{\text { BEAMGASor }}$ \\
4 & BG & BEAMGASor \\
5 & BGDM & BEAMGASc \\
6 & DMsingle & DMsingle \\
7 & DMBeamgas & BGc $\odot$ DMsingle \\
8 & DML & DMLikeLow \\
9 & DMU & DMUnlikeLow \\
10 & DMMBSingle & MB1 $\odot$ DMSingle \\
11 & DMMBLike & MB1 $\odot$ DMLikeLow \\
12 & DMMBUnlike & MB1 $\odot$ DMUnlikeLow \\
13 & Photon1 & MB1 $\odot$ LOPHOSMB \\
14 & Photon2 & MB1 $\odot$ L0PHOSLE \\
15 & Photon3 & MB1 $\odot$ LOPHOSHE \\
16 & Photon4 & MB1 $\odot$ L1PHOSIP \\
\hline
\end{tabular}




\subsubsection{Trigger inputs and classes}

The trigger inputs and classes were introduced in the previous section. Trigger inputs are pulses provided by the trigger detectors and synchronised to the LHC clock cycle, as distributed by the RD12 Trigger Timing and Control (TTC) system [199, 200].

Trigger inputs are sent as LVDS signals using twisted pair cables, and are put in time by delays in the CTP input circuits. The trigger outputs are returned to the detectors using either similar twisted pair cables or signals sent on the TTC system for L0, and the TTC system only (using optical fibre) for the other levels. Trigger data are transmitted with each successful L1 and L2 trigger, as described in the next section.

The purpose of the past-future protection circuit is to ensure that the events selected for readout are not spoilt by pile-up. As pile-up will be frequent, or, in some cases, inevitable at expected LHC luminosities (e.g. for $\mathscr{L}=10^{30} \mathrm{~cm}^{-2} \mathrm{~s}^{-1}$ in pp collisions), the pile-up protection must do more than simply reject events if any other event occurs within a specified time window. In $\mathrm{Pb}-\mathrm{Pb}$ collisions, the circuit makes use of a classification of events into two groups, peripheral and semicentral, and specifies separate limits for each. The time windows are set to be equal to the sensitive windows for the detectors, i.e. the (nominal) maximum time over which two signals from two different events could overlap. Thus, for instance, the sensitive window for the TPC is $\pm 88 \mu \mathrm{s}$, so for clusters in which the TPC is included a possible past-future protection specification, for example, to demand no more than 2 additional peripheral events and no additional semi-central events to take place in an interval of twice $88 \mu$ s centred on the event under consideration.

A slightly different approach is required for proton-proton interactions, where the increased luminosity makes pile-up a certainty, but where the multiplicities are much lower than in ion-ion interactions, and therefore some degree of pile-up is tolerable. Here, what is important is to ensure that all detectors in a cluster read out in a satisfactory manner. For example, pile-up in the ITS is more serious for reconstruction than pile-up in the TPC. For this reason, in this case the past-future protection does not select on multiplicity, but offers checks in two different time windows (e.g. $\pm 10 \mu$ s for the ITS and $\pm 88 \mu$ s for the TPC) so as to ensure that pile-up is not excessive in either.

In general, the input data handled by the ALICE CTP is global, in the sense that the CTP does not correlate specific geometrical regions in different detectors. However, for certain applications it might not be necessary to record data from all regions of the detector, but only from certain azimuthal sectors, with an obvious saving in the overall data volume. The boundaries between these different azimuthal sectors, which define 'Regions-of-Interest' (RoI), line up in the larger central detectors TPC, TRD and TOF, and equivalent boundaries could be imposed in software in the ITS. If a given trigger detector can identify an azimuthal sector as being the one carrying the information giving rise to the trigger (e.g. the presence of a high- $p_{\mathrm{t}}$ electron), it is foreseen that a flag can be set identifying the sectors of interest. These can then be treated in a special way, e.g. by selecting only those sectors for readout to DAQ, or by treating them differently in the HLT.

\subsubsection{Trigger data}

The CTP must be able to process triggers for all trigger clusters concurrently, and therefore has a tight time budget for collecting and distributing data. For this reason these data are kept to a minimum; for example, all information concerning how a trigger signal was generated must be 
obtained from the detector which produces it. Several types of data are recorded concerning the operation of the trigger, as listed below.

Event data. For each accepted event, information is sent to each detector specifying the event identifier (orbit number and bunch crossing number), trigger type (physics trigger, software trigger, calibration trigger), detector set to be read out (given as a cluster list for physics data) and the list of active trigger classes. These data are sent in the L2 accept (L2a) message, which goes to every TTCrx (TTC receiver chip) on each detector.

Interaction record. All interactions that occur in a given orbit are recorded (giving bunch crossing number, and, for $\mathrm{Pb}-\mathrm{Pb}$ events, a classification into centrality categories: peripheral and semicentral). The principal purpose of this record is to aid pile-up correction. Out-of-time events recorded in the TPC will have different apparent origins from triggered events owing to their different drift times. Their apparent origin can be computed using the interaction record, thus aiding their removal from the event data. The data are sent as special records in the data stream, and are collected in the trigger Local Data Concentrator (LDC).

Scalers. A large number of scalers are recorded. In particular, all inputs are counted, and for each trigger class the number of events passing each stage of the trigger (L0, L1, L2, after physics selections, if any, and again after the corresponding BUSY and past-future protection constraints have been applied.) These data are essential both for on-line monitoring and for subsequent offline luminosity and cross-section calculations. Scalers are read at regular intervals (currently every minute) and their values are broadcast using a Distributed Information Management system (DIM) server [201]. The values are recorded in the 'ProzeßVisualisierungs und SteuerungsSystem' (PVSS) [202], where ratios can be monitored to check the correct operation of the trigger. At the end of a run, a file containing both the individual scaler readings and their sum for the run just ended is transmitted from PVSS to DAQ. Notice that the use of a DIM server to transmit from the CTP to PVSS opens the possibility of defining other DIM clients, should this be required.

Snapshots. For completeness, we note that it will also be possible to run 'snapshots' in which all steps in the trigger (input and output patterns, busy status, etc.) are recorded on a bunch-crossing by bunch-crossing basis for a period of about $30 \mathrm{~ms}$. The purpose originally foreseen for this facility is for diagnostic tests of the CTP itself. Snapshots can also be used for checks of correlations between CTP inputs, since an unbiased record of all input data received during the $30 \mathrm{~ms}$ snapshot period can be analysed. For example, time correlations between a pulse on one input and pulses in other input channels in adjacent bunch crossings can be studied; trigger efficiencies for different inputs can also be studied.

\subsubsection{Event rates and rare events}

When several trigger classes are running concurrently, it becomes necessary to adjust the rates at which they are read out to reflect the physics requirements and the overall DAQ bandwidth. These factors may dictate rates quite different from the natural interaction rates. 
There are two main mechanisms for controlling readout rates. Downscaling factors may be applied to each trigger class, specifying, for example, that only every $n$th event should be read out. This mechanism allows gross adjustments to be made, but is inflexible because the downscaling factors remain in place throughout a physics run. This means that the change in interaction rate as the luminosity decays following each fill cannot be followed. For this reason, in addition to downscaling factors, a second method is used. Studies of data flow through the front end and DAQ systems show that with the current choices for numbers of front end buffers, saturation of front end data storage can be avoided, but without further action temporary data storage in the DAQ can become saturated, with a relaxation time of the order of seconds. This phenomenon would particularly affect rare processes, as these would find the available bandwidth for data recording utilized by more common processes. To avoid this problem, all trigger classes are classified into two groups: those corresponding to rare processes and those corresponding to common processes. Initially all activated trigger classes can generate triggers. On a signal from the DAQ, sent when the occupied temporary storage exceeds some preset 'high water mark', the common classes are temporarily disabled, thus ensuring continued available bandwidth for rare processes. When the available temporary storage has gone below some corresponding 'low water mark' the common classes are again enabled. Owing to the long relaxation time, timing is not critical, and software signals are adequate for toggling the suppression of common classes. Details of the model used to study the system response, and a description of a simple example, are given in section 6.2.4).

\subsection{Data AcQuisition (DAQ) System}

\subsubsection{Design considerations}

ALICE will study a variety of (physics) observables, using different beam conditions. A large number of trigger classes will be used to select and characterise the events. These trigger classes belong to two broad categories depending on whether they are frequent or rare.

Several triggers (central, semi-central and minimum bias) are so frequent that the limiting factor is the performance of the data acquisition system. These triggers will use a very large fraction of the total data acquisition bandwidth. On the other hand, rare triggers such as dimuon or dielectron events, use less bandwidth and are limited by the detector livetime and the luminosity. The experiment will use the data-taking periods in the most efficient way by acquiring data for several observables concurrently following different scenarios. The task of the ALICE Trigger, Data AcQuisition (DAQ) and High-Level Trigger (HLT) systems is therefore to select interesting physics events, to provide an efficient access to these events for the execution of high-level trigger algorithms and finally to archive the data to permanent data storage for later analysis.

The trigger and DAQ systems were designed to give different observables a fair share of the trigger and DAQ resources with respect to DAQ bandwidth for frequent triggers and detector livetime for rare triggers. The trigger and DAQ systems must balance the capacity to record central collisions which generate large events with the ability to acquire the largest possible fraction of rare events.

In the ALICE Technical Proposal [4-7], it was estimated that a bandwidth of $1.25 \mathrm{~GB} / \mathrm{s}$ to mass storage would provide adequate physics statistics. While the current estimate of event sizes, 
trigger rates and, in particular, the number of physics observables (and therefore trigger classes) has increased considerably, it is still possible to satisfy the physics requirements with the same total bandwidth by using a combination of increased trigger selectivity, data compression and partial readout [203]. Detailed scenarios of use of this bandwidth were elaborated in the ALICE Technical Design Report on Trigger, Data Acquisition, High-Level Trigger and Control System [17]. This bandwidth is consistent with constraints imposed by technology, cost and storage capacity, including the cost of the media needed to store the data and the computing needed to reconstruct and analyse these data. The Tier-0 of the LHC Computing Grid project, that is being assembled at CERN, will provide a bandwidth to mass storage higher than the needs of ALICE for data acquisition. However, efforts will be continued to reduce the bandwidth without jeopardising the physics performance.

\subsubsection{System architecture}

\section{System architecture}

The architecture of the data acquisition is shown in figure 6.1. The detectors receive the trigger signals and the associated information from the Central Trigger Processor (CTP), through a dedicated Local Trigger Unit (LTU) interfaced to a Timing, Trigger and Control (TTC) system. The Front-End Read-Out (FERO) electronics of the detectors is interfaced to the ALICE-standard Detector Data Links (DDL). The data produced by the detectors (event fragments) are injected on the DDLs using the same standard protocol. The fact that all the detectors use the DDL is one of the major architectural features of the ALICE DAQ.

At the receiving side of the DDLs there are PCI-X based electronic modules, called 'DAQ Readout Receiver Card' (D-RORC). The D-RORCs are hosted by the front-end machines (commodity PCs), called Local Data Concentrators (LDCs). Each LDC can handle one or more DRORCs. The D-RORCs perform concurrent and autonomous Direct Memory Access (DMA) transfers into the LDCs' memory, with minimal software intervention. The event fragments originated by the various D-RORCs are logically assembled into sub-events in the LDCs.

The CTP receives a busy signal from each detector. This signal can be generated either in the detector FERO's or from all the D-RORCs of a detector. These two options are respectively shown on the left and right detectors sketched in figure 6.1. The CTP also receives a signal from the DAQ enabling or disabling the most common triggers. It is used to increase the acceptance of rare triggers by reducing the detector dead-time. This signal is generated from the buffer occupancy in all the LDCs.

The role of the LDCs is to ship the sub-events to a farm of machines (also commodity PCs) called Global Data Collectors (GDCs), where the whole events are built (from all the sub-events pertaining to the same trigger). Another major architectural feature of the ALICE DAQ is the event builder, which is based upon an event-building network. The sub-event distribution is driven by the LDCs, which decide the destination of each sub-event. This decision is taken by each LDC independently from the others (no communication between the LDCs is necessary), but it is synchronized among them by a data-driven algorithm, designed to share fairly the load on the GDCs. The Event-Destination Manager (EDM) broadcasts information about the availability of the GDCs to all LDCs. The event-building network does not take part in the decision; it is a standard commu- 


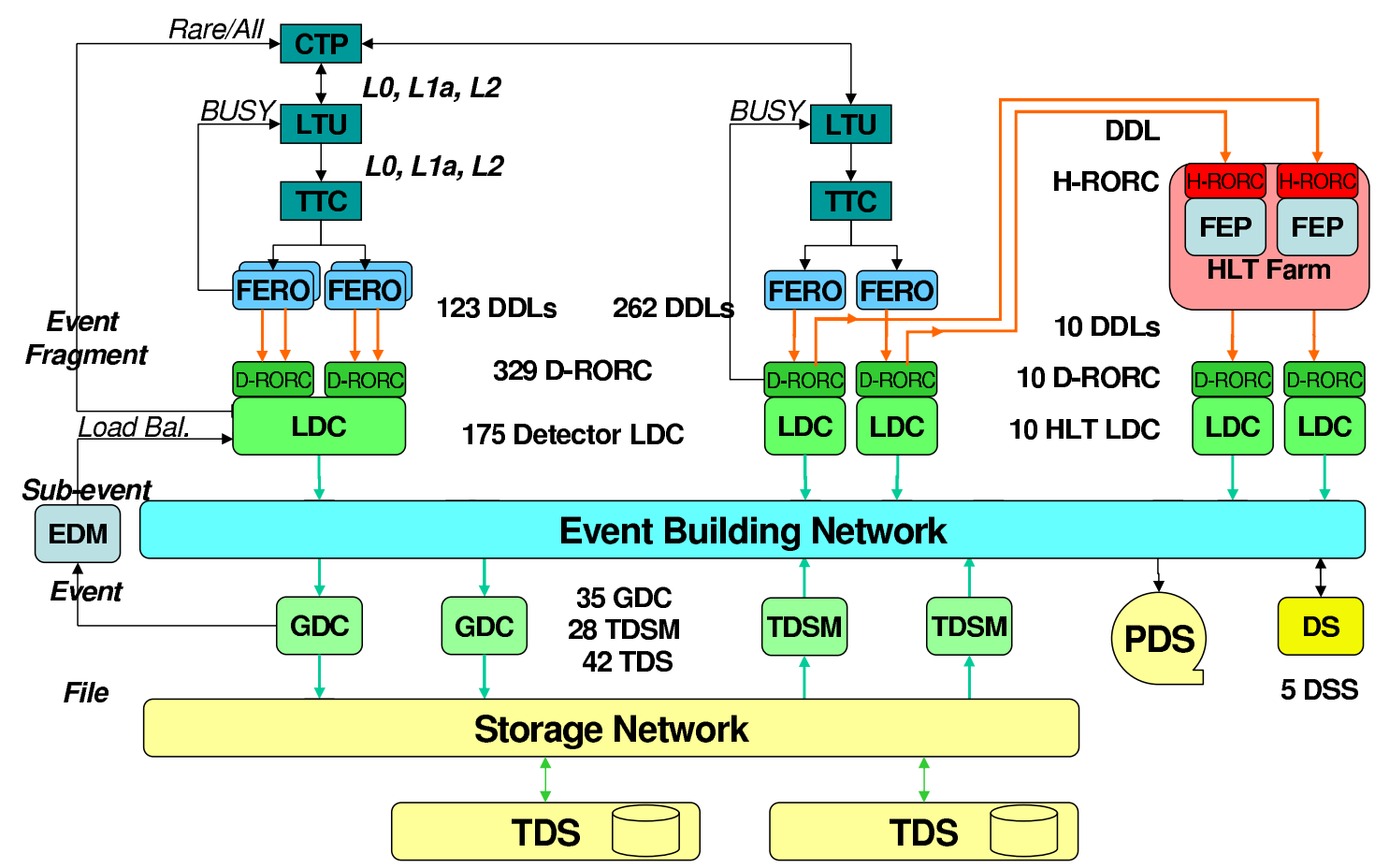

Figure 6.1: The overall architecture of the ALICE DAQ and the interface to the HLT system.

nication network (commodity equipment) supporting the well-established TCP/IP protocol. The role of the GDCs is to collect the sub-events and assemble them into whole events. The GDCs also feed the recording system with the events that eventually end up in Permanent Data Storage (PDS).

The HLT system receives a copy of all relevant raw data via DDLs and the 'HLT Readout Receiver Card' (H-RORC) into the Front-End Processors (FEP). The generated data and decisions are transferred to dedicated LDCs.

\section{Data transfer}

The Detector Data Link (DDL) is the common hardware and protocol interface between the frontend electronics and the DAQ system. The transmission medium is a pair of optical fibres linking sites several hundred metres apart. The DDL is able to transmit data in both directions with a sustained bandwidth of $200 \mathrm{MB} / \mathrm{s}$. It can be used to send commands and bulky information (typically pedestals or other calibration constants) to the detectors [204, 205].

The D-RORC is the readout board of the DDL link. It interfaces the DDL to the PCI bus of the host LDC computer. In addition, the D-RORC grants to the LDC full control of all the features of the DDL. The D-RORC technology is based on a FPGA. The card is able to transfer the event fragments from the link directly into the PC memory at $200 \mathrm{MB} / \mathrm{s}$, without on-board buffering; this bandwidth fulfils (and far exceeds) the original requirement of the ALICE dataacquisition system. The Direct Memory Access (DMA) transfer is carried out by the firmware in co-operation with the readout software running on the LDC. In this closely coupled operation, the role of the software is limited to the bookkeeping of the data-block descriptors. This approach 
allows sustained autonomous DMA with little CPU assistance and minimal software overhead. The data formatting introduced by the DDL allows the D-RORC to separate the event fragments in memory, without interrupting the host CPU. Performance measurements and long-term tests show that the system fully exploits the PCI bandwidth and that the transfer rate is largely independent of the block size.

The D-RORC includes two DDL interfaces. For the detectors whose data are analysed by the HLT system, one of the DDL interfaces is used to transfer a copy of the raw data to the HLT computers. RORC prototypes were built based on the PCI and PCI-X standards [206, 207]. These prototypes were used to develop the DAQ system to integrate the DAQ with the detector electronics.

\section{Event building}

The sub-events prepared by the LDCs are transferred to one GDC where the full event can be assembled. The event building is managed by the Event Building and Distribution System (EBDS). The EBDS distributed protocol runs on all the machines, LDCs and GDCs, participating as data sources or destinations. The goals of the EBDS are to synchronise all the LDCs concerning the GDC used as destination and to balance the loads on the different GDCs. The aim is to keep up with the data-flow while keeping the EBDS protocol overhead as low as possible.

The de-facto standard TCP/IP is used as the transport mechanism for the EBDS protocol and for the data transfer. This protocol is the standard to which the networking industry has converged and it runs on a wide range of hardware layers. Ethernet is the dominant, ubiquitous Local Area Network (LAN) technology. It has therefore become the baseline choice for the event-building network. However, as long as we can rely on a standard protocol such as TCP/IP, the hardware layer used in the event-building network could be modified if a better technology would become available during the experiment lifetime.

\section{Data storage}

The GDCs archive the data over the storage network as data files of a fixed size to the Transient Data Storage (TDS). During a run period, each GDC produces a sequence of such files and registers them in the ALICE Grid software (AliEn) [208]. The data files are then read by the TDS movers (TDSM) over the storage network and exported to the computing centre where they are recorded to the Permanent Data Storage (PDS). The TDS is made of storage arrays connected to the storage network. The storage network itself is based on Fibre Channel 4 gigabit/s (FC 4G). The shared access from all the nodes (GDCs and TDSMs) to the TDS is performed through a commerical cluster file system (Quantum StorNext).

\section{DATE: the DAQ software framework}

The Data Acquisition and Test Environment (DATE) [209, 210] is the DAQ software framework. It is a distributed, process-oriented system. It is designed to run on Unix platforms connected by an IP-capable network. It uses the standard Unix system tools available for process synchronisation and data transmission. The system configuration is realised with MySQL. 
The dataflow is realized in two steps. The LDC collects event fragments and reassembles them into sub-events. The LDC is also capable of local data recording, if used in stand-alone mode, and online sub-event monitoring. The GDC puts together all the sub-events pertaining to the same physics event, builds the full events and sends them to the mass storage system. The GDC is also capable of online event monitoring.

The DATE controls and synchronises the processes running in the LDCs and the GDCs. It can run on an LDC, a GDC or another computer. The monitoring programs receive data from the LDC or GDC streams. They can be executed on any LDC, GDC or any other machine accessible via the network.

\section{AFFAIR: the DAQ performance monitoring software}

The perfomance of a system as large as the ALICE Data Acquisition, including several processes distributed on many processors, needs to be continuously and closely monitored. The fundamental requirement for a detailed, real-time assessment of the DAQ machines (LDCs and GDCs), for the usage of the systems resources, and for the DATE performance is addressed by the AFFAIR package (see p. 217 in [17]). AFFAIR gathers performance metrics from the LDCs and GDCs and performs the centralised handling of them. Statistics and trends are stored in HTML format and can be viewed using any Web browser.

\section{MOOD: the DAQ framework for the Monitoring Of Online Data}

To monitor the quality of the data stream created by any of the ALICE detectors, the MOOD [211, 212] toolkit has been developed. MOOD is a data visualisation and data quality monitoring tool. It includes a generic part which implements the interface with DATE and a detector-specific part that can be tailored to detector-specific requirements and setups. MOOD is fully integrated with the ROOT development toolkit, the AliROOT environment, and uses the ALICE common event data format. MOOD can handle online and offline data streams, available on the LDCs and on the GDCs.

\section{AMORE: the DAQ framework for the Automatic MOnitoRing Environment}

The data quality monitoring is automatized. Each detector defines a set of physics plots which have to be continuously filled and checked against reference ones. The AMORE framework [213] includes three components: the client part which collects the data, the server part which accumulates the plots and archives them, and the display program which provides an interactive distributed access to the plots archives. In addition, alarms are raised as soon as collected plots do not conform any more to the expected reference. These alarms are displayed on the operator screens and initiate automatic recovery actions.

\subsubsection{System flexibility and scalability}

The requirements for the DAQ system evolve with data taking and with new ideas of high-level triggers. Currently, the only hard limit present in the design of the DAQ system is the number of DDLs used to read out the TPC and the TRD. The number of links has been fixed so that these two 
detectors can be completely read out at a maximum rate of $200 \mathrm{~Hz}$ at the highest anticipated multiplicity for central collisions. The next stages in the data flow chain (computers and event-building network) are completely scalable and their number can be adapted to the needs. No architectural change would be needed in the DAQ system to scale to a total throughput varying by a factor 4 or 5. This flexibility also authorise a staged deployment of the system and the implementation of new scenarios on short notice.

\subsubsection{Event rates and rare events}

The full data flow behaviour is modelled, using an interaction rate of $8 \mathrm{kHz}$ at nominal luminosity and realistic event size distributions of different trigger classes. The model consists of the Central Trigger Processor (CTP), the detectors, the DAQ, the High-Level Trigger (HLT) and the Permanent Data Storage (PDS) [214-216]. Events are characterised by different trigger classes, peripheral (PE), semi-central (SC) or central (CE), according to their multiplicity. In addition, an event can also be of the electron (EL) or muon (MU) trigger class based on an assigned probability. This model is based on the public-domain tool Ptolemy [217].

A rate control mechanism has been included in the DAQ such that no rare events (electron and muon) are lost due to back-pressure, while at the same time as many frequent events (peripheral, semi-central or central) are accepted as possible with the installed bandwidth.

This rate control is based on a back-pressure from the DAQ that detects the amount of data filled in each computer, and informs the trigger whenever this level reaches a high fraction of the buffer size. When this happens, the frequent events, as the main bandwidth contributors and events of lower priority, would then be blocked by the trigger.

Figure 6.2 gives the $\mathrm{L} 2$ rates as a function of time when the LDC high level is set to 0.3 , the low level is at 0.2 , and the downscaling factor for PE, SC and CE events is set at 0.2. The periodic behaviour of the PE, SC, and CE events is apparent, and so is the constant rate of the EL and MU events. The rate control increases the rates of rare events with $22 \%$ of EL events (it was 2\%) and $78 \%$ of MU events (it was $22 \%$ ) accepted. This is a large performance improvement and is close to the maximal rates possible, after past-future $(\mathrm{P} / \mathrm{F})$ rejection. The $\mathrm{PE}, \mathrm{SC}$, and $\mathrm{CE}$ events fill up the remaining bandwidth to the PDS.

Figure 6.3 shows the LDC occupancy as a function of time, from which it is clear that the LDCs are never full, and hence there is no loss due to LDC back-pressure. Figure 6.4 shows the fraction of time a detector is busy. The events lost due to busy detectors are only caused by detector readout dead times and by transfer limitations through the detector buffer chain. These are hardware properties of the detector and cannot be influenced by the DAQ or CTP.

In summary, an effective and robust method to maximise the L2 rates of rare and interesting events can be achieved by controlling the rates of peripheral, semi-peripheral and central events with feedback from the LDC occupancy and with downscaling. No difficulty in either messaging the information from the LDCs, or the stability of the system with different interaction rates or envisioned physics is anticipated.

\subsubsection{Data challenges}

The anticipated performances of the ALICE DAQ were verified one year before data taking. 


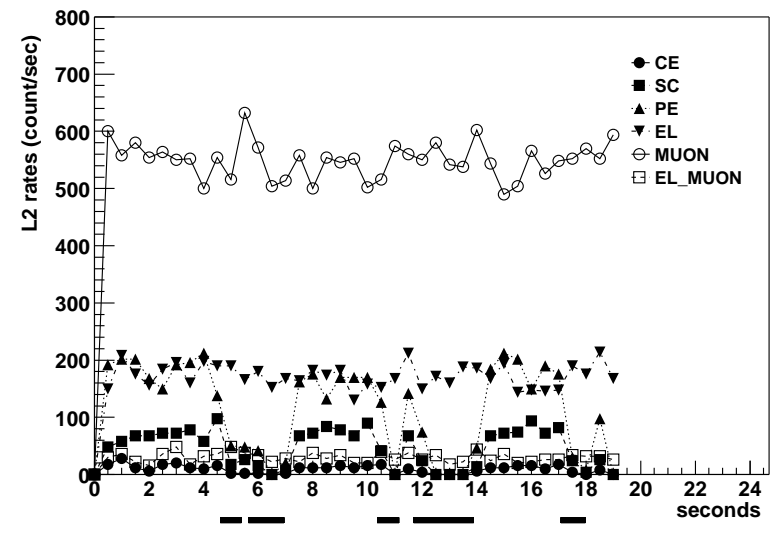

Figure 6.2: L2 rates as a function of time. The bold lines under the plot indicate the periods of activation of rare triggers only.

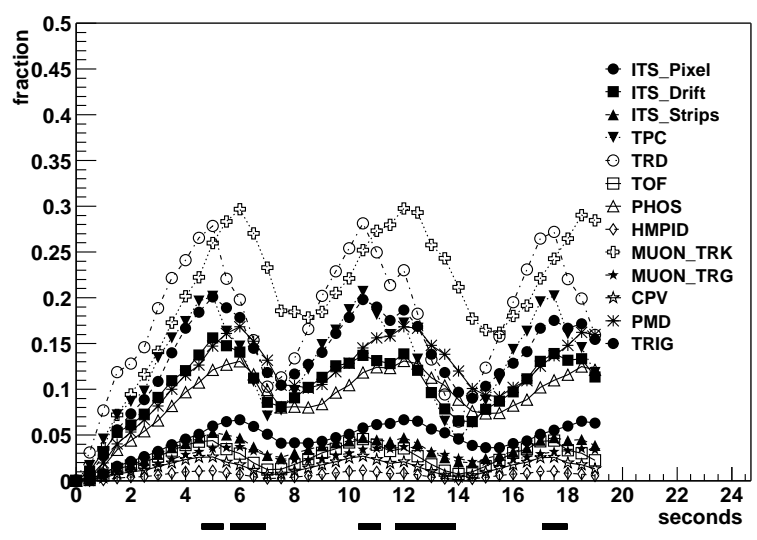

Figure 6.3: Fraction of the LDC buffer filled as a function of time. The bold lines under the plot indicate the periods of activation of rare triggers only.

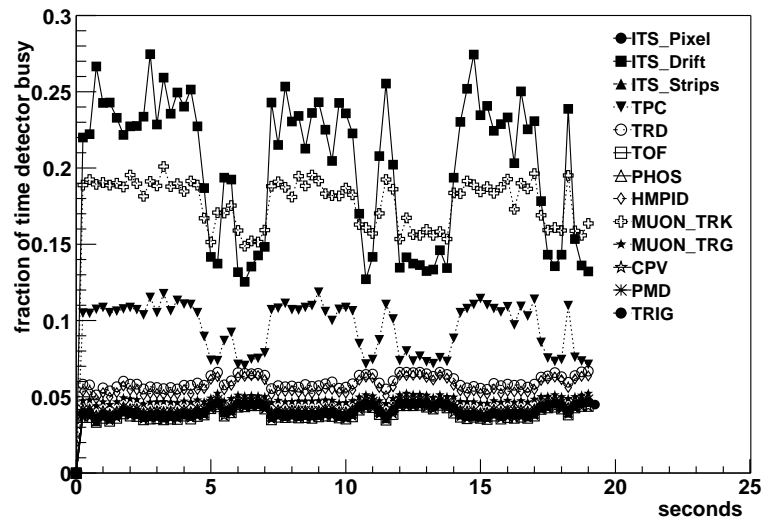

Figure 6.4: Fraction of time a detector is busy as a function of time. The bold lines under the plot indicate the periods of activation of rare triggers only.

The two curves of figure 6.5 show respectively the aggregate bandwidth to and from the event-building, and the bandwidth of the data archived to the MSS in the computing centre.

Owing to the unprecedented quantity of data to be stored in the MSS, the volume of data 


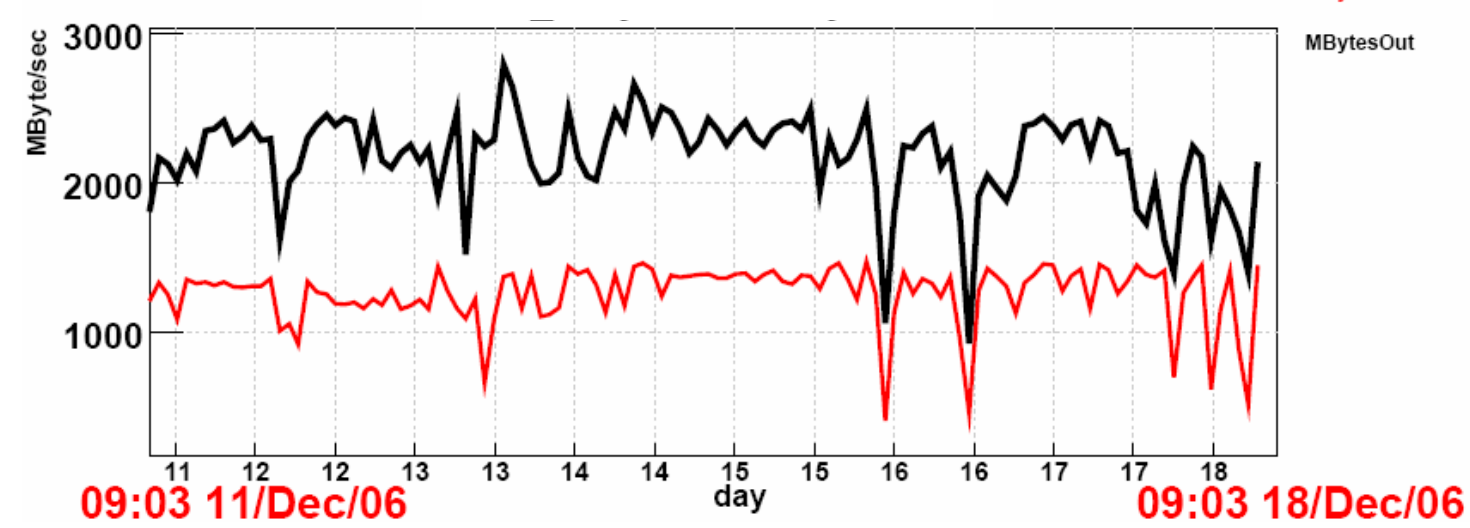

Figure 6.5: ALICE Data Challenge bandwidth results.

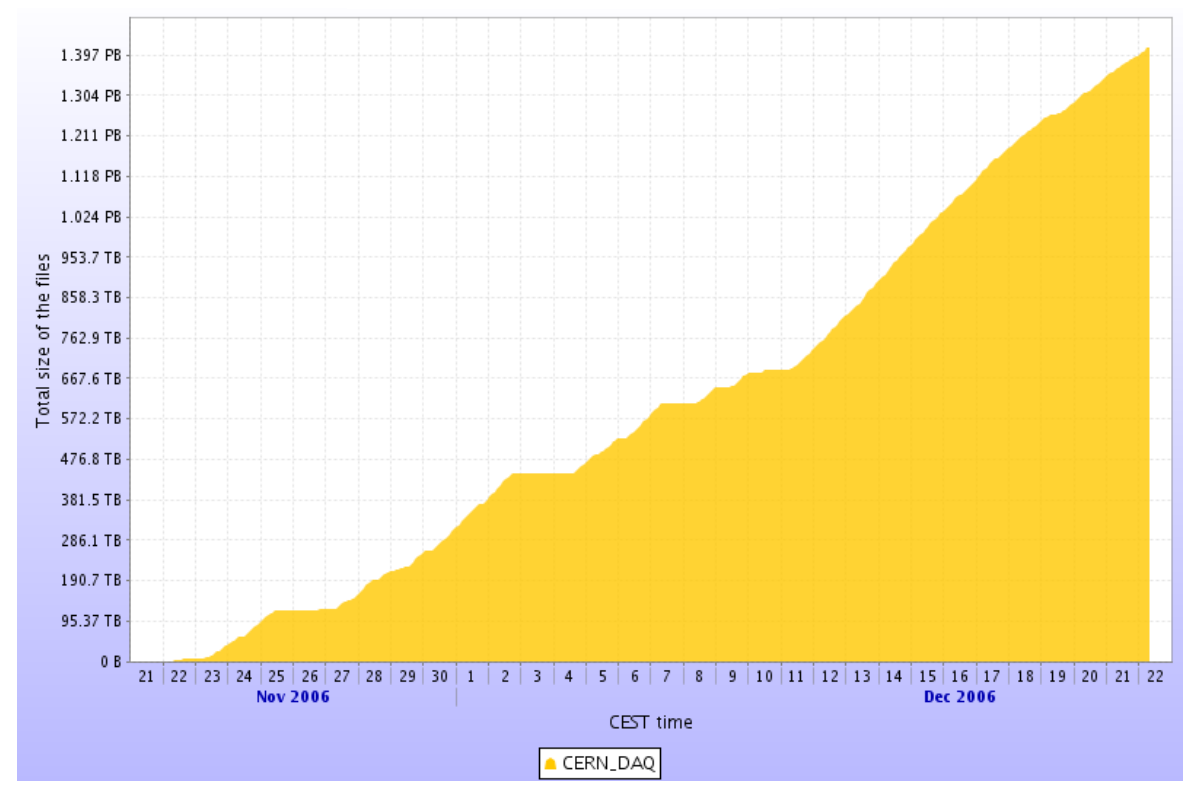

Figure 6.6: ALICE Data Challenges data to Mass Storage planning.

recorded during the Data Challenge have reached a volume equivalent to the total amount of data that the ALICE experiment will archive during a heavy-ion run as shown in figure 6.6.

\subsection{High-Level Trigger (HLT)}

\subsubsection{Introduction}

According to the simulation studies [4], the amount of data produced in the TPC alone, in a single central nucleus-nucleus collision, corresponds to about $75 \mathrm{MB}$ assuming $\mathrm{d} N_{\mathrm{ch}} / \mathrm{d} \eta=8000$ at midrapidity. The data rate for all detectors, resulting after a trigger selection, can easily reach $25 \mathrm{~GB} / \mathrm{s}$, while the physics content of a large number of events might be small and the DAQ archiving rate is about $1 \mathrm{~GB} / \mathrm{s}$. Therefore on-line processing is advised in order to select relevant events or 


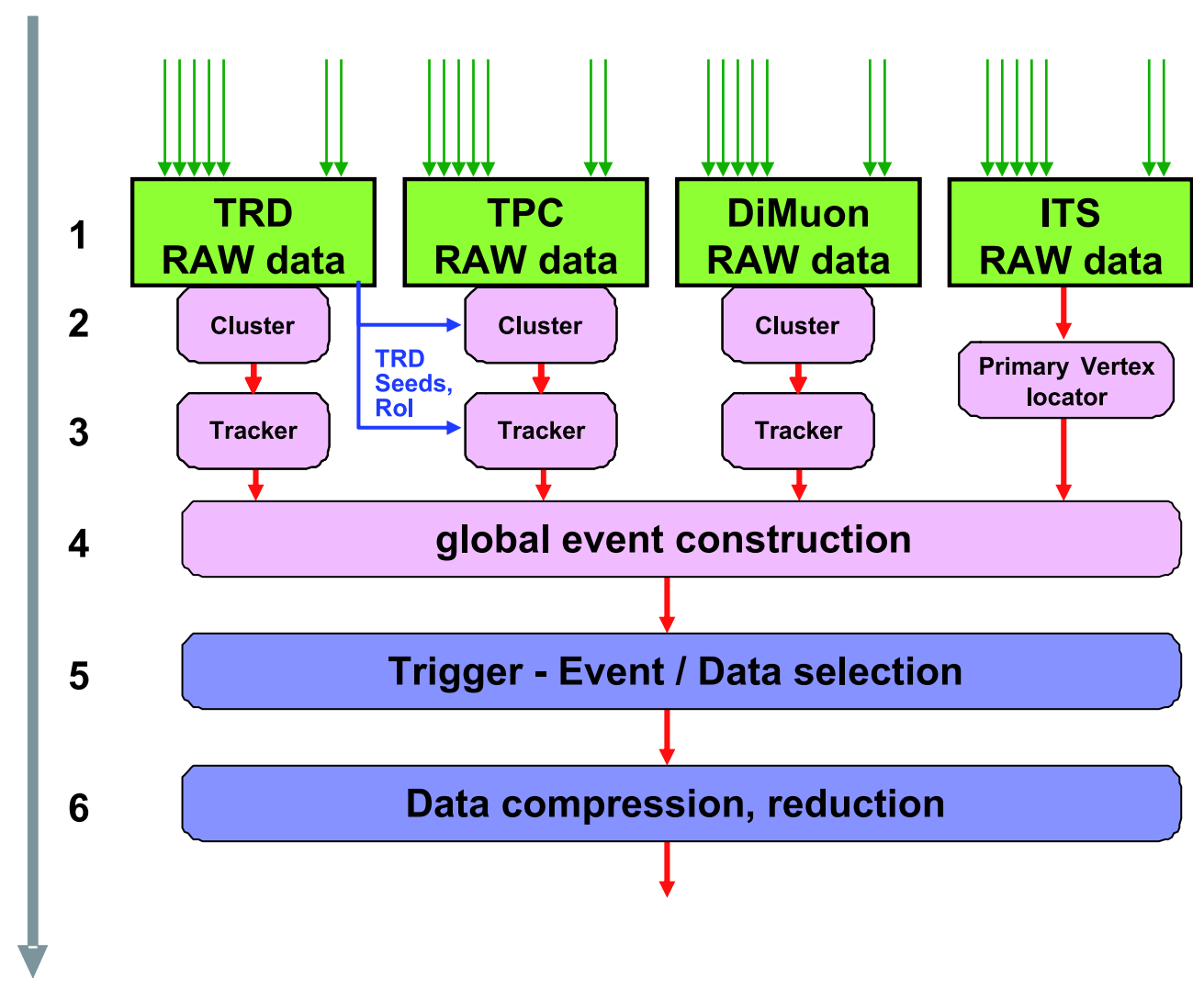

Figure 6.7: The six architectural layers of the HLT.

sub-events and to compress the data without loosing their physics content. The overall physics requirements of the High-Level Trigger [218] are categorised as follows:

Trigger Accept or reject events based on detailed online analysis.

Select Select a physics region of interest within the event by performing only a partial readout.

Compress Reduce the event size without loss of physics information by applying compression algorithms on the accepted and selected data.

\subsubsection{Architecture}

The HLT implements a processing hierarchy as outlined in figure 6.7. The raw data of all ALICE detectors are received via 454 Detector Data Links (DDLs) at layer 1. The first processing layer performs basic calibration and extracts hits and clusters (layer 2). This is done in part with hardware coprocessors (see section 6.3.2.2) and therefore simultaneously with the receiving of the data. The third layer reconstructs the event for each detector individually. Layer 4 combines the processed and calibrated information of all detectors and reconstructs the whole event. Using the reconstructed physics observables layer 5 performs the selection of events or regions of interest, based on run specific physics selection criteria. The selected data is further subjected to complex data compression algorithms. 
In order to meet the high computing demands, the HLT consists of a PC farm of up to 1000 multi-processor computers. The data processing is carried out by individual software components running in parallel on the nodes of the computing cluster. In order to keep inter-node network traffic to a minimum and for the means of parallelisation, the HLT data processing follows the natural hierarchical structure. Local data processing of raw data is performed directly on the FrontEnd Processors (FEPs), hosting the H-RORCs. Global data processing, with already reduced data, is done on the compute nodes. The trigger decision, Event Summary Data (ESD) of reconstructed events and compressed data are transferred back to the DAQ via the HLT output DDLs.

\subsubsection{Physical cluster layout}

The HLT physical cluster layout consists of 51 19" racks located in two counting rooms (CRs) at the ALICE experimental area at Point 2 at CERN. The first installation stage includes 81 FEP nodes and 15 infrastructure nodes, each equipped with one CHARM (Computer Health and Remote Management) PCI card for Cluster Monitoring.

All the nodes are off-the-shelf PCs with two AMD Dualcore Opteron $2 \mathrm{GHz}$ CPUs, $8 \mathrm{~GB}$ RAM and two Gigabit Ethernet connections. Each FEP contains two H-RORCs with two DDLs receiving the detector data. The infrastructure nodes consist of four server nodes, two gateway nodes and 9 portal nodes. The gateway nodes connect the cluster to the CERN network. Two server nodes are used as monitoring servers for the Cluster Management Framework SysMES (see section 6.3.3) and two as AFS file-servers. Each file-server implements a net storage capacity of 2 TB on a hardware Raid 6. The portal nodes are used for connections to various ALICE subsystems (see section 6.3.5). In addition, three multipurpose portal nodes and two nodes of every type were installed for redundancy reasons. All the nodes are connected via 1-to-1 Gigabit Ethernet switches, while the FEPs use two Gigabit Ethernet ports. The infrastructure nodes and racks, housing the FEPs and compute nodes, are interconnected via an InfiniBand backbone network.

All ALICE detectors are connected to the HLT, following the read-out granularity (number of DDLs) of each sub-detector: TPC 216, TRD 18, PHOS 20, DiMUON 23, ITS (SPD and SSD) 36, EMCAL 24, HMPID 20, FMD 3, and ACORDE, Trigger, ZDC, T0 and V0 1 DDL each. 12 DDLs transfer the results of the HLT to DAQ.

\subsubsection{HLT-Readout Receiver Card (H-RORC)}

The H-RORC (figure 6.8) is a Virtex-4 FPGA based PCI card designed for both (i) receiving and pre-processing of the detector RAW data of all ALICE detectors and (ii) transmitting processed events out of the HLT computing farm to the DAQ. The H-RORC therefore implements the interface between the HLT system and the ALICE data transport links DDL.

In order to receive these data, the H-RORC is able to host two Destination Interface Units (DIUs). Each link has a maximum bandwidth of $160 \mathrm{MB} / \mathrm{s}$ giving a maximal total incoming data rate of $320 \mathrm{MB} / \mathrm{s}$. Incoming data can be transferred directly into the main memory of the Front-End Processors via PCI DMA burst transfers with a rate of up to $370 \mathrm{MB} / \mathrm{s}$ per H-RORC. The FEPs are capable of handling two H-RORCs with two DDL interfaces each, running at full speed. In order to send processed events out of the HLT, the H-RORC can host up to two Source Interface Units (SIUs) to send data via DDL fibres to the DAQ. All transactions are PCI DMA based. 


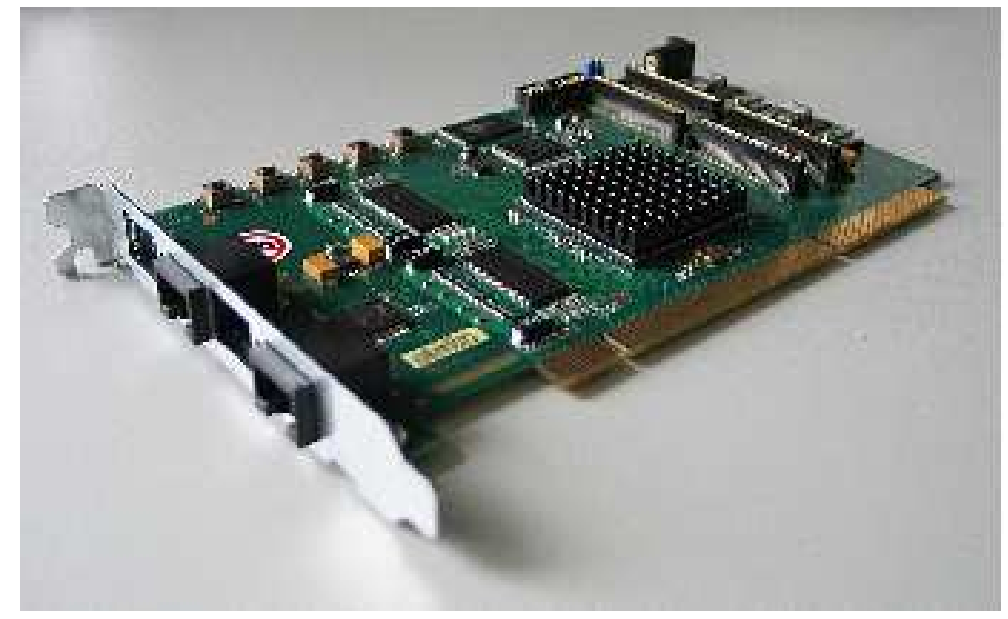

Figure 6.8: H-RORC data pump and FPGA coprocessor.

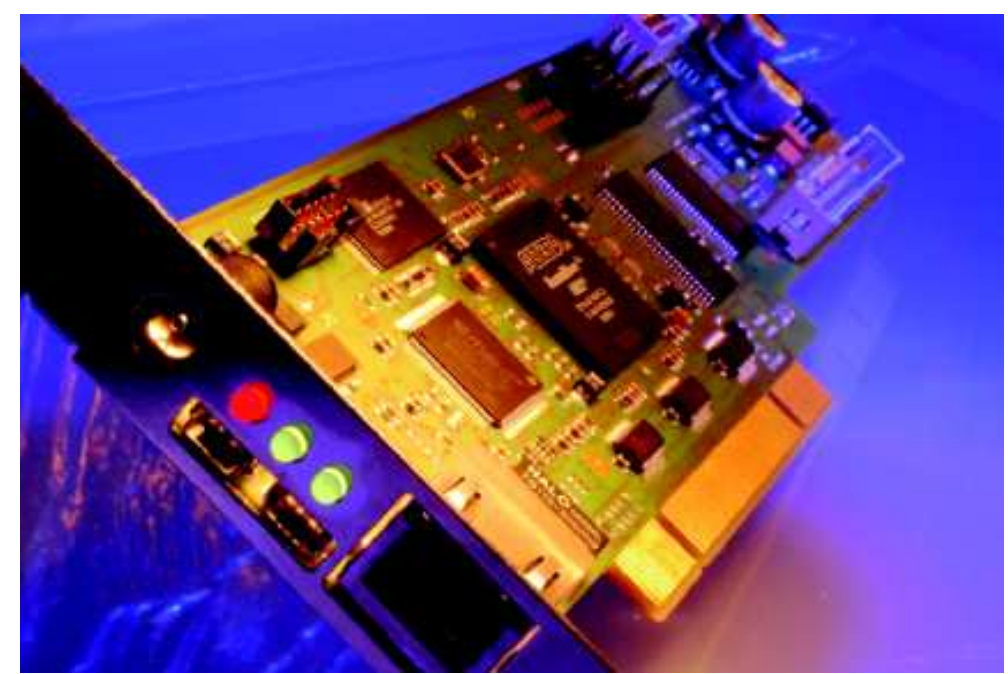

Figure 6.9: CHARM remote management card.

Data can also be pre-processed inside the FPGA [219]. This is supported by four independent Double Data Rate Synchronous Dynamic Random Access Memory (DDR-SDRAM) modules of $32 \mathrm{MB}$, each allowing data storage with up to $2.3 \mathrm{~GB} / \mathrm{s}$, and two fast full-duplex serial links connect each H-RORC to its neighbours. There are two on-line-processing modules being prepared, the TPC and the DiMuon Cluster Finder.

\subsubsection{Cluster management}

The HLT Cluster is managed using the SysMES Framework (System Management for Networked Embedded Systems and Clusters) and Lemon (LHC Era Monitoring). SysMES is a scalable, decentralised, fault tolerant, dynamic and rule-based tool set for the monitoring of networks of target systems based on industry standards. The resources to be monitored are the applications running on the computer nodes as well as their hardware, the network switches and the RMS (Rack Moni- 
toring System). In addition each computer implements a remote administration sensor and actuator (CHARM card figure 6.9), allowing full remote control of the individual node, also implementing autonomous error detection and correction functionality.

The system monitoring data is visualized by Lemon servers and the SysMES servers will react accordingly. The data transportation framework is designed to handle dynamic re-routing of the data flow during run-time. This is utilised to keep the analysis chains running even if severe problems (like hardware failure) occur, such that no data loss or damage of the online analysis will happen. Therefore an intermediate layer between SysMES and the PubSub framework (see section 6.3.4.1) is being developed, consisting of two applications, the Configuration Manager and the Resource Manager.

\subsubsection{Software architecture}

The HLT software is divided into two functional parts, the data transportation framework and the data analysis. The first part is mainly of technical nature, it contains the communication of the components, steering and data transfer between components/nodes. The second part contains the physics analysis. Development of Analysis Components is independent from data transportation which allows for a high degree of flexibility. The Analysis Components can be run in the offline analysis without changes, making a direct comparison of results possible.

\subsubsection{Data transportation and Publisher-Subscriber framework}

Based on the Publisher-Subscriber (PubSub) principle, a generic data transportation and steering framework was developed, the HLT PubSub framework [220-223]. The PubSub framework carries out the task of communication, data transportation and load distribution within the HLT. Figure 6.10 illustrates key principles and components of the PubSub framework.

Communication takes place in a pipe-lined data flow push architecture. Each component receives data, processes it and forwards new output data to the next component in the chain. The entire communication mechanism is designed for a low processing overhead, primarily through the use of shared memory and the exchange of descriptors. Figure 6.10 also depicts key components of the Publisher Subscriber Framework used to shape the data-flow in the system. An event merger component can merge data streams consisting of data blocks of the same event into one stream with multiple blocks per event. Communication between different nodes is supported by dedicated network bridge components. These connect to other components using the common interface and tunnel data over the network. The scatterer and gatherer pair of components provide capabilities for load balancing and distribution among multiple nodes or one multiprocessor node having multiple physical and/or logical processors. The scatterer splits a single data stream consisting of multiple events into several data streams, each transporting a correspondingly smaller number of events. Each event is forwarded by the scatterer unchanged. On the other end the gatherer collects those streams again into a single stream. An interface that allows access to data at any point in a processing chain, e.g. for monitoring, is described in section 6.3.5.4. 

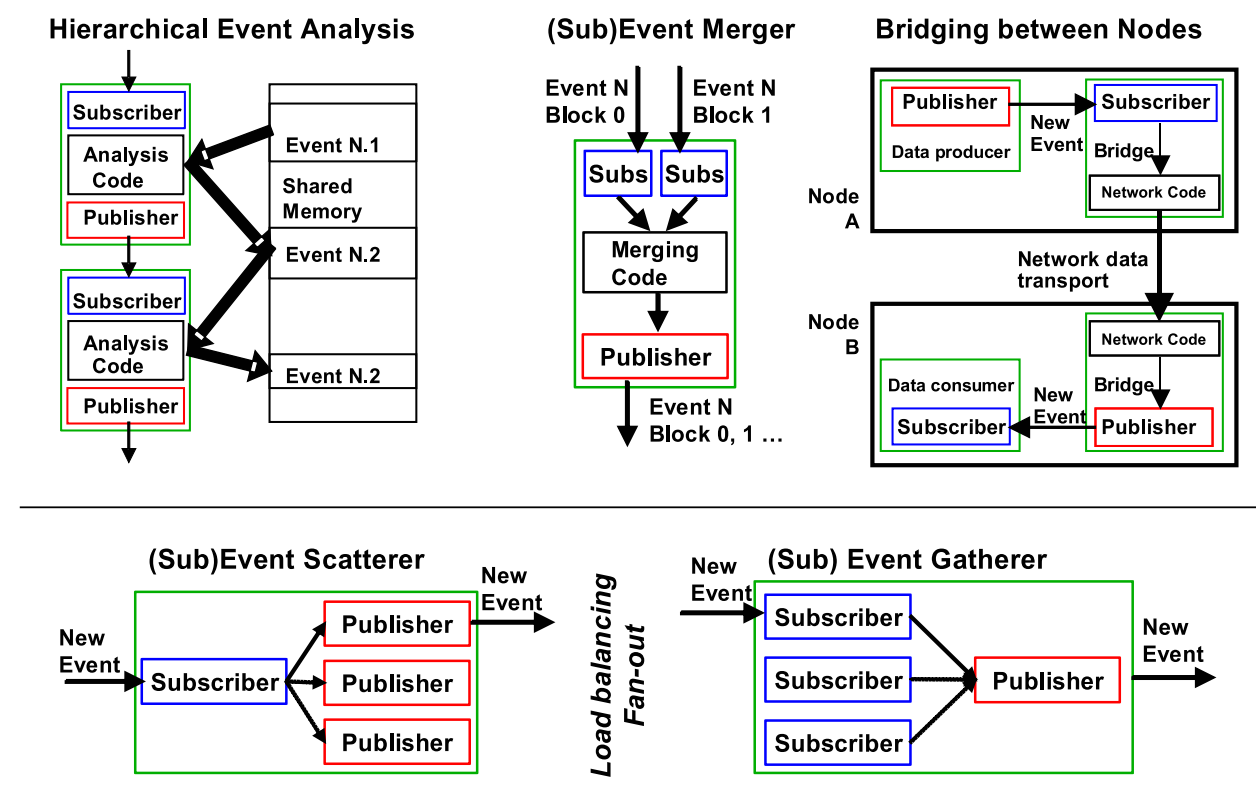

Figure 6.10: Illustration of key principles and components of the HLT Publisher Subscriber framework.

\subsubsection{HLT analysis framework}

The HLT analysis framework [224] encapsulates the data and physics analysis and separates it from the data transportation. A class hierarchy hides the complex interface logic from the derived component classes implementing the actual physics analysis algorithms. The framework integrates the HLT analysis components into the offline AliRoot reconstruction and models a behaviour similar to the PubSub Framework for the components. A C interface layer provides access to the analysis components for external applications, which include a PubSub interface wrapper program as well as a simple standalone run environment for the components.

The online and offline environment are two different running modes of the HLT analysis. The first is the main running mode, while the latter one is frequently used for the evaluation of the HLT algorithms and analysis. Therefore, binary compatibility is considered to be a necessary feature of the system. The same shared library has to run in AliRoot and PubSub. In the online HLT, a special processing component, the AliRootWrapperSubscriber, can load an analysis module and instantiate the actual analysis component via the external $\mathrm{C}$ interface and implements all the other online functionality which is necessary to plug the analysis code into a HLT processing chain. As a result, the component libraries compiled in the AliRoot framework can directly be loaded and used from the PubSub system. 


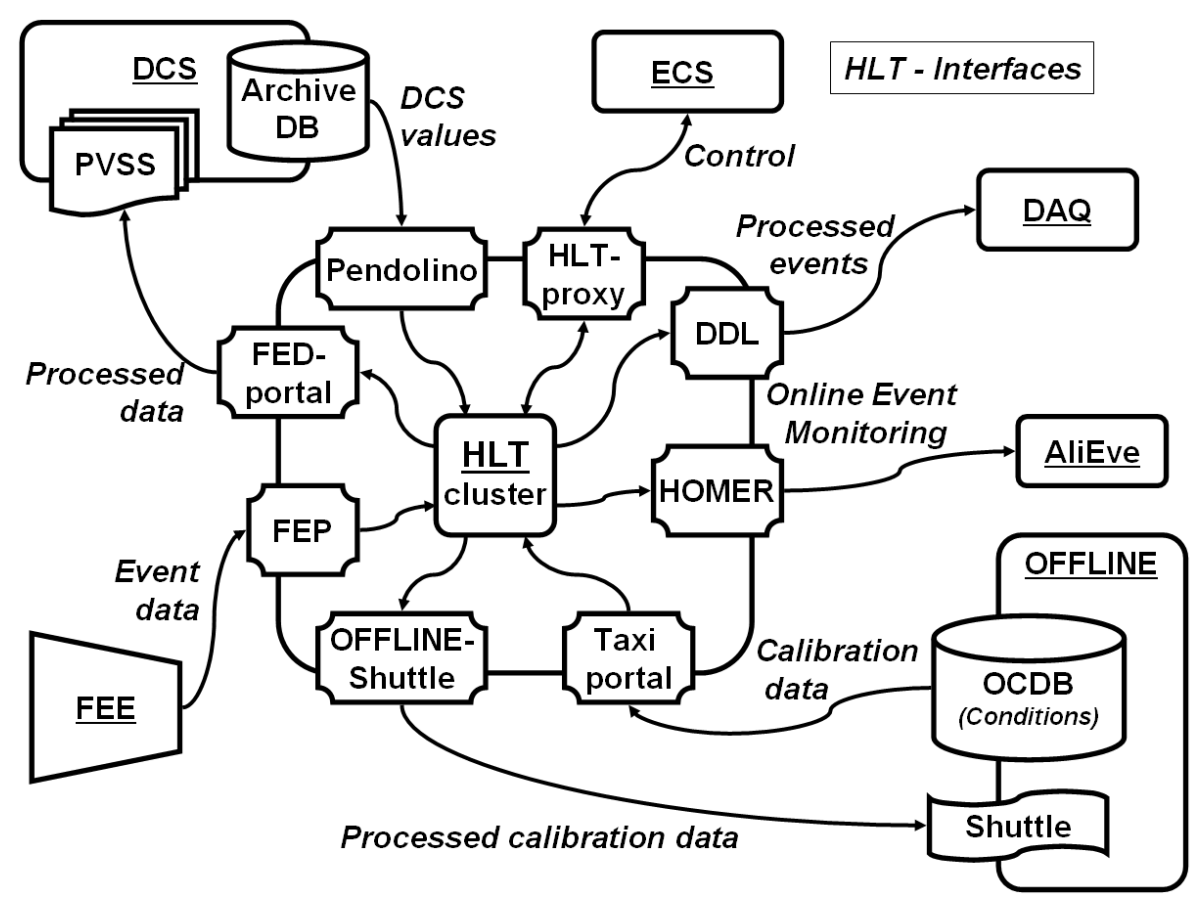

Figure 6.11: HLT interfaces to the other systems of ALICE.

\subsubsection{HLT interfaces to other online systems and offline}

The HLT communicates with the other ALICE systems through various external interfaces and portal nodes (figure 6.11, [225]). Fail safety and redundancy in the design of these interfaces will avoid single points of failure and reduces the risk of time delays or data loss.

\subsubsection{Offline interface}

The Offline Conditions Data Base (OCDB) stores all produced calibration and condition settings. A special application, the Taxi, requests new calibration data in regular time intervals from the OCDB and synchronises them with the local HLT copy of the database which is available on all processing nodes and allows Detector Algorithms to access the calibration data of previous runs in the same way as in the offline environment. After each run, updated calibration data is shipped back to the OCDB via a portal. A special PubSub data sink component collects the produced calibration data inside the HLT cluster and saves them to the File Exchange Server (FXS). A MySQL DB is used for storing additional meta data. The offline Shuttle will contact both after each run to fetch the updated data.

\subsubsection{Detector Control System (DCS) interface}

Permanently monitored values like temperatures are requested in regular time intervals by the Pendolino software from the DCS Archive DB through the DCS interface during the run. These fetched values are then provided to the Detector Algorithms on the HLT cluster nodes. Vice versa, the HLT writes back data to the DCS via the Front-End-Device Application Programming Interface 
(FedAPI), which is common among all detectors. It consists of a DIM-server (Distributed Information Management) on the HLT-side, and a DIM-client, embedded in the PVSS (Process Visualisation and Steering System) of the DCS. The server publishes monitored data and the client includes them into the DCS.

\subsubsection{Experiment Control System (ECS) interface}

All the interactions with the other systems are governed by states and state transitions issued from the ECS. For this purpose a finite state machine runs on the corresponding interface portal. It accepts transition commands and returns the current state of the HLT through an HLT proxy interface software. In addition, ECS hands in running conditions like run number and trigger settings before the start of each run.

\subsubsection{Monitoring tools}

In order to export data from the HLT at any point in the analysis chain, a tool called HOMER (HLT On-line Monitoring Environment including ROOT) was developed. It provides a general access method via two user transparent mechanisms of connecting to PubSub components: via a TCP/IP port or shared memory. A reader library allows access to data blocks and can directly be used from the interactive ROOT prompt.

The open concept of HOMER makes detector monitoring independent of the specific monitoring back-end. The offline monitoring AliEve is also used for general detector online monitoring applications. AliEve is part of AliRoot and combines an event display including 3D visualisation with tools for investigation and browsing of ROOT data structures and histograms. Specific monitoring components run on the HLT which accumulate results of the analysis of many events. The data can be in any format, also a complex ROOT data structure, e.g. a histogram. This makes the HLT a versatile monitoring tool which allows not only examination of the quality of the raw data, but also the monitoring of analysed data online.

\subsubsection{Online data processing}

The studies described in the following subsections were all performed on data simulated with the ALICE offline software AliRoot.

\subsubsection{Event reconstruction}

The Time Projection Chamber (TPC) is read out by 557568 channels, delivering event sizes up to $75 \mathrm{MB}$ for central $\mathrm{Pb}-\mathrm{Pb}$ collisions. Fast online reconstruction algorithms consisting of a Cluster Finder and a Track Follower were developed [219, 226-231]. By using the 2D position in the pad-row plane and the drift time in the gas, the Cluster Finder reconstructs 3D space-points using the centre of gravity approach. The Track Follower maps the space-points into conformal space where helical tracks can be fitted by a linear parametrisation. A helix-fit of the tracks provides the kinematic properties of the particles. The tracking is done on the sector level. If tracks cross more than one sector, they are merged [226]. The algorithms were originally developed for the STAR L3 trigger [232]. Beyond multiplicities $\mathrm{d} N_{\mathrm{ch}} / \mathrm{d} \eta$ of 4000 the Cluster Finder efficiency deteriorates 
due to a significant number of overlapping clusters. A fast Hough-transform tracker was developed for this scenario, using the raw data directly [231].

The Transition Radiation Detector (TRD) reconstruction on HLT was designed to directly use the algorithms implemented for the offline analysis. The offline HLT-TRD cluster finder and the HLT-TRD tracker are able to handle a full non-zero suppressed TRD event (load of $43.2 \mathrm{MB}$ ) in real time. In the case of zero-suppressed data the volume of a raw $\mathrm{Pb}-\mathrm{Pb} \mathrm{d} N_{\mathrm{ch}} / \mathrm{d} \eta=8000$ event does not exceed 7.1 MB including a 20\% coding overhead. Given the design rate of the HLT-TRD event processing chain as of $200 \mathrm{~Hz}$ the HLT cluster allocates 6 processing nodes per TRD super-module to fulfill the timing requirements. In addition to the components responsible of the reconstruction the TRD-HLT processing chain incorporates the offline model to produce the calibration reference data and delivers a collection of relevant histograms to the interface to OCDB.

For the Photon Spectrometer (PHOS) detector the main objective is to measure the exact timing and energy information of an electromagnetic shower in a typical $3 \times 3$ crystal matrix of the calorimeter. Therefore the signal will be oversampled in order to measure the pulse shape. Consequently, at a trigger rate of $1 \mathrm{kHz}$ the 5 PHOS modules will deliver a maximum of $3 \mathrm{~GB} / \mathrm{s}$ for non-zero-suppressed and non-baseline-corrected events. Based on the Peak Finder algorithm, processing components were developed to analyse the pulse shape and reduce the data to the relevant time and energy information. Furthermore, algorithms for cluster and shower reconstruction will be run on the HLT.

In the Muon Spectrometer the primary goal of the dimuon High-Level Trigger (dHLT) is to improve the sharpness of the $p_{\mathrm{t}}$ cut compared to what is obtained by the L0 trigger. dHLT performs fast, online, partial event reconstruction [17] on stations 4 and 5, which allows it to improve the $p_{\mathrm{t}}$ resolution by more than a factor of two. Thus a better separation between background and interesting signal events is possible reducing the background data accepted by the spectrometer. The dHLT can handle at least a $1 \mathrm{kHz}$ event rate with a data rate of $500 \mathrm{MB} / \mathrm{s}$. Event reconstruction starts with hit reconstruction, which uses a simple 3 pad cluster finding algorithm. The efficiency achieved is above 98\%, and a resolution of $100 \mu \mathrm{m}$ and $1 \mathrm{~mm}$ along the $y$ and $x$ directions (perpendicular and horizontal to the ground respectively) is reached respectively. For tracking, a track following algorithm [233] is used, giving a single muon reconstruction efficiency above $97 \%$ and is independent of the event size. From the found tracks the $p_{\mathrm{t}}$ is estimated using a simple calculation involving the particle's deflection angle [12]. This gives a $p_{\mathrm{t}}$ resolution of about $0.13 \mathrm{GeV} / c$ and $0.18 \mathrm{GeV} / c$ around the $1 \mathrm{GeV} / c$ and $2 \mathrm{GeV} / c p_{\mathrm{t}}$ cuts respectively. The total processing time for a central event is about 2.5 ms. Further details about the algorithms can be found in the dHLT Project Review [234].

\subsubsection{Online data compression}

For the TPC detector, the data rate is expected to reach up to $15 \mathrm{~GB} / \mathrm{s}$ after zero suppression. Any means for reducing the data size online is therefore desirable in order to increase the number of events which can be collected and written to mass storage. Lossless and lossy data compression techniques like entropy encoding and vector quantisation were evaluated using simulated TPC data [235]. The results indicate that standard compression algorithms achieve compression factors of about 2. However, HLT online pattern recognition results in track and cluster information which effectively models the TPC data and can be utilised to compress such data more efficiently and 
reduce the data size to about $11 \%$, with a negligible loss of tracking performance for the expected particle multiplicities at LHC [226, 236].

In PHOS, after zero-suppression and skipping of empty channels in the front-end electronics, the data rate into HLT is about $300 \mathrm{MB} / \mathrm{s}$. Online processing of the pulse shapes results in an energy and a time-of-flight information per channel. This information is forwarded to DAQ for achieving at a data rate of about $20 \mathrm{MB} / \mathrm{s}$ which corresponds to a data volume reduction factor of 15 .

\subsubsection{Calibration procedure}

Detector algorithms are implemented by detector groups in order to create useful calibration data, which is archived in the OCDB. These algorithms are designed to run in both offline and HLT framework. In the HLT, the detector algorithms are embedded in normal HLT processing components. A special base class for those calibration components was added to the HLT analysis framework, as calibration algorithms mainly accumulate data of several events and deliver the result at the end of run. The calibration data can be shipped via 3 parallel output streams namely the OCDB, to TCP ports (HLT online monitoring) and to the DAQ via the data stream. Calibration components publish results at the end of the run via the offline interface to the OCDB (see section 6.3.5).

For the TPC Calibration the general off-line calibration classes for signal and noise were integrated in the HLT framework. They were successfully tested, processing more than one million raw data events recorded during the TPC commissioning phase.

Benchmarks indicate less than $16 \%$ of the total time is spent inside the HLT framework.

The calibration parameters for PHOS calibration mainly consist of energy and time-of-flight distribution for each detection channel, for relative calibration as well as $\pi^{0}$ invariant mass histograms for absolute calibration. Using simulated data, the calibration components were successfully tested at the HLT cluster.

\subsection{Offline computing}

\subsubsection{Introduction}

The role of the Offline Project is the development and operation of the framework for data processing. This includes tasks such as simulation, reconstruction, calibration, alignment, visualisation and analysis. These are the final steps of the experimental activity, aimed at interpreting the data collected by the experiment and at extracting the physics content. In an experiment of the complexity and dimension of ALICE, this implies the development and operation of a quite diverse set of environments. In particular we can distinguish three main areas:

- Distributed computing: The computing resources required to process the ALICE data are such that they cannot be concentrated in a single computing centre. Therefore data processing is distributed onto several computing centres located worldwide. Grid Middleware allows treatement of this heterogeneous collection of distributed computing resources as an integrated computing centre. 
- Offline data processing: This includes the typical offline activities of calibration, alignment, reconstruction, simulation and analysis, both centrally organized and end-user driven. The offline data processing typically uses the framework for distributed computing and only in some rare cases runs on local resources.

- Quasi-online operations: During proton-proton collisions, the data is reconstructed and analysed quasi-online. Nucleus-nucleus events is reconstructed during the accelerator shutdown following the nucleus-nucleus run. This implies a tight synchronisation with the more "classical' online activities of DAQ and HLT. It is a new area for offline computing, since it dispenses with the old practice to process the data usually many months after it was collected.

The coordinated operation of all these elements, to realise timely and reliably the physics discovery potential of the data collected by the ALICE experiment, is called the ALICE computing model. The realisation of this model, elaborated during the last ten years by the ALICE collaboration under the coordination of the ALICE Offline Computing Project, constitutes the subject of this section.

\subsubsection{Computing model}

The inputs to the ALICE Computing Model are the computing resources needed to process the data, the amount of data foreseen and the time lapse between the moment data are recorded and the moment results are needed. The constraints are the foreseeable amount of resources available, their location and the offered service level.

The amount of resources required to process the data coming from the ALICE experiment is of such magnitude that it is not feasible to concentrate it in a single place. While Funding Agencies do realise that this investment in computing equipment is necessary to harness the physics potential of the experiment and to realise the investment made in the hardware, they can only afford the related investments in their own countries. Moreover, even if the computing resources could be all brought to CERN, the maintenance and operation of this complex system would still remain a problem due to manpower availability. The solution to this problem is to provide the necessary computing resources in centres distributed around the world. In the case of ALICE the number of such centres is around sixty, spread in more than thirty countries.

The amount of different tasks to be performed, the necessity to perform them in a timely and documented fashion, and the number of users involved require that this heterogeneous distributed system works as a single integrated computing centre. This is by no means a small challenge, for which there is no ready-made solution to be found. This problem is the focus of current computing science research and development that goes under the name of Grid [239].

The distributed computing infrastructure serving the LHC experimental programme is coordinated by the Worldwide LHC Computing Grid (WLCG) project. The WLCG computing infrastructure is, by nature, highly hierarchical. All real data originate from CERN, with a very large computing centre called Tier-0. Large regional computing centres, called Tier-1, share with CERN the roles of a safe storage of the data on high reliably storage media (at present, magnetic tapes) and to perform the bulk of the organised processing of the data. Smaller centres, called Tier-2, are 
logically clustered around the Tier-1's. The main difference between the two kind of centres is the availability of high-reliability mass-storage media at Tier-1's. Tier-2's use the 'closest' Tier-1 to store the data that they produce. The major role of Tier-2's is simulation and end-user (sometimes also called chaotic) analysis.

Within the WLCG structure, a centre, to qualify as a Tier-1 or Tier-2, has to sign and follow up the corresponding, morally binding, Service Level Agreement (SLA), which specifies Quality -Of-Service (QoS) and intervention delays.

Smaller centres, corresponding to a departmental computing centre and sometimes called Tier-3's, contribute to the computing resources but there is no definite role or definition for them.

\subsubsection{Computing needs}

In determining the computing needs of the ALICE experiment, we distinguish two modes of operation, the proton-proton and the nucleus-nucleus data taking. The amount of data collected depends on the running conditions (such as luminosity, triggers, available detectors, settings in the detectors' hardware, DAQ and HLT mode, etc.) and on their evolution during the life of the accelerator. The time to process these data and the amount of secondary data produced depends on the offline programs, which are in constant evolution following the requirements of the physicists. This makes it very difficult to provide a firm estimate of the needs, particularly in the startup phase, as we have witnessed in these years a constant redefinition of the accelerator commissioning scenario.

We present here the estimation of the computing resources needed in a Standard Data Taking Year (SDTY). These data are a reasonable compromise between the figures determined at the time of writing the Physics Performance Report [20,21] and the current values, which are larger, but still subject to optimisation, both as far as the data size and the computing time are concerned.

In table 6.4 we show the parameters for a SDTY and in table 6.5 we report the computing needs derived using the WLCG [237] standard efficiency factors. The figures for the initial periods, before standard running conditions are reached, are more difficult to calculate as they depend on the exact accelerator schedule.

\subsubsection{Data processing strategy}

The data processing strategy and the Tier computing centres hierarchy derive from the Monarch model [238]. The strategy varies according to the type of collision. During proton-proton collision the data, recorded at an average rate of $100 \mathrm{MB} / \mathrm{s}$, are written by the DAQ on a disk buffer at the CERN (Tier-0) computing centre, where the following four activities proceed in parallel on the RAW data:

- Copy to the CASTOR tapes;

- Export to the Tier-1 centres to have a second distributed copy on highly-reliable storage media and to prepare for the successive reconstruction passes that will be processed in the Tier-1 centres;

- First pass processing at the Tier-0 centre. This includes: reconstruction, production of calibration and alignment constants and scheduled analysis; 
Table 6.4: Parameters of the ALICE Computing Model wherefrom the computing needs are determined. The proton-proton RAW event size is an average figure. The real pile-up factor will change during the run as a function of the luminosity. The $\mathrm{Pb}-\mathrm{Pb}$ data rate is an average figure. The actual event size depends on the trigger and the event multiplicity. What is important to retain is that the 'bandwidth budget' of $1.25 \mathrm{~GB} / \mathrm{s}$ will be fully exploited, irrespectively of the chosen trigger.

\begin{tabular}{|l|l|r|r|}
\hline Category & Item & Value \\
& & $\mathrm{pp}$ & $\mathrm{Pb}-\mathrm{Pb}$ \\
\hline \hline \multirow{5}{*}{ Real Data } & RAW single pp & $200 \mathrm{kB}$ & \\
\cline { 2 - 4 } & pp pile-up & 5 & \\
\cline { 2 - 4 } & Rate & $100 \mathrm{~Hz}$ & $100 \mathrm{~Hz}$ \\
\cline { 2 - 4 } & RAW size per event & $1.1 \mathrm{MB}$ & $13.75 \mathrm{MB}$ \\
\cline { 2 - 4 } & ESD size per event & $40 \mathrm{kB}$ & $3 \mathrm{MB}$ \\
\cline { 2 - 4 } & Tag catalogue per event & $11 \mathrm{kB}$ & $11 \mathrm{kB}$ \\
\cline { 2 - 4 } & Yearly running time & $10^{7} \mathrm{~s}$ & $10^{6} \mathrm{~s}$ \\
\cline { 2 - 4 } & Events per year & $10^{9}$ & $10^{8}$ \\
\hline \hline \multirow{5}{*}{ Simulated Data } & RAW size per event & $400 \mathrm{kB}$ & $300 \mathrm{MB}$ \\
\cline { 2 - 4 } & ESD size per event & $90 \mathrm{kB}$ & $6 \mathrm{MB}$ \\
\cline { 2 - 4 } & Tag catalogue per event & $11 \mathrm{kB}$ & $11 \mathrm{kB}$ \\
\cline { 2 - 4 } & Events per year & $10^{9}$ & $10^{7}$ \\
\cline { 2 - 4 } & Signals per underlying event & \multicolumn{2}{|c|}{3} \\
\hline \hline \multirow{5}{*}{ Statistics } & Reconstruction passes per year & \multicolumn{2}{|c|}{3} \\
\cline { 2 - 4 } & RAW replication factor & \multicolumn{2}{|c|}{20} \\
\cline { 2 - 4 } & ESD replication factor & \multicolumn{2}{|c|}{3} \\
\cline { 2 - 4 } & Scheduled analysis passes per reconstruction & \\
\cline { 2 - 4 } & Chaotic analysis passes per year & \\
\hline
\end{tabular}

- Fast processing of selected sets of data, mainly calibration, alignment, reconstruction and analysis on the CERN Analysis Facility (CAF) that will be described in section 6.4.4.7.

During nucleus-nucleus runs, the model is slightly different, as the rate of data acquisition is so high that an excessive amount of computing resources and network bandwidth would be necessary for quasi-online processing. Therefore the processing of the nucleus-nucleus RAW data proceeds as follows:

- Registration of the RAW data in CASTOR;

- Partial export to the Tier-1 centres to allow remote users to examine the data locally;

- Partial first pass processing at the Tier-0 centre to provide rapid feedback on the offline chain;

- Fast processing, mainly calibration, alignment, reconstruction and analysis on the CAF.

At the end of the period of nucleus-nucleus data taking, there will be a four-months shutdown of the accelerator. During this period the nucleus-nucleus data are read back from tapes and are 
Table 6.5: Computing needs during a standard data taking year.

\begin{tabular}{|c|c|c|c|}
\hline \multirow[t]{2}{*}{ Category } & \multirow[t]{2}{*}{ Item } & \multicolumn{2}{|c|}{ Value } \\
\hline & & $\mathrm{pp}$ & $\mathrm{Pb}-\mathrm{Pb}$ \\
\hline \multirow{5}{*}{$\begin{array}{l}\text { CPU Resources / year } \\
\text { (MSI2k s) }\end{array}$} & Reconstruction of real and simulated data & $4.6 \times 10^{7}$ & $5.7 \times 10^{8}$ \\
\hline & Generation of of simulated data & $4.9 \times 10^{7}$ & $2.1 \times 10^{8}$ \\
\hline & Scheduled analysis & $2.3 \times 10^{8}$ & $3.4 \times 10^{8}$ \\
\hline & End-user analysis & $5.8 \times 10^{7}$ & $8.7 \times 10^{7}$ \\
\hline & Total & \multicolumn{2}{|c|}{$1.6 \times 10^{9}$} \\
\hline \multirow{6}{*}{$\begin{array}{l}\text { Mass storage / year } \\
\text { (TB) }\end{array}$} & RAW data & $1.1 \times 10^{3}$ & $1.4 \times 10^{3}$ \\
\hline & Reconstructed data & $5.8 \times 10^{2}$ & $3.7 \times 10^{3}$ \\
\hline & Simulated RAW data & $4.0 \times 10^{2}$ & $3.0 \times 10^{3}$ \\
\hline & Reconstructed simulated data & $5.8 \times 10^{2}$ & $3.7 \times 10^{3}$ \\
\hline & Calibration & \multicolumn{2}{|c|}{$1.0 \times 10^{1}$} \\
\hline & Total & \multicolumn{2}{|c|}{$1.2 \times 10^{4}$} \\
\hline \multirow{4}{*}{$\begin{array}{l}\text { Disk } \\
\text { (TB) }\end{array}$} & Output buffer at CERN & \multicolumn{2}{|c|}{$2.4 \times 10^{2}$} \\
\hline & Disk at Tier-1 centres & \multicolumn{2}{|c|}{$1.2 \times 10^{4}$} \\
\hline & Disk at Tier- 2 centres & \multicolumn{2}{|c|}{$4.3 \times 10^{3}$} \\
\hline & Total & \multicolumn{2}{|c|}{$1.6 \times 10^{4}$} \\
\hline
\end{tabular}

processed in a way similar to proton-proton data (export to the Tier-1 centres, first pass processing, fast processing of selected data) but at a rate four times lower than the actual nucleus-nucleus data acquisition. This requires a large-scale resource deployment that is still affordable within the budgetary and infra-structural constraints.

The quasi-online data processing scenario is more complicated, and it will be described in somewhat greater detail in section 6.4.4.7.

During the first pass reconstruction, high-precision alignment and calibration data are produced, as well as a first set of Event Summary Data (ESD) and Analysis Object Data (AOD). The feedback derived from the first pass, including analysis, is used to tune the code for the second pass processing. In our computing model, we have foreseen three processing passes. However this has to be interpreted as an estimation of the computing resource budget needed to extract the physics content from the data. We expect the second pass reconstruction to be composed of a large number of jobs processing only a fraction of the data, to tune the chain in order to perform one more complete pass on the data, which constitutes the third pass.

One full copy of the raw data is stored at CERN, and a second one is shared among the Tier-1's outside CERN. Reconstruction is shared by the Tier-1 centres, CERN being in charge of processing the first pass. Subsequent data reduction, analysis and Monte Carlo production is a collective operation where all Tier's participate, with Tier-2's being particularly active for Monte Carlo and end-user analysis. 


\subsubsection{Distributed computing}

\subsubsection{ALICE Grid}

The implementation of the above model relies on a distributed computing infrastructure enabled by Grid Middleware. Since 2001, ALICE developed a set of Middleware services, AliEn [240], which implements the above model. With the development of various large Middleware projects, it became possible to replace some of these services with the services offered by these projects. In the resulting architecture, the user interacts with the Grid via the AliEn User Interface (UI), and the services are offered by a combination of AliEn Middleware, providing high-level or ALICEspecific services, and the Middleware installed on the computing centre, providing basic services.

This architecture allows a seamless distribution of high-level services, offering uniform functionality and UI across different Grid domains, thus implementing one of the most characteristic features of the 'Grid vision'. The system has been in continuous operation since 2001 with periodic large scale exercises called 'data challenges' performed to test the evolution and scalability of the system. During the last year before the data taking, these data challenges were replaced by a continuous 'production mode' in order to verify the operational stability of the system.

The AliEn system is built around Open Source components, uses Web Services model and standard network protocols. AliEn Web Services play the central role in enabling AliEn as a distributed computing environment. The user interacts with them by exchanging SOAP (Simple Object Access Protocol) [241] messages and they constantly exchange messages between themselves behaving like a true Web of collaborating services. AliEn consists of the following key components and services:

Authentication. AliEn supports various authentication schemes, implemented using the extensible Simple Authentication and Security Layer (SASL) protocol, in particular GLOBUS GSI/GSSAPI that makes it compatible with the WLCG security model.

Authorisation. The authorisation in AliEn is similar to any UNIX-like system. Files have read, write and execution permissions, and they can be set for the owner of the file, group, or world.

Auditing services. Every job (task) submitted and executed in AliEn is tracked with a unique job identifier. The AliEn Task Queue database holds complete information on all active jobs: the job owner, the job scripts, input and output files, processing stage, execution site and host. All completed jobs are moved to an archive database. The job auditing is possible at any stage of a job and tools are available to do per-job and summary audit of both the active and archived jobs.

Workload management. The AliEn workload management system is based on a pull approach. A central service manages all the tasks, while computing elements are seen as 'remote queues' and can provide access to a single machine dedicated to run a specific task, a cluster of computers, or even an entire foreign Grid. When jobs are submitted, they are sent to the central queue. The workload manager optimises the queue taking into account job requirements based on input files, CPU time, architecture, disk space, etc. The remote queues 
advertise their capabilities to the Job Broker. If there are free resources, the Job Broker signals the remote queue to start Job Agents. At this point, the real job is still not assigned to the site. Once the Job Agents start running on the Worker Nodes (WN), they do a set of sanity checks of the environment, and advertise to the Job Broker their capabilities. The Job Broker compares these with the requirements of the jobs in the Task Queue and in case of a successful match, assigns the job to a Job Agent. The Job Agent will then execute the job. If at the end of the job there are still sufficient resources, in particular lifetime, the Job Agent will ask again the Job Broker for tasks. The AliEn user interface uses the Condor ClassAds [242] as a Job Description Language (JDL). The Workload Management service can modify the job's JDL entry by adding or elaborating requirements based on the detailed information it gets from the system like the exact location of the dataset and replicas, client and service capabilities. When a job requires several files, the workload management systems 'splits' the job in several sub-jobs, each of them dealing with files that are co-located at the same Storage Element (SE). The workload management system still handles the job as a single entity, and keeps track of each individual sub-job.

API services. The API (Application Programming Interface) services provide a SOAP interface to the AliEn command set. This interface allows the building of very thin clients, requiring only one library (gsoap) to be completely Grid-enabled. The API services are used to provide an AliEn interface in ROOT.

Data management. Real files are managed by an AliEn SE. Access to the data is obtained via a POSIX interface and mediated by the xrootd program [243]. When a file is requested by an application running on a worker node, the following chain of events is triggered:

1. The File Catalogue is queried with the Logical File Name (LFN) or the Globally Unique IDentifier (GUID) of the file to obtain permission to perform a given operation in the form of a security envelope.

2. The local xrootd redirector is contacted to get access to a copy of the physical file. The Physical File Name (PFN) is obtained from the File Catalogue, but it may also be generated on the fly from the GUID.

3. The local xrootd redirector provides the location of the file. At this point two options are possible:

(a) The file is opened directly at the location provided by xrootd via a POSIX call;

(b) The file is copied on the local disk of the WN and then opened there.

The xrootd redirector can also offer access to a file that is not local at the computing centre where the job is running. This possibility is not currently exploited because batch jobs are executed where the files are stored. The xrootd program was interfaced with the major storage systems in use in HEP, and can of course run 'natively' on a set of Unix disk servers. Bulk transfer of data between Tier- 0 and Tier-1's is performed via FTS [244], under control of an AliEn element called File Transfer Daemon (FTD), which takes care of the needed interaction with the File Catalogue. Data transfer other than Tier- $0 \Leftrightarrow$ Tier-1's is done via xrootd itself. 
File and meta-data catalogues. Input and output associated with any job is registered in the AliEn File Catalogue, a virtual file system in which a file or a file collection (data set) is identified by a GUID. Meta-data can be assigned to files, and in particular the file's 'logical name', i.e. its Unix-like name in the virtual distributed file system.

The file catalogue does not own the files; it only keeps an association between the LFN and (possibly more than one) PFN on a real file or mass storage system. PFNs describe the physical location of the files and include the name of the AliEn storage element and the path to the local file.

The system supports file replication and caching and uses file location information to schedule jobs for execution. The File Catalogue supports Unix-like permissions for files and directories. These are automatically checked when accessing the File Catalogue through the xrootd interface with the security envelope [245] developed by the ARDA group [246].

The File Catalogue supports regular files as well as DataSets and information about running processes in the system (in analogy with the /proc directory on Linux systems). Each job sent to AliEn for execution gets a unique ID and a corresponding / proc/id directory where it can register temporary files, standard input and output, as well as all job products. In a typical production scenario, only after a separate process has verified the output, will the job products be renamed and registered in their final destination in the File Catalogue.

The data in the File Catalogue are organized by year, accelerator period and run. The data that are common to several runs are stored in a logical partition of the File Catalogue called Offline Condition Data Base (OCDB). The files contained in this portion are selected via a special combination of meta-data and support versioning.

Information service. The information service (IS) keeps track of the status, type and capabilities of all AliEn services, central and at the sites. For instance, all storage elements register themselves in the IS. They also publish the protocols they support. This way, the clients can figure out the best place to store files according to their needs.

Monitoring service. Monitoring in AliEn is based on the MonALISA framework [247]. A hierarchic architecture with selective aggregation at each level is employed for the monitoring, before sending the information upstream. This is a determinant factor in reducing the volume of raw monitoring information as much as possible while preserving important details. All AliEn services and the job wrapper are instrumented with a monitoring module (ApMon). Extensive information is collected about CPU and memory usage, consumed and wall-clock kSI2k time, open files and network traffic per service and job. A global view of the entire AliEn Grid, as well as long term persistence of the data is assured by the MonALISA repository. This service subscribes to general interest data and stores it into a database. It offers both near real-time and history views, with different levels of detail, down to individual user jobs.

AliEn provides a complete Grid solution for the ALICE experiment. It is, however, in our interest to minimise the amount of ALICE code and to maximise the use of common Middleware and 
services. This is in order to reduce the maintenance load to be performed by ALICE members and to rely as much as possible on common infrastructures.

This is possible only to a certain extent, insofar as the various Grid projects do not provide all the services needed by the ALICE Computing Model. To alleviate this situation, AliEn services are interfaced to the Grid systems present on the computational resources which are part of the ALICE Grid. This represents a good compromise, offering to the user a seamless interface to the heterogeneous components of the ALICE Grid, and minimising the amount of proprietary services and components.

AliEn is interfaced with the WLCG and ARC [248] Grid systems, where it is running in production, while the interface with the OSG [249] is at the moment of writing at the prototype stage.

To minimise the intrusiveness of the ALICE-specific Grid services, these are all installed on a single computer at each computing centre, the so called 'VO-Box'. This machine is the contact point between the Grid implemented via the common Middleware and the ALICE Grid. This setup allows us to realise a sort of 'Grid of Grids', where different Grids can seamlessly inter-operate.

During several 'data challenges', AliEn has shown its capability to implement the ALICE Computing Model as described in the Computing Technical Design Report [19] for simulation and reconstruction.

The AliEn capability to run analysis was tested to a lesser degree. At the moment of writing there are many millions of simulated events that were produced and reconstructed, and on which the analysis algorithms are exercised. This part of the framework is the one evolving most rapidly.

As the first step, the analysis framework extracts a subset of the Datasets from the File Catalogue using meta-data selection. Then the framework negotiates with dedicated Grid services the balancing between local data access and data replication. Once the distribution is decided, the analysis framework creates sub-jobs. The framework collects and merges available results from all terminated sub-jobs on request. An analysis object associated with the analysis task remains persistent in the Grid environment so the user can go offline and reload an analysis task at a later date, check the status, merge current results, or resubmit the same task with a modified analysis code.

\subsubsection{AliRoot framework}

The ALICE offline framework, AliRoot [250], is shown schematically in figure 6.12. Its implementation is based on Object-Oriented techniques for programming and, as a supporting framework, on the ROOT system [251], complemented by the AliEn system which gives access to the computing Grid. These fundamental technical choices result in one single framework, entirely written in $\mathrm{C}++$, with some external programs (hidden to the users) still in FORTRAN.

The AliRoot framework is used for simulation, alignment, calibration, reconstruction, visualisation and analysis of the experimental data. AliRoot has been in continuous development since 1998. It was used to perform simulation studies for the Technical Design Reports of all ALICE subsystems and to optimise their design. In the preparation phase, before the start of data taking, it was used to evaluate the physics performance of the full ALICE detector and to assess the functionality of the framework towards the final goal of extracting physics from the data.

The role of the AliRoot framework is shown schematically in figure 6.13. The kinematics tree containing, for example, the physics processes at the parton level and the results of the 


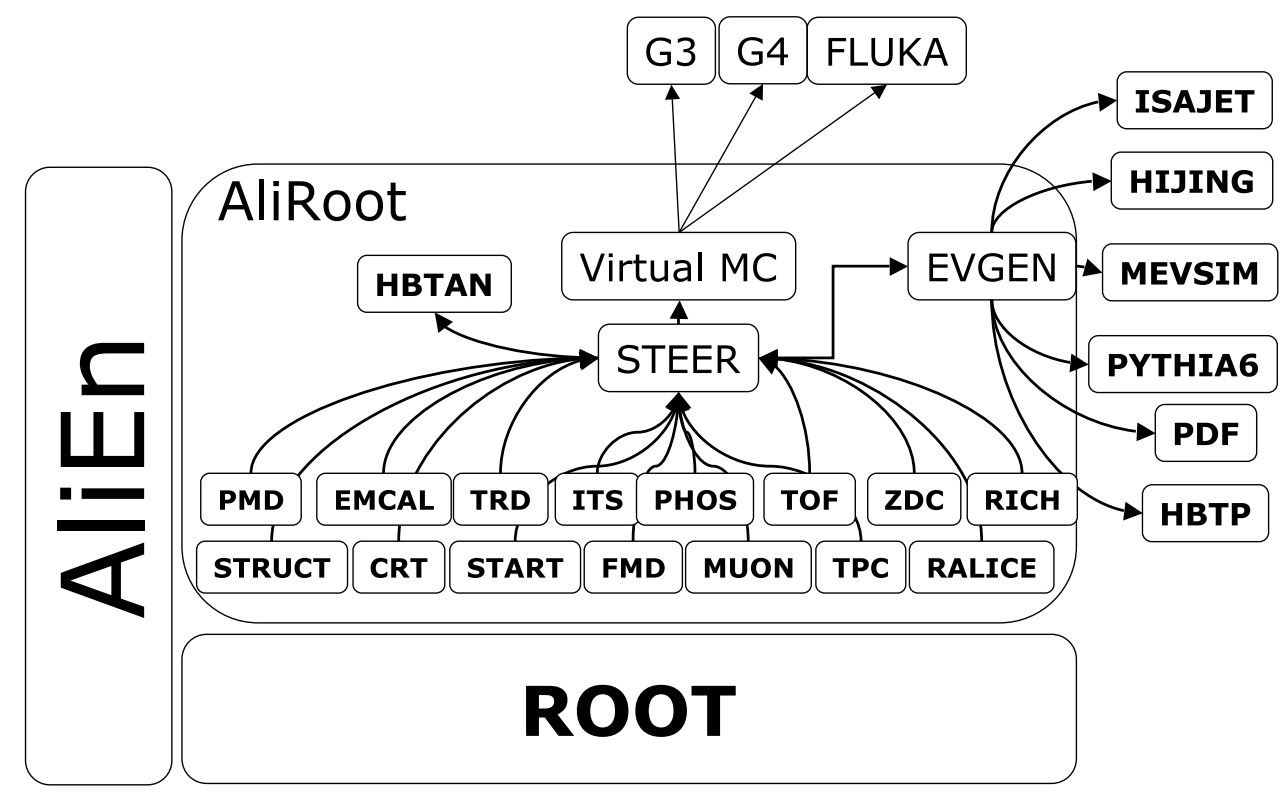

Figure 6.12: Schematic view of the AliRoot framework.

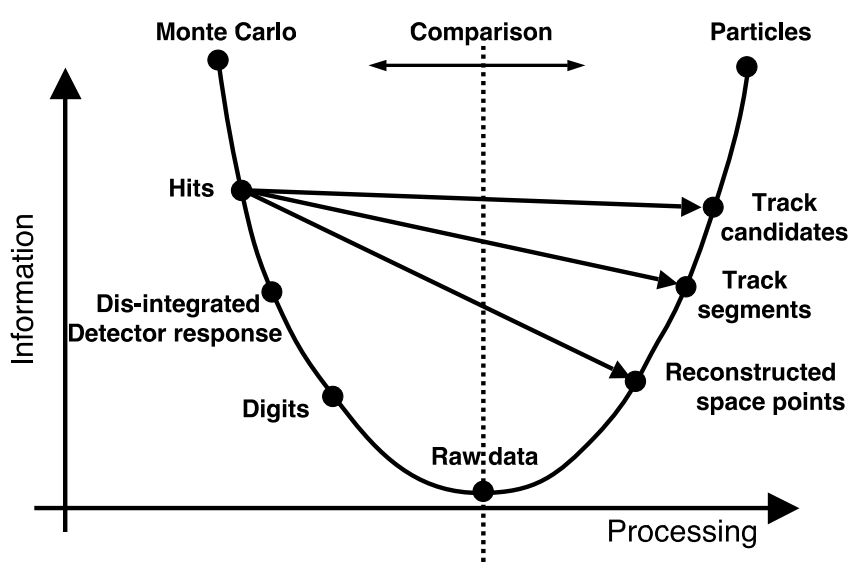

Figure 6.13: Data processing framework.

fragmentation (primary particles) is created by event generators. The data produced by the event generators contain full information about the generated particles: type, momentum, charge, and mother-daughter relationship. During the transport, the response of the detectors to each crossing particle is simulated. The hits (energy deposition at a given point and time) are stored for each detector. The information is complemented by the so called 'track references' corresponding to the location where the particles are crossing user defined reference planes. The hits are converted into digits taking into account the detector and associated electronics response function. Finally, the digits are stored in the specific hardware format of each detector as raw data. At this point the reconstruction chain is activated. Typically the detectors perform local reconstruction such as clusterisation, then a seeding procedure is used to start a Kalman filter [252] tracking. To evaluate 
the software and detector performance, simulated events are processed through the whole cycle and finally the reconstructed particles are compared to the Monte Carlo generated ones.

'Shortcuts' are possible in the interest of saving computing time, for instance using 'hits' directly for physics studies, instead of producing digitised data and then reconstructing them.

The users can intervene in this cycle provided by the framework to implement their own analysis of the data or to replace any part of it with their own code.

The basic design features of the AliRoot framework are re-usability and modularity. Modularity allows replacement of well defined parts of the system with minimal or no impact on the rest. For example, it is possible to change the event generator or the transport Monte Carlo without affecting the user code. Elements of the framework are made modular by defining an abstract interface to them. The codes from the different detectors are independent so that different detector groups can work concurrently on the system while minimising the interference. The adopted development strategy can handle design changes in production code for cases when new elements are introduced. Re-usability is the protection of the investment made by the programming physicists of ALICE. This investment is preserved by designing a modular system and by making sure that the maximum amount of backward compatibility is maintained while the system evolves.

The ROOT framework, upon which AliRoot is developed, provides an environment for the development of software packages for event generation, detector simulation, event reconstruction, and data analysis. It offers, among other features, integrated I/O with class schema evolution, an efficient hierarchical object store with a complete set of object containers, $\mathrm{C}++$ as a scripting language and a $\mathrm{C}++$ interpreter, advanced statistical analysis tools (multidimensional histograms, several commonly used mathematical functions, random number generators, multi-parametric fit, minimisation procedures, cluster finding algorithms etc.), HTML documentation tools and advanced visualisation tools. The ROOT system was extended with ALICE specific classes and libraries grouped in modules. These libraries are loaded dynamically and the contained classes share the same services with the native ROOT classes, including object browsing, $\mathrm{I} / \mathrm{O}$, dictionary and so on.

The ROOT system is interfaced with the Grid Middleware in general and, in particular, with the ALICE-developed AliEn system. In conjunction with the PROOF [253] system, which extends ROOT capabilities on parallel computing systems and clusters, this provides a distributed parallel computing platform for large-scale production and analysis.

\subsubsection{Event simulation}

The offline framework was developed to allow for efficient simulations of nucleus-nucleus, protonnucleus and proton-proton collisions and to provide a predictive and precise simulation of the detector response. It includes the following options:

- Interfaces to several external generators, like for example HIJING [142].

- A simple event generator based on parametrised $\eta$ and $p_{\mathrm{t}}$ distributions can provide a signalfree event with multiplicity as a parameter.

- Rare signals can be generated using the interface to external generators like PYTHIA [254] or simple parameterisations of transverse momentum and rapidity spectra defined in function libraries. 
- Tools to combine underlying events and signal events on the primary particle level (cocktail) and on the digit level (merging).

- Generators for realistic beam-gas and beam-halo event simulations.

- 'After-burners' are used to introduce predefined particle correlations.

To facilitate the usage of different generators, we developed an abstract generator interface that allows the study of full events or signal processes, and a mixture of both, i.e. cocktail events.

Several event generators are available via the generic generator interface class, TGenerator. Via this abstract base class we wrap FORTRAN Monte Carlo codes that are thus accessible from AliRoot.

In many cases, the expected transverse-momentum and rapidity distributions of particles are known. In other cases the effect of variations in these distributions must be investigated. In both situations it is appropriate to use generators that produce primary particles and their decays by sampling from parametrised spectra. The corresponding parameterisations are stored in independent function libraries wrapped into classes that can be plugged into the generator.

The modularity of the event-generator framework allows easy integration of objects that are responsible for changing the output of event generators or for assembling new events making use of the input of several events. These processors are generally called 'after-burners'. They are especially needed to introduce a controlled (parameterised) particle correlation into an otherwise uncorrelated particle sample.

\subsubsection{Detector response simulation}

For the detector-response simulation different transport Monte Carlo packages are available, namely GEANT 3 [141], GEANT 4 [255] and FLUKA [139]. They have a very different user interface for signal scoring and geometry definition. To be able to use them to simulate the ALICE detector via the AliRoot framework, they were interfaced to the Virtual Monte Carlo abstract interface in ROOT. Moreover, their native geometry modellers were replaced by TGeo, the geometry modeller provided by ROOT. Thanks to this, it is enough to instantiate the appropriate class in the simulation configuration file to simulate ALICE with any of these three codes.

For special studies, usually requiring very large sample sizes, the detector response is simulated via appropriate parameterisations or other techniques that do not require the full particle transport. These application are known as 'fast simulations'. Several were implemented in AliRoot. The systematic error introduced by the parameterisations is in general small compared to the reduction of the statistical error.

The ALICE detector is described in great detail, see figure 6.14, including services and support structures, absorbers, shielding, beam pipe, flanges, and pumps. The TGeo package is used for particle transport during simulation, and for alignment and reconstruction. The magnetic field of the solenoid and the dipole magnet is described by a parametrisation of detailed measurements obtained with an accuracy of the order of 1.0 Gauss. 


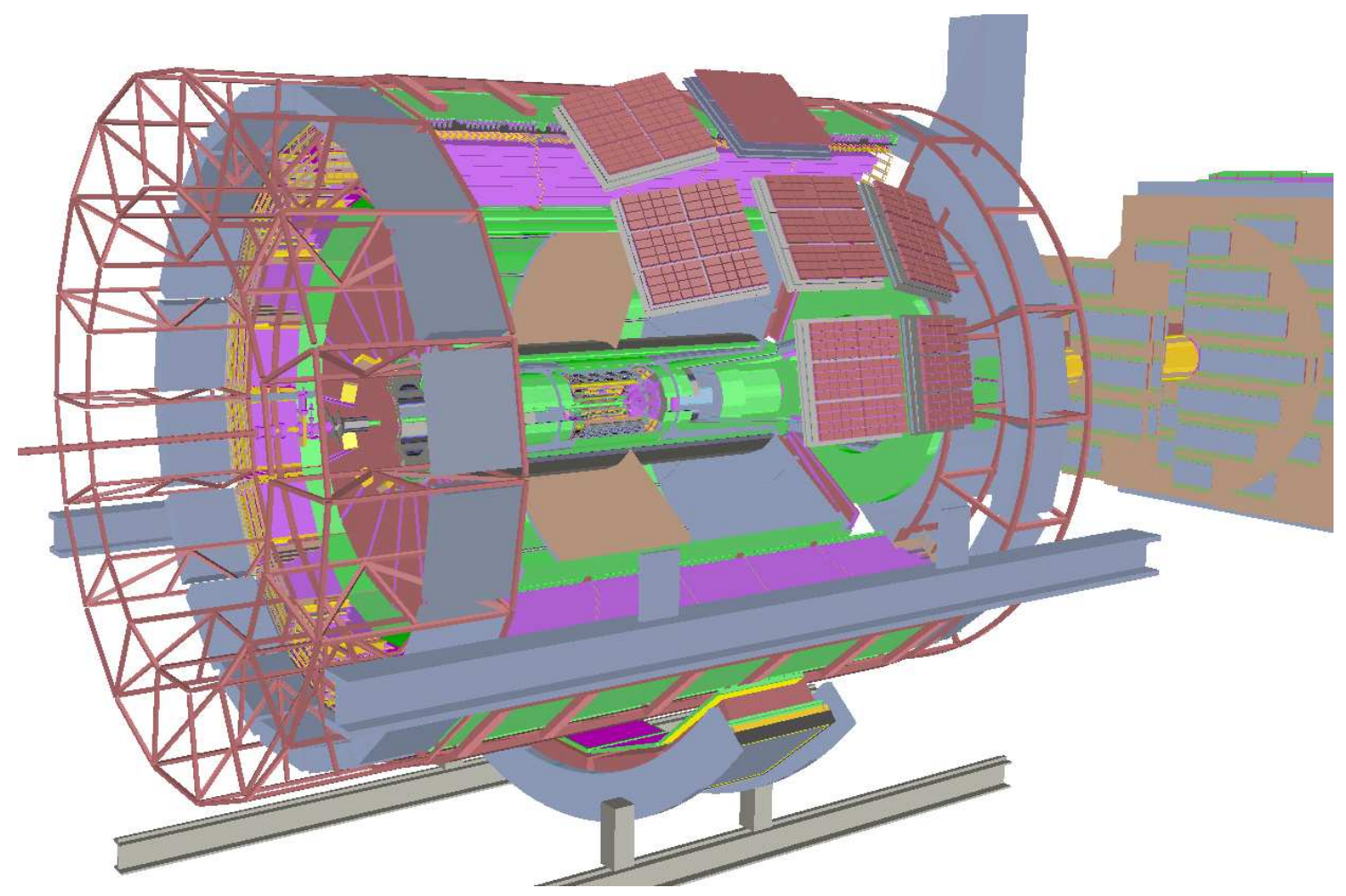

Figure 6.14: Geometry of the ALICE detector in the AliRoot simulation.

\subsubsection{Alignment framework}

Thanks to the use of the TGeo package, it is possible to perform detailed alignment studies for the ALICE detector. The parts of the detector that are subject to relative positioning different from the ideal one, are called 'aligneable volumes'.

When the simulation program is started, the ideal geometry is generated via compiled code or read from the OCDB where it was saved in a previous run. Several objects are marked as 'aligneable', that is the geometrical modeller is ready to accept modifications to their position, even if they were obtained by replication. The framework then reads the 'alignment objects' which contain the 'adjustments' in the position of the aligneable objects. The particle transport is then performed in the modified geometry.

The same procedure can be repeated for reconstruction and, therefore, the effect of detector misalignment and the performance of the alignment algorithms can be tested. During reconstruction of real data, the 'best' alignment objects are loaded from the OCDB. For the first pass reconstruction these are the alignment objects produced from survey data. During subsequent reconstruction passes, the alignment objects are produced by the alignment algorithms optimizing the reconstruction quality during processing of the raw data.

Survey data are automatically loaded on the Grid in a standard text format for automatic parsing and conversion to alignment objects. Alignment objects are stored in the OCDB and accessed via meta-data. 


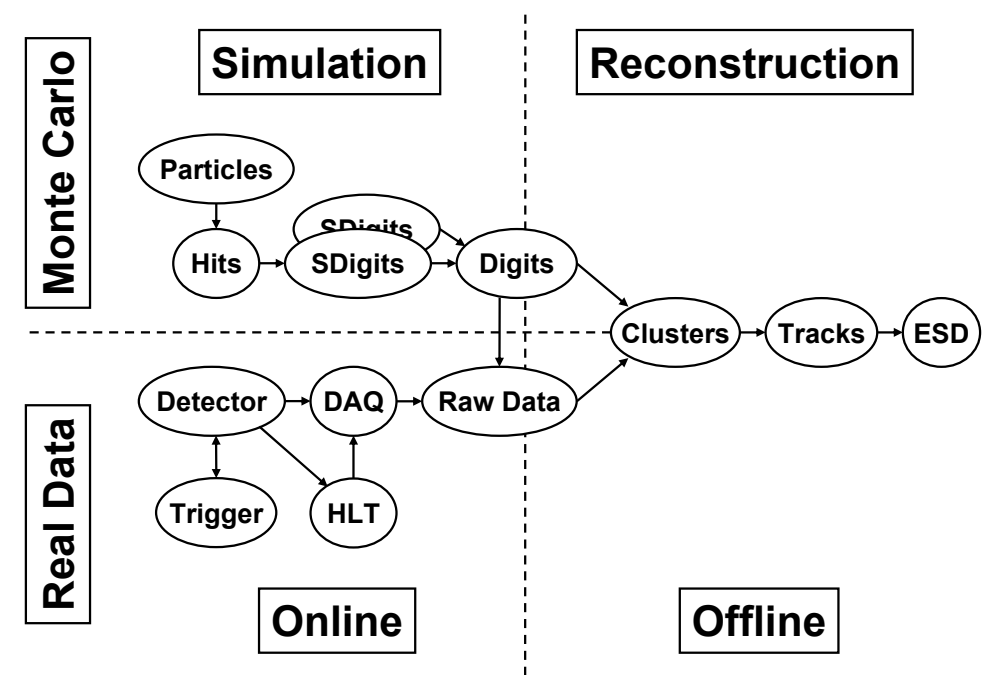

Figure 6.15: Interaction of the reconstruction code with the other parts of AliRoot.

\subsubsection{Calibration framework}

This framework is very similar to the alignment one. The initial calibration constants come either from the detector properties as measured during construction, or from algorithms running online during data-taking aimed at providing a partial calibration sufficient for the first-pass data reconstruction. During the reconstruction itself, better calibration constants can be calculated and stored in the OCDB.

\subsubsection{Reconstruction framework}

The ALICE reconstruction code is part of the AliRoot framework. Its modular design allows its code to be compiled into separate shared libraries and executed independently of the other parts of AliRoot. As an input, the reconstruction uses the digits together with some additional information like module number, readout channel number, time bucket number, etc. The reconstruction can use both digits in a special ROOT format, more convenient for development and debugging purposes, and digits in the form of raw data, as they are output from the real detector or can be generated from the simulated special-format digits above (see figure 6.15). The output of the reconstruction is the ESD containing the reconstructed charged particle tracks (together with the particle identification information), decays with the $\mathrm{V}^{0}$ (like $\Lambda \rightarrow \mathrm{p} \pi$ ), kink (like charged $\mathrm{K} \rightarrow \mu \nu$ ) and cascade (like $\Xi \rightarrow \Lambda \pi \rightarrow \mathrm{p} \pi \pi$ ) topologies and particles reconstructed in the calorimeters.

The main steering reconstruction class, AliReconstruction, provides a simple user interface to the reconstruction. It allows users to configure the reconstruction procedure, include or exclude a detector from the run, and ensure the correct sequence of the reconstruction steps:

- reconstruction steps that are executed for each detector separately (typical example is the cluster finding);

- primary vertex reconstruction; 
- track reconstruction and particle identification (PID);

- secondary vertex reconstruction $\left(\mathrm{V}^{0}\right.$, cascade and kink-decay topologies).

The AliReconstruction class is also responsible for the interaction with the AliRoot I/O sub-system and the main loop over the events to be reconstructed belongs to this class too.

The interface from the steering class AliReconstruction to the detector-specific reconstruction code is defined by the base class AliReconstructor. For each detector there is a derived reconstructor class. The user can set options for each reconstructor in the form of a string parameter. Detector-specific reconstructor classes are responsible for creating the corresponding specific cluster-, track- and vertex-finder objects and for passing the corresponding pointers to the AliReconstruction. This allows one to configure the actual reconstruction process using different versions of the reconstruction classes at the detector level.

The detailed description of the reconstruction and particle identification in all the ALICE detectors can be found in ref. [21] and it is reviewed in the chapter 8. Here we shall only outline briefly the software structure.

The space points are reconstructed by a detector-specific cluster-finding procedure. For each space point we also calculate the uncertainty of the space-point position estimation. All of the central tracker detectors (ITS, TPC, TRD) have their own detailed parametrisation of the space-point position uncertainties. The space-point coordinates together with the position uncertainties are then passed to the track reconstruction. If, in addition to the space-point position, the detector is also able to measure the produced ionization, this information can be used for the particle identification.

Offline track reconstruction in ALICE is based on the Kalman filter approach. The detector specific implementations of the track-reconstruction algorithm use a set of common base classes, which makes it easy to pass tracks from one detector to another and test various parts of the reconstruction chain. For example, we can use smeared positions of the simulated hits instead of the ones reconstructed from the simulated detector response, which is very useful for testing purposes. In addition, each hit structure contains the information about the track that produced it. Although, this implies the storage of extra information, it was proved to be very useful for debugging the track reconstruction code.

Combining (using a Bayesian approach) the particle-identification information coming from the outer detectors (TRD, TOF, HMPID) and $\mathrm{d} E / \mathrm{d} x$ measured by TPC and ITS, ALICE is able to identify charged particles. The neutral particles in the central-rapidity region are identified by the calorimeters (PHOS and EMCAL). There is also the possibility to count photons in the forward region using the PMD.

The muon arm is designed to precisely measure the muon momenta behind the absorber. The muon track-finding algorithm uses the Kalman-filter technique as well.

The algorithms in preparation for the High-Level Trigger (HLT) reconstruction code are implemented as a last step within the AliRoot simulation chain. This allows for studying the HLT track-finding performance and replaying the online trigger decisions during the offline data analysis. The code that reads the data produced by HLT is organised as a 'virtual' detector reconstructor class which derives from the base AliReconstructor class and is called by the steering class AliReconstruction. The output of the HLT reconstruction is stored in an ESD object using the same format as the offline reconstruction, which facilitates the comparison between offline and HLT reconstruction results. 


\subsubsection{Analysis}

Analysis is the final operation performed on the data and the one finally destined to extract physics information. In the ALICE Computing Model, the analysis starts from the ESD produced during the reconstruction step. Analysis tasks produce AOD with standard content condensed from the ESD as well as AODs for specific analyses. Further analysis passes can start from condensed AODs.

We distinguish two different analysis activities, scheduled (or ordered) and end-user (or chaotic) analysis. Scheduled analysis will be performed in a way that sometimes is indicated as 'freight train'. ALICE generic analysis framework 'attaches' a number of 'official' (at the level of the collaboration) algorithms (the 'coaches') and 'carries' them through the data. The advantage is that each event is read only once and the different algorithms are applied to it. Care has to be taken that one coach does not derail the whole train. Scheduled analysis has a very predictable resource consumption and data access pattern, and this makes it an ideal activity for large batch job runs at Tier-1's centres.

End-user analysis comprises all the activities performed by users in the framework of a specific physics analysis activity. Its peculiarity is the unpredictability of both the data access pattern and the resource consumption. End-user analysis is prototyped on local systems with a limited amount of data and then performed interactively on local clusters using the parallel PROOF system, or it is submitted as a batch job to the AliEn system, which will execute on Tier-2 and Tier-1 systems, according to resource availability.

We developped an analysis framework, AliAnalysis that can be used for scheduled and end-user analysis. Based on TSelector and TTask, it was implemented such that the user code is independent of the used computing scheme (local, PROOF or Grid). It also allows us to include of Monte Carlo truth information into the analysis chain so that it can be used for efficiency and acceptance correction studies. The analysis framework permits the splitting of each analysis into a tree of dependent tasks. Each task is data oriented: it registers the required input data and publishes its output. The optimization of the execution chain is done after the registration of all tasks included in the analysis.

\subsubsection{CERN Analysis Facility (CAF)}

The need for a very fast feedback during data taking lead to the deployment of a large cluster for interactive parallel processing of the data with PROOF as the enabling technology. The CAF allows physicists to perform, very rapidly, various operations on the data acquired with the full offline framework, providing information on the quality of the data itself, and on the used calibration algorithms. CAF is also intended for early discovery of important features of the data, in order to direct and refine the 'freight train' analysis passes.

The general architecture of the CAF is shown in figure 6.16 .

Most of the activity is on the raw data and intermediate reconstruction output (e.g. clusters). However, the final reconstruction output (ESD and AOD) is also available, to e.g. exercise AOD production on the CAF. The local disk space of the CAF acts as a cache for files stored in other persistent storage systems. The CAF is enabled to stage files from AliEn SE identifying files via the AliEn File Catalogue. Furthermore files can be retrieved from CASTOR directly. 


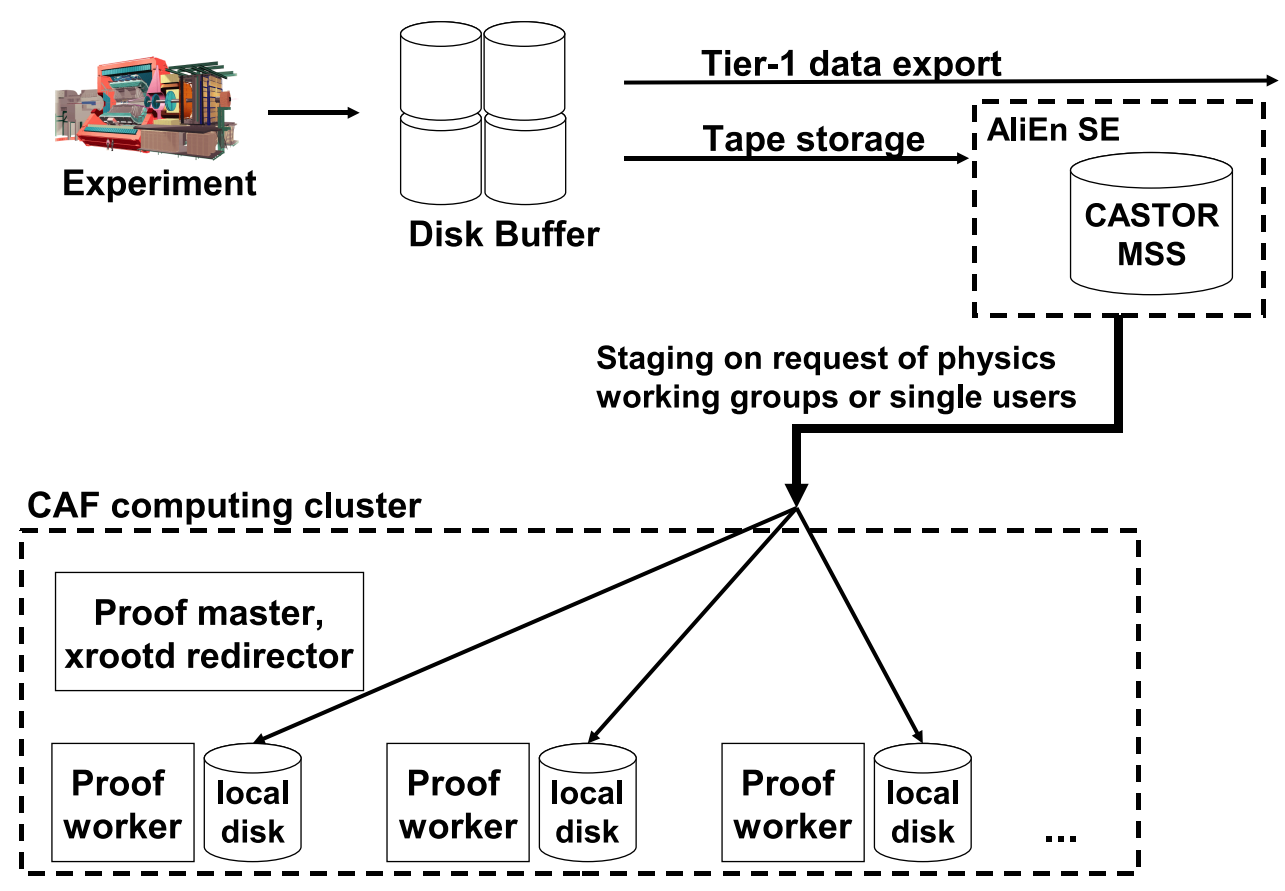

Figure 6.16: Architecture of the CERN Analysis Facility.

Staging is triggered by user's requests to the head node of the system. The system chooses a disk server, and the file is staged to its local disk. As the access is done via the AliEn File Catalogue, the user is able to chose to stage a remote file, located on a SE not necessarily at CERN. All the file staging and access is achieved via xrootd plus a plugin that contains the staging logic from AliEn and CASTOR. The CAF also allows to access files from AliEn and CASTOR without staging them.

The above functionality is part of xrootd and was tested on the CAF. This functionality is however insufficient for the intended use of the CAF. We foresee different groups, subsystems and Physics Working Groups, accessing the system at the same time. It is therefore necessary to introduce a system of CPU and disk quotas in order to regulate the usage of the CAF.

The performed tests indicate that with a reasonable mix of queries of different lengths, each user obtains a performance which is very close, or even larger, than their quota of resources (number of machines per user), because of the necessary pauses between queries in interactive usage. The overall utilisation of the system in this case stays above $75 \%$. These tests were conducted with normal batch nodes which were removed from the CERN lxbatch system. This is very important because the possibility to use standard lxbatch nodes reduces the cost of the CAF.

The CAF is monitored on different levels with the help of MonALISA. Apart from hostmonitoring, query statistics is recorded after each query. This includes the amount of data processed, the number of events, the CPU time consumed and the time the user waited for the query to finish. This information is used to visualize the utilization of the cluster as well as for the CPU fairshare mechanism. 


\subsubsection{Software development environment}

The ALICE software community is composed of small groups of 2-3 people who are geographically dispersed and who do not depend hierarchically from the Computing Coordinator. This affects not only the organisation of the computing project but also the software development process itself as well as the tools that have to be employed to aid this development.

This is a novel situation for HEP quantitatively if not qualitatively. The experiments' offline programs have to be developed by truly distributed teams. Traditional software engineering methods are not meant to cope with this reality. The development of AliRoot has therefore followed some empirical principles.

Requirements are necessary to develop the code. ALICE users express their requirements continuously with the feedback they provide. The core offline team redirects its priorities according to the user feedback. In this way, we make sure that we are working on the highest priority item at every moment, maximising the efficiency of our work by responding to user's need and expectations.

Design is good for evolving the code. AliRoot code is re-designed continuously since the feedback received from the users is folded into the continuous design activity of the core offline team. At any given time there is a design of the system considered as a short-term guide to the next development phase and not a long-term static objective. This design activity is essential since it rationalises the development and indicates how best to include the features demanded by the users.

Testing is important for quality and robustness. While component testing is the responsibility of the groups providing modules to AliRoot, full integration testing is done nightly to make sure that the code is always functional. The results of the tests are reported on the Web and are publicly accessible [256]. The problems are reported on a Savannah server and assigned to the responsible developer.

Integration is needed to ensure coherence. We have a single code base handled via the SVN [257] version management tool where one or two developers for every module store their code. The different modules are largely independent to allow parallel development. A HTML version of the code is also extracted and created every night thanks to the ROOT documentation tools [258].

Discussions are valuable for users and developers. In ALICE, the discussion list is a valid place to express requirements and discuss design, extending the discussions we have during offline meetings. It is not infrequent that important design decisions are taken on the basis of an email discussion thread where all interested users participate. Redefinition of planning and sharing of work is also very frequent, adding flexibility to the project and allowing it to address both shortterm and long-term needs. A specialised Web-based tool is used to track the current planning tasks and the available FTE resources. 
The ALICE offline code is composed of one single framework, Object Oriented in design and implemented in $\mathrm{C}++$ written by multiple authors. This requires a high degree of uniformity in the code as its structure has to be readable and easily understandable. We have therefore adopted a limited set of coding and programming rules [259]. To effectively enforce them, we have developed, together with IRST, a code analysis tool [260] able to check compliance of the code with our coding rules. This now runs nightly and a table of the violations in all modules is published on the Web [261].

This development requires a specific release policy. It is based on the principle of fixed release dates with flexible scope. Given that the priority list is dynamically rearranged, it is difficult to define the scope of a given release in advance. Instead, we decided to schedule the release cycle in advance. The current branch of the SVN repository is 'tagged' approximately once a week with one major release every couple of months.

A release is announced a fortnight before. If a conflict arises, the scope of the release is reduced but the date is not moved. On the date, a branch is created. Usually before the announced date a large amount of code is checked into the head branch, so once the branching is performed, the code tend to be rather unstable. Then the code in the release branch is stabilised and corrected for one more fortnight and finally the release is tagged.

We have tried to make installation as fast and reliable as possible. AliRoot is one single package that links only to ROOT, and of course to the transport Monte Carlo. Thanks to this, we have not needed configuration management tools. We try to be as independent of the specific version of the operating system and compiler as possible. To ensure easy portability to any future platform, one of our coding rules states that the code must compile without warning on all supported platforms. At this time, these are Mac OS X, Linux on IA32 and IA64 machines, and Solaris.

\subsubsection{The quasi-online environment}

One of the requirements to the ALICE Offline framework is to provide physics results as early and as reliably as possible. The amount of data produced both in proton-proton and in nucleus-nucleus running and the limitation on the amount of resources available (disk spindles, tape drives, CPUs) sets a minimum time for a full reconstruction pass through the data. To obtain meaningful physics results timely, the data have to be processed quasi online for proton-proton and at a very high pace after the nucleus-nucleus run during the shutdown, as explained in the computing model section 6.4.2.2.

To perform such a reconstruction, it is necessary to have 'reasonable' calibration information available immediately after the end of each run. This is achieved via a quasi-online framework developed jointly with the Data Acquisition (DAQ) and High Level Trigger (HLT) project, which is shown in figure 6.17 .

The main idea is to provide calibration information that can be used at the end of the run for the first reconstruction pass. This information comes from different sources.

- DAQ: The first source of calibration are the data themselves. The data for calibration are collected on the Local Data Concentrators (LDC), Global Data Concentrators (GDC) and the monitoring farm local disks. The various run conditions also participate to the calibration process, and these are collected from the DAQ logbook or from the Experiment Control System (ECS) which is part of the DAQ. 


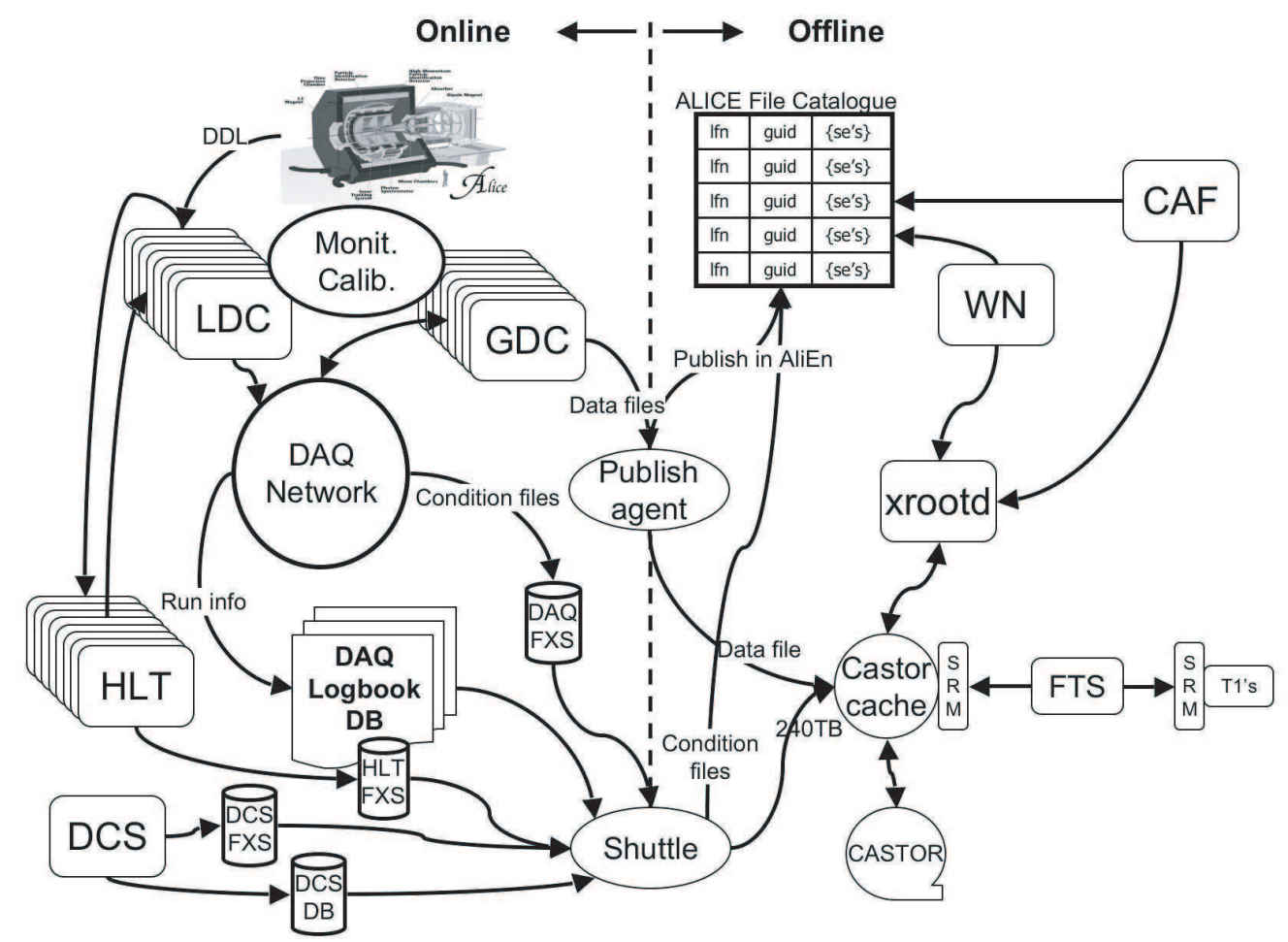

Figure 6.17: The quasi-online framework.

- DCS: A large part of the information necessary for calibration comes from the Detector Control System. A fraction of the DCS data consists of parameters monitored continuously and unasinchronously with respect to data taking, and archived in an Oracle database (DCS archive). A special server called AMANDA provides an API to the DCS archive and allows to recuperate these data. DCS may also produce calibration data which cannot be archived on the Oracle database: these data are shipped to Offline through a file exchange server.

- HLT: The High-Level Trigger is of course a very important source of calibration information. It is also a user of calibration data for its own reconstruction.

- Trigger: The trigger scalers can also provide information needed.

- DCDB: Some calibration information is static and it is measured once and for all in the laboratory and registered in the Detector Construction Data Base (DCDB). The subset of these data which are needed for Offline reconstruction are transferred to the OCDB before the start of the data taking.

These data are collected during the run, but only after they are processed by the detectorspecific algorithms that are used to calculate the calibration parameters. The original data from which the parameters are calculated are called 'reference data' and some of them are stored and catalogued as the rest of the data. The processed data are put on shared storage areas, called File eXchange Servers (FXS). A program called Shuttle runs at the end of each data-taking run. Its task is to collect all the data from the FXSs and from the DCS archive, further process them if necessary, 
and then catalogue them in the OCDB, a special portion of the AliEn File Catalogue. These data will be used for the first pass reconstruction. 


\section{Chapter 7}

\section{Control System}

\subsection{Detector Control System (DCS)}

\subsubsection{Introduction}

The primary task of the ALICE Control System is to ensure safe and correct operation of the ALICE experiment [17]. It provides remote control and monitoring of all experimental equipment in such a way that the ALICE experiment can be operated from a single workplace, the ALICE Control Room (ACR) at LHC point 2, through a unique set of operator panels. The system provides the optimal operational conditions so that the data taken with the experiment is of the highest quality. The ALICE control system was designed to reduce the downtime of the experiment and hence contribute to a high running efficiency. It also maximises the number of channels operational at any time, and measures and stores all parameters necessary for efficient analysis of the physics data.

\subsubsection{Design strategy and system architecture}

The ALICE control system is coordinated and built by the ALICE Control Coordination (ACC) team in collaboration with the sub-detector groups, the online systems groups and the various CERN services groups.

\subsubsection{Requirements}

Although covering a wide variety of components, and being developed by various groups in parallel, the DCS is a coherent and homogeneous system, and allows for easy integration of components. The DCS is flexible and scalable, to accommodate the changes of the experiment during its lifetime. As the control system is supposed to be operational throughout all operational phases of the experiment (data taking, shutdown etc.), it is able to cope with different operational modes, and allows independent and concurrent operation of each sub-detector, or any part of it.

Since the shift crew is not necessarily expert in controls or in the operation of a detector, special attention was given to the presentation of the system to the operator. Remote access to the DCS is needed and a strict access control mechanism was put in place, based on the origin of the access and the user profile. All data required to configure the experiment equipment is stored in databases. All relevant data for the operation of the experiment is archived and is available at 
any time. All data relevant for the analysis of the physics data is stored in a database and is easily accessible from the offline system.

Ensuring the integrity of the detector equipment is largely the task of the control system. The control system is reliable and allows for both hardwired and software actions in case of hazardous situations. It is easily maintainable, also in the long term when some of the original expertise might have disappeared.

\subsubsection{Methods}

Given the large number of sub-detectors and sub-systems that need to be controlled the only way to come to an integrated control system for the ALICE experiment is to use common solutions for common requirements. The ALICE Controls Coordination (ACC) was set up to coordinate all the controls activities within the experiment. The Joint COntrols Project (JCOP, [262]), a collaboration between CERN and the four LHC experiments, provided a discussion forum and developed a set of tools and components used to implement the control system. The ACC team systematically collected the controls requirements for each sub-system involved and established light-weight User Requirements Documents (URDs) in order to enable the identification of communalities. Each URD is accompanied by an 'overview drawing' that depicts the hardware structure of the control system of each sub-detector.

\subsubsection{Architecture}

The ALICE control system is responsible for configuring, monitoring and controlling the equipment of the experiment. These are commercial hardware devices such as power supplies and crates, as well as sub-detector specific equipment like front-end chips, etc. It also covers computing devices such as PCs and PLCs as well as the software processes running on them. This task is accomplished mainly by sending commands and settings to and reading information back from the equipment.

The control system is able to take pre-programmed decisions and automatic actions without operator intervention such as recovering from errors. The operator is able to interact with the control system through a user interface that presents the information and allows issuing of commands.

\section{Hardware architecture}

The hardware architecture of the control system can be divided into three layers; a supervisory, control and field layer as shown in figure 7.1. The supervisory layer consists of a number of computers (Operator Nodes, ON) that provide the user interfaces to the operators. The supervisory level interfaces to the control layer, where computers (Worker Nodes, WN) and PLC or PLC-like devices interface to the experimental equipment. These devices collect and process information from the lower, so called field layer, and make it available for the supervisory layer. At the same time it receives information from the supervisory layer to be processed and distributed to the field layer. The field layer comprises all field devices such as power supplies and fieldbus nodes, sensors, actuators, etc. 


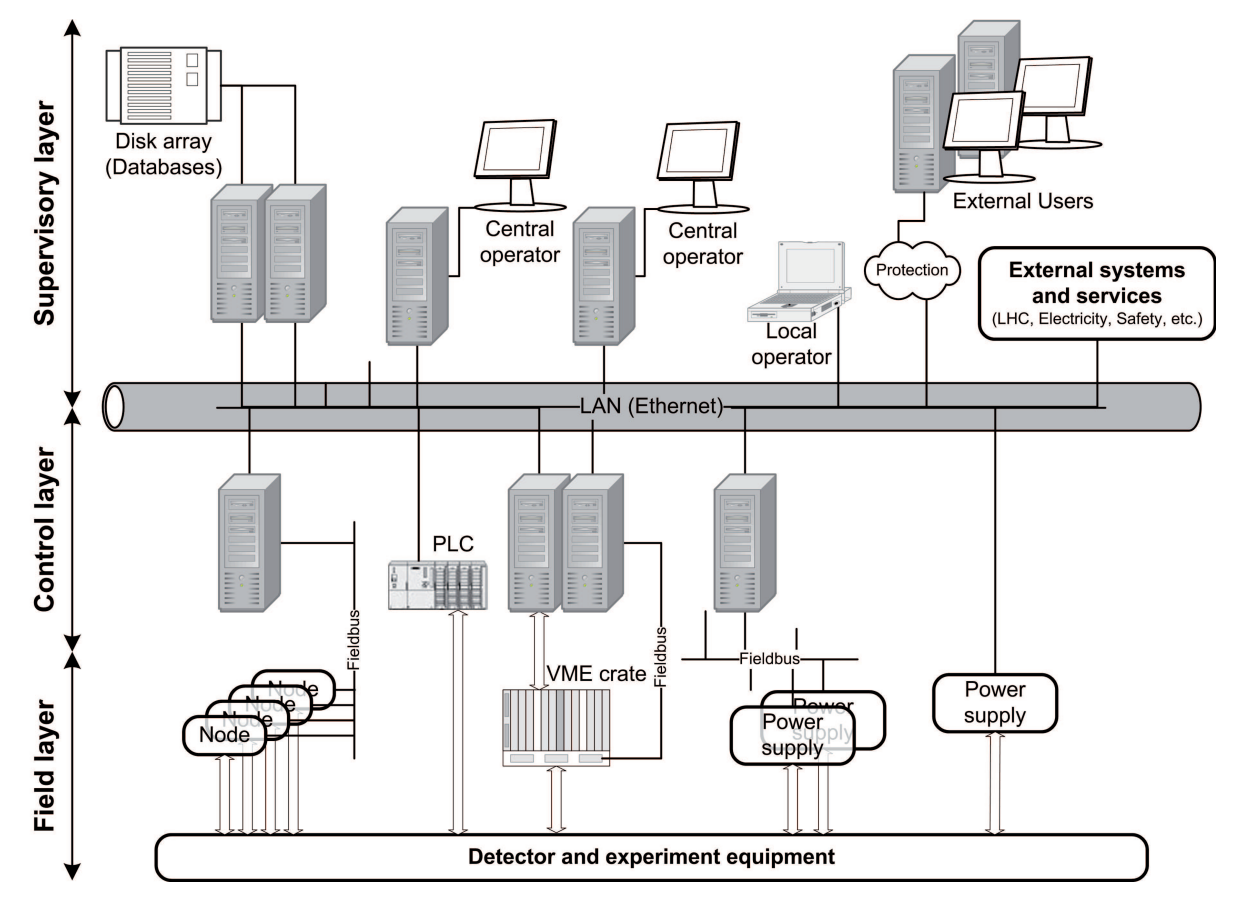

Figure 7.1: ALICE DCS hardware arcitecture.

\section{Software architecture}

The software architecture (figure 7.2) is a tree-like structure that represents the structure of subdetectors, their sub-systems and devices. The structure is composed of nodes which each have a single 'parent' except for the top node called the 'root node' that has no parent. Nodes may have zero, one or more children. A node without children is called a 'leaf', and a subset of a tree's nodes is called a 'sub-tree'.

\section{Control, Logical and Device Units}

There are three types of nodes, a Control Unit (CU), a Logical Unit (LU) and a Device Unit (DU) that serve as basic building blocks for the entire hierarchical control system. The CU and LU model and control the sub-tree below it and the device unit 'drives' a device. The hierarchy can have an arbitrary number of levels to provide the sub-detectors with as many abstraction layers as required.

\section{Partitioning}

The hierarchy also offers a high degree of independence between its components and allows, by means of the concept of 'partitioning', for concurrent use. Partitioning is the capability of independently and concurrently controlling and monitoring parts of the system, typically sub-trees of the hierarchical control tree. This functionality is essential during the installation and commissioning phase, where parts of the control system might not yet be available but sub-detectors need to control the installed equipment. The feature is also essential during operation for debugging purposes or sub-detector test or calibration running. During longer shutdown periods, sub-detectors might want 


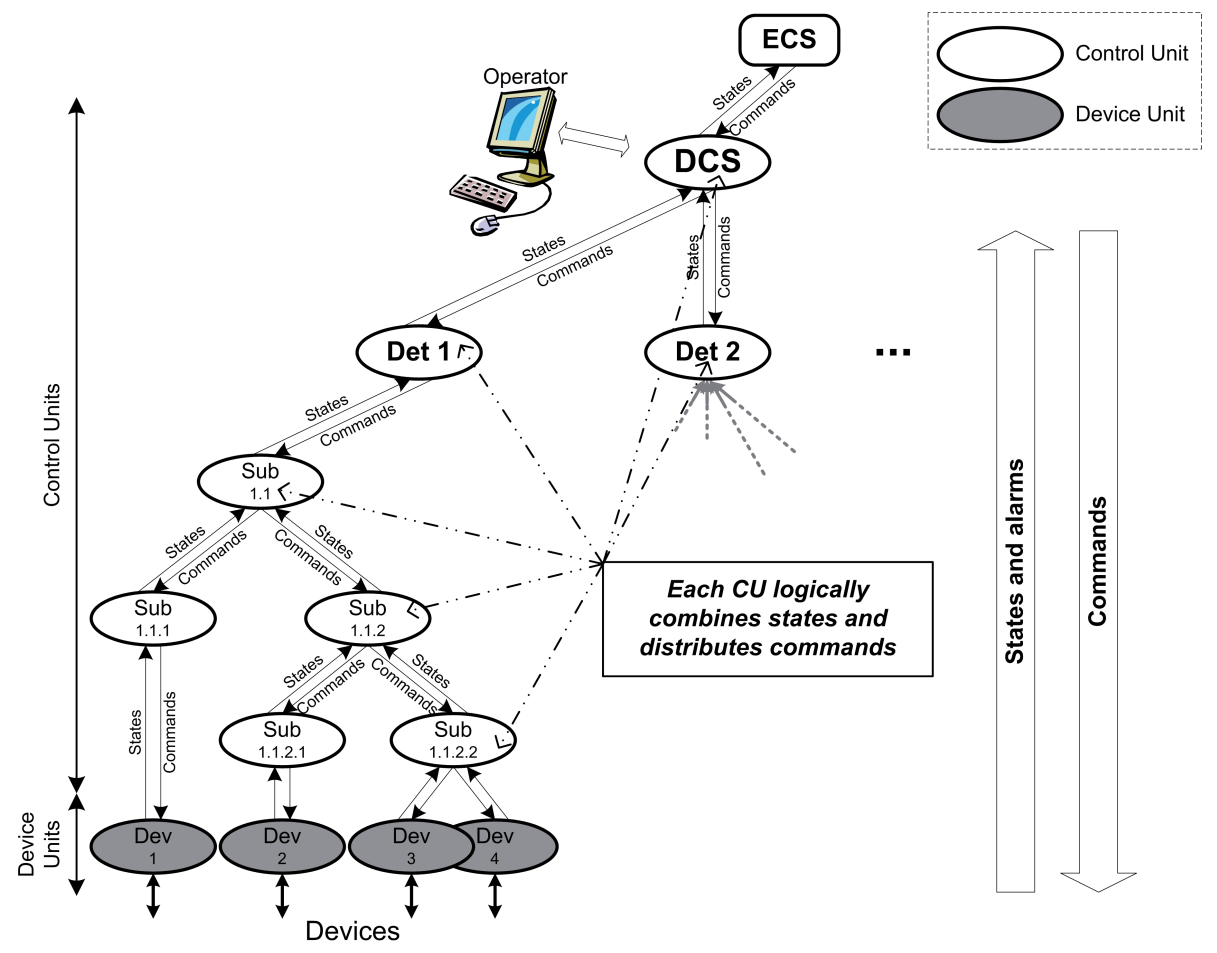

Figure 7.2: DCS hierarchical control arcitecture.

to run their sub-detector control system while other parts of the control system are still switched off. Only the control units in the control tree can become the root node of a partitioned control tree.

\section{Finite-State Machine}

The behaviour and functionality of each unit in the control tree is modelled and implemented as a finite-state machine (FSM). The finite-state machine concept is a fundamental component in the control system architecture. It is an intuitive, generic mechanism to model the functionality of a piece of equipment or a sub-system. The object to be modelled is thought of as having a set of stable 'states'. It can transit between these states by executing 'actions' that are triggered either by commands from an operator or another component or by other events such as state changes of other components. Two types of objects can be defined in the FSM concept: abstract objects, represented by a control or logical unit, and physical objects, represented by a device unit in the control tree. This concept allows for distributed and decentralised decision making and actions can be performed autonomously, even when controlled centrally. This will naturally lead to parallelism in automated operations such as error recovery, thus increasing the efficiency of the system. 


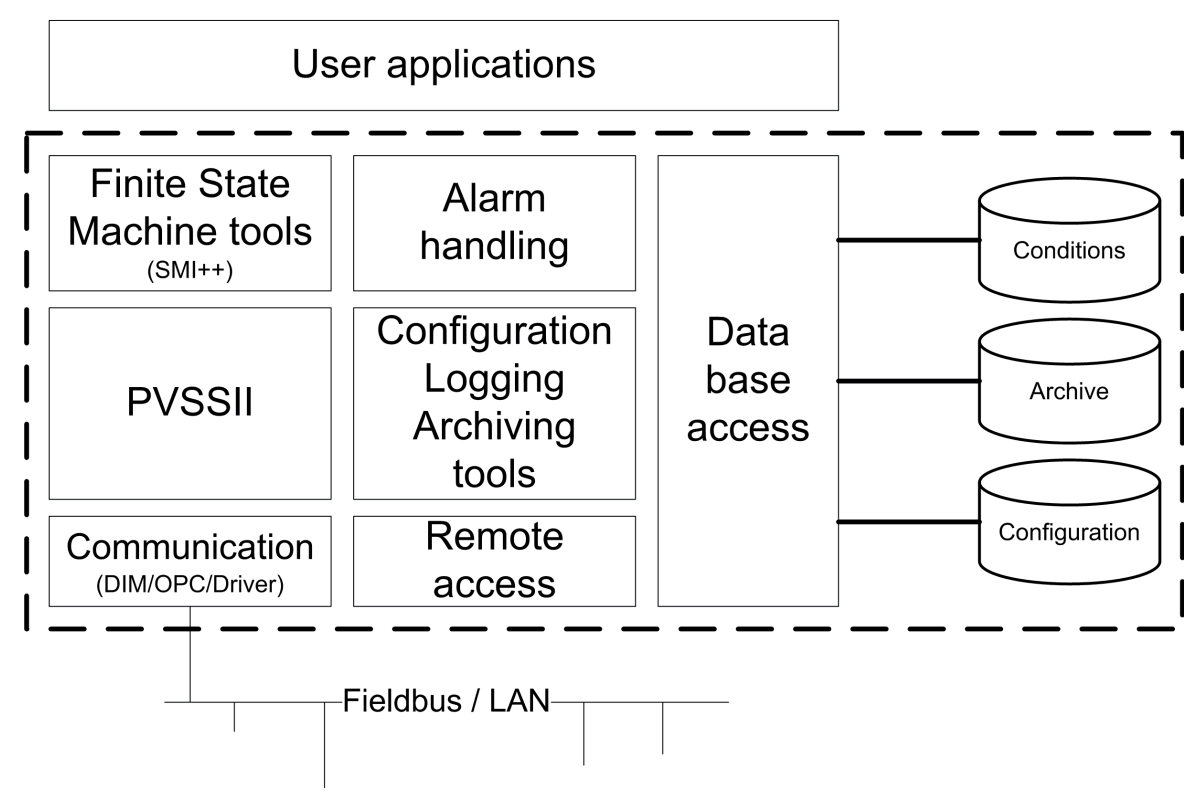

Figure 7.3: Overview of control system components.

\subsubsection{System implementation and operation}

\subsubsection{System components}

\section{PVSSII}

The core software of the control system is a commercial SCADA (Supervisory Controls And Data Acquisition) system: PVSSII. This package was selected after an extensive evaluation performed by the CERN-wide Joint Controls Project (JCOP). PVSSII is used by all four LHC experiments, as well as by several of the services such as gas, safety, etc. It offers many of the basic functionalities needed by the control system.

\section{JCOP and ALICE frameworks}

Around PVSSII a framework was built as a joint effort between the four LHC experiments (figure 7.3). This framework provides tools and components for the implementation of all the common tasks that are expected from the control system such as FSM, database access, access control, basic user interfaces, configuration, etc. The JCOP-framework also implemented interfaces to several hardware devices that are commonly used and that hide much of the PVSSII internals from a non-expert end-user. In the same context an ALICE framework was developed to cater for ALICE specific needs.

These tools are used by the sub-detector expert to build their applications. The tools are accompanied by a set of rules and guidelines (naming conventions, user interface look and feel, etc.) to ensure the homogeneity and to simplify the integration of independently developed components. 


\section{Operating systems}

All PVSSII applications (projects) are running on the Windows operating system on the Worker Nodes (Windows XP) and the Operator Nodes (Windows Server 2003). Specific worker nodes (those interfacing to the Front End Electronics) run the Linux (SLC4) operating system.

\section{Communication and hardware access}

Communication with the hardware is restricted to a limited set of communication protocols. Two different protocols cover all the needs. OPC (OLE for Process Control) is a widely accepted and an industry standard to communicate with commercial devices. It implements a client-server mechanism, where the supplier of the device usually delivers the OPC server. PVSSII is a generic OPC client. The second recommended protocol is DIM (Distributed Information Management) that is used to communicate with custom built equipment. DIM is available for many platforms and libraries are available for several computer languages. DIM implements a client-server mechanism over TCP/IP. When a DIM-server is developed for a given piece of equipment, PVSSII can be used to access it through the DIM-client integrated in the framework. In addition PVSSII can also directly communicate with equipment through drivers. PVSSII drivers exist for Modbus devices and some other (mainly industrial) devices.

DIM is also the underlying communication layer for the Data Interchange Protocol (DIP) that is used to exchange data between the control system and LHC as well as with the various services such as gas, electricity, cooling etc.

\section{Finite-State Machine}

The hierarchical tree-like control structure, as described earlier, relies heavily on finite-state machines. PVSSII itself does not provide any FSM functionality, but this was added in the framework (SMI++, [263]). There is a very close integration between PVSSII and the FSM tools.

\section{Databases}

A large variety of data, needed for the operation of the experiment, has to be stored. The amount and the use of the data vary widely and it is therefore stored in a collection of databases, each optimised for its particular use, all housed in a dedicated Oracle, RAC database server at the experiment site.

PVSSII has its own internal proprietary run-time database which is used to store the values that are read from the devices, information on the configuration of PVSSII itself and any information that is needed for the operation of the PVSSII system. This database is optimised for fast access, as it is an essential part in the operation of the PVSSII system. Data archiving is an integral part of PVSSII and is the mechanism to store the history of any data available in the system that the user decides to archive. PVSSII allows archiving to be done to a file based archive or to a RDB (Oracle). During first commissioning and tuning of the sub-detector a file based system is used, in the final production system data is archived into the database.

The configuration database holds the data needed for the configuration of the whole control system; this includes the configuration of the control system itself, configuration of hardware 
devices such as power supplies but also chips in the front-end electronics, configuration of processes, etc.

The PVSSII archive described above is an essential tool for system debugging. It contains all information acquired during operation; however retrieval of this data is complicated when attempted from outside of PVSSII. The main reason is that the control system is distributed and accessing of data requires detailed knowledge about its structure. To overcome these limitations the so called Offline Conditions DataBase (OCDB) is used. It mirrors data essential for offline analysis and provides an easy access and retrieval mechanism. The interface between the OCDB and the DCS archive was implemented by the central team as a dedicated PVSSII manager.

\subsubsection{Applications}

The ALICE experiment consists of a fairly large number $(\sim 130)$ of sub-systems and controls slices that need to be developed. The aim was to standardise and use common solutions as far as possible. At the device level the sub-detector users were encouraged to use similar types of devices whenever possible, and common specifications were developed for devices to be purchased, such as high and low voltage power supplies, VME crates and VME single board computers. Manufacturers were in this way asked to provide standard control interfaces based on OPC and CERN standard fieldbuses. For the Front-End Electronics (FEE), which is custom made for each sub-detector, a standard software interface for its control and configuration was defined.

Nearly all requirements for High and Low Voltage could be satisfied by three manufacturers (CAEN and Iseg for HV; CAEN and Wiener for LV). This already significantly reduced the development effort.

General purpose monitoring comprises any parameter that is not acquired through any of the other sub-systems. These parameters are typically temperatures measured on the detector. To avoid the dispersion of solutions for this task, a general purpose monitor device was adopted: the ELMB [264]. The ELMB is a general purpose microcontroller (with 64 analogue inputs and 16 digital I/Os) connected via CANbus. An OPC server is available to control the ELMB and access the acquired data. A major feature of the ELMB is that it can work in radiation environments and therefore be used directly on the detector.

Common solutions however not only consist of standard equipment and standard control interfaces, but wherever possible also implement standard logical behaviour of the equipment. A control unit for a class of devices implements a unique FSM state diagram describing the behaviour and commands for that class of devices. This naturally leads to not only uniformity in names of states and commands, but also a 'non-expert' operator is presented with coherent behaviour for similar sub-systems throughout the experiment. The behaviour includes standard operation features such as automatic recovery of anomalies.

\section{Front-End Electronics}

The control of Front-End Electronics (FEE) is a complex and delicate task. It involves control of voltage regulators, power switches, error registers, etc., and monitoring of temperatures, voltages, currents, status registers, etc. of the FEE boards. It also involves configuration and initialization of FEE controllers and of all the various custom chips on the detector boards. It requires a very 
close interaction between the DCS and DAQ, as in the majority of the cases, both systems share the access path to the FEE or use the FEE concurrently. A further challenge is that the architecture and implementation of the FEE is different for each sub-detector and based on custom chips. Many different techniques are deployed to communicate with these chips such as JTAG, Ethernet, GOL, Profibus, CANbus, etc., each requiring a different access strategy. Also the Detector Data Link (DDL) provides functionality for downloading configuration information. In addition, large amounts of data, unusually large compared to other areas of the control system, are involved; especially for configuration of the FEE.

In order to achieve maximum commonality between all different FEE architectures a FrontEnd Device (FED, [265]) was defined. The FED represents a hardware abstraction layer allowing DCS transparent access to the FEE. It responds to standard commands and performs requested tasks such as loading configuration registers, resetting the chips, etc. If the FEE provides data which needs to be monitored, this is gathered by the FED and made available to the supervising software.

The FED is built as a package of software and hardware with a standardized software interface. A common FED client-server model was adopted for the FED which hides the implementation details to higher software layers. The server communicates with the hardware and publishes data as services. A client can subscribe to services and send commands to the server. Several clients can subscribe to the same server in parallel allowing for distributed monitoring. The DIM protocol was chosen as the underlying communication layer.

\section{Services}

The DCS interfaces to various services in order to keep the control system up to date with the experiment operation environment. Some of the services allow active control from DCS, however the majority of interactions are monitoring only. For communication with the services the Data Interchange Protocol (DIP) was defined, which allows a transparent way to exchange information between the systems involved.

Eight sub-detectors in ALICE have a gas system, with their associated control system. The operation and maintenance of the gas system is taken care of by an LHC-wide operations team. However, any information needed by the sub-detectors from the gas system for their operation is made available through DIP. A central application subscribes to the DIP parameters and makes them available in the distributed PVSSII system, for use by the sub-detectors.

Sub-detector cooling systems with their control systems are developed ALICE-wide by Cooling and Ventilation group of the Technical Support Department (TS/CV). These cooling systems (and their controls) are designed, built and installed by the TS/CV team; the control is based on PLC technology. DCS exchanges information and commands through a 'concentrator PLC', using the Modbus TCP/IP protocol.

Both gas and cooling systems generate, as backup to the software interface, hardware interlocks allowing sub-detectors to take actions and protect their equipment in case of serious anomalies.

The power distribution racks that deliver power to the individual equipment racks are installed and maintained by Electrical Engineering group (TS/EL), including a control system. This control 
system, which is PLC based, reads status information (on/off, error, etc.) and provides on/off control for each outlet. The DCS interfaces with these PLCs, via the Modbus TCP/IP protocol.

Each rack is equipped with a monitoring system that allows monitoring of the environment inside the rack and the operational state of the rack. This monitoring system was developed as a common solution for the four LHC experiments and is based on the ELMB. The protection of each rack is ensured by a thermo-switch and a smoke detector; in case of anomaly the power to the rack will be cut.

DCS also exchanges information with the LHC machine, the magnet control system, safety systems and the primary services, and the DIP protocol is used for this. A central service subscribes to the DIP parameters and republishes the information inside the distributed PVSSII system, for use by the sub-detectors.

\section{Detector Safety System and Interlocks}

The Detector Safety System (DSS, [266]) is a robust part of the ALICE DCS, designed for highavailability and is based on a redundant PLC system. It is designed to monitor the experiments environment (temperature, presence of cooling, water leaks) and to take automatic protective actions (cut power, close water valves) in case of anomalies.

Hardware interlocks are implemented at several levels. Sub-detectors have implemented various protection mechanisms on their detector equipment; high temperature detected on the electronics and automatically switches off that piece of electronics. Also the DSS is used as an interlock system where independent sensors are available to detect anomalous conditions; the DSS is then programmed to take protective action.

\subsubsection{Operation}

All interaction with the experiment is done through graphical user interfaces (figure 7.4) and this is the only part the end-user will see from the control system. In order to facilitate the operation of the various parts of the experiment, a major effort has been made to achieve the same 'look and feel' for all user interfaces. This is essential for an efficient operation of the experiment, as a small shift crew will operate a large set of different sub-detectors. This crew should therefore be able to rapidly diagnose problems in any of the ALICE sub-detectors; a task that is greatly facilitated by a high level of uniformity across the user interfaces. Therefore a standard ALICE user interface was developed as a framework tool used by all sub-detectors to implement their graphical user interfaces.

As the control system controls the often delicate and unique equipment of the sub-detectors the potential danger of serious and irreversible damage imposes a need for an advanced access control mechanism to regulate interactions of the users with the control system components. The system must be protected against inadvertent mistakes by operators or experts as well as against malicious attacks from outside. 


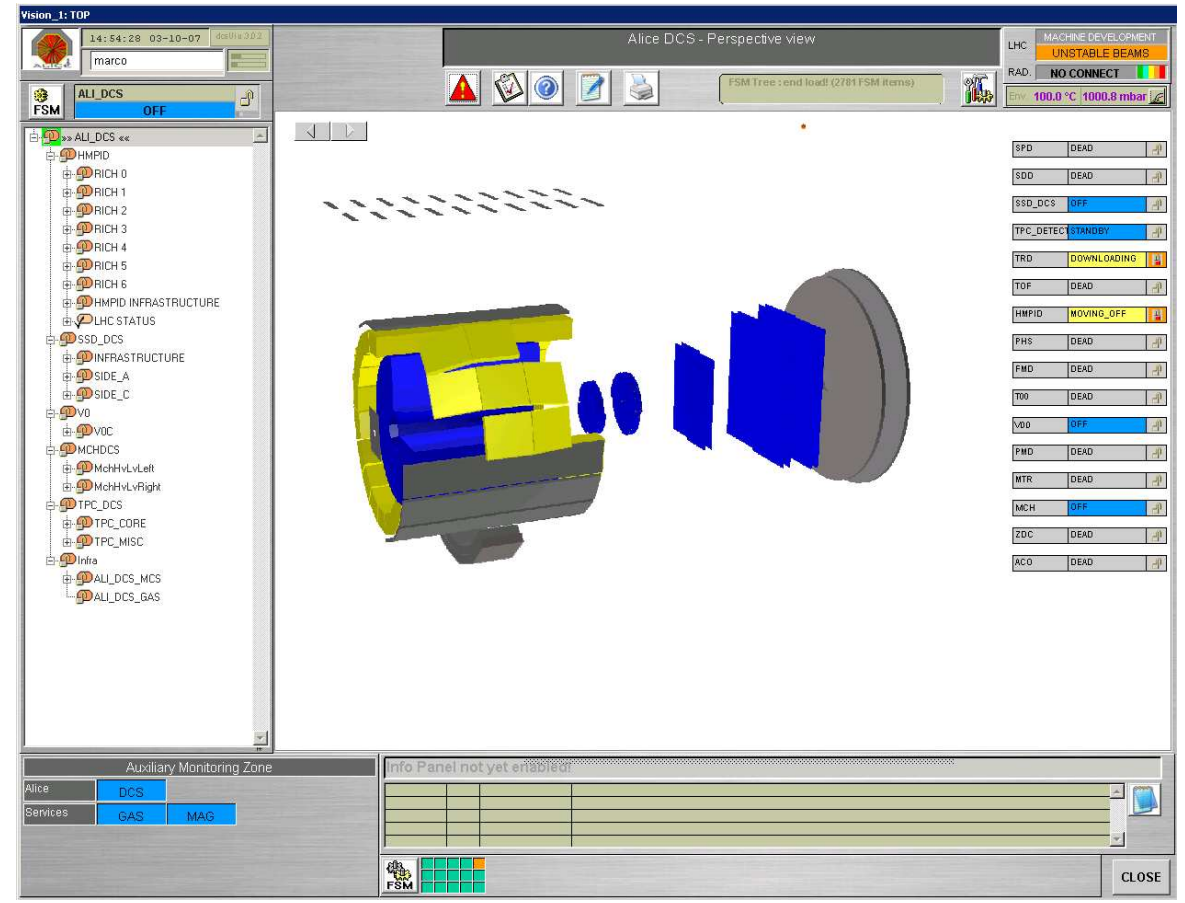

Figure 7.4: ALICE DCS user interface.

\subsection{Experiment Control System (ECS)}

\subsubsection{Requirements}

The control of the ALICE experiment is based on several independent 'online systems'. Every 'online system' controls operations of a different type belonging to a different domain of activities: Detector Control System (DCS), Data Acquisition (DAQ), Trigger system (TRG), and High-Level Trigger (HLT). The 'online systems' are independent, may interact with all the particle detectors, and allow partitioning. Partitioning is the capability to concurrently operate groups of ALICE detectors. In the final setup the detectors will mainly work all together to collect physics data. In the commissioning phase, however, detectors are debugged, and tested as independent objects. While this mode, called 'standalone mode', is absolutely vital in the commissioning and testing phase, it will also be required during the data-taking phase to perform calibration procedures on individual detectors. It will therefore remain essential during the whole life cycle of ALICE.

The Experiment Control System (ECS) coordinates the operations controlled by the 'online systems'. It permits independent, concurrent activities on part of the experiment by different operators and coordinates the functions of the 'online systems' for all the detectors and within every partition. The components of the ECS receive status information from the 'online systems' and send commands to them through interfaces based on Finite-State Machines (FSM). The implementation of these interfaces is based on the SMI++ package [263]. The interfaces between the ECS and the 'online systems' contain access control mechanisms that manage the rights granted to the ECS: the 'online systems' can either be under the control of the ECS or be operated as independent systems. In the second case the 'online systems' provide status information to the ECS, but do not receive commands from it. 


\subsubsection{System architecture}

\section{Partitions and standalone detectors}

A partition is a group of particle detectors. From the ECS point of view, a partition is defined by a unique name that makes it different from other partitions and by two lists of detectors: the list of detectors 'assigned' to the partition and the list of detectors 'excluded' from the partition. The first list, called 'assigned' detectors list, contains the names of the ALICE detectors that are members of the partition and can be active within it. This static list represents an upper limit: only the detectors included in the list can be active in the partition, but they are not necessarily active all the time. The lists of detectors assigned to different partitions may overlap: the same detector can appear in different lists. The lists of assigned detectors cannot be empty. The second list, called 'excluded' detectors list, contains the names of the ALICE detectors that have been assigned to the partition, but are currently not active in it. This dynamic list is a subset of the assigned detectors list and can be empty. Although a given detector may appear in the assigned detectors list of many partitions, it can only be active in one at any time. The excluded detectors list of a partition contains the names of the detectors that are not active because they are active in another partition, because they are running in standalone mode, or because of an explicit operator request. Explicit operator requests are subject to restrictions: during the data-taking phase the structure of a partition cannot be changed.

Two types of operations can be performed in a partition: those involving all the active detectors, called global operations, and those involving only one active detector, called individual detector operations. The ECS handles the global operations by monitoring the DCS status of all the active detectors. It interacts with the DAQ and HLT processes that steer the DAQ and HLT activities for the whole partition. It sends commands to the Trigger Partition Agent (TPA) that links the partition to the Central Trigger Processor (CTP). When a global operation starts, the ECS inhibits all the individual detector functions. The ECS handles an individual detector operation by monitoring the DCS status of the detector, interacting with the DAQ and HLT processes that steer the DAQ and HLT activities for that particular detector, and sending commands to the Local Trigger Units (LTU) associated to it. When an individual detector task starts, the ECS inhibits the global operations for the partition, but it does not inhibit individual detector tasks on the other detectors. These individual detector operations, such as calibration procedures, can be concurrently performed within the partition.

A standalone detector is a detector operated alone and out of any partition. The tasks performed by a standalone detector are equal to the individual detector operations that are allowed when the detector is active in a partition. The ECS handles these functions watching the DCS status of the detector and interacting with the DAQ and HLT processes that steer the DAQ and HLT activities for that detector and with the LTU associated to it.

The major difference between a standalone detector and a partition with only one single detector is that this last partition is linked to the CTP by a TPA, whereas the standalone detector only interacts with its LTU. 


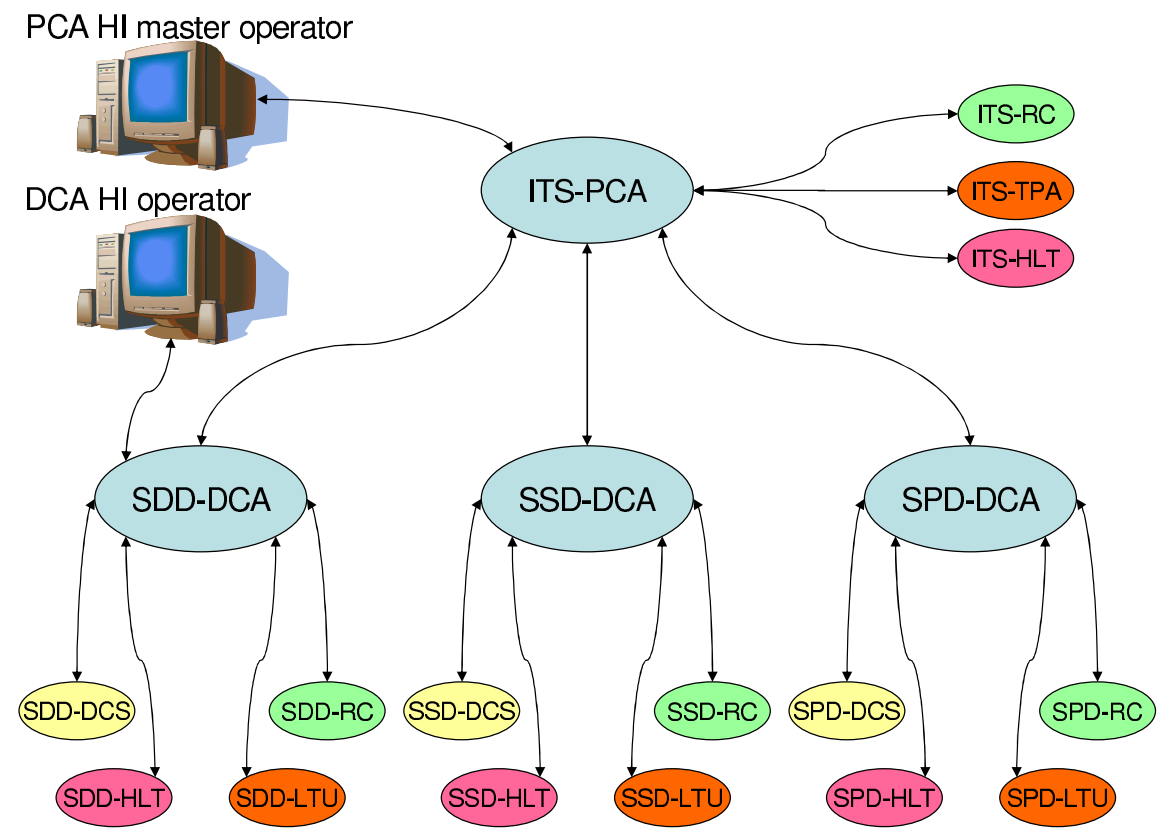

Figure 7.5: The overall architecture of the ALICE ECS applied to the three ITS detectors.

\section{ECS architecture and components}

Every detector in standalone mode or assigned to a partition is controlled by a process called a Detector Control Agent (DCA) and every partition is controlled by a process called a Partition Control Agent (PCA). When a detector is in standalone mode, its DCA accepts commands from an operator via a DCA Human Interface (DCAHI). At any time several DCAHIs can coexist for the same DCA, but only one can send active commands: the others can only get status information. When a detector is active in a partition, its DCA accepts commands only from the PCA controlling the partition. Operators can still invoke DCAHIs, but only to get information and not to send active commands. A PCA Human Interface (PCAHI) provides to an operator full control of a partition. At any time, many PCAHIs can be active for the same PCA, but only one has the control of the partition and can be used to send commands. DCAs and PCAs get status information from the 'online systems' and eventually send commands to components of these systems through interfaces based on Finite-State Machines. An example of the ECS architecture with three detectors (SDD, SPD, and SSD), all active in a partition called ITS, is given in figure 7.5. For each detector, there is a DCS object (xxx-DCS), an LTU control object (xxx-LTU), a DAQ RC object (xxx-RC), and a HLT object (xxx-HLT).

The major components of the ECS, described below, are the Detector Control Agent (DCA), the Partition Control Agent (PCA), the Detector Control Agent Human Interface (DCAHI), and the Partition Control Agent Human Interface (PCAHI).

\section{Detector Control Agent (DCA)}

There is one DCA for every detector running in standalone mode or assigned to a partition. The main tasks performed by this process are the following: 
- It handles standalone data-acquisition runs for the detector working alone.

- It handles electronics setup procedures. This function and its implementation are detector dependent.

- It handles calibration and test procedures. These procedures are by definition detector dependent as well as their implementation.

The DCA accepts commands from one master operator at a time: either a PCA or a DCAHI.

\section{Partition Control Agent (PCA)}

There is one PCA per partition. The main tasks performed by this process are the following:

- It handles data-acquisition runs using all the detectors active in the partition.

- It delegates individual detector functions to the DCAs controlling the detectors active in the partition.

- It handles the structure of the partition allowing the inclusion/exclusion of detectors whenever these tasks are compatible with the data-taking runs going on for individual detectors or for the whole partition.

The PCA accepts commands from one PCAHI at a time.

\section{Human Interfaces}

An operator can run a detector in standalone mode with a DCAHI having the mastership of a DCA. One can send commands to the DCA, change the rights granted to the DCA, and send commands directly to objects in the DCS, DAQ, HLT, and TRG 'online systems'. Without the mastership of the DCA, the DCAHI can only get information and cannot issue active commands.

An operator can run a partition with a PCA Human Interface having the mastership of a PCA. One can send commands to start global and individual detector tasks, can change the rights granted to the PCA, can change the structure of the partition excluding or including detectors, and can send commands directly to objects in the DCS, DAQ, HLT, and TRG 'online systems'. Without the mastership of the PCA, the PCAHI can only get information and cannot issue active commands.

\subsubsection{Interfaces to the online systems}

The main components of the ECS receive status information from the 'online systems' and send commands to them through interfaces based on Finite-State Machines. The interfaces between the ECS and the 'online systems' contain access control mechanisms that manage the rights granted to the ECS. The 'online systems' can either be under the control of the ECS or be operated as independent systems where the 'online systems' provide status information to the ECS but do not receive commands from it. 


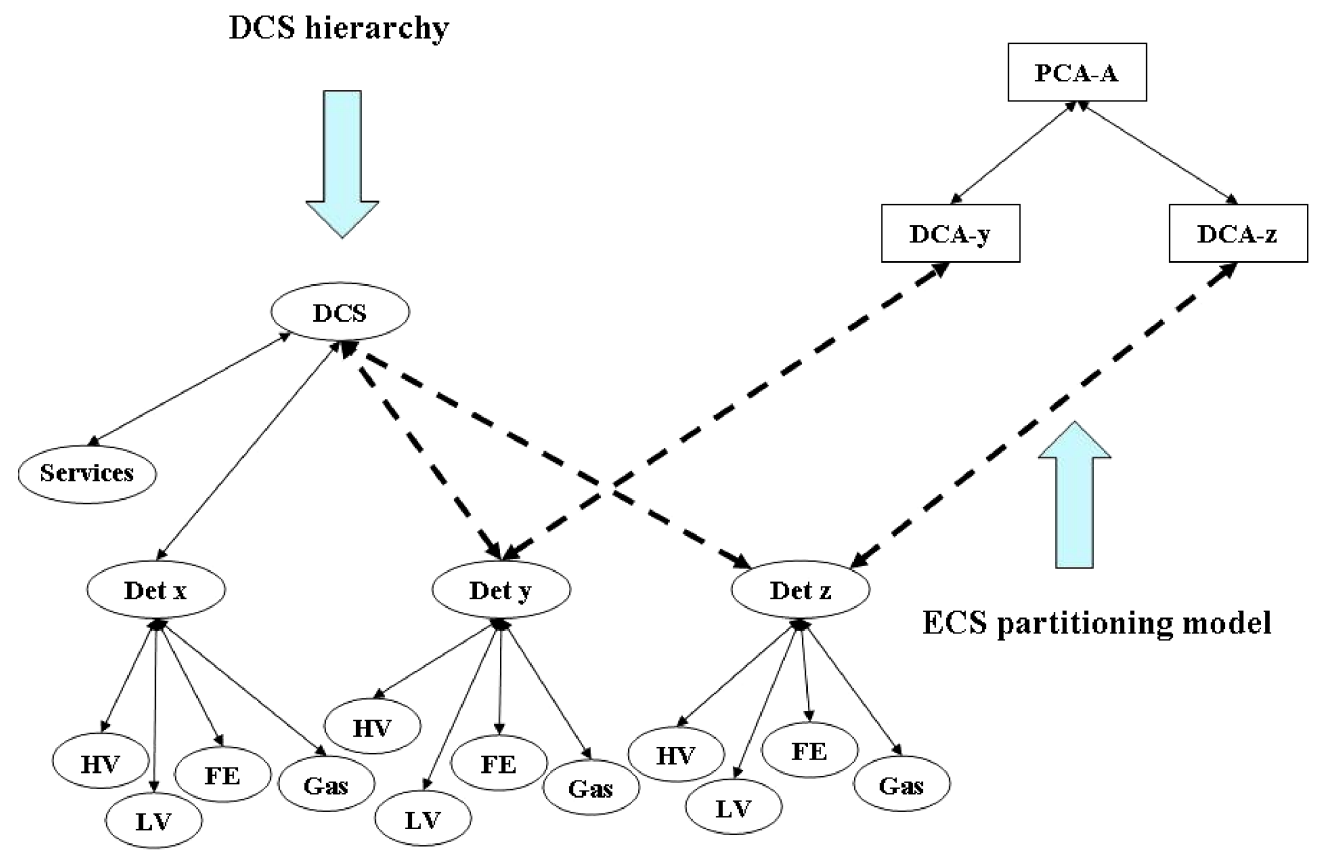

Figure 7.6: The interface of the ECS with the DCS.

\section{ECS/DCS interface}

The interface between the ECS and the DCS consists of one object per detector: the roots of the sub-trees described above and representing the detectors within the DCS. These objects can provide status information to the DCS and, at the same time, to the ECS. Every object, depending on the rights granted to the ECS and to the DCS, accepts commands either from the DCS or from the ECS but not from the two systems at the same time.

Figure 7.6 shows an example where two detectors, named ' $y$ ' and ' $z$ ' are active in an ECS partition named 'A'. The figure shows the double role of the SMI++ objects that provide status information for the two detectors both to the DCS and to the ECS. The figure does not specify if these objects are under the DCS or the ECS control.

\section{ECS/TRG interface}

Figure 7.7 shows the ECS/TRG interface. A SMI++ domain named 'TRIGGER' contains the objects describing the basic trigger components: the LTUs associated to the detectors and the CTP. These SMI++ objects are associated to processes, called proxies, which actually drive the LTUs and the CTP. When a global operation is performed, all the detectors active in a partition produce raw data: the generation of raw data by the detectors is performed under the control of their associated LTUs. These LTUs are synchronized by the CTP. There is only one CTP, but many partitions can be operated at the same time and all of them need access to the CTP. The Trigger Partition Agents (TPAs) associated to the different partitions handle the access conflicts. There is one TPA per partition. The TPA interacts with CTP and LTUs. 


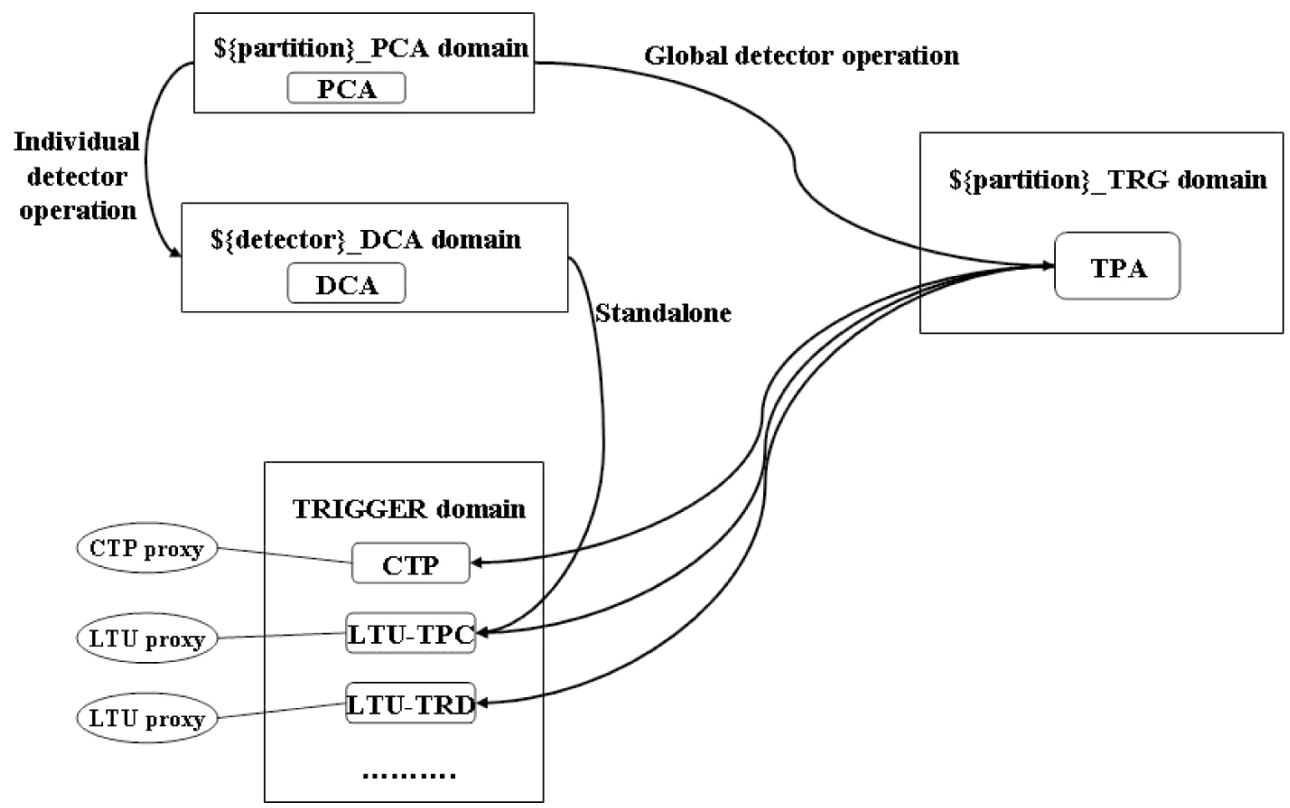

Figure 7.7: The interface of the ECS with the TRG.

When a detector is operated in standalone mode, the DCA controlling it interacts directly with the LTU associated to the detector and the CTP is ignored. When a detector is active in a partition and an individual detector operation is executed on it, the PCA delegates the task to the DCA controlling that detector. The DCA again interacts with the LTU associated with the detector. The CTP is ignored. When a global operation is performed in a partition, the PCA controlling the partition interacts with the TPA that in turn interacts with CTP and LTUs. The PCA has no direct interaction with the CTP and the LTUs.

\section{ECS/DAQ interface}

The interface between the ECS and the DAQ is made of SMI++ objects representing RC processes. These are: (a) a RC process per detector, which steers the data acquisition for a given detector and for that detector only. (b) a RC process per partition that steers the data acquisition for the whole partition with data produced by all the active detectors.

\section{ECS/HLT interface}

The interface between the ECS and the HLT is made of SMI++ objects representing the state of a part of the HLT as used by a detector or a partition.

\subsection{Online Detector Calibration}

The condition data including parameters such as detector response calibration, bad channel maps, pedestal values and so on, are evaluated on line from data collected during normal data taking or during special runs. So-called Detector Algorithms (DA) running on DAQ LDCs, GDCs or 
dedicated Data Monitoring Machines subscribe to the raw data stream and produce the input for the subsequent computing of the desired conditions data. Furthermore, for certain detectors DAs run in DCS and HLT. The DA output is stored on File Exchange Servers (FXS) in the system running the DA, i.e. DAQ, DCS or HLT. Upon completion of a run, the Experiment Control System end-of-run signal triggers a special program called SHUTTLE. This program in turn activates one preprocessor per subdetector which collects the data from the FXSs as well as data from the DCS Archive DB which contains constantly monitored parameters by PVSS, e.g. high voltages and temperatures. From these inputs the preprocessor calculates the conditions, transforms them in "calibration objects" and saves them as ROOT files into a special portion of the AliEn catalogue called Offline Condition DataBase (OCDB). The reconstruction program retrieves the calibration files by querying the OCDB portion of the AliEn catalog. 


\section{Chapter 8}

\section{Performance}

In this chapter we discuss the basic performance of the ALICE detector for track finding and reconstruction, charged particle identification and neutral particle detection as expected from Monte Carlo simulations. For a comprehensive review of the physics performance we refer the reader to $[20,21]$.

The ALICE detector was designed to cope with the highest predicted charged-particle multiplicity densities for central $\mathrm{Pb}-\mathrm{Pb}$ collisions at $\mathrm{LHC}\left(\mathrm{d} N_{\mathrm{ch}} / \mathrm{d} \eta\right.$ at mid-rapidity up to 8000 ; see section 1.3.1 of [20]). In addition, the ALICE experiment will also study collisions of lower-mass ions, as a means of varying the energy density, and of protons (both pp and pA). Besides primarily providing a reference for the study of nucleus-nucleus interactions, pp data will also allow for a number of genuine pp-physics studies.

One of the most challenging tasks for ALICE is that of track finding in an unprecedented particle multiplicity density environment. Track finding begins with the reconstruction of the primary vertex using the correlation of the hit positions in the innermost detector (SPD). The position of the primary vertex is then used as a constraint to initiate the tracking of primary particles in the TPC and for the subsequent track following. Secondary tracks are found during a further tracking pass. Finally, the reconstruction of secondary vertices is carried out.

Charged hadrons are identified combining information provided by the ITS, TPC, TRD, TOF, and HMPID detectors. Electrons are identified using information from the TRD and the TPC. The information from the EMCal will improve the purity of electron identification, especially at higher momenta. The PHOS spectrometer detects and identifies photons. Below about $20 \mathrm{GeV} / c$ the direct-photon spectrum is obtained by subtracting, from the overall identified-photon spectrum, the contribution from decay photons, mainly from $\pi^{0}, \eta$, and $\omega$ decays. The yields of these mesons are measured by an invariant-mass analysis. At higher momenta, up to about $100 \mathrm{GeV} / c$, direct photons are identified on an event-by-event basis. The EMCal will enlarge the photon-identification acceptance, albeit with a performance not as high as that of the PHOS. In the forward pseudorapidity region $(2.3<\eta<3.5)$ the photon multiplicity is measured by the PMD.

In the forward muon spectrometer, tracks are reconstructed behind the absorber, starting from the last stations and proceeding towards the interaction point. In this way we identify muons in the pseudo-rapidity region $-4.0<\eta<-2.5$. 


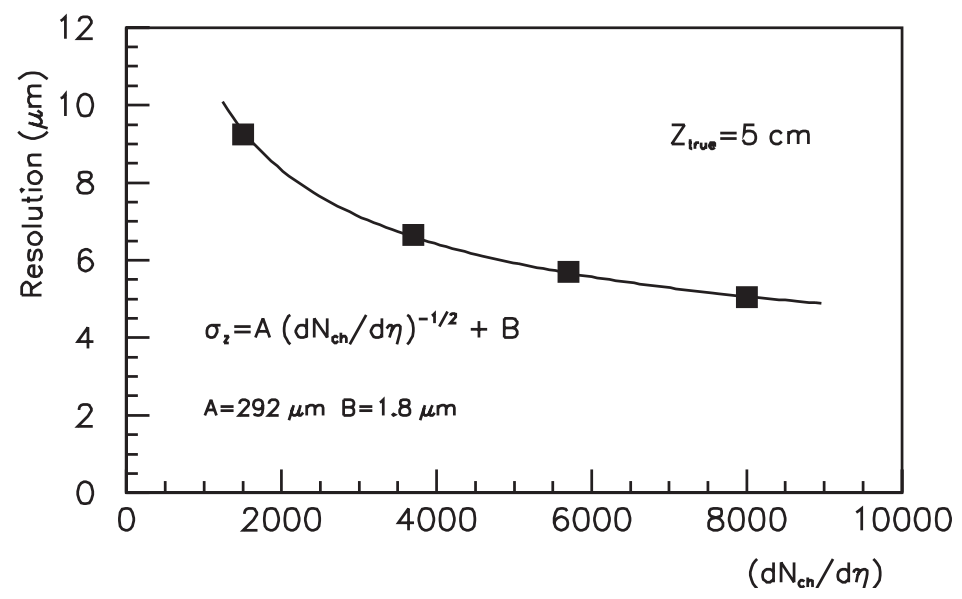

Figure 8.1: Resolution of the reconstructed vertex position as a function of the charged-particle density. The solid line is the result of a fit using the parametrization given by eq. (8.1).

\subsection{Track and vertex reconstruction}

\subsubsection{Primary vertex determination}

The reconstruction of the primary vertex is based on the information provided by the Silicon Pixel Detectors (SPD), which constitute the two innermost layers of the ITS. We select pairs of reconstructed points in the two layers, which are close in azimuthal angle in the transverse plane. Then from their $z$-coordinates we estimate the $z$-position of the primary vertex using a linear extrapolation. Finally we perform a similar procedure in the transverse plane. Here, due to the bending in the magnetic field, the linear extrapolation is a somewhat crude approximation; however, thanks to the short distances from the interaction point, the $x$ - and $y$-coordinates of the primary vertex are determined with a sufficient precision to be used as constraints in the first tracking pass. This estimate of the primary vertex position is then used to correct the measurement of the $z$-coordinate, for effects due to an off-axis position of the interaction point in the transverse plane. For well focused beams we can determine the transverse position of the interaction point averaging over many events, provided that the beam position is sufficiently stable in time.

The resolution on the position of the primary vertex depends on the track multiplicity, i.e. on the charged-particle density. In figure 8.1 this dependence is shown for the resolution in the $z$-coordinate $\sigma_{z}$ and is fitted by the expression

$$
\sigma_{z}=\frac{A}{\sqrt{\mathrm{d} N_{\mathrm{ch}} / \mathrm{d} \eta}}+B,
$$

where typically $A=290 \mu \mathrm{m}$ and $B$ is a few microns, depending essentially on the residual misalignment of the silicon pixel layers. For heavy-ion charged-particle densities we thus obtain a vertex-position resolution on the $10 \mu \mathrm{m}$ level, and for the average pp event $\left(\mathrm{d} N_{\mathrm{ch}} / \mathrm{d} \eta=6-7\right)$ we get $150 \mu \mathrm{m}$. This measurement of the primary-vertex position is used as an input for the tracking.

After track reconstruction, the position of the primary vertex is recalculated using the measured track parameters. In figure 8.2 the expected final resolutions in the $z$-coordinate, and in the 


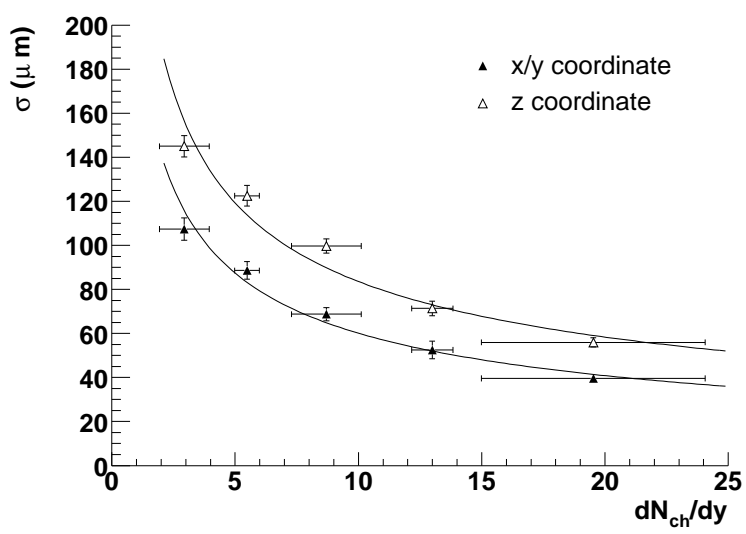

Figure 8.2: Resolution on the primary-vertex position as determined using reconstructed tracks, as a function of the charged-particle density for pp events.

transverse $x / y$-coordinates, are shown as a function of the charged-particle density (for pp collisions). When fitting these dependencies to eq. (8.1) we get $A=270 \mu \mathrm{m}$ for the $z$-coordinate, and $A=210 \mu \mathrm{m}$ for the $x / y$-coordinate (again with a few microns for $B$ ). For the average pp multiplicity, the precision on the measurement of the primary vertex position after the tracking step is improved to $110 \mu \mathrm{m}$ in the $z$-coordinate, and to $70 \mu \mathrm{m}$ in the transverse coordinate.

\subsubsection{Track reconstruction}

The basic method employed for track finding and fitting is the Kalman filter as introduced to this field by P. Billoir [252]. This method depends critically on the determination, for each track, of a set of initial seed values for the track parameters and their covariance matrix. This seeding is done using the space points reconstructed in the TPC. The space-point positions are calculated from the centre of gravity of the two-dimensional clusters (in the pad-row and time directions). At high particle densities, due to the large occupancy, we use a more sophisticated cluster unfolding which takes into account the cluster structure. The seeding is done twice: the first time assuming that the track originated from the primary vertex and the second assuming that the track originated elsewhere (decay, secondary interaction, etc.). We start to combine the space points from a few outermost pad rows using, in the first pass, the primary-vertex position as a constraint. The procedure is repeated several times, choosing a set of pad rows closer and closer to the centre of the TPC. From every seed we start the track following, pad row by pad row inside the TPC. The Kalman filter essentially consists of the following steps: a) we propagate the state vector of the track parameters and their covariance matrix to the next pad row, b) we add to the inverted covariance matrix (which represents the information matrix of our knowledge of the track parameters at that point) a noise term (representing the information loss due to stochastic processes like multiple scattering and energyloss fluctuations), c) if the filter finds in the new pad row a space point compatible with the track prolongation, we add this measurement, i.e. we update the track parameters and the covariance matrix, increasing the information. We repeat the seeding a second time, now without the primaryvertex constraint. We then proceed again with track following as outlined above. The track-finding 


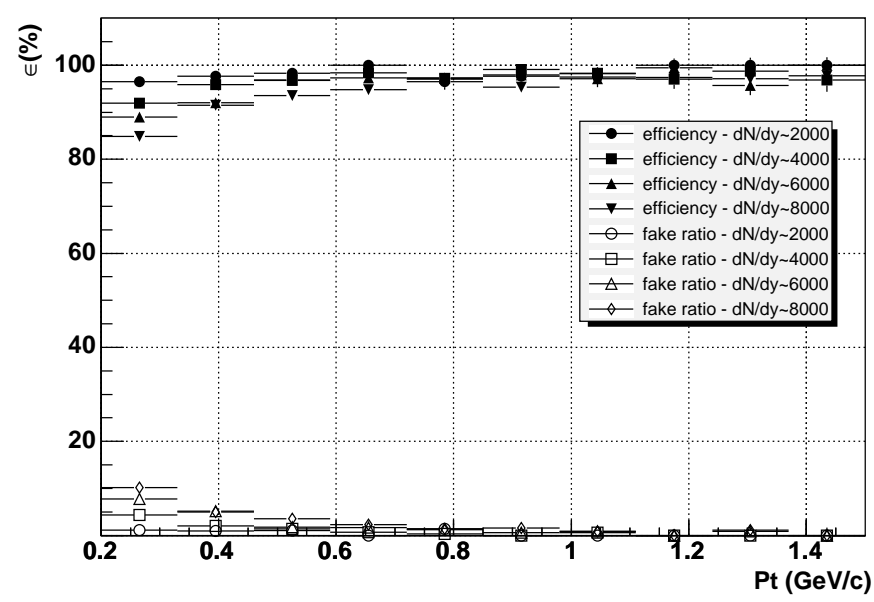

Figure 8.3: TPC and ITS track-finding efficiency and fraction of fake tracks as a function of transverse momentum for different track multiplicities.

efficiency after this stage, if we normalize to the number of tracks which are potentially findable (i.e. without taking into account decays in flight, detector acceptances, etc.) is nearly $100 \%$.

After this step, the tracks are propagated to the outer layers of the ITS. We start with the highest-momentum tracks and then continue with the lower-momentum ones in order to make the most precise (i.e. less ambiguous) track-space point assignments first. For highly ionizing tracks in the TPC, this knowledge is used in the ITS tracking for energy-loss corrections and multiplescattering noise estimates. Tracks found in the TPC as primaries are followed by the Kalman filter in two independent passes, first imposing the primary-vertex position as a constraint, and then without this condition. Both sets of track parameters are stored for further analysis. The tracks found during the second TPC pass (i.e. without vertex constraint) are followed in the ITS only without imposing the primary-vertex constraint. Whenever more than one space-point candidate is found within the search window around the prolongation of a track (a half-width of four standard deviations is typically used), all possible assignments are used as different hypotheses and are followed independently towards the innermost ITS layer. In this way each TPC track can have several candidate paths throughout the ITS. A decision is made only at the end, based on the sum of the $\chi^{2}$ along the track-candidates' path in the ITS. Optionally, we allow for layer skipping and cluster sharing between tracks, in which case a weight factor is introduced in the $\chi^{2}$ sum. The results for the combined TPC-ITS tracking are shown in figures 8.3 and 8.4. In figure 8.3 the expected track-finding efficiency for primary tracks and the relative frequency of fake tracks are presented as a function of transverse momentum for different charged-particle densities. The efficiency for primary tracks is normalized to the number of tracks which are potentially findable. Fake tracks are defined here as tracks that have more than one space point incorrectly assigned. For very high densities ( $\mathrm{d} N_{\mathrm{ch}} / \mathrm{d} \eta=6000$, well above the current theoretical predictions), the efficiency for tracks with $p_{\mathrm{t}}=200-300 \mathrm{MeV} / \mathrm{c}$ is about $90 \%$, and increases to values above $95 \%$ at higher momenta. The fake-track probability at the same time is below $10 \%$ in the lower momentum bin, and decreases below $1 \%$ at high momenta. For the same value of the charged-particle density figure 8.4 shows the expected quality of the TPC-ITS track finding, for different definitions of properly found and of fake tracks. 


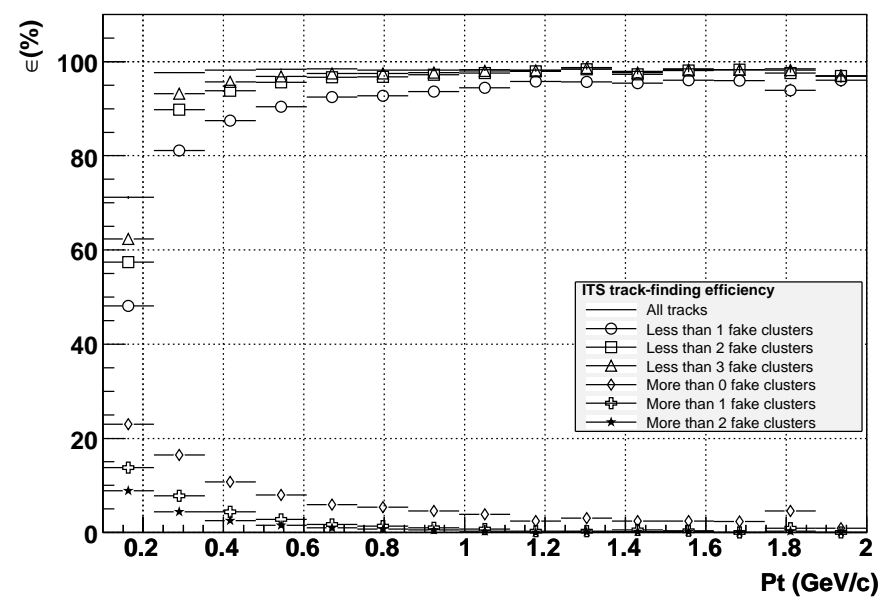

Figure 8.4: TPC and ITS track-finding efficiency and fraction of fake tracks for different amounts of wrongly associated ITS clusters $\left(\mathrm{d} N_{\mathrm{ch}} / \mathrm{d} \eta=6000\right)$.
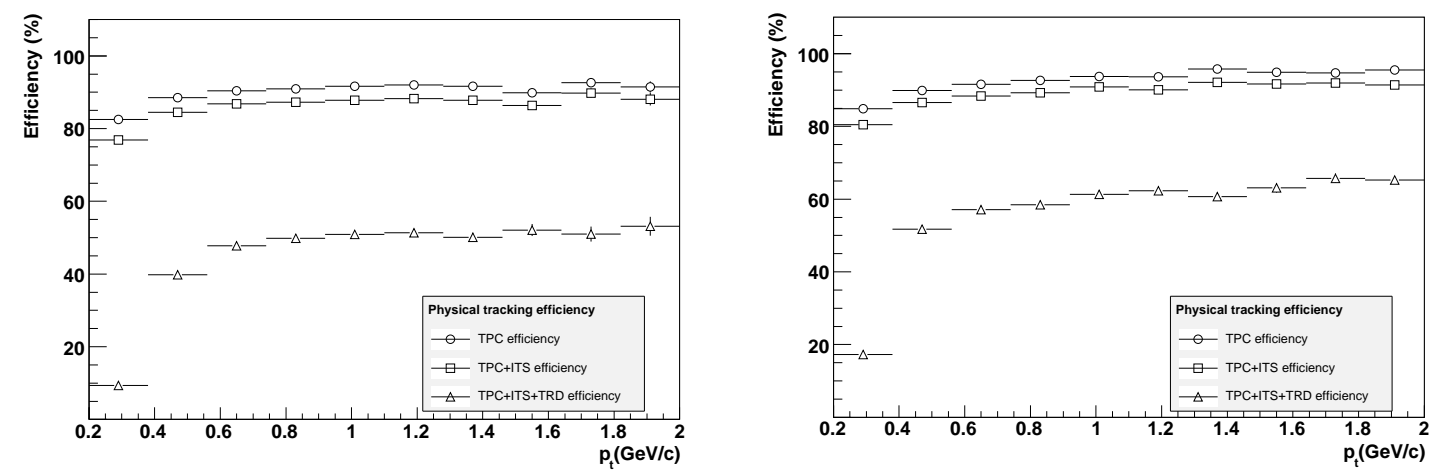

Figure 8.5: Physical track-finding efficiency for different combinations of the tracking detectors. Left: Central $\mathrm{Pb}-\mathrm{Pb}$ collisions $\left(\mathrm{d} N_{\mathrm{ch}} / \mathrm{d} \eta=6000\right)$. Right: pp collisions.

When the ITS tracking is completed, we reverse the Kalman filter and follow the track from the inner ITS layers outwards. Starting with much more precise track parameters than during the first step, we now try to eliminate improperly assigned points (outliers). We then continue following the tracks beyond the TPC, assigning space points in the TRD, and matching the tracks with hits in the TOF, minimum-ionizing clusters in the HMPID and space points in the CPV (located in front of the PHOS). Finally, we reverse the Kalman filter one last time and we refit all tracks from the outside inwards, in order to obtain the values of the track parameters at or nearby the primary vertex. Optionally, we proceed with an additional track-finding step using only points from the ITS, after having removed all the ITS space points already assigned to tracks. This is useful for finding tracks that have not been seeded in the TPC because they went through a non-sensitive area (e.g. in between the readout chambers).

In figure 8.5 the track-finding efficiencies at different stages, this time normalized to the total number of primary charged tracks in the acceptance (i.e. taking into account decays and insensitive areas in the detectors) are presented as a function of transverse momentum, again for high 

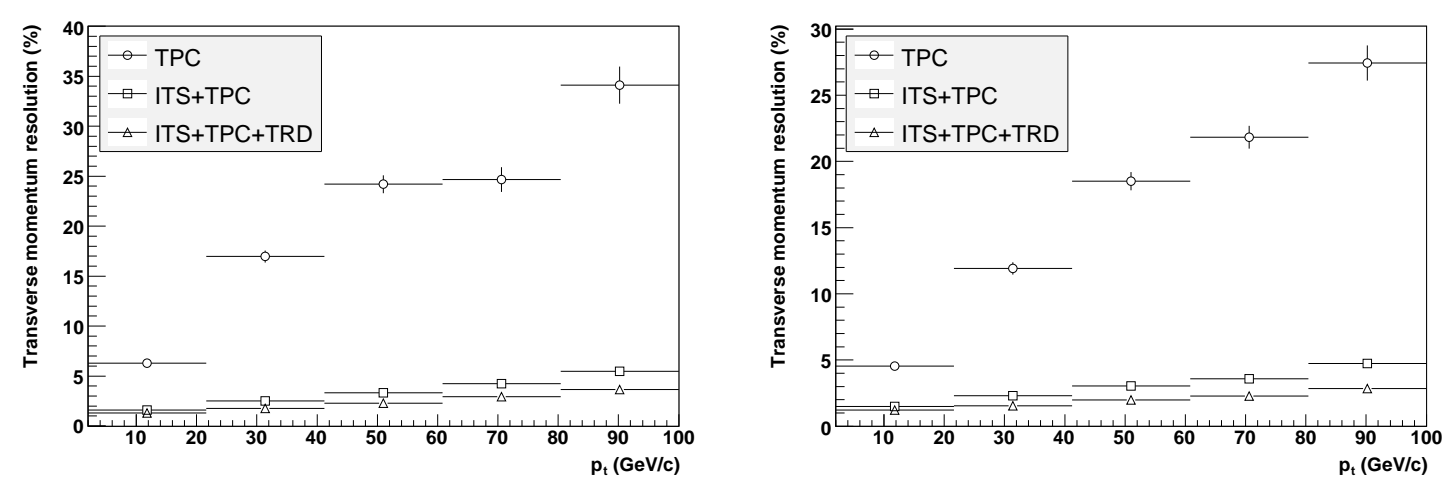

Figure 8.6: Transverse-momentum resolution for different combinations of the tracking detectors. Left: Central $\mathrm{Pb}-\mathrm{Pb}$ collisions $\left(\mathrm{d} N_{\mathrm{ch}} / \mathrm{d} \eta=6000\right)$. Right: pp collisions.

charged-particle density $\left(\mathrm{d} N_{\mathrm{ch}} / \mathrm{d} \eta=6000\right)$ and for pp collisions. The efficiency for the TPC and the TPC-ITS track finding goes to $90 \%$ at very high momenta; this value is essentially determined by the size of the TPC dead zones, which cover about $10 \%$ of the azimuthal angle. The large drop after inclusion of the TRD is because of interactions in the material and decays as well as additional dead zones. The TRD, therefore, is used in the tracking optionally, only for those tracks for which it improves the precision. Figure 8.6 shows the momentum precision obtained with different detector combinations, again for two different values of the charged-particle density. The relative momentum precision for the TPC stand-alone shown in figure 8.6 is calculated without imposing the primary-vertex constraint. The significant improvement at high transverse momenta when we combine the measurements from the TPC and ITS is basically due to the increase in tracking length. The best relative momentum precision, limited by multiple scattering to about $0.7 \%$, is achieved for low-momentum tracks with $p_{\mathrm{t}}$ about $500 \mathrm{MeV} / c$. For both the track-finding efficiency and the momentum precision, only a slight deterioration is seen when going from low to high charged-particle density.

The track parameters obtained both with and without the primary-vertex constraint are stored for all tracks, in order to allow for the subsequent analysis of short-lived particle decays (such as charm and beauty decays) taking place very close to the primary vertex. The main performance parameter for such studies is the resolution in the impact-parameter (the distance between the primary vertex and the track prolongation to the point of closest approach to the primary vertex). This resolution depends both on the precision of the primary-vertex-position determination and on the precision of the determination of the track-parameters. In figure 8.7 the impact-parameter resolution in the transverse and longitudinal directions is shown for different particle species as a function of $p_{\mathrm{t}}$. These results were obtained for the case of central $\mathrm{Pb}-\mathrm{Pb}$ collisions (i.e. with very high precision on the primary-vertex position). Figure 8.8 shows the impact-parameter resolution expected for two values of the charged-particle multiplicity in pp collisions (only for pions, and in the transverse direction). In all cases the impact-parameter resolution in the transverse direction (the quantity we rely upon for heavy-flavour physics) is significantly better than $100 \mu \mathrm{m}$ at $p_{\mathrm{t}} \sim$ $1 \mathrm{GeV} / c$, a necessary condition to study e.g. charm decays. 


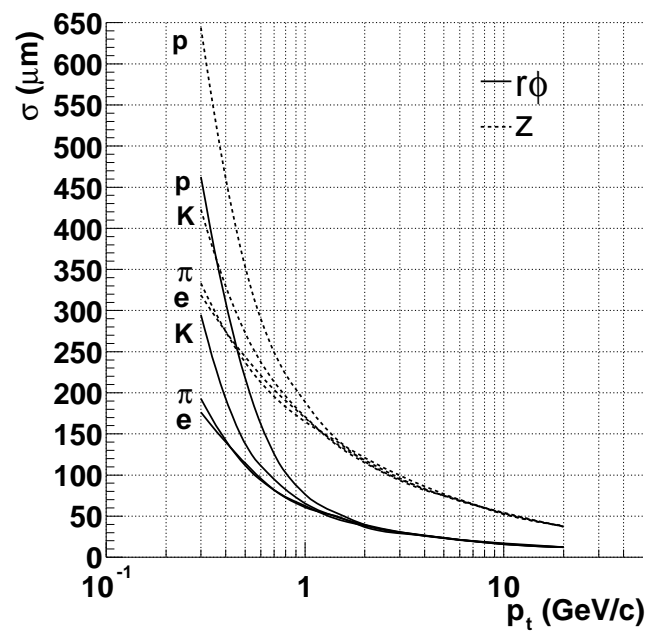

Figure 8.7: Impact parameter resolutions in central $\mathrm{Pb}-\mathrm{Pb}$ collisions for electrons, pions, kaons and protons as a function of the transverse momentum. An assigned cluster in each of the six ITS layers is required.
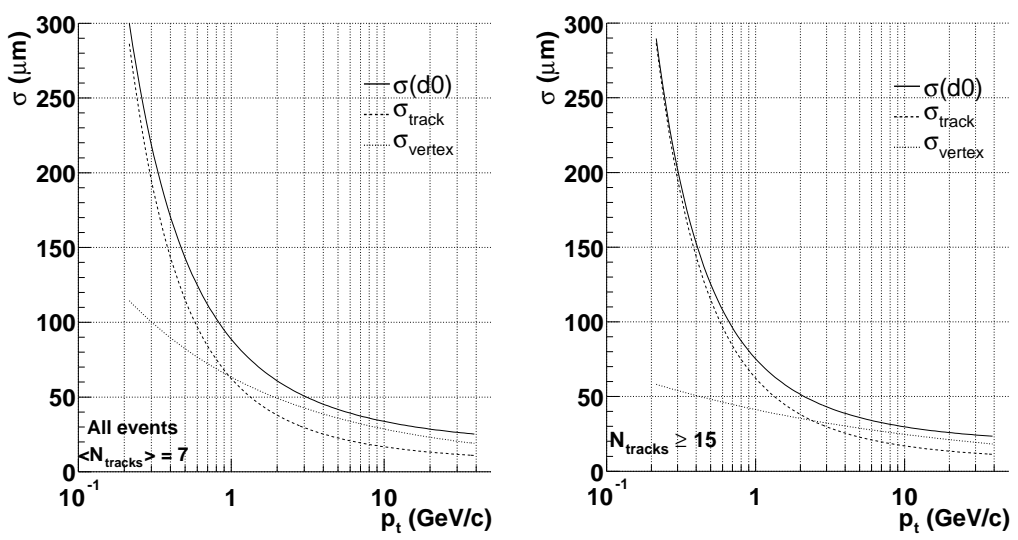

Figure 8.8: Impact-parameter resolution in the transverse plane as a function of the transverse momentum in pp collisions. The dotted lines show separately the contributions from the track extrapolation and the primary vertex accuracy.

\subsubsection{Secondary-vertex finding}

Vertices from strange particle decays are searched for at the reconstruction level. Only secondary tracks, i.e. tracks which have a large enough impact parameter, are used for this purpose. We combine opposite-sign secondary tracks and calculate their distance of closest approach. If this distance is below some predetermined value, and the point of the closest approach is located before the first measured points on both tracks, we retain the pair as a candidate for a secondary decay vertex. Additional cuts are then imposed in the subsequent analysis phase. An example of such an analysis is shown in figure 8.9 where the invariant mass distribution of $\Lambda \rightarrow \pi^{-}$p decay candidates is plotted. Tight cuts were applied on the distance of closest approach and on the pointing angle (the angle between reconstructed $\Lambda$ momentum and the line joining the primary and secondary vertices), in order to maximize the signal-to-background ratio. 


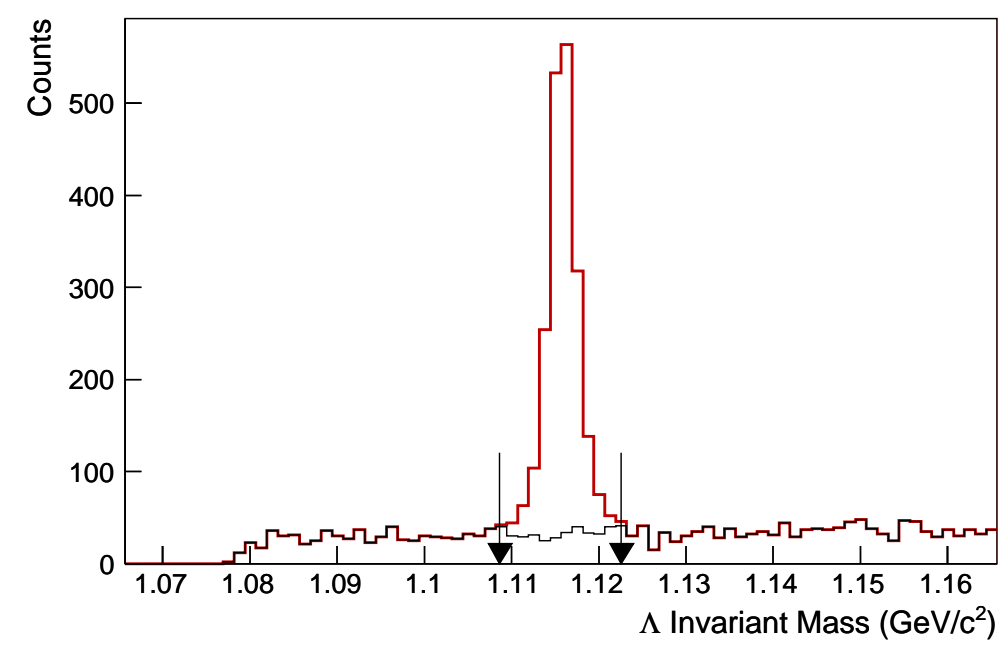

Figure 8.9: $\Lambda$ invariant-mass spectrum corresponding to the reconstruction of 300 HIJING events $\left(\mathrm{d} N_{\mathrm{ch}} / \mathrm{d} \eta=6000\right)$, using tight cuts on the distance of closest approach and the pointing angle.

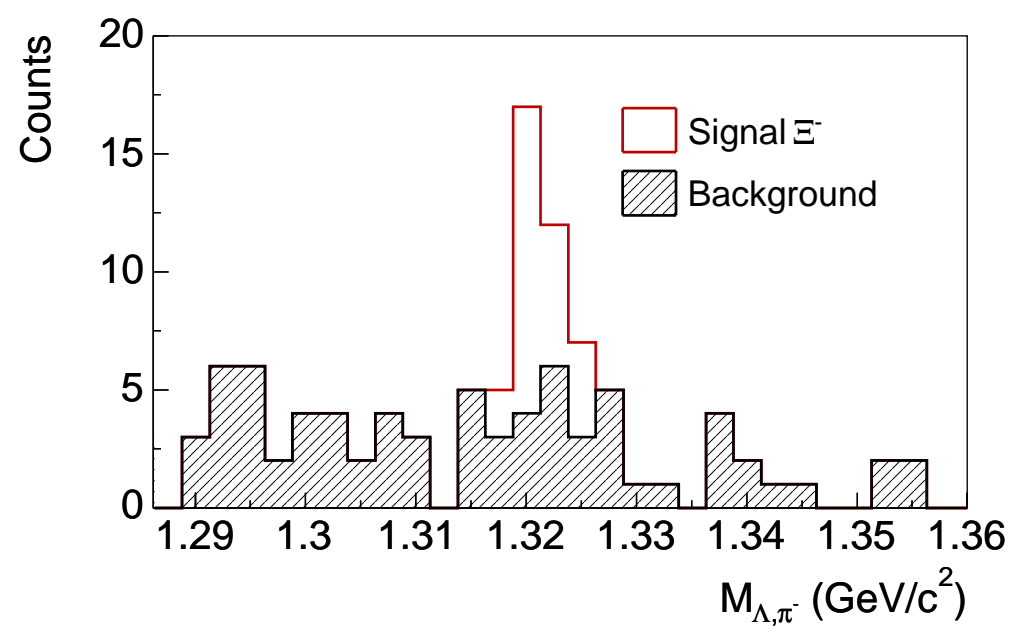

Figure 8.10: $\Xi$ invariant mass spectrum obtained with the reconstruction of 300 HIJING events $\left(\mathrm{d} N_{\mathrm{ch}} / \mathrm{d} \eta=6000\right)$.

The selection on the value of the pointing angle is relaxed during the cascade reconstruction, in order not to bias the sample against cascade decays. As an example, we show in figure 8.10 the effective-mass plot obtained at the analysis level by combining reconstructed $\Lambda$ candidates with a pion track to form another secondary vertex. A $\Xi^{-}$signal is clearly visible. Such a $\Lambda$ would not in general point towards the primary vertex (the parent $\Xi^{-}$would).

At the reconstruction level, we also search for decays in flight within the TPC fiducial volume by combining a primary track disappearing before the end of the TPC, with a secondary track of the same sign. The track pair has to be closely matched in space and to have correct space ordering of the last measured point on the primary track and the first measured point on the secondary track. The efficiency for track finding for such decays is presented in figure 8.11 as a function of the radial position of the decay point in the TPC. The fiducial region to study charged-kaon and -pion decays is $120<r<210 \mathrm{~cm}$. 

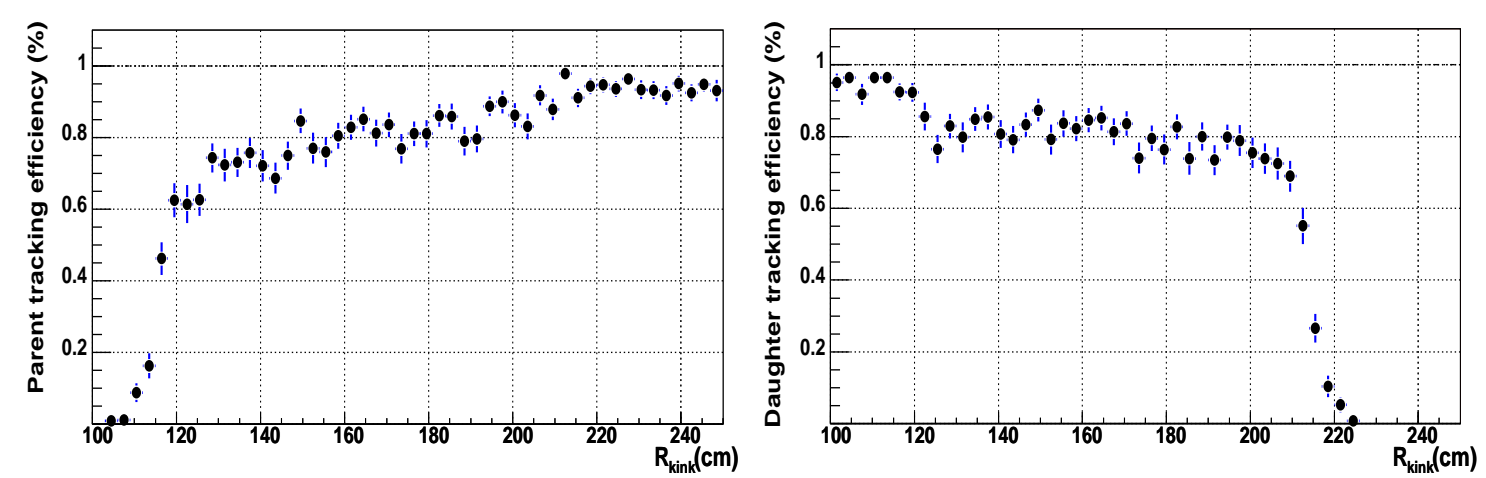

Figure 8.11: Left: Tracking efficiency for parent tracks as a function of the radial coordinate of the decay vertex. Right: Tracking efficiency for daughter tracks as a function of the radial coordinate of the decay vertex.

No attempt to reconstruct secondary vertices from decays of heavy-flavour particles is made at the reconstruction stage. Such decays are only dealt with at the analysis stage.

\subsection{Particle identification}

\subsubsection{Charged-hadron identification}

Several detectors, the ITS, TPC, TRD, TOF and HMPID, participate in the charged-Particle IDentification (PID), each with a different momentum-dependent performance. The best results are achieved by combining the information they provide individually. Particle identification is performed in two steps: during the first step, the information from each detector is used to assign to every track a set of probabilities, one for each particle type. The sum of these probabilities is normalized to unity. For example, if a detector has no identification power for a given track, it assigns equal probabilities to all particle types. On the other hand, if the detector unambiguously decides that the track is of a given type, it assigns probability 1 to that type, and 0 to the others. The procedure for combined PID must guarantee that, in the first of these two extreme cases (no identification power) the detector in question does not influence the result, and in the second (an unambiguous identification) the decision of the detector in question is final and cannot be altered by the information from other detectors. The information from the individual detectors is then combined in the second PID step, described later in the section.

Four layers of the ITS (two silicon-drift and two silicon-strip detector layers) provide signalamplitude information, which can be used for PID in the low-momentum range by measuring ionization energy loss $\mathrm{d} E / \mathrm{d} x$. This is estimated as a truncated mean (using the two or three lowest amplitude signals out of four) in order to minimize the influence of Landau fluctuations. The resulting correlation plot is shown in figure 8.12. The resolution of the ITS $\mathrm{d} E / \mathrm{d} x$ measurement is about $11 \%$, which allows for good $\pi / \mathrm{K}$ separation up to $450 \mathrm{MeV} / c$ and for good $\mathrm{p} / \mathrm{K}$ separation up to about $1 \mathrm{GeV} / c$. A typical ITS response function, for the momentum bin $400-425 \mathrm{MeV} / c$, is shown in figure 8.13. The tails at lower $\mathrm{d} E / \mathrm{d} x$ values for kaons and protons are due to the incorrect assignment of a cluster during track reconstruction. Such a cluster is with the largest probability 


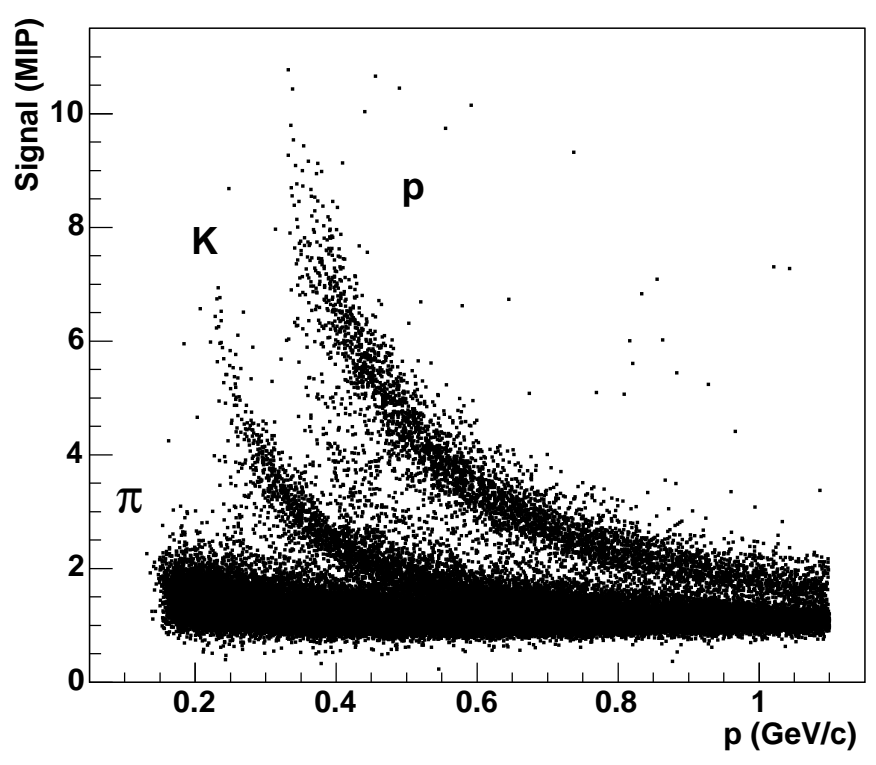

Figure 8.12: Plot of the correlation between the specific SDD/SSD signals (MIP units) calculated by the truncated mean method and the particle's momentum obtained from the TPC and ITS tracking for different particle species.

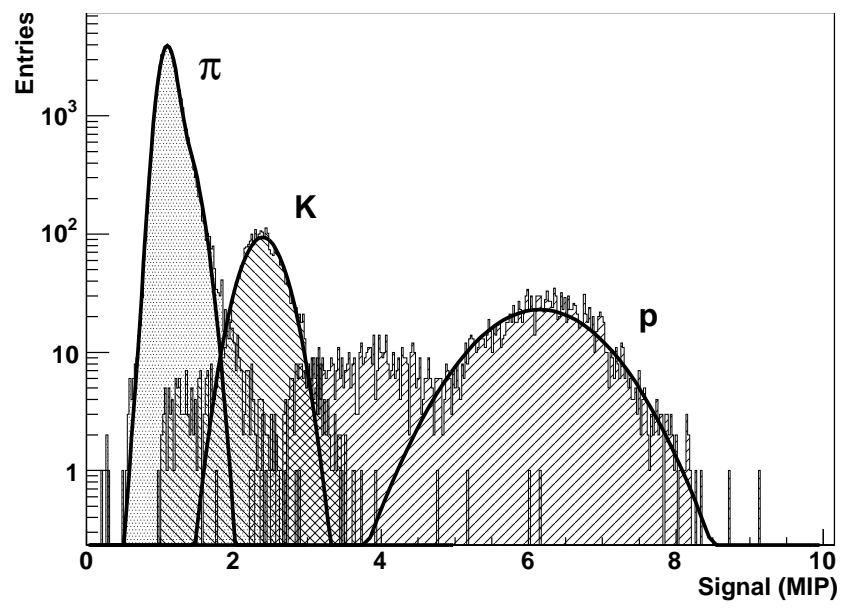

Figure 8.13: Distributions of the truncated mean SDD and SSD signals (MIP units) obtained with the HIJING generator for pions, kaons and protons for a reconstructed momentum of 400$425 \mathrm{MeV} / c$. The curves are the result of Gaussian approximations.

due to a pion, therefore lowering the truncated mean amplitude. The size of this effect will depend on the track-quality selection criteria which will have to be optimized on the real data sample and on the experimental particle-species ratios. The track probabilities for different particle types are calculated using such detector response functions.

The $\mathrm{d} E / \mathrm{d} x$ measurement in the TPC is treated in a similar way, using the truncated mean of the $65 \%$ lowest-amplitude pad-row samples. The estimated resolution of the $\mathrm{d} E / \mathrm{d} x$ measurement depends slightly on the charged-particle density; it changes from $5.5 \%$ for pp events to $6.5 \%$ for central $\mathrm{Pb}-\mathrm{Pb}$ collisions. The simulated response function in the latter case is shown in figure 8.14 


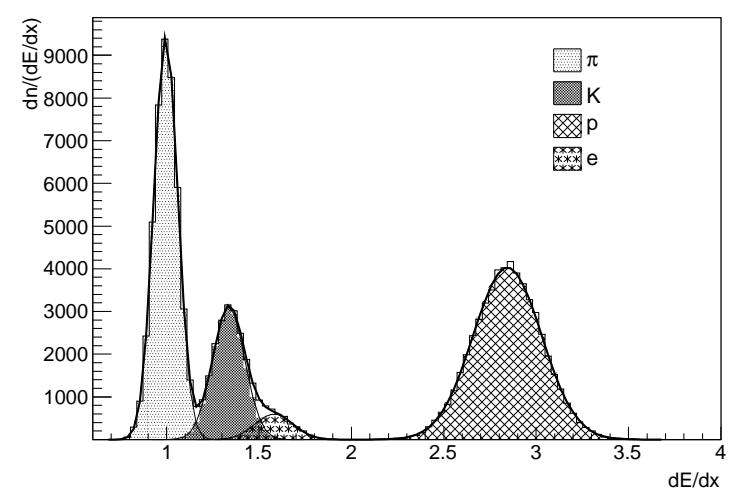

Figure 8.14: Simulated TPC $\mathrm{d} E / \mathrm{d} x$ distribution for electrons, pions, kaons, and protons with $p=0.5 \mathrm{GeV} / c$. The solid line indicates the result of the fit to a sum of four Gaussian distributions. The shaded areas correspond to the contributions from different particle species.
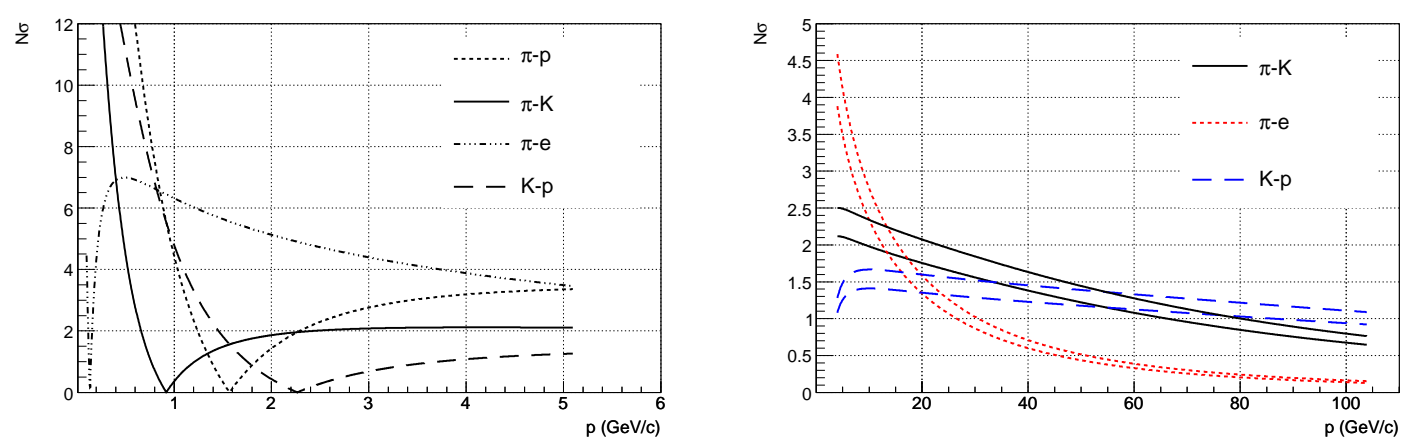

Figure 8.15: Momentum dependence of the $\langle\mathrm{d} E / \mathrm{d} x\rangle$ separation for different particle combinations in units of the energy loss resolution. Left: In the low-momentum range assuming a constant resolution of 6.5\%. Right: In a wide momentum range, for a resolution of 5.5\% (upper curves) and $6.5 \%$ (lower curves).

for a narrow momentum bin around $0.5 \mathrm{GeV} / c$. The separation power (expressed as number of standard deviations) for the different pairs of particle species is plotted as a function of momentum in figure 8.15. In the lower momentum range (left panel), one starts with excellent separations in the $1 / \beta^{2}$ region (below the particle masses). Increasing the momentum, the separations progressively worsen, decreasing to zero, at the value of momentum where the Bethe-Bloch curves for the two particles cross. At still higher momenta, the particle separation increases again thanks to the relativistic rise of the $\mathrm{d} E / \mathrm{d} x$ in the TPC gas, up to the Fermi plateau, before a final gentle decrease towards very high momenta. It is actually possible to measure separately the average abundances of the different particle species up to several tens of $\mathrm{GeV}$ of momentum, as can be seen from the right panel of figure 8.15 .

The measurement of $\mathrm{d} E / \mathrm{d} x$ in the TRD contributes to charged-particle PID in the same momentum range as for the TPC. Although the ionization in the TRD gas (based on xenon) is larger than in the TPC (based on neon), the TRD $\mathrm{d} E / \mathrm{d} x$ measurement is only a complement to the TPC measurement, because of the limited TRD track length (the resolution scales as $\sqrt{L}$ ). The precision on the $\mathrm{d} E / \mathrm{d} x$ measurement in the TRD is estimated to be $18-20 \%$. 
Pion Identification
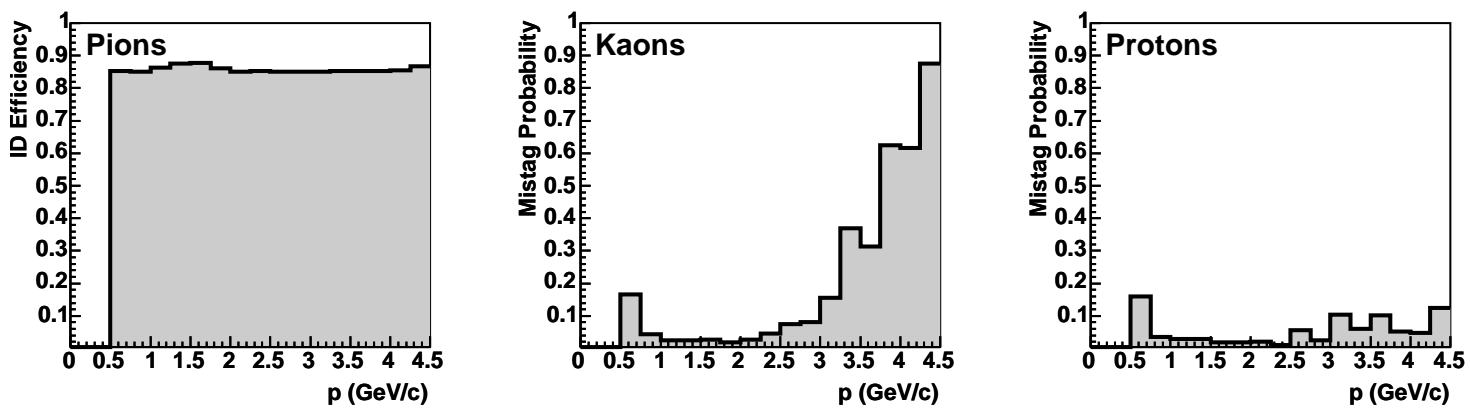

Kaon Identification
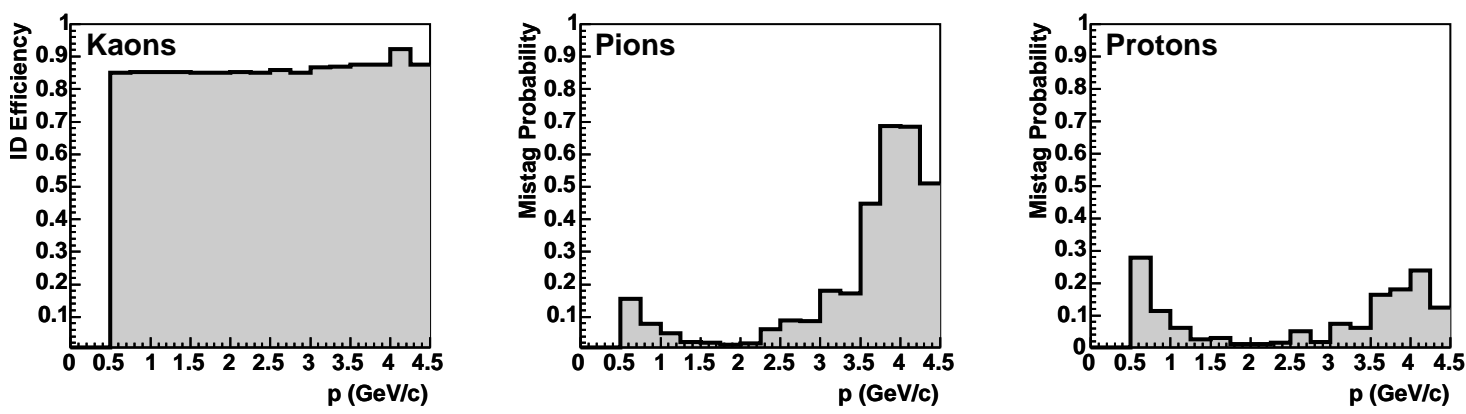

Proton Identification
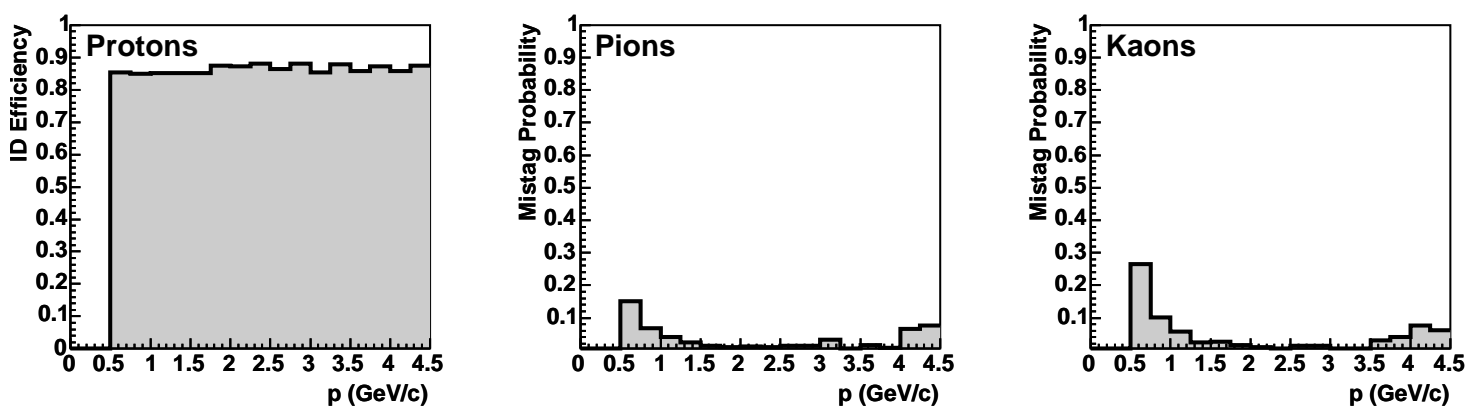

Figure 8.16: PID efficiencies (tuned at about $85 \%$, see text) and mistag probabilities in the TOF for the case of pion (top), kaon (middle) and proton identification (bottom), for pions, kaons and protons in central $\mathrm{Pb}-\mathrm{Pb}$ collisions.

As we also saw above, charged-particle identification based on a $\mathrm{d} E / \mathrm{d} x$ measurement performs well in the $1 / \beta^{2}$ region and, for gas-based detectors, in the multi-GeV region. However, this technique inevitably leaves a hole in the momentum range around the minimum of the ionizing losses (i.e. between 0.9 and $3 \mathrm{GeV} / c$, see figure 8.15). In the ALICE experiment this range is covered by the TOF detector, that is able to measure a particle's arrival time with a precision of about 80 ps. During the last tracking pass in the Kalman filter we compute the integral of the particle's time-of-flight for different mass hypotheses, and compare it with the TOF measurement smeared by the response function. In this way, we obtain the TOF track probabilities for the different particle species. Figure 8.16 shows, for the central $\mathrm{Pb}-\mathrm{Pb}$ collisions, the efficiency-contamination matrix for pion, kaon, and proton identification by the TOF, obtained tuning the correct-particle-identification 

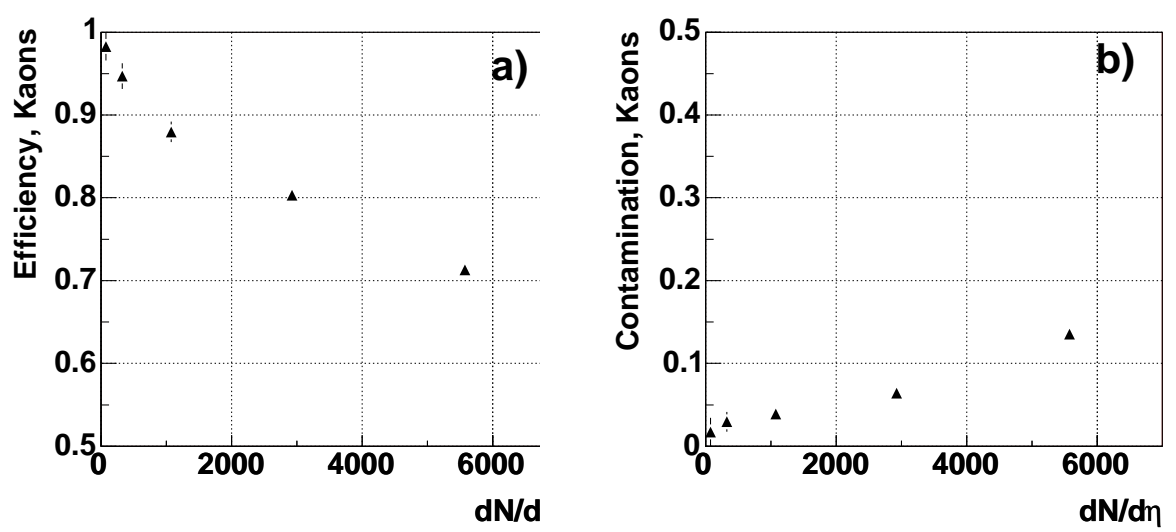

Figure 8.17: TOF PID efficiency (a) and contamination (b) for kaons, integrated over the momentum interval $0.5<p<3 \mathrm{GeV} / c$, as a function of the charged-track multiplicity per unit rapidity.
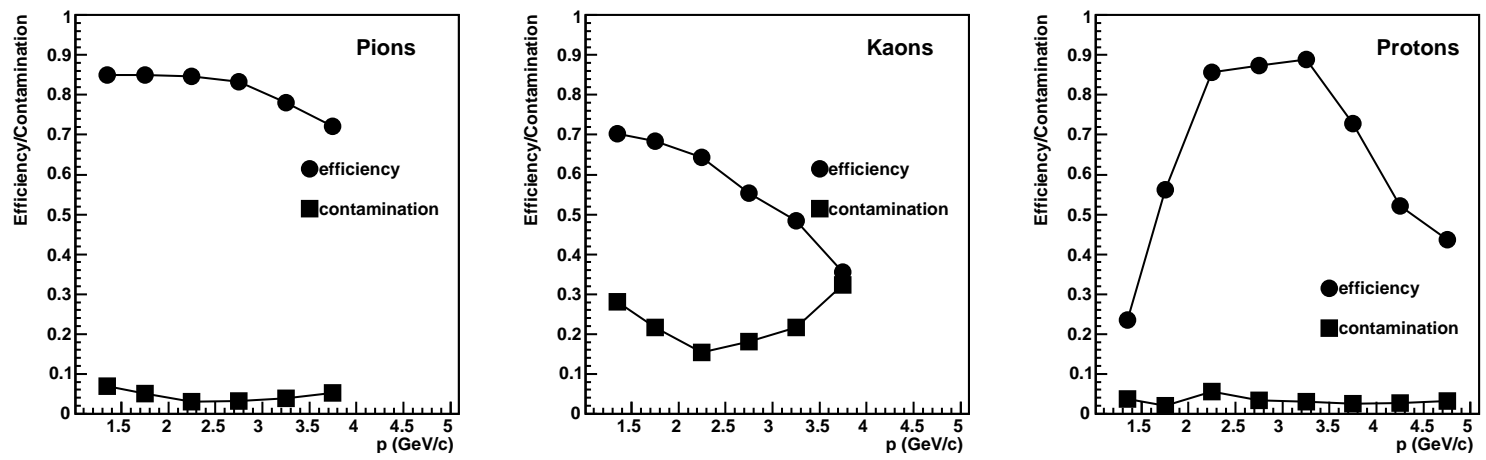

Figure 8.18: Efficiency and contamination for pions, kaons, and protons in the HMPID.

probability $\approx 85 \%$. The TOF detector allows us to extend, on a track-by-track basis, the kaon/pion separation out to $2.5-3 \mathrm{GeV} / \mathrm{c}$ and the proton/kaon separation out to $3.5-4 \mathrm{GeV} / c$. The dependence of the TOF performance on the charged-particle density is shown in figure 8.17 for kaons.

The HMPID is used to further extend the momentum range for charged-particle identification, albeit only within a limited acceptance. The expected performance is shown in figure 8.18, where the momentum dependence of the identification efficiency and the contamination probability are plotted for different particle species. As can be seen, the HMPID is able to identify protons up to $5 \mathrm{GeV} / c$.

The various PID measurements are translated into probabilities for each track to belong to the different particle types. At the level of the measurements from the individual detectors, we do not attempt to take into account the differences in the different particle-species populations (as they will in general be different for each analysis, due to the different applied selection cuts). Such 'a priori' probabilities are taken into account at the combined PID stage (i.e. after the analysis cuts have been applied), using an iterative procedure. Simulation studies have shown that such a procedure converges to the correct values of the particle yields, in at most three iterations, even when starting with equal a priori probabilities for all particles. The combined particle identification performance of the ITS, TPC and TOF is shown, for the example of kaons in figure 8.19. 

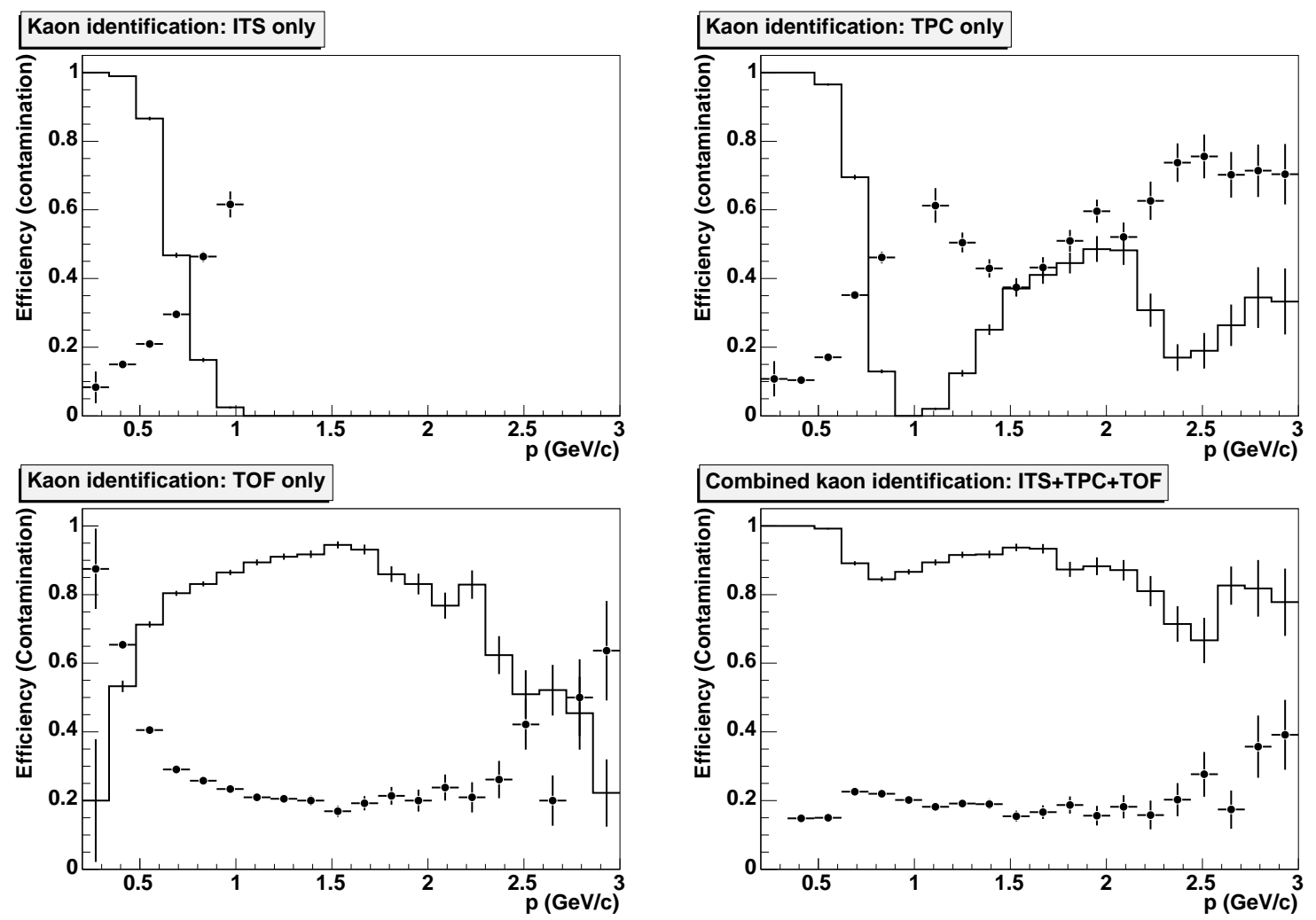

Figure 8.19: Single-detector efficiencies (solid line) and contaminations (points with error bars) for charged-kaon identification with the ITS, TPC and TOF stand-alone and combined.

\subsubsection{Electron identification}

The main source of information for electron identification in ALICE is the TRD (TransitionRadiation Detector), which can also provide electron-identification information at the trigger level. The TRD is expected to reject pions by a factor of 100 or better, for $90 \%$ electron detection efficiency. This is shown in figure 8.20 for particle momentum of $2 \mathrm{GeV} / c$. The charged-hadron rejection power is expected to deteriorate only very slowly as the momentum increases, making it possible to discriminate between pions and electrons up to a few tens of GeV. Figure 3.44 in section 3.3 shows an analysis of the pion rejection power as a function of the momentum employing different methods for particle identification. At low momenta the TPC also contributes to electron identification via the $\mathrm{d} E / \mathrm{d} x$ measurement (cf. figure 8.14).

Additional electron-identification capability, in particular at high momenta, will be provided by the ALICE electromagnetic calorimeters: the PHOS, in a restricted acceptance; and later the EMCal, expected to provide another two orders of magnitude of hadron rejection power.

\subsection{Neutral-particle detection}

Excellent photon-detection capability is provided by the PHOS. The expected energy resolution is shown in figure 8.21 as a function of energy. In order to extract the signal from the direct photons 


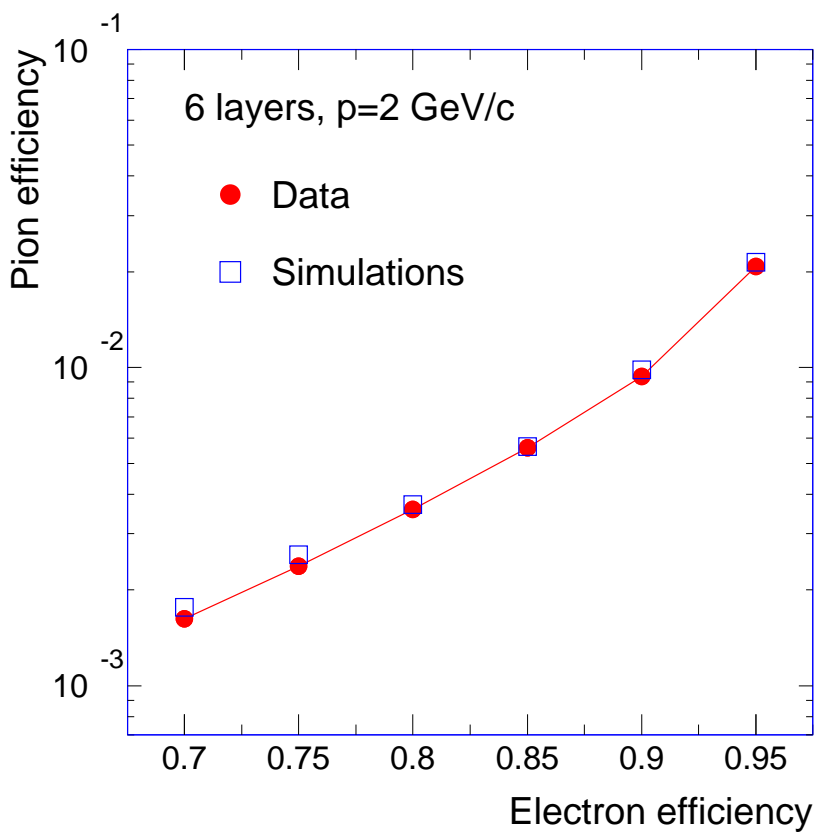

Figure 8.20: Pion efficiency as a function of electron efficiency in the TRD for a momentum of $2 \mathrm{GeV} / c$. Simulations are compared to data measured in test beams.

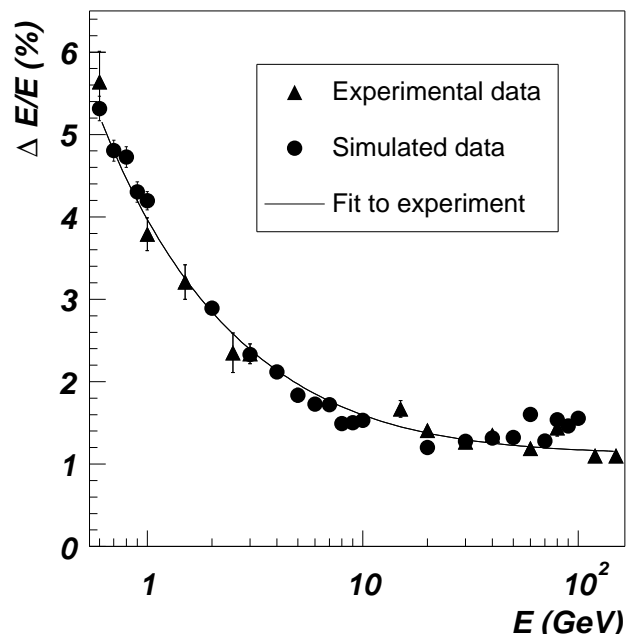

Figure 8.21: Energy resolution of a $3 \times 3 \mathrm{PbWO}_{4}$ array measured in response to mono-energetic electrons $(\boldsymbol{\Lambda})$ compared with simulations of mono-energetic photons $(\bullet)$. The continuous line represents the result of the fit to the experimental data.

produced in heavy-ion collisions, it is necessary to measure the production of $\pi^{0}, \eta$, and other resonances in as wide a transverse-momentum range as possible. In figure 8.22 the performance of PHOS for the extraction of the $\pi^{0}$ signal is shown in bins of increasing $p_{\mathrm{t}}$. The measured yields of $\pi^{0}, \eta$, etc. will then be used to subtract from the observed $\gamma$ transverse momentum spectrum the contribution due to particle decays. 

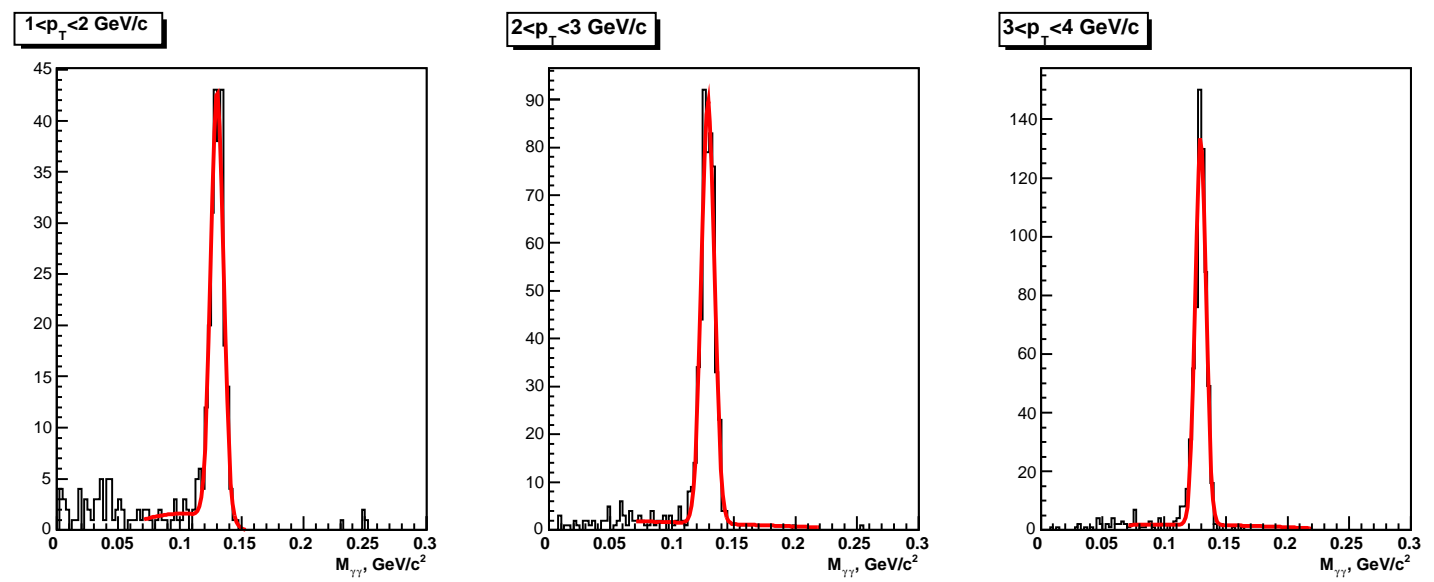

Figure 8.22: $\gamma \gamma$ invariant-mass spectra for different $p_{\mathrm{t}}$ bins.

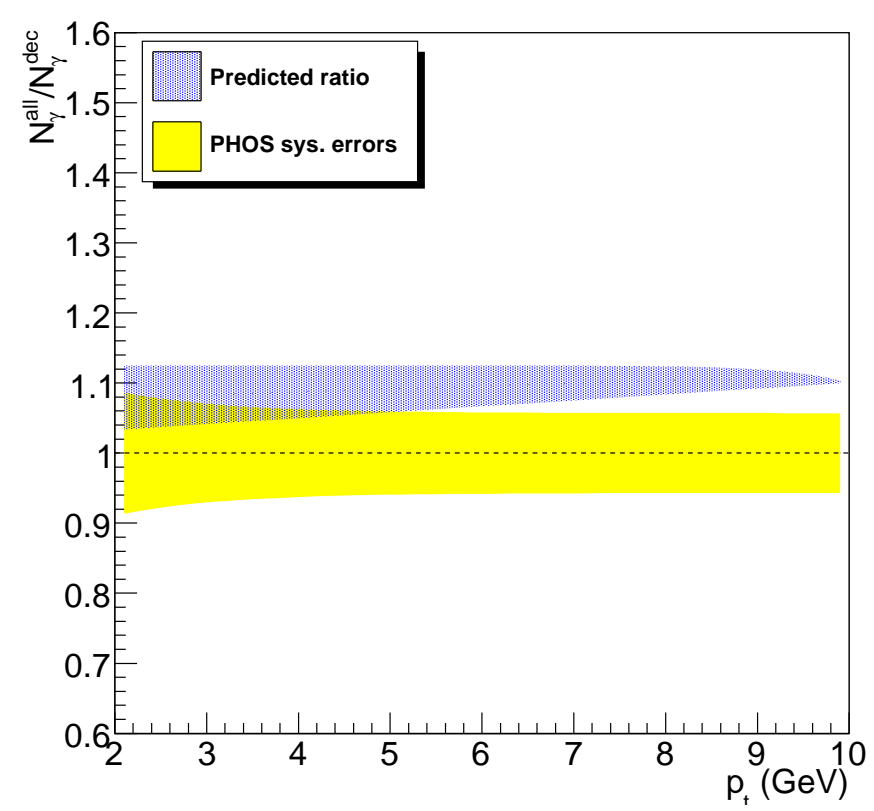

Figure 8.23: Error band, including systematic uncertainties, for the ratio of all photons to decay photons as a function of $p_{\mathrm{t}}$.

In figure 8.23 the estimated error band, including systematic uncertainties, for the ratio of all photons to decay photons is shown. The excess above unity represents the direct photon signal. Above $10 \mathrm{GeV}$ this signal is dominated by the production of QCD photons, which is expected to be well described by perturbative QCD calculations. In the few $\mathrm{GeV}$ region additional photon radiation from the initial high-density phase of the heavy-ion collisions is expected. As illustrated in figure 8.23 , such a signal should be observable, if it amounts to at least $6 \%$ of the contribution from $\pi^{0}, \eta$, etc. decays.

Additional capabilities for the detection of neutral particles will be provided by the EMCal detector over a larger acceptance, but with lower energy resolution (by a factor 2.5-3) than PHOS. 


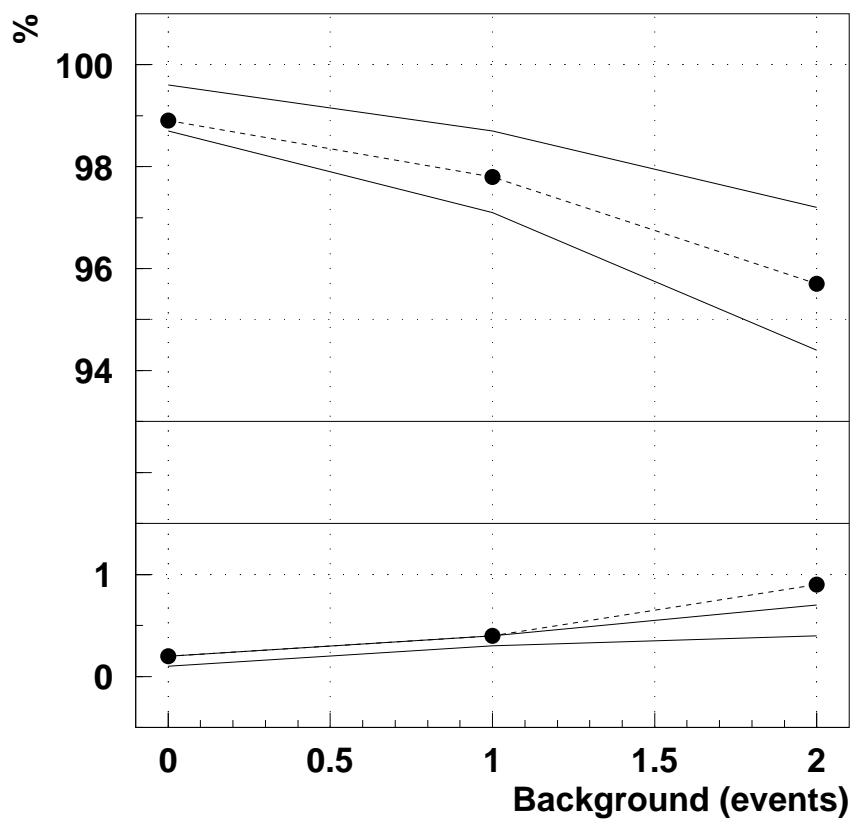

Figure 8.24: Track-finding efficiency (upper part) and percentage of fake tracks (lower part) versus occupancy, expressed in terms of the number of superimposed nominal background events. The closed points show results of the tracklet matching method. The bands show the results for the Kalman filter for different algorithm parameters. Lines are drawn to guide the eye.

The EMCal will also open the possibility of triggering on high-transverse-momentum jets and will allow us to improve the measurement of the jet energy including the neutral component. Finally, another photon detector, the PMD, will allow us to count the number of photons produced in the forward region $(2.5<\eta<3.5)$.

\subsection{Muon detection}

Muons are detected in the muon spectrometer in the pseudo-rapidity range $-4.0<\eta<-2.5$. The muon tracks are reconstructed by five tracking stations located behind the absorber. The measurement of their deflection in the muon dipole magnet allows the determination of their momenta. Two different methods have been developed for track finding in the spectrometer. The first combines tracklets found in the muon tracking stations (each station measures two space points on the track); the second is based on the Kalman filter. The two methods give compatible results. A comparison of the two methods is shown in figure 8.24; the muon-finding efficiency and the fake-track probability are shown as a function of the background level in the tracking stations, expressed in terms of number of superimposed central $\mathrm{Pb}-\mathrm{Pb}$ events. A tracking efficiency above $95 \%$ is obtained, even at a level of background twice that expected in central $\mathrm{Pb}-\mathrm{Pb}$ collisions.

The momentum resolution for the reconstructed tracks is shown in figure 8.25 as a function of momentum. We achieve a relative momentum precision of about $1 \%$, which then results in a contribution to the invariant-mass resolution for dimuons of about $0.7 \%((1 / \sqrt{2} \times \Delta p / p)$. The remaining contribution to the dimuon invariant-mass resolution, due to the error on the determination of the 


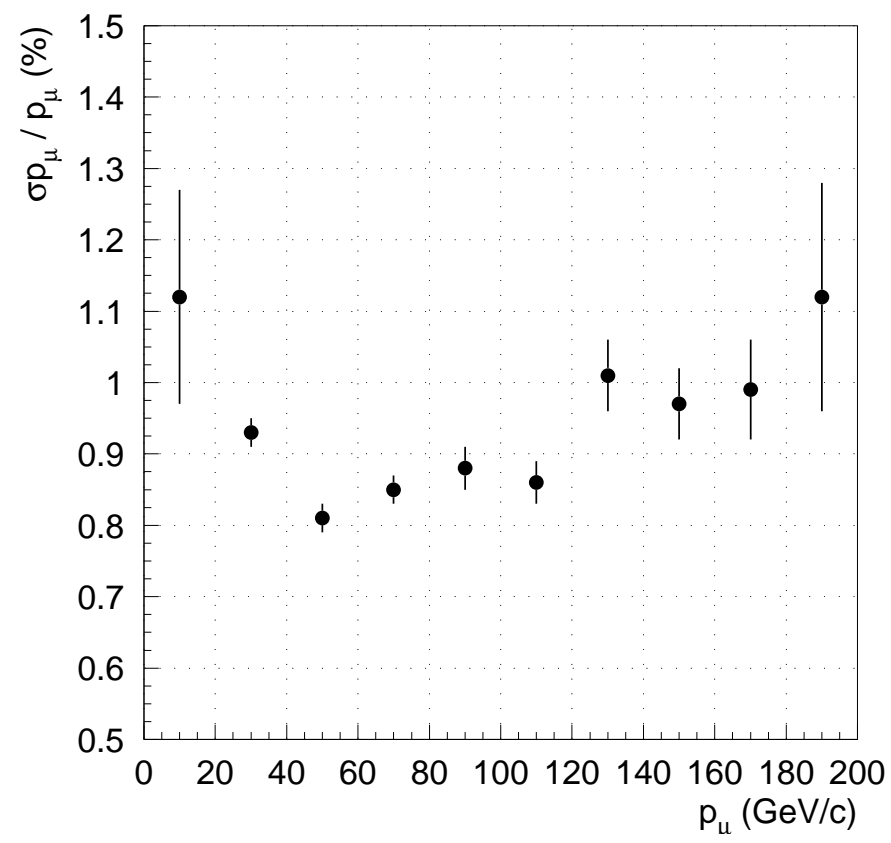

Figure 8.25: Relative momentum resolution as a function of momentum for muons from $\Upsilon$ decays.

angle between the two muons, is lower. This is due to the fact that the primary-vertex constraint is used in the muon reconstruction, which allows to determine the angle using the positions of the primary-vertex and of the first measured point behind the absorber, and not by just relying on the measured track direction in the tracking stations. The precision on the angle is therefore determined essentially by the resolution on the first measured point. As a consequence, the mass resolution for the $\Upsilon$ reconstruction is better than $100 \mathrm{MeV} / c^{2}$, which allows for unambiguous separation of the different $\Upsilon$ states. 


\section{Acknowledgements}

The collaboration wishes to acknowledge contributions given by: D. Anstett, P. Bouvier, B. Cantin, M. Connor, F. Dalla Santa, C. David, C. Decosse, A.Ferrand, D. Gabriele, U. Genoud Prachex, C. Hervet, P. Ijzermans, S. Junod, X. Lagrue, Y. Lesenechal, S. Maridor, D. Meunier-Picard, B. Moris, E. Paulat, S. Philippin, L. Radulescu, J. Van Beelen, M. Van Stenis, D. Williams.

We acknowledge the support of the following funding agencies:

- Swiss Fonds Kidagan and the Calouste Gulbenkian Foundation from Lisbon, Armenia

- CERN

- National Natural Science Foundation of China (NSFC) Ministry of Education of the People's Republic of China

- Croatian Ministry of Science and Technology

- Cubaenergia (Cuba)

- Ministry of Education, Youth and Sports of the Czech Republic

- Danish National Science Research Council (DNSRC) The Carlsberg Foundation

- Academy of Finland Helsinki Institute of Physics (HIP)

- Centre National de la Rcherche Scientifique (CNRS) Institut National de Physique Nucléaire et de Physique des Particules (IN2P3) Commissariat à l'Énergie Atomique (CEA) "Region Pays de Loire," "Region Alsace," "Region Auvergne"

- Federal Ministry of Education and Research (BMBF) Helmholtz Association

- University of Athens-Greece Program PYTHAGORAS II / Support of University Research Groups

- Gesellschaft fur Schwerionenforschung (GSI)

- Hungarian Scientific Research Fund (OTKA) National Office for Research and Technology (NKTH)

- Department of Atomic Energy (DAE) and Department of Science and Technology (DST) of the Government of India

- Instituto Nazionale di Fisica Nucleare (INFN) 
- Ministry of Education, Culture, Sports, Science and Technology (MEXT) Japan Society for the Promotion of Science (JSPS)

- Joint Institute for Nuclear Research (JINR)

- Korea Foundation for International Cooperation of Science and Technology (KICOS)

- Consejo Nacional de Ciencia y Tecnología (CONACYT) Dirección General de Asuntos del Personal Académico (DGAPA) ALFA-EC in the HELEN program (High Energy Physics Latinoamerican-European Network)

- Foundation for Fundamental Research on Matter (FOM) Netherlands Organisation for Scientific Research (NWO)

- The Research Council of Norway

- Ministry of Science and Higher Education, Poland

- National Authority for Scientific Research (Autontatea Nationala pentru Cercetare Stiintifica) and Romanian National Agency for Scientific Research (NASR)

- Ministry of Education and Science of the Russian Federation Russian Federal Agency of Atomic Energy International Science and Technology Center CERN-INTAS

- Ministry of Education of the Slovak Republic

- Xunta de Galicia (Conselleria de Educacion)

Centro de Aplicaciones Tecnologicas y desarrollo Nuclear (CEADEN)

Ministerio de Educación y Ciencia (MEC)

IGFAE, Universidade de Santiago de Compostela

Research Center for Energy, Environment and Technology (CIEMAT)

IAEA (Atomic International Energy Agency)

EELA

- Department of Science and Technology, South Africa

- The Swedish Research Council

The Knut and Alice Wallenberg Foundation

- Texas Learning and Computation Center at the University of Houston

- Ministry of Education and Science of Ukraine

- The Science and Technology Facilities Council (STFC)

- Department of Energy (DOE)

National Science Foundation (NSF) 


\section{ALICE acronym list}

ACC

ACORDE

ACR

ADC

ADULT

AFFAIR

AGS

ALABUF

ALCAPONE

ALICE

AliEn

AliRoot

ALTRO

AMBRA

AMORE

AMS

AOD

APD

API

ApMon

ARC

ARDA

ARM

ASIC

BCAM

BCF9929A

BC404

CAD

CAF

CARLOS

CASTOR

CCD

CCIU

ALICE Control Coordination

ALICE Cosmic Ray Detector

ALICE Control Room

Analog-to-Digital Converter

A DUaL Threshold

A Fine Fabric and Applications Information Recorder

Alternating Gradient Synchrotron

SSD Analogue driver and switch

SSD Regulator and control chip

A Large Ion Collider Experiment

Alice Grid Environment

Alice ROOT

ALICE TPC Readout

SDD Digital event buffer

Automatic MOnitoRing Environment

Austriamicrosystems AG

Analysis Object Data

Avalanche Photodiodes

Application Programming Interface

Application Monitoring API

Advanced Resource Connector

A Realisation of Distributed Analysis for LHC

Advanced RISC Machine

Application-Specific Integrated Circuit

Boston CCD Angle Monitors

Bicron shifting fibres

Bicron scintillator

Computer Aided Design

CERN Analysis Facility

SDD Zero suppressor

CERN Advanced STORage manager

Charge-Coupled Detector

Channel Concentrator Interface Unit 


\begin{tabular}{|c|c|}
\hline CDB & Configuration DataBase \\
\hline CERN & European Organization for Nuclear Research \\
\hline CFC & Carbon Fibre Composite \\
\hline CFSS & Carbon Fibre Support Sector \\
\hline CHARM & Computer Health ad Remote Management \\
\hline CIU & Channel Interface Unit \\
\hline CM & Control Modules \\
\hline CMOS & Complementary Metal-Oxide-Semiconductor \\
\hline CMRW & Column-Memory and Read-Write ( protocol) \\
\hline CompactPCI & Compact Peripheral Component Interconnect \\
\hline CPDM & Clock and Pulser Distribution Module \\
\hline CPU & Central Processing Unit \\
\hline CPV & Charged Particle Veto \\
\hline CROCUS & Cluster Read Out Concentrator Unit System \\
\hline CRRC & Signal shaper \\
\hline CRT & ConcentRaTor \\
\hline CSA & Charge Sensitive Amplifier \\
\hline CsI & Cesium Iodide \\
\hline CT1 & Central Trigger of level 1 \\
\hline CT2 & Central Trigger of level 2 \\
\hline CTE & Coefficient of Thermal Expansion \\
\hline CTP & Central Trigger Processor \\
\hline CTTM & Cosmic and Topology Trigger Module \\
\hline $\mathbf{C U}$ & Control Unit \\
\hline DA & Detector Algorims \\
\hline DAC & Digital-to-Analog Converter \\
\hline DAQ & Data Aquisition \\
\hline DATE & Data Acquisition and Test Environment \\
\hline DCA & Detector Control Agent \\
\hline DCAHI & Detector Control Agent Human Interface \\
\hline DCDB & Detector Construction Data Base \\
\hline DCS & Detector Control System \\
\hline DDL & Detector Data Link \\
\hline DIM & Distributed Information Management system \\
\hline DMA & Direct Memory Access \\
\hline DRM & Data Readout Module \\
\hline D-RORC & DAQ RORC \\
\hline DSP & Digital Signal Processors \\
\hline DSS & Detector Safety System \\
\hline DU & Device Unit \\
\hline EBDS & Event Building and Distribution System \\
\hline ECFA & European Committee for Future Accelerators \\
\hline ECS & Experiment Control System \\
\hline
\end{tabular}




$\begin{array}{ll}\text { EDM } & \text { Event Destination Manager } \\ \text { ELMB } & \text { Embedded Local Monitor Board } \\ \text { EM } & \text { Electromagnetic } \\ \text { EMCAL } & \text { Electromagnetic Calorimeter } \\ \text { EMC } & \text { ElectroMagnetic Calorimeter } \\ \text { EMS } & \text { External Monitoring System } \\ \text { ENC } & \text { Equivalent Noise Charge } \\ \text { ESD } & \text { Event Summary Data } \\ \text { FC } & \text { Fibre Channel } \\ \text { FC } & \text { File Catalog } \\ \text { FC4G } & \text { Fibre Channel 4 Gbits/s } \\ \text { FEA } & \text { Front-End Analog card } \\ \text { FEC } & \text { Front-End Card } \\ \text { FEE } & \text { Front-End Electronics } \\ \text { FED } & \text { Front-End Device } \\ \text { FEP } & \text { Front-End Processor } \\ \text { FERO } & \text { Front-End Read-Out } \\ \text { FEROM } & \text { Front-End Read-Out Module } \\ \text { FFT } & \text { Frontal Fan-out Trigger } \\ \text { FMD } & \text { Forward Multiplicity Detector } \\ \text { FORTRAN } & \text { FORmula TRANslation } \\ \text { FPGA } & \text { Field-Programmable Gate Array } \\ \text { FRT } & \text { FRonTal } \\ \text { FSM } & \text { Finite State Machine } \\ \text { FTD } & \text { File Transfer Daemon } \\ \text { FTD } & \text { Frontal Trigger Dispatching } \\ \text { FTE } & \text { Full Time Equivalent } \\ \text { FTS } & \text { File Tranfer Service } \\ \text { FWHM } & \text { Full Width Half Maximum } \\ \text { FXS } & \text { File eXchange Server } \\ \text { GB } & \text { Gigabyte (10 }{ }^{9} \text { bytes) } \\ \text { GDC } & \text { Global Data Collector } \\ \text { GIF } & \text { Gamma Irradiation Facility } \\ \text { GMS } & \text { Geometry Monitoring System } \\ \text { GOL } & \text { Gigabit Optical Link } \\ \text { GSI } & \text { Grid Security Infrastructure } \\ \text { GSSAPI } & \text { Grid Security Sercice Application Programming Interface } \\ \text { GTL } & \text { FEE readout bus } \\ \text { GTU } & \text { Global Tracking Unit } \\ \text { GUID } & \text { Global Unique IDentifier } \\ \text { HAL25 } & \text { SSD front-end chipc } \\ \end{array}$


HLT

HMPID

HPTDC

H-RORC

HTML

HV

IA32/64

I/O

IP

IR

IRST

IS

ITS

JCOP

JDL

JTAG

LAA

LAN

LDC

LED

LEP

LFN

LHC

LMS

LNN

LSB

LTM

LTU

LU

LVDS

LV

LO

L1

L2

MANAS

MANU

MARC

MB

MCM

MIP

MM

MonALISA

MOS
High-Level Trigger

High Momentum Particle Identification Detector

High-Performance Time-to-Digital Converter

HLT RORC

Hyper Text Markup Language

High Voltage

Intel Architecture, 32/64-bit

Input/Output

Interaction Point

Infrared

Institute of Scientific and Technological Research

Information Service

Inner Tracking System

Joint Control Project

Job Description Language

Joint Test Action Group

Lepton Asymmetry Analyser (an R\&D project started in 1986)

Local Area Network

Local Data Concentrator

Light Emitting Diode

Large Electron-Positron Collider

Logical File Name

Large Hadron Collider

Longitudinal Monitoring System

Logical File Name

Least Significant Bit

Local Trigger Module

Local Trigger Unit

Logical Unit

Low-Voltage Differential Signaling

Low Voltage

Level-0 trigger

Level-1 trigger

Level-2 trigger

Multiplexed ANALogic Signal processor

MAnas NUmérique

Muon Arm Readout Chip

Minimum Bias

Multi-Chip Module

Minimum Ionizing Particle

Master Modules

MONitoring Agents using a Large Integrated Services Architecture

Metal-Oxide Semiconductor 


\begin{tabular}{|c|c|}
\hline MRPC & Multi-gap Resistive-Plate Chamber \\
\hline MSS & Mass Storage System \\
\hline MT & Multiplicity Trigger \\
\hline MWPC & Multi-Wire Proportional Chamber \\
\hline NN & Neural Network \\
\hline NTD & Neutron Transmutation Doped silicon \\
\hline OCDB & Offline Conditions Data Base \\
\hline ODH & Oxygen Deficient Hazard \\
\hline OLE & Object Linking and Embedding \\
\hline ON & Operator Node \\
\hline OPC & OLE for Process Control \\
\hline ORI & Optical Readout Interface \\
\hline OS & Operating System \\
\hline OSG & Open Science Grid \\
\hline PASCAL & SDD Multi purpose ASICs \\
\hline PASA & Preamplifier / Shaping Amplifier \\
\hline PATCH & Protocol for ALICE Tracking Chambers \\
\hline PC & Personal Computer \\
\hline PCA & Partition Control Agent \\
\hline PCAHI & Partition Control Agent Human Interface \\
\hline PCB & Printed Circuit Board \\
\hline PCI & Peripheral Component Interconnect \\
\hline PCI-X & Peripheral Component Interconnect eXtended \\
\hline PDS & Permanent Data Storage \\
\hline PFN & Physical File Name \\
\hline PG & p-Gas Trigger \\
\hline PHENIX & Pioneering High Energy Nuclear Interaction Experiemnt \\
\hline PHOS & Photon Spectrometer \\
\hline PID & Particle Identification \\
\hline PIN & Positive Intrinsic Negative \\
\hline PLC & Programmable Logic Controller \\
\hline PMD & Photon Multiplicity Detector \\
\hline PMT & Photomultiplier Tube \\
\hline PFN & Physical File Name \\
\hline POSIX & Portable Operating System for Computer Environment \\
\hline PROOF & Parallel ROOt Facility \\
\hline PS & Proton Synchrotron \\
\hline PVSS & Prozessvisualisierungs- und Steuerungs-System \\
\hline PWO & $\mathrm{PbWO}_{4}$ \\
\hline PX24 & $23 \mathrm{~m}$ diameter access shaft \\
\hline QCD & Quantum Chromo Dynamics \\
\hline QE & Quantum Effeciency \\
\hline QoS & Quality Of Service \\
\hline
\end{tabular}




\begin{tabular}{|c|c|}
\hline QRL & Cryogenic Ring-Line \\
\hline $\mathbf{R C}$ & Run Control \\
\hline RCU & Readout Control Unit \\
\hline RHIC & Relativistic Heavy Ion Collider \\
\hline RICH & Ring Imaging Cherenkov detector \\
\hline RORC & Read-Out Received Card \\
\hline RPC & Resistive Plate Chamber \\
\hline SASL & Simple Authentication and Security Layer \\
\hline SBU & Sequential Build-Up \\
\hline SCADA & Supervisory Controls And Data Acquisition \\
\hline SDD & Silicon Drift Detector \\
\hline SDTY & Standard Data Taking Year \\
\hline SE & Storage Element \\
\hline SEU & Single Event Upset \\
\hline SFP & Small Form-Factor Pluggable \\
\hline SG2 & Building for primary gas circulation equipment \\
\hline SI2K & Specification Integer 2000 \\
\hline SIU & System Interface Unit \\
\hline SLA & Service Level Agreement \\
\hline SMI & State Management Interface \\
\hline SMP & Symmetrical Multi Processing \\
\hline SMT & Symmetrical Multi Threading \\
\hline SOAP & Simple Object Access Protocol \\
\hline SPD & Silicon Pixel Detector \\
\hline SPS & Super Proton Synchrotron \\
\hline SSD & Silicon Strip Detector \\
\hline STAR & Solenoidal Tracker At RHIC \\
\hline STU & Summary Trigger Unit \\
\hline SVN & SubVersioN \\
\hline SX2 & Assembly hall covering the underground access shaft \\
\hline SXL2 & Assembly surface building \\
\hline TAB & Tape Automatic Bonded \\
\hline TCI & Trigger Crocus Interface \\
\hline TCP/IP & Transmission Control Protocol/Internet Protocol \\
\hline TDC & Time-to-Digital Converter \\
\hline TDS & Transient Data Storage \\
\hline TDSM & Transient Data Storage Manager \\
\hline TID & Total Ionizing Dose \\
\hline TMAE & TriMethylAminoEthyl \\
\hline TMS & Transverse Monitoring System \\
\hline TMU & Track Matching Unit \\
\hline TOF & Time-Of-Flight \\
\hline TOT & Time-Over-Threshold \\
\hline
\end{tabular}


TPA

TPC

TR

TRAP

TRD

TRG

TRM

TRU

TS/CV

TS/EL

TTL

TTC

TTCrx

T0

UI

UMC

UNIX (UNICS)

UP25

UPC

UPS

URD

UV

UX25

VME

Vo

VO

WLCG

WLS

WN

WWW

ZEM

ZDC

ZN

ZP
Trigger Partition Agent

Time Projection Chamber

Transition Radiation

Tracklet Processor

Transition Radiation Detector

Trigger

TDC Readout Module

Trigger Region Unit

CERN Cooling and Ventilation Group

CERN Electronic Engineering Group

Transitor-Transistor Logic

Timing, Trigger and Control

Timing, Trigger and Control receiver

Time 0 detector

User Interface

United Microelectronics Corporation

UNiplexed Information and Computing System

Connection tunnel between the UX25 area and the LHC by-pass tunnel

Ultra Peripheral Collisions

Uninteruptible Power Supply

User Requirements Document

Ultraviolet

Main underground cavern

Versa Module Eurocard

Vertex 0 detector

Virtual Organisation

Worldwide LHC Computing Grid

Wavelength Shifting

Worker Node

World Wide Web

ZDC ElectroMagnetic detector

Zero Degree Calorimeter

ZDC Neutron detector

ZDC Proton detector 


\section{Bibliography}

[1] H.J. Specht, Experimental aspects of heavy ion physics at LHC energies, CERN-90-10-v-2, http://doc.cern.ch/cernrep/1990/90-10/90-10_v2.html.

[2] J. Schukraft, A dedicated heavy ion experiment at the LHC, ECFA-92-146.

[3] ALICE collaboration, Letter of intent for a large ion collider experiment, CERN-LHCC-93-016, http://cdsweb.cern.ch/record/290825;

ALICE collaboration, Debye screening in heavy-ion colisions with the ALICE detector, CERN-LHCC-95-24, http://cdsweb.cern.ch/record/303751.

[4] ALICE collaboration, Technical proposal for A Large Ion Collider Experiment at the CERN LHC, CERN-LHCC-95-71, http://cdsweb.cern.ch/record/293391.

[5] ALICE collaboration, The forward muon spectrometer of ALICE : addendum to the technical proposal for a Large Ion Collider experiment at the CERN LHC, CERN-LHCC-96-032, http://cdsweb.cern.ch/record/314011.

[6] ALICE collaboration, ALICE: a transition radiation detector for electron identification within the ALICE central detector - an addendum to the Technical Proposal, CERN-LHCC-99-013, http://cdsweb.cern.ch/record/401988.

[7] ALICE collaboration, ALICE electromagnetic calorimeter: addendum to the ALICE technical proposal, CERN-LHCC-2006-014, http://cdsweb.cern.ch/record/932676.

[8] ALICE collaboration, ALICE high-momentum particle identification: Technical Design Report, CERN-LHCC-98-019, http://cdsweb.cern.ch/record/381431.

[9] ALICE collaboration, Technical design report of the photon spectrometer, CERN-LHCC-99-004, http://cdsweb.cern.ch/record/381432.

[10] ALICE collaboration, ALICE Zero-Degree Calorimeter (ZDC): Technical Design Report, CERN-LHCC-99-005, http://cdsweb.cern.ch/record/381433.

[11] ALICE collaboration, ALICE Inner Tracking System (ITS): Technical Design Report, CERN-LHCC-99-012, http://edms.cern.ch/file/398932/1.

[12] ALICE collaboration, ALICE dimuon forward spectrometer: Technical Design Report, CERN-LHCC-99-022, http://cdsweb.cern.ch/record/401974; ALICE dimuon forward 
spectrometer: addendum to the Technical Design Report, CERN-LHCC-2000-046, http://cdsweb.cern.ch/record/494265.

[13] ALICE collaboration, ALICE Photon Multiplicity Detector (PMD): Technical Design Report, CERN-LHCC-99-032, http://cdsweb.cern.ch/record/451099; ALICE Photon Multiplicity Detector (PMD): addendum to the technical design report, CERN-LHCC-2003-038, http://cdsweb.cern.ch/record/642177.

[14] ALICE collaboration, ALICE time projection chamber: Technical Design Report, CERN-LHCC-2000-001, http://cdsweb.cern.ch/record/451098.

[15] ALICE collaboration, ALICE Time-Of-Flight system (TOF): Technical Design Report, CERN-LHCC-2000-012, http://cdsweb.cern.ch/record/430132; ALICE Time-Of Flight system (TOF): addendum to the technical design report, CERN-LHCC-2002-016, http://cdsweb.cern.ch/record/545834.

[16] ALICE collaboration, ALICE transition-radiation detector: Technical Design Report, CERN-LHCC-2001-021, http://cdsweb.cern.ch/record/519145.

[17] ALICE collaboration, ALICE trigger data-acquisition high-level trigger and control system: Technical Design Report, CERN-LHCC-2003-062, http://cdsweb.cern.ch/record/684651.

[18] ALICE collaboration, ALICE forward detectors: FMD, TO and VO: Technical Design Report, CERN-LHCC-2004-025, http://cdsweb.cern.ch/record/781854.

[19] ALICE collaboration, ALICE computing: Technical Design Report, CERN-LHCC-2005-018, http://cdsweb.cern.ch/record/832753.

[20] F. Carminati et al., ALICE: physics performance report, volume I, J. Phys. G 30 (2004) 1517.

[21] B. Alessandro et al., ALICE: physics performance report, volume II, J. Phys. G 32 (2006) 1295.

[22] L. Betev et al., Definition of the ALICE coordinate system and basic rules for sub-detector components numbering, ALICE-INT-2003-038, http://edms.cern.ch/file/406391/1.

[23] Code E - Fire protection, EDMS Id: 335728, http://edms.cern.ch/file/335728/2.01/; Code F Rev. Radiation Protection, EDMS Id: 335729, http://edms.cern.ch/file/335729/2/; IS 5 Emergency stop, EDMS Id: 335742, http://edms.cern.ch/file/335742/2/; IS 37 Alarms and alarm systems, EDMS Id: 335802, http://edms.cern.ch/file/335802/4/.

[24] A. Morsch and B. Pastircak, Radiation in ALICE detectors and electronic racks, ALICE Internal Note ALICE-INT-2004-017, http://edms.cern.ch/file/358706/1.

[25] B. Adeva et al., The construction of the L3 experiment, Nucl. Instrum. Meth. A289 (1990) 35 . 
[26] D.K. Swoboda et al., Results from the ALICE dipole magnet commissioning, Proceedings of the $19^{\text {th }}$ International Conference on Magnet Technology, Genua Italy, IEEE Trans. Appl.Sup., June 2006, IEEE Trans. Appl. Supercond. 16 (2006) 1696.

[27] http://alignment.hep.bradeis.edu/Devices/BCAM/Index.html.

[28] S. Coli et al., The cooling system of silicon drift layers of the ALICE inner tracking system, ALICE Internal Note ALICE-INT-2008-008.

[29] O.N. Godisov et al., Concept of the cooling system of the ITS for ALICE: Technical Proposals, Theoretical Estimates, Experimental Results, Proceedings of the $1^{\text {st }}$ International Workshop on Electronics and Detectors Cooling, Lausanne Switzerland (1994).

[30] A. van den Brink et al., Conductive cooling of SDD and SSD front-end chips for ALICE, Proceedings of the $7^{\text {th }}$ Workshop on Electronics for LHC Experiments, Stockholm Sweden (2001).

[31] A. Morsch and B. Pastircak, Radiation in ALICE detectors and electronics racks, ALICE Internal Note ALICE-INT-2002-028, https://edms.cern.ch/file/358706/1.

[32] F. Antinori et al., Experience with a $30 \mathrm{~cm}^{2}$ silicon pixel plane in CERN experiment WA97, Nucl. Instrum. Meth. A 360 (1995) 91;

G. Alexeev et al., First results from the 1994 lead beam run of WA97, Nucl. Phys. A 590 (1995) 139.

[33] F. Faccio et al., Estimate of the Single Event Upset (SEU) rate in CMS, Proceedings of the $4^{\text {th }}$ Workshop on Electronics for LHC Experiments, Rome Italy (1998).

[34] W. Snoeys et al., Pixel readout electronics development for ALICE PIXEL VERTEX and LHCb RICH detector, Nucl. Instrum. Meth. A 465 (2001) 176, also in Proceedings of the PIXEL 2000 Workshop, Genova, Italy (2000), http://cdsweb.cern.ch/record/516874.

[35] K. Wyllie et al., Front-end pixel chips for tracking in ALICE and particle identification in LHCb, Proceedings of the PIXEL 2002 Conference, SLAC Electronic Conference Proceedings, Carmel U.S.A. (2002).

[36] P. Riedler et al., Overview and status of the ALICE silicon pixel detector, Nucl. Instrum. Meth. A 565 (2006) 1.

[37] P. Riedler et al., The ALICE Silicon Pixel Detector: System, components and test procedures, Nucl. Instrum. Meth. A 568 (2006) 284.

[38] See http://morel.web.cern.ch/morel/cgi-bin/alice.htm for a detailed description of the pixel bus structure and technologies.

[39] A. Kluge et al., The ALICE on-detector pixel PILOT system - OPS, Proceedings of the $7^{\text {th }}$ Workshop on Electronics for LHC Experiments, Stockholm, Sweden (2001) http://cdsweb.cern.ch/record/528583. 
[40] A. Kluge et al., The read-out system of the ALICE pixel detector, Proceedings of the PIXEL 2002 Workshop, Carmel, U.S.A. (2002).

[41] A. Kluge, The ALICE silicon pixel detector front-end and read-out electronics, Nucl. Instrum. Meth. A 560 (2006) 67.

[42] V. Manzari et al., Assembly, construction and testing of the ALICE silicon pixel detector, Nucl. Instrum. Meth. A 570 (2007) 241.

[43] R. Santoro et al., Status of the ALICE silicon pixel detector, Nucl. Instrum. Meth. A 581 (2007) 330 .

[44] M. Caselle et al., Assembly procedure of the ALICE silicon pixel detector module, Proceedings of the New Frontiers in Subnuclear Physics, Milano Italy (2005).

[45] S. Moretto et al., The assembly of the first sector of the ALICE silicon pixel detector, J. Phys. Conf. Ser. 41 (2006) 361.

[46] A. Pepato et al., The mechanics and cooling system of the ALICE silicon pixel detector, Nucl. Instrum. Meth. A 565 (2006) 6.

[47] F. Scarlassara et al., Cooling tests for the silicon pixel detectors, ALICE Internal Note ALICE-INT-2000-18, https://edms.cern.ch/file/112976/1.

[48] I.A. Cali et al., The ALICE silicon pixel detector control system and on-line calibration tools, 2007 JINST 2 P04008.

[49] J. Conrad et al., Minimum bias triggers in proton-proton collisions with VZERO and pixel detectors, ALICE Internal Note ALICE-INT-2005-025, http://cdsweb.cern.ch/record/960438.

[50] ALICE Central Trigger Processor, User Requirement Document (URD), http://epweb2.ph.bham.ac.uk/user/pedja/alice/urd10.ps.

[51] G. Aglieri Rinella et al., The level 0 pixel trigger system for the ALICE experiment, 2007 JINST 2 P01007.

[52] G. Aglieri Rinella, Characterization of two Zarlink $1310 \mathrm{~nm} 12$ channels optical receiver modules, ALICE Internal Note ALICE-INT-2006-025, https://edms.cern.ch/document/793128/1.

[53] P. Riedler et al., Recent test results of the ALICE silicon pixel detector, Nucl. Instrum. Meth. Phys. A 549 (2005) 65.

[54] P. Nilsson et al., Test beam performance of the ALICE silicon pixel detector, Nucl. Instrum. Meth. A 535 (2004) 424.

[55] D. Elia et al., Study of the ALICE Silicon Pixel Detector performance in a beam test at the SPS, ALICE Internal Note ALICE-INT-2005-007, https://edms.cern.ch/document/567094/1. 
[56] D. Elia et al., Sensor thickness dependence of the ALICE Silicon Pixel Detector performance, ALICE Internal Note ALICE-INT-2005-011, https://edms.cern.ch/file/591633/1.

[57] P. Riedler et al., First results from the ALICE silicon pixel detector prototype, Nucl. Instrum. Meth. A 501 (2003) 111.

[58] A. Rashevsky et al., Characteristics of the ALICE Silicon Drift Detector, Nucl. Instrum. Meth. A 461 (2001) 133.

[59] D. Nouais et al., Drift velocity monitoring of SDDs using MOS charge injectors, Nucl. Instrum. Meth. A 450 (2000) 338.

[60] D. Nouais et al., The ALICE silicon drift detector system, Nucl. Instrum. Meth. A 501 (2003) 119, ALICE-PUB-2001-59, http://cdsweb.cern.ch/record/531804.

[61] P. Cerello et al., The detailed simulation of the ALICE Silicon Drift Detectors, ALICE Internal Note ALICE-INT-2001-34, https://edms.cern.ch/file/324376/1.

[62] E. Lopez Torres and P. Cerello, Efficiency and spatial resolution improvement in the ALICE SDDs via cluster unfolding, ALICE Internal Note ALICE-INT-2001-35, https://edms.cern.ch/file/324334/2.

[63] A. Kolozhvari et al., Measurement of the SDD two track resolution, ALICE Internal Note ALICE-INT-2004-016.

[64] H. Gulkanyan, S. Grigoryan and H. Karayan, Exploiting light beams of different wavelength in the optical monitoring systems, ALICE Internal Note ALICE-INT-2001-25, https://edms.cern.ch/file/322121/5.

[65] G. Mazza et al., A 32-channel, $0.25 \mu \mathrm{m}$ CMOS ASIC for the readout of the silicon drift detectors of the ALICE experiment, IEEE Trans. Nucl. Sci. 51 (2004) 1942.

[66] A. Rivetti et al., A low-power 10-bit ADC in a $0.25 \mu \mathrm{m}$ CMOS: design considerations and test results, IEEE Trans. Nucl. Sci. 48 (2001) 1225.

[67] A. Rivetti et al., The front-end system of the silicon drift detectors of ALICE, Nucl. Instrum. Meth. A 541 (2005) 267.

[68] S. Antinori et al., Design and test of the final ALICE SDD CARLOS end ladder board, Proceedings of the $12^{\text {th }}$ Workshop on Electronics for LHC and Future Experiments, Valencia Spain (2006), http://cdsweb.cern.ch/record/1027438.

[69] S. Antinori et al., A rad-hard 2D-compressor ASIC for ALICE SDD experiment, Nucl. Inst. Meth. A 524 (2004) 295.

[70] P. Moreira et al., A radiation tolerant gigabit serializer for LHC data transmission, http://edms.cern.ch/file/906036/1/. 
[71] S. Antinori et al., Design and test of the ALICE SDD data concentrator card CARLOSrx, IEEE Nucl. Sci. Symp. Conf. Rec. 1 (2006) 316, also in Proceedings of the 2006 IEEE Nuclear Science Symposium and Medical Imaging Conference, San Diego U.S.A. (2006).

[72] W. Carena et al., Specification document for the potential degrader rods of the ALICE TPC field cage, ALICE Internal Note ALICE-INT-2001-47, https://edms.cern.ch/document/333020/1.

[73] L. Toscano et al., Mass production testing of front-end ASICs for ALICE SDD system, Proceedings of the LECC05, $11^{\text {th }}$ Workshop on Electronics for LHC Experiments, Heidelberg Germany (2005), http://cdsweb.cern.ch/record/920430.

[74] S. Beolé et al., Production and assembly of the ALICE silicon drift detectors, Nucl. Instrum. Meth. A 570 (2007) 236.

[75] E. Crescio et al., Results from beam tests of large area silicon drift detectors, Nucl. Instrum. Meth. A 539 (2005) 250.

[76] D. Nouais et al., Correction of dopant concentration fluctuation effects in silicon drift detectors, Nucl. Instrum. Meth. A 461 (2001) 222.

[77] M. Germain et al., Irradiation of a silicon-strip detector for the ALICE experiment at LHC, Nucl. Instrum. Meth. A 482 (2002) 634; Radiation damages in double-sided silicon strip module, Nucl. Instrum. Meth. A 485 (2002) 121;

I. Rachevskaia et al., Test and quality control of double-sided silicon microstrip sensors for the ALICE experiment, Nucl. Instrum. Meth. A 530 (2004) 59;

L. Bosisio et al., Experience with the test and qualification of double-sided silicon microstrip sensors for the ALICE inner tracking system, IEEE Nucl. Sci. Symp. Conf. Rec. 3 (2006) 1429;

I. Rashevskaya et al., Qualification of a large number of double-sided silicon microstrip sensors for the ALICE inner tracking system, Nucl. Instrum. Meth. A 572 (2007) 122.

[78] J.R. Lutz et al., Tab bonded SSD module for the STAR and ALICE trackers, Proceedings of the $5^{\text {th }}$ Workshop on Electronics for LHC Experiments, Snowmass Colorado U.S.A., CERN-ALICE-PUB-99-47, http://cdsweb.cern.ch/record/426377;

J.R. Lutz et al., Front-end modules for ALICE SSD, Proceedings of the $9^{\text {th }}$ Workshop on Electronics for LHC Experiments, Amsterdam The Nederlands (2003), http://cdsweb.cern.ch/record/720612;

M. Bregant et al., Assembly and validation of the SSD silicon microstrip detector of ALICE, Nucl. Instrum. Meth. A 566 (2006) 18;

M. Bregant et al., The ALICE vertex detector: focus on the micro-strip layers, Nucl. Instrum. Meth. A 569 (2006) 29;

M. Bregant et al., Assembly and validation of the ALICE silicon microstrip detector, Nucl. Instrum. Meth. A 570 (2007) 312. 
[79] C. Hu-Guo et al., The HAL25 front-end chip for the ALICE silicon strip detectors, Proceedings of the $7^{\text {th }}$ Workshop on Electronics for LHC Experiments, Stockholm, Sweden (2001), http://cdsweb.cern.ch/record/528581;

Test and evaluation of HAL25: the ALICE SSD front-end chip, Proceedings of the $8^{\text {th }}$ Workshop on Electronics for LHC Experiments, Colmar, France (2002)

http://cdsweb.cern.ch/record/593263;

C. Colledani, C. Hu and J.D. Berst, HAL25 V3 user manual v0.1, LEPSI-IN2P3-CNRS/ULP, Strasbourg France.

[80] A.P. de Haas et al., Very low mass microcables for the ALICE silicon strip detector, Proceedings of the $5^{\text {th }}$ Workshop on Electronics for LHC Experiments, Snowmass Colorado (2002), http://cdsweb.cern.ch/record/426351;

$\mathrm{V}$. Borshchov et al., Aluminium microcable technology for the ALICE silicon strip detector: a status report, Proceedings of the $8^{\text {th }}$ Workshop on Electronics for LHC Experiments, Colmar France (2002), http://cdsweb.cern.ch/record/593907.

[81] G.A. Feofilov et al., Inner tracking system for ALICE conceptual designed mechanics, cooling and alignment, Proceedings of the CERN Workshop on Advanced Materials for High Precision Detectors, Geneva Switzerland (1994);

A. van den Brink et al., A lightweight 3D support structure for precise tracking system, Proceedings of the $11^{\text {th }}$ European Conference on Composite Materials, Rhodes Greece (2004).

[82] J. Manschot et al., Cooling of the SSD, ALICE Internal Note ALICE-INT-2003-021, https://edms.cern.ch/document/387009/1.

[83] R. Kluit et al., Design of ladder endcap electronics for the ALICE ITS SSD, Proceedings of the $7^{\text {th }}$ Workshop for LHC and Future Experiments, Stockholm Sweden (2001), http://cdsweb.cern.ch/record/528423;

R. Kluit et al., Realization of ladder EndCap electronics for the ALICE ITS SSD, Proceedings of the $10^{\text {th }}$ Workshop for LHC and Future Experiments, Boston U.S.A. (2004), http://cdsweb.cern.ch/record/814045.

[84] M.J. Rossewij et al., FEROM, the ALICE SSD read out system, Proceedings of the $9^{\text {th }}$ Workshop on Electronics for LHC experiments, Amsterdam The Netherlands (2003), http://cdsweb.cern.ch/record/720606.

[85] M. Oinonen et al., ALICE silicon strip detector module assembly with single-point TAB interconnections, Proceedings of the $11^{\text {th }}$ Workshop on Electronics for LHC and Future Experiments, Heidelberg Germany (2005), http://cdsweb.cern.ch/record/920152.

[86] A. van den Brink et al., Performance measurements of Storage Area Network in the CASPUR computing centre, ALICE Internal Note ALICE-INT-2004-004, https://edms.cern.ch/file/441371/1/ALICE-INT-2004-004.pdf.

[87] S. Plumeri et al., In-beam tests of silicon strip detectors for the ALICE experiment at the LHC, ALICE Internal Note ALICE-INT-2005-028; 
O. Sokolov et al., In-beam performance of the ALICE silicon strip detectors, Nucl. Instrum. Meth. A 562 (2006) 110;

O. Sokolov, Protoyping of silicon strip detectors for the inner tracker of the ALICE experiment, Thesis Utrecht University, Utrecht The Nederlands (2006);

S. Plumeri, Characterisation des modules de detection silicium double-face a micropistes pour le trajeto graphe de l'experience ALICE, Thesé de l'Université Louis Pasteur, Strasbourg, Octobre 2006.

[88] D. Antończyk et al., Performance studies with an ALICE TPC prototype, Nucl. Instrum. Meth. A 565 (2006) 551.

[89] C. Garabatos, The ALICE TPC, Nucl. Instrum. Meth. A 535 (2004) 197.

[90] J. Wiechula et al., High-precision measurement of the electron drift velocity in $\mathrm{Ne}-\mathrm{CO}_{2}$, Nucl. Instrum. Meth. A 548 (2005) 582.

[91] H. Stelzer et al., The ALICE TPC readout chamber: from prototypes to series production, ALICE Internal Note ALICE-INT-2003-017, https://edms.cern.ch/document/384259/2.

[92] U. Frankenfeld et al., The ALICE TPC inner readout chamber: results of beam and laser tests, ALICE Internal Note ALICE-INT-2002-030, https://edms.cern.ch/document/359622/1.

[93] R. Campagnolo, Readout electronics for the ALICE time projection chamber, Proceedings of the HEP-EPS 2005, Lisbon Portugal (2005), POS (HEP2005) 373.

[94] G. Renault, B.S. Nielsen, J. Westergaard and J.J. Gaardhøje, The laser of the ALICE time projection chamber, Int. J. Mod. Phys. E 16 (2007) 2413.

[95] B. Mota et al., A low-power 16-channel AD converter and digital processor ASIC, Proceedings of the ESSCIRC, Florence Italy (2002).

[96] R. Esteve Bosch et al., The ALTRO chip: a 16-channel A/D converter and digital processor for gas detectors, IEEE Trans. Nucl. Sci. 50 (2003) 2460.

[97] B. Mota et al., Performance of the ALTRO chip on data acquired on an ALICE TPC prototype, Nucl. Instrum. Meth. A 535 (2004) 500.

[98] C. Gonzáles Gutiérres et al., The ALICE TPC readout control unit, IEEE Nucl. Sci. Symp. Conf. Rec. 1 (2005) 575.

[99] M. Richter et al., The control system for the front-end electronics of the ALICE time projection chamber, IEEE Trans. Nucl. Sci. 53 (2006) 980.

[100] C. Adler et al., Electron/pion identification with ALICE TRD prototypes using a neural network algorithm, Nucl. Instrum. Meth. A 552 (2005) 364.

[101] A. Andronic et al., Energy loss of pions and electrons of 1-6 GeV/c in drift chambers operated with Xe, $\mathrm{CO}_{2}(15 \%)$, Nucl. Instrum. Meth. A 519 (2004) 508. 
[102] A. Andronic et al., Transition radiation spectra of electrons from 1 to $10 \mathrm{GeV} / \mathrm{c}$ in regular and irregular radiators, Nucl. Instrum. Meth. A 558 (2006) 516.

[103] C.W. Fabjan and W. Strucyinski, Coherent emission of transition radiation in periodic radiators, Phys. Lett. B 57 (1975) 483.

[104] C. Adler et al., Position reconstruction in drift chambers operated with $\mathrm{Xe}, \mathrm{CO}_{2}(15 \%)$, Nucl. Instrum. Meth. A 540 (2004) 140.

[105] H.K. Soltveit et al., Final version of the analog front-end electronics for the ALICE TPC-detector and ALICE TRD-detector, GSI Sci. Rep. (2003) 244, http://www.gsi.de/informationen/wti/library/scientificreport2003/files/213.pdf.

[106] M. Gutfleisch, Digitales frontend und preprozessor im TRAP1-chip des TRD-triggers für das ALICE-experiment am LHC, Diploma Thesis, University of Heidelberg, HD-KIP-02-24, Heidelberg Germany (2002), available at http://www.kip.uni-heidelberg.de/ti/publications/diploma/2002MarcusGutfleisch.pdf.

[107] F. Lesser, Entwurf und realisierung eines vierfach MIMD prozessor mikrochips für eine anwendung in der hochenergiephysik, $\mathrm{PhD}$ Thesis, University of Heidelberg,

HD-KIP-02-34, Heidelberg Germany (2002), available at http://www.kip.uni-heidelberg.de/ti/publications/phd/2002FalkLesser.pdf.

[108] A. Akindinov et al., Particle identification with the ALICE TOF detector at very high particle multiplicity, Eur. Phys. J. C 32 (2004) s165.

[109] A. Akindinov et al., Study of QGP signatures with the $\phi \rightarrow K^{+} K^{-}$signal in $\mathrm{Pb} P b$ ALICE events, Eur. Phys. J. C 45 (2006) 669.

[110] E. Cerron-Zeballos et al., A new type of resistive plate chamber: the multigap RPC, Nucl. Instrum. Meth. A 374 (1996) 132.

[111] A. Akindinov et al., The multigap resistive plate chamber as a time-of-flight detector, Nucl. Instrum. Meth. A 456 (2000) 16.

[112] A. Akindinov et al., Space charge limited avalanche growth in multigap resistive plate chambers, Eur. Phys. J. C 34 (2004) s325.

[113] A. Akindinov et al., A study of the multigap RPC at the gamma irradiation facility at CERN, Nucl. Instrum. Meth. A 490 (2002) 58;

A. Akindinov et al., Operation of the multigap resistive plate chamber using a gas mixture free of flammable components, Nucl. Instrum. Meth. A 532 (2004) 562;

A. Akindinov et al., Latest results on the performance of the multigap resistive plate chamber used for the ALICE TOF, Nucl. Instrum. Meth. A 533 (2004) 74;

A. Akindinov et al., Study of gas mixtures and ageing of the multigap resistive plate chamber used for the Alice TOF, Nucl. Instrum. Meth. A 533 (2004) 93. 
[114] A. Akindinov et al., The MRPC detector for the ALICE time of flight system: final design and performances, Nucl. Phys. 158 (Proc. Suppl.) (2006) 60;

A. Akindinov et al., Quality assurance procedures for the construction of ALICE TOF detector, Nucl. Phys. B 158 (2006) 78.

[115] F. Anghinolfi et al., NINO: an ultra-fast and low-power front-end amplifier/discriminator ASIC designed for the multigap resistive plate chamber, Nucl. Instrum. Meth. A 533 (2004) 183.

[116] M. Mota, J. Christiansen, S. Debieux, V. Ryzhov, P. Moreira and A. Marchioro, A flexible multichannel high-resolution time-to digital converter ASIC, IEEE Trans. Nucl. Sci. 2 (2000) 9/155;

J. Christiansen, HPTDC High Performance Time to Digital Converter version 2.2 (March 2004), for HPTDC version 1.3, available at https://edms.cern.ch/file/599848/2.2/hptdc_manual_ver2.2.pdf;

A. Akindinov et al., Design aspects and prototype test of a very precise TDC system implemented for the Multigap RPC of the ALICE-TOF, Nucl. Instrum. Meth. A 533 (2004) 178.

[117] F.Q. Wang and N. Xu, Baryon phase-space density in heavy-ion collisions, Phys. Rev. C 61 (2000) 021904.

[118] Y. Andres et al., R\&D in ALICE: the CsI-based RICH high momentum particle identification detector, Eur. Phys. J. Direct. C 4 (2002) 25.

[119] F. Piuz, CsI-photocathode and RICH detector, Nucl. Instrum. Meth. A 371 (1996) 96;

E. Nappi, CsI RICH detectors in high energy physics experiments, Nucl. Instrum. Meth. A 471 (2001) 18.

[120] A. di Mauro et al., The HMPID circulation system, ALICE Internal Note ALICE-INT-2007-003 v.2, https://edms.cern.ch/document/813849/2.

[121] A. Di Mauro, A study of the angular resolution of the ALICE HMPID CSI-RICH detector, CERN Internal Note CERN-EP-2000-058, http://cdsweb.cern.ch/record/436404.

[122] H. Hoedlmoser et al., Production technique and quality evaluation of CsI photocathodes for the ALICE/HMPID detector, Nucl. Instrum. Meth. A 566 (2006) 338.

[123] H. Hoedlmoser et al., Photo-current scanner system for in situ quality assessment of large area CsI photocathodes, Nucl. Instrum. Meth. A 566 (2006) 351.

[124] J.C. Santiard and K. Marent, The Gassiplex0.7-2 integrated front-end analog processor for the HMPID and the dimuon spectrometer of ALICE, Proceedings of the $5^{\text {th }}$ Conference on Electronics for LHC Experiments, Snowmass U.S.A. (1999), CERN Internal Note CERN-ALICE-PUB-2001-49, http://cdsweb.cern.ch/record/523473. 
[125] H. Witters, J.C. Santiard and P. Martinengo, Dilogic-2: a sparse data scan readout processor for the HMPID detector of ALICE, Proceeding of the $6^{\text {th }}$ Workshop on Electronics for LHC Experiments, Krakow Poland (2000), CERN Internal Note CERN-ALICE-PUB-2000-010, http://cdsweb.cern.ch/record/439170.

[126] M.Yu. Bogolyubsky, Yu.V. Kharlov and S.A. Sadovsky, Direct photon identification with artificial neural network in the photon spectrometer PHOS, Nucl. Instrum. Meth. $\mathbf{A} 502$ (2003) 719.

[127] D.V. Aleksandrov et al., A high resolution electromagnetic calorimeter based on lead-tungstate crystals, Nucl. Instrum. Meth. A 550 (2005) 169.

[128] M. Ippolitov et al., Studies of lead tungstate crystals for the ALICE electromagnetic calorimeter PHOS, Nucl. Instrum. Meth. A 486 (2002) 121.

[129] A.M. Blick et al., A charged-particle detector based on proportional tubes with a segmented cathode and cathode readout, Instrum. Exp. Tech. 44 (2001) 339.

[130] M.Yu. Bogolyubsky et al., Methods of coordinate reconstruction in gaseous detectors with cathode readout, Instrum. Exp. Tech. 45 (2002) 327.

[131] F.D. Brooks, Development of organic scintillators, Nucl. Instrum. Meth. 162 (1979) 477.

[132] H. Gray, The reconstruction of high-pT photons with the electromagnetic calorimeter of the ALICE experiment at the LHC, $\mathrm{PhD}$ Thesis, University of Capetown South Africa (2005).

[133] H. Muller et al., Trigger region unit for the ALICE PHOS calorimeter, Proceedings of the $11^{\text {th }}$ Workshop on Electronics for LHC and Future Experiments, Heidelberg Germany (2005), CERN-OPEN-2006-012, http://cdsweb.cern.ch/record/929391.

[134] A. Fernández et al., Cosmic ray physics with the ALICE detectors, Czech. J. Phys. 55 (2005) B801;

A. Fernández et al., ACORDE a cosmic ray detector for ALICE, Nucl. Instrum. Meth. A 572 (2007) 102.

[135] O. Adriani et al., The L3 + C detector, a unique tool-set to study cosmic rays, Nucl. Instrum. Meth. A 488 (2002) 209.

[136] CDF collaboration, A. Sansoni et al., Quarkonia production at CDF, Nucl. Phys. A 610 (1996) 373.

[137] CDF collaboration, D. Acosta et al., Measurement of the $J / \psi$ meson and $b$-hadron production cross sections in p $\bar{p}$ collisions at $\sqrt{s}=1960 \mathrm{GeV}$, Phys. Rev. D 71 (2005) 032001.

[138] F. Guerin et al., ALICE Muon Trigger Performance, ALICE Internal Note ALICE-INT-2006-002, http://cdsweb.cern.ch/record/960471. 
[139] A. Fassò et al., FLUKA: present status and future developments, Proceedings of the IV International Conference on Calorimeters and their Applications, World Scientific, Singapore (1994).

[140] E.N. Donskoy et al., VNIIEF status report private communications.

[141] R. Brun, F. Bruyant, M. Maire, A.C. McPherson and P. Zanarini, GEANT3 user guide, CERN data handling division DD/EE/84-1 (1985), http://wwwinfo.cern.ch/asdoc/geantold/GEANTMAIN.html;

M. Goossens et al., GEANT detector description and simulation tool, CERN program library long write-up W5013 (1994), http://cdsweb.cern.ch/record/1073159.

[142] X.N. Wang and M. Gyulassy, HIJING: a Monte Carlo model for multiple jet production in pp, pA and AA collisions, Phys. Rev. D 44 (1991) 3501;

M. Gyulassy and X.N. Wang, HIJING 1.0: a Monte Carlo program for parton and particle production in high-energy hadronic and nuclear collisions, Comput. Phys. Commun. 83 (1994) 307;

The code can be found in http://www-nsdth.lbl.gov/ xnwang/hijing.

[143] R. Guernane et al., Machine induced Background in the ALICE Muon Trigger System in p-p Data taking, ALICE Internal Note ALICE-INT-2003-041, https://edms.cern.ch/document/407450/1.

[144] S. Grigoryan et al., Contribution of Secondary Pi/K Mesons, produced in the Absorber, into the Dimuon Background in Pb-Pb Collisions, ALICE Internal Note ALICE-INT-2002-06, https://edms.cern.ch/document/338221/2.

[145] A. Rimai et al., Spatial resolution studies in cathode pad and in cathode strip chambers, ALICE Internal Note ALICE-INT-1997-23, https://edms.cern.ch/document/106754/1.0.

[146] R. Wurzinger, Y. Le Bornec and N. Willis, MonteCarlo studies on cathode strip / pad chambers for the ALICE di-muon arm, ALICE Internal Note ALICE-INT-1997-10, https://edms.cern.ch/document/106932/1.0.

[147] G. Chabratova et al., Development of the Kalman filter for tracking in the forward muon spectrometer of ALICE, ALICE Internal Note, ALICE-INT-2003-002, https://edms.cern.ch/document/371480/1.

[148] A. Zintchenko et al., A new approach to cluster finding and hit reconstruction in muon chambers of ALICE, ALICE Internal Note, ALICE-INT-2003-006, https://edms.cern.ch/document/373848/1.

[149] J. Peyré, B. Genolini and J. Pouthas, A full-scale prototype for the tracking chambers of the ALICE muon spectrometer. Part I: mechanics, anode and cathode plane design, assembly and construction, ALICE Internal Note ALICE-INT-1998-28, https://edms.cern.ch/document/106760/1.0; 
M. Arba et al., Assembly of a large slat chamber prototype for the ALICE Muon

Spectrometer, ALICE Internal Note ALICE-INT-2002-17,

https://edms.cern.ch/document/347613/1.

[150] L. Kharmandarian, Etude de chambres de trajectoire du spectrometere dimuon de l'experience ALICE aupres du LHC, PhD Thesis PHD-IPNO-T-00-02, Institut de Physique Nucleaire (IPN), Orsay France (1999);

M. Boudjemline et al., Results of Slat CPC Prototype Test for ALICE Dimuon Spectrometer, ALICE Internal Note ALICE-INT-2002-023, https://edms.cern.ch/document/350787/1.

[151] N. Willis et al., Tests and simulations results on a mockup of the ALICE Dimuon tracking chambers, ALICE Internal Note ALICE-INT-2003-030,

https://edms.cern.ch/document/404445/2.

[152] Open Source Instruments, http://www.opensourceinstruments.com.

[153] R. Tieulent et al., The geometry monitoring system of the ALICE dimuon spectrometeroverview, ALICE Internal Note ALICE-INT-2005-009, https://edms.cern.ch/document/588193/1.

[154] P. Pillot et al., The geometry monitoring system of the ALICE Dimuon Spectrometer simulation results, ALICE Internal Note ALICE-INT-2005-020, https://edms.cern.ch/document/652635/1.

[155] R. Tieulent et al., Results from the test bench of the geometry monitoring system of the ALICE muon spectrometer, ALICE Internal Note ALICE-INT-2007-010, https://edms.cern.ch/document/831890/1.

[156] P. Courtat et al., The electronics of ALICE dimuon tracking chambers, ALICE Internal Note ALICE-INT-2004-026.

[157] A. Charpy et al., Test of the tracking readout electronics of the dimuon forward spectrometer, ALICE Internal Note ALICE-INT-2006-016, https://edms.cern.ch/document/771807/1.

[158] M.P. Comets et al., Results of the in-beam tests performed with the quadrant 0 of station 1 for the ALICE dimuon arm, ALICE Internal Note ALICE-INT-2003-035, https://edms.cern.ch/document/406119/3.

[159] D. Guez, Modélisation et simulation des paramètres critiques de la première station du spectromètre dimuons d'ALICE, PhD Thesis, PHD-IPNO-T-03-05, Institut de Physique Nucleaire (IPN), Orsay France (2003).

[160] C. Suire et al., Radiation studies for the readout electronic of the ALICE dimuon forward spectrometer, ALICE Internal Note ALICE-INT-2005-008, https://edms.cern.ch/document/582311/1. 
[161] C. Suire et al., Radiation studies for the ALICE dimuon forward spectrometer tracking $D A Q$, ALICE Internal Note ALICE-INT-2007-001.

[162] R. Arnaldi et al., Design and performance of the ALICE muon trigger system, Nucl. Phys. 158 (Proc. Suppl.) (2006) 21.

[163] G. Blanchard, P. Crochet and P. Dupieux, The local trigger electronics of the ALICE dimuon trigger, ALICE Internal Note ALICE-EN-2003-010.

[164] R. Arnaldi et al., Spatial resolution of RPC in streamer mode, Nucl. Instrum. Meth. A 490 (2002) 51, and references therein.

[165] R. Arnaldi et al., Overview on production and first results of the tests on the RPCs for the ALICE dimuon trigger, Nucl. Phys. 158 (Proc. Suppl.) (2006) 83.

[166] R. Arnaldi et al., Ageing tests on the low-resistivity RPC for the ALICE dimuon arm, Nucl. Instrum. Meth. A 508 (2003) 106.

[167] R. Arnaldi et al., Beam and ageing tests with a highly-saturated avalanche gas mixture for the ALICE pp data taking, Nucl. Phys. 158 (Proc. Suppl.) (2006) 149.

[168] R. Arnaldi et al., A dual threshold technique to improve the time resolution of resistive plate chambers in streamer mode, Nucl. Instrum. Meth. A 457 (2001) 117.

[169] P. Gorodetzky et al., The zero degree hadronic sampling calorimeter for the NA50 experiment at the CERN SPS, Proceedings of the $4^{\text {th }}$ International Conference on Calorimetry in High Energy Physics, World Scientific, Singapore (1994);

G. Anzivino et al., Quartz fibers: a new calorimetry for multi-TeV, very forward physics, Nucl. Phys. 44 (Proc. Suppl.) (1995) 168;

O. Ganel and R. Wigmans, Quartz fiber calorimetry for LHC experiments, Nucl. Instrum. Meth. A 365 (1995) 104.

[170] R. Arnaldi et al., The Zero Degree Calorimeters or the ALICE experiment, Proceedings of the $8^{\text {th }}$ International Conference on Calorimetry in High Energy Physics, Lisbon Portugal (1999).

[171] R. Arnaldi et al., Performances of Zero Degree Calorimeters for the ALICE experiment, Nucl. Instrum. Meth. A 456 (2001) 248.

[172] P. Gorodetzky et al., Radiation physics and chemistry, Pergamon Press, Oxford U.K. (1992); P. Gorodetzky et al., Quartz fiber calorimetry, Nucl. Instrum. Meth. A 361 (1995) 161.

[173] R. Arnaldi et al., The quartz-fiber zero-degree calorimeter for the NA50 experiment at CERN SPS, Nucl. Instrum. Meth. A 411 (1998) 1.

[174] R. Arnaldi et al., The neutron Zero Degree Calorimeter for the ALICE experiment, Nucl. Instrum. Meth. A 564 (2006) 235. 
[175] R. Arnaldi et al., Test beam results on the proton Zero Degree Calorimeter for the ALICE experiment, Proceedings of the $12^{\text {th }}$ International Conference on Calorimetry in High Energy Physics, Chicago Illinois U.S.A. (2006),

[176] N. De Marco et al., Performance of the Zero Degree Calorimeters for the ALICE experiment, IEEE Trans. Nucl. Sci. 54 (2007) 567.

[177] C. Oppedisano et al., Centrality measurement in the ALICE experiment with the Zero Degree Calorimeters, PhD Thesis, Universitá degli Studi di Torino, ALICE Internal Note ALICE-INT-2002-08, https://edms.cern.ch/document/339254/1.

[178] C. Oppedisano et al., An electromagnetic calorimeter prototype for the ALICE experiment, Proceedings of the International Europhys. Conference on High Energy Physics (HEP 2001), Budapest Hungary (2001) [PoS (hep2001) 257].

[179] T. Sjöstrand, S. Mrenna and P. Skands, PYTHIA 6.4 physics and manual, JHEP 05 (2006) 026.

[180] Ideas ASA, VA1_prime2, http://www.ideas.no/products/ASICs/VAfamily.html.

[181] A. Yokoyama et al., Radiation-hardness of VA1 with sub-micron process technology, IEEE Trans. Nucl. Sci. 48 (2001) 440.

[182] R. Esteve Bosch et al., The ALTRO chip: A 16-channel A/D converter and digital processor for gas detectors, IEEE Trans. Nucl. Sci. 50 (2003) 2460.

[183] C.H. Christensen et al., The ALICE forward multiplicity detector, Int. J. Mod. Phys. E 16 (2007) 2432.

[184] J.R. Alfaro et al., Rejection of beam gas interactions in pp collisions and timing requirements, ALICE Internal Note ALICE-INT-2004-021, https://edms.cern.ch/document/494149/1.

[185] B. Cheynis et al., For a V0 detector..., ALICE Internal Note ALICE-INT-2000-29, https://edms.cern.ch/document/303914/1.

[186] B. Rapp, Production de dimuons en réactions pp et PbPb avec ALICE: le détecteur V0 et les résonances de basse masse, Thesis, Lyon University, Lyon France (2004), http://lyoinfo.in2p3.fr/alice/BR/these-rapp.pdf,

[187] B. Cheynis et al., Test results for the V0 detector in ALICE, ALICE Internal Note ALICE-INT-2003-040, https://edms.cern.ch/document/407398/2.

[188] J.R. Alfaro et al., Simulation of the V0A detector, ALICE Internal Note ALICE-INT-2006-018, https://edms.cern.ch/document/773744/1.

[189] B. Cheynis et al., Radiation effects on ALICE V0 detector components, Nucl. Inst. Meth. A 569 (2006) 732. 
[190] M. Mota and J. Christiansen, A four channel, self-calibrating, high resolution, time to digital converter, Proceedings of the ICECS98, CERN-EP-98-049 http://cdsweb.cern.ch/record/354075.

[191] B. Cheynis et al., Radiation effects on V0 detector elements, ALICE Internal Note ALICE-INT-2005-052, https://edms.cern.ch/document/684409/1.

[192] V. Grigoriev et al., A start trigger detector for the ALICE spectrometer, Instrum. Exp. Tech. 43 (2000) 750.

[193] M. Bondila et al., ALICE T0 detector, IEEE Trans. Nucl. Sci. 52 (2005) 1705.

[194] V. Grigoriev et al., Beam tests of the second prototype of a Cherenkov counter for the ALICE T0 detector, ALICE Internal Note ALICE-INT-2000-17, https://edms.cern.ch/document/111907/1.

[195] V. Grigoriev et al., ALICE T0 detector based on Cherenkov counters: R\&D principal results, ALICE Internal Note ALICE-INT-2001-38, https://edms.cern.ch/document/325382/1.

[196] D. Evans et al., ALICE trigger system, Proceedings of the $10^{\text {th }}$ Workshop on Electronics for LHC and Future Experiments, Boston U.S.A. (2004), CERN 2004-010, http://cdsweb.cern.ch/record/814257.

[197] D. Evans et al., The ALICE central trigger processor, Proceedings of the $11^{\text {th }}$ Workshop on Electronics for LHC and Future Experiments, Heidelberg Germany (2005), CERN 2005-038, http://cdsweb.cern.ch/record/921038.

[198] A. Bhasin et al., Recent developements on the ALICE central trigger processor, Proceedings of the $12^{\text {th }}$ Workshop on Electronics for LHC and Future Experiments, Valencia Spain (2006), CERN 2007-001, http://cdsweb.cern.ch/record/1027489.

[199] B.G. Taylor, Timing distribution at the LHC, Proceedings of the $8^{\text {th }}$ Workshop on Electronics for LHC Experiments, Colmar France (2002), CERN-2002-003, http://cdsweb.cern.ch/record/592719.

[200] S. Baron and A. Monera Martínez, Status of the TTC upgrade, Proceedings of the $12^{\text {th }}$ workshop on electronics for LHC and future experiments, Valencia Spain (2006), CERN-2007-001, http://cdsweb.cern.ch/record/1027471

[201] C. Gaspar, M. Dönszelmann and P. Charpentier, DIM, a portable, light weight package for information publishing, data transfer and inter-process communication, Comput. Phys. Commun. 140 (2001) 102.

[202] P.C. Burkimsher, JCOP experience with a commercial SCADA products, PVSS, Proceedings of the ICALEPCS 2003, Gyeongju Korea (2003), http://cdsweb.cern.ch/record/924942. 
[203] G. Paic et al., Physics requirements for the ALICE DAQ system, ALICE Internal Note ALICE-INT-2000-30, https://edms.cern.ch/document/250015/2.

[204] G. Rubin, ALICE Detector Data Link (DDL) - hardware guide for the front-end designers, ALICE Internal Note ALICE-INT-1998-021, https://edms.cern.ch/document/112129/1.4.

[205] E. Denes, ALICE detector data link, pRORC library routines, version 2.2,ALICE Internal Note ALICE-INT-2002-09, https://edms.cern.ch/document/340457/3.

[206] F. Carena et al., PCI-based readout receiver card in the ALICE DAQ system, Proceedings of the $8^{\text {th }}$ Workshop on Electronics for LHC Experiments (LECC), Colmar France (2002), CERN-2002-003, http://cdsweb.cern.ch/record/594314.

[207] W. Carena et al., PCI based read-out receiver card in the ALICE DAQ system, Proceedings of the $18^{\text {th }}$ JINR International Symposium on Nuclear Electronics and Computing, Varna Bulgaria (2001).

[208] P. Buncic et al., The architecture of the AliEn system, Proceedings of the Computing in High Energy Physics (CHEP 2004), Interlaken, Switzerland (2004), http://cdsweb.cern.ch/record/865533.

[209] CERN ALICE DAQ Group, ALICE DAQ and ECS user's guide, ALICE Internal Note ALICE-INT-2005-015, http://cdsweb.cern.ch/record/960457.

[210] J.P. Baud et al., ALICE data challenge III, ALICE Internal Note ALICE-INT-2001-36, https://edms.cern.ch/document/336659/1.

[211] Ö. Çobanoğlu et al., Development of an on-Line data quality monitor for the relativistic heavy-ion experiment ALICE, Proceedings of the IEEE RT2005, Real Time Systems conference, Stockholm Sweden (2005), http://dx.doi.org/10.1109/RTC.2005.1547409.

[212] Ö. Çobanoğlu et al., The ALICE-LHC online data quality monitoring framework: present and future, Proceedings of the IEEE RT2007, Real Time Systems conference, Fermilab, Batavia Illinois U.S.A. (2007).

[213] F. Roukoutakis et al., The ALICE-LHC online data quality monitoring framework, Proceedings of the Computing in High Energy Physics (CHEP 2007), Victoria BC Canada (2007), http://cdsweb.cern.ch/record/1066450.

[214] G. Di Marzo et al., Specification and simulation of the ALICE DAQ system, ALICE Internal Note ALICE-INT-2002-01, https://edms.cern.ch/document/333766/2.

[215] T. Anticic et al., Trigger and DAQ simulation, ALICE Internal Note ALICE-INT-2003-001.

[216] T. Anticic et al., Specification and simulation of the ALICE trigger and DAQ system, Proceedings of the Computing in High Energy Physics (CHEP 2001), IHEF Beijing China (2001).

[217] Ptolemy project, http://ptolemy.eecs.berkeley.edu/ptolemyclassic/pt0.7.1/index.htm. 
[218] A. Vestbø et al., The ALICE high level trigger, J. Phys. G 30 (2004) s1097.

[219] ALICE collaboration, G. Grastveit et al., FPGA coprocessor for the ALICE high level trigger, Proceedings of the CHEP03, 2003 Conference for Computing in High energy and Nuclear Physics, La Jolla, California U.S.A. (2003) http://cdsweb.cern.ch/record/619518, [physics/0306017].

[220] T.M. Steinbeck, A modular and fault-tolerant data transport framework, $\mathrm{PhD}$ Thesis, Ruprecht-Karls-University Heidelberg, cs.DC/0404014.

[221] T.M. Steinbeck et al., An object-oriented network-transparent data transportation framework, IEEE Trans. Nucl. Sci. 49 (2002) 455.

[222] T.M. Steinbeck et al., New experiences with the ALICE high level trigger data transport framework, http://cdsweb.cern.ch/record/865834, Proceedings of the Computing in High Energy Physics (Chep 2004), Interlaken, Switzerland (2004).

[223] T.M. Steinbeck et al., A framework for building distributed data flow chains in clusters, in Applied Parallel Computing, Springer-Verlag Berlin Heidelberg Germany (2002), http://dx.doi.org/10.1007/3-540-48051-X_45.

[224] M. Richter et al., High level trigger applications for the ALICE experiment, IEEE Trans. Nucl. Sci. 55 (2008) 133.

[225] S.R. Bablok et al., ALICE high level trigger interfaces and data organisation, Proceedings of the Computing in High Energy Physics (CHEP 2006), Mumbai, India (2006).

[226] A. Vestb $\varnothing$, Pattern recognition and data compression for the ALICE high level trigger, $\mathrm{PhD}$ Thesis, Institute for Physics and Technology, University of Bergen, Bergen Norway (2004).

[227] R. Bramm et al., Online pattern recognition for the ALICE high level trigger, Nucl. Instrum. Meth. A 502 (2003) 443.

[228] V. Lindenstruth et al., Real-time TPC analysis with the ALICE high-level trigger, Nucl. Instrum. Meth. A 534 (2004) 47.

[229] V. Lindenstruth et al., Online pattern recognition for the ALICE high level trigger, IEEE Trans. Nucl. Sci. 51 (2004) 383.

[230] T. Alt et al., Benchmarks and implementation of the ALICE high level trigger, IEEE Trans. Nucl. Sci. 53 (2006) 854.

[231] C. Cheshkov, Fast Hough-transform track reconstruction for the ALICE TPC, Nucl. Instrum. Meth. A 566 (2006) 35.

[232] C. Adler et al., The STAR level-3 trigger system, Nucl. Instrum. Meth. A 499 (2003) 778.

[233] F. Manso, A first algorithm for a dimuon high level trigger, ALICE Internal Note ALICE-INT-2002-04, https://edms.cern.ch/document/337419/1. 
[234] B. Becker et al., ALICE dimuon high-level trigger: project review, ALICE Internal Note ALICE-INT-2007-022, https://edms.cern.ch/document/878756/1.

[235] D. Röhrich and A. Vestbø, Efficient TPC data compression by track and cluster modeling, Nucl. Instrum. Meth. A 566 (2006) 668.

[236] J. Berger et al., TPC data compression, Nucl. Instrum. Meth. A 489 (2002) 406.

[237] The worldwide LHC computing grid project, http://lcg.web.cern.ch/LCG/.

[238] Models of networked analysis at regional centres, http://www.cern.ch/MONARC/.

[239] I. Foster and C. Kesselmann, The grid: blueprint for a new comuting infrastructure, Morgan Kaufmann Publishers, U.S.A. (1999).

[240] P. Saiz et al., AliEn - ALICE environment on the GRID, Nucl. Instrum. Meth. A 502 (2003) 437;

AliEn home page, http://alien.cern.ch/.

[241] A simple object access protocol, http://www.soaplite.com.

[242] Condor classified advertisements, http://www.cs.wisc.edu/condor/classad/.

[243] The Scalla software suite: xrootd/olbd, http://xrootd.slac.stanford.edu.

[244] http://egee-jra1-dm.web.cern.ch/egee-jra1-dm/FTS/.

[245] D. Feichtinger and A.J. Peters, Authorization of data access in distributed storage systems, Proceedings of the $6^{\text {th }}$ IEEE/ACM international workshop on grid computing Seattle, U.S.A. (2005), http://dx.doi.org/10.1109/GRID.2005.1542739.

[246] A realisation of distributed analysis for $L H C$, http://lcg.web.cern.ch/LCG/peb/arda/Default.htm.

[247] I.C. Legrand et al., MonALISA: an agent based, dynamic service system to monitor, control and optimize grid based applications, Proceedings of the CHEP 2004 conference, Interlaken, Switzerland (2004), http://cdsweb.cern.ch/record/865735.

[248] Advanced resource connector, http://www.nordugrid.org/middleware/.

[249] Open science grid, http://www.opensciencegrid.org/.

[250] The ALICE experiment offline project, http://www.cern.ch/ALICE/Projects/offline/aliroot/Welcome.html.

[251] An object-oriented data analysis framework, http://root.cern.ch.

[252] P. Billoir, Track fitting with multiple scattering: a new method, Nucl. Instrum. Meth. A 225 (1984) 352;

P. Billoir et al., Track element merging strategy and vertex fitting in complex modular detectors, Nucl. Instrum. Meth. A 241 (1985) 115; 
R. Frühwirth, Application of Kalman filtering to track and vertex fitting, Nucl. Instrum. Meth. A 262 (1987) 444;

P. Billoir, Progressive track recognition with a Kalman like fitting procedure, Comput. Phys. Commun. 57 (1989) 390.

[253] M. Ballintijn, R. Brun, F. Rademakers and G. Roland, Distributed parallel analysis framework with PROOF, http://root.cern.ch/twiki/bin/view/ROOT/PROOF.

[254] H.-U. Bengtsson and T. Sjöstrand, The Lund MonteCarlo for hadronic processes: Pythia version 4.8, Comput. Phys. Commun. 46 (1987) 43.

[255] S. Agostinelli et al., Geant4, a simulation toolkit, Nucl. Instrum. Meth. A 506 (2003) 250, CERN-IT-2002-003, http://cdsweb.cern.ch/record/602040; see also http://wwwinfo.cern.ch/asd/geant4/geant4.html.

[256] Night builds of ALICE off-line software, http://pcaliweb02.cern.ch/Offline/AliRoot/Night-Builds.html.

[257] Subversion, http://svnbook.red-bean.com/.

[258] Hyperised version of the AliRoot, http://aliceinfo.cern.ch/static/aliroot-new/html/roothtml/USER_Index.html.

[259] AliRoot coding conventions, http://aliceinfo.cern.ch/Offline/AliRoot/Coding-Conventions.html.

[260] A. Potrich and P. Tonella, $C++$ code analysis: an open architecture for the verification of coding rules, Proceedings of the CHEP'2000, International Conference on Computing in High Energy and Nuclear Physics, Padova, Italy (2000).

[261] Violated rules table, http://aliceinfo.cern.ch/static/aliroot-new/log/violatedRules.html.

[262] A. Daneels and W. Salter, Selection and evaluation of commercial SCADA systems for the controls of the Cern LHC experiments, Proceedings of the ICALEPCS99 conference, Trieste Italy (1999), http://cdsweb.cern.ch/record/532627; JCOP, http://itco.web.cern.ch/itco/Projects-Services/JCOP/welcome.html.

[263] B. Franek and C. Gaspar, SMI++ object oriented framework for designing and implementing distributed control systems, IEEE Trans. Nucl. Sci. 45 (1998) 1946.

[264] B. Hallgren et al., The embedded local monitor board (ELMB) in the LHC front-end I/O control system, $7^{\text {th }}$ Workshop on Electronics for LHC Experiments, Stockholm Sweden (2001), http://cdsweb.cern.ch/record/530675.

[265] P. Chochula, L. Jirdén and A. Augustinus, Control and monitoring of front-end electronics in ALICE, Proceedings of the $9^{\text {th }}$ Workshop on Electronics for LHC Experiments, Amsterdam, The Netherlands (2003), http://cdsweb.cern.ch/record/722075.

[266] S. Lüders et al., The Cern detector safety system for the LHC experiments, Proceedings of the ICALEPCS03 Conference, Gyeongju Korea (2003). 